rcin.org.pl 



\section{LES}

\section{SOCIETES I'INSECTES}

LEUR ORIGINE - LELR ÉYOLUTION

PAR

William Morton WHEELER

Phoressentra l'Université Harvand (Camibidge. Mass.)

Avec 61 figures dans le texte

\section{PARIS}

LIBRAIRIE OCTAVE DOIN

GASTON DOIN C CDITEURS

8, place de l'odéon, 8 
TOUS DROITS RÉSERNÉS

COPYRIGBL By gaston doIN \& $c^{1 e}, I \eta^{26}$

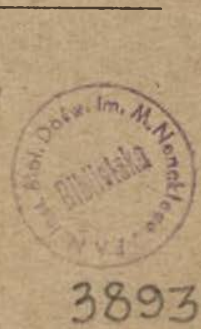

rcin orgyt 
Gaston DOIN \& $\mathrm{C}^{\mathrm{i} e}$, Éditeurs, 8, place de lOdéon, Päris̄-6º

\section{ENCYCLOPEDIE SCIENTIFIQUE}

Publiée sous la direction du $D^{\prime}$ TOULOUSE

BIBLIOTHEQQUE H. Honokiogo (N. H. W.,

I) E

\section{BIOLOGIE GENERALE}

Directeur : Maurice CAULLERY

Professeur à la Faculté des Sciences de Paris

Le développement et le progrès des Sciences ont, tout à la fois, pour condition et pour résultat, leur fractionnement de plus en plus grand en spécialités qui, dans la pratique, deviennent, non seulement inde. pendantes, mais même étrangènes les unes aux autres. C'est là une fâcheuse nécessité. Elle dérive de ce que la méthode scientifique est analytique par essence. Mais, l'analyse faite, il faut en rapprocher les résultats : plus la spécialisation est poussée loin, plus ce besoin eit impérieux et en mème temps plus il est difficile de le satisfaire.

Les sciences biologiques, par la complexité même de leur objet, ont subi au plus haut degré cet émiettement inéluctable et, plus que toutes les autres, elles exigent cette synthèse: car l'organisme est un et les divers 
points de vue auxquels on le considère, dans les divers compartiments de la Biologie, n'ont de valeur véritable que confrontés les uns aux autres et agencés, en quelque sorle, pour reconstituer la Vie, dans la mesure où cela est prossible. La liste des Bibliothèques composant l'Encyclopédic illustre sulfisamment la multiplicité des sciences partielles auxquelles donnent lieu les Etres vivants; la bibliothèque de Biologie générale doit être le lien entre toutes; elle a la lourde tâche d'en assurer la synthèse.

Par là même, elle est plus malaisée à concevoir et surtout à exécuter. 11 est assez facile d'inventorier et de découper le domaine d'une science spéciale; on trouve, sans trop de peine, des spécialistes qualifiés pour fournir une mise au point de chacun des fragments ainsi délimités. Il n'en va pas de mème pour la Biologie générale. Pour en traiter les problèmes d une façon satislaisante, il faut unir une connaissance précise et critique des faits et des techniques diverses à la vue d'ensemble qui permet de dominer cos laits et dien extraire la signilication générale.

On s'efforcera d'atteindre' ce but dans les livres de la présente série. La liste et les titres, qui figurent cidessous, n'en sont pas donnés ne varietur. Ils expriment simplement le plan conçu.

La Biologie générale étant comprise comme la synthèse des disciplines particuliènes : zoologie, botanique, paléontologie, physiologie, chimie et physique biologique, etc., elle doit envisager les manifestations et le lonctionnement des organismes d une façon globale. 
Il faut donc extraire tout d'abord doe ces sciences particulières les caraclères généraux des phénomènes vilaux el préciser leurs rapports avec ceux-qu'offre la matière inanimée. Cie sera l'objel d un volume d'introduction.

Iyant ainsi dégagé ce qu'on peut, à l'heure actuelle, considérer comme le propre de la Vire et ajusté à nos connaissances modernes le vieux problème du mécanisme et du vitalisme, nous envisagerons le fonctionnement vital dans son substralum, l'Organisme. Mais cet examen peut et doit se faire à une série d'échelles différentes, si l'on peut dire.

Il y a une vie élémentaire, dont la Biologe du $\mathrm{xix}^{\mathrm{e}}$ siècle a mis en évidence l'abolue généralité, c’est la vic cellulaire; pour beaucoup d organismes inférieurs, c'est mèmi toule li vie; la ccllule est l'un té fondamenlale en matière d'organismos. Sa connaissance est la base sur laquelle doit ètre construite lit Biologie géné. rale.

Une seconde étape est l'élude de l'individu considéré comme édifice pluricellulaire. Une série de volumes formant la seconde partie de la Bibliothèque, serrml consacrés aux lois généralies de la réalisation, de la reproduction et du fonctionnement synergique de ces édifices. It s'en dégagera la notion si complexe et parfois a fugrilive de l'individualité, qui sera étudiée et disculée spéciatement.

La vie de l'organisme ne se conģoit que dans le milieu, et même les frontières dię liorganisnte d du milieu sont beaucoup plus malipsées à lracer quion ne limagine 
communémbent. La Lroisième partie de la Bibliothègut: sera faite de volumes où ces rapporls généraux seront étudiés. Certains se rattachent plus intimement à la Phys:ologie, mais en ce cas, ou bien ils tnvisigent des fonctions extrèmement généraless, telles que lïrritabilité ou l'assimilation el alors ils rentrent dans l'élude générale des rapports de l'organisme et du milieu; ou bien ils traitent de fonction- (comme la luminosité, par exemple) qui, - tout en ayant une grande valeur biologique, pleinement reconnue par les physiologistes el se rattachant intimement aux conditions londamentales du fonctionnement vital - échappent cependant à peu près complètement, en lait, au cadre de la physiologie classique. Celle-ci est.en effel, délimilée surtout, en réalité, par l'expérimentation sur les Vertébrés, nì ces fonctions sont rudimentaires et font pratiquement défaut; si elles sont bien représentées, c'est en tout cas, sur des types qui ne font pas parlie de ce quion pourrait appeler as ice irrévérencieusement la laune des laboratoires physiologiques.

Dans celte partie de la Bibliothèque, on voudrait aussi laire à l'Ethologie la part qui lui est due et qui $n$ 'est pas suffisimment reconnue.

Ia dernière parliu de la série envisage les organis. mes à une échelle supéricure à l’individu, celle de li lignée ou de l'expèce. Est-il brsoin de souligner que, depuis Darwin, ce point de vue, qui n'est autre que le problème de l'Evolulion, domine loute la Biologie générale. Pour le traiter atutrement que d'une manière philosophique et spéculative il faut considérer les rapports 
de l'organisme el du milieu dans la succesion des générations : c’est-à-dire éludier, par les méthodes positives: I'Hérédilé ; la Variat on sous ses diverses formes; la combinaison des lignées hétérogènes, c'est-à-dirè l'Hybridation; le problème de l'établissement de la conformité de l'organisme aux conditions du milieu, c'est-à-dire l'Adaplation ; les transformations successives des lignées; c'est-à-dire la Phylogénie; enfin envisager les mécanismes par lesquels nous pouvons nous représenter ces transformations, c'est-à-dire les théories évolutionnistes. Lä, plus quailleurs, il serait fructueux de réaliser des livres courts, clair: suffisamment documentés et diune critique judicieuse.

Il esi dans la nature de choses que la section de Biologie générale chevauche parfois sur les bibliothèques spéciales. Dans son intégralité, elle ast une mise en weuvre des matériaux de celles-ci, mais à un point de vue différent et qui évitera tout double emploi véritable. Elle est, d'autre part, nécessairement dégagée du caractère strictement technique et souvent pratique, qui convient à beaucoup de volumes de ces bibliothèquns particulières.

Elle ne vise cependant pas moins à l'ulilité. Nous espérons qu'elle rencontrera un accueil favorable auprès de catégories très variées de lecteurs : biologistes, médecins, philosophes, esprits simplement cultivés, et aussi spécialistes divers.

La spécialisation enlève le plus communément le loi-ir de coordonner les notions partielles el cependant il y a là une nécessité essentielle pour la culture de 
l'esprit et même pour la conduite judicieuse des travaux particuliers.

La Bibliothèque de Biologie générale s'efforcera de répondre à ce besoin, et, sans demander aux auteurs d'abdiquer leur personnalité, elle lâchera de conservier. dans son ensemble, une unité correspondant à celle de son objet: La Vie.

Les volumes ont publiés dans le format in-18 jésns ; ils forment chacuin de 300 à 600 pages avec ou sans figures dans le texte. Chaque ouvrage se vond séprarément.

Voir, à la fin du volume, la liste des bibliothèques de l'ENCYCLOPÉDIE SCIENTIFIQUE et celle des volumes publiés. 


\section{TABLE DES IOLUMES \\ ET LISTE DES COLLABORATEURS}

\section{Introduction}

r. Les Phénomènes vitaux.

\section{I. - La Vie élémentaire}

2. La Cellule (Morphologie), par M. HeNefguy, Vembir de l'Institut, Professeur au Collège de France.

3. La Gellule (Physiologie), par M. E. Fav aE்-Finut, Préparateur au Collège de France.

\section{I. - L'Individu}

4. L'Guf et les facteurs de l'Ontogénèse, par M. A. Brachet, Correspondant de l'Institut, Professeur à l'Université de Bruxelles.

5. La Tératogénèse, par M. Et. Rabald, Professeur i la Faculté des Sciences de Paris.

6. Les Formes larvaires et les Métamorphoses, pat M. Ch. Púrez, Professeur à la Faculté des Sciences dè Paris.

7. La Reproduction asexuée.

8. La Régénération et la Greffe, par V. Chlltehr, Professeur à la Sorbonne.

9. Organisme et Sexualité, par M. Cauldieny.

10. Les Corrélations organiques et l'Individualité. 


\section{III. - L'Organisme et le Milieu}

I I. L'Irritabilité et les Tropismes.

12. Les mutations matérielles dans les êtres vivants (aliment et milieux nutritifs).

13. Les mutations énergétiques dans les êtres vivants (luminosité, chaleur, électricité; etc.).

I4. La Biologie des Pigments, par M. J. Verne, Professeur agrégé à la Faculté de Médecine de Paris.

15. Les Sociétés d'insectes ; leur origine, leur évolution, par M. W.-M. Whencen, Professeur à l'Université Harvard.

16. Parasitisme et Symbiose, par M. M. Caullery, Professeun à la Faculté dels Sciences de Paris.

17. Les Milieux biologiques marins, par M. P. Marais de Beauchamp, Maître de Conférences à l'Universilé de Strasbourg.

I8. La Biologie des eaux douces, par M. A. Vander, Maître de Conférences à l'Université de Toulouse.

19. Les principaux faciès biologiques terrestres.

20. La Concurrence vitale.

\section{IV. - L'Esspèce et l'Évolution}

21. L'Hérédité, par M. E. GuÝ́Not, Prol'esscur à l'Université de Genève.

32. La Variation, par M. E. Gứ́xor.

23. L'Hybridation.

2\%. L'Espèce.

2j. L'Adaptation, par M. L. Cuḱnor, Correspoisdanl de l'Instilu't, Professeur à l'Universi!é de Nancy.

2(i. La Phylogénie.

27. Les Théories évolutionnistes. 


\title{
QUELQUES APPRÉCIATIONS
}

\author{
sur les Ouvrages parus
}

\section{dans la Bibliothèque de Biologie de l'"Encyclopédie Scientifique"}

\section{L'HEREDITÉ}

\author{
par E. Guxúxot
}

II ny a pas de livre a notre connaissance, dit M. S. J. IIontes, Professeur a líniversité de Californic, qui donnc en un espace aussi restreint, un exposi aussi clair et satis. faisant des faits et principes de l'héréditi. Ce livre est bien au courant el temoigne l'une compréhension el d'une penetrattion philosophiqute complète du sujet.......

\section{LA TÉRATOGENĖSE Élude des variations de l'organisme par E. Rabaun}

L. ibule de la Teralogenèse dépasse le cirdre un pen restreint de tembryologie morphologique fure; les donnies quelle fomrnit projettent quelque clarté sur les phenomenes de varialion et d'herédité fonc sur l'origine cl lievoiulion des ilres. Cest ce qui est apparu constamment it litulrur ant cours de ses itudes el. cela est l'idie londamentale qui lui a inspire ce volume donl on ne siturait fitire un suflisanl bloge......

\section{L'ADAPTATION}

par L. Cúnot

.... I. adiphation implique une idéc dubilite on mienx de convenance. C'est une des questions les plus complexes. les plus difficiles, tant par son etendue qui embrasse le (hamp presque enlier de la Biologie que par les problemes de fait el de milaphysique rquelle souleve. Le lecteur lira ces ouvrage avec le plus grand interét el sera etonne de vile ce frobleme passionnant traile d'une ficon alussi complele, anssi claire dans un nombre de pages aussi restreint pour un sujet aussi considerable, 


\title{
PARASITISME ET SYMBIOSE
}

\author{
par M. Callelery
}

.....Concu diun point de vue très general, appuyé sur une documentation considerable, justifié par une abondante bihliographie, ce livre trouve beaucoup de lecteurs, tant frarmi ceux curieux de Biologie generale que parmi les spécialistes des divers ordres gui sont appelés à rencontrer des fails de parasilisme dans la science pure ou dans ses ipp.ications.......

\section{LES SOCIÉTÉS D'INSECTES}

\section{par Williay Morton Wheeler-}

.....Non seulement ies zoologistes, mais encore tous ceux qui suntéressent allx problemes généraux de la Biologic 1rouveront dans ce livre un puissant intérêt. sans parler des Philosoplies et Sociologues qui ne manqueronl pas dien tirer le plus grand profit.

Cet ouvrage, quii est le fruit des recherches propres rle liauleur, est profondément original danis ses conceptions el nouveau dans sa rlocumentation......

\section{LES PIGMENTS DANS L'ORGANISME ANIMAL}

\section{par J. VtrRse}

....1l est inutile dinsister sur limportance de l'etude de: pigments. Quelle quen soit la branche te la Biologie flue fion cultive, on est lenu de s'intéresser ì eux.

Entre autre mérile, cet ouvrage a celui de grouper les resultals d'un grand nombre de faits nouveaux qui ont ile acquis ces dernières annees sur divers pigments. II coordonne ces fails avec ce que l'on connaissail déji el en dégage des conceptions générales dłu plus haut interit......

\section{L'CUUF ET LES FACTEURS DE L'ONTOGENÈSE}

\section{par A. Braciet}

....Cel ouvage contient lanalyse minulieuse dles diver:acles que comporte la fecondalion el la signification hio:sirque de chacun dentre eux y est tres clairement digagée. liee un litlent dexpmsition remarquble lauleur s'esi foul specialement efforce de demminer les caparites evo. lutives duspermatozoule, puis de lieruf inmir en riservan! une tres large part allx point- de vue nouveaux yula fiti natre lapplication de la methole experimentale at me science qui était restec purement morplologique jisquen ces dernieres années,.... 


\section{ENCYCLOPÉDIE SCIENTIFIQUE}

PLBLIÉE SOUS LA diRBction DU D' TOULOUSE Secrétaire général: H. PIÉRON

\section{BIBLIOTHĖQUE DE BIOLOGIE GÉNÉRALE}

\section{Directeur: M. CAULLERY}

Prolesseur à la Faculté des Sciences de Paris

$$
\text { - LES }
$$

\section{SOCIÉTÉS D'INSECTES}




\section{DU NEVE AUTEUR}

Ants, their structure, development and behavior. New-York (Columbia University Press) I 9 Iо, $8^{\circ}$ i $63 \mathrm{p}$., 286 fig.

Social Life among Insects, New-York, (Harcourt-Brace

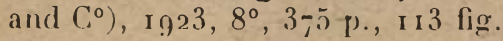




\section{PRÉFACE}

Si ce, livre ne devait avoir pour public que de purs zoologistes, toute préface y serait inutile, car il serait superflu de présenter aux lecteurs M. W. M. WheELer, dont le nom est un des plus connus parmi la brillante pléiade des biologistes américains contemporains. Avant d'être un des maîtres de l'entomologie générale, M. WHEELER avait marqué sa place en zoologie par d'importantes recherches sur des sujets variés, - il suffit de rappeler ici ses travaux sur la sexualité des Myzostomes et sur l'embryogénie des Poissons Cyclostomes. Depuis plus d'uni quart de siècle, il s'est de plus en plus exclusivement consacré à l'étude des Insectes et particulièrement des sociétés d'Insectes, surtout dı groupe des Fourmis. Les spécialistes savent quelle œuvre considérable il a publiée sur ces animaux, et il a donné, en dehors de très nombreux mémoires, la mesure de son esprit de synthèse par des articles d'ensemble et des livres dont le succès a été considérable ( 1 ).

(1) Ants, their structure, development and behavior. NewYork (Columbia University Press, 1910, $8^{\circ}$, 663 p., 286 fig.). Social life among the Insects, New-York (Harcourt Brace and $C^{\circ}, 1923,8^{\circ}, 375$ p., 113 fig.). 
Je suis donc très reconnaissant à mon ami WiIEeLER d'avoir bien voulu confier à la section de "Biologie générale " de l'Encyclopédie scientifique le présent ouvrage, que les circonstances ont fait naître en France. De cellesci il y a lieu de nous féliciter. Ce livre est, en effet, un fruit précieux des échanges universitaires franco-américains; le promoteur de ce mouvement, M. James $H$. Hyde, peut s'en réjouir une fois de plus. M. W. M. WheELER a été, en r 924-1925, l'exchange-professor envoyé par l'Université Harvard à l'Université de Paris. Voici la trace solide qui reste de cette mission.

Pour le cours qu'il devait faire, il a choisi l'Evolution des Insecles sociaux, sujet où il disposait d'une information personnelle des plus étendues et d'une autorité indiscutable. Les douze leçons qu'il a professées, de mars à mai ${ }_{925}$, sont reproduites ici sans changement. Les nécessités de l'enseignement commandaient, en effet, à l'exposé un esprit de synthèse qui est comme la marque essentielle de cette collection. Ce livre est loin d'être le premier qui soit écrit sur les sociétés d'Insectes, mais il est profondément original dans les conceptions - fruit des recherches propres de l'auteur, - nouveau dans sa documentation, qui s'étend aux travaux des plus récentes années et, il faut le dire, tout à fait différent de ceux que M. Wheeler a précédemment écrits.

En dehors des zoologistes, il s'adresse à tous ceux qui s'intéressent aux problèmes généraux de la Biologie. Dans un sujet qui se rattache à la philosophie et à la sociologie, il apporte, pour la discussion des analogies avec les sociétés humaines, une base étend!se de faits scientifiques, envisagés d'un point de vue strictement positif et expérimental et discutés avec une critique pénétrante, à la lu- 
mière des connaissances les plus modernes. Philosophes et sociologues y trouveront donc certainement grand profit. Aux naturalistes il fournit une mise au point d'une extrême compétence sur des problèmes très complexes et où présentement foisonnent les recherches. Au point de vue du progrès de la science, M. Wheeler met fortement en évidence combien l'étude des groupes sociaux d'Insectes, Hyménoptères Aculéates et Termites, est un champ fécond, surtout dans la faune des tropiques, devenue aujourd'hui très accessible. Il a illustré d'ailleurs cette vérité par son effort personnel et par les beaux résultats qu'il a rapportés de ses voyages en Guyane et à Panama. On en trouvera maints échos dans les pages qui suivent. On peut donc espérer que son exemple, les idées qu'il suggère, la documentation qu'il a rassemblée, susciteront, à la lecture de ce livre, des, vocations dans la voie qu'il trace.

II. CAULLERY, Professeur à la Sorbonne. 



\section{TABLE DES MATIÈRES}

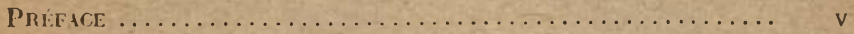

Intronucrion. - Caractères généraux des sociélés dinsectes

Sommare. - Extension des tendances sociales che\% les animaux. I.eur caractère dappétition. - Les sociètés d'insectes sont des familles; degrés divers de réalisation (insectes infrusociaux, subsocimux et sociunx). - ImporLance de laugmentation de la durée de la vie. - Trente cas de sociètés d'insectes. - Exemple : Coccidotrophus (Coléoptères silvanides) ; rapports avec les Coccides. l.es melhodes de reconstitution de la phylogénie et les sociètés d'insectes. - Létiologie de ces sociétés. La socièté considéree comme un tout. - Programme du livre.

ChAPITRE PREMLn. - L'origine des lérebrants et des acu-

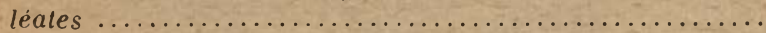

Sommane. Divisions principales et origine des Ifyménoptères. - Documents paléontologiques. - Phytophages, Térébrants (Trigonalidés, histoire des Périlampidés, Eucharides). - La forme primitive des larves d'Hyménoptères. - Modes d'alimentation de la progéniture : formes atrophaptique, dystrophaptique, eutrophaptique. 
Charitre: II. - L'évolution des V'espidés ...............

Sommafe. - Indication chez des Térébrants de caractères propres aux Aculeates. - Subdivisions des Aculéates et rapports des familles. - Les Bethylidx. - Leurs facons de traiter la proie, types béthyliné, sclérodermatiné, dryinine. - Les Vespides, solitaires subsociaux el formesociales.

Chapitre 11I. - L'écolution des Abeilles ................

Sommen, - Aftinites avec les Sphecida ; discussion, parallisme des mours avec les Vespidx. - Les Allodape, le: Halictus (nombre de generations et polymorphisme), les Bombince Meliponinie et Apinit. -- Formes fossiles. Progrés de la nidification. - La production de la cire. - Hodes divers de constitulion des colonies el les formes sociales superieures.

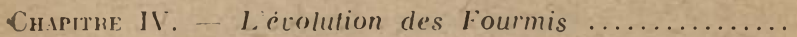

Sommnn: - Principales subdivisions; leurs rapports. Origine monophylétique ou polyphylétique. - Origine des fourmis parmi les Aculéates. - Les Tiphiidæ. Documents paleontologiques. - Thermophilie et xérophilie des fourmis. - Apparition probable au Trias. Ancienneté et longue durée de l'évolution du groupe.

Chaprim: 1. - Levolution des Termiles ..............

Soumunt: - Composition bisexuelle des colonies de termiles. - Caractère tropical du groupe, ses subdivisions, ses allinités. - Docuinents paléontologiques. - Origine mésozoïque ou permienne. - Distribution géographigue. - Affinités avec d'autres Orthoptères. - Mours nupliales. - Hahiludes alimentaires (xylopharjie). - Toile des Prolozoaires intestinaux. - Ennemis des termites. 
Chaptre: VI. - Le polymorphisme .............. 189

Sommane. - Cas divers. - La parthénogenèse et le sexe. Difierenciation d'une caste ouvrière chez les fourmis; son evolution. - Caracteres adaptatifs des castes. Polymorphisme des termites. - Différenciation congenilale ou epigénélique des castes. - Les diverses formes de soldats. - Soldats (fourmis ou termites) gardant - lentree du nid (phragmose).

Chapitae VII. - Le polymorphisme (Suite) $\ldots \ldots \ldots \ldots$

Sommare. - Prédétermination ou épigenèse? - Rỏle de lalimentation: guêpes, bourdons, abeilles, fournis. Action des parasites: mermithergates, ròle des Lomechusina. - Parasitisme de fourmis sur d'autres fourmis. - Principe diEmery et de Vienueyer. - Le gradien! axial de ChiLv. - Discussion des hypothèses trophogeniquel et blaslogénique (WEISMAxix).

Chipitie VIII. - Le milieu social el la trophallaxis ....

Sommare. - Importance préponclérante de la caste ouvriére dans la constitution du milieu social. - Champ trophoporique. - Trophallaxis (échange de nourrilure). Exemples de ce processus dans les divers groupes dinsectes sociaux. - Ròle des perceptions gustatives el olfaclives.

Chipitre. IX. - Evolution des associés el des parasites des

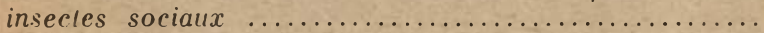

Sommane. - Calégories diverses: Sphécophiles, Mélitıphiles, Myrmécophiles, Termitophiles. - Exemples de synnecèles et de synechthres. - Associés vrais ou symphiles. - Parasites. - Termitophiles. - Parallélisme de l'évolution des associés des fourmis et des termites (phusogastrie, etc.). - Critique des interpretation:- de WVASMANN. 
Chupren: X. - Licolution des parasiles sociaux ...... 337

Sommane. - Guejpes et abeilles parasites. - Fourmis parasites. - Cleplobiose, Leslobiose, Parabiose, Xénobiose, etc. - Esclavage (dulose), ses varieles : cas rle Formica sanguinea, des Polyergus (fourmis amazones), des Strongylognalhus, des Harpagoxenus. - Parasilisme temporaire. - Parasitisme permanent.

Chipitu: XI. - Conclusions .................... 369

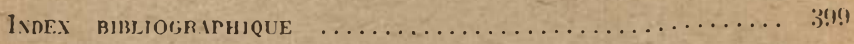

INOEX ALPHABETQLE DES IUTETIS $\ldots \ldots \ldots \ldots \ldots \ldots \ldots \ldots$ 14.

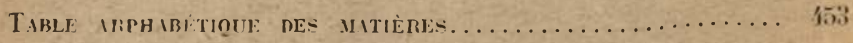




\section{LES SOCIÉTÉS D'INSECTES IEUR ORIGINE, LEUR ÉVOLUTION}

\section{INTRODUCTION}

\section{CARACTERES GENERAUX DES SOCIETES D'INSECTES}

Sommanz. - Extension des tendances sociales chez les animaux. Leur caractère d'appétition. - Les sociétés d'insectes sont des familles; degrés divers de réalisation (insectes in- irasociaux, subsociaux et sociaux. - Importance de l'augmentation de la durée de la vie. - Trente cas de sociétés d'insectes. - Exemple : Coccidotrophus (Coléoptères silvani(les); rapports avec les Coccides. -- Les méthodes de reconstitution de la phylogénie et les sociétés d'insectes. L'étiologie de ces sociétés. - La société considérée comme un tout. - Programme du livre.

J'ai choisi pour plusieurs raisons l'évolution des insectes sociaux comme sujet de celte série de conférences. D'abord, il m'a paru éminemment approprié au laboratoire fondé par l'illustre Giard pour l'étude de l'évolution des êtres organisés. En second lieu, je ne pouvais trouver un sujet qui intéressât davantage les jeunes biologistes d'un pays qui a produit un Réaumur, un Latreille, un Lepeletier de Saint-Fargeat, un Dufour, un Fabre, un Pérez, un Ferton, un Paul Marchal et un Bouvier, sans parler d'une foule d'autres brillants hyménoptéristes. Troisièmement, j'ai moi-même consacré un quart de siècle à l'étude d'un groupe d'insectes sociaux; j”ai eu de 
nombreuses occasions de voyager; j'ai reçu l'aide d'un grand nombre d'entomologistes enthousiastes de toutes les parties du monde, j'ai donc pensé que je pourrais suggérer ou souligner quelques directions de pensées dignes de votre considération. Il m'a paru possible aussi que vous ne fussiez pas familiers avec tous les travaux qui ont été accomplis par mes compatriotes; en les mentionnant, autant que mon sujet le comporte, je puis, dans une faible mesure, servir celte " entente cordiale " intellectuelle que nous désirons maintenir entre la France et les Etats-Unis. Enfin, je crois que l'étude des insectes sociaux a actuellement un intérêt particulier pour les travailleurs sérieux, à la fois en philosophie, en sociologie, en zoologie et dans l'étude des mœurs des animaux. Celui qui incline à la philosophie ne peut manquer de trouver un aliment pour la pensée dans les étranges analogies avec les sociétés humaines qui se révèlent continuellement parmi les guêpes, les abeilles, les fourmis et les termites, puisque nous sommes nous-mêmes des animaux - j'allais dire des insectes 1 - sociaux, et le zoologiste et l'éthologiste noteront qu'ils suggèrent un cortège surprenant de faits et de problèmes fascinanıs. En outre, la vie sociale très remarquable des insectes, par le fait qu'elle est presque exclusivement déterminée par les réflexes, les tropismes et ce qu'on appelle les instincts, et non par l'intelligence, prend une grande significalion théorique, si nous considérons les tendances et les courants présents anti-intellectualistes et relativistes de la pensće européenne et américaine.

Je me servirai en partie de matériaux rassemblés ell vie de conférences faites à l'Institut Lowell, à Boston, sur "La vie sociale chez les insectes ". J'y avais insisté 
sur le rôle fondamental de la nutrition dans le développeinent des diverses sociétés d'insectes; ici, je voudrais trailei le sujet d'une façon différente et plus technique, et développer certaines matières qui avaient été simplement esquissées ou indiquées.

Je crois devoir essayer d'abord de donner des réponses au moins partielles à quatre questions générales :

$r^{\circ}$ Que sont les insectes sociaux ?

$2^{\circ}$ Peut-on montrer qu'ils ont eu une évolution ?

$3^{\circ}$ S'il en est ainsi, quelles sont les particularités de cette évolution, et à quelles méthodes devrons-nous faire appel pour les élucider?

$4^{\circ}$ A quelles causes ou conditions générales pouvonsnous rapporter cette évolution?

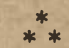

Que sont les insectes sociaux? Les mots "sociaux ", "qui s'associent" sont, il va de soi, un peu vagues et teintés d'anthropomorphisme. Leur sens vague est dû à la multiplicité extraardinaire et au caractère protéiforme des phénomènes, même chez les Insectes, sans parler des diverses classes de Vertébrés. Ces phénomènes s'échelonnent le long du chemin qui mène d'un grégarisme ou d'une collectivité íaibles, ou de la simple union des sexes, à des sociétés permanentes et hautement conslituées, qui rappellent, de loin, la nôtre. L'anthropomor. phisme qui s'attache a! mot "sociaux " s'explique aisément, et le critique peut le condamner ou le pardonner selon ses convictions philosophiques générales (I).

(1) Je dirai seulement que je pense que, dans la discussion de matières psychologiques, - et les phénomènes en question sont psychologiques en partie, - nous ne pouvons éviter un certain anthropomorphisme, ou une certaine téléologie. 
DeEgener (igi 8) a essayé récemment, dime façon hardie mais peu satisfaisante, de classer el de décrire toules les formes variées d'association qu'on rencontre dans le règne animal. Il distingue quelque quatre-vingt douze catégories différentes, dont cinquante-trois sont représentées chez les Insecles. Il les a toules dotées de noms scientifiques, dérivés surtout du grec, et dont quelquesuns, comme " hétérosýmphagopaedium ", "amphotérosýnhesmium " et "héiérosynépileium ", ont une lourdeur bien germanique. Les'quatre-vingt douze catégories se partagent en deux groupes; les sociétés ou associations accidentelles, chez lesquelles la réunion des organimes n'a pas de valeur intrinsèque, c'est-à-dire n'a pas une fin utile à l'individu, et les sociétés ou associations essentielles, chez lesquelles la réunion des organismes a une valeur intrinsèque et sert en partie des fins utiles à l'individu. Chacune de ces calégories principales se subdivise, suivant que l'association comprend des individus de la même espèce ou d'espèces différentes. Le " principium divisionis" finaliste, suivant lequel les principaux groupes sont constitués, est très douteux, pour ne pas dire plus; car, dans les sociétés animales les plus hautement constituées, le fait d'être membre de la société implique parfois inévitablement la destruction de l'individu. Et, indépendamment du fait que le même animal (par exemple Necrophorus) est placé par DeEGEser dans différentes catégories, quelques-unes de ces catégories frisent la fantaisie; par exemple, son "hétérosymporium ", qui comprend les insectes de différentes espèces charriés ensemble par une crue, ou les différents animaux fuyant devant un feu de prairie, ou son "symphotium. ", qui comprend l'assortiment hétérogène d'in- 
sectes altirés par une lampe. Sn laisser aller à un tel verbalisme conduirait à donner ì la biologie une réputation pire que celle de la scolastique niédiévale.

11 semble bien évident que, pour prendre une figure, le comportement de tout animal gravite autour de deux axes, dont l'un est agressif et individualiste, l'autre coopératif ou social. Les darwiniens tenaient le premier mode de comportement pour amplement établi, et le soulignaient beaucoup, de telle sorte que le dernier paraissait être exceptionnel et demander une explication spéciale. Aujourd'hui, on demanderait plutôt une explication du mode de vie solitaire, tellement tous ceux qui étudient les animaux dans leur milieu vivant compliqué, sont profondément impressionnés par leurs inclinations sociales ou associatives. La chose est apparente, même dans les biocoenoses, puisque tout organisme, peu importe qu'il soit égocentrique, prédateur ou parasite, est social ou coopératif, an moins en tant que membre de quel(que bioconose (r).

Si l'on nous demandait d'indiquer un groupe d'animaux lypiquement solitaires, nous choisirions peut-être les Araignées, mais, lorsque nous les étudions sous les tropiques, nous trouvons que plusieurs d'entre elles sont positivement grégaires, ou vaguement sociales. Récemment, à Panama, Nathan Bıккs et moi, nous avons élé frappés par les moeurs de plusieurs de ces Arachnides.

Une des araignées it loile orbiculairc les plus grandes et les plus connues, Nephilla plumipes, construit régulièrement ses toiles, pendant la saison hmmide, de façon que celles des

(1) La vérité de ce que nous venons de dire a été exprimée il $y$ a longtemjs et d'une façon admirable par Espivis, dans l'introduction de son ouvrage "Des sociétés animales 》 (1877). 
différentes femelles se réunissent et forment parfois des édifices énormes et remarquables. Un nid composé, que nous avons observé dans les arbres, au sommet de la colline d'Ancon, près de la ville de Panama, avait bien vingt pieds de large et plus de vingt-cinq pieds de haut. Il s'y suspendait au moins deux cents femelles et presque autant de mâles de Nephilla, outre un nombre considérable d'Argyrodes nephilla, petite araignée Thomiside parasite, qui vit uniquement sur les toiles de Nephilla. Nous avons aussi rencontré plusieurs espèces de Theridion et d'Uloborus, qui unissent leurs nids d'une manière semblable, et une espèce particulièrement sociale, non décrite apparemment, mais alliée à Anelosimus socialis Simon. Dans les nids de cette araignée, des douzaines de femelles de tout âge se promenaient librement et, selon toute probabilité, se nourrissaient en commun, au moins des plus grandes proies. Plusieurs de ces araignées portaient sur leur ábdomen les larves d'un hyménoptère parasite (probablement Polysphincla). Des insectes étrangers eux-mêmes peuvent vivre impunément dans les nids de quelques-unes de ces araignées de Panama. Dans l'île Barro Colorado, nous avons trouvé de nombreuses nymphes rouges et des adultes noirs d'une petite Nabide à allure de fourmi (Arachnocoris albomaculatus Scott), immobile, ou se promenant sur les toiles d'Uloborus et de Theridion, et se nourrissant probablement des proies abandonnées par les araignées (voir MYers 1925). A deux reprises, nous avons observé des douzaines de petites mouches Cécidomyides, pacifiquement stationnaires sur les toiles de Nephilla et d'Uloborus. Quand on secouait les toiles, les chétifs insectes ne s'envolaient que pour revenir imınédiatement à leur première place.

Simon a fait, en 1893, des observations analogues sur les mœurs sociales d'araignées du Vénézuéla et en cite de nombreux exemples dans d'autres contrées tropicales. Il donne une exceliente histoire d'Epeira bandelieri, Anelosimus socialis et Uloborus republicanus. Dans les circonstances ordinaires, E. bandelieri est solitaire, mais, quand approche l'époque de la ponte, plusieurs femelles se réunissent et construisent ensemble une grande capsule elliptique, dans laquelle elles se retirent el filent leurs cocons ovigères. $A$. socialis vit en colonies de plusicurs milliers d'individus, qui construisent une toile commune. Les araignées s'y promènent librement, se rencontrent en se palpant, comme feraient des fourmis avec leurs antennes, et se mettent quelquelois at plusieurs pour dévorer une proie un peu volumineuse. 
L'association de $U$. republicanus est de beaucoup la plus parfaite, car elle offre, sur la même toile, un travail coınmun, auquel contribuent tous les associés, en même temps qu'un Iravail individuel propre i chacun d'cux. Plusieurs centaines d'Uloborus vivent enseinble; ils filent entre les arbres une toile immense, formée d'un réseau central assez serré, sur lequel se liennent côte à côte beaucoup d'individus des deux sexes, mais principalement des mâles; ce réseau est suspendu par de longs fils, divergeant dans loutes les directions et prenant attache sur les objets environnants. Dans les intervalles des mailles, formés par ces grands fils, d'autres Uloborus tissent des toiles orbiculaires à rayons et à ccrcles, qui ne sont alors hąbitées que par un seul individu. C'est dans le réseau central que doit avoir lieu l'accouplement, autant que nous avons pu en juger par la quantité de mâles qui y étaient réunis. C'est certainement là que s'effectue la ponte.

La ponte parait être presque simultanée pour loutes les femclles d'une même colonic; à ce moment, les mâles ont disparı, les femellés ont cessé de filer cies toiles régulières, elles se retiennent sur le réseau central, à quelques centimètres les unes des autres, gardant chacune son cocon daus une immohililé comjlete (1).

D'après Bouvifr (1918), "SÉmichon (1909) a constaté le même parlage fraternel des proies que chez $U$. republicanus, dans une araignée sociale mexicaine, le Cœnolhele gregalis, rapporlée au Muséum par Léon Diguet (1909 a-1909 b). Celte espèce établit sur les arbres de vastes toiles sociales, qui se développent concentriquement, all moyen de fils cardés où viennent se prendre les insectes; des milliers d'individus vivent en bonne harmonie dans cet immense sac alvéolaire et n'en sortent ja-

(1) Sim.ox (1892-95), Distant (1898), M.insillle (1898), Pogock (1903), et Jамимітин (1908) ont donié des récits des mœurs d'une intéressante araignée sociale du genre Stegodyphus, représentée par plusieurs espéces dans l'Afrique du Sud. Walsinghim (1903), МarshiLl et Рососк, décrivent également une teigne particuliere (Batrachedra stegodyphobius) qui vit, à tous ses stades, dans les nids de ces araignces. M. J. II. Emerton m'informe que cerlaines de nos araignces de la Nouvelle-Angleterie. elles-mèmes, peuvent filer des toiles élroitement contiguës, par exemple Diclyna muraria et Amaurobius ferox, et qu'il a vu jus d'une centaine de petils moucherons-tranquillement immobiles sur la toile de linyphia maryinata. Voir aussi Sсишанz (1904) et Banis (1904). 
mais, sauf après la saison pluvieuse, où ils émigrent, - j'allais dire essaiment, - pour laisser place aux jeunes. Ces nids peuvent se diviser et, suspendus au plafond, servent de piēges à mouches dans certaines régions du Mexique; l'un d'eux, exposé dans les galeries du Muséum, atleint plusieurs mètres de longueur » (1).

La plupart d'entre nous seront probablement d'accord avec Petrucci (igo6), qui a fait il y a quelque temps une revue des diverses formes de sociétés chez les Vertébrés et a montré leurs dérivations polyphylétiques, quand il dit: "la seule chose héritée dans les activités sociales de l'animal, c'est la tendance au groupement, tendance affirmée de façon prédominante dans tout le domaine biologique, et qui se réalise en phénomènes concrets dès que les circonstances extérieures lui laissent la possibilité de le faire ". Perricci ramène naturellement sa " tendance associative " à des phénomènes tels que la constitution cellulaire des Métazoaires et des Métaphytes et même que les équilibres atomiques et les associations moléculaires du monde inorganique. Mais cette conception, bien qu'elle ne soit pas sans impliquer des considérations philosophiques, devient très vague.

(1) Diguet (1909 a) a écrit les noles suivantes; qui concerment dedix commensaux gu'il trouva habilant les nidis de Conothele: "Dans toutes les parties de l'inlérieur du nid, on rencontre en très grande abondance, vivant en commensal, un Latridide du genre Melanophthalma; le rôle qui paraît incomber à ce Coléoptère d'infime dimension est de présider à la propreté du nid, en faisant disparaître et en transportant les détritus qui peuvent encombrer ou souiller les galeries; sa principale nourriture parait être les restes du rejas de l'araignée. Comme commensale, on rencontre encore, vivant en parfaite harmonie avec la colonie du Mosquero, une araignée errante, qui s'est fait l'hôte de ce logis où elle trouve une vic facile et assurée. " Cette araignée a été reconmue par Sımox (1909) comme étant la Pacilochroa convictrix Simon. 
Dans le but de rendre la matière plus concrète et plus intelligible, au moins dans le domaine biologique, je préférerais considérer la "tendance associative " comme une "appétition ", au sens dans lequel Foullé́e, (1920) et les psychologues anglais et américains Drever ( 1917 ), Craig (igi8) et Tuunstone (1924) ont employé ce terme. Elle se placerait ainsi près des autres appétitions, comme la faim, et le sexe, bien qu'elle soit plus faible, plus continue, c'est-à-dire moins spasmodique et, par conséquent, moins évidente.

Les animaux sociaux élevés, lorsqu'ils sont séparés des stimuli continuels et ordinaires de leur espèce, manifestent une inquiétude qui montre de la façon la plus frappante cet appétit social (I).

Il est peu douteux que cette appétition sociale se manifeste très clairement et très généralement chez certains insectes, mais il sera avantageux pour nous de rétrécir cette conception, si nous voulons l'utiliser ici, par l'introduction d'autres considérations. On a insinué que les sociétés de fourmis et d'abeilles elles-mêmes peuvent être dérivées phylogénétiquement d'associations fortuites de femelles de la même espèce. Mais c'était avant que la constitution effective et la genèse des sociétés d'insectes fussent connues. Tout le monde admet aujourd'hui que toutes les sociétés d'insectes dignes de ce nom et quelle que soit leur population, sont des familles, c'est-à-dire des affiliations entre les parents, et, dans la

(1) Il va de soi que, pour una part, je n'adhère aucunement aux vies de Becher (1917), qui posc en poslulat l'existence chez certaines plantes et cerlains animaux, d'un penchant altruiste -distinct à servir d'autres organismes tout à fait étrangers. Celte vue a été très justement critiquée par Bequant (1924) et d'autres auteurs. 
plupart des cas, la mére scule, avec la descendance. Cette vue est abondamment confirmée par l'étude de l'ontogénie des sociétés d'insectes existantes et par les indications fragmentaires de leur phylogénie que nous pouvons obtenir.

Il y a eu, évidemment, une longue évolution, passant par des stades nombreux d'intimité croissante entre la mère et sa progéniture, à parlir du stade le plus rudimentaire d'indifférence complète ou presque complètc jusqu'à la coopération mutuelle constante. Nous pouvons construire une série telle par exemple que la suivante, sans nous arrêter à énumérer des exemples concrets, dont la plupart vous viendront immédiatement à l'esprit :

$1^{\circ}$ La mère insecle dissémine simplement des oufs dans le milieu général dans lequel vivent normalement les individus de son espèce (insectes atrophaptiques). - Dans quelques cas, les œufs sont placés près de la nourriture des larves (insectes dystrophaptiques).

$2^{\circ}$ Elle place ses œuls dans une portion déterminée du milieu (feuilles, etc...), qui servira de nourriture aux larves venant d'éclore (insectes eutrophaptiques).

$3^{\circ}$ Elle pourvoit les œuls d'un revêtement protecteur. Ce stade peut se combiner avec (1) ou (2)..

$4^{\circ}$ Elle reste avec les œufs et les jeunes larves et les protège elle-même.

$5^{\circ}$ Elle dépose les œuis dans un emplacement sûr ou spécialement préparé (nid), avec une provision de nourriture aisément accessible allx jeunes qui viennen! d'éclore (approvisionnement en masse).

$6^{\circ}$ Elle resle avec les oufs et les jeunes, protège et nourrit constamment ces derniers arec de la nourriture préparée (approvisionnement progressif).

$7^{\circ}$ La progéniture est non seulement protégée et nourrie par la mère, mais coopère éventuellement avec elle pour l'élevage de nichëes additionnelles de jeunes, de telle sorte que le parent et sa descendance vivent ensemble en sociélé annuelle ou durable. 
Les insectes qui rentrent dans les catégorics I à 5 peuvent être appelés " infrasociaux ."); ceux de la $6^{\circ}$, qui sont plus intéressants pour nous, "quasi - " ou " subsociaux ". Il n'y a que la dernière $\left(7^{\circ}\right)$ catégorie qui comprenne des formes vraiment sociales. Le stade final de la série est atteint primitivement par le développement d'un intérêt croissant de la mère pour le destin futur de sa descendance. Il va de soi qu'il est rendu possible par une augnentation de la durée de sa vie individuelle. Si ceux qui étudient la sociologie humaine s'étaient rendu comple de cette condition évidente, ils nous auraient épargné quelques spéculations qui se répètent constam. ment dans la littérature sociologique. John Fiske, dans sa "Philosophie cosmique " (1874), soutenait que l'augmentation de la durée du premier âge et de l'enfance humaine avaient conduit à l'association définitive des parents avec la lignée. C'était là, pour lui, une nouvelle interprétation de l'origine de la famille. Mais Loveroy (1922) a montré récemment que la notion était déjà familière à plus d'un penseur du xviri ${ }^{\ominus}$ siècle. Il mentionne particulièrement le poète Pope et les philosophes Locre et Roussfau, et montre que Rousseau a détruit l'argument de Lоскг, qui était essentiellement celui de John Frske, dès 1755 , dans son "Discour's sur l'origine 'de l'inégalité ". Roussiau faisait ressortir que, si l'habitude de la vie de famille n'avait pas été établie par l'homme primitif durant les mois qui précèdent la naissance du premier enfant, le mâle humain serait difficilement venu en aide à la femelle après l'accouchement: "Comment l'aiderait-il à élever un enfant qu'il ne sait pas toujours être sien, et dont il n'a ni désiré ni prévu la naissance? ". Il va de soi que nous devons aujourd'hui, 
nous orienter ver's l'étude des Anthropoïdes, si nous désirons quelque lumière sur les origines lointaines et nébuleuses de la famille humaine. Toutefois, il est évident qu'aucune augmentation simple de la durée du premier âge et de l'enfance ríaurait de valeur, sans une augmentation précédente ou concomitante de la durée de la vie des parents. Cette considération, si clairement indiquée par les Insectes, paraît avoir complètement échappé aux auteurs mentionnés ci-dessus.

Nous ne savons rien des causes physiologiques de la longévité accrue des insectes sociaux adultes. Il est probable, comme l'a suggéré PearL (1924), que la durée de la vie d'un animal est en raison inverse de l'activité de son métabolisme. La durée de la vie, dans les trois castes de fourmis et d'abeilles sociales, semble être grossièrement proportionnelle à leur dépense respective d'énergie. Mais le véritable problème est celui de l'augmen. tation de la vie de l'adulte après le commencement de la reproduction. La longue vie que mènent certain insectes avant de se reproduire (par exemple Cicada seplemdecim; les larves d'Ephémérides, de Cérambycides, elc.) ne peut pas conduire au développement de sociétés. Et il va de soi aussi que la longévité de l'adulte donne simplement au parent des possibilités, mais ne fait rien pour l'intérêt porté à la progéniture, ou le soin pris à son éducation.

Il me semble que la diminution de l'activité métabolique que l'on peut supposer, selon Peari, augmenter la durée de la vie, spécialement chez les femelles fécondes, peut être dûe au fait que tous les insectes sociaux ou subsociaux vivent dans de petites cavités du sol ou du bois, ou, dans le cas le plus exceptionnel des guêpes so- 
ciales ou de certaines fourmis tropicales, à l'intérieur de nids en carton. Le milieu, par conséquent, est de ceux qui restreignent ou inhibent les mouvements musculaires. Il est sombre, pauvre en oxygène et de température uniforme et plutôt basse. Toutes ces conditions favorisent nécessairement un métabolisme et une activité réduits, et une accumulation de graisse dans le corps de l'insecte. Les reines ou mères, des sociétés d'insectes donnent bien l'impression d'avoir acquis leurs particularités physiologiques et quelques-unes de leurs particularités morphologiques en réponse à un tel milieı, car clles sont très apathiques et tendent à perdre la faculté de voler (Meliponina), ou même les ailes (fourmis et lermites) et à acquérir un anabolisme accentué, comme le montrent l'accumulation de graisse, et la ponte d'œufs riches en vitellus. Aussi est-il très probable que l'aug. mentation de la vie de l'adulte et la fécondité de ces insectes ont été graduellement acquises en raison du milieu réduit dans lequel non seulement ils se développent, mais continuent à vivre quand ils sont adultes. Leur fécondité est, dans une certaine mesure, fonction de leur longévité, et s'exprime clairement par la grandeur de la colonie adulte dans toutes les espèces d'insectes sociaux, spécialement chez les termites les plus élevés (Termes), chez les fourmis comme les Eciton et les Atta, et chez l'abeille domestique. D'un autre còté, la petitesse des colonies complètes de plusieurs fourmis primitives (Ponerince) et de termites (Calotermilid(c) paraît être la conséquence de la vie plus courte de la reine mère.

J'ai dressé, pour en parler rapidement, la liste suivante des insectes que nous avons appelés subsociaux et sociaux. 


\section{COLEOPTERA}

1. Silvanida (Coccidotrophus, Eunausibius).

2. Scarabridæe (Copris, Minotaurus).

3. Passalidæ (Passalus, etc.).

4. Tenebrionidx (Phrenapates).

5. Ipida (Xyleborus, etc.).

6. Platypodidx (Platypus, etc.).

HYMENOPTERA (Aculeala).

7. Bethylidic (Scleroderma).

8. Masaridinie (Ceramius).

9. Eumenina (Synagris, Odynerus).

10. Zethinæ (Zethus).

-11. Stenogastrinæ (Stenogaster).

12. Epiponinæ (Belonogaster, Chartergus, etc.).

-13. Ropalidiinæ (Ropalidia).

-14. Polistinze (Polistes).

-15. Vespina (Vespa).

16. Sphecinæ (Ammophila).

17. Trypoxylonina (Trypoxylon).

18. Bembicina (Bembex).

-19. Halictinx (Halictus).

20. Ceratinina (Allodape).

-21. Bombinæ (Bombus).

-22. Meliponinæ (Melipona, Trigona).

-23. Apinæ (Apis).

-24. Formicidæ (Formica, etc.)

\section{AUTRES ORDRES}

25. Blattoidea (Dasypoma).

26. Dermaplera (Forficulida).

27. Orthoptera (Gryllotalpa).

28. Embidaria (Embiid $x)$.

29. Zoraptera (Zorolypus).

-30. Isoptera (Termitida).

Il n'est pas douteux que celte liste soit incomplète, puisque notre connaissance des mœurs de beaucoup d'insectes, surtout tropicaux, est encore très défectueuse. J'aurais du joindre à la liste les espèces australiennes de Perga, appartenant aux Tenthredinidæ, et un certain nombre d'Hétéroptères, dont les femelles gardent les œufs et les jeunes qui viennent d'éclore. Mais ces cas et d'autres similaires conduisent insensiblement aux formes infrasociales. 
On a donc reconnu une organisation sociale au moins dans trente cas distincts, et dans huit ordres naturels très différents. Douze au moins de ces groupes (désignés par des astérisques) sont devenus définitivement sociaux. l.es autres n'ont pas dépassé une ébauche très imparfaite. Quelques-uns de ces insectes subsociaux, surtout les Coléoplères, sont très intéressants, mais leurs mœurs sont si variées que je dois vous renvoyer au bref récit que j'en ai donné dans mon livre "Social life among the Insects " (La vie sociale chez les insectes). Une esquisse des mœurs du Silvanidé social que j'ai observé d'abord pendant l'été de 1920 , à la Guyane anglaise, servira d'exemple à toute la série.

Ces Coléoptères, que Schwarz et Barber ont nommés Coccidotrophus socialis et Eunausibius wheeleri, ont moins d'un quart de pouce de longueur, un corps allongé, grêle, subcylindrique, d'une couleur rouge ou chatain, avec des paltes courtes et des antennes en massuc. On les trouve senlement dans les pétioles creux d'un arbre très intéressant, Tachigalia paniculata, et seulement chez les spécimens jeuncs, d'une hauteur de 1 l/2 ì 7 pieds, pendant qu'ils poussent à l'ombre des grands arbres de la jungle.

Les arbres plus âgés, qui peuvent alteindre quarante pieds el plus, ont tous leur pétioles habilés nar des fourmis qui mordent ou piquent désngréablement (l'sendorn!yrma maligna et crucians, Azteca foveolata). Chaque co!onic de Coléoplères est élablic par un mâle et une femelle, qui perforent la paroi du pétiole, le débarrassent de toute moelle ou de tous les restes d'occupants antérieurs qu'il peut contenir, et commencent à se nourrir d'un tissu particulier, riche en protéines, qui est disposé en bandes longitudinales parallèles sur la paroi du pétiole. En rongeant ce tissu, ils creusent peu a peul des sillons et cntassent leurs excréments dans les espaces non rongés, de telle sorte que l'intérieur de chaque pétiole prend un aspect parliculicr. Pendant que les Coléoptères s'occupent ainsi, de nombreuses petites cochenilles du genre Pseudococeus ( $P^{2}$. brevipes), couvertes d'une cire d'un blanc de neige, pénètrent dans le pét:nle par l'orifice percé par les Colénptères, s'inslallent dans les sillons, enfon- 
çent leurs suçoirs délicals dans le tissu nourricier el se gonflent de leurs sucs.

Bientòt, les Coléoptères commencent à pondre leurs ceuls, petits, élliptiques et b!ancs, le long des bords des sillons, et les larves qui éclosent, joliment translucides, se promènent dans la cavité et se nourrisent du même tissu que les parenls. Quelque incroyable que cela puisse paraître, les adultes, comme les larves de tout âge, ont appris à caresser les cochenilles avec leurs antennes, absolument comme nos fourmis communes caressent des cochenilles et des pucerons analogues el se nourrissent des goutteleltes de miellée que ces pucerons donnent, quand on leur titille convenablement le dos. Les Silvanides sont si friands de ce nectar que j'ai vu des Coléoptères et des larves caresser des

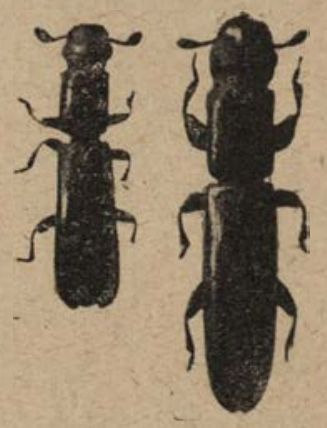

Fig. 1. - 1, Eunausibius wheeleri. Schwarz el Barher. 2, Coccidolrophus socialis. Shhwarz et Barber.

cochenilles pendant une heure el plus, recevant et avalant le liquide à des intervalles de quelques minules. Iorsqu'il arrive que deux ou plusieurs Coléoptères, ou deux ou plusieurs larves, ou un groupe d'adultes et de larves se mettent à caresser la mème cochenille, ils se placent en cercle autour d'ellc, commne des porcs autour d'une auge. Les individus les p'us grands ou les plus forts essaient de repousser les autres avec leur lìle. Mais les individus écartés cherchent a revenir et à recommencer leurs caresses, jusqu'à ce que les coups deviennent trop durs, ou jusıu'il ce que les individus les plus forts s'en aillent caresser une autre cochenille. Les Coléoptères et leur progéni- 
fure frouvent ainsi une nourriture substantielle, formée en parlie des lissus riches en protéine du Tachigalia et en partie de l'enu sucrée émise par les cochenilles, lesquelles se gorgent à leur tour de la sève de l'arbre. Les Colćopteres échelonnent leur ponte, de telle sorte qu'on trouve, dans la même colonic, des larves à tous les slades. Quand elle est mùre, chaque larve se construit un cocon à l'aide de petites particules qu'elle détache des tissus de la plante, s'y glisse, ferme l'orifice de l'intérieur et se transforme en chrysalide. Quand les imagos éclosent, ils restent avec leurs parents, et commencent bientôt à pondre des œufs, de telle sorte que la colonie peut se monter à plusieurs douzaines d'adulles, larves, pupes et cochenilles a tous les stades. Tous vivent pacifirfuement ensemble, exceplé pendant les petiles scènes de faınille qu'amène la traile de leur palient troupeau blanc de neige. Quand le pétiole devient trop peuplé, des couples de Coléoptères le quittent, entrent dans d'autres pétioles du même Tachiyalia, ou de pieds voisins, et fondent de nouvelles colonies. Lorsque l'arbre croît et, sortant du sous-bois, émerge à la lumiere dı soleil, les fourmis, qui prennent mainlenant complètement possession de l'arbre, expulsent les Coléopteres de leurs cavités pétiolaires, mais adoptent leurs cochenilles, de mène que les Allcmands, pendant l'invasion, s'appropriaient le bétail français. La description précédente s'applique a Coccidotrophus socialis, mais liunousibius, plus rare, a des mours tres voisines (1).

\section{**}

Il m'a fallu un lemps si long pour répondre à la premic̀re des quatre questions que je posais au début de cette conférence, que je devrai ètre très bref dans mes réponses aux trois aulres. Notre seconde question élait peuton montrer que les insectes sociaux ont eu une évolulion? Je considere que nous acceplons lous la théoric de l'évolution, au moius dans le serıs du "transformisme ",

(1) Je Dr W. M. Nux o, plus récemment, découvert une seconde espece de Coccidolrophus (C. cordix, Barher), qui vit essentiellement de la même façon, avec les mêmes cochenilles, dans les renflements de la ligge d'une autre myrmécophyte, Coldia alliodora, de la Bolivie. 
et que nous répondrions tous affirmativement à la questions posée.

D'ailleurs toute cette série de conférences corroborera, je pense, cette manière de voir et en précisera les contours. Nous devons admettre, en mème temps, que notre connaissance très maigre des différents groupes auxquels appartiennent la plupart des insectes subsociaux nous empêche actuellement de donner une idée de leur évolution. Ils sont tropicaux pour la plupart, et ce sont des représentants, ou imparfaitement connus, ou isolés et sporadiques, de grands groupes, qui n'ont jamais été l'objet d'une étude éthologique comparée étendue. Il nous est donc impossible de tracer le cours probable de leur développement phylogénique. Par exemple, les Coccidotrophus et Eunausibius décrits plus haut appartiennent à la famille de Coléoptères des Silvanidés, dont la plupart des espèces ont des mœurs simples, non sociales. Mais, si nous connaissions les espèces tropicales qui sont étroitement alliées à Coccidotrophus et Eunausibus, nous pourrions projeter quelque lumière sur leur comportement particulier. Nous sommes dans la même situation difficile vis-à-vis des Ipidés (Scolytidés), Platypodidés, etc., , Cette pénurie de documents nous oblige à nous restreindre à l'évolution des groupes vraiment sociaux, qui appartiennent exclusivement aıx Hyménoptères aculéates et aux Isoptères. Ceux-ci ont été lièreusement l'objet d'études pénétrantes et étendues de la part d'un tel nombre de chercheurs que nous pouvons édifier un corps remarquable d'observations et d'expériences précises.

Les conférences qui suivront répondront aussi à la première partie de la troisième question, savoir les particularités de l'évolution des insectes sociaux. Mais nous de- 
vons discuter brièvement ici la deuxième partie, qui a trait aux méthodes à employer pour rechercher cette évo. lution. Notons bien que nous nous occupons de la phylogénie d'insectes caractérisés par un type particulier de comportement. Par conséquent, comme le comportement ne se fossilise pas, nous devrons, pour le passé, l'induire des particularités morphologiques et des modes de comportement qui sont liés à des particularités analogues chez les insectes actuels. C'est dire que nous sommes contraints de procéder historiquement (I).

Mais, du point de vue du biologiste " pur sang ", qui considère que la science n'est pas autre chose qu'une continuation de la physique et de la chimie, et n'est donc capable de se développer que par l'observation directe, l'expérimentation et les mathématiques, celui qui s'occupe de phylogénie, ou de l'aspect historique des organismes, est dans une position fâcheuse. Il ne peut pas observer le fonctionnement des formes éteintes, ni combiner d'expériences sur elles. Tous ses essais de reconstruire ce que fut la vie passée des animaux et des plantes sont donc nécessairement plus ou moins indirects, hypothétiques et problématiques. Il ne peut pas même recourir aux méthodes des archéologues, parce que, excepté dans des cas extrêmement rares, il ne reste pas d'objets cons-

(1) Il y a eu depuis quelque temps des discussions considerables, surtout en Allemagne, sur les relations précises de la biologie et de l'histoire, et de l'histoire et de la philosophie, et l'on parait accepter à nouveau ce que pensaient la plupart des anciens chercheurs, savoir que la biologie au sens large, englobant l'anthropologie et la psychologie, a ceci de particulier qu'elle est à la fois une science naturelle et une branche de l'histoire (phylogénie). [Voir spécialement les travaux érudits de iI. Rickert (1921), Kroner $(1913,1919)$, Mehlis (1915) el Schixel (1922)]. 
truits par les animaux disparus. Les seuls exemples que je connaisse sont des nids fossiles de guèpes Euménides, décrils par Handlinscu (I9Io), de l'Oligocène supérieur de Florsheim et les monticules des plus grandes fourmis Allides, que Branver (rgoo) pense avoir persisté dans quelques cas à travers les époques géologiques. Si l'on me permet d'employer le langage des psychoanalystes actuels, celui qui étudie la phylogénie ne peut que compenser son infériorité par rapport au biologiste expérimentateur ou statisticien, en épuisant el mème surmenant les maigres ressources méthodiques dont il dispose. Ces ressources, qui sont certainement moins limitées en entomologie que dans maint autre domaine, sont les suivantes :

$I^{\circ}$ Les documents paléontologiques. Ils sont nombreux et ont ćté récemment l'objet de révisions critiques et d'additions de la part d'entomologistes compétents, familiers avec les espèces vivantes roisines. Et, bien que ce qui reste de certaines périodes géologiques soit d'une maigreur déplorable, un nombre suflisant de formes ont été conservées pour donner une image cohérente de l'évolution de la plupart des ordres et de beaucoup de familles d'insectes ailés (Plerygogenea). Ces documents constituent. évidemment, les sources les plus précieuses pour celui qui s'occupe de phylogénie.

?. La morphologie comparée, c'est-i-dire l'anatomie comparée et l'ontogénie des insectes actuels.

$3^{\circ}$ I.a grande masse de documents taxonomiques ou biotaxiques, codlifiés et classés, et qui sont solidement fondés sur la morphologic externe des espècrs acluelles.

$4^{\circ}$ Les documents sur la distribution géographique, ou"chorologie, des espèces fossiles et actuelles.

$5^{\circ}$ L'éthologie comparée, ou comportement des formes vivantes (1).

(1) Sсиях:L (1922) a publié récemment un réquisitoire serré contre la phylogénie. Mais sa discussion révèle une connaissance singulièrement insuffisante de la technique actuelle de la phylogénie. 
Dans des conférences comme celles-ci, il est facile de présenter certains documents, surtout des faits relatifs aux fossiles et à la morphologie des formes vivantes. Mais il est impossible de présenter d'une façon satisfaisante, même à un auditoire de biologistes, les innombrables documents taxonomiques et chorologiques enchevêtrés, qui, évidemment, tirent toute leur force et toute leur signification en grande partie par leur accumulation. C'est à l'entomologiste spécialiste seul qu'il est possible d'évaluer ces documents. Essayer de citer un grand nombre de noms spécifiques et génériques à un public qui n'a jamais manié ni même aperçu les insectes désignés, c'est, dans les circonstances les plus favorables, provoquer la somnolence et, dans les plus défavorables, un véritable coma. J'ai peur de ne pouvoir écarter une masse considérahlc de celte matière soporifique. La présenlation de fails étholog̣iques se heurte également à des conditions défavorables. Bien que ces faits soient passionmants, ils ont actuellement une valeur secondaire. En effet, bien qu'il nous soit possible fréquemment d'inférer de la structure d'un organe sa fonction physiologique, nous passons sur un terrain dangereux, lorsque nous essayons d'inférer le comportement, qui implique l'organisme tout entier, de ses composants morphologiques. En outre, il faut tellement de mots pour donner une bonne description de faits éthologiques, surtout chez des formes si hautement spécialisées que les insectes sociaux, que ces faits se prêtent peu à être exposés oralement.

$$
\text { ** }
$$

Notre quatrième question concernait les causes ou les 
conditions générales de l'évolution chez les insectes sociaux, ou l'étiologie du processus, comme aurait dit HuxLEY. Elle se résout naturellement elle-même en un certain nombre de problèmes intriqués. Il est évident que l'évolution des insectes sociaux ne peut être séparée des problèmes de l'évolution organique en général, mais le seul comportement de ces insectes comme organismes sociaux suggère des problèmes d'intérêt plus spécial. Les insectes sociaux descendent, ce n'est pas douteux, de formes solitaires qui ont acquis leurs caractères fondamentaux de structure et de comportement en réponse à un milieu analogue à celui des insectes non sociaux. Mais le développement d'un mode de vie sociale permanente a créé un milieu nouveaư et exceptionnel. C'est une réponse multimillénaire continue à ce milieu qui a produit cer tains caractères nouveaux, qui ont pu modifier le patrimoine présocial physiologique, éthologique et morphologique acquis, se superposer à lui et quelquefois le masquer. La vie sociale, comme il serait facile de le montrer, si le temps le permettait, doit influencer profondément les activités organiques tout à fait fondamentales de reproduction, nutrition et protection, car toutes ces activités se règlent d'une manière très différente chez une espèce sociale comparée à une espèce non sociale. On a quelquefois exprimé des doutes sur le fait de savoir si l'homme montre des effets acquis de la vie sociale, puisque le langage lui-même doit être appris à tous les enfants. Mais la société humaine est quelque chose de très jeune comparée aux sociétés d'insectes les plus récentes.

Un autre problème plus général est suggéré par la société d'insectes ou la colonie considérée colmme un tout; celle-ci, comme je l'ai montré autre part (I9II), est 
si remarquablement analogue au corps d'un métazoaire considéré conıme un ensemble de cellules, ou, même à n'importe quel organisme vivant considéré comme un tout, qu'elle doit être soumise aux mêmes lois. Mais le biologiste, avec ses méthodes actuelles, est impuissant à donner une explication de l'organisme considéré comme un tout. Il ne peut faire appel à l'entéléchie, ou "élan vilal ", quel(que suggestives et satisfaisantes que de telles entités puissent être pour des philosophes.ı Il ne lui sert de rien, non plus, de dire qu'un essaim d'abeilles ou une colonie de fourmis ou de termites a une superentéléchie, une " âme de la ruche ", pour employer l'expression de Réaumur el de Mréterlinck, cette âme de la ruche étant conçue comme contrôlant les entéléchies des différents individus. C'est simplement une aulre façon de poser le prohlème, une façon très vague et illusoire. Nous pouvons seulement considérer le caractère organique de la colonie considérée comme un tout, comme un mode d'expression de ce fait qu'elle n'est pas équivalente à la somme des individus qui la constituent, mais qu'elle représente un " niveau émergent ", différent et actuellement inexplicable, dans le sens de C. Lroyd Morgan (i $\left.9_{23}\right)$, Sellars (i 922$)$, Parker (1924) et d'autres.

$$
\text { ** }
$$

Pour finir, j'énumérerai brièvement le sujet des conférences suivantes, que nous restreindrons aux insectes vraiment sociaux, c'est-à-dire, comme nous l'avons vu, à une seule grande subdivision des Hyménoptères, les Aculéates, et à l'ordre des Isoptères, ou Termiles. Dans la scconde conférence, j'ahorderai la question de la phylo- 
génie générale des Aculéates; de la troisiême à la sixième l'évolution plus spéciale des guêpes, des abeilles, des fourmis et des termites. La septième et la huitième scront. consacrées au problème de polymorphisme ou pléomorphisme, phénomène qui a résulté d'une division du travail poussée très loin, et impliquée dans la vie sociale. Jans la neuvième, je me propose de discuter les activités réciproques, ou intercommunications, des stimuli et de la nourriture (trophallaxie), chez les individus sociaux, et dans les dixième et onzième conférences les types variés de parasitisme qui se sont développés par suite de ces actions réciproques, à la fois chez les insectes sociaux euxmêmes, et entre eux et les insectes étrangers. Dans la conférence finale, j'indiquerai le cours probable du développement futur des sociétés d'insectes, ou leur destin sur une planète dont le balancement naturel des faunes et des llores est en train de se troubler rapidement sous l'influence d'un animal social plus jeune et plus puissant, l'Homme. 


\section{CHAPITRE PRENIER}

\section{L'ORIGINE DES TEREBRANTS \\ ET DES AGULEATES}

Sommare. -- Divisions principales el origine des llyménoptères. - Documents paléontologiques. - Phytophages, Térébrants : (Trigonalidès, histoire des Périlampidés, Eucharidés). - La forme primitive des larves d'Hyménopteres. - Nodes d'alimentation de la progéniture : formes atrophaplique, dystrophaptique, eutrophaptique.

A la seule exception des Termites, tous les groupes d'insectes vraiment sociaux, que nous avons reconnus tels dans notre dernière conférence, sont restreints non seulement à un seul ordre, les Hyménoplères, mais même à un sous-ordre unique, les Aculéates. Comme ces organismes sociaux dérivent indubitablement d'espèces solitaires, représentées, d'une façon plus ou moins étroite, dans la faune actuelle par des formes voisines, nous som. mes amenés à nous poser le problème de l'origine phỵlogénique de l'ordre des Hyménoptères daus son ensemble, et des Aculéates et de leurs onze types sociaux en pariiculier. On a accumulé, dans ces dernières années, tant de matériaux intéressants sur cette question, que cette conférence et les trois suivantes seront nécessaires pour les considérer même brièvement.

Les Hyménoptères constituent un ordre d'insectés sin- 
gulier, nellement caractérisé, et d'une élendue immense. Sclon Handemscri, on a déjà décrit 70.000 espèces el le nombre d'espèces existantes est estimé à 200.000. Il comprend plus de roo familles, renfermant plusieurs centaines de genres. Cet ordre est donc, tout de suite après les Coléoptères, le plus vaste de la classe des Hexapodes. Aucun de cenx qui se rendent compte de l'amplitude, de la complication et de la difficulté de la tâche, ne s'étonnera qu'un tel ordre ait été étudié de façon très inégale, bien qu'une armée d'entomologistes de valeur y aient consacré leur vie pendant plus d'un siècle.

Il n'avait pas échappé à ce merveilleux génie taxonomique que fut Latreille, que la division la plus naturelle de l'ordre des Hyménoptères était une coupure en trois sous-ordres. Il les appela Phytophages, Térébrants et Aculéates. Le premier comprenait les mouches à scie et les perce-bois, le second les espèces parasites, et celles qui produisent les galles, et le troisième les guêpes, les abeilles et les fourmis. Ces groupes ont persisté dans leurs grandes lignes jusqu'à nos jours, bien que masqués par l'introduction de noms nouveaux el par une conviction croissante, el, je pense, bien fondée, que les Térébrants et les Aculéates sont beaucoup plus étroilement unis entre eux qu'aux Phytophages ( $\mathrm{r}$ ).

(1) D'autres entomologistes ont appelé ce dernier sous-ordre Sessiliventres, Symphyles ou Chalastogastres, el ont employé les termes Pétioliventres, Apocrites ou Clistogastres pour les Térébrants plus les Aculéales. Je crois qu'il faut loucr Hannumscu d'ère revenu en 1924, dans le "Handbuch der Entomologie s) de Scurönfn, a la division ternaire et a la terminologie de LAtnetr.t. Mais l'entomologiste autrichien a toutefois ajouté un quatriène ordre, les "Pélécinoïdes », pour le seul genre américain Pelecinus, que l'on a toujours considéré comme un Térébrant aberrant. Ce n'est pas très heureux, et l'on peut appliquer 
Tous les auteurs sont d'accord pour placer les trois sous-ordres en série ascendante, les Phytophages étant le groupe le plus primitif et le plus près du type ancestral, les Aculéates le plus récent et le plus hautement spécialisé par sa structure et ses mœurs.

\section{$*^{*} *$}

Laissons de côté pour le moment les relations mutuelles des trois groupes et passons à la question de l'origine phylogénique des Hyménoplères en tant que groupe naturel. Jusqu'à une époque très récente, ce groupe se trouvait dans une position isolée, à cause de la morphologie des espèces et du manque de fossiles significatifs. Tandis que, de la plupart des autres ordres, on pouvait remonter, en suivant les documents paléontologiques, avec une probabifité plus ou moins grande, à des ancêtres Paléodictyoptéroïdes ou Protoblattoïdes, les Hyménoptères restaient suspendus dans le vide. Les vieux entomologistes, et même Ashmead ( 1896 ) plus récemment, avaient tendance à relier les Hyménoptères aux autres Métaboles supérieurs (Lépidoptères, Diptères, etc.). Mais HANDLIRSCH (r go8), dans son grand travail sur les insectes fossiles, entreprit de les faire dériver des Protoblattoïdes, et les plaça près des Coléoptères, qu'il supposait avoir un ancêtre similaire. Toutefois, on n'a généralement pas

le même jugement à l'essai récent de Rohwer (1917) et de BRADLEy (CoMstock, 1924), de faire un sous-ordre des Idiogastres, avec la vieille famille des Oryssidés, qui appartient évidemment aix Phylophages. On a promené des Térébrants aux Aculćates alternativement, un petit nombre de familles à caractères anormaux : les Trigonalidés, les Chrysidés, les Cleptidés, les Béthylidés et les Rhopalosomatidés. 
accepté ses arguments. Handurinscil passait en revue tous les matériaux paléontologiques, qui consistaient en quelque quinze espèces de Pseudosirex du Jurassique (Malm de Solenhofen; (Bavière) et Purbeckien inférieur d'Angleterre), un Térébrant unique (Ephialtites jurassicus, Meunier) du Malm de Catalogne (Espagne), et un grand nombre d'espèces apparlenant aux familles les plus diverses du tertiaire d'Europe et de l'Amérique du Nord Lés matériaux du tertiaire, mème les plus anciens, de l'Eocène et de l'Oligocène inférieur, monlraient que l'ordre s'épanouissait déjà à cette époque avec sa magnifique diversité acluelle, landis que les seize espèces citées du jurassique étaient les seules formes à qui l'on pùt demander quelque lumière sur la premièré hisloire des Hỵménoptères (I).

Tillyard (rgi 8 ), dans ses travaux imporlants sur le complexe des Panorpoïdes, diverge des vues de HaxDurrscir et incline à faire dériver les Hyménoptères de formes Mécoptéroïdes.

Les éludes récentes de Tillyano (1924) sur les fossiles permiens récoltés par l'Université Yale dans les schistes argileux de Wellington, près de Elmo (Kansas), ont projeté beaucoup de lumière sur l'origine probable des Hyménoptères. On a extrait quelques 4.000 spéci-

(1) L'espèce de Pseudosirex est très analngue anx Siricidés récents; elle montre la haute antiquité de cette famille, déja haulement spécialisée, de Phylophages perceur's de bois. L.'Ephialtites est indubitablement un vrai Térébrant, mais il est si mal conservé qu'on ne sait à laquelle de plusieurs familles actuelles le rapporter. Ce maigre matériel fossile ne nous donne, on le voit, aucune indication qui nous permette de faire dériver les Hyménoptères d'insecles plus primitifs. Il permet sculement de conclure que la première évolution de l'ordre esl antérieure au Jurassique. 
mens dinsectes de ce calcaire argileux tendre, qui représente le dépòt d'un lac d'eau douce (Duvian 192/, Tillyand I g24). Bien que les échantillons soient conslitués surtout par des ailes, ils sont souvent si bien conservés quion peut même y distinguer les couleurs.

Dans ce matériel, qui a déjà donné un ordre nouveau, les Protodermaptè es, un Palcodictyoptère particulier, I)unbaria fascialipennis Tilly, plusieurs Éphémérides, de petites blattes, de grands Prolodonates et de vrais Odonates, Tillyand a découvert les ailes de trois espèces et genres d'un ordre nouveau, quil appelle les Prolohyniénoplères. Il place dans deux sous-familles les trois especes Prolohymen permianus, Permohymen schucherti et Asthenohymen dunbari. Prolohymen est la forme la plus primilive; elle a une nervation alaire comparable a celle des ryélidés et rles Siricidés, parmi les Phylophages vivants. Toutefois, il y a de profondes différences, qui montrent non seulement que les Prolohyménoptères se relient étroitement aux Mécoptères et aux Névroplères, mais qu'ils n'ont aucune affinité avec les Protoblattoïdes, ou blatles primitives. Les deux paires d'ailes sont plus semblables et sans tornus ou termen, la paire postérieure étant seulement légèrement plus courte et plus large que la paire antérieure et n'étant pas reliée a elle pendant le vol. Le membrane alaire est rigide et translucide, comme chez les Hyménoptères vivants. Protohymen et Permohymen ont un stigma alaire distinct, mais cette formation manque chez Asthenohymen.

Les ailes décrites par Tillyano sont donc si évidemment intermédiaires entre celles des Mécoptères et celles des Hyménoptères actuels, que l'hypothèse de HaxdLrnsci sur l'origine protoblattoüde de ce dernier ordre doit être abandonnée et que nous devons placer les Hyménoptères à la tête de la série des Hexapodes, parmi les membres actuels du complexe panorpoïde: les Lépidoptères, les Diptères et les Mécoptères (Panorpes). Une telle position s'harmonise mieux avec les données ontogéniques, et annule le postulat malheureux de Haxd- 
Lriscr, que l'holométabolie se serait développée d'une façon autonome chez les Hyménoptères.

$$
* *
$$

Revenons maintenant à l'évolution à l'intérieur de l'ordre des Hyménoptères. Bornons nos remarques à une étude brève des trois sous-ordres et de leurs relations mutuelles. Handursci a certainement raison, quandi il affirme que les formes actuelles sont toutes si hautement

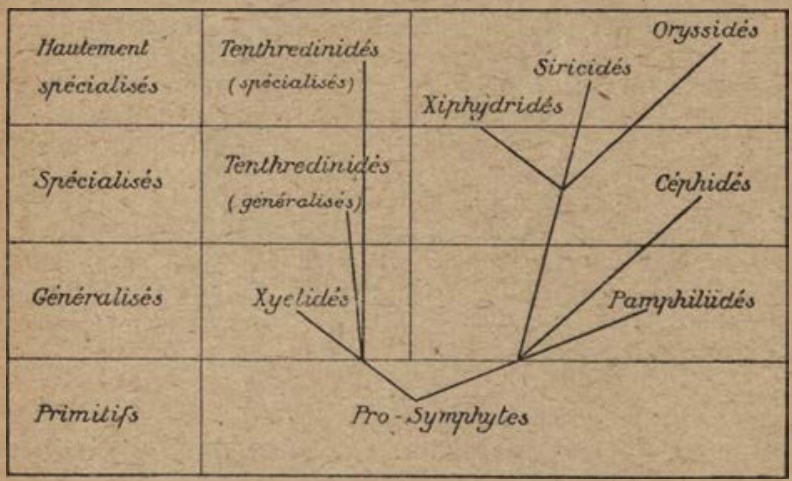

Arbre phylogénique, indiquant les affinités probables des diverses familles de Phytophages' (d'après YuAs.).

spécialisées dans des direclions particulières, que l'établissement de lignées phylétiques n'a que la valeur d'une spéculation. Le premier groupe à considérer, les Phytophages, ou Symphytes, est considéré unanimement comme le plus primitif On est de même d'accord sur les relations des familles dans ce sous-ordre. 
Les Pamphiliidés et les Xyélidés, mangeurs de fenilles, yeuvent être considérís comme les plus anciens non seulement de fous les Phytophages vivants, mais de tous les Hyménoptères vivants. A partir des Xyélidés, semble avoir pris naissance une serie, qui a conduit aux Tenthrédinidés el aux Cimbicidés; it partir des Pamphiliidés, une aulre série, -qui a mené aux Siricidés mangeurs de bois, aux . Xyphydriidés et aux Céphidés, et a la famille particulière des Oryssides, qui parait ètre un rejeton des Siricidés. L'allure de celle évolution, indiquée sur le tableau ci-joint de YUASA (1922), est confirmée par l'étude des larves comme des adultes. Les larves des Pamphiliidés sont considérées par Havolınsch comme les plus primitives des larves dHyménoptères (1). Elles sont cylindriques, avec trois paires de pattes thoraciques bien développées et une paire de cerques, mais sans fausses pattes abdominales (2).

Enfir, chez les Oryssidés, tous les appendices thoraciques et abrominaux ont disparu, et la larve est vermiforme comme celle d'un Térébrant. Celle condition s'explique par le fait que la larye d'Oryssus, comme l'a récemment montré Bumke (1917), parasite la larve de Coléopteres buprestides variés (voir également Rohiver et Cushmin (1917) et Comstock (1924).

Chez les Phytophages, on voit apparaître sporadiquement certaines particularités éthologiques qui s'accentuent et deviennent plus largement représentées chez les Térébrants et les Aculéates. Ainsi le parasitisme des Oryssus est le seul cas connu dans le sous-ordre; il annonce clairement l'existence universelle du phénomène chez les Térébrants. Bien que tous les Phytophages soient végétariens à l'état de larves, les adultes de quelques-

(1) Pour de bonnes éludes dı mode de vie des Pamphiliidés voir II. Schmint (1910) el II. C. Sirmin (1920).

(2) Toutefois, Yussi (1922) considère coinme l'archétype une larve éruciforme, avec dix paires de fausses pattes abdominales, type dont s'approchent les ryélidés et les Tenthrédinidés. Chez les Céphidés (Mundeton, 1917), Xiphydriidés el Siricidés (Yuts.t) perceurs de bois, les fausses paltes addominales disparaissent et les pattes thoraciques sont très réduites: Toutefois, les larves de Céphidés conservent yeux et paltes. 
unes des mouches à scie, spécialement des genres Tenthredo et Tenthredella (Mrazer igog, Rohwer igi3, el d'autres), sont franchement entomophages. En outre, la parthénogenèse, très fréquente chez les Térébrants et les Aculéates, paraît être bien établie chez les Tenthrè$\operatorname{des}(\mathbf{I})$.

Des mœur's grégaires et subsociales sont fréquentes (hez les: Tenthrédinidés, surtout durant les stades de larve et de pupe (2).

Tandis qu'on distingue facilement des Phytophages les Clistogastres, qui comprennent les Térébrants et les Aculéates, par leur abdomen pétiolé, la nervation plus ou moins réduite de l'aile, les larves tout à fait vermiformes, et les différences de mœurs, ils est beaucoup moins facile d'établir une ligne de démarcation netle et tranchée entre les Térébrants et les Aculéates. Un des

(1) Les mâles d'un grand rombre l'espèces sont beaucoup plus rares que les femelles. Selon Mac Gillivray (1914), von Sicbold a trouvé qu'on ne connaissait les mâles que d'un cinquième des espèces d'Allemagne, el Camenon ne put reconnaítre les måles que d'un tier's des espèces d'Angleterre. Quand la parthénogenèse survient, elle est probablement mixte ou indéterminée, puisque, chez quelques mouches à scie, les œufs non fécondés ne produisent que des mâles (arrhénoloquie), chez d'autres que des femelles (thélyloquie), et chez d'autres encore les deux sexes (amphéroloquie). Aulant qu'on sache, à peu d'exceptions près, les œufs non fécondés des Térébrants et des $\Lambda$ culéates produisent seulement des inales.

(2) Voir, pour une partie de la bibliographie du sujet, mon livre "Social Lile among the Insects ", 1923, p. 290-292. Les travaux de Cuntis (1845), F. Smitn (1866) et Dicke (1916) contiennent aussi des études et des figures intéressantes des cocons sociaux de mouches à scie brésiliennes du genre Dielocerus. Les singulières larves sociales observées par Mann et par moimême à la Guyane anglaise et en Bolivie (Wheeler et Masix, 1923), peuvent aussi appartenir à ce genre. 
meilleurs caractères distinctifs réside dans le fait que la tarière possède chez les Térébrants des apophyses séparées et est cmployée pour déposer les œufs dans ou sur d'autres insectes, ou dans des tissus de végétaux. Chez les Aculéales, au contraire, la tarière a ses apophyses soudées et est modifiée pour former un aiguillon, organe d'allaque ou de défense et non de ponte (I).

Les hyménoptéristes sont d'accord pour considérer que les Clistogastres dérivent des Phytophages primitifs et pour penser que les Térébrants sont plus intimement alliés aux Phytophages que ne le sont les Aculéates. Mais l'accord cesse quand il s'agit de savoir quelle est la famille des Térébrants qui est la plus primitive, et si les Aculéates descendent des Térébrants, ou tirent indépendamment d'eux leur origine directement des Phytophages. Hınmirnscir (rgo8) considérait les Ichrieumonides comme le groupe de Térébrants le plıs primitif, celui qui aurait donné naissance aux Aculéates. Brues (rg2I) rejette cette conception, en tant qu'elle concerne l'origine des Térébrants et considère les Stéphanidés comme la famille qui se relie le plus naturellement aux Phytophages. Il souligne aussi l'association singulière des familles de Térébrants primitives avec des insectes perce-bois. C'est ce qu'on observe chez les Oryssidés parmi les Phytophages. Le fait est visible également dans

(1) Il y a toutefois des exceptions. Ainsi les Sapygidés, que l'on place parmi les Aculéates, emploient la tarière comme les Térébrants, tandis que les Trigonalidés, que la plupart des hỵménoptéristes placent dans les Térébrants, ont la tarière tellement réduite qu'elle ne peut pas être utilisée dans la ponle. On connait également un certain nombre d'Aculéates (Chrysididés, un bon nombre de fourmis et quelques abeilles) chez lesquels, de mêne, l'aiguillon est vestigial.

SOCIÉTÉS D'INSECTES 
le groupe plus généralisé des Evaniidés, les Braconidés les plus primitifs, beaucoup d'Ichneumonidés de struclure primitive et chez les Capitoniidés.

A l'exception de l'Ephialtites du jurassique, dont nous avons parlé, tous les Térébrants fossiles connus sont du tertiaire. (On ne connait qu'un petit nombre d'espèces de l'éocène, mais on en a trouvé beaucoup, représentant des genres et des familles nomhreuses, dans l'ambre de la Baltique, d'àge oligocène inférieur et dans les schistes argileux d'OEningen, Radoboj et Florissant, que l'on rapporte au Miocène. Les fossiles de l'ambre et ceux de Florissant ont été éludiés d'une manière critique par BRUEs dans une série de travaux (1906, 1908a, 1910a, 1910b, 1923). Sa liste des Térébrants tertiaires connus, publiée en 1910b, comprend 212 espèces réparties en 101 genres el 21 familles, matériaux suffisants pour donner une idée convenalo'e de l'étendue et de la diversité du sous-ordre pendant le Tertiaire ancien et moyen. Les espèces diffèrent toules de celles qui vivent actuellement; elles appartiennent toutefois pour la plus grande partie à des genres actuels bien connus el toules les familles lertiaires connues existent encore aujourd'hui. Pourtant, quelques-uns des genres disparus sont particulier's, ou uniques. Kinsey (1919) a décrit trois espèces dı genre primitif de Cynipides Aulacidia, de l'ambre de la Baltique, el Cockenfic (1915, 1916a, 1920a, 1921a, 1921b) a décrit un certain nombre d'autres Térébrants du Niocène de Florissant et de l'Oligocène supérieur de l'Ile de WVight, et un petit nombre de formations encore plus anciennes, l'Eocène de Green River. Il rapporle ces derniers à deux genres d'Ichneumonicles et à un genre de Braconides. Mais tous les genres et toutes les espèces tertiaires décrites jusqu'à présent sont absolunent aussi spécialisés dans leur structure et étaient probablement aussi spécialisés dans leurs mœurs que leur's congénères actuels. Ils ne jetlent donc aucune lumière sur la phylogénie des familles acluelles de Térébrants et d'Aculéates.

On applique souvent aux Térébrants le nom de "Parasites "), parce que, à l'exception des Cynipides et de certains Chalcidoïdes qui s'attaquent aux plantes, ils déposent tous leurs oufs sur ou dans diautres insectes, dont les tissus servent de nourriture à leurs larves. Le sujel 
demande à être examiné brièvement, car on a souvent prétendu que le mot de "parasites ") appliqué aux Térébrants, à beaucoup de Diptères et à quelques autres insectes qui ont des mours analogues, est impropre. Ces insectes diffèrent des parasites proprement dits de trois points de vue. D'ahord, ils attaquent d'autres insectes, et les vrais parasites, à l'exception de quelques Crustacés et de quélques Infusoires, n'attaquent pas des membres de leur propre groupe. En second lieu ils causent presque toujours la mort de leur hôte. Enfin, ils ne vivent avec leur hôte que pendant le stade larvaire tout au plus nymphal, et, quand ils sont adultes ils ne présentent aucun effet " dégénératif " de l'association (parasites protéliens de CaulI.ERY (I 922). Ce sont, par conséquent, des prédateurs très raffinés.

On a essayé de matérialiser cette distinction par un mot. On pourrait accepter " pseudoparasitisme » s'il n'avait pas été employé par R. Blanciand dans un autre sens (1889).

RuLEY, en 1893 , a clairement reconnu le besoin d'un terme différent quand il appelait les Ilyménoptères et les Diptères entomophages "parasites mortels ), pour les distinguer des "parasites vrais " et des "parasites inquilins ». O. M. Reuter (1918, p. 53) a introduit le terme " parasitoüdes" pour ces prédateurs particuliers, el moi-mème (1923) et plus récemment Roor (1924) avons employé ce terine. II est probable, cependant qu'on ne l'adoptera pas et que "parasile " continuera a être employć. Si l'on sous-entend clairement la distinction, il n'y a aucun inconvénient à en faire usage. Remarquons pourtant qu'il a nalurellement amené des confusions dans les discussions phylogéniques, particulièrement dans le cas de l'opinion de HandLunscr, qui fait dériver les Aculéates d'ancêtres Térébrants.

Le phylogéniste est, naturellement, si convaincu de la haute spécialisation adaptative de lous les parasites qu'il se garde de s'en servir comme d'ancêtres hypothétiques. 
Mais cette objection ne tient pas dans l'exemple de HavnLiRsch, si nous considérons les Térébrants comme une classe particulière de prédateurs, ou de parasiloödes, surtout puisque les Aculéates inférieurs ont les mêmes mours. C'est ce qu’à bien clairemenl étahli F. Picino, dans son solide traviail sur les Insectes du figuier (ı!) r!̣, ए. 167$)$.

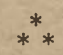

Il ne me sera pas possible de discuter tout au long les nombreuses modifications extraordinaires que montre l'ontogénie des Térébrants, par exemple la polyembryonic, si soigneusement étudiée dans ces dernières années en France, en Italic et aux Etats-Unis. Toutefois, le phénomène Je plus fréquent de l'hypermétamorphose demande à être examiné partiellement, dans une famille particulière et très primitive, les Trigonalidés. Au sujet des affinités de celte famille, les hyménoptéristes manifestent la plus grande diversité d'opinions. Quelques-uns (Westwood, Ashmead, Schmiedeknecht), considèrent ces insectes commé des Aculéates primitifs, tandis que d'autres (Börner, Haxdlirscir, Bradley, etc.) les considèrent comme des Térébrants primitifs. Des attributions si différentes montrent que la famille doit se placer près du point de bifurcation à la fois des Térébrants et des Aculéates, à partir du tronc phỵtophagien ancien de l'ordre des Hyménoptères.

W. A. Scrulz, qui a fait en 1907 une monographie des Trigonalidés, a reconnu quarante-deux espèces, réparties entre dix-sept genres et cinq sous-familles. Ils ont. une distribution cosmopolite. Mais les espèces sont toutes 
très rares et locales et apparemment en voie d'extinction. Ils sont plutòt petits, souvent vivement marqués de points ou de bandes. Leur aspect est très archaique, montrant III alliage déconcertant de caractères de Phytophages, de Térébrants et d'Aculéates ( 1 ).

On connait Irès imparfaitement les mours des Trigonalidés, el, comme j'essaierai de le montrer, on ne les a pas inlerprétees correctement. Ces insectes se rencontrent d'ordinaire volant près du sol et prenant le soleil sur la végétation basse, a des endroits oủ les guêpes sociales sont communes. Ces documents, el ceux qui ront suivre ont conduit les auteurs a les cull-idérer comme parasites dans les nids des Vespides. $\Lambda u$ Brilish Vluseum, il est sorti diun nid de Polisles canridensis qu'on seconait, un specimen unique de Seminola depressu, espèce sudamericane; el, all Nuséum de Paris, d'un nid de Paracharlergus apicalis, un spécimen de S. mejicana. G. W Tarlon (1898) prit vingt-trois màles et quatre femelles de Bareo!/onalos canadensis sur des plantes a quelques pierls des entrées d'un nid de Vespa occidentalis. Il en deduisit que les parasites devisent s'être développés dans ce nid. Schulz (1905) a décrit le màle de Nomadina cisandina d'après un spécimen capturé pur (O. Dreher dans un nid de Polyjbia dimidiata, à Sào-Paulo (Brésil). De ces fails, Schutry conclut que les Trigonalidés doivent en-

(1) Lialenne mulliarticuléc et le nombre de segments abdominaux sont lérébrants, mais la tête rappelle les mouches à scie, la forıne générale du corps, les Béthylidés, et la forme de labdomen, les Mutillidés. Dans quelques espèces (Bareogonalinæe el Vomadinina), la portion antéricure de la tête se prolonge en museau, comme celle a'un certain nombre de Coléoplères archaïques et celle des Mécoptères. Il y a des cerques, mais l'oviscapte est réduit à un vestige minuscule. BönNen (1919) découvrit que les máchoires possédaient des appendices paraglossaux bien développés et qu'elles étaient beaucoup plus primitives que celles des alltres lérébrants. Il créa pour la famille une section spéciale, les "Archiglossala ". La nervation alaire est très primitive, el ressemble beaucoup a celle des Aculéates, en ce qu'elle possède trois cellules cubitales ferinées. Les tarses on des vésicules plantaires hien développées. Ces formations, dit Börner, manquent chez les Térébrants, mais se rencontrent souvent chez les Aculéales. 
Irer dans les nids de guêpes et pondre sur la couvée. Il inlerpréta mème les articulations dilatées de leurs palpes comme des adaptations spéciales destinces à leur faire trouver leur chemin dans le dédale obscur des nids.

Deux observations, loulefois, montrent que les Trigonalidis peuvent avoir occasionnellement des hôtes différents. Dès 1891, Riley et Howard obtinrent Tapinogonalos pulchella, de l'Amérique du Nord, de la pupe d'une mouche Tachinaire (Exorisia lobeliæ), qui vit dans la chenille de Acronycta lobeliæ, et en 1909, Bıscuorf a élevé un spécimen de l'espèce nord-américaine Lycogaster pullala à partir du cocon d'un Ichneumonide, Ophion macrurus, parasite lui-mème de la chenille de Telea polyphemus. Dans ces deux cas, donc, les Trigonalidés se conduisaient comme des hyper-parasites. Outre le fait que les pupes de ces insectes sont nues, c'est-à-dire non enveloppées d'un cocon, les faits que nous avons exposés constituent lout ce que nous savons de leurs mœurs. Bugniox (1910), a publié un travail sur l'anatomie de l'espèce européenne Pseudogonalos hahni, sur laquelle je reviendrai, et Cochenerc $(1916 \mathrm{~b})$ a décrit un Trigonalis ( $T$. pervelus) de l'ambre de Burma, que l'on suppose être d'âge tertiaire.

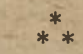

Je pense maintenant qu'on peut, en faisant une revue brève de ce que nous savons du développement des Eucharidés et Périlampidés, familles de Térébrants, projeter quelque lumière, non seulement sur les Trigonalidés, mais aussi sur quelques points obscurs du développement ontogénique des Hỵménoptères en général.

I. $y$ a quelques dix-huit ans ( $190^{-7}$ a), j'ai trouvé, dans les nids d'une fourmi dı Texas (Pheidale instabilis), un Eucharide d'un vert métallique (Orasema viridis) qui, si j'en juge par des dissections de la femelle, produit un nombre énorme d'neufs minuscules. Je n'ai pas vu les œufs après la ponte, mais seulement. les larves primaires, minuscules el actives, longues d'environ $o^{\mathrm{mm}} \mathbf{r} 6$, d'une couleur foncée, avec une face dorsale fortement chiti- 
nisée, une lête bien développée, |reize segments postcéphaliques et une paire d'appendices (cerques) à l'extrémité postérieure. Ces larves, que j'ai appelées plus tard "planidia ", s'attachent elles-mèmes au cou des larves et
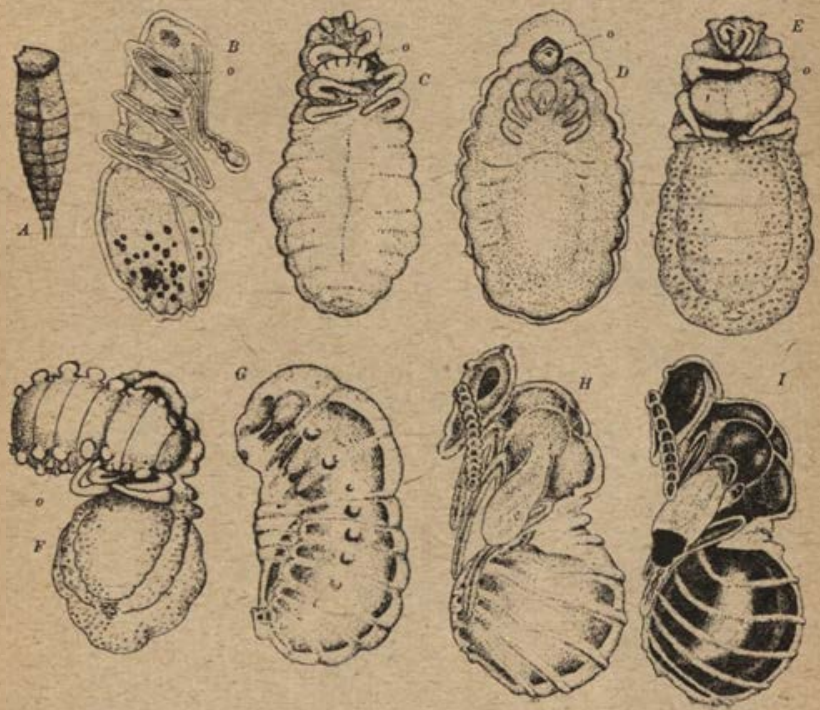

Fig. 2. - Développement d'Orasema viridis: A, premier stade larvaire; B - F, larves d'Orasema o, à des stades divers, aftachées au cou de pupes de Pheidole instabilis. (La pupe $F$, domnera une femelle phthisogyne); G. - I. Stades de pupaison d'Orasema.

des nymphes de fourmis el, apress sitre nourries el avoir mué, passent à un stade comparable à la larve ordinaire des IIymenoptires, vermiforme et lourde. Quand elles ont achevé toute leur croissance, elles subissent la nym- 
phose in silu, sans lisser de cocon, tombent de leur hòte, et, bientòt après, sortent, à l'élat d'Orusema adultes, qui sont transportés el nourris par les fourmis, mais peuvent éventuellement sortir du nid et passer dans le monde extérieur.
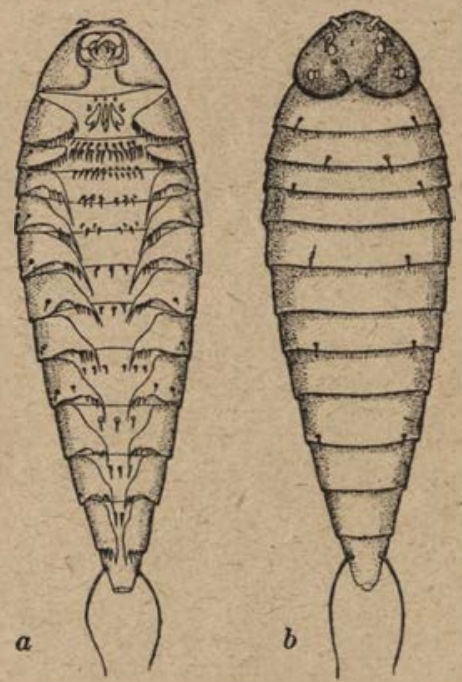

Fig. 3. - Planidium de Perilampus hyalinus; a, face ventrile; $b$, face dorsale (Gir. 2īo environ). (d'aprés HI. S. Sмттн).

H. S. Smitu a fait faire ultérieurement un pas notable à notre connaissance du planidium et de son développe-

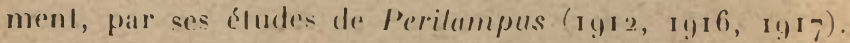
Dans son premier travail, il montra que le planidium de $P$. hyalinus se rencontre quelquefois rampant à la surface de chenilles fileuses d'automne (Hyphantria textor); 
et qu'il se fraic une voie à travers la chenille. Il ne subit alors aucun changement jusqu'à ce qu'il rencontre, à J'intérieur de l'hòte, la larve d'un de ses parasites com-
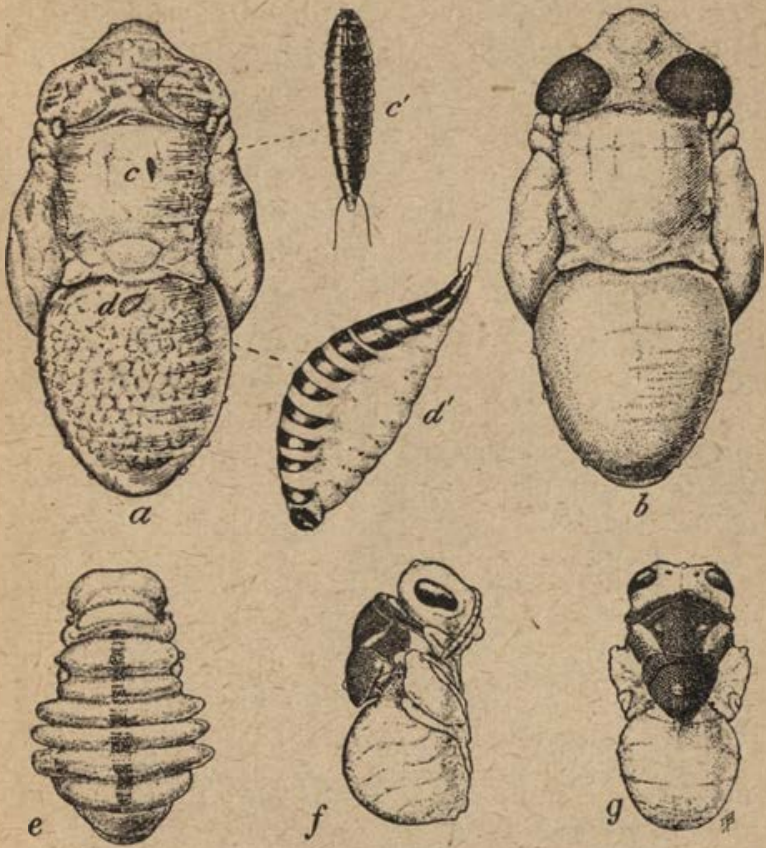

Fig. 4. - P'upe de Varichiela aldrichi; a, parasitée par Perilampus hyalinus; b, non parasitée; $c, d$, planidia (plus grossis en $\left.e^{\prime}, d^{\prime}\right) ; e$, larve avancée, $f-g$ pupe de $P$. hyjalinus (d'apre- II. S. Simru).

muns, c'est-à-dire, ou bien le Tachinide Varichnela aldrichi, ou bien l'Tchneumonide Lumtnerium validum. Dans le 
cas où le planidium rencontre un asticot de Varichoetn, il pénètre à son intérieur, même s'il est Irès petit, et ne bouge plus jusqu'à ce que l'asticot se change en pupe. Si c'est une larve de Limnerium qu'il rencontre, le planidium y pénètre de même et reste inactif jusqu'au printemps suivant. Varichœta subit la nymphose en automne, de sorte que le planidium y effectue sa sortie dans cette mème saison et hiverne à l'extérieur. Limnerium validum hiverne à l'état de larve et se métamorphose au printemps. Le planidium reste donc, dans ce cas, interne jusqu'au printemps, époque à laquelle il sort au moyen de ses mandibules efficaces et de sa tête épineuse, et achève son développement précisément de la même manière que les individus qui avaient choisi des Varichota pour hôtes. Le développement ultérieur du planidium de Perilampus sur les parasites de la chenille fileuse est tout à fait semblable à celui d'Orasema: le minuscule parasite, après s'être nourri, donne extérieurement une larve vermiforme avant la nymphose. Ainsi Perilampus hyalinus est un hyperparasite. On l'a élevé, lui et d'autres espèces du genre, à partir de plusieurs autres parasites de chenilles, dont Sirtir donne une liste. Mais on connaît des cas de Perilampus élevés à partir d'autres insectes (Chrysopa, Anthonomus grandis et les parasites Sarcophagides des sauterelles). En 1915 , W. R. Tномpson a publié une étude de l'anatomie interne d'un planidium indéterminé, qu'il a trouvé sur des chenilles de Nochuelle qui se nourrissaient dHamamelis virginica, à Ithaca, New-York. En 1923, Norma Ford a décrit un planidium de Perilampus qu'elle a trouvé attaché à une sau. terelle de prairie (Conocephalus fasciatus). Dans ce cas, l'hôte vrai est probablement la larve d'une Sarcophaga, 
cspèce que Swenk (IgII), Kelley (IgI4) et d'autres, ont montré être parasites de sauterelles.

Dans ses travaux de 1916 et 1917 , Sмтти a aussi résolu le problème de l'endroit où la femelle de Perilampus pond ses œufs. Après avoir élevé $P$. chrysopa à partir de cocons de Chrysopa, il a réussi à observer la ponte du parasite. Les œufs sont pondus simplement sur les feuilles, près de colonies de pucerons infestées de larves de Chrysopes. La femelle dépose environ cinquante de ses œufs minuscules en un seul jour. "L'œuf est très faiblement attaché à la surface de la feuille par un de ses bouls. L'éclosion a lieu au bout de sept à dix jours, et le premier stade larvaire est du type planidium décrit dans le travail indiqué plus haut (1912). Pendant plusieurs jours avant l'éclosion, le planidium, de couleur foncée, se distingue parfaitement à travers la coque transparente de l'œuf. Le planidium est actif immédiatement après l'éclosion, rampant rapideınent de-ci, delà. Bientôt, il s'attache à la feuille par sa ventouse caudale et se dresse à angle droit sur celle feuille. Dans cette position, i. demeure, pendant des journées entières, immobile, excepté quand quelque insecte vient à sa portée. Il se prend alors d'une activité frénétique, s'allongeant, se balançant dans toutes les directions, pour essayer de s'altacher à l'hôte en perspective (la larve de Chrysope). Si cette (lernière a le malheur de trop s'approcher, le planidium s'attache avec la rapidité de l'éclair à un poil ou à une soie de l'hôle. Il passe ensuite à loisir des poils au corps de l'hôte et s'y attache par ses crochets buccaux. Il est très fréquent qu'on trouve les planidia attachés au pédoncule de l'œuf de Chrysope, dans une position perpendiculaire à ce pédoncule. Ceci montre un instinct iritéressant du planidium; il peut barrer la route, et effectivement le fait, comme l'auteur l'a observé, à la jeune larve de Chrysope, quand elle quitte l'œuf et rampe le long du pédoncule. La préscience de la mère Chrysopa, qui place ses œufs à l'extrémité d'un long pédoncule pour parer au penchant cannibale de ses larves est ici sa perte, car le planidium, attaché au pédoncule de l'œuf de Chrysope, est sủr d'alteindre son hôle, alors que ceux qui se trouvent à la surface de la feuille peuvent aussi bien s'accrocher à un Aphide, ou à un autre insecte $»$. SMuLyan (1916) a, en fait, trouvé un planidium de Perilampus attaché à l'antenne d'un Aphide. En étudiant le développement ultérieur de $P$. chrysopx, Sмıти a trouvé que le planidium reste accroché à la larve de Chrysopa sans se nourrir, jusqu'à ce que celte dernière soit prêle à se 
changer en pupe, bien qu'occasionnellement il puisse être délaché de l'hôte par les mues. Après que la larve de Chrysope a filé son cocon et a subi la nymphose, le planidium commence a se nourrir et se développe à la manière déja décrite de P. hyalinus.

On a fait, dans ces dernières années, d'autres observations intéressantes et importantes sur les Eucharidés. Ce sont tous, comme Orasema, des parasites des fourmis.

En I89o, Forel a trouvé un Eucharis my'rmecine adulte dans le cocon d'une fourmi bull-dog d'Australie (Myrmecia forficuta). Brues, plus récemment (1 gra a), a décrit le planidium, les stades larvaires avancés, el la "ymphe de Psilongaster fascuiventris, trouvés par Tillyarn sur des nymphes d'une aulre fourmi bull-dogg, Myrmecin gulosa. G. H. et E. H. Wheeler ont, en 192!, décrit Schizaspidia polyrhachicida, des cocons d'une fourmi des Philippines, Polyrhachis (Myrmhopla) dives.

Mais les observations les plus complètes, et qui comblent à peu près complètement les lacunes de mes observations sur Orasema, ont été faites par Cuausex (r923) sur Schizaspidia tenuicornis, qui parasite la fourmi charpentière japonaise commune, Camponolus japonicus.

A la fin d'août, on trouva $S$. tenuicornis insinuant par paquets des œufs minuscules dans les bourgeons des divers arbres (Morus alba, Castanea sativa, Cladrastis amurensis el floribunda). L.e nombre des œufs déposés par une seule femelle variait de 940 à 1.230 ; en moyenne, il élait de 1.050 . Plusieurs femelles peuvent pondre dans un seul bourgeon, et il peut $y$ avoir un tel nombre de bourgeons utilisés, que nous avons calculé qu'un seul buisson pouvait porter 4.320 .000 œufs ! II y a, parmi ces œufs, une grande mortalité, qui est due à ce que, dans certains cas, les écailles des bourgeons lombent, exposant les œufs à l'humidité, aux moisissures, elc... Le planidium se développe dans l'œuf pendant l'hiver et il éclot en juillet. I] a, à peine plus de $0^{\infty}, 1$ de longueur et est capable de mouve- 

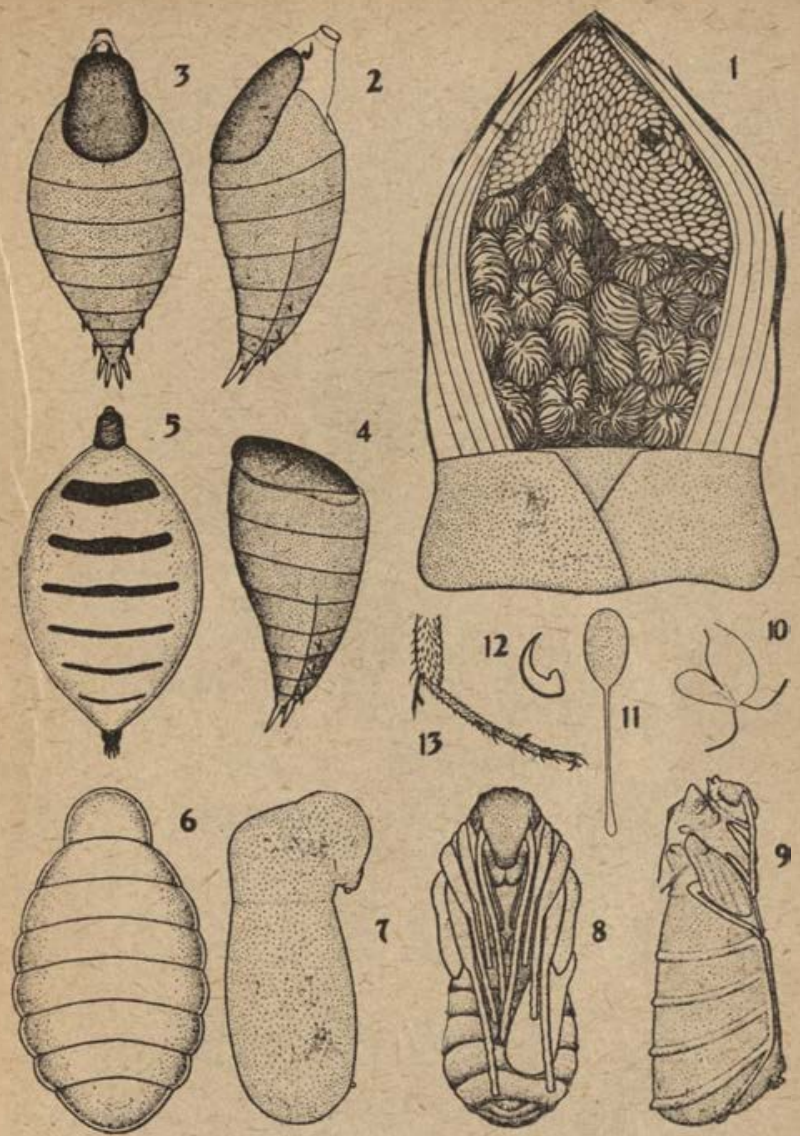

Fig. 5. - Développement de Schizaspidia tenuicornis, Ashm, 1 , bourgeon de mûrier ouvert, pour montrer en place deux paquets d'oeufs; $2-4$, première lârve (latéralement, dorsalement et la tête rétractée) (Gr. 400); 5, première larve, stade avancé vue dorsalement (Gr. 160). 6, - 7, $2^{\circ}$ et $3^{\circ}$ stade larvirires, $8,3^{-}$stade, en place sur un male de Cimponotus; 9. fupe $q ; 10$, Coupe sagittale des pieces buccales; 11, auf ovarien; 12 , mandibules (1er slade); 13,3 larves (1 er slade) sur une patte d'une ouvrière de Camponotus. 
ments de flexion, mais assez limités, grâce à sa bouche qui fait ventouse et à un groupe de fortes épines qui, à l'extrénité postérieure du corps, remplacent les cerques des autres planidia. Les larves se fraient un chemin hors du bourgeon et déambulent sur les branches et les feuilles. Là, elles arrivent
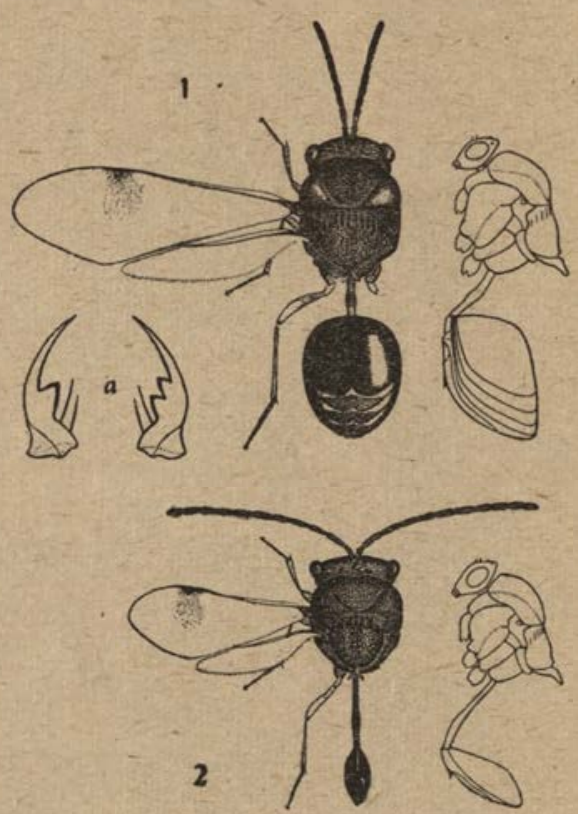

Fig. 6. - Schizaspidia tenuicornis, Ashın. 1, femelle adulte (face dorsale et prolil) $a$, mandibule ; 2 , mâle adulte (face dorsale et profil).

au conlact dés ouvrières de Camponotus, qui visitent les pucerons et recherchent les fruits des arbres, et elles s'atlachent aux poils des tarses des fourmis. C'est ainsi qu'elles se font charrier dans les nids de celles-ci. Là, elles passent de leurs 
porteurs au couvain des fourmis. "Si la chance les favorise, elles parviennent à s'altacher aux larves des fourinis. Après setre promenées quelque temps sur tout le corps, elles finissent par se lixer, dorsalement, i l'une des articulations soit de la lète el du premier segment thoracique, soit des deux preniers segments du thorax. Elles enfoncent alors solidement les inandibules dans le derme de l'hôte, puis ne font plus aucun mouvement. " Ainsi se passent deux stades, pendant lesquels le plánidium se nourrit el devient vermiforme. Après que l'hôte a filé son cocon et, ayant mué, est devenu une semi-chrysalide, ou même une chrysalide, le parasite reprend sa prise. Le point d'attache à la nymphe de fourmi est presque invariablement situé sur le métathorax, jusle au-dessous des pattes ou des bourrelets alaires. Le développemeitt, maintenant, s'accélère beaucoup et la nutrition s'achève ell quelques jours après la deuxième mue. La nymphose a lieu a l'intéricur du cocon de l'hôle, et les adultes, quand ils apparaissent dans le nid, ne sont pas nourris el soignés par les fourmis, comme c'est le cas pour Orasema. Ils sortent du nid, et l'accouplement a lieu à l'extérieur. Les mâles flànent aulour des nids et saisissent les femelles, dès qu'elles arrivent à la lumière, avant même qu'elles puissent prendre leur vol. Lo vie de l'adulte de $S$. tenuicornis est extrêmement courte, comme le montre le tableau de Cunsin, que nous reproduisons ici :

Stade $œ u f \ldots \ldots \ldots \ldots \ldots \ldots \ldots \ldots$.

jer stade larvaire.

$2^{e}$

$3^{\ominus}$

Stade nymphe.

Adulte (à l'intéricur du cocon).....

Adulle (hors du nid)..............
11 mois.

- 20 jours.

3 jours.

4 jours.

6 jours.

1 jour.

2 à- 3 jours.

Dans mon travail de 1907 , je considérais comme assuré que la femelle de Orasema viridis devait pondre ses œufs dans le nid des fourmis. Mais Swith et Clausen ont donné des raisons de penser que cet Eucharide pond prohablement ses œufs sur les feuilles des buissons, comme Perilampus, on dans des hourgeons, comme Schizaspidia. Toutefois, nous appelons l'attention sur le fait qu'Or'isema est in hôte des fourmis bien plus que Schizaspidia, puisque les ouvipies de Pheidole les nourrissent réellement, !es entourent de soins et les véhiculent. En outre, en novembre 1923, le Dr W. M. Mixi a mis la main sur une autre espèce d'Orasema, dans les nirls d'une petite Pheidole de la Basse Californie, et il a vu, dans les chambres 
de ces nids, cinq des mâles réellement accouplés avec des nymphes de femelles. Celte observation semble bien indiquer, sans le prouver évidemment, que les œufs dorasema sont pondus dans les nids des fourmis.

$$
\text { ** }
$$

Nous pouvons, après cette revue du développement compliqué des Périlampidés et des Eucharidés, tel que l'ont établi les entomologistes américains, revenir aus Trigonalidés. Notons que le fait que ces insectes se rencontrent très rarement, et d'une façon sporadique à l'état adulte comme à l'état larvaire, et que la larve esl, soit parasite, soit hyperparasite d'hôtes très variés, amène à penser à un développement qui débute par une larve primaire petite et active, comme celle des Périlampidés et des Eucharidés. Ceci devient presque cerlain lorsque nous considérons des observations anatomiques de $\mathrm{Bu}$ gNion sur la femelle de Psendogomalos hahni, espèce européenne. Il dit: " La femelle n'a ni tarière, ni aiguillon. On remarque seuTement, à l'entrée du conduit génilal, quelques petites pièces chitineuses, qui, dans l'acte de l'accouplement, sefvent probablement à maintenir le pénis 1 . Et sa description de chaque ovaire montre qu'il se compose de 300 à 400 ovarioles grêles, contenant chacume une série d'ueufs très petits, ayant sculement $\mathrm{o}^{\mathrm{mm}}, \mathrm{I} 8$ de long. Si nous comptons 5 œufs par ovariole, comme l'indiquent ces figures, nous voyons qu'une seule femelle doit produire de 3.000 à 4.000 œufs ! Bugnion ne paraît. pas avoir remarqué la signification de cette fécondité extraordinaire, beaucoup plus grande que celle de Schizaspidia, dı Japon, décrit par Cunusex. Lorsque nous considérons la grande rareté des Trigonalidés à tous les 
stades, nous ne pourons que conclure à un gaspillage d'œufs énorme, et ce gaspillage ne peut résulter que d'un mode de ponte et de développement tel que celui que nous avons décrit chez les Perilampida et les Eucharida. Les petits œufs sont probablement pondus sur les feuilles des buissons ou des herbes. Les larves qui en sortent sont probablement très semblables à des planidia, et doivent s'attacher aux guêpes et aux chenilles qui passent. C'est ainsi qu'elles arriveraient au couvain des guêpes et aux parasites hyménoptères ou diptères des chenilles.

Le premier stade larvaire des Trigonalidés, quand il sera connu, aura certainement un grand intérêt, car, si nous en jugeons par l'allure archaïque de l'adulte, il peut fort bien être d'un tỵpe plus primitif et plus généralisé que les planidia des Chalcidoïdes que nous avons décrils. Peut-être a-t-il effectivement des pattes thoraciques et est-il, ainsi, un vrai triongulin ! La ressemblance frappante, à la fois de structure et de comportemenl, qu'il y a entre le planidium des Hyménoptères, la larve triongalin des Coléoplères Méloïdes et Rhipiphorides et les Strep. siptères a élé remarquée par plusieurs observateurs (I).

Il y a, au surplus, à la fois chez les Térébrants et les Diptires, un certain nombre de premières formes larvaires, qui ressemblent à des planidia et d'autres, comme les larves cyclopoïdes et eucoïliformes de certains Platygaslridés et Cynipoïdes, qui paraissent représenter des modifications particulières du type planidial. Iracuandson ( 19 I 3 ) et E. H. Wheeler $\left(9_{2} 3\right)$ ont distingué, parmi les

(1) Pour les études récentes sur les larves des Méloïdes américains et des listes hibliographiques, voir les travaux de WII-mims et Hringerforn (1914), Mir.tikex (1921), Pinken el BöVING (192/) et BnuEs (192/h). 
Térébrants, aı moins une dizaine ou une douzaine de premiers stades larvaires. Chez les IDiptères, on rencontre des larves à allure de planidium parmi les Acrocéridés et des slades larvaires actifs, qui se comportent cemme des planidia, sont présents chez les Némestrinidés, les Tachinidés qui pondent sur des feuilles, et les Bombylidés ( $\mathrm{I}$ ).

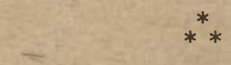

On connaît des types de développement avec triongulin actif ou larve campodéiforme, également chez certains Névroptères, par exemple Mantispa (Brauer, I 869) et chez certains Coléoptères, par exemple le carabide Liebia scupularis (SiLvestri, I 9o/4), qui est parasite de la

(1) Les Acrocéridés et les Némestrinidés sont d'un intérêt particulier, si on les compare aux Trigonalidés, parce que ces deux familles de Diptères sont également composées d'insectes rares, sporadiques et archaïques, qui pondent un grand nombre d'œufs tout petits à clistance de leurs hôtes, mais dans leur entouràge. King (1916) a montré que la femelle de Pterodontia flavipes, stationne au vol devant un tronc d'arbre et projette ses œufs sur l'écorce, à laquelle ils se collent. Les larves à allure de planidia, actives, s'attachent à des araignées qui passent, s'enfoncent dan's leur corps, puis deviennent des larves vermiformes, lourdes. Les Némestrinidés pondent leurs œufs très grêles dans des galeries creusées dans les piquets de bois et abandonnées par des Coléoptères, et les minusculés larves primaires pourvies de soies courbes, sortent en rampant. Arrivées cuehors, elles se dressent de façon à être emportées par le vent. Elles s'attachent probablement à des Scarabéidés et pellvent devenir parasites de leurs larves (voir Adam HANnLInsci (1882), Brauer (1883 a, 1883 h), et Bruch (1917). Les Bombylidés sont des insectes heaucoup plus abondants que les Acrocéridés et les Némestrinidés, parce que la femelle pond ses œufs très près de l'hôte de la larve (voir Shelronn (1913), FarRe, RiLey, Chapunn, etc...). 
Galéruque de l'orme. On en connaît encore chez plusieurs Staphỵlins du genre Aleochara, qui parasitent les pupes de divers Muscides (Wadsworth rgi5, Scott r 920). Dans tous les cas, les entomologistes parlent d'hypermétamorphoses, parce qu'il y a au moins deux formes larvaires différentes: une petite, active et sobre, qui effectue la recherche de l'hôte, et une seconde, gloutonne et obèse, qui se développe après que le contact avec l'hôte s'est ieffectué et assuré. Brauer (I869) et plus récemment Hermoss (1907), ont fait quelques objections à un tel emploi du terme hypermétamorphoses. On devrait le réserver, selon eux, aux cas analogues à celui des Méloés, décrit originellement par FABRE, c'est-à-dire aux cas où un stade larvaire immobile (pseudo-chrysalide ou larve coarctée) s'intercale entre le stade larvaire trophique et la vraie chrysalide. Il est probable, d'ailleurs, que les entomologistes continueront à employer le mot comme ils l'ont fait jusqu'à prézent, dans les cas de di ou de trimorphisme larvaire successif. L'opinion de Braver et de Heynoss, que de tels cas ne sont pas essentiellement différents des cas de métamorphoses ordinaires, aurait plus de poids qu'elle n'en a, si les phénomènes biologiques, en général, se prêtaient mieux à une discrimination logique précise.

Une question plus importante est de savoir si nous devons considérer des types de développement tels que ccux des Pćrilampidés, des Eucharidés et des Trigonalidés (probablement) comme plus primitifs que le type ordinaire de développement des Hyménoptères, ou au contraire, comme secondairement acquis. Cette dernière opinion est soutenue par quelques auteurs. Brues (rgrgh) exprime clairement la chose quand il dit : "Il est évident 
que le planidium et le triongulin se sont intercalés dans le développement larvaire, tout de mème que le stade larvaire lout entier s'est ajouté au cycle évolutif des insectes mélaboles. Leur présence, spécialement chez les $\mathrm{Hy}$ ménoptères est tout à fait secondaire. "Je doute beaucoup que cette vue soit exacte. Des cas tels que ceux de Manlispa, d'Aleochara, de Lebia, des Véloïdes, des Acrocéridés, etc..., me paraissent indiquer très clairement que le slade larvaire vermiforme obèse s'est intercalé secondairement, et que le triongulin ou planidium, si semblable à la larve typique et évidemment primitive de la plupart des Mégaloptères, des Névroptères et des Coléoplìres adéphages, est réellement un stade archaïque, bien que souvent considérablement modifié. Ce stade aurait disparu chez la plupart des Térébrants et chez tous les Aculéates, à l'exception possible du premier stade larvaire actif des Chrysididés. Par le fait qu'elle possède des pattes thoraciques et des cerques, la jeune larve des Pamphilidés, parmi les Phytophages, peut être rapprochée du triongulin. Il est possible qu'on comble la lacune qui sépare une telle larve d'un planidium, quand on aura découvert le premier stade larvaire des Trigonalidés. La larve ćruciforme des Mécoptères actuels, des Trichoptères, des Lépidoptères et des Tenthrédinidés dérive évidemment de la larve carnpodéiforme, et on admet généralement que la larve vermiforme peut dériver soit de la larve éruciforme, soit de la larve campodéïforme.

Par conséquent, si je ne me trompe, c'est sous la forme de larves actives que les ancêtres éloignés des Térébrants recherchaient leurs proies ou leurs hôtes, puisque leurs mères n'avaient pas encore acquis l'habitude de pondre teurs oufs directement sur on dans la nourriture de la 
larve. Ces formes anciennes étaient ce que j’ai appelé des insectes " atrophaptiques " ou " dystrophaptiques ". L'existence, même chez quelques Ichneumonides et Braconides, d'un premier stade larvaire plutôt actif, qui rappelle de loin un planidium est significative à cet égard (I).

Si le temps le permettait, il serait intéressant de retracer l'intérèt croissant que la femelle des Térébrants porte à sa progéniture, en passant des conditions atrophaptique et dystrophaptique à la condition eutrophaptique. Dans cette dernière mème, il y a deux phases: une, dans laquelle la femelle ne fait pas de nid, mais recherche l'hôte ou la proie et pond sur elle ou dans son intérieur ; et une autre, dans laquelle la femelle fait un nid et l'approvisionne en proies ou en nourriture pour le jeune.

La première méthode est caractéristique de la plupart des Térébrants et de quelques Aculéates. La femelle peut même entrer dans l'eau pour chercher la proie on l'hòte. Brues (I92I, p. I5 r) a fait une revue rapide des cas connus de ce comportement singulier, qu'on rencontre dans plusieurs groupes différents de Téréhrants. "Dans plusieurs cas bien connus, le comportement du parasite adulte s'est si profondément modifié que, non sculement les femelles pénètrent dans l'eau pour rechercher leurs hôtes, mais qu'elles peuvent être, au moins dans quelques cas, accompagnées par les mâles. La première observation de ce genre a été faite, il y aura bientôt un șiècle,

(1) C'est le cas par exemple dans des espèces telles que Calliephialtes messor, selon Cushmax (1913) et Ephedrus incompletus, selon E. H. WheELer (1923). L'étude future des preiniers stades du développement larvaire des familles les plus primitives, telles que les Stéphanidés, les Lvanidés et les Míga lyridés pout nous réserver quelques decouvertes intéreseantes. 
par Francis Walker (36) sur Agriotypus, et l'observateur bien connu Sir John Lubbock ('63), a fait plus tard une ćtude des mœurs de deux Chalcidiens, dans laquelle il décrit le mode de natation. Une espèce, le "Mymaride" (Cataphractus cinctus), utilise pour nager des ailes ciliées et en palettes, tandis que l'autre, un Trichogrammide (Prestwitschia aquatica), se meut au moyen de ses patles. De nombreuses autres contributions, notamment celles de von Siebold ('58), W. Müller ('8g), Marchal ('oo), Rousseau ('o 7 ), Scullz ('o 7 , 'ioa, 'iob), et Matulson et Crosby ('r 2), en ont accru l'intérêt, non seulement en mettant en lumière les membres aquatiques de plusieurs familles, mais en déterminant quelques-uns des hôtes auxquels elles s'attaquent. Dans beaucoup de cas, l'adaptation à la vie aquatique n'est pas si parfaite que dans ceux dont nous venons de parler. Mais beaucoup d'autres espèces nagent rapidement, utilisant, soit leurs pattes, soit leurs ailes, qui montrent ordinairement des modifications adaptées à un tel comportement ( $\mathrm{r}$ ).

Le stade final, eutrophaptique, dans lequel la femelle apporte au jeune, dans une cellule ou un nid préparé d'avance, les proies ou la nourriture, se rencontre seulement chez les Aculéates, et seulement, même chez les membres les plus hautement spécialisés de ce groupe.

(1) A la liste des formes donnée par Brues on peut ajouter un Braconide aquatique, Giardinaia urinator, découvert par P. de Stefani Pérez (1902). 


\section{L'EVOLUTION DES VESPIDES}

Indication chez des Térébrants de caractères propres aux Aculéales. - Subdivisions des Aculéales et rapports des familles. - Les Bethylidæ. - Leurs façons de traiter la proie, types béthyliné, sclérodermatiné, dryininé. - Les Vespidés, formes solitaires, subsociales et sociales.

Nous avons attiré l'attention sur les liens qui unissent les Térébrants, ou Hyménoptères parasites, et les Aculéates, ou Hyménoptères à aiguillon; liens si étroits que la plupart des auteurs modernes fondent ces groupes en un sous-ordre unique, les Clistogastres, et que les Aciléates sont supposés dériver d'ancêtres Térébrants; cependant, d'autres auteurs donnent aux deux groupes des ancêtres communs et très voisins, qui auraient fait partie des Phytophages primitifs, Quelle que soit notre opinion sur ce point, et bien que, chez les Térébrants, l'oviscapte soit employé à déposer les œufs, tandis qu'il est transformé en aiguillon chez les Aculéates et ne sert pas pour la ponte, (sauf chez les Sapygidés, les Chrysididés et les Dryinidés), il nous faut admettre que les Térébrants possèdent déjà certains caractères qui se retrouvent, plus ou moins modifiés et élaborés, chez les Aculéates supérieurs. Parmi ces particularités, les suivantes méritent notre altention.

1. Chez les Térébrants eux-mêmes, il existe une tendance sporadique au polymorphisme dans un sexe ou dans les deux. 
Ainsi, chez un Braconide, Sycosoler lavagriei, suivant Lichrenstein el Picard (1917) et Pichrd (1919), on trouve delix formes pour chaque sexe, l'une ailée, l'autre aptère. On connait hien, éga lement deux formes de femelles chez cerlains Chalcidides, Harmolita par exemple (Webster et Reeves, 1909), et certains Cynipidés; landis que, chez des insectes de la tribu des Chalcidoides, Idarnini (Philotrypesis), Grandi (1921, 1923) a découvert l'existence d'un polymorphisme particulier, tout à fait semblable à celui de certaines fourmis ouvrières, quoique limilé all sexe mảle. Chez Pezomachus flavocinctus, Strickilivd (1912) a décrit un polymorphisıne spécial du mâle, lequel peut ètre ailé, subaptère ou aptère. Après avoir ainsi noté, chez cerlains Térébrants, la disparition des ailes dans les deux sexes, et particulièrement chez la femelle, comme aussi, dans un mème sexe, une grande diversité dans la taille de la tête et des mandibules, suivant les individus, nous sommes un peu moins surpris de retrouver ces caractères avec une plus grande fréquence chez crtains groupes d'Aculéates sociaux.

2. Chez cerlains Térébrants, l'oviscaple peut fonclionner comme l'aiguillon des Aculéates, et introduire dans la proie une sécrétion qui provoque une paralysie au moins temporaire des muscles. Ceci a élé observé dar Silvestu (1907), chez un Chalcidide, Elasmus flabellalus, qui paralyse entièrement la larve d'un Tinćide, Prays oleellus, dans laquelle il pond. DoTrx (1911) a découvert que Microbracon juglandis paralyse totalement les chenilles de Ephestia liühniella, si bien que celles-ci restent fraîches de deux à quatre semaines; et Picaro (1919) a noté une paralysie analogue, mais plus faible, des larves de Hypoborus ficus, alleintes par Sycososter lavagnei.

$3^{\circ}$ On sait que certains Térébrants déposent leurs œufs à l'inlérieur du corps de la proie, tandis que d'autres les déposent a la surface ; ce dernier procédé est le seul qu'utilisent les Iculéates, à l'exception des Dryinidés. Plusieurs auteurs, et, en demier lieu, Picanı) (1919), ont fait remarquer que les Térébrants déposent leurs œufs à l'extérieirr, quand la proie est dissimulée dans une plante ou une cavité du sol, ou bien dans un cocon ou une chrysalide; la ponte à l'intérieur de la proie est, par contre, de règle, lorsque celle proie est à découvert ou vil librement (1).

(1) Parmi les rares exceptions, nous pouvons citer Polysphinela, qui parasite des araignées (Lichtenstein el RiBiud (1!22) il un Chalcide, Schizonotus sieboldi, qui, suivant Cushmu (1917) déjose ses œuls sur les nymphes, exposées à nu, 
$4^{\circ}$ Chez les Aculéates qui paralysent leur proie, on a souvent constaté qu'ils malaxent et ingèrent des portions de cette proie. Un tel phénomènc est fort naturel ; car, ce faisant, l'Aculéate adulte continue simplement à s'alimenter avec des portions d'un insecte de mènic espèce que celui sur lequel il s'est développé au stade larvaire. Celle lendance se manifeste chez beaucoup de Térébranls, mais sous une autre forme: ces insectes absorbent le suc exsudant des piquires que l'oviscapte a faites dans les neufs, larves, nymphes, ou adultes de l'espèce parasitée, et immédialement apris la ponte; à moins que l'oviscapte ail servi simplement d'outil pour procurer l'aliment. Ces mœurs singulières ont été observées en premier par ManCHAL (1905, 1909), chez Tetrastichus xunthomelsense qui pond dans les oufs de la Galéruque de l'orıne (Galerucella xanthomelrena) et chez Aphelimus mytiluspidis, qui pond dans ın Coccide (Aspidiotus ostreicformis (l).

d'un Chrysomélide (Melasoma interruplum). Le même auteur signale un Eulophide. Euplectrus comstocti, qui pond sur le ver du coton. Silvestri (1911) a alissi décrit des mœurs identiques dans une espèce européenne. Euplectrus bicolor, qui parasite les cheni'les de Plusia gamma et Mamestra brassicie. Chez les Aculéates, c'est aussi une loi générale de pondre a la surface de la proie lorsque celle-ci se trouve placée dans une cavité ; cependant, on connaît les Rhopalosomatidés et quelques Pompilidés qui fixent leurs orufs sur des insectes vivant librement.

(1) Plusieurs entomo'ogistes américains les ont aussi observées chez Telrastichus asparagi, qui pond dans les œufs du Criocère (Crioceris asparagi); de même: Saınders, chez Aphelinus luscipennis, qui parasite un Coccide, Aspidiolus rapax (Howino (1910); Silvestri (1910) chez Telrasticluls xamthomelana; Dotex (1911), chez Pleromalus puparum el chez une espece de Meraporus, deux parasites des nyınphes du papillon du chou (Pieris rapæ) et chez Microbracon juglanais, parasite de la mite de farine (Ephestia kühniella);Rocisud (1917), chez Nasonia brevicornis, qui pond dans les nymphes des Nuscidées; Lichtrastein (1921), chez un Chalcide, Habrocylus cionicida, qui dépose ses aeufs sur les nymphes d'un charançon (Cionus thapsi); Trouverot (1921) chez un Braconide Habrobracon johanseni; Whitivg (1921a) chez $H$. brevicornis, qui allaque. le s vers de farine; CAfrrey (1921) chez Anaslatus semillavidus, parasile des ceufs de la mite d'olivier, Hemilenca olivax; T. B.ı- 
Le cas le plus curieux est celui de l'Habrocytus decr't par Lichtrnstein; en eflet, le Chalcide coule Poviscapte à travers le cocon du Charançon, puis entre le cocon et la nymphe, et enfin à l'intéricur de celle-ci, et il le laisse dans cette position pendant une demi-heure; durant ce lemps, un produit secrété se durcit autour de l'organe, et forme un tube délicat, à travers lequel le parasite aspire le sang de sa proie, dès qu'il retire l'oviscapte. On remarquera que, dans tous les exemples cilés, l'insecle n'emploie pas ses mandibules pour s'alimenter, parce que sa proie est Irop grosse el a une peau trop résistante; tandis que les. Aculéates, plus puissants et de plus grande taille, utilisent sans peine leurs mandibules dans ce but. Cependant, je vous montrerai, par 'a suite, que de petits Aculéates (Béthylidés) se servent de l'aiguillon comme les Térébrants, pour prélever leur nourrilure.

$5^{\circ}$ Peut-être pouvons-nous discerner le germe d'une faible sociabilité, parmi les Térébrants, chez ccux qui se développent à plusieurs dans le mème hòte, el qui, la nymphe ure fois formée, ont leurs cocons groupés en masses compactes. Dans un genre de Chalcididés, Cratolechus, ou Comedo, comme on l'appelle actuellement (Howann, 1891), les nymphes sont nues, et sont fixées côte à côte sur une feuille, formant ainsi une ellipse réguliere autour de quelques-uns des individus; cette disposition laisse supposer cliez les larves une particulière "Fühlung ", pour employer le terme allemand. Nous devons cependant admeltre que la sociabilité est bien moins prononcée chez les larves de Térébrants que chez les Phytophagę plus anciens ou primitifs; et il est bien prouvé que des jeunes larves sont souvent détruites (fuand la proie, trop réduite, ne pout nourrir plus d'un parasile.

Leo diverses particularités signalées dans les cinq paragraphes précédents, peuvent être considérées comme représentant des tendances éthologiques très anciennes et primitives, qui réapparaissent, en se développant parallè-

Four-Brown (1922) el Picakn (1923) chez un Chalcicle, Melillobin acasta, qui dépose ses œufs sur les larves et les nymphes d'un grand nombre d'llyménoplères, Diptères et Coléoptères, et Voukassovitch (1924), chez un Ichneumonide, Dicielotus en!! throstoma, un parasite de Polychrosis botrana, 
lement ou de façon convergente, et sous des formes plus prononcées et plus spécialisées, chez les Aculéates. Par contre, la plupart de ceux-ci s'écartent des Térébrants, lout d'ahord par l'art qu'ils ont acquis de creuser des cavilés dans le sol ou le bois et de s'édifier des cedlules avec de la terre, des matériaux d'origine végétale ou même des produits sécrétés par leur propre corps et solidifiés, et aussi par l'habitude de mettre en réserve, dans ces constructions, un aliment (insecte ou végétal) destiné à leurs jeunes ou à eux-mêmes. La lente évolution de ces deux caraclères peut être parfaitement suivie, dans le sous-ordre des Aculéates et spécialement chez les guêpes sociales et les abeilles; mais ce sujet est si vaste, et a été si souvent traité dans les ouvrages de vulgarisation, que je ne lui accorderai pas longtemps mon attention.

$$
* *
$$

En ce qui concerne la façon dont on peut grouper les diverses familles d'Aculéates, les relations de ces groupes entre eux, et l'ordre dans lequel on peut les ranger, les divergences d'opinions sont actuellement assez grandes, entre les divers hỵménoptéristes, pour laisser les éthologistes dans l'incertitude. Des deux plus récentes classifications, celle de Handuirsch (1924) et celle de Bradley, Rohwer et Bequaert (Comstock, r924), la premiere, plus conservatrice, porte plus fortement l'empreinte des travaux des savants européens ; tandis que la seconde, qui résume quelques-unes des façons de penser d'Ashmfad, est mieux acceptée par les hyménoptéristes américains. Pour ma part, je préférerais plutôt 
la classification de Havdursch, dans la mesure où elle conserve la plupart des anciens groupements de Latreille, bien que ceux-ci demandent à être en partie modifiés. Pour le bit que je me propose, il me suffira dadopter la disposition suivante:

\section{SOUS ORDRE IES ACULEATES}

(VESPOINÉS OU VESPIFORMIÉS)

Superfanille des Bethyloïdées: Familles des Dicrogéniidés. Béthyllidés, Dryinidés, Embolémidés, Sclérogibbidés, Rhopalo. somatides.

Superfamille des Tubulifères : Familles des Cleptidés, Chrysididés.

Superfamille des Hétérogynes: Familles des Sap!gidés, Scoliidès, Taiphiidés (inc. Méthocidés et Myrmasidés). Mutillidés, Anthoboscidés. Thynnidés, Plumariidés, Formicidés.

Sıperfamille des Pompiloïdés : Famille des Pompilides (1).

Superfamille des Diploptères: Famille des V'espidés.

\section{(SPHÉCOMĹ OU sPHÉCIFORMĹs)}

Superfamille des Fouisseurs : Familles des Ampulicidés, Sphecidés.

Superfamille des Anthophiles: Famille des Apidés.

La paléontologie ne nous apporte aucune lumière sur l'origine des diverses familles groupées ci-dessus. Comme les familles des Térébrants, beaucoup d'entre elles sont représentées, dans le Tertiaire inférieur et moyen, par des genres identiques à ceux qui vivent à l'époque ac-

(1) La position exacte des Pompilidés est sujelte à discussion; Börner el Havilirsch, comme bien d'autres hyménoptérisles avant eux, les placent parmi les Fouisseurs; d'autres, tels Ashmeid, Rohwer, Briduer, Baiks, etc..., parmi les Vespoïdes. Tant que leurs affinités ne seront pas mieux précisćes, je crois qu'il n'y a aucun inconvénient al les considerer comme ine superfamille indépendante. 
tuelle, ou qui leur sont très étroitement alliés. Et la morphologie, comme l'éthologie, montre clairement que toutes les familles éteintes d'Aculéates étaient hautemenl différenciées ; aussi est-il impossible (à de rares exceptions près), de les faire dériver les unes des autres. Néanmoins, on peut trouver, dans l'étude du développement, de la structure et des mours, qualques exemples qui indiquent clairement certaines tendances d'évolution. Celles-ci paraissent très nettes dans le groupe des Béthỵloïdés, placé en tête du sous-ordre, et qui mérite une attention particulière, à cause des rapports morphologiques qu'il a avec toutes les autres superfamilles, rapports nombreux et souvent difficiles à saisir.

Qunique Hulmay (I838) ait considéré Bethylus comme un Fouisseur, ce genre, et les genres voisins, furent placés au nombre des Proctotrypidés, jusqu'à ce qu'en igo2, Asnmean les eût transportés parmi les Vespoïdés. Actuellement, on ne peut douter que ce soit là un groupe d'Aculéates cosmopolite et primitif, quoique s'étant spécialisé d'une façon particulière et très diverse. On en a décrit plus de r 200 espèces, la plupart de petites dimensions, et plus de oo genres, répartis entre quelque six familles.

Les spécialistes leur ont attribuć les affinités les plus diverses avec les autres Aculéates. KierFen, qui publia des monographies des Béthylidés (1908) et des Dryinidés (1907), considère les preıniers comme " très ètroitement rattachés aux genres Meria et Tiphia, des Scoliidés sensu lato "), et le genre Mesilius comme les rattachant aux Tubulifères (Chrysididés). En 1909, il a décrit un genre Parachrysis, qui peut être assigné, soit aux Béthylidés, soit aux Chrysididés. Westwood (1881) remarque la ressemblance du genre de Béthylidés Apcnesia avec les Mutillidés. Pr.Rkivs (1905) note. la parenté des Béthylidés avec la série des anciens Fouisscurs d'une part et avec les Proctolrypidés d'au- 
tre part. Brues (1910b) signale la parenté des Béthylidés el des Dryinidés, spécialement des genres Epyris el Pristocera, avec les Ampulicidés, et du genre Mystrocnemis, des Sclérogibbidés, avec les Tiphiidés et avec la famille des Cosilidés d'Ashmeal) (Anthoboscidés). Il pense que les Embolémidés sont un groupe ancien, se rapprochant des formes ancestrales des Proclotrypidés. BRIDWELL (1917a) considère comme purement superficielle la parenté des Béthylidés et des Proctotrypidés; mais comme " éminemment naturelle " celle des Scoliidés et des Chrysididés. Bonnen (1919) range les Béthylidés comine les Chrysididés parmi les Térébrants, mais les croit liés aux Cleptidés. Brufs (1922, 1923c) note la ressemblance du genre Algoella, qui parait être un Béthylide, avec les Anthoboscidés (Sierolomorpha) et les Mutillidés, et décrit un genre particulier de l'ambre de la Baltique, Palæobethylus, qui rappelle de façon frappante les Ampulicidés, les Rhinopsis spécialement. Hannlınscu (1924) fait des Béthyloïdés la première superfamille des Aculéates, tandis que Bhidley (Comstock, 1924), intercale la famille des. Béthylidés entre les Formicidés et les Vespidés el la famille des J)ryinidès parmi les Sphécoïdés, après les Ampulicidés.

La famille des Rhopalosomatidés demande une discussion spéciale, à cause de l'incertitude de sa place dans la classificalion. Elle ne comprend que trois genres et espèces connus: Rhopalosoma poeyi, présent des Antilles au Maryland et au Missouri; Paniscomima erlangerianä, dans l'Afrique-du Sud, el Hymenochimæra abnormis, aux Indes. Hoon (1913) a découvert la larve de $R h$. poeyi fixée au troisième segment abdominal diun criquet sauteur alerte (Orocharis saltator), à PlummersIsland, près de Washington, D. C. Le Rhopalosoma adulte est noclurne; il rappelle fortement, par la couleur et la texlure de son tégument, les Vespidés nocturnes du genre Apoica et les malles nocturnes de certaines fourmis (Eciton, Diacamma, Lobopelta, etc.).

Westwoon, (1874), qui étudia le premier avec soin, les affinilés de cet insecte, dit qu'il existe " quoad affinitales animum excrucians ", mais pense que ce doit ĉtre un Vespidé. Nylannen le considère comıne une fourmi, HaLıDıx comme un Fouisseur; Fred Smith comme un Ichneumonide Ophionine, Cresson comme un Braconide, Shanr, comme allié aux Scoliidés et aux Sphécidés. Pour tout concilier, Ashmead (1902-03), fonde pour lui une famille spéciale qu'il situe entre ses Cosilidés el les Thynnidés. Monely (1910) ne pouvant le placer nulle part parmi les Térébrants, se contente d'en faire un Aculćate allié aux Scoliidés, mais avec la nervation d'un Ichneumonide. Rонiver (1913) pense 
que " les Rhopalasumatidés el les Dryinidés ont peut-être ume urigine commune, ce que semble indiquer la larve \#, Tumen el Wirtinstox (1917) affirment que l'organe génital màle des Rhopalosomatidés " présente des rapports très nets avec celui des Euménides et diffère fortement de celui des autres IIyménoptères. Ainsi, de loutes les lenlatives failes pour fixer une place is ce groupe, la meilleure est celle de Westwoon, qui le rattache aux lespides. (e grompe descend presque surrement de la mine soluche que les Eumenirlés, mais il a été fortement modifié par la vie nocturne el sanr doute aussi par le parasitisme. La vasle extension de la lamille indique une très grande anciemnete, quoi qu'elle paraisse absente en Australie. HANDI.IRsch (19:4) place Rhopalosoma dans les Hétérogynes, entre les Sapygrides et les Inthoboscinés, Branley, (Constock, 192.1) entre les Vespidés et les Béthylidés. Brues a suggéré verbalemenl, qu'il pourrait se situer au voisinage des Béthylidés, position qu'indiqueraient aussi les affinités, soupçonnces par ROHWER, avec les Dryinidés.

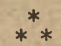

Mais, au lieu d'énumérer les opinions opposées, concernant les rapporls taxonomiques des Béthylidés, il nous paraît plus intéressant, et plus conforme à nos desseins, d'examiner les moeurs de ces insectes. D'après les faits connus, et si nous exceptons les Rhopalosomatidés, il sem. ble que la façon de traiter la proie, que la ponte et le développement correspondent, dans la superfamille, à trois méthodes ou types différents, qu'on peut désigner respectivement comme: type béthyliné, type sclérodsrmatiné et type dryininé.

$I^{\circ}$ Lee type bélhyliné a été observé dans les genres $B e$ Ilyylus (Perisemus), Pristocert, Epyris et Holepyris. Jusqu'à ces lemps derniers, on ne savait que très peu de choses de ces insectes, hormis les observations, très fragmentaires, de Hilidiy (1835), Ferton (igor) et Nielsen (igo3). 
Huabuy a vu une femelle de Bethylus fuscicornis trainer sur le sol une chenille paralysée de Tinéide, vers un roseau creux, pénćtrer dans le roseau comme pour faire une reconnaissance, ressortir et tircr la chenille à l'intérieur de la cavité. Dans une ronce creuse, Ferton a trouvé quatre larves vertes, de la mème espèce de Bethylus, dévorant une chenille verte. Avec elles, était un Bethylus adulte, femelle, que l'auteur considère comme la sœur des larves, mais qui évidemment était leur mère. En fendant ur morceau de ronce dans lequel un Pemphredon avait niché auparavant, Nielsen découvrit, à quelques pouces de 'l'entrée, une chenille de Tortrix qu'un Bethylus fulvicornis entraînait justement vers l'intérieur de la cavité, où l'auteur trouva une deuxieme chenille, en partie dévorée déjà par quatre larves de Bethylus; la première chenille avait été paralysée, et Nielsen conclut que $B$. fulvicornis est à un stade plus primilif que les guépes fouisseuses vraies, parce qu'il s'abslient de faire un nid, et parce qu'il laisse les chenilles libres dans son abri, sans les séparer les unes des autres par des cloisons; landis que toutes les guêpes fouisseuses vraies, vivant dans des troncs, construisent des alvéoles. Le Bethylus diffère en outre des Fouisseurs, en ce qu'il dépose plusieurs œufs sur la même chenille, coutume très répandue chez les Térébrants. Près du Cap (Afrique du Sud), Bridwell (1917 b) a observé un Epyris femelle trainant sur le sol une larve de Ténébrionide. Mais les observations les plus complètes ont été faites sur Ep!rris extraneus, aux îles Hawaï, par F. X. Wrlliams (1918). Celle petile guêpe noire, qui ressemble à une Tiphia, capture la larve, beaucoup plus grosse qu'elle, d'un Ténébrionide (Gonocephalum seriatum), la pique jusqu'à ce qu'elle l'ait immobilisée, la saisit, par un palpe semble-t-il, et la transporte sur son dos jusqua quelque fissure du sol; là, elle façonne autour de sa proie un grossier alvéole et colle un œuf unique sur le milieu de la face ventrale du premier segment abdominal. La larve, une fois éclose, repose d'abord à la surface de la proie; mais elle se redresse ensuite, formant un angle droit avec l'axe longitudinal de sa victime. Quand elle est entièrement formée, elle abandonne ce qui reste de sa proie, et file un cocon. Des inceurs presque indentiques ont été découvertes par BridweI. (1917 c, 1919) chez Holep!ris hawaïensis el par Hyslop (1916) chez Pristocera armifera de l'Mmérique du Nord. L'aul isolé de celte dernière espèce a été trouvé dans le sol, fixé à la surface ventrale d'une larve d'Elatéride (Limonius agonus). Suivant Brubwer.l, Silvestri a décrit le développeinent d'une espèce italieme d'Holepyris. 


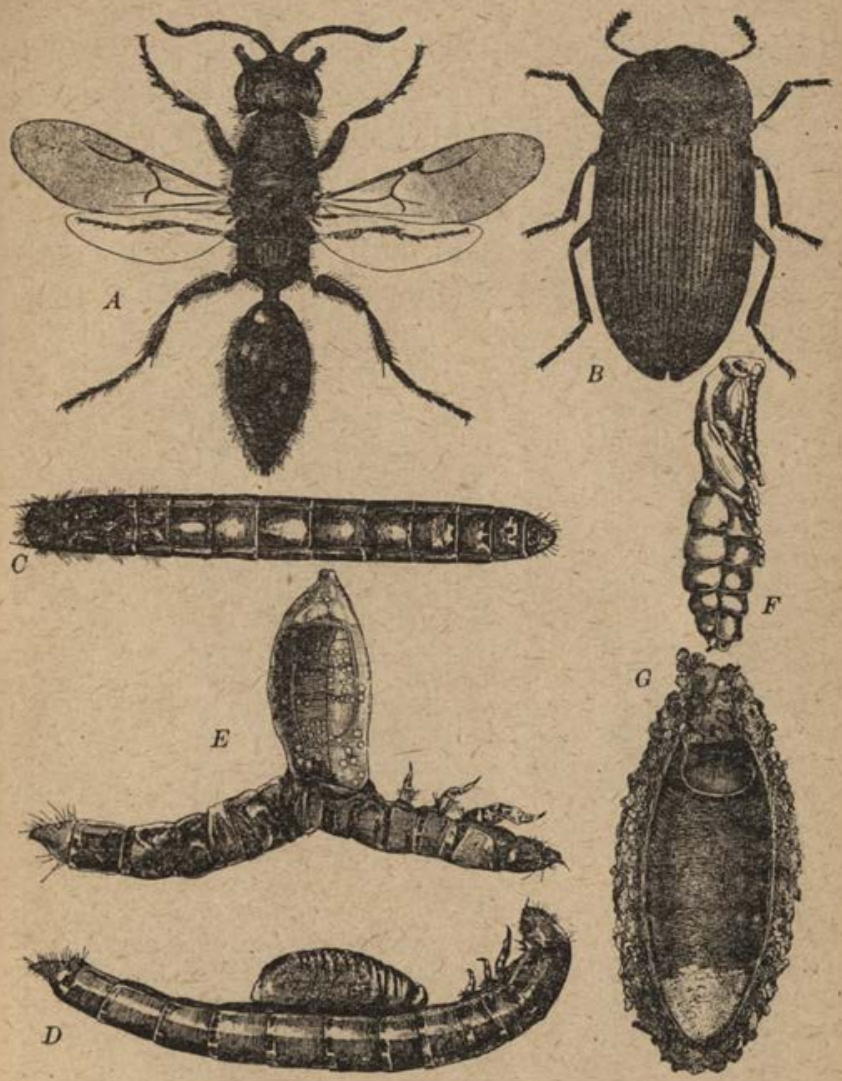

Fig. 7. - Evolution d'Epyris extraneus A, aux dépens de la larve de Gonocephalum seriatum (Ténébrionide B); C, larve de Gonocephalum avec auf d'Epyris au milieu de la face ventrale; $\mathrm{D}$, jeune larve d'Epyris se nourrissant sur celle de Gonocephalum; E, stade larvaire plus avancé d'Epyris; $F$, pupe d'Epyris; $\mathbf{G}$, cocon (d'après F. X. Willians).

SOCIÉTÉS D'INSECTES 
$2^{\circ}$ Le type de développement dit sclérodermatiné a élti observé dans les genres Ciephalonomia, Lolius, Goniuzus, Scheradierma, Neoscleroderma, Anoxus, Apenesia; Siero'a, et Parasierola. Dans ce cas, le Béthylide va en quête d'une proie qui est une larve de Coléoptère, d'un Lépidoptère ou même, quelquefois, dune fourmi ; il la paralyse par des piqûres répétées, et dépose sur elle plusieurs œufs. Après l'éclosion, les larves consomment la proie et les nymphes restent auprès d'elle, après que les cocons aient été filés.

Parmi les observations anciennes se rapportant à ce type de développement, les plus completes sont celles de Busck sur Lælius trogodermatis, telles qu'elles sont publiees dans le "Insect Book" d'Howird (1905). La femelle de Lielius allaque et paralyse la larve de Dermestes, arrache les longs poils de la surface ventrale, sur laquelle elle fixe ensuite un à six cufs. Le cycle vital complet n'exige que trente i trente-cinq jours. Lil nymphe passe l'hiver, les cocons étant, en cette saison, plus compacts et de couleur plus foncée que ceux de la génération d'été. D'après Busck, le Lielius paralyse toutes les larves de Dermestides qu'il rencontre, nf́ais il ne pond pas sur toutes; d'autre part, la larve, quoique paralysée, peut évacuer ses déjections et même peut muer. Dans ce dernier cas, les œufs fixés sur la cuticule périssent. Busci a également Irouvé que la femelle pond plus de deux fois son volume d'œufs, et qu'elle peut parfois être parthénogénélique, mais ne produisant alor's que des mâles.

Des descriptions succinctes ont été clonnées des mœurs de plusieurs espèces de Goniozus et de leurs proies par AudourN (1842), Popenoe el Marlatt (1889), Ashmean (1893), Picard (1919) et autres; et de celles de Parascleroderma et Cephalonomia par Picard. Ashmead (1893) a publié la liste d'un grand nombre d'hôtes dans sa monographie des Proctotrypidés de l'Amérique du Nord; de méme, Kiffrén (1908) dans sa monographie des Béthylidés.

Les genres Scleroderma, Sierola, Parasierola et Cephalonomia furent étudiés de façon plus étendue par Bnidwer.l (1917, 1919,1920 ), aux fles Hawaï, ou ces interessants insectes se trouvent en si grand nombre. qu'en un seul mémoire, Fullawas (1920(1) a pu décrire 171 nouvelles espèces du seul genre 
Sierola. Swezer (1915) a lui aussi publié quelques séries d'hôles des Bèthylidés hawaïens.

Seleroderma immigrans, espèce des Philippines introduite aux Hawaï, a été étudiée en très grand détail par Bribwell (1920); plus récemment, il m'a été possiblo d'élever une espèce de nos Etats du Sud, S. macrogaster. Les mœurs de celle-ci ressemblent beaucoup à celles de $S$. immigrans; commo d'aillcurs toutes les espèces paraissent avoir essentiellement les mèınes mœurs, je puis décrire celles de $S$. macrogaster comme paradigme du genre. Ainsi que l'ont remarqué Westwood (1881) el Binwell, pour d'autres espèces du genre Scleroderma, S. macrogaster a deux formes de mâles et deux formes de femelles, ailées et aptères, les femelles ailées et les mâles aptères étant d'ailleurs plutôt rares. Le dimorphisine des sexes a donc heaucoup d'analogies avec celui fu'a observé Picano chez. Sycosoler lavagnei. Les deux formes de chaque sexe se comportent de la même façon. Bintver. moflrit un lot de cocons de $S$. maciogaster du Texas et m'indigua que je pourrais probablement élever celte espèce sur les larves de quelque Cérambycide Clytine. Je ne connaissais pas l'hote originel, mais j'ai retrouvé ce que Bunwer.. avait justement montré dans lo cas des Sclérodermes des Hawaï, c'est que cette espèce du ' $\mathrm{c} x a s$ pouvait se développer sur des nymphes et des larves tres diverses. L.e résultal fut parfait avec des larves et les nymphes d'un Cérambycide de Hicorea (Cylene pictus) et d'un autre Cérambycide (Liopus cinereus); très bon avec d'autres Coléoptères (nymphes de Pissodes strobi, larves de Cléridés, nymphes de Thymalus fulgidus) et avec des larves d'Ichneumons; moirs satisfaisant avec les nymphes et les larves molles de fourmis (Camponotus americunus el Lasius americanus). Lorsqu'on place dans un petit flacon une femelle de Scleroderma, qui est longue de 2,5 à $3 \mathrm{~mm}$. seulement, avec une larve bien vivante de C!lllene, qui est plusieurs milliers de fois plus grosse, le Scleroderma l'évile au débul, mais à la fin il grimpe sur elle, agrippe la cuticule avec ses mandibules, et pique la larve, en débutant généralement par la région des muscles mandibulaires. La femelle va, de place en place, continuant è mordre el à piquer la larve, aussi longtemps que les muscles solis-cutanés, saisis dans ses mandibules, donnent quelque signe de contraction, et jusqu'a ce que la larve soit totalement paralysée. Cela exige'quelçues jours, do un à quatre, suivant la taille de la victime. Les piqùres sont faites au hasard et ne se suivent pas de façon régulière. Buıwell considère le fait de s'atlaquer d'ahord aux inuscles mandibulaires comme une 
adaptation, destince is empècher que la proie saisisse et broie le Sclèroderme, mais ceci se produit très rarement, méme quand lattaque débute en une autre région. Quand la proie est immobilisce, le Scléroderme s'en nourrit pendant plusieurs jours; pour cela, il comprime la larve en divers endroits et il aspire le sang qui exsude en fines goutlelelles ax points ou furent preccedemment pratiquées les piquires. Son abdomen grossit iriors rapidement, en mime temps que se développent les ovaires et bientòt, il se met a pondre un grand nombre dicufs blancs, brillants, el qui sont plutót gros par rapport à la dibiension de 'a femelle; celle-ci les depose a la surface de la proie, et de prélérence dans les sillons intersegmentaires. Lia proie, quoique vivante encore, demeure parfaitement immobile mais ni mues, ni développement daucume sorte ne surviennent jamais par la suile. Toulefois, si on a fourni me nymphe, le développement peut n'ètre pas arrêté, bien que l'abdomen reste paralysé; et dans quelques cas exceptiomels, la peau peut se durcir, si bien que les larves de Scléroclerme, écluses, meurent et l'imago de thote apparait, mais mourante. Les œufs dejosés sur une lapve ou sur une nymphe jeune produisent de petiles larves qui, au débul, gisent à la surlace ; elles devicitnent ensuite fuselées et se redressent; si bien que l'hote cu parail hérissé comme un porc-épic.

L.e Sclóroderme mère reste avec les larves; il se tient souvent au-dessus d'elles, parfois, les prenant entre ses paltes allléricures, il les lèche. I l'occasion, la mère aussi continue ì hoire le sang de l'hòle qui exsude entre les têtes, profondciment inplantées, de ses jeunes larves. Il lui arrive parfois de dévorel ses ouls, mais jamais je ne l'ai vue s'allaquer à ume de ses larves. En dévorant quelques-uns de ses œufs, elle parait obéir à un instinct qui lui fait régler le nombre des œufs suivant le volume de la proie. Quand les larves sont mùres, elles abandonnent les débris ridés et désséchés de la proie, et lilent des cocons d'un blanc ncigeux, réunis en une seule masse. La nymphose dure de quatorze à trente jours. Les mâles sortent les premiers de leurs cocons; ils percent les cocons femelles et fécondent les pupes. Les femelles mûres fécondées aussi sans délai, s'accouplent quelquefois avec le mème individu cinq à huit lois, à courts intervalles. La même fernelle peut aussi s'unir avec plusieurs mâles à la suite. Ces derniers sont d'ailleurs si ardents qu'ils tentent souvent de s'accoupler entre eux. La mere ayant une longue existence, peut s'accoupler avec un de ses fils, paralyser aussitôt une autre larve de Coléoptère, élever une autre couvée et s'accoupler encore avec un de ses 
petils-fils. Les màles meurent aussilor apri-s laccouplement. Si Hre femelles vierges sont isolées, el quon leur fournisse une proie, elles la paralysent parlatement, mids leur ponte est exlicumement relardée. Les culs et les larves se developpent rependant de façon normale, mais ne produisent que des mâles.

$3^{\circ}$ Le lype dryininé a éte ohserve rhez les Dryinidés qui sunt des parasites bien commus des Homoptìes (Fulgorides, Jassides, Membracides) ef des fourmis. Les femelles de Dryinidés, sauf celles du petit gemre Aphelopus, nut III larse antérieur très particulier, en forme de pince, avec lequel clles maintiennent lhote immobile tandis quielles introduisent un neuf dans son corps. La larve qui cul provient sort finalement sur le còté de l'abdomen, dans une poche spéciale constituée, semble-l-il, dans quelques formes par les dépouilles de la larve, et dans dautres formes par lhypoderme civagine de lhoic, une fois mûre, la larve se détache sjontanément, et file son cocon sur une feuille où plus rarement pémitre dans le sol pour s'enpuper.

Les marurs des Dryinides ont elé décriles par un grand nombre dob=ervateur's: Penms (1857), Mik (1882), SWEzE (1903), Plinkins (1905), Kiefren (1907), Jeinivi. (1913), Keilin et Thompson (1915), et d'autres, Ginno (1889 $a, 1889 b)$ a décrit les larves d Aphelopus melaleucus sur Tuphlocyba rosie et a conslaté que les individus ainsi infectés sont alleints le "castration parasilaire ". Mitauscu $(1909,1911)$ a décril des altérations analogues produites par les larves d'Aphelopus sur des Membracides américains, el KonNhiusen (1919) a publié une importante étude sur Ins modifications que subit un gros Membracide, très commun chez nous, Thelia bimaculata, lorsquil est parasite par $A$. Thelice. Le temps me manque pour examiner l'eflet particulier du farasite sur lapprareil génital male et femelle de l'hote; mais je somlignerai le fait, prouvé par Konnhusen, que $A$. thelie est polyembryonique. It dépose dans la nymphe du Membracide un ouf unique, qui produil de quarante il soixanle parasites. Quand ils ont allcint leur maturilé dans l'hòte, ils s'en écharpent, pas- 
sent ì l'élal de nymphe el demeurent pendant tout l'hiver dans le sol.

1. theliac est également intéressant à un autre point de vue, car il constitue un exemple d'alimentation-par l'oviscapt comnie nous en arons décrit dans la première partie de chapitre. Suivant Konnhuusen, après avoir choisi une nympl de Thelia, la femelle d'Aphelopus " saisit entre ses manc hules la partic caudale de l'abdomen de la nymphe, et, se re-

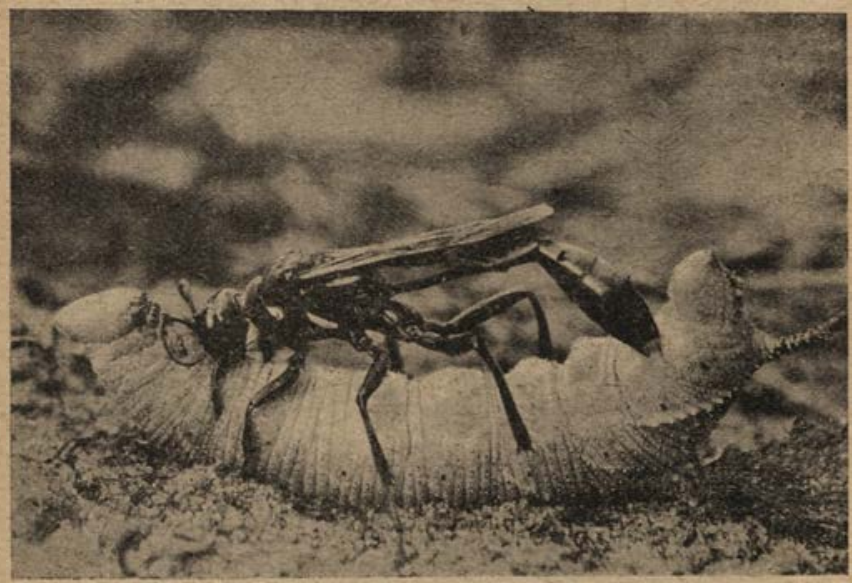

Fig. 8. - Sphex procerus Iransportant une chenille de Sphinx à son lerricl (Phot. C. Hartmak).

tenant solidement par les pattes à l'abdomen de l'hòte, elle tente d'enfoncer la pointe de son oviscapte, semblable à un stylet, à travers les membranes intersegmentaires de chaque paire de terga ahdominaux. La nymphe se débat lorsque l'oviscaple pénètre, et de l'orifice anal exsude une goutte de liquinide; l'Aphelopus saisit celte goutle avec ses mandibules et la fait disparailre ell une seconde. $₫$ Dans d'autres cas, Kornhnusen a noté que l'Aphelopus, après avoir pondu, grimpe sur l'abdo- 
men de la nymphe pour s'emparer d'une goutte d'excréments (niellée).

Les descriptions ci-dessus montrent que les Béthyloïdés se comportent de façon très hétérogène, les Dryinidés

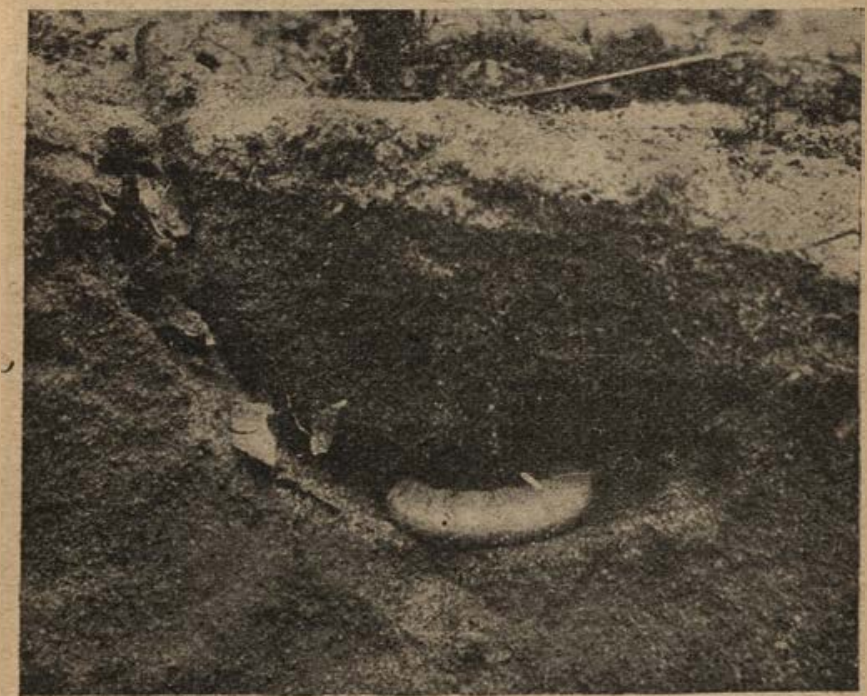

Fig. 9. - Terrier de Sphex procerus (en coupe). Le lunnel dentrée est obstrué de débris. Dans le terrier, une chenille de sphinx paralysce, al laquelle est fixé $u$ ceuf de Sphex (d'apres C. Hantmix).

rappelant essentiellement dans leurs moeurs les Térébrants ; quelques Béthylidés ressemblant aux Ampulicidés, Sphécidés, et Pompilidés, qui poursuivent leur proie, la paralysent et la traînent en lieu sûr, et d'autres ressem- 
blant aux Scoliidés, Tiphiidés et Thynnidés qui vont quérir leur proie dans sa propre retraite et l'y paralysent. Bien mieux, le Scléroderme fournit l'exemple d'une méthode généralisée, combinant celle des Térébrants, celle des Tiphiidés et, pour une faible part, celle des Formicidés. Cependant, les procédés des Sphécidés et des Tiphiidés ne sont pas nettement séparés, puisque, d'après quelques observalions publices, les Tiphiidés s'empareraient, à l'occasion, de leur proie à la surface du sol, la transporteraient à une courte distance el, là, lenfouiraient (I).

Les Scléroderme sont encore d'un intérêt exceptionnel, comme présentant les rudiments essentiels des moeurs sociales; en effet, lïnsecte mère vivant longtemps, subsiste sur la mème nourrilure que les jeunes, šintéresse ì ses larves, les lèche mème, et peut élever plusieurs couvées successivement. Quand la mère et ses descendants femelles, on plusicurs femelles de convées différentes, sont enfermées dans le mime récipient avec.une larve de Cyllene, il ny a point entre elles de rivalite, comme on pourrait sy attendre, mais an contraire, elles cooperent pour paralyser la proje ef toules pondent sur elle en commun. Effectivement, nous pourons admettre à l'origine des moeurs sociales des gruèpes, des abeilles et des four-

(1) Ainsi, d'apres Bnmwkt. Gosse auraft, autrefois observe un Elis t-notala, thatriant dans sa bouche une litrve dun groLamellicorne; el, suivant FonBes (1908) et Davis (1919), lorsque des larves de Lachnosterna sont placées sur le sol auprès d'une femelle de Tiphia, celle ei les pique el les paralyse puis se met en devoir de les enterrer; tandis que, dans les conditions normales, elle va en quete de sil proie sous terre et pond sur elle in situ. Dans le nord de l'Ifrirgue, Fentox (1911) a observé une petite Myzine, chassant sur le sable une larve d'un Ténébrionide du genre Tent!ria, la piquant une fois et l'enterrant alıssitôt au roisinage de la surface. 
mis, des conditions analogues à celles qui sont réalisées pour les sclérodermes (I).

$$
\text { ** }
$$

Les Guêpes sociales appartiennent loutes à une famille unique, les Vespides, qui constilue à elle seule la section des Diploptères, ainsi nommée parce que, au repos, les ailes sont repliées longitudinalement ; c'est là un groupe très homogène et très nettement défini. Beaucoup d'espèces sont solitaires, et, comme l'a montré Dicke (19) Io, I 914 ), les espèces sociales ne possèdent pas de caractìres morphologiques que l'on puisse raltacher à leur mode de vic, ou qui-permettent de les séparer, en un groupe à part, des espèces solitaires. C'est pourquoi les particularités éthologiques, et surtout les méthodes de nidification, prennent une signification taxonomique considérable. I'après les autorités plus anciennés, on peut distinguer deux familles: les Vasarides et les Vespides, les premières étant solitaires et strictement anthophiles, approvisionnant leurs nids avec du pollen et du miel ; les secondes étant solitaires ou sociales, plus ou moins anthophiles comme adultes, mais fournissant généralement à leurs larves des insectes comme aliment. On sait que quelques espèces sociates accumulent du miel dans leur's nids; les unes comme Vecturina lecheguana d'une façon

(1) Bien entendu, je ne crois pas que les insectes sociaux soient dérivés d'ancêtres Béthylidés. Quoique les Béthylidés actuels soient cerlainement très anciens, ils sont à plusieurs égards haulenent spécialisés et il n'y a aucune raison de supposer qu'ils aient donné naissance à l'une quelconqque des autres familles d'Aculéates. 
régulière, quelques autres, plutòt rares, (Polistes, Polybir occidentalis) à l'occasion seulement. Suivant H. de SAUsSure (i 853), firaud (1871), Ferton (igor, etc), et Bratis (1910, igir), les espèces solitaires des genres européens et africains Masaris, Caramius et Celonites, accumulent bien du miel dans les alvéoles de leurs nids de terre ; mais, suivant Davidson ( $\mathrm{I}_{9} \mathrm{I} 3$ ), les espèces du genre voisin Pseudomasaris, qui vit dans les Etats de l'Ouest, approvisionnent leurs jeunes avec des chenilles paralysées, comme font les Euméninés. Les observations de Brauvs sur Ceramius lichtensteini du désert de Karoo (Afrique du Sud) sont d'un intérêt particulier ; elles montrent en effet que cet insecte, adapté lui-même à la vie dans des conditions de grande sécheresse, est en outre devenu subsocial ( $\mathrm{I}$ ).

(1) Les habitudes des nidification sont decrites comme suit: " J'ai souvent mis au jour les nids de $C$. lichlensleini. La galerie descend perpendiculairement, en ligne droite ou courbe suivant la nature du sol, el souvent jusqu'à une profondeur de qualre ou cing pieds quand la terre est molle. Le long do celte galerie principale, la guêpe crense de petites cavités verticillées et y construil des alvéoles ovales, en argile. Ces alvéoles rappellent ceux de Sceliphron spirifex et autres formes voisines, mais sont d'un ovale plus arrondi. Il est certain que la guêpe nourrit ses larves avec du mie] de fleur, jusqu'à leur iroissance complète. Je n'ai jamais trouvé de masses de miel ou de pollen accumulées dans les alvéoles avec les larves, inême lorsque celles-ci sont toutes jeunes. Dès que la larve est entièrement formée, la guépe ferme l'alvéole. Pour passer à l'état de nymphe, la larve édific, à l'intérieur de l'alvéole d'argile un cocon d'aspect parcheminé, qui ressemble, par sa forme arrondie et sa base très aplatie, à une petite fiole pharmaceutique. Dans un tel alvéole, la larve peut vivre plusieurs années, suivant les périodes de sécheresse, el sans former de chrysalide. Actuellement, je conserve un certain nombre de ces alvéoles maintenus à sec pendant trois ans el reniermant encore des larves vivantes non transformées. ” 
Bequaert ( 1918 ), dans sa récente révision des guêpes solitaires et sociales du Congo, et BradLey (1922), dans sa monographie des guêpes Masaridés, ont abandonné la famille des Masaridés et considèrent les Diploptères comme constituant une seule famille, les Vespides, comprenant neuf sous-familles, étroitement liées entre elles. Celles-ci peuvent ètre classées de la façon suivante :

Solitaires ou au plus

\section{VESPIDES}

\section{Subsociales}

Euparaginés.

Masaridinés.

Euménidinés.

Zéthinès.

\section{Sociales}

Slenogastrinés.

Ropalidines.

Epiponinés (Polybiinés).

Polistinés.

lespinès.

Il ny a point de spécialisation marquée, dans la struclure du corps qui nous conduise de la première à la dernière de ces sous-familles; mais il y a un développement graduel, très défini et une spécialisation des tendances sociales, montrant que les Vespidés ont très amplement évolué, sur les plans fonctionnels (physiologiques et étho. logiques, c'est-à-dire instinctifs) (I).

(1) Voir Ducle (1914, p. 319) : "J'ai insisté déja (en opjposition avec Asumsan) sur le fait que les Euménidinés et les Vespinés sont seulement des sous-familles de la famille des Vespidés, puisque, mẻme à l'époque actuelle, les deux sont reliées par des formes de transition très nelles et puisque nous ne connaissons aucun caractere morphologique qui puisse les distinguer complètement. En fait, ces deux sous-familles sont étahlies uniquement par la présence, chez les Vespidés, de femelles ayant un appareil génital avorté (dites ouvrières), tandis que de tels individus ne se rencontrent pas chez les Euménidinés, ces derniers possédant tout au plus des femelles fécondes, qui, uccasionnellement, édifient un nid commun. ” 
Nous ne savons rien des mours des Euparaginés, groupe ne comprenant que trois espèces rares des Etats

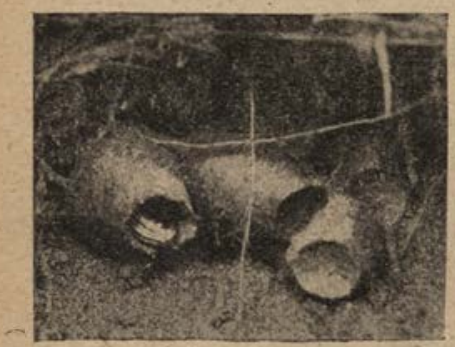

A

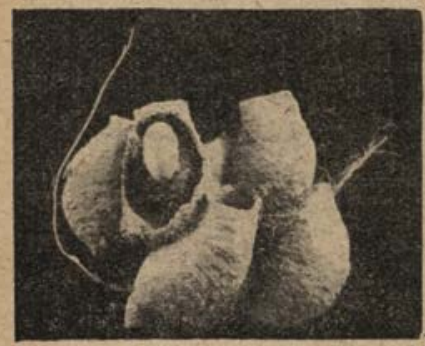

C

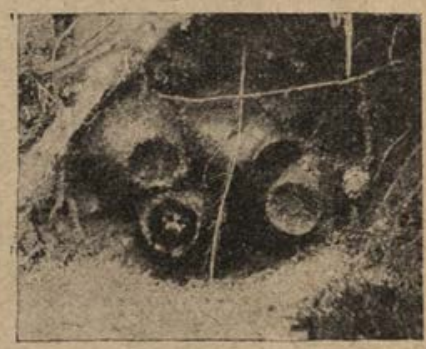

B

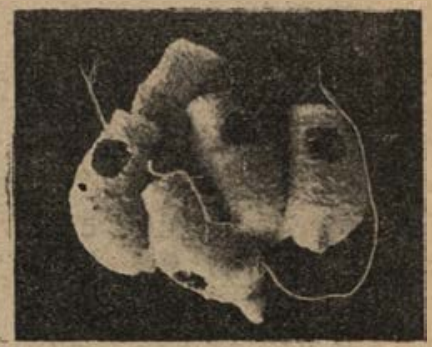

D

Fis. 10. - Quatre elals du nid argileus dodynerus dorsalis : A, ine des cellules est ouverle et aljprovisionnee de petiles chenilles; 13, mème nid, le lendemain (lat guêp)e s'est retirén dans une cellule faile la veille). (, une des cellules est ouverte pour montrer la larve se nourrissant des chenilles. D), inème nid avec les trous par wil se soml échajpeces les guêjes (d'apris: C. Humpais).

du Sud-Ouest, el itroitement relie: aux Masaridinces. Le groupe primilif el central de toute la série esl sans aucun 
doute celui des Euméninés, de beaucoup le plus riche en espèces ; la plupart, appartenant aux genres Odynerus et Eumenes, sont familières à tous ceux qui étudient les Hyménoptères des régions tempérées. Toutes creusent des

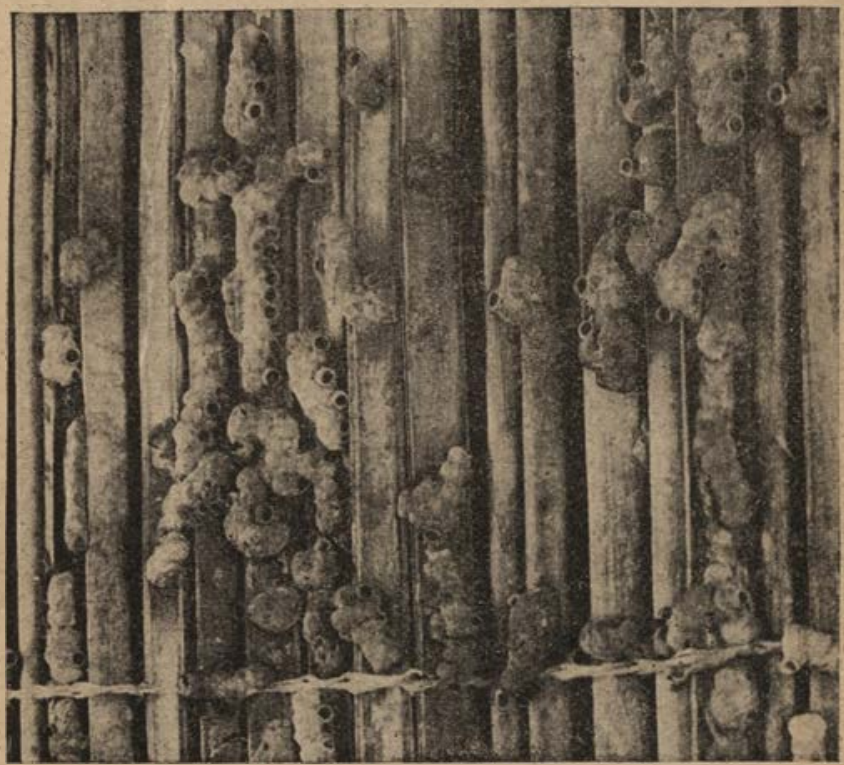

Fig. 11. - Nids d'argile de Synagris cornuta, sur le chaume d'une hutte au Congo (d'après Bequaert, photo H. O. Lang). Certains de ces nids montrent un orifice pédiculé, par oú les larves sont nourries.

alvéoles dans le sol, occupent les cavités des troncs d'arbres ou édifient, en des endroils nus, d’úlégants alvéoles en argile. Toutes pondent d'abord, puis apportent, pous 
la larve attendue, des provisions consistant en chenilles paralysées, renversànt ainsi l'ordre habituellement suivi par la plupart des autres guêpes solitaires: la proie d'abord, l'œuf ensuite. C'est de ce groupe commun des Euméninés que, d'après Ducke (1914), descendent les oinq sous-familles d'espèces sociales, suivant au moins cinq ou six lignes indépendantes.

Certains genres sociaux dérivent d'ancêtres analogues au genre Eumenes, d'autres d'ancêtres analogues au genre Odynerus. Mais, même chez les espèces actuelles de la sous-famille des Euméninés, quelques pas, très significatifs, ont été faits dans la direction de la vie sociale. RouBAUD a trouvé que certaines espèces africaines d'Odynerus et de Synagris ne suivent pas la méthode habituelle d'approvisionnement en masse, - la quantité de chenilles paralysées nécessaire à la larve étant placée auprès de l'œuf, puis l'alvéole étant fermé, - mais qu'elles ont adopté effectivement la méthode de l'approvisionnement progressif, nourrissant jour après jour la larve qui se déreloppe. Ceci se rapproche, de façon nette et significative, des mœurs sociales. Cette manière de se comporter paraît trouver sa source dans une pénurie saisonnière die proies, ainsi que l'a montré Roubacd (1908, 1910, 1916), dans le cas de Synagris spiniventris et calida.

La socialisation est un peu plus avancée chez les Zéthi. nées, qui ont été étudiées récemment par Ducke ( 191 q), Howes $\left(\mathrm{I}^{\mathrm{I}} \mathrm{7}\right.$ ) el F.-X. Williams (IgIg). D'après H. de SAussure (1852, 1875), les alvéoles de Zethus (Didymogastra) romandinus, de l'Amérique du Sud, sont faits, non d'argile comme ceux des Eumeninés, mais d'um mélange de fibres ligneuses et de gommes. Suivant FonBEs (1885), Zethus cyanopterus, de l'Inde Orientale, fait 
ses alvéoles avec de pelits morceaux de feuilles. Un travail sur cette même espèce, que Williams a étudiée aux Philippines, a paru dans ma "Social Life among the Insects » (1922). Il montre que la femelle nourrit sa larve, jour après jour, avec de petites chenilles qu'elle a partiellement dévorées. Elle veille sur sa larve avec soin, et, tant que cette dernière est de petite taille et que l'espace est suffisant, elle dort dans l'alvéole. Quand la larve est complètement formée, elle l'enferme dans l'alvéole et commence à en bâtir un autre.

Le mémoire de Ducke est si intéressant qu'il faut en signaler la majeure partie. Ducre a souvent trouvé dans le Parà le nid de Zelhusculus lobulatus qui "consiste en alvéoles presque cylindriques, bâtis en lianes, les alvéoles étant ouverts d'un côté, en partie de l'autre el étant faits de débris arrondis de feuilles, irréguliers et paraissant cimentés par une matière analegue à la gomme. Ces alvéoles ont un aspect particulier, galeux pourrait-on dire; au début, ils sont verts; mais en peu de jours, ils deviennent bruns et durcissent. Aussitot que l'alvéole en construction a atteint la moitié de sa hauteur, la guêpe pond un æuf sur le plancher et poursuit la construction qui s'achève juste au moment oì la jeune larve éclot. CelleCı est nourrie avec des larves d'insectes, très petites au début, puis plus grosses, larves telles que des chenilles, et qui ont été paralysées par la guêpe. Sur la larve développée, la mère ferme l'alvéole, avec les mêmes matériaux qui ont servi à la construction, la substance résineuse étant toutefois plus abordante. Les nids sont presque toujours construits par plusicurs guêpes réunies; souvent plus d'une douzaine. Je vis une fois deux femelles travaillant à peu de distance l'une de l'autre; checune construisait des alvéoles qui furent réunis plus tard par l'addition d'alvéoles intermédiaires, et ne formèrent plis qu'un seul édifice commun. Dans les nids les plus grands, on trouve males et femelles et il y demeure au moins une partie de la nouvelle génération, dont les jeunes femelles agrandiront. l'édifice. La femelle qui bătit un nid, passe les nuits et les jours de pluie dans l'alvéole ouvert sur le plancher duquel git la larve qui se trouve ainsi protégée par le corps de la inère. L'abdomen est dirigé vers le tias, tandis que la téte ê 
les antennes sortent de l'alvéole. Les mảles prennent une position semblable dans les alvéoles vides. ”

Les travaux de Ducke et Williams montreni que quelques-uns au moins des Zéthinés sont, à tous égards des guêpes sociales. Ceux-ci sont nettement aussi avancés que certains Vespidés sociaux de la première sous-famille, les Sténogastrinés d'Orient. Williams, qui a étudié de façon un peu fragmentaire quatre espèces des Philippines appartenant à ce groupe et présentant une extraordinaire diversité dans la nidification, a montré par là que ces insectes seraient très primitifs. Trois de ces espèces emploient de la pulpe de bois ou de carton pour faire leur nid, et sont donc à ce point de vue, au mème degré que les quatre autres sous-familles de guêpes sociales; mais ane espèce (Stenogaster varipictus) fait ses alvéoles, comme beaucoup d'Euméninés, on grande partie avec de la terre.

Les Epiponinés ou Polybiince sont un groupe tronıal, vaste et hétérogène, comprenant un nombre de genres (23) et d'espèces beaucoup plus grandi qu'aucune autre sous-famille de guêpes sociales, et s'étageant depuis des formes socialement très primitives, comme les Belonogaster d'Afrique, jusqu'à des formes hautement spécialisées, comme les Nectarina et les Chartergus néotropicaux. On observe également de grandes différences dans l'architecture du nid; celui-ci, dans les genres plus primitifs, consiste en un rayon unique, non enveloppé; formé de cellules hexagonales, et fixé à un support quelconque par un pédoncule; dans les formes plus avancées, il comprend un seul ou plusieurs rayons superposés et enfermés dans une envcloppe munie d'un orifice pour l'entrée et la sortie. Dans certains cas, les rayons sont pédon- 


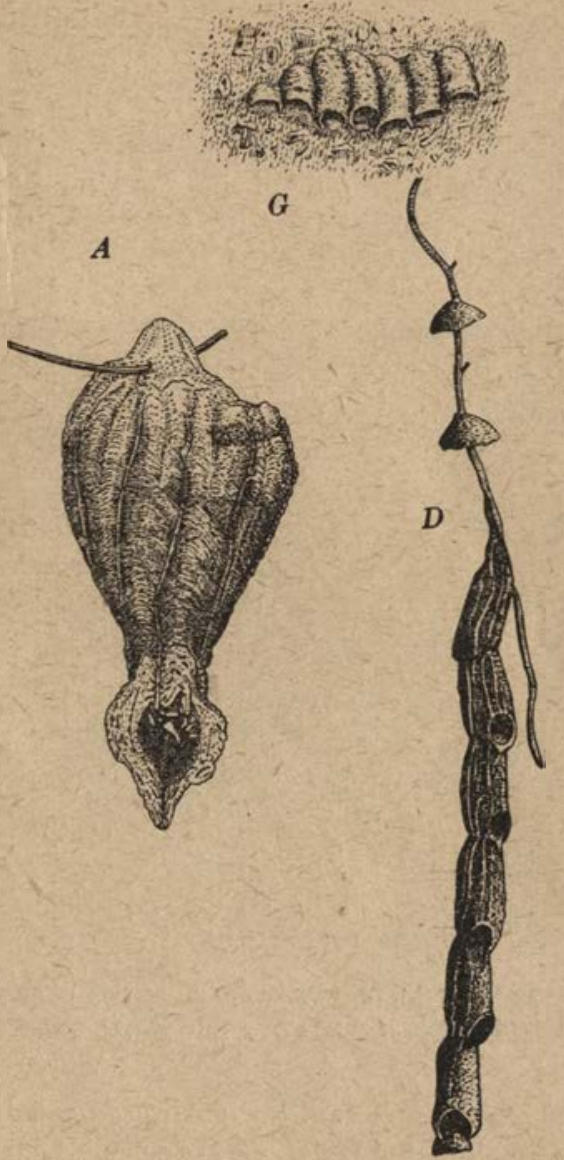

Fig. 12. - Nids de Sténogastrinés des Philippines (d'après F. X. Williams). Nid de Stenogaster micans, var. luzonensis; D, nid de Stenogaster sp.; G, nid de St. varipictus sur l'écorce d'un arbre. 
culés (stélocy'llaires); dans d'autres, ils sont fixés directement sur le support ou sur l'enveloppe (phragmocyttaires). Dans presque tous les cas, le nid est entièrement fait en papier. Mais un petit nombre d'espèces américaines de Polybia emploient de l'argile. Sauf celui de Polybia atra, le nid est toujours au-dessus du sol et fixé

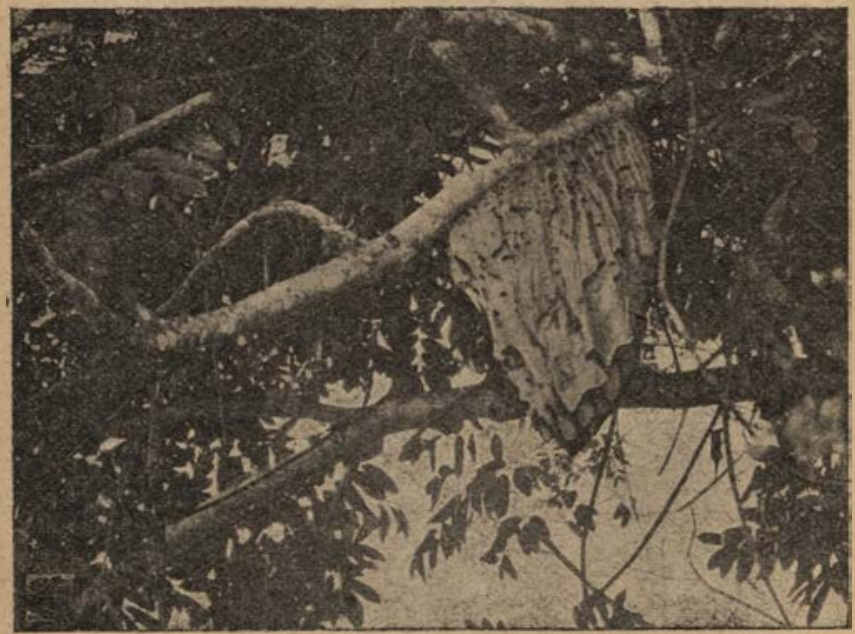

Fig. 13. - Nid de Polybioides melæna, au Congo (d'après Bequaert, phot. H. O. Lang).

aux branches ou aux feuilles d'arbres, ou à la face inférieure de quelqu'abri (toits, terrasses, etc.). D'après RouBAUD ( 1916$)$, il est de règle chez les formes primitives comme Belonogaster, qu'une seule femelle fécondée commence à bâtir le nid en construisant une alvéole unique, 
pédonculée; puis elle accroît le rayon, en ajoutant de nouvelles cellules périphériques, disposées en cercles concentriques ; mais il n'est pas rare que d'autres femelles viennent se joindre à la fondatrice, avant que le travail soit poussé très loin. Chaque larve est nourrie avec des houlettes de chenilles triturées, durant tout son développement; ensuite elle ferme sa cellule, en filant sur l'orifice un houchon convexe, el elle forme son cocoll. Les femelles qui sortent des cocons sont, comme la mère, toutes en possession d'ovaires bien développés, et prêtes à être fécondées. Autrement dit, toutes les femelles de la colonie sont physiologiquement égales, et, même si l'on observe entre elles quelque différence de taille, céla n'a rien à voir avec leur fécondité. Les colonies sont peu f́tendues, les nids n'ayant pas, en général, plus de 5 ò fio alvéoles, rarement ils arrivent jusqu'à 200 ou 300 . bans les colonies plus vastes, il y a une division rudimentaire dı travail, les femelles les plus âgées étant consacrées à la ponte, tandis que les plus jeunes vont en quête de la nourriture et des matériaux destinés au nid, et celles qui vienuent d'apparaître nourrissent les larves et entretiennent le nid. Les màles aussi restent au nid; mais ils se comportent comme des parasites, s'emparant de la nourriture aussitôt que les femelles en apportent. Belonogaster est considéré comme une guêpe polygyne, parce que chacune de ses colonies contient plusieurs femelles fécondées. Quand la colonie a atteint son développement maximum, les femelles l'abandonnent par petits groupes, et fondent de nouveaux nids, soit ensemWe, soit séparément. Ce phénomène est connu sous le nom d' " essaimage ", et ne se produit que chez les guê- 
pes des tropiques, où il paraît être une adaptation à des conditions climatiques favorables.

Dans les genres d'Epiponinés plus élevés de l'Amérique du Sud, au contraire, les femelles ne sont pas toutes semblables; elles sont diflérenciées en femelles vraies, ou reines (qui ont des ovaires bien développés et qui peuvent être fécondées), et des ouvrières, aux ovaires imparfaitement développés, et qui sont stériles ou qui produisent des ocufs non fécondés et donnant des mâles. D'après H. et R. von hierixg ( $189^{6}$, Igno) et Ducke (1910, 1914), beaucoup de ces guèpes sont polygynes et elles forment des nidrs el des colonies nouvelles en envoyant au loin des essaims d'ouvrières avec une ou deux douzaines de reines. Les colonies deviennent souvent Irès populcuses et comprennent des centaines ou'même des milliers d'individus.

Certaines espèces (Nectinina, Polybia) ont lhahitude d'accumuler du miel en quantité considérable dans leur: rayons; pour d'autres, on sait qu'elles capturent et tuent de nombreux lermites mâles et fomelles, et des fourmis màles, et qu'elles les emmagasinent dans les parois du nid, ou même dans les rayons, formanł ainsi une réserve de nourriture qui est reprise quand cela devient nécessaire.

Les Ropalidinés sont un petil groupe comprenant seulemenl trois genres de guêpes paléotropicales, le micux connu des trois étant le genre Ropalidia. Ce sont des formes primitives, qui construisent un rayon unique, nu, comme celui des Belonogaster, et qui nourrissent leurs jeunes avec des insectes malaxés et mis en boulettes. Les colonies polygynes, sont peut étendues; mais, d'après Roubaun (igi6), on peut y tistinguer de vraies nuvrieres, 
quoique celles-ci soient en nombre très restreint en conıparaison des femelles vraies. Dans quelques espèces, il semble que se produisent des essaimages.

Les Polistinés ne sont représentés que par deux genres. L'un d'eux, Polistes, est cosmopolite et, comme Ropalidia el Belonogaster, construit un seul rayon nu, suspendu à la face inférieure de quelqu'abri, par un pédoncule cenIral ou excentrique. Comme plusieurs espèces vivent è Furope et aux Etats-Unis, les mœurs du genre sont trìs bien connues. Ine seule femelle, ou reine, fonde le nid el le construit au début. Ses descendants soni des ouvrières, mais elle parait pondre aussi des oufs produisant des mâles. Les femelles vraies sont généralement plusieurs dans les colonies des espèces tropicales, qu'on peut regarder par suite comme polygynes, et peul-être alors ces colonic's essaiment-elles. Vais, dans les régions tempéríes, la colonie de Polistes a un dévelnppement annuel el n'est généralement pas très populeuse. Les jeunes femelles, fécondées l'été précédent, passent l'hiver éparses el cachées sous l'écoree d'un arbre ou dans les creux des murailles, d'où elles ressortent au printemps, pour forider les colonies nouvelles. On connaît plusieurs espèces, mème dans les régions tempérées, qui emmagasinenl dans leur: rayous de petites quantités de miel.

Comme les Polistince, la sous-famille des lespince ne comprend que deux genres: Vesp: el Provespa. Le premier genre, le seul, en dehors des Polistes, qui se rencontre dans la zone tempérée norl, est formé d'espèces comprenant les gruèpes sociales les plus grandes et les plus typiques. Autant qu'on sache, elles sont strictement monogyres. Le nid est fondé par une seule grosse 
femelle, el consiste au début en un petit rayon pendant, comme celui des Polistes; mais, tant qu'il n'est encore formé que d'un petit nombre de cellules, il s'entoure d'une enveloppe plus ou moins sphérique. Les premiers aufs pondus donnent des ouvrières, qui sont beaucoup plus petites que la mère et sont infécondes. Elles restent a vec la mère, agrandissent le nid, rayons et enveloppe ; et, pour installer la couvée qui s'accroît rapidement, elles construisent des rayons additionnels, en une série dirigée du haut vers le bas, chaque nouveau rayon étant fixé au rayon qui le surmonte, par un ou plusieurs pédoncules (stélocyttaire). Au début, il naît un grand nombre d'ouvrières. Mais quand l'été s'avance, apparaissent les mâles et les femelles. Les femelles étant de plus grande taille que les ouvrières, les alvéoles où elles se développent sont beaucoup plus vastes que ceux des ouvrières. Après l'accouplement des femelles et des mâles, la colonie disparaît, à l'exception des femelles fécondées, qui hivernent comme les femelles de Polistes, it qui fondent de nouvelles colonies au printemps suivant. II s'est. donc formé, chez les Vespinés, une caste distincte d'ouvrières, ses membres pouvant, toutefois, occasionnellement ou peutêtre d'une façon générale, pondre des œufs qui donneront des mảles. On divise habituellemient les espèces de guêpes en deux groupes, l'un ayant les joues allongées, l'autre les ayant très courtes. Dans l'Europe et l'Amérique du Nord, les espèces de la première forme construisent de façon régulière des nids aériens, au-dessus du sol ; celles du second type font leurs nids dans des cavités qu'elles creusent dans le sol. A la fin de l'été, les colonies sont souvent très populeuses ( 3 à 5.000 individus).

Sur cerlaines particularités des Vespidés sociaux, telles 
que leurs habitudes d'alimentation, leurs castes et le polymorphisme rudimentaire qu'elles présentent, - tous sujets que j'ai à peine effleurés dans les précédents paragraphes, - j'aurai l'occasion de revenir encore dans les chapitres suivants, en liaison avec des phénomènes simi. laires que présentent d'autres insectes sociaux. 


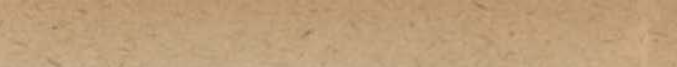

a. 


\section{C.HAPITRE III}

\section{L'EVOLUTION DES ABEILLES}

Vlinités avec le: Sphecidar ; discussion, parallélisme des moeurs ivec les Vespida. - Les Allodape, les Halictus (nombre de générations of polymorphisme), les Bombinæ, Meliponinie, ef Apince. - Formes fossiles. - Progrès de la nidification. La production de la cire. - Modes divers de constitution des co'onies chez les formes sociales supérieures.

Les Vespidés, succinctement étudiés dans la leçon précédente, forment un groupe d'Aculéates bien délimité, renfermant un nombre considérable d'espèces solilaires, et un nombre comparativement restreint d'espèces sociales, principalement tropicales. Néanmoins, les différentes sous-familles, telles qu'elles sont établies, présentent une évolution progressive des moeurs sociales, depuis les types très primitifs, comme les Synngris, les Zéthinés et les Sté. nogastrinés, jusqu'au genre lespa, qui est netlement spécialisé. La suite du développement est caractérisée pat l'apparition graduelle et la différenciation d'une caste d'ouvrières, par la complication croissante de l'architecture du nid, par l'augmentation de population des colonies, et par la plus grande taille de la femelle féconde, ou reine, comme on le voit dans le genre Vespu. Certes, les colonies de quelques Polistinés, squécialement des Epi- 
poninés et des Vespinés, ont atteint un stade d'intégration sociale très définie; nous sommes cependant obligés de reconnaître qu'il n'est pas d'un ordre très élèvé.

La différenciation de la caste ouvrière est extrêmement faible chez les Epiponinés, et les colonies de Vespinés et de Leaucoup de Polistinés ont un développement annuel; c'est là une condition qui fixe des limites très définies à l'éiendue de la population dans la colonie, et qui, en outre, retarde beaucoup, ou même empêche, tout progrès ultérieur dans le développement social. Il est probable qu'un tel progrès n'est désormais possible parmi les Vespidés, que pour quelques-uns des genres les plus primitifs d'Epiponinés de l'Afrique et de l'Amérique tropicales.

Si nous considérons maintenant la seconde famille des tculéales, chez laquelle se sont aussi développées les mueurs sociales, les Abeilles on Apidés - Anthophiles ou Mellifères de Latreille - nous nous trouvons en présence d'une lâche bien plus difficile. L'existence ríelle de ce groupe - (quelque 20.000 espèces, d'après l'eslimation de Friese (in23)) - est mal définie, et, comme l'élude allentive de leurs mours n'a été faite que pour quelques rares genres, surtout européens, toute généralisation élhologique est prématurée et provisoire. A l'époque acluelle, il n'y a parmi les Aculéates, aucune autre famille qui déploie une aussi splendide floraison d'espèces sur notre planète; elle est toutefois surpassée par les Formicidés, quant au nombre des individus. La majeure parlie des espècés de Mellifères est confinée dans deux contrées étroitement limitées, correspondant aux parties les plus chaudes des zones tempérées nord et sud. La faune dabeilles tropicales est bien plus réduite, sur- 
tout dans le vieux continent, et le nombre des espèces diminue constamment à partir des zones tempérées chaudes et vers les pôles.

Les difficultés de notre tâche sont accrues par l'embarras où sont placés actuellement les taxonomistes, du seul fait des conditions éthologiques. La famille des Apidés est entièrement formée, d'une part d'espèces qui recherchent activement le nectar et le pollen, donc intimement associées aux fleurs, d'autre part, d'espèces qui ont perdu cette hahitude, et sont devenus parasites d'autres espèces plus ou moins voisines. Dans le premier groupe, apparaissent des modifications remarquables des organes et de l'anatomie, de la langue par exemple, et des pattes postérieures; en même temps se développe la pilosité du corps et des appendices, qui facilite la récolte d'un aliment si spécial. Le second groupe, au contraire, présente une dégénérescence de ces mêmes modifications ou leur suppression totale. D'autres organes sont d'une extraordinaire uniformité dans les deux groupes, telles les ailes et leur nervation. Rien ne peut être plus défavorable pour les taxonomistes ; car on admet comme loi générale qu'on définit et caractérise très mal les sous-familles, tribus et genres, au moyen de structures d'origine adaptative; d'autre part, il est, tout au moins, déconcertant de voir ces mêmes caractères adaptatifs faire défaut dans les espèces parasites, qui, dians beaucoup de cas, sont certainement dérivées, phylogénétiquement, des genres auxquels appartiennent leurs hôtes (voir WheEler, rgrga) et doivent donc être placées à leurs côtés dans une classification naturelle. Considérons en outre qu'il existe plusieurs grenres d'abeilles, cosmopolites, ou à très large extension, comprenant de très nombreuses espèces fort étroitement 
allices entre elles (Megachile, Halictus, fudrenn, Anthophora, Prosopis, Sphecodes, Colletes, etc...) ; que beaucoup dautres genres particuliers ou locaux sont monoIypiques, ou sont formés d'un petit nombre d'espèces aux caractères instables, ou difficiles à apprécier ; et nous comprendrons pourquoi nul n’a pu établir, parmi les sous-familles el les fribus d'Apidés, une classification qui ait été acceptée par lout le monde. En fait, de telles tentatives sont prématurées ; iar les taxonomistes eux-mênes connaissent incomplètement les grandes faunes d'abeilles de lous les continents, sauf celles de l'Europe. Ceci est particulièrement vrai pour les faunes de l'Australie et de l'Amérique du sud, oi l'on trouve de nombreux types primilifs, qui, en demier ressorl, projeiteraient une grande clarté sur les relations des genres confinés actuellement dans les autres parties du monde.

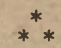

Depuis longtemps, les avis sonl parfaitement unanimes en ce qui concerne les affinités structurales très étroites des Abeilles et des Sphécidés ou Fouisseurs (I). Ces affinités sont si accentuées, que, dans la classification de

(1) I.c seul auteur qui, à ma connaissance, ait repoussé celle opinion est Börner (1919). Il place les Wheilles il colté des Vespidés, parce qu'elles ne possedent pas de brosse aux palles poslérieures. Evidemment, l'absence de cel organe ne prouve pas que les Ipidés ne sont pas étroilement liés a des formes inalogues aux Sphécidés, ou qu'ils n'en dérivent pas. IInNt.mscu (1924) a suggéré que la structure très parliculière du lihia el du mélatarse des palles poslérieures de l'abeille repićsenterait un strigile hauteınent modifić et aclapté pour recueillir lo pollen sur la surface du corpus; s'il était élahli, ce fait répondrait à l'ohjection de Bönnfr. 
BraDley (CoMstock, r924), les Apidés sont considérés comme l'une des familles de la section des Sphécoïdés, et que des hyménoptéristes fort avertis ont décrit parfois les abeilles comme des Fouisseurs (I).

Il esl très frappant de voir heaucoup d'abeilles possćder des habitudes qui sont nettement celles des Fouisseurs ; tels les genres parasites, à système pileux moins développé (Sphecodes, Vomada, Epeolus, etc), ou les types, conme prosopis, qui ingurgitent le nectar ef le pollen et chez lesquels fail défaut lappareil collecteur habituel.

C'est pourquoi les lyménoptéristes ont été amenés à faire dériver les abeilles des Fouisseurs et à leur supposer des ancètres entomophages. Dans la seconde moitié du siècle dernier, pendant la période romantique de spéculations darwiniennes, cette hypothèse fut envisagée dans deux mémoires pleins d'idées de H. Mürlen ( $18-2$, I $88 \mathrm{r}$ ), l'auteur bien connu d'un bel ouvrage sur les relations des abeilles et des fleurs. Les Sphécidés adultes sont d'adroits explorateurs des fleurs, et MüLțer suggère qu'ils ont acquis plus d'habileté à découvrir et à extraire le nectar des fleurs, en poursuivant les insectes dont ils font leur proie, en creusant et en édifiant des alvéoles, tandis que s'accroissait, en même temps, la stature de leur corps.

La conception, suivant laquelle les abeilles dérivent des Fouisseurs, a été envisagéc à plusieurs reprises par

(1) Ainsi, l'espèce type de Neolarra (pruinosa) a élé regardćc comme une guẻpe alliée aux Bembicinés, ou Larrinés, par A Ámitu (1890)) el Fox (1893); mais Bulien (1896) a reconnu ensuite que c'était une abeille, voisinc du genre Phileremulus. Un genre d'abeilles du Chili, Lipanthus, (Psrenythia) était aussi placé au début parmi les Fouisseurs. 
Friese, et d'une façon très complète dans son récent oivvage (1 $\left.9_{2}^{2} 3\right)$. Il affirme que les Apidés ont une origine diphylétique et peut-ètre même triphỵlétique, et il présente trois arbres généalogiques de la famille, l'un établi par lui-même, et basé sur la nervation des ailes, un autre par Tosi (1 895$)$ et basé sur la structure anatomique du grésier, ou proventricule, et un autre par Lavghofer (18.97), basé sur la structure de la langue. Ces trois schémas ont élé conçus indépendamment les uns des autres, et tous trois s'accordent à faire descendre les abeilles de deux souches indépendantes hypothétiques de Fouisseurs, l'une ayant conduit aux genres Prosopis, Colletes, etc., l'autre aux genres Sphecodes, Halictus, elc... (I).

Friese indique un troisième noyau ancestral possible pour les abeilles, parmi les Vespidés (Orlynerus), à partir du genre australien très particulier Hylıodes, qui, aulant qu'on le sache, n'a domné naissance à aucune autre forme. En attribuant ainsi aux Apidés une origine polyphỵlétique, on fait naître l'idée d'une convergence, peutêtre masquée ou dissimulée, ou d'un parallélisme, dans le développement, qui serait dù à l'existence d'habitudes anthophiles chez certains groupes aberrants de Sphécidés ou même de Vespidés.

Quoique des opinions analogues à celles de MüLLEn et de Friese aient été soutenues par von Alten (igro) et d'aulres, on pourrait même aller plus loin et affirmer que

(1) Friese considère les Sphecodes comme une espèce très primilive d'aheilles non parasites, mais prédatrices. Ses arguments, fort intèressants mais nullement convaincants, renouvellent le débat concermant les mœurs des Sphecodes, qui paraissaient ètre bien établies après les recherches de Nibi. six, Fentox, Breitenich el aulres (voir Wheeler, 1919 a). 
les Apidés ne dérivent nullement de la famille des Sphécidés, telle qu'elle est actuellement constituée, mais qu'ils sont eux-mèmes simplement l'équivalent taxonomique d'une sous-famille ou (s'ils sont diphylétiques) de deux sous-familles anthophiles de Sphécidés, au mème degré que n'importe quelle autre sous-famille entomophage acluelle, par exemple les Benbicinés, Crabroninés, Sphécinés, elc... Il n'est pas parfaitement prouvé que les Sphécidés nourrissent tonjours leurs jeunes avec des insectes morts ou paralysés. Si lés Aculéates descendent des Phỵtophages directement, el non à travers les Térébrants, less hahiludes régétariennes des Abeilles seraient primitives, et rien ne nous prouve le contraire. C'est la position qu'a prise actuellement Roubsun ( 1918 ).

Deux groupes de faits semblent en quelque mesure appuyer celte conception. D'ume part, les Sphécidés actucls sont forurés d'un ensemble de sous-familles, olı, suivant KoHL (1896) et Hinnlinscir (1924), de groupes de genres, qui sont tous, autiul que nous sachions, trop haulement spécialisés, tant au point de vue structural qu'au point de vue éthologique, pour servir d'ancèlres aux Apidés; et, d'autre part, les données pileontologiques prouvent que, pendant tout le Tertiaire au moins, les rapports des Sphécidés et des $\Lambda$ pidés sont essentiellement les mêmes qu'à l'éporque actuelle. Cockeñer. (1906, 1909, 1917) a trouvé dans l'ambre de la Baltique (Oligocène inférieur) des Sphécidés appartenant aux genres. bien commus Crabro el l'ison, et en même lemps des abeilles nombreuses el hautement spécialisées (I:lectrapis, Prolobombus, ('halcobombus, Sophrobombus, Ctenoplectrella et Glyplapis); dans les schistes de Florissant (Miocene), il a décrit des Fouisseurs des sous-familles Crabronina, Pemphredoninx, Philanthillie, Nyssonina et Sphecina, contemporains de genres hautement spécialisés d'abeilles solitaires tels que Ceratina, Anthiclium, Dianthidium, Heriades, Andrena, Calyplapis (voisin d'Andrenu); et dans le - schistes dOEningen (Miocène), il a décrit des. abeilles des genres actuels Lithurgus, Xylocopa et Andrena. II nous faut donc chercher les ancêtres des abeilles et des Sphéci- 
dés dans les lormalions crétacées ou peut-être mème prêcrétacées (1).

Il faut admettre cependant qu'il y a quelque vérité dans l'opinion ancienne d'une transformation des abeilles ancestrales, qui d'entomophages seraient devenues anthophiles, tout au moins lorsqu'on considère les Masaridinés parmi les Vespidés (2).

J'ai insisté dans ma dernière leçon, sur le fait que le genre primitif Pseudomasaris, des Etats du Sud-Ouest, emmagasine des insectes comme nourriture pour ses jeunes; tandis que les genres moins primitifs Masaris, Celonites, Ceramius, de l'Europe et de l'Afrique, sont, de l'avis généra!, strictement anthophiles comme les abeilles. Il est bien évident, comme le laisse supposer Ribaud (1922), que l'insecte adulte impose à ses descondanls le genre-de l'aliment; et-que le Sphécirle adulte, difforrant en cela de beaucoup de Masaridinés et des abeilles, a en réalité un régime double, puisqu'il se nourrit, et de nectar, el d'insectes. Dans la plupart des cas, le second régime est limité simplement à une malaxation de la proie paralysée clont ils goûtent à peine; c'est là, pourrait-on dire, une réminiscence du régime larvaire qu'a eu ce même Sphécide.

Si nous considérons les mœurs au point de vue de la reproduclion, mœurs qui ont amené le développement des sociétés, nous trouvons un parallélisme extraordinaire

(1) Etant donné que les abeilles n'ont pu se développer qu'en symbiose avec les plantes à fleurs, la paléobotanique pourrait fournir quelque lumière sur l'époque la plus probable de l'évoJution des $\Lambda$ pidés. Le seul travail récent à retenir que j'aic rencontré à ce sujet est celui de IVieland (1924).

(2) II annlinsch $(1924$, p. 781$)$ soutient netlement celle opinion: "Il cst tris probable, dit-il, que les Apides remonlent ì des formes plus anciennes de Sphegides primitifs, les strigiles des palles postérieures de ceux-ci étant peut-ètre converlis elı un organe compliqué ressemb'ant à une brosse. Dans ces formes, le rigime végétarien ne dérive certainement pas de la phytophagie primitive des Symphytes, mais du mode de vie des formes zoophages, qui prennent soin de leur.couvée." 
entre l'ensemble des Apidés et les Vespidés tels que nous les avons étudiés dans notre précédente leçon. En étu. diant la progression qui va de la vie solitaire, telle qup l'ont $9^{5} \%$ des espèces, aux habitudes de la forme la plus hautement socialisée, l'abeille domestique, nous retrouvons, pour ainsi dire, les mèmes motifs divers que chez les guêpes, mais transposés dans un autre ton. Chacune des millier's d'espèces solitaires a, dans ses mœurs, quelque particularité qui lui est propre; mais la différence est généralement si minime que, dans l'ensemble, les habitudes sont très uniformes. A l'exception d'abeilles parasites qui dérivent secondairement de formes non-parasites, toutes les abeilles solitaires font leurs nids, soit dans le sol, soit dans des cavités de plantes ou des crevasses de murailles, etc..., ou bien construisent des alvéoles en terre ou en résines. Quelques espèces revêtent les creux de leurs nids avec des morceaux de feuilles ou de pétales de fleurs, avec des poils végétaux ou des particules ligneuses, ou avec des lamelles d'une substance qu'elles secrètent et qui ressemble au celluloïde ou à la baudruche. Comme on le voit, ces matériaux proviennent en majeure partie de végétaux. Le nid est, en général, formé de quelques alvéoles cylindriques ou elliptiques, disposés en séries linéaires ou, plus rarement, en une masse compacte ; dès qu'un alvéole est achevé, il est muni d'une houlelte, ou d'une particule conique de pollen, ou de pollen imbibé de miel (et appelé pain d'abeille); un œuf est déposé à la surface, et l'alvéole est clos. Nous retrouvons là ce tỵpe de l'approvisionnement en masse, qu'emploient les guêpes solitaires, analogue à celui des Euméninés ou des Sphécidés, sauf que les provisions du jeune sont faites de matières végétales et non plus animales. 
Cependant, le pollen et le miel sont une nourriture idéale, car le premier est riche en protéines et en huiles, le second en sucre et en eau, et tous les deux renferment des sels divers,-en quantité suffisante, pour la croissance des
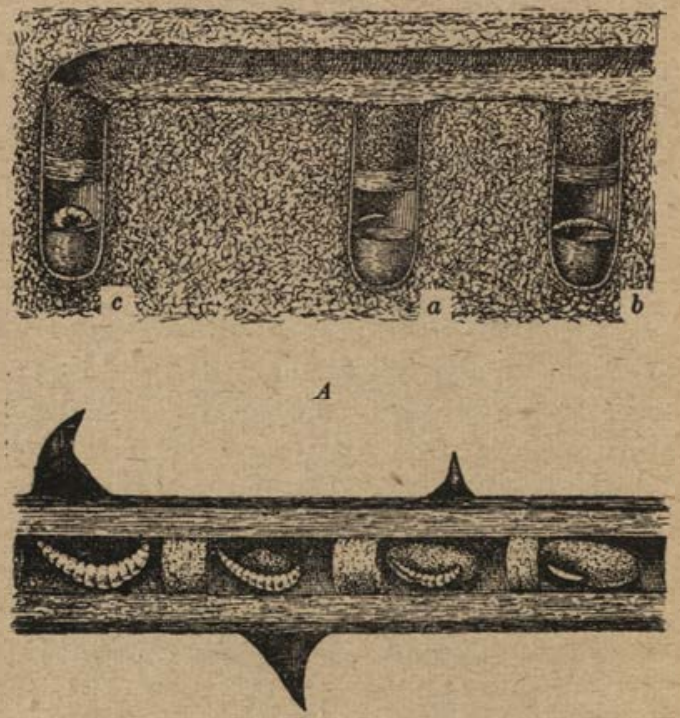

$B$

Fig. 14. - Nids d'abeilles solitaires. A, nid de Colletes succinctus, dans le sol (d'après Valéry MAYET); $a$, cellule approvisionnee, contenant un œuf ; $b$, cellule renformant une jeune larve ; $c$, larve plus avancée ; B, nid de Ceratina cucurbitacea, dans une tige de ronce, montrant. l'œuf et divers stades larvaires approvisionnés de pain d'abeilles (d'après Dufour et PERRIS).

larres. Comme dans le cas des guêpes solitaires, la mère abeille meurt avant que naisse sa progéniture. 
Chez beaucoup de Spliécidés solitaires (Bember, Stizus, PhiInnthus, etc...), qui font leurs nids dans lo sol, los femellos inslallent leurs terriers très près les uns dos autres, mais chacune s'occupe seulement de sa propre couvée et s'oppose d lintrusion de ses roisines. Il en est de même absolument che\%. les abeilles de nombreux genres (Panurgus, Eucera, Osmia, Andrena, Spinoliella, Melitoma, Pachycentris, etc...), ainsi que l'ont vu bien des observateurs. Dans certains de ces cas, plusieurs femelles utilisent une seule entrée commune, pour les alvéoles, mais rien n'indique qu'un tel "parocisme " soit un pas fait dans le sens de la vie sociale. C'est en général l'expression d'une vague tendance grégaire, d'une tendance à l'association; à moins que ce ne soit le résultat d'une attraclion exercée sur plusieurs abeilles femelles de la même espéce par un ensemble particulier et limité de conditions ambiantes définies. Il est très probable que c'est un cas d'association de ce type qui est constitué par les espèces du genre Euglossa, souvent mentionnées dans lit littcrature et dont on sait qu'elles construisent des groupes d'alvéoles ellipsoïdaux avec du fumier, de la terre et de la résine. Litreille (1809) plaça près des Bombinés ce genre de grands et beaux insectes néotropicaux, qu'il a le premier décrit, en indiquant le développement de la corbula aux tibias postérieurs, structure qu'on ne retrouve chez aucune autre abeille, sauf les espèces sociales supérieures; et c'est pourquoi cet auleur, avec Lucas (1878), et d'auIres, ont été conduits à supposer que les Euglossa devaient être sociales. Lepeletier (1827), qui ne put trouver les brosses a cire aux pattes postéricures, a une opinion toute différente. Möвıus (1856) a mème revendiqué la découverte d'un revêtement cireux dans les alvéoles de E. surinamensis. Plus récemment, Schulz (1902), Duck: (1902, 1903, 1905), R. von Ihering (1904) et Schrotriy (1922), qui ont observé les Euglossa dans l'Amérique du Sud, conviennent qu'ils ne sont point sociaux, bien que certaines espècos, par exemple $E$. nigrita, édifient de vastes nids de plus de 200 alvéoles. Dans ce cas, le nid ost fait par plusieurs lemelles, mais chacune ne s'occupe que do sa propre couvée; l'entrée seule est commune. On n'a point confirmé l'existence de cire dans les alvéoles, quoique Frisse (1923, p. 76) ait élabli que les basilarses postérieurs portent des brosses à cire chez Euglossa, Eumorpha et Eulæma.

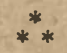

Sauf de rares cas imparfaitement décrits et par suite 
douteux, toutes les abeilles sociales connues appartienment à cinq groupes, comprenant certaines espèces des genres Allodape et Halictus et toutes les espèces des trois familles hautement différenciées: Bombinés, Méliponinés, et Apinés. Les trois dernières sont depuis longtemps connues comme éminemment sociales, et leurs mœurs ont été si souvent décrites que j'en indiquerai seulement quelques rares aspects. Il nous suffira de dire pour l'instant qu'une origine commune n'est nullement certaine, pour les habitudes sociales de ces sous-familles, D'ailleurs, il n'y a pas de connexion phylogénétique entre les genres Allodape et Halictus, qui appartiennent à des groupes d'abeilles très éloignés l'un de l'autre, le premier aux Tarsilégidés, le second aux Podilégidés, et aucun n'est l'ancêtre direct des Bombinés, Méliponinés et Apinés. Nous pouvons donc conclure, tout au moins jusqu'à preuve du contraire, que les mours sociales ont apparu dans la famille des Apidés en cinq occasions différentes, an moins, tout comme nous avons trouvé cinq sources indépendantes du développement social chez les Diploptères ou Vespidés. Etant donné l'extraordinaire intérêt qui s'attache aux genres 1llodipe et Halictus, je consacrerai une grande partic de celte leşon à la discussion de leurs mœurs.

Les abeilles sud-africaines du genre Allodape forment une remarquable transition entre les modes de vie solitaire et social, mais d'un type qui diffère absolument de celui du genre Halictus ou des trois sous-familles supérieures. Les espèces sont petites et se rapprochent plutôt fortement de nos abeilles menuisières du genre Ceratina; de l'Europe et de l'Amérique du Nord; mais leurs larves se signalent par la présence de paires d'appendices avec 
lesquels elles se maintiement sur leur nourriture. Bien quayant déjà publié ( 1923 ) la conmmnication sur ces insectes que le $D^{r}$ Hans Bratiss a eu l'obligeance de m'adresser, il y a guelques années, je me permets de la reproduire ici, tant elle est suggestive :

"Les espèces du genre Allodape nichent dans les tiges sèches et creuses de végétaux, très rarement dans des galeries souterraines. Dans les deux cas, elles creusent des cavités, ou s'installent dans celles qui existent déjà. Flles choisissent de préférence les tiges qui contiennent de la moelle, comme celles des ronces, des Liliacées, d'aloès, des Amaryllidacées, d'Aspanagus, d'acacia. Survant la méthode suivie pour approvisionner le jeune, on peut distinguter trois groupes différents d'espèces:

$1^{\circ}$ lispeces les plus rimitives, rarement observées. - La mere amasse dans le creux du nid du pain d'abeilles, en petils cones ou petits paquets isoles, et autant qu'en exigeront les larves juscju'i la nymphose, exactement comme le fonl d'auIres aheilles, par exemple les Ceratina, qui sont les plus proches des Allodape. Les petits las isolés de nourriture sont disposés les uns au-dessus des autres, dans la tige creuse et chacun est pourvu d'un wuf. I.a larve s'accroche à son paquet d'aliment, au moyen de longs appendices segmentaires très particuliers, que j'ai provisoirement appelés " pseudopodes \#, et clle absorbe son propre paquet jusqu'au moinent de la nymphose. la dimension de ce petit paquet est en rapport avec la dimension de l'espece, comme chez les Ceratina, et chaque paquet nourrit une seule larve. La larve tient ses appendices allongés comme des pattes d'araignées. et est solidement fixéc sur son paquet, comme les larves des abeilles solitaires telles que les Ceratina. Jusque là, il n'y a pas de grandes différences entre ces habitudes et celles des abeilles solitaires. Toutefois, il y en a une, et fondamentale: C'est que les Ceratına, après avoir mis des provisions et pondu dans un alvéole, le ferment aussitôt avec une cloison de matćriaux obtenus en rongeant les plantes, et construisent donc une série de cellules individuelles, tandis que les Allodape ne eonstruisent absolument aucune cloison. Les petits paquets de nourriture, chacun 
jusit: suffizant pour une seule larve el chacun muni d'un seul cuf, bien que disposés comme chez Ceratina, Osmia, etc..., en une série linéaire, l'un au-dessus de l'autre, dans le creux du nid, sont libres, l'un couromnant l'autre, el non séparés par des cloisons de matériaux tels que ceux que nous mentionnions ci-dessus. Le paquet inférieur est le plus àgé ; aussi le trouveton généralement porteur dine larve, alors que chacun de ceux qui le surmontent porte un œuf. Vous conviendrez que celte diflérence peut être considérée comme d'une importance capitale. Dans ces espèces plus primitives, la mère n'approrhe pas de la larve, puisque celle-ci a été fournie d'aliments une fois pour toutes et en assez grande quantité pour alteindre le moment de la nympliose; ceci, comme chez les guepes et los abeilles solitaires.

20 Espéces plutiol communes, de petite el moyenne laille. La mère adulte co'le un certain nombre d'œufs, chacun par un de ses pôles, et suivant une demi-spirale, déterminée par la courbure de la cavité tubulaire du nid, sur la paroi, en général vers le milieu, soit un peu au-dessus, soit un peu au-dessous. J'ai rencontré une espèce commune qui occupait des cavités souterraines en tube, el qui disposail ainsi ses œufs. Les larves, après l'éclosion, s'agrippent fortement à la paroi du tube, gràco a leur's pseudopodes, ot sont toutes au mème niveau, la léte lournée vers l'enlrée de la cavité. De lemps en temps, la mère apporte un petit morceau de pain d'abcilles et le dépose au milieul des larves affamées. Les larves mangent donc loutos simullanément de la méme masse de pain. A leur dernière mic, les larves perdent leurs pseudopodes el deviennent des nymphes qui restent l'une derrière l'autre dans la cavilé du nid. Ainsi, dans ces espèces, la mère reste en contact continuel avec les larves.

$3^{\circ}$ La majorilé des espèces, des plus pelites comme taille aux plus grandes. - La mère pond des œufs isolés et libres sur le fond du tube qui constitue le nid. Au regard de la tatle de la mère, les oufs sont très gros, on pourrait dire anormalement gros, et ils paraissent ètre pondus à de longs intervalles. La mère nourrit individuellement chaque larve; celle-ci serre entre ses deux gros pseudopodes les particules de pain d'abeille et elle garde ainsi pour elle-meme toute la nourriture. Quand on examine un nil qui a été occupé quelque temps par une mère abeille, on y trouve une ou plusieurs larves, dans la position que j'ai décrite, chacune avec sa propre pelote do pain 
dabeille. Plus tard, les filles aident li mère a approvisionner les larves. Quand la colonie est devenue populeuse, on trouve la cavité du tube comblée par les larves et les nymphes à tous les stades. Cependant le dernier œuf git presque toujours sur le plancher du tube. Et, comme la mère doit toujours parvenir jusqu'au fond du tube pour nourrir les plus jeunes larves, le contenu du tube est souvent mélangé; pourtant les larves les plus grosses et les nymphes sont le plus souvent tout près de l'orifice, donc tout au-dessus. Ainsi, dans ces espèces, les larves perdent leurs pseudopodes à la dernière mue » (1).

Les observations de Bracns sur les Allodape sont d'un Irès grand intérêt, car elles révèlent, dans les limites d'un seul genre, loule une série de degrés, débutant par l'approvisionnement en masse du jeune, comme chez les abeilles et les guìpes solitaires, et finissant par l'approvisionnement progressif. Et dans ce dernier cas, il y a non seulement des rapports étroits qui s'établissent entre la mère et ses descendants, mais dans le troisième groupe d'espèces décrit par Bracxs, une association des descendants avec la mère pour former une famille ou une société qui travaille en commun. L'origine de ces conditions particulières doit ètre tout simplement, commé le suggère Bracxs, l'absence de cloisons telles que celles qu'édifient les autres abeilles solitaires entres leurs alvéoles, pleins de provisions. Le degré final, celui des larves nourries individuellement, jour par jour, par la mère et ses filles avec de petites boulettes d'aliments, ne diffère pas dans son essence de ce que nous trouvons chez les bourdons et chez certaines fourmis (2).

(1) Brauns a publié, en 1902, une note brève sur ce troisieme type de mœurs

(2) A ce propos, notons que chez les abeilles Gastrilèges $d u$ yenre Lithurgus, - qui sont liées aux Megachile, et qui creusent dans le bois des funnels digitiformes. - on trouve certains 
Le genre Halictus est le plus étendu des genres d'abeilles; il se rencontre dans le monde entier, et comprend environ r.000 espèces décrites, la plupart différant entre elles par des caractères si légers et si subtils, qu'elles font le désespoir des taxonomistes. On n'a étudić les mours que d'un petit nombre, les résultals étant presque toujours fragmentaires et souvent contradictoires (I).

A ce qu'on sait, les Halictes nichent dans le sol ou, plus rarement, dans les bois pourris, en agrégations comprenant des centaines de nids. Chaque nid débute par une

indices qui les rapprochent, d'une part des inœur's des Allodape, d'autre part de celles des abeilles snciales supérieures. GuTBIER (1914) a découvert qu'une espèce paléarctique, $L$. luscipennis, pond plusieurs œufs sur la même ınasse de pain d'abeille, et que colle-ci est dévorée en commun par les larves naissantes. Quand elle attcint sa croissance complète et avant la nymphose, chaque larve s'isole cependant elle-même par des cloisons faites avec ses excréments. Dans une espèce australienne, $L$. dentipes, qu'on connait aussi aux Carolines et dans d'autres îles du Pacilique, Lunwig (1904) et Friese (1905, 1923) ont observé que certaines cavités digitiformes du nid sont employćes comme magasins pour conserver du pollen.

(1) Les espèces curopéennes ont été examinées par les auteurs suivants: WilckeNaEn (1817), Eversmaix (1816), F. SMith (1855), Bneitenbich (1878), Fabre (1879-80, 1903), VerhoefF (1891. 1892, 1897), Friese (1891, 1923), J. Pérez (1895), Aurivillius (1896), Ferton (1898), von Buttel-Reepen (1903), Semichon (1912), FinriNger (1914), Armbrister (1916), Legeivie (1922) el Stölihert (1923). Aux Etats-Unis, très peul de savants ont étudié ces abeilles si communes. Les seuls mémoires importants que je puisse signaler sont, l'un, de J.-B. Sммтн (1901) sur un Haliclus non identifié et sur H. (Augochlora) humeralis, et l'autre, de Melander el Brefs (1903) sur H. (Chloralictus) pruinosus. R. von Ihering (1904) a publié une description des nids de Augochlora graminea du Brésil, el Lünenwiz.nt (1911), de courtes notes sur les alvéoles d'une espèce brésilienne, Neocorṭnura erinnys. Plus récemment, Ilenbst (1922) nous a donné quelques indications intéressantes sur les mœurs de trois sous-genres du Chili: Augochlora, Ayapostemon et Corynura. 
galerie étroite, plus ou moine verticale, ayant en général moins de deux pieds de long, mais pouvant atteindre, dans le cas de l'Augochlora humeralis, une profondeur de six pieds. Les cellules ellipsoïdales sont creısées isolément ou en groupes le long de la galerie. Dans certaines espèces ( $H$ : sexcinctus), elles forment un grossier rayon et, chez $H$. quadricinclus, ce rayon est entouré d'une cavité qui l'isole particllement. Beaucoup d'Halictes, comme l'a observé FABre, revètent leur galerie et leurs alvéoles d'une fine couche de sécrétions qui durcissent et constituent comme un vernis hydrofuge. Pendant bien des années, on a discuté avec persistance sur le nombre de générations annuelles de ces abeilles, leur composition sexuelle et leur parthénngénèse. Il n'y aurait aucun intérêt pour nous à nous engager dans la discussion complète des diverses opinions émises par différents auteurs à se sujet; je choisirai seulement, pour les examiner rapidement, les interprétations de Fabre et d'Armbresten et surtout celle de STöknert dont les investigations récen. les et très exactes ont donné à cette question un aspect lout nouveau et d'un intérêt intense.

Depuis l'époque de Frederick Sмrтr, tous les mélittologues sont d'accord sur le fait qu'une couvée d'Halictus/se développe pendant l'été et produit à l'automne des mâles et des femelles, qu'après l'accouplement et la mort des mâles, les femelles hivernent dans les terriers maternels ou aux alentours, et rétablissent, au printemps suivant, le vieux nid, nu en creusent de nouveaux, puis commencent à aller en quète de provisions pour leurs couvées. FABRE, qui a étudié surtout $H$. calcentus $(=$ cylindricus $)$ el, en une certaine mesure, scahioso (= sexcinctus) et fodiens, affirme que les femelles fécondées avant l'hiver 
ne produisent que des descendants femelles et que ceux-ci produisent, parthénogénétiquement, la génération d'automne possédant des représentants des deux sexes. Il pense donc qu'il y a deux générations par an, l'une de l'automne au printemps, avec des femelles fécondées, l'autre, en été, parthénogénétique. Il est faux, on l'a prouvé, que cette dernière génération comprenne exclusivement des femelles parthénogénétiques, et que les deux sexes de la génération automnale s'accouplent dans le nid; mais il est exact, comme nous le verrons, que les femelles qui passent l'hiver survivent plusieurs mois et restent les gardiennes du nid. Mais cela n'est pas la seule fonction, ni la plus importante, des femelles âgées.

Armbruster a entrepris de déterminer le nombre et le caractère des générations annuelles, en faisant la statistique des époques du vol des Haliclus d'Allemagne, et il conclut que mâles et femelles s'accouplent à l'automne, mais que, tout au moins dans le Nord de l'Europe, les femelles, comme les mâles, meurent après avoir approvisionné leurs alvéoles. Ainsi, la couvée seule passe l'hiver et produit uniquement des femelles au printemps suivant. Celles-ci donnent, sans fécondation, une génération d'été de fomelles, qui, à leur tour, produisent parthénogénétiquement la génération d'automne, avec mâles et femelles. Il y aurait donc, d'après Anmbruster, trois générations par an, puisque les femelles de l'automne et du printemps ne sont pas les mêmes individus, comme le croyaient FABRE et d'autres auteurs, et il y aurait deux générations parthénogénétiques, la première ne produisant que des femelles (thélytoque), la seconde donnant des représentants des deux sexes (amphérotoque). Armbruster croit qu'il en est de méme chez le Sphérode parasite de 
l'Haliclus, el ra jusqu'à parler d'un type de génération spécial a l'Halictus, unique chez les abeilles, puisque les œufs non fécondés n'obéissent pas à la loi de Dzierzon et produisent des femelles à l'été et des mâles et femelles à l'automne.

Stöcknert s'élève avec un certain mépris contre cette opinion, qu'il traite de présomptueuse; erronée et appuyée seulement sur des données statistiques. Ses observations personnelles ont été exécutées pendant sept ans dans la Haute-Bavière, et ont porté principalement sur $H$. malachurus, maculatus, sexcinctus ,immarginatus et puncti. collis, qui sont les représentants de la plupart des groupes européens de ce genre. Les observations relatives à la reproduction et à la parthénogenèse ont été soigneusement contròlées par Zaxder, au moyen de dissections des organes reproducteurs femelles. Il semble que le nombre. des générations annuelles varie d'une espèce d'Halictus à l'autre. Ainsi certaines formes septentrionales, par exemple H. lineolatus, ont seulement une génération comme Leaucoup d'espèces d'Andrena; d'autres (H. lucasius, $H$. clavipes el $H$. soror) en ont deux, chacune possédant des individus des deux sexes, également comme certaines Andrena. En outre, quelques Halictus, qui accomplissent des vols de longue durée, ont une troisième génération qui s'intercale en automne, surtout quand l'hiver est tardif (H. morio, puncticollis, villosulus). Mais qu'il y ait une, deux, on trois générations, les mâles meurent à l'automne; il arrive toutefois, mais rarement, dans quelques espèces du sud de l'Europe, qu'un petit nombre de mâles survivent et s'envolent au printemps suivant ; tandis que les femelles hivernent toujours.

Stücknert a donc bien élabli que Farre et Arvbri ster 
ont eu tort de parler d'une ou de plusieurs générations parthénogénétiques; cependant, il existe, chez certains Halictus, un țpe de reproduction qui est même plus intéressant parce qu'il éclaire l'évolution des mours sociales. On peut prendre comme exemple $H$. malachupus.

Cette abeille niche dans la terre argileuse durcie, et forme des congrégations de plus de mille terriers. Les femelles qui ont passé l'hiver reparaissent du début au milieu de mars; elles commencent à neltoyer les terriers, à les vernir et à visiter les fleurs des saules et des dents de lion. Bien que plusieurs femelles aient hiverné paisiblement dans le même terrier, il n'en reste plus à ce moment que trois, deuxx, ou le plus souvent une seule, à la suite des lulles qu'elles se livrent entre elles pour la possession du terrier. Les individus expulsés doivent chercher des nids inoccupés, ou en creuser de nouveaux. Les alvéoles des jeunes sont édifiés directement à partir de la galerie principale ; ils reçoivent des provisions et un œuf. S'il reste dans le nid plus d'une femelle, ces filles d'une mème mère construisent néanmoins des groupes d'alvéoles séparés. Vers la mi-juin, la couvée éclot; elle consiste presque exclusivement en femelles. Celles-ci sont de plus petite taille que leur mère, et d'un autre type; les taxonomistes les ont décrites sous le nom d'H. longulus. Les premiers individus formés sont anormaleınent pelits. Il n'y a pas de màles longulus. La mère est encore en vie el ses filles du type longulus restent auprès d'elle et commencent a édifier des alvéoles. Ce travail s'exécute surtout la nuit, comme Fabie l'a observé chez II. calceatus. Les œufs de ces alvéoles sont pondus par la mère et non par les longulus, qui cependant récoltent les provisions, consistant surtout en pollen de Hieracium et Leontodon. La mere reste au logis et en garde l'entrée, comme l'ont observé Fume et d'autres. Les femelles longulus vont ainsi en fourrageurs jusque vers la fin de septembre, quoique chaque individu ne vive prohablement pas plus de quatre à six semaines. Leur nombre auginente jusqu'en aout; il y en a alors environ une douzaine dans chaque nid. Vers le début d'aout, commencent à apparaitre les femelles du type malachurus, loujours à partir d'aufs pondus par la méme mère. Ces femelles sont très engourdies et lèthargiques, en comparaison des femelles longulus si aclives et remuantes, et elles ne récoltent point le pollen, mais explorent les fleurs pour chercher le nectar. Les males, egalement fils de la vieille mère, ap- 
paraissent en mème temps et deviennent de plus en plus nombreux; ils ne s'occupent point des femelles longulus, inais ils sont prompts à s'unir, sur la surface du nid, avec les jeunes femelles malachurus, lorsque celles-ci sortent du terrier à l'aurore de quelque jour ensoleillé. C'est une sorte d'esquisse d'un vol nuptial. A la dissection, on ne trouve jamais de femelles longulus fécondées. Après avoir survécu durant tout l'hiver précédent et avoir produit les longulus en été et les malachurus à l'automne, la vieille mère, tout usée et les ailes à demi détruites, survit jusqu'à la fin de la saison. Les dissections de ZANDER ont montré que ses ovaires peuvent contenir des oufs mûrs et son spermathèque être rempli de sperme, jusqu'à la fin d'août. Un peı plus tard, elle cesse de pouvoir voler, elle s'éloigne en rampant et meurt. Meurent aussi les femclles longulus et les mâles, mais les jeunes femelles malachurus fécondées s'apprêtent à hiverner dans leur nid malerinel.

Stöcrihent a retrouvé chez. $H$. maculalus des mnur's très voisines de celles de malachurus. l.es femelles d'été sont également plus petites que leur mère, mais elles ont le même dessin, et n'ont pas reçu des taxonomistes, un nom différent. Chez. $H$. immarginalus, au contraire, les femelles d'été diffèrent de leur mère par leur taille, leur poncluation et leur rugosité, el sont connues sous le nom de pauxillus. La forme estivale de $H$. puncticollis, quoique ayant la même taille que la mère, en diffère assez pour avoir mérité des systématistes le nom de villosulus.

Dans ce bref résumé des observations de STöcknert, vous n'avez pu manquer de remarquer la ressemblance très frappante et très suggestive de $H$. malachurus et des genres Vespa et Bombus. Les femelles longulus stériles correspondent parfaitement aux ouvrières des Vespa et Bombus, tandis que la mère malachurus représente la reine, guêpe ou bourdon, qui passe l'hiver. En fait, l'organisation sociale de $H$. malachurus et des espèces voisines est presque aussi hautement développée que chez Bombus, sauf en ce qui concerne le nombre des individus collaborant. La ressemblance s'accroît encore du fait que, chez quelques espèces voisines $(H$. sexcinctus et quadricinctus), les cellules des jeunes ont la forme d'un rayon 
grossier, et que, suivant FaHringer (1914), $H$. scabiosie utilise certains alvéoles pour emmagasiner du pollen. Vous remarquerez, en outre, qu'il n'y a, dans le genre Halictus, aucune infraction à la loi de Dzierzon, aucune femelle ayant une origine parthénogénétique; les affirmations opposées de Fabre et d'Armbruster proviennent d'une erreur d'interprétation des faits. Les observations de Stöcrнert, sur lesquelles j'aurai l'occasion de revenir, nous montrent quelles nombreuses surprises nous sont réservées, lorsqu'on abordera, dans d'autres continents et surtout en Amérique du Sud et en Australie, l'étude expérimentale précise des mœurs de ces abeilles en apparence si monotones et dépourvues d'intérêt.

$$
\text { ** }
$$

Les abeilles sociales supérieures, les Bombinés, Méliponinés et Apinés, qui représentent les formes les plus hautement différenciées dans la section "podilège " de la famille des Apidés, offrent aux phỵlogénistes un si grand nombre de problèmes embarrassants, que toute une série de leçons suffirait difficilement à les examiner comme il convient. On a souvent supposé que ces trois familles étaient assez étroitement unies entre elles, en partie à cause de leurs ressemblances morphologiques, et en partie parce que toutes secrètent de la cire et s'en servent pour édifier leurs rayons et leurs alvéoles; mais il y a de telles divergences dans leurs mœurs et la genèse de leurs sociétés que nous sommes conduits à soupsonner l'exístence de trois développements convergents à partir de plusieurs sources ancestrales indépendantes, plutôt que trois branches divergentes d'un tronc commun. Cette 
idée est bien suggérée par les colonies de formes telles que Halictus malachurus, dont nous avons vu qu'elles res. semblaient tant it celles des Bombinés. Mon temps est si mesuré, que je me limiterai à vous fournir un petit nombre d'indications sur la paléontologie, la secrétion de la cire, le degré de polymorphisme et les méthodes suivant lesquelles s'établissent les colonies, dans ces trois sousfamilles.

On a décrit des abeilles sociales dans l'ambre de la Baltique (Oligocène inférieur), dang l'ambre de la Sicile (Hiocène) et dans les divers schistes de l'Oligocène supéricur et du Miocène d'Europe, et von Buttel-Reepex (I 9o6, I 9 15) a tenté d'interpréter certains de ces fossiles comme les ancêtres effectifs de notre moderne Apis mellifica. Nais une étude récente d'un spécialiste des abeilles, Cockerell (rgoga, i gog b), a montré que ces espèces, placées parmi les entomologistes antérieurs dans les genres modernes-Bombus, Meliponi, Trigona et Apis, appar. tiennent en majeure part à des genres éteints presque tous, et très différents.

Parmi les especes de Bombinés trouvées dans l'ambre de la Baltique, Bombus pusillus Menge a seulement trois millimètres de long et ne peut donc être un Bombus; B. carbonarius Menge et Bombusoides mengei Motschulsky sont, suivant Cockenell, absolument méconnaissables. Cockenill (1909a) a montré que, parmi les cinq espèces de Bombus de l'Oligocène supérieur et du Miocène qu'on a décrites, deux (B. jurinei et abacus) appartiennent au genre Xylocopa et on ne peut s'empêcher de conserver quelque doute sur l'identification générique des aulres, antiquus Heyden, crassipes Novat et grandrevs Heer.

Cockerel. $(1909 b)$ a cepenclant trouvé trois genres distincls voisins des Bombus, dians l'ambre de la Baltique, à savoir: Protobombus avec deux espèces, Chaleobombus avec deux et Sophrobombus avec une seule. Il decrit Prolobombus comme beaucoup plus proche de Bombus que d'Apis, et peut-etre est- 
il l'ancêtre direct du premier de ces genres, Les autres genres représentent des lignées collatérales, ne conduisant en aucune façon à nos abeilles modernes. Nous pourrions en conclure que les Bombinés étaient, pendant le Tertiaire, un groupe plus vaste, plus diversifié qu'à l'époque actuelle, où ils ne comptent qu'un seul genre.

Brischike et Burmeister (Handeinsch, 1908) ont cilé des espèces de Melipona et Trigona trouvées dans l'ambre de la Baltique, mais Cockenell regarde comme dénués de toute valcur ces rapporls génèriques. Cependant, Tosı (1896) a décrit et figuré très suffisamment deux Méliponinés appartenant à un genre éteint, Meliponoryctes succini et sicula, trouvées dans l'ambre de la Sicile, qui est plus récent. Celà est d'un grand intérêt, car celà montre que cette sous-famille, actuellement limitée aux régions néotropicales, Ethiopie, Indes, Australie, a été autrefois représentée en Europe. Il peut donc se trouver des Méliponinés authentiques dans l'ambre de la Baltique, qui contient de nombreux genres d'insectes confinés maintenant en Australie el dans les Indes Orientales.

Les deux espèces fossiles d'Apis, dont von Burtel-Reepen lit la base de ses vues sur l'origine germanique de l'abcille domestique, ont eu un triste sort. Suivant Cockerell (1909 a), Apis adamitica Heer, du Miocène d'OEningen, se trouve être un Lithurgus, c'est-à-dire une abeille gastrilège voisine du genre Megachile, et, toujours d'après le même auteur, l'Apis meliponoides de von Butrel-Reepen, trouvé dans l'ambre de la Baltique, est, non un Apis, mais le type d'un genre nouveau, Electrapis. Une seconde espèce,lornquisti, rapportée sans certitude par Cockerell au genre Electrapis, est plus voisine encore du genre Bombus, L'Apis proava de Mrons, provenant aussi de l'ambre de la Baltique, s'identifie difficilement d'après la description donnée et ajppartient sans soule à quelque autre genre. Ainsi, nous demeurons pratiquement privés de loule donnée paléontologique permeltant de dresser un arbre généalogique, analogue à celui de von Buttel-Reeren, pour les Bombinés, Méliponinés el Apinés.

$$
\text { ** }
$$

Dans ma dernière leçon, je n'ai peut-être pas assez in. sisté sur les progrès très frappants que fait la nidification chez les guêpes diploptères, à partir des nids construits 
dens gu avec la terre et l'argile, et en passant par les nids faits de petits morceaux de feuilles (Zethinés), pour arriver enfin aux nids des cinq sous-familles sociales, qui fabriquent un véritable papier mâché, consistant en fines fibres végétales agglutinées avec une secrétion buccale. Une évolution parallèle se retrouve chez les Abeilles; la plupart des formes solitaires inférieures nichent dans le sol ou font des alvéoles en terre, tandis que les formes supérieures utilisent des substances végétales très diverses, quelquefois même une pâte de feuilles, comme chez l'espèce européenne, Osmia inermis, et enfin, dans les trois sous-familles sociales les plus hautes, le matériel nécessaire à la construction des alvéoles pour les jeunes et pour les réserves est obtenu exclusivement sous la forme d'une sécrétion unique en son genre, connue sous le nom de cire des abeilles. Il y a donc, dans les deux groupes, une évolution similaire el très significative, depuis les mœurs les plus primitives, terricoles ou humicoles, jusqu'aux mæurs arhoricoles plus élevées.

Il n’a jamais été prouvé qu'aucune des abeilles solitaires sécrite de la cire; mais von Buttel-Refepen (rgo3) et Friese ( $19^{23}$ ) ont fait remarquer que les spécimens femelles récemment tués de plusieurs genres (Tetralonia, Eucera, Anthophora, Pachymeius et Xylocopa) laissent exsuder, en séchant, entre les segments abdominaux et sur leur face dorsale, une substance qui rappelle beaucoup la cire, quoique l'analyse chimique, faite sur Tetralonia, n'ait rien révélé que la présence de graisse. Mais la cire a une composition analogue, étant aussi formée de li. poïdes. Friese ne croit pas que ces exsudats graisseux puissent être utilisés par les abeilles solitaires, puisqu'elles ne sont munies à leurs pattes postérieures d'aucun appa- 
reil pouvant servir à les récolter entre les segments; mais tout ceci a été très peu étudié, et il nous est impossible d'affirmer qu'il n'existe point, chez certaines espèces solitaires, des caractères phy̧siologiques définis, qui conduisent graduellement à la sécrétion de cire telle qu'elle se produit chez les abeilles sociales supérieures (I).

Les régions qui sécrètent la cire chez les Bombinés, Méliponinés et Apinés, sont, on le sait, distribuées très différemment sur l'abdomen et suivant les individus des differentes castes; chez les Bombus, la cire esl sécrétée aussi bien par les reines que par les ouvrières, et du second au quatrième segments abdominaux, dorsalement et ventralement; chez les Méliponinés, elle est sécrétée par les reines, les ouvrières et les màles, du second aul quatrième segments, mais seulement sur la face dorsale; et chez les Apinés, seules les ouvrières en produisent et seulement sur la face ventrale, dir second au cinquième segments. Dans tous les cas, la sécrétion n'a lieu que chez les individus jeunes. Les Bombinés seraient donc les représentants du degré le plus primitif et le plus généralisé, c'est-à-dire le degré qui aurait donné naissance aux conditions réalisées chez les Méliponinés, par la perte des

(1) Heselhaus (1922) a montré que, sous les larges aires veloutées qui bordent l'orbite inlerne des Andrena, el dans certains segments abdominaux, chez les Osmia, il y a des glandes qui ressemblent beaucoup, par leur structure, aux vraies glandes à cire des Apinés, Bombinés et Méliponinés, telles que les a décrites Dreyling (1905). Ces organes ne produisent certainement pas de la cire typique, chez Osmia et Andrena. Chez Andrena, la sécrétion produite est peut-ètre une huile essentielle volatile et les glandes faciales seraient donc des organes odorifères; toujours, celles-ci sont bien proches des organes olfactifs de l'antenne, et ce fait est, comme l'indique Hesfi.h.us, pou favorable à uno telle interprétation. 
glandes de la face ventrale de l'abdomen, el, chuz los Apinés, par la perte de celles du côté dorsal. La façon dont la cire est utilisée présente aussi des différences intéressantes d'une sous-famille à l'autre ; les Apinés l'emploient pure; les Bombinés la mélangent avec une grande quantité de jollen [voir les analyses de Suxpwick citéas par Armbruster ( 1914$)$ ] el de substances grasses; et les Véliponinés la mélangent largement de terre, de résines (propolis) et d'autres substances d'origine végétale.

$$
*_{* * *}^{* *}
$$

Comme j'ai l'intention de discuter assez longuement la question du polymorphisme, ou développement de castes chez les insectes sociaux, dans deux leçons qui suivront, je ne ferai que l'effleurer, en ce qui concerne les abeilles. Chez certains Halictus et chez les Bambus, la femelle présente deux phases, mais elle n'offre que des différences minimes de structure, en comparaison de celles que nous montrent les guêpes sociales, les ouvrières étant, chez celles-ci, de plus petite taille que la reine, non fécondahles, et par suite stériles ou pondant des œufs ne produisant que des mâles. La différenciation est quelque peu plus poussée chez les Méliponinés et les Apinés ; ici, les différences de mœurs entrainent une inversion significalive des conditions observées chez les Halictus et Bomhus ; la reine, tout en conservant et en exagérant même la fécondité des reines de ces dernières formes, subit une dégénérescence, réductrice de l'appareil de récolte du pollen aux pattes postérieures, et de certaines parties de la tète (plus petite taille dé la tête chez les Véliponinés, réduction des parties buccales chez Apis). 
Pour lerminer, je vous dirai quelques mots des différentes façons dont se forment les colonies chez les Bombinés, Méliponinés et Apinés, et sur la question de la monogynie (" monogamie " ou " monométrose ") et de la polygynie (" polygamie " ou "pléométrose "), que j'ai effleurée dans la leçon précédente. Dans les régions tempérées nord, la colonie de Bombus a un développement annuel; elle est fondée par une seule reine fécondée, ayant passé l'hiver, tout comme les colonies de Vespa;
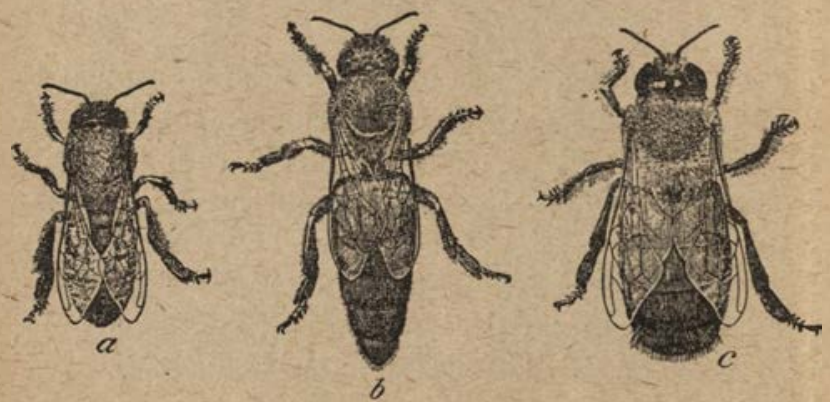

Fig. 15. - Abeille domestique (Apis mellifica). a, ouvrière ; $b$, reine ; $c$, male

mais, d'après R. vox Inerixg (rgo3), les colonies de $B$. carbonarius et cayennensis du Brésil sont pérennanles et contiennent plusieurs reines fécondées, qui fondent de nouvelles colonies en essaimant, comme beaucoup de guê. pes sóciales tropicales, étudiées par Ducke, par R. et H. von Ihering et par Roubacd. Suivant Silvestri (igo2) et II. von Inering ( $g_{9}$ o3), les colonies de Méliponinés se fondent aussi par essaimage. Il y a seulement une reine dans ('haque colonie, et, comme elle ne peut voler, étant alour. 
dio par son abdomen trop volumineux, et ses ailes, relativement petites, étant souvent mutilées, les essaims d'ouvrières quittent le nid avec de jeunes reines, comme dans le cas des guẻpes tropicales et des bourdons. Chez l'abeille domestique, on sait parfaitement qu'il n'y a quine seule reine, mais elle conserve son aptitude au vol, et elle part avec un essaim d'ouvrières, aussitôt qu'une jeune reine esı sur le point de naitre dans la ruche. Par anticipation, je puis vous dire que, chez les Formicidés, la grande majorité des espèces, même tropicales, fondent indubitablement leurs colonies comme les Vespa et les Bombus des régions tempérées; quoique des colonies puissent aussi se former par essaimage, c'est-à-dire au moṿen de femelles lécondées, abandonnant les colonies vastes, en compagnie d'ouvrières et parfois aussi en association avec un petit nombre de jeunes socurs reines (" pléométrose primaire m.).

Si nous mellonș à part, pour les considérer ultéricurement, les cas aberrants de certains parasites sociaux, nous pouvons done distinguer quatre types dans le mode de formation des colonies chez les Aculéates sociaux, silvoir :

1. Le type Vespa, observé aussi dans les especes septentrio. nales de Bombus et Polisles, chez. Ilaliclus el chez beaucoup de fourmis. La colonie naissante est concentrée en une reine fécondée unique.

$2^{\circ}$ Le type Belonogaster, rencontré aussi parfois chez les $\mathrm{Ha}$ lictus et quelques fourmis (Lasius, Myrmecocystus, WheELEn, 1917, elc.). Ia colonie débute avec plusicurs reines fécondées. probablement søurs:

$3^{\circ}$ Le type Melipona, représenté aussi par beaucoup de Vespidés tropicaux (Polisles, Polybia, elc.) el par Bombus. I.a colonie ne renferme d'abord qu'une seule reine fécondée et un certain nombre d'ouvrieres qui sont ses sœurs. 
$4^{\circ}$ Le type Apis mellifica, qui ne parait exister chez aucun autre insecte. La nouvelle colonie est formée d'une vieillo reine fécondée et d'un certain nombre d'ouvrières qui sont ses filles.

Le quatrième type n'est peut-être qu'une variante du troisième, cela n'a pas été démontré de façon satisfaisante ; sans doute pourrait-on élucider quelque peu ce point litigieux par une étude sérieuse d'autres espèces d'Apis, $A$. dorsala et $A$. florea de la région indo-malaise. Le type Vespa est monogyne, tandis que les types Belonogaster et Melipona sont polygynes, avec la différence que, dans le premier, les individus fondant la nouvelle colonie sont tous fertiles, alors que, dans le second, un seul est fertile, tous les autres étant stériles.

\section{$\stackrel{* *}{*}$}

R. et H. von Ihering, Ducke el Roubaud admettent qué les types polygynes sont plus primitifs, le type monogyne étant un type dérivé, qui a paru chez les abeilles et les guêpes septentrionales comme conséquence de conditions climatiques défavorables. Mais ceci ne peut s'appliquer à la monogynie quasi-universelle des fourmis. D'un autre cỏlé, nous pourrions dire que les colonies de Vespa et de Bombus septentrionaux produisent chaque année un essaim de femelles et d'ouvrières, mais l'apparition du froid fait périr les ouvrières inoins résistantes et ne laisse survivre que les reines fécondées éparses qui hivernent jus qu'au printemps suivant. Il me paraît que la question de la plus ou moins grande ancienneté de l'une ou l'autre méthode est purement scholastique ; que la méthode suivie ne doit dépendre que du degré de tolérance mutuelle et d'animosité des reines. Ceci semble prouvé par les sociétés primitives d'Halictus (Stöcknent, I 923 ). Un certain nombre de reines sœurs fécondées hivernent paisiblement dans le même terrier maternel; mais, à l'arrivée du printemps, des conflits éclatent entre elles et cela conduit 
à une colonie monogyne. Pourtant, dans certains cas, deux ou trois de ces soeurs peuvent calmer leur hostilité et. etablissent une colonie polygyne. Nous retrouverions une imprécision analogue dans le type de formation des colonies chez les guêpes et chez les Bombus de nos régions, si les reines fécondées avaient pour habitude d'hiverner dans le nid maternel, ou, chez les fourmis, si les reines, au lieu de se disperser au cours du vol nuptial, se groupaient dans des cavités appropriées. Cela se produit parfaitement pour plusieurs reines chez les Lasius, Farmica ou Myrmecocystus, et vox Butrel-ReEpes (I go5) a assisté à la naissance d'une colonie digyne de Lasius niger. Pendant douze mois, deux reines de cette espèce vécurent très amicalement côte à còte, soignant en commun leurs couvées, mais, dès qu'apparurent les premières ouvrières, elles se livrèrent, l'une à l'autre, de violents combats. On les sépara, et l'une d'elles, cruellement blessée par sa søur, fut tuée par les ourrières; ainsi la colonie devint monogyne. Les instincts des deux reines subirent ainsi un brusque changement au moment où la couvée devint mûre. Un tel changement survient chez Halictus, mais à une phase bien plus précoce de la formation des colonies, tandis qu'il ne paraît pas se produire chez Belonogaster ( $\mathbf{r}$ ).

(1) D'après-les observations de JANEr (1904), on voit que dans les colonies polygynes avancées de Solenopsis et.de Tetramorium, conservées dans des nids artificiels, les reines sont peu à peu tuées par les ouvrières, si bien qu'une seule subsizte. Comme cotte élimination ne se produit pas dans les colonies sauvages, JANET suppose, avec juste raison, semble-t-il, que cola provenait sans doute d'un défaut de nourriture dans les nids artificiels. 


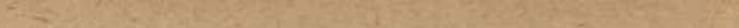

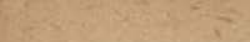


CHAPITRE IV

\section{L'EVOLUTION DES FOURMIS}

Principales subdivisions; leurs rapports. - Origine mon phylétique ou polyphylétique. - Origine des fourmis parmi les Aculéates. - Les Tiphiidær. - Documents paléontologiques. - Thermophilie et xérophilie des fourmis. - Apparition probable au Trias. - Ancienneté et longue durée de l'évolution du groupe.

Le troisième et dernier groupe d'Aculéates sociaux, le plus intéressant de maint point de vue, comprend la famille des Formicida, ou fourmis. C'est un groupe cisenliellement tropical. Jusqu'à une époque récente, la connaissance que nous en avions se restreignait au petit nombre d'espèces de la zone tempérée nord ; aucune description compréhensive n'était possible. Toutefois, durant la seconde moitié du siècle dernier, Forel, Emery, Ern. André, Santschi, Vienmeyer, Donisthorpe, Chawley, Mann, moi-même, et d'autres, bâtissant sur les fondations posées par Latreilie, Frederick Smith et Gustave Mayr, avons entrepris de compléter l'édifice taxonomique de la famille, et d'acquérir à l'occasion quelque connaissance des mœurs des différentes espèces, autant qu'il était possible. Il reste encore évidemment beaucoup à faire, mais la masse de faits accumulés est déjà imposante.

Ces faits ont servi, et servent, il l'élaboration d'ouvra. 
ges succincts, dont plusieurs sonl déjà à la portée des tra. vailleurs. Durant les vingt dernières années, des ouvrages généraux, semi-populaires, ont été publiés par Fonkl en français, par Escuericir ( $19 r_{7}$ ) et Brun (192/) en allemand, par EMERY en italien ( $1915 a$ ) et par DoxisthohPE (r 915 ) et moi-même, (rgro, r $9^{2} 3$ ), en anglais. Les "Fourmis de la Suisse " de Forel (nouv. édit. I920), et surtout son "Monde Social des Fourmis " (192I-23), embrassent si bien le sujet que je suis sans excuse de consacrer cette conférence à la structure et aux mœurs des fourmis. Je suis bien sûr que vous avez quelque connaissance des fourmis, aussi ne m'occuperai-je, aujourd'hui, autant que le temps le permettra, que d'un petit nombre de sujets généraux, tels que la phylogénie de la famille, ses restes fossiles, et sa distribution géographique.

\section{$* *$}

Le fait que la famille des Formicidés, à l'inverse de celles des Vespidés et des Apidés, se compose uniquement d'insectes sociaux, est un obstacle sérieux à la recherche de leur phylogénie. On en a décrit jusqu'à présent 6.000 formes différentes: espèces, sous-especes, el variétés, de toutes les parties du monde, et il est probable que uns jeunes myrmécographes porteront le nombre à 10.000 , avant la fin du siècle présent. On connaît à peine la moilié de ce nombre de formes de termites : les fourmis sont donc de beaucoup le plus vaste groupe d'insectes sociaux. Selon Forfi el Every, la famille des Formicidés comprend cinq sous-familles : les Ponerinc, Dorylince, Myrmicirue, loulichaderinas et Formicina (Campanatince). Les Ponerina sont un groupe primitif mais assez héléro. 
gène. Les Dorylince comprennent les fameuses fourmis d'Afrique, et les fourmis militaires ou fourmis visiteuses de J'Amérique tropicale. Les Myrmicince sont un assemblage vaste et assez hétérogène de genres. On les reconnlâ̂t toutefois aisément aux deux petits segments pétiolaires de l'abdomen, présents dans toutes les casles. Les Dolichoderince et Formicince se caractérisent toutes deux par un petit et unique segment pétiolaire, mais différent par la forme du gésier, celle de l'orifice anal et le caractère des glandes venimeuses. J'ai récemment reconnu deux autres sous-familles : les Cerrapachyinoe, incluses primitivement dans les Ponerince, et les Pseudomyrmina, placées autrefois parmis les Myrmicina. Il me scmble qu'on devrait encore créer une huitième sous-famille pour la tribu très aberrante ef Irès spécialisée des Leptanillini, qu'EnEnY place parmi les Dorylince. Il est probable que les Myrmicince seront dans l'avenir scindées en plusieurs sous-familles.

Voici donc quelles sont les principales séries de sousfamilles qu'on peut reconnaitre à l'heure actuelle. J'indique en regard leur distribution géographique essentielle :

Dorylinæ. - Tropicales et subtropicales.

Cerapachyinæ. - Tropicales et subtropicales.

Ponerinæ. - Très largement tropicales.

Leptanillina. - Tropicales et subtropicales.

Pseudomyrminæ. - Tropicales.

Hyrmicinæ. - Cosmopolites.

Dolichoderinæ. - Largement tropicales.

Formicina. - Cosmopolites.

On roit que presque toutes les fourmis nord-américaines et européennes appartiennent à deux sous-familles seulement: les Myrmicince et les Formicino. On a beau- 
coup discuté lés relations des familles entre elles. Ln certain nombre de sous-familles sont dans leur ensemble très primitives (Dorylina, Cerapachyina, Ponerina), landis que d'autres sont très hautement spécialisées (Dolichoderina, Formicina). Il y a en outre des groupes annexes comme les Cerapachyino, qui unissent les Ponerina aux Dorylince; Rhopalomastix, qui, selon FoneL, marque le passage des Ponerino aux Formicino et Aneu. retus qui semble relier Dolichoderina et Ponerinoe. En outre, on peut reconnaître un certain nombre de tendances morphologiques et éthologiques: la réduction progressive de l'aiguillon dans les familles les plus spécialisécs (quelques Myrmicince, les Dolichoderince et les Formici$n(x)$, le passage de la vie terricole à la vie arboricole, dont je parlerai plus tard, et l'évolution d'un régime entomophage prononcé à un régime aphidicole et végétarien (spermatophage et mycétophage). Cette dernière évolution ressemble étrangement à celle de l'Homme, telle que la concevait, le premier, Coxdorcet, du stade chasseur el pastoral au stade agricole; elle ressemble aussi aux trois stades de l'histoire humaine reconnus par Auguste Coмve : conquète, défense, industrie.

Je ne crois donc pas du tout, contralrement à Havininsch (1924) que la discussion des relations mutuelles des sousfamilles de Formicidés soit inutile. On peut pardonner à Havnu.risch, anteur de maint arbre phylétique, de rejeter ceux des autres hymenoptéristes. Mais que penser de lui quand on le voit proclamer inulile une occupation à laquelle il s'est si souvent livré? Après tout, ces arbres phṛlétiques ne sont que des traductions graphiques des relations phylogéniques concevihles. Ils n'ont ni plus ni moins de valeur que les autres manières de présenter en raccourci des notions que nous savons provisoires, dans des domaines encore obscurs. Si nous osons donc, en dépit do l'éminent entomologisle autrichien, essayer de 
construire larbre généalogique des sous-familles de Formicidés, nous hii donnerons l'allure suivante:

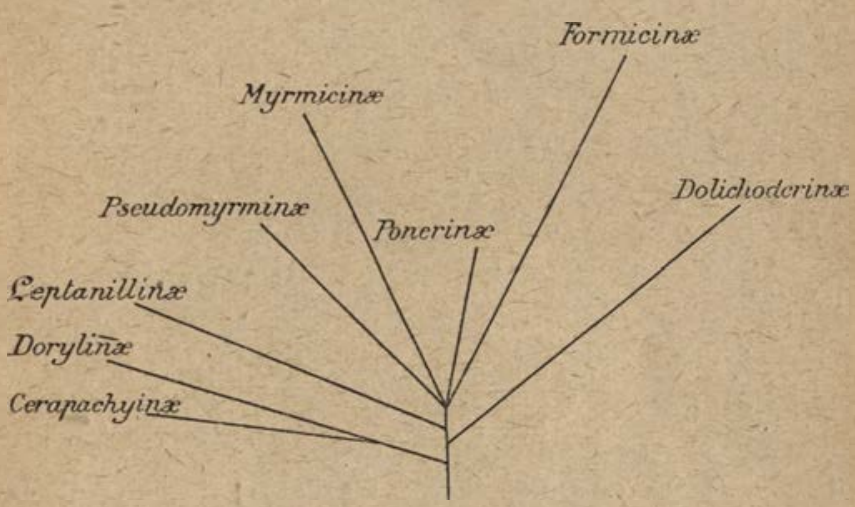

Las Ponerinæ représentent le fond primitif, d'où ont divergé les autres sous-familles. Le degré de specialisation que ces sous-familles ont alteint est indiqué grossierement par le nivean de leur nom.

Il y a une question que les myrmécologistes n'ont pas discutée explicitement, bien que notre étude des Vespidés et des Apidés la suggère. C'est la suivante. Les différentes familles de fourmis dérivent-elles réellement d'un fond commun, où sont-elles polyphylétiques, comme les différentes sous-familles sociales de guêpes et d'abeilles ? Nous les avons considérées, dans le diagramme qui précède, comme monophylétiques ; c'est l'opinion soutenue par Forel en igro, et par moi-méme en r920. Mais Emery croit à une origine polyphylétique des sous-familles, telle que le schéma ci-dessous (publié en r 920 ) l'indique : 
Prodorylinz Cerapachyinz

Dorylin.7
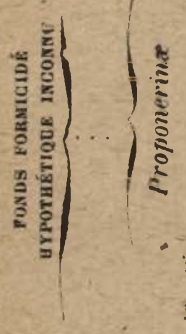

Euponering

Amblyoponini, Eclalommini, Proceralini

| Myrmicinii / prolongeant le fouds avec le minimum

Platylhyreini ! de différenciation Myrmicinx

Dolichoderina

Formicinze (amponotinx)

EMEny suggère donc que, du fond commun d'ancêtres hypothétiques, ont pris naissance quaire séries parallèles d'évolution. Ce fond commun remonterait beaucoup plus haut que les Proponerince, ancêtres des Ponerince. Par conséquent, si nous adoptons les vues d'EsenY, nous sommes dans un cas tout à fait semblable à celui des Vespidés; les ancêtres hypothétiques ont dû être très primitifs. Ce sont très probablement des Aculéates solitaires et subsociaux. Il est très clair que, si tous les Vespidés et les Apidés solitaires avaient disparu, nous considérerions très probablement les formes sociales, seules survivantes, comme des familles monophylétiques. Mais, d'un autre còté, les différentes sous-familles de Formicidés ont loutes des relations très étroites et, comme nous l'avons dit, elles semblent être, unies par des groupes annexes. Il est donc plus probable que la famille dans son ensemble, est bien monophylétique.

Nous avons maintenant à chercher le point d'origine des fourmis parmi les Aculéates solitaires, autant que nous le pouvons en faisant appel à la morphologie, la paléographie et l'étholngie. Dans l'ensemble, on a l'impression 
d'une famille étroitement circonscrite et très archaïque. On trouve cel aspect archaïque dans les nombreux genres et especes qui combinent des caractères évidemment

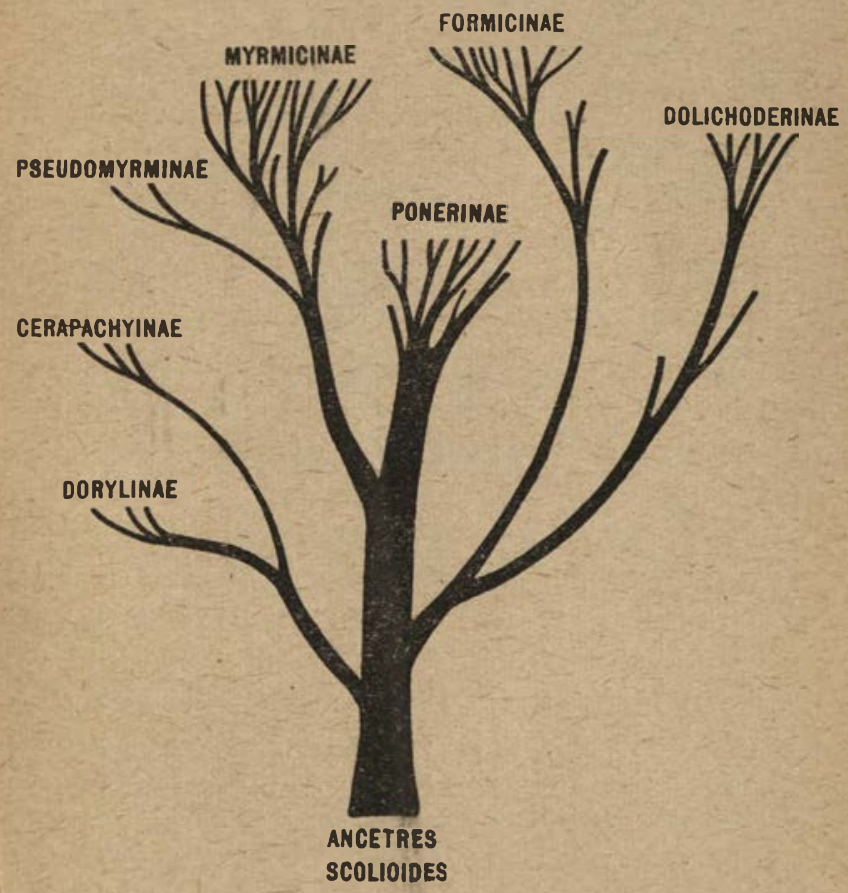

Fig. 16 .

Irès anciens avec des caractères de haulè spécialisation: dimorphisme sexuel prononçé, puisque les différences ensre males et femelles sont, a part les ailes, plus pronon- 
cées que dans tous les autres Aculéates, caste d'ouvrières extrêmement spécialisées, non seulement aptères, mais différant beaucoup plus de la femelle fertile, ou reine, que chez les Apidés ou les Vespidés solitaires. Toutes ces différences indiquent une évolution très longue, plus longue apparemment que celle des guêpes ou des abeilles sociales. Il est évident qu'il ne faut pas chercher les ancêtres des Formicidés parmi les Spluecidoe, ou Fouisseurs, mais, à cause de la structure du thorax, l'absence des strigiles aux pattes postérieures, la nervation de l'aile, etc..., parmi les Vespoïdés ou Vespiformes. Les Diploptères (Vespida:) et Pompilidre doivent être éliminés comme ancêlres, à cause de leurs caractères morphologiques. Nous restreignons donc la recherche à la série de dix familles qui constitue la section Heterogyna, la plus ancienne du sous-ordre des Iculéates: les Sapygidar, Scoliida, Tiphiidie, Thynnidce, Methocidae, Plumariida, Mutillide, Apterogynida, Fedschenkiidce et Anthoboscida. Le type de comportement de ces insectes, pour autant qu'on le connaisse, est très uniforme, et plus primitif que celui des Pompilidés, des Vespidés solitaires et des Sphécidés, puisque ils se conduisent précisément comme des parasitoïdes, cherchent et paralysent leur proie insecte, et pondent sur elle in situ, ou, dans de rares cas (Sapygidoe), se conduisent comme parasites d'abeilles ou de Sphécidés.

Les auteurs n'ont pas été longs à tirer parti du fait que nous venons de mentionner, pour soulenir des opinions différentes sur la famille à laquelle se relient le plus étroitement les Formicidiés. La plupart d'entre eux, à la suite de Latreille, se sont arrêtés aux Mutillidés, mais d'autres ont montré des caractères de fourmis chez pres- 
que toutes les autres familles d'Heterogyna. (Superfamille "Formicinà ", de Börner) (I).

Selon BöHXER (1919), on pourrait construire une fourmi idéale en combinant l'aile postérieure du mâle de Mutilla, les mâchoires d'un Myrmoside, les épines tibiales d'un Méthocide et le crâne d'un Sapygide. Il considère réellement les Plumariidære comme uno sous-famille de Formicidés et Plumarius comme "peut-être la plus ancienne des formes de fourmis". Mais si sa suggestion que le cràne des fourmis est plus primitif que celıi des Scoliidés, Thynnidés et Mutillidés est intéressante, il est d'autre part évident que les Sapygidés, et très probablement les Plumariidés, sont, ḋ maint égard, moins analogues aux fourmis que beaucoup d'autres familles d'Heteroryyna. Les Sapygidés sont connus comme parasites d'abeilles solitaires. Les mœurs de Sapyga quinquepunctata, telles que les décrit FaBre (1890), el celles de $S$. similis, telles que les décrit NielseN (1902), montrent quils se conduisent comme des Térébrants et utilisent leur long aiguillon en oviscapte, pour percer les parois de la cellule de l'abeille et déposer l'œuf près de l'œuf de l'hôte, ou sur lui. La jeune larve de Sapyga, après avoir dévoré l'œuf de l'hơte, s'attaque aux provisions qui lui élaient destinées (pollen et miellée). A cause de ce régime végétal, Nielsev voudrait placer les Sapygidés parmi les Apidæ, où Curtis les avait une fois placés (près des abeilles parasites du genre Nomada), "ou même dans une famille alliée indépendante » (2).

(1) Bönner (1919), comme Handirsch, a opéré beaucoup de fusions dans ce groupe. Ce dernier aute!ır (192/), ne reconnait que quatre familles: les Sapygida, les Rhopalosomatidæ (que j'ai placée dans les Bethyläldea), les Mutillidæe et les Formicidx, et traite comme sous-familles les autres groupes dont nous avons parlé plus haut. Les Mutillidx englobent donc, comme sousfamilles, les Mutillinæ, les Anthoboscinæ, les Scoliinæ, les Fedschenkiinx, les Plerombrinx, les Thynninie, les Methocinæ, les Myrmosinæ, les Apterogyninæe et les Radobæninæ. Il considère que les Plumariidæe constituent une superfamille indépendante, qu'il place au commencement de la série des Aculéates.

(2) Si ce qui dit Rонwen dans les " Hymenoptera of Conneclicut " $(1916$, p. 620) est correct, ¿̀ savoir que l'une des Sapygidés Nord-Américaines a été élevée sur les cellules d'une guêpe crépineuse (Sceliphron cementarium), le genre contiendrait au moins queluues especes entomopliages. 
Des singuliers Plumariidés, on ne connait que des mảles. If n'y a que deux genres; Plumarius (Konowiella), de l'Argentine et du Chili et Myrmecopterina (Archihymen) de l'Afrique du Sud (voir BrLes, 1924c), distribution qui indique que la famille est très ancienne. La nervation alaire est heaucoup plus primitive que celle des fourmis et les antennes et l'abdomen sont tout ì fait différents. La femelle est probablement aptère.

On connaît peu de choses en ce qui concerne les Fedschenkiida et les Anthoboscidx, chez lesquelles les femelles possèdent des ailes, comme les mâles. On ne connaît que deux espèces de Fedschentia, toutes deux du Turkestan (Ernest AXDRÉ, 1903). La seule espèce d'Anthoboscidæ que j'aie vue, notre Sierolomorpha ambigua d'Imérique du Nord, alors qu'elle est très primitive, parait être trop simple pour qu'on la regarde comme proche de l'ancêtre hypothétique des Fourmis. Les Mutillidx, Apterogynidæ, Thynnidæ, Myrmosidæ et Methocidx ont des femelles aptères; on ne peut donc les considérer comine des groupes ancestraux. En ceci, je m'accorde avec Handirsch et je m'écarte de Fonel et d'Emery. Dans son travail important sur les Dorylinie (1895), EMERy rejette les Thynnidés, que Fonel avait inclus parmi les ancêtres possibles des Fourmis, mais il dit: "Dans l'ensemble, je suis d'accord avec Forel, mais je soutiens avec plus de force. l'opinion que les ancètres des fourmis étaient ètroitement reliés aux formes les plus anciennes de Mutillidés et même que leur morphologic était celle de cette famille \%. Il regarde la relation avec les Thynnidés comme "simplement collatérale, et fondée sur leur dérivation commune des Mutillidés ». Ce sont évidemment les organes génitaux mâles très généralisés des Mutillidés, la condition aptère de la femelle des fourmis Dorylines et le pédicule abdominal particulier d'Apterogyna, rappelant celui des Fourmis, qui ont conduit EMEnY à sa conception. Mais la nervation alaire de ce genre, que E. ANDRÉ et EMERY incorporent aux Mutillidés, est particulièrement spécialisée, très différente de celle des Formicida. Entry a vu parfaitement que la condition aptère des femelles de Mutillidés est un obstacle; aussi soutient-il hardiment que les femelles de fourmis étaient originellement aptères, mais auraient secondairement acquis des ailes parfaitement développées par héritage des mảles. Mic Clendon et moi (1903) avons critiqué il y a longtemps cette opinion, mais, en 1920, le myrmécologiste italien affirmait qu'ıl n'en avait pas changé, bien que, dans le paragraphe suivant, il inontrât que son opinion actuelle était différente; les fourmis ancestrales auraient possédé deux sortes de femelles fertiles, 
une ailée et une aptere, opinion que je suis heureux de parlager (vide infra, p. 132). Il ne nous reste ainsi que deux familles, les Scoliida et les Tiphiidæx, que l'on réunit souvent en une famille unique. Haxdinsch (en 1893 et en 1908), les considérait comme extrèmement voisines des fourmis.

Il me semble que le groupe qui mérite l'allention la plus séricuse, au point de vue de l'origine des fourmis, est celui des Tiphiidre, el spécialement le genre Elis (Myzine), car il ressemble aux fourmis par la forme des yeux, la nervation alaire et la tendance générale à posséder une constriction entre tous les segments abdominaux, fait qui rappelle si bien ce qu'on trouve chez certains Cerapachyinx (Sphinctomyrmex, Zasphinctus, Eusphinctus) et Dorylinæ (Leptanilloides) (1).

Il est intéressant de noter que celte vraie famille des Tiphiidæ a des affinités de structure et de mœurs avec les Béthylidés, considérés dans une conférence précédente. Il est probable que les trois groupes convergents: Belhylidæ, Tiphiidx et Formicidæ, ont une origine commune parmi les formes éteintes, formes ancestrales dont les mœurs auraient ressemblé à celles des Bethylidx disparus. Il est probable qu'ils chassaient des proies insectes, qu'ils les paralysaient el les trainaient dans leurs lerriers, pour pondre leurs œufs sur elles. La mère insecte serait restéc avec ses jeunes jusqu'à ce qu'ils fussent mùrs. Nous passerions aisément d'un tel état subsocial à un état social, tel qu'il existe chez toutes les fourmis, en imaginant que les filles, à l'éclosion, soient restées attachées à leur mère el aient amené leurs proies dans le terrier maternel. Une division physiologi-

(1) Nul taxonomiste, apparamment, n'a noté que les fourmis seules possèdent une paire de grandes glandes à la partie poslérieure du thorax (voir J ANET 1898b). Elles paraissent exister chez. loutes les espèces, et dans toutes les castes, et s'ouvrent près des insertions des cuisses postérieures. Chez quelques fourmis, par exemple chez les especes de Cremalogaster du sous-genre Physocrema, des Indes Orientales, les chambres et les tissus de ces glandes sont si développés que tout le métathorax est visiblement enflé. Je n'ai pu retrouver aucune trace distincte de ces formations chez les autres Aculéates, ni dans les sous-ordres inférieurs d'Hyménopteres. Avant qu'on puisse se prononcer sur leur signification taxonomique, il est nécessaire de faire une ctude comparative étendue, avec des préparations totales du légument et des coupes de la région mélastermale, ctans des lamilles variées d'Hyménoptères. 
que du travail a pu se développer ensuite graduellement chez ces individus associés, la femelle fondatrice de la colonie ayant appris à nourrir sa première couvée avec sa salive au lieu de proies, tandis que ses filles seraient devenues les nourrices slériles des portées suivantes. Nous pouvons aussi penser que ces ancêlres des fourmis, Bèthyloïdés ou Tiphioïdés, avaient à la fois des femelles ailées et aptères, comme Scleroderma. Cette conception nous fournirait le point de départ nécessaire des trois formes de femelles; fertile et ailée, fertile et aptère, et stérile et aptère (ouvrières), formes que nous trouvons chez les fourmis actuelles. Nous éviterions ainsi la dérivation très improbable que soutient EMEıY : dérivation de femelles aptères avec héritage des ailes des màles. Laptérisme actuel des femelles de Mutillidx, Thumnidx, etc., pourrait se rapporter aussi au dimorphisme des femelles des ancêtres Béthylö̈dés éloignés, mais nous devrions concevoir qu'il n'y aurait eu aucun progrès dans le type de comportement, pas même jusqu'à un stade subsocial comme celui de Scleroderma.

Le seul résultat de cette discussion, qui a été poussée, je le crains, au point de devenir ennuyeuse, est peut être le suivant: les Formicida ont les nuêmes relations phylogéniques avec les familles les plus inférieures des Aculéates, spécialement avec les Tiphiida et les Bethylida, que les Vespidés sociaux ont avec les Eumenina et les abeilles avec la famille des Sphecida.

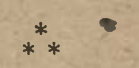

Notre perspective des origines lointaines des Formicidés, telle que nous la découvrent leur morphologie et celle des autres familles d'Heterogyna, s'approfondit si nous faisons appel aux faits paléontologiques. Ici, nous sommes sur un sol un peu plus sûr que pour l'étude paléogégraphique des autres familles d'Aculéates, parce que les fourmis sont les insectes les plus abondants dans les dépôts tertiaires. Nous les retrourons jusqu'à l'Eocène, 
mais, de celte dernière période, on ne connait qu'un petit nombre de spécimens. Par contre, le nombre de fourmis oligocènes el miocènes est considérable. Si le nombre d'es. pèces décrites est moins important que celui de toutes les autres familles d'llyménoptères réunies, les spécimens sont tellement plus abondants que notre connaissance des formes individuelles est notablement plus satisfaisante. Ainsi, jusquà présent, on a examiné Ir. - I I fourmis, représentant seulement g3 espèces, de l'ambre de la Ballique. Sur ce nombre, $1.46 \mathrm{r}$ ont été étudiées par Marr (1868), 6go par Ernest Axdné (1 895$)$ et 9.560 par moimême (1914). On a accumulé dans les musées beaucoup) de matériel d'autres formations, sans l'étudicr. H y a pas mal d'années, plusicurs musées américains m'ont envoyé, pour en faire l'étnde, quelque 8.000 fourmis des argiles schisteuses miocènes de Florissant (Colorado), mais la pression incessante d'aulres travaux m'a empêché d'en faire même une détermination générique satisfaisante. J'espère toujours que quelqu'un de mes étudiants reprendra le travail avec mon aide, et le mènera à bien. Il y a soixante-seize ans (1849), Heer a décrit un certain nombre de fourmis fossiles conservées de la niême façon, et également d'âge mincène, des argiles schisteuses de OEningen (Bade) et Radolooj (Croalie), mais ses déterminations génériques sont si douteuses qu'elles sont inutilisables. Heureusement, un certain nombre de ces spécimens ont été examinés à nouveau plus tard, et réinterprétés par le célèbre myrmécologiste Guslav MıYn (I867). Une série d'espèces restreinte mais extrèmement intéressante, a été décrite en I 89 I par Emery, de l'ambre de Sicile (Miocène moven). Plus récemment, Cockerele (igr5, ig20 a) et Donistuonpe $(\mathbf{1} 920)$ ont décrit plusieurs espèces de l'Eo- 
cène et de l'Oligocène moyen de l'Angleterre. Dans son livre nonumental sur les insectes fossiles, IAnntinscir (1908) a dressé une liste de près de 300 espèces de fourmis tertiaires, mais je n'arrive pas à y trouver, pas plus que dans les espèces décrites jusqu’à présent, plus de 169 ayant des déterminations génériques valables et mon esti. mation est encore probablement trop libérale.

Bornons-nous d'abord an Tertiaire d'Europe. On connait seulement deux espéces de l'Eocene(couches de Bagshot de Bournemouth). Elles ont été décrites par Cockinell (1920a) sous les noms de Formica heteroptera et de OEcophylla bartoniana, loutes deux à partir d'ailes antérieures (1).

Vous nolerez que les 169 espèces se répartissent entre 54 genres, dont 27 , c'est-à-dire la moitié, sont éteints, tandis que la moitié restante est divisée à peu près également entre genres existants représentés par des espèces en Europe et dans d'autres continents, et genres qui sont actuellement confinés à la région indo-australienne. Beaucoup de genres éteints étaient également de celte région. C'est pour l'Oligocène inférieur (ambre de la Baltique) que le tableau montre le plus clairement ces faits. C'est la plus ancienne formation qui ait donné beaucoup de fourmis. te fait est dù largement, sans aucun doute, au grand nombre de spécimens et à leur très belle conservation. C'est dans l'ambre de la Sicile (Miocène moyen), qu'on trouve le plus grand nombre de genres indo-australiens. Sur les

(1) Cócrenell compare l'aile de $F$ heteroptera à celle de Colobopsis stricta Jerdon, de l'Inde, je n'arrive pas à imaginer pour quelle raison, car Colobopsis, comme tous les autres sousgenres de Camponotus, $n^{\circ}$ il pas la cellule discoïdale qui est présente chez le fossile.

Bien que les spécimens soient des ailes de fourmis indubitables, ils ne se prêtent pas à une identification générique. Tout ce que nous pouvons dire en ce qui les concerne, c'est que, pendant les temps éocènes, de vraies fourmis, appartenant probablement a la famille la plus hautement spécialisée des Formieinx, vivaient dans ce qui est actuellement l'Angleterre. Pour être aussi bref que possible, j'ai condensé dans le tableau cidessous tous les documents valables de fourmis fossiles de l'Eocène, de l'Oligocène et du Miocène d'Europe. 
seize espèces, représentant qualorze genres, huit sont indoaustraliens, trois seulement sont éteints el trois seulement sont

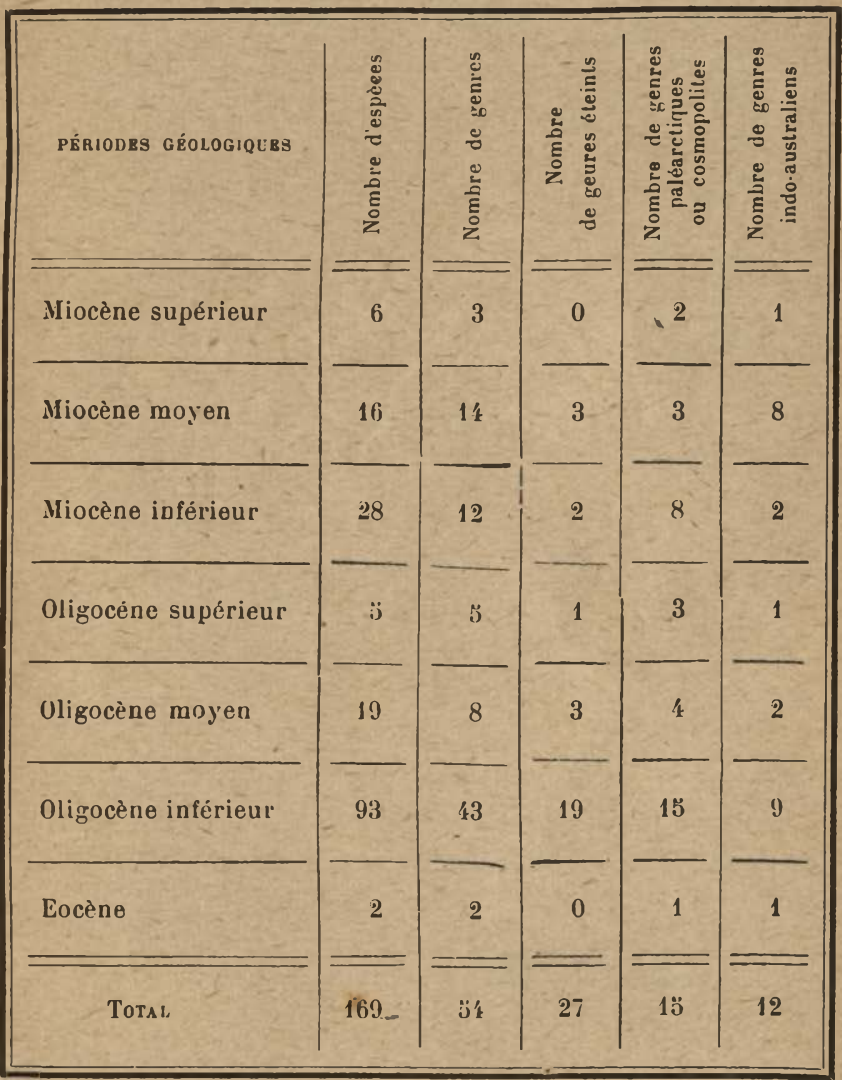

encore représentés en Europe et dans d'autres régions. Par Inalheur, les lourmis et les autres insectes sont loin d'etre 
aussi abondants dans l'ambre de la Sicile que dans celui de la Baltique.

A part le Miocène de Florissant, auquel j'ai déjà fait allusion, les formations lertiaires de l'Amérique du Nord ont fourni très peu de fourmis. Scconer (1890) rapporte quatre espèces de l'Oligocène de Quesnel (Colombic britannique) aux genres actuels bien connus Aphicnogaster, Myrmica, Dolichoderus et Formica, et Cockerell (1906a) a décrit une Ponera hendersoni de Florissant, mais la lecture atfentive des descriptions et l'étude des figures me laisse des doutes sur la valeur de l'identification générique de tous ces inscetes. On connait six espèces
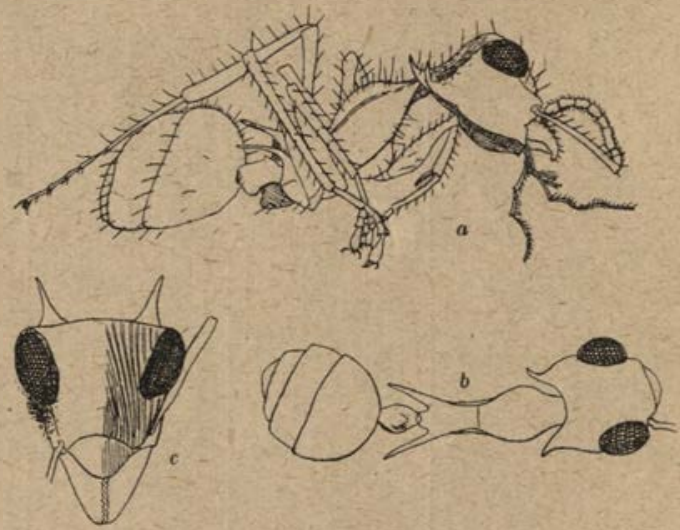

Fig. 17. - Sicilomymmer corniger, de lambre de sicile. (d'apu's EMFKY).

de l'Eocène. Elles ont un intérèt particulier, parce qu'à l'exception des deux de Bournemouth (Angleterre), ce sont les fourmis les plus anciennes quion connaisse. Trois dentre elles ont été décrites par Sccnner, à savoir Camponotus velus et Liometopum pinque de l'Eocene de White River (Colorado) et Lasius. terreus de l'Eocène de Green River (Vyoning). Je considère les identifications génériques comme très douteuses. Les trois autres espèces, décrites récenment par Cockenfli. (1921á, 1923a et. 1923b) sont Eolormica eocenica et Archimyrmex rostratus de la formation de Green River, ef Formica eoplera, de lEocine supérieur (Jackson) du Texas. Eolormica et Archimyrmex sont 
décrits comme genres éleints. Le premied, que Cockerelt pense pouvoir ètre identique a Liomelopum pinque de Scudner, n'd certainement aucun rapport avec Formica ou Liometopum. I,a restóuration de la tête faite par Cockerell, telle qu'on la voit de lace, ne peut être identifiée, au moins en ce qui concerne les yeux et ocelles, à la tête de profil dans la photographie du fossile. Tout ce qu'on peut dire, à l'examen rle la figure, c'est que le spécimen est un mâle, probablement de quelque Formicidé. Cockerell pense qu'il se relic a CEcophylla, mais c'est une queslion de le savoir. Il pense aussi que son Archimyrmex rostralus est un Ponériné voisin de Prionomyrmex de l'ambre de la Baltique et du genre actuel Myrmecia, d'Australie. Mais, a en juger par la figure, le spécimen est plus probablement un Myrmiciné. C'est ce que suggèrent la forme du pétiole, la pointe émoussée ou brisće (?) de l'épinotum e! la forme de la tête, qui est différente de celle des Ponerinæ actuelles. Eoformica eocenica est décrite à partir d'une simple aile antérieure, magniliquement conservée, qui est nettement celle d'une fourmi et probablement d'une Formiciñs, mais une détermination générique plus précise n'est pas possible.

Bien que la position générique précise du petit nombre de fourmis connues de l'Eocène d'Europe et de l'Amérique du Nord, soit si incertaine, il est sûr que ces fourmis ne sont en aucune manière plus primitives ou plus généra. lisées que les fourmis de l'ambre, et ces dernières sont presque aussi haulement spécialisées que nos formes vivantes. Toutes les huit sous-familles, à l'exception des Dorylince et des Leptanillinue, - sont représentées dans la faune de l'ambre, et si bien qu'il n'a nullement élé nécessaire de modifier leur définition pour les accommoder aux espèces tertiaires. Non seulement les trois castes étaient aussi nettement différenciées chez les fourmis de l'ambre que chez les fourmis actuelles, mais les ouvrières de quelques genres (Pheidologelon, I'seudolmsius, Dimorphomyrmex) étaient déjà pléo- ou polymorphes. Les larves et pupes également, qui sont quelquefois conservées, sont précisément semblables à 
celles des espèces vivantes. Comme je l'écrivais en I $9^{1} 4$ : "Il y a donc des indications cerlaines que les mocurs et les instincts des fourmis de l'ambre étaient presque aussi évolués, sinon autant que ceux des fourmis vivantes. Plusieurs d'entre clles avaient appris à visiter les pucerons, étaient en conséquence devenues "trophobiotiques ", comme le montre un bloc d'ambre de la collection de Königsberg, contenant un certain nombre d'ouvrières de Iridamyrmex goepperli mélangées à un lot de pupilles pucerons. On peut difficilement douter que les fourmis de l'ambre eussent des myrmécophiles dans leurs nids, puisque Klebs mentionne, dans ca liste des Coléoptères de l'ambre (igro), trois genres de Paussida (Cerapterus, Pleuropterus et un genre non décrit). Ces fourmis avaient aussi des Acariens parasites, comme le montrent deux ou. vrières de Lasius schiefferdeckeri de la collection de Königsberg, chacune portant un $\Lambda$ carien altaché à la base de l'un des tibias postéricurs. Ces spécimens montrent également que les Acariens avaient déjà acquis l'habitude particulière de se fixer dans des régions défunies du tégument de leur hôte "). La faune de l'ambre de la Baltique est, en vérité, si semblable à la faune vivante que nul myrmécologiste ne serait surpris de trouver n'importe lesquels de ces genres éteints encore vivants à l'heure ac. tuelle dans les forêts des Indes Orientales ou de l'Australie, ou de trouver dans l'ambre les genres bizarres el archaïques de ces régions. En fait, cela est arrivé deux fois. Gesamyrmex a été décrit par Mark comme genre éteint en 1868, mais plus tard, Ern. ANDRÉ ( 1892 ), en décrivit une espèce vivante ( $G$. chaperi) de Bornéo, et j'en ai rícemment indiqué un autre du sud de la Chine (Ig2i). Er. nest Avdré a décrit le genre Dimorphamy'rmex de Bor- 
néo, el, en 1.g0j, EmERy en trouva une espèce dans l'ambre. J'en ai trouvé une seconde espèce dans la mème formation (1914) et une autre virante dans les Philippines $(\operatorname{Ig} 6)(\mathrm{r})$.

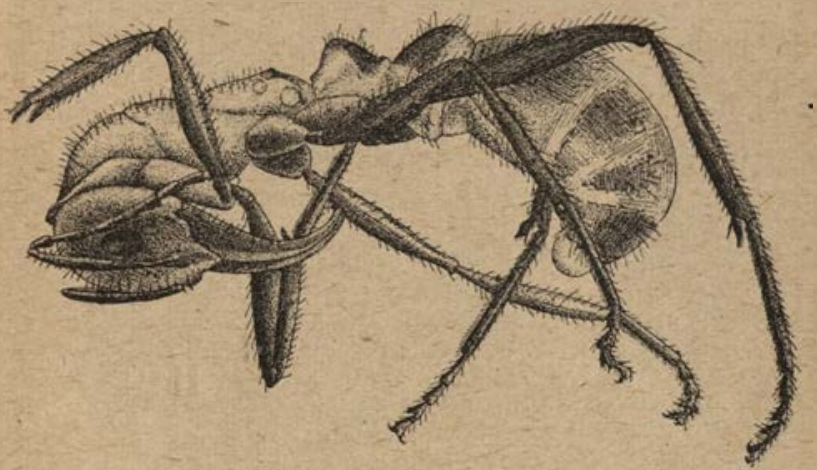

Fig. 18. - Ouvrière de Prionomyrmex longiceps (Ponériné) de l'ambre de li Baltique.

$a$, profil ; b, face dorsale ; $c$, tête vue dorsalement

(1) Il est à peine nécessaire d'indiquer qu'on ne peut fonder, dans deg spéculations sur la distribution géographique d'un genre particulier à l'époque actuelle, aucune conclusion sur l'absence de certains genres dans l'ambre ou d'autres formations géologiques. Mais les zoogéographes ignorent continuellement un truisme si évident. Tous les documents paléontologiques positifs sont extrêmement précieux. Au contraire, les assertions sur l'absence de formes particulières dans des parties du monde diverses durant les périodes géologiques sont absolument sans valeur. Nulle part lexactitude de cette vue n'est plus clairement illustrée que chez les insectes, et spécialement les fourmis fossiles. La nature des formations dans lesquelles se rencontrent les fossiles peut avoir empèché la conservation de portions significatives de la faune. Ainsi, l'ambre a englué seulement les insectes petits el délicats, et, parmi les 


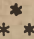

Avant d'entamer la discussion de certains points en rapport avec la distribution géographique actuelle des fourmis, je dois appeler l'attention sur certaines de leurs particularités générales que personne ne me semble avoir suffisamment soulignées. Nous avons vu que les Formicida sont l'une des familles étroitement reliées d'une série, celle des Heterogyna, dont plusieurs ont des mœurs si exclusivement terrestres, que leurs femelles ont complètement perdu leurs ailes. Ces familles, dans leur en-

fourmis, surtout les espèces arboricoles, ou celles qui montent à l'occasion sur les arbres. Nous n'y trouvons par conséquent qu'un petit nombre de Myrmicina, Cerapachyina et Ponerinæ, qui sont surtout terricoles, et, au contraire, un grand nombre de Dolichoderinæ et Formicinæ qui, ou bien vivent dans les arbres, ou bien aiment visiter, dans leur feuillage, les Aphides, les Coccides, etc. L'absence complete des deux sous-familles Jorylinx et Leptanillina de l'ambre peut s'expliguer de la mème façon, parce que la plupart des espèces actuelles de la première famille, et loutes les espèces actuelles de la dernière sont souterraines, ou hypogées. On peut aussi concevoir, il va de soi, que ces sous-familles peuvent ne pas avoir été représentées dans les forêts de l'ambre. L'action sélective du milieu est encore plus manifeste dans le cas des fossiles miocènes de Florissant, Oiningen et Radoboj. Presque tous ces fossiles, contrairement à ceux de l'ambre, sont des femelles et des mâles ailés, ou qui viennent de perdre leurs ailes récemment, et les arguments géologiques montrent que les argiles schisteuses dans lesquelles on les trouve ont été déplosées dans des lacs peu profonds; les fourmis mâles et femelles y sont tombés en descendant du vol nuptial, et les ouvrières n'y ont été entrainées que rarement par des crues. Faute d'avoir remarqué cello explication très simple, un éminent géologue américain Joseph LECONTE (1884) a conclu que les mœurs sociales, les instincts et la caste nuvrière se sont développés chez les fourmis depuis le Viocène, ador's qu'un regard, mème hatif, sur los fourmis de l'ambre de la Baltique (Oligocène infèrieur) l'aurait conviancu du contraire. 


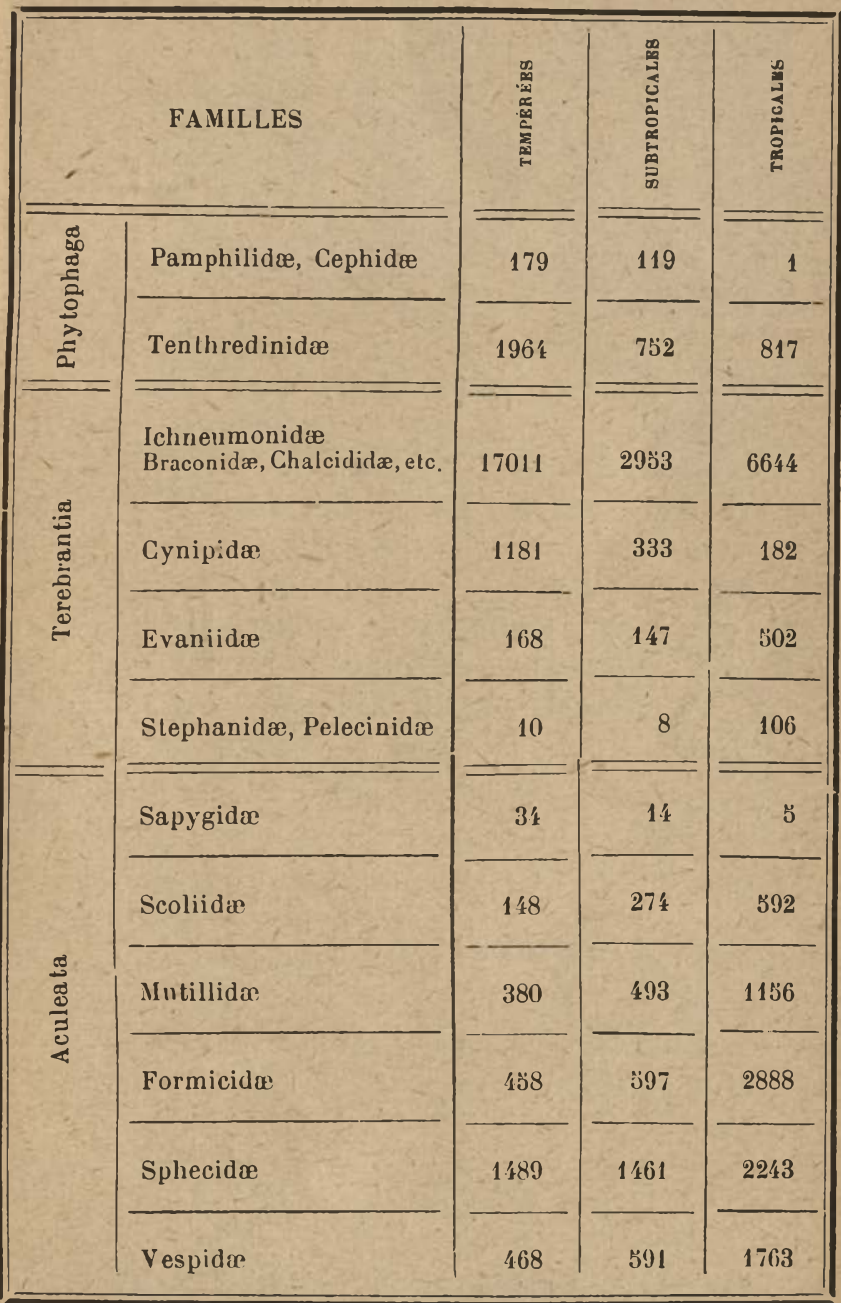


semble, dépendent beaucoup des conditions du sol (édaphiques) et du climat, et presque toutes sont nettement thermophiles. Le tableau (p. 141) de Handulascil ( $1913 b$ ) montre que ce n'est pas vrai des Hyménoptères en général. Dans ce tableau est indiqué le nombre d'espè̀ces de plusieurs grandes familles des trois sous ordres dans les zònes tempérées, subtropicales et tropicales.

On observera que les Phytophages abondent dans la zône tempérée mais diminuent de mombre vers les tropiques, - que quelques familles de Térébrants montrent la même particularité, tandis qu'on observe une augmentation vers les tropiques des autres familles, et que les Aculéates, à la seule exception des Sapygida, ont une thermophilie similaire prononcée, qui se manifeste le plus netlement chez les Formicidoe et les familles alliées: Scoliidoe ot Mutillidoe (qui sont évidemment prises ici sensu lutiore). Les Vespidés montrent une thermophilie presque aussi marquée que celle des autres Vespoïdés. Si les abeilles avaient été comprises dans le tableau, nous aırions observé un développement maximum dans les zones subtropicales. Par conséquent, nous pouvons conclure que les Heterogyna, dans l'ensemble, constituent un groupe d'Aculéates fortement thermophile.

II serait intéressant de construire un autre tableau pour marquer les degrés de xérophilie des Aculéates, mais ce serait plus difficile. La grande majorité des espèces de beaucoup de familles d'Heterogyna, notamment des Mutillida, Apterogynidre, Thynnidee, Plumariider, Methocidce, My'rmosida et F'edschenliiidae préfèrent indubitablement un milieu sec. Tous ces groupes abondent donc dans les déserts, quelques-uns y sont confinés. La xéro- 
philie des Formicida est moins évidente, parce qu'elle est masquée par certaines autres tendances. Alors qu'il est vrai que les espèces de fourmis sont très nombreuses sous les tropiques humides des deux hémisphères, il est également vrai que les individus de fourmis sont presque aussi abondants dans les déserts, dans des régions telles que les garrigues de New-Jersey et d'autres endroits, qui portent une végétation plus ou moins xérophyte. Par con. tre, elles sont pauvrement représentées, en espèces comme en individus, dans les régions froides et humides, comme la Nouvelle-Zélande, le sudi du Chili, la Patagonie, le nord de l'Europe et les Selkirk-Mountains de l'Imérique britannique. Le fait que la grande majorité des fourmis nichent dans la terre, montre que la famille était originellement terricole, et cet habitat l'oblige à éviter tous les sols qui ne sont pas bien drainés. D'où leur rareté dans les prairies humides et les marécages, et leur prédilection pour les pentes des montagnes et des collines, spécialement celles qui font face à l'est et au sud dans l'hémisphère nord et au nord et à l'est dans l'hémisphère sud. Dans les portions pluvieuses des tropiques, où nous trouvons plusieurs grands genres arboricoles, elles ont évidemment choisi la nidification dans ou sur les arbres pour éviter l'humidité excessive et néfaste du sol. Mais, même dans ces régions, comme je l'ai observé dans l'Amérique centrale, et ailleurs dans les régions tropicales humides, les fourmis têrricoles préfèrent les talus, le sol sous les pierres ou les troncs d'arbres abattus, ou le bois décomposé qui absorbe peu d'eau de pluic.

J'ai insisté sur ces faits, parce qu'ils me semblent indiquer en définitive, chez les fourmis, une xérophilie profondément enracinée et une origine très prohable, - en 
même temps que les Scoliides, les Mutillides, les Thynnides, etc... - , sur les grandes plaines et les plateaux continentaux intérieurs, durant l'époque Mésozoïque; dans les régions même, en fait, où quelques botanistes pensent que les Angiospermes ont pris naissance. Il faut noter à ce propos que, mème les forêts de l'ambre, dans lesquelles les fourmis doivent avoir été extrêmement abondantes, étaient probablement plutôt xérophytiques. L'ambre lui-même était produit par une espèce de pin, et la grande quantité de poils étoilés disséminés dans les morceaıx de résine fossile montre que les chênes étaient abondants. En fait, la faune dı Samland doit avoir élé assez semblable à celle des garrigues de New-Jersey, mais avec une adjonction plus grande d'éléments tropicaux. Ulumen (1912), dans sa monographie des Trichoptères (Phryganes) de l'ambre de la Baltique, a ingénieusement montré que la région était monlagneuse. Il trouva que, sur les trente genres actuels de Phryganes qui étaient représentés dans la résine fossile, les larves de r $3 \%$ devaient avoir vécu dans des eaux fortement agilées, $8 \%$ dans des eaux stagnantes, et $4 \%$ daus des cours d'eau à cours lent. II conclut que 35 genres, avec 73 espèces, connus seulcment de l'ambre, passaient leur vie larvaire dans des torrents, que 7 genres avec 14 espèces vivaient dans des eaux tranquilles, et que 7 genres avec 7 espèces étaient probablement indifférents pour leur habitat aqueux. Parmi les aires continentales existantes, l'Australie me semble présenter les conditions les plus rapprochées de celles dans lesquelles je considère que les fourmis mésozö̈ques doivent avoir évolué. Ce continent possède une végétation plus ou moins xérophyte, excepté dans l'extrême nord, où il y a un apport considérable d’immigrants papous dans 
la flore et la faune. Le grand développement des Thynnidés en Australic est aussi significatif du même point de vue.

Nous pouvons conclure, par conséquent, que les fourmis sont primitivement une famille d'insectes terricole et thermophile, qui a pris naissance dans un milieu sec, et qui préfère toujours un tel milieu. A partir de cette condition, nous pouvons tracer trois lignes de rayonnement et de spécialisation adaptatifs. Un certain nombre de genres, dans toules les sous-familles, exceplé les Pseudomyrmina et les Dolichoderince, unt abandonné la vie à la surface du sol et sont devenus souterrains, ou hypogés. D'autres ont renforcé leur tolérance naturelle pour la sécheresse et sont devenus déserticoles, et il est intéressant de noter que celle catégorie comprend plusieurs genres primilifs, snécialement parmi les Myrmicince (Messor, Vovomessor, Veramessur, Pogonomyrmex). Finalement, un cerlain nombre de genres et de sous genres, dans les régions à grandes pluies, sont devenus arboricoles. Il est intércssant de noter que cette dernière catégorie est représentée à peu près exclusivement par les genres les plus spécialisés des quatre sous familles les plus élevées, les Myrmicince, les Pseudomyrmince, les Dolichoderince et les Formicince, et n'a pas de représentant parmi les Dorylino, ni les Leptanillina, et seulemenl un trìs petit nombre (par exemple Neoponera), parmi les Ponierina. En outre, même des genres, aussi exclı. sivement arboricoles que Pseudomyrma et Crematogaster contiennent quelques formes terricoles. Pseudomyrma elegans, par exemple, comme l'a montré Fones, niche toujours dans le sol, et il y a plusieurs Cremutogasler 
qui nichent dans un sol plutôt Eec dans l'Amérique du Nord, l'Australie et ailleurs ( $\mathbf{I}$ ).

(1) Si mes conclusions sont correctes, EMERY (1920) doit s'ètre trompé dans son inlerprétation des successions d'habitat dans la section des Eucamponotinæ, groupe qui comprend le genre Camponotus, avec plus de 1.000 espèces, sous-espèces el variétés décrites, et plusieurs genres alliés.

Tous les groupes arboricoles cités par EMERY se rencontrent en Australie, excepté Gesomyrmex, qui est indo-malais et Gigantiops, qui est néotropical. Mais Gigantiops, comme je l'ai montré (1922b), n'est pas arboréal. Dans la Guyane britannique, je l'ai trouvé nichant dans de vieux troncs d'arbres sur le sol, et le Dr W. M. Mann m'informe qu'il l'a pris sous des pierres en Bolivie. Gesomyrmex, également, est probablement terricole. Les lypes de $G$. howardi m'ont été adressés mélangés à un lot de fourmis bien connues comme terricoles. Selon mes observations, quelques-unes au moins des espèces d'Opisthopsis et de Calomyrmex nichent dans la terre. Parmi les nombreux sous-genres de Polyrhachis, toutes les espèces ausitraliennes de Campomyrma et Chariomyrma sont terricoles et, en outre, comme Calomyrmex, distinctement xérophiles. En Afrique, certains membres du sous-genre Myrma sont terricoles et il en est probablement de même de quelques de ses espèces des Indes Orientales. On ne connait rien des mœurs de l'Echinopla, mais il est probablement arboricole. Et, quand nous bornons notre attention à Camponotus, nous trouvons que les sous-genres terricoles Myrmoturba, Dinomyrmex, Myrmosericus, elc..., sont évidemment beaucoup plus primitifs que les sous-genres Myrmorhachis, Colobopsis et ses alliés, etc..., qui ont la tête et les mandibules de la reine et des soldats plus ou moins spécialisées pour vivre dans le bois. Ici encore, il faut noter que Myrmoturba, qui embrasse un nombre considérable d'espèces, sous-espèces et variétés, est hautement xérophile, car la plupart de ses formes sont confinées à des régions quelque peu arides ou désertiques. Dans l'Amérique du Nord, Myrmoturba se rencontre seulement dans les portions les plus sèches des états du SudOuest et de l'Ouest. Par conséquent, à mon avis, l'évolution des Eucamponotini a été exactement l'inverse de celle qu'à indiqué EMERY. Je considère comme également fallacieuse son opinion, que les fourmis ancestrales, qui ont donné naissance a OEcophylla, Camponotus et Polyrhachis, avaient l'habitude d'employer leurs larves à unir en filant des feuilles et des détritus 
L'existerice, dès l'Oligocène inférieur, d'une grande série, remarquable par la diversité des genres, - dont beaucoup, représentant presque toutes les sous familles modernes de Formicidce, existent toujours et comprennent même des espèces comme Formica flori et Lasius schiefferdeckeri, identiques pratiquement à $F$. fusca et $L$. niger, espèces actuelles communes d'Eurasie, et de l'Amérique du Nord, - indique que la faune actuelle, même sous les tropiques, peut avoir diminué de nombre d'espèces et de genres depuis le début du Tertiaire. Il n'y a certainement aucun indice d'évolution progressive durant l'ère cœnozoïque: Nous sommes donc amenés à penser que l'origine et les premiers stades d'évolution de la famille, les plus significatifs, se placent à l'ère mésozoïque. Mais nous ne pouvons que conjecturer ce développement; tout fossile nous manque.

Havdlirsch (1go8) el Emery (1920) diffèrent quant à la période d'origine des Formicidae. Selon le premier, " nous devons nous rallier à l'opinion que le foyer d'évolution ("Fntwicklungsher( ") de toute la famille des Formicidce (au sens large) doit être cherché dans les aires continentales eurasiatiques du Tertiaire le plus ancien ou du Crétacé supérieur. Les formes qui auraient pris naissance là, auraient atteint l'Amérique du Nord par les connections continentales orientales et occiden.

pour construire le nid. Cette habitude, que l'on connait actuellement si bien chez OEcophylla, se rencontre seulement chez deux especes néotropicales de Camponolus, parmi plusieurs centaines, et chez quelques sous-genres du genre palcotropical Polyrhachis (Cyrtomyrma, Myrmolhrinax, M!yrmhopla), et il n'y a rien qui indique qu'il n'ait pu être acquis indépendamment dans tous les trois genres, longlemps après qu'ils ont passé à la vie arboréale. 
tales de l'hémisphère nord, et auraient émigré vers le sud, à partir de l'Amérique du Nord, aussi bien que de l'Europe et de l'Asie. Emery conçoil une origine mésozoïque plus lointaine de la famille ; il pense que les Ponerince remonteraient au moins au Crétacé inférieur, les Dorylince aux temps précétacés, et la famille peut être à la fin du Jurassique. Pour ma part, j'estime que ce n'est pas encore assez haut. Ce que nous avons dit de la thermoxérophilic des Formicidae, rend probable leur apparition au Trias, sinon à la fin du Permien, périodes qui, selon les paléoméléorologistes (ScHucuert igrá, HuxTivgtox rgr 4 et d'autres), se caractérisent par une température élevée et une grande aridité. Les mœurs terrestres des fourmis et leurs relations très intimes et très plastiquas avec leurs larves et leurs pupes, qui s'opposent aux moeurs des guêpes et des abeilles sociales, d'une évolution plus récente, semblent indiquer une origine dans des conditions plutôt arides : celles qui ont probablement existé sur les grandes masses continentales, durant le Trias et le début du Jurassique. Les adaplations arboréales de certains genres pourraient ainsi être conçues comme ayant pris naissance durant le Crétacé, période de température continuellement élevée, mais d'humidité plus grande, comme celle des forèts tropicales actuelles.

Si nous postulons un passé géologique si lointain pour les fourmis, nous leur accordons le temps nécessaire pour acquérir, non seulement une grande diversité générique et spécifique, mais encore une distribution cosmopolite, avant le début du Terliaire. In certain nombre de problèmes suggérés par la distribution géographique actuelle des genres prennènt aussi un aspect différent. Ces problèmes sont l'origine de controverses nombreuses et nous 
ne pouvons ici les examiner en détail. EMERY, influencé, il semble bien, par H. von Inering et ScharfF, a fait un usage considérable de communications continentales transallantiques et antarctiques, pour les genres représentés à l'heure actuelle seulement dans l'Amérique du Sud, l'Afrique du Sud, l'Australie et la Nouvelle Zélande, landis que Havnurascir (Igr3b) et moi-même (IgI ze), inclinons à adopler, comme explication satisfaisante de la distribution actuelle de lels genres "résiduels", la vue plus ancienne d'une origine circumpolaire et probablement eurasiatique des Formicida, et leur migration par la communication terrestre bien élablie de la mer de Behring, ainsi que leur migration vers le sud dans les deux hémisphères. Cette dernière opinion est appuyće par les statistiques soignées de la distribution actuclle de I6. Ino genres et I 80.000 espèces d'insectes. Ces statistiques montrent que les communications continentales diverses, donl voy Imerixg, Scharff, Emery et d'autres postulent l'existence, ne sont rien moins qu'assurées. Handirscil montre en outre que, sur une carte du monde indiquant seulement les plus importantes des communications continentales que réclame l'école analylique des zoogéographes, les océans sont réduils à $\mathbf{m}$ petit nombre de pelites lagunes. Ceci nécessiterait une distribution des organismes marius passés et présents différenle de celle qu'on observe. Dautre part, dans cette hypothèse, les faunes tropicales de lous les continents et des iles seraient homogìnes, toin donc de montrer les diflérences considérables qu'on constate actuellement.

L'étude de notre faune néarctique prédispose naturellement les naturalistes américains it defendre l'hypothise d'une origine boréale des organismes, et leur migration 
vers le Sud, dans d'autres parties du globe, sans l'aide de communications continentales autres qu'entre l'Alaska et l'Asie Orientale et entre l'Est de l'Amérique du Nord et l'Europe. Actuellement, le plus valeureux champion de cette vue, et le plus énergique adversaire des " constructeurs de ponts " est le $\mathrm{D}^{\mathrm{r}} \mathrm{W}$. D. Matrhew (igi5), autorité bien connue en matière de Mammifères vivants et fossiles. Mais, même en Australie, où des communications continentales antarctiques ont trouvé beaucoup de défenseurs, le courant d'opinions semble changer, si nous en jugeons par un intéressant travail récent de Loxgmax ( 1923 ), qui ne trouve que peu d'indications en faveur des opinions de Hedley, Haswell et d'autres ( $\mathrm{r}$ ).

Ce sont évidemment les géologues à venir qui décideront en dernier ressort, si les communications continentales des anciens géologues et des zoogéographes de l'école. de von Ihering ont réellement existé. Si nous en jugeons par des travaux récents très intéressants comme celui de KoBer (I $9^{2}$ I) sur la structure de la lithosphère, il n'y a aucune preuve géologique de l'existence, ni des ponts construitspar von Ineruxg pour le Tertiaire, ni de communica-

(1) Kolne (1913) et moi, avons eté pris à partie par EMERr, parce que nous considérions la faune entomologique d'AusIralie (fourmis comprises), comme essentiellement mésozoïque. Alors qu'il est bien connu que cette faune comprend également un certain nombre d'éléments de la Papouasie et de l'Inde Orientale, il n'y a pas de preuve qu'ils n'aient pénétré en Australie avant les temps tertiaires. Schucher'r (1916) dit: a Nous devons conclure que l'Australie a été un continent insulaire, au moins depuis l'Eocène supérieur, puisque c'est depuis celte période que les Mammifères placentaires ont pris partout la prééminence sur toute la vie terrestre ». Et Longman apporte de bonnes raisons de penser que les Marsupiaux ont eu une évolution. prétertiaire longue el particulièrement locale, en Australie. 
tions intercontinentales similaires durant l'ère mésozoïque. Les conclusions de Koвer semblent bien fondées. Elles se résument en cette phrase : "Nous voyons, par conséquent, que, sans aucun doute, les six grandes masses continentales, à savoir: l'Amérique du Nord, l'Eurasie, l'Amérique du Sud, l'Indo-Australie et l'Antarctide ont eu une existence permanente depuis l'ère mésozoïque ". Et on peut tirer la même conclusion de sa description condensée des continents durant l'ère mésozoïque. Toutefois, il indique l'existence de deux masses continentales, "hypothétiques \#, dans le Pacifique, l'une dont le centre actuel est dans les Hawaï et l'autre dans l'hémisphère sud, mais toutes deux sont petites et loin de pouvoir constituer des ponts continentaux. 

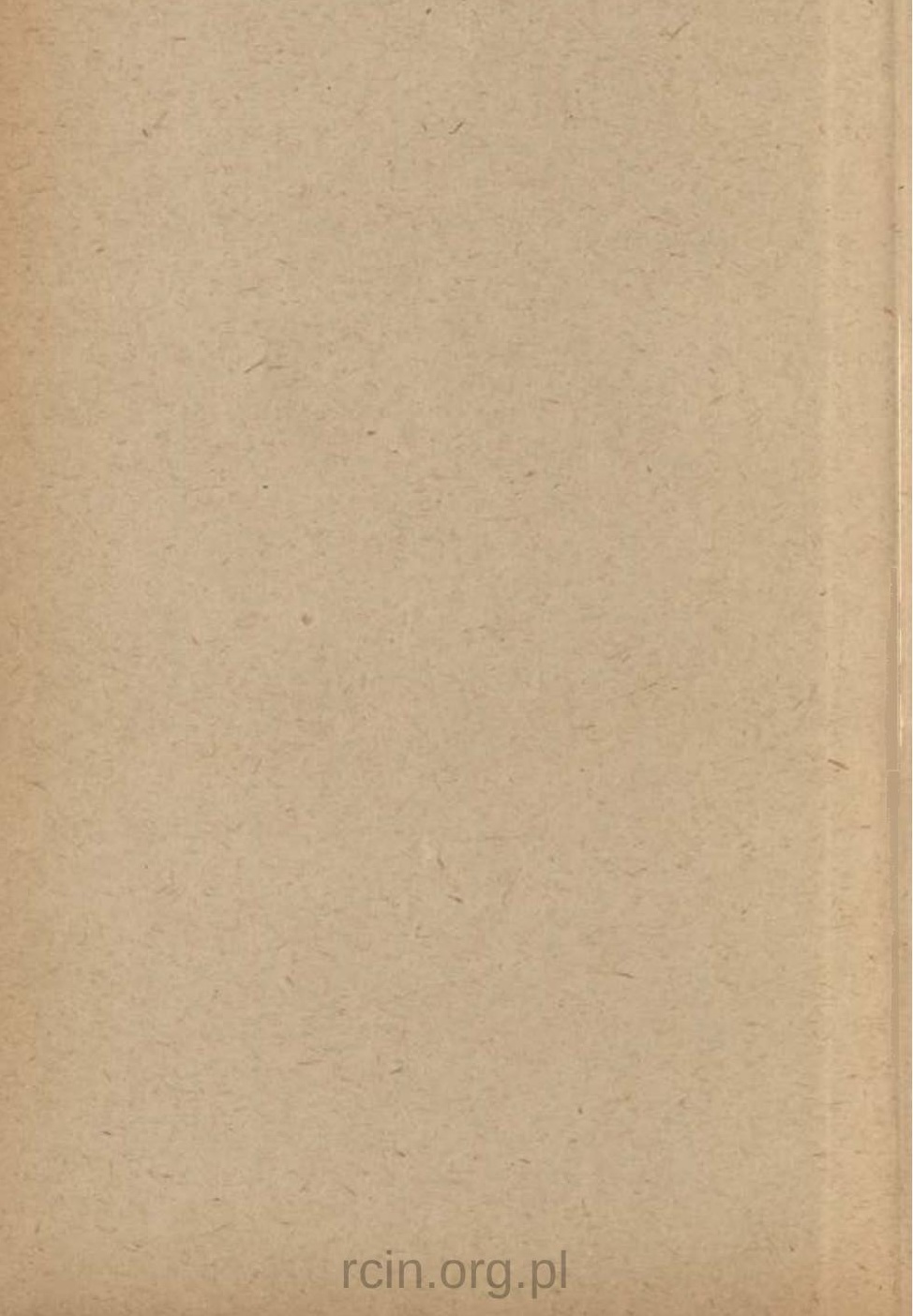


\section{CHAPITRE $\checkmark$}

\section{L'EVOLUTION DES TERMITES}

Composition bisexuelle des colonies de termites. - Caractère tropical du groupe, ses subdivisions, leurs affinités. - Documents paléontologiques. - Origine mésozoïque ou permienne. - Distribution géographique. - Affinités avec d'autres Orthoptères. - Mœurs nuptiales. - Habitudes alimentaires (xylophagie). - Rôle des Protozoaires intestinaux. Ennemis des termites.

Au nombre des caractères présentés par les divers groupes de fourmis, d'abeilles et de guêpes sociales que nous avons examinés dans les leçons précédentes, il en est un particulièrement frappant: c'est le retour constant, dans les mœurs de tous ces groupes, du même type général, du même motif, avec des modifications déterminées par le genre de nourriture et par d'autres nécessités ambiantes. Cette répétition est sans aucun doute provoquée en partie par des ressemblances morphologiques et éthologiques entre les ancêtres solitaires immédiats de ces insectes, el, en partie, par l'ancêtre commun le plus lointain de tous ces groupes. Comme nous l'avons vu, tous les Aculéates sociaux sont dérivés de Sphécoïdes et de Vespoïdes solitaires et j'ai essayé de rattacher ces deux groupes à des Béthyloïdes éteints hypothétiques, qu'on peut supposer ètre la souche primitive du sous-ordre des Acu- 
léates tout entier. Que les abeilles sociales, quoique descendant de Sphécoïdes, soient néanmoins, et à beaucoup d'égards, plus proches des Vespidés sociales que ne le sont les Formicidés descendant des Vespoïdés, ce fait remarquable peut sans doute s'expliquer par une évolution de ce dernier groupe plus longue et plus fertile en événements.

Ce que l'on pourrait appeler l'héritage ou le fonds "solitaire " des Aculéates sociaux est recolivert naturellement par les caractères, de structure ou d'habitudes, que ces insectes ont acquis, à une époque plus récente, comme conséquence de la vie sociale:

$1^{\circ}$ Le dimorphisme sexuel prononcé, qui, chez les Aculéates solitaires comme chez les Térébrants, se manifeste plus dans leurs mœurs que dans leur struclure. - Les exemples complexes d'instinct sont patents chez la femelle uniquement et le inâle en est réduit au simple rôle d'agent fécondateur. Gràce ì la perfection du spermathéque de la femelle, qui peut enmagasiner le sperme du male et te conserver vivant très longtemps, cette femelle se comporte comme si elle était bisexuce ou hermaphrodite, et elle exerce un controle fonctionnel sur toute la reproduction. Elle seule est donc soumise à la pleine incidence des stimuli ambiants, et, en réagissant vis-ì-vis d'eux, elle peut s'avancer dans la voie de la spécialisation ou de l'adaptation progressive, aussi loin que le lui permet la plasticilé de sa constitution. Le mâle, à part quelques légères modifications spécifiques, est condamné à la stabilité dans sa struclure, et à la monotonie dans sa manière de vivre. Il constitue le sexe conservateur, le sexus sequior.

$2^{\circ}$ La mise en réserve, comme aliment pour les jeunes, d'insectes ou de produits d'origine végétale, par les Vespoïdes ou les Sphécoïles solitaires. - Cette prévoyance peut être considérée comme une activité sociale potentielle, car les conditions du développement social sont présentes implicitement et dans le temps, et la société deviendrá effective ou explicite, dès que les conditions spatiales nécessaires seront réalisées, c est-à-dire lorsque les parents coexisteront avec leur's descendants adultes. 
$3^{\circ}$ Le nid, qui va du simple terrier creusé dans le sol, ou de la cavité toule faite, aux alvèoles soigneusement bâties en terre ou en matériaux empruntés aux plantes.

$4^{\circ}$ Le développement des réactions de défense chez la mère. Ces réactions sont la suite naturelle des habitudes de chasse et de lutte des ancêtres, les Béthyloïdes primitifs, et aussi de la transformation de l'oviscapte en aiguillon. Certains Térćbrants eux-mêmes (Ichneumons) piquent quand on les touche.

Les Aculéates solitaires usent aussi de leurs mandibules comme arme de défense, car ces organes ont peu à peu acquis plus d'efficacité, en servant à prendre et à transporter la proie, à creuser le terrier, etc. Au début, ils sont employés en mème temps que l'aiguillon, mais ils peuvent se substituer à lui comme organe principal de défense, ainsi que l'a montré Roubaun (1918).

Comme conséquencès du contact réel, effectif, entre l'Aculéale mère et ses enfants, et aussi de l'association entre elle et ses semblables, d'où naît la division physiologique du travail, on observe, dans la vie en sociétés, des modifications et des additions dont les plus significatives sont les suivantes :

$1^{\circ}$ La différenciation des femelles en deux castes, ou deux formes, l'une fertile (reines), l'autre stérile (ouvrières). En outre, la reine se distingue du mâle, stationnaire, conservateur, par sa longévité accrue, sa taille et des différences morphologiques. De plus, sa fécondité est beaucoup plus grande que celle de la.femelle solitaire; tandis que, les ouvrières, surtout chez les fourmis, tendent à se différencier de plus en plus de la reine et finissent par être dimorphiques, par dissociation de leur double fonction primitivement cumbinée: alimentation el protection (ouvrières proprement dites et soldats).

$2^{\circ} \mathrm{La}$ réunion d'individus qui forment la colonie voit se développer diverses méthodes de transmission des stimuli ou de distribution de la nourriture ou des sécrétions (trophallaris).

$3^{\circ}$ La récolte de la nourriture, sa mise en réserve, son utilisation se font en commun. 
$4^{\circ}$ Le nid, qui est l'œuvre combinée el conrdonnco de lit communauté, s'agrandit, se perfectionne et devient permanenl.

$5^{\circ}$ Les fonctions de protection et de défense deviennent aussi beaucoup plus efficientes et formidables, étant réservées a loute la caste ouvrière ou à une partie de celle-ci, tout particulièrement spécialisce, la caste des soldats.

II faut remarquer qu'avec l'apparition de la vie sociale, les mœurs deviennent non seulement plus compliquées, mais aussi cumulatives. C'est là un point important. Auguste Comte a montré il y a longtemps, et I) GRANGe également ( 1923 ), tout récemment et très nettement, que la meilleure définition à donner d'une société est de la considérer comme une collectivité cumulative. Nalurellement, ces sociologistes, et d'autres qui ont exprimé des opinions semblables, pensaient avant tout à la société humaine, qui est, par essence et de façon manifeste, caractérisée par une accumulation extraordinaire de pensées et d'actes et de tout ce que celà implique : mours, institutions, langages, inventions, capital, art, science, etc.

Dans son livre intitulé "The Manhood of Humanity ", le comte Korzybsкy ( $192 \mathrm{I}$ ), a également insisté sur les aspects cumulatifs de la société humaine, et emploie pour les désigner le terme de "time binding ». Pour lui, c'est là une activité exclusivement humaine ; mais, comme l'a montré De Grange en plusieurs endroits, c'est en réalité un caractère commun à toutes les véritables sociétés, qu'elles soient animales ou humaines. Chez les Aculéates sociaux, les cumulations se révèlent très clairement, bien qu'elles soient généralement très primitives et rudimentaires, en comparaison de celles quo présente l'humanité, et qu'elles soient en grande mesure confinées dans la 
sphère de ce qu'on appelle les instincts et des effets de ces instincts. Mais il a dù en être également ainsi aux tous premiers degrés des sociétés humaines. Au point de vue matériel, les communautés sauvages sont bien plus éloignées, économiquement, du stade cumulatif, que ne le sont les abeilles des ruches ou les fourmis et les termites les plus élevés.

Au cours de lcur passage de l'état solitaire à l'état social, les Aculéates se sont trouvés, semble-t-il, dans l'impossibilité de modifier un caractère très particulier: les conditions d'exislence du mâle. Dans toutes leurs sociétés, ce sexe n intervient pas dans les actes sociaux ou cumulatifs comme lel, mais il demeure dans un état qui est, par essence, identique à celui du mâle chez les ancêtres solitaires. Je dis "par essence ", parce que, chez certaines guêpes et abeilles sociales, il paraît se socialiser légèrement, au moins dans la mesure où il sent sa dépendance vis-à-vis du nid ou de la ruche, vers lesquels il revient après être allé en quête de nourriture ou à la recherche de femclles vierges. Mais cette lueur de sociabilité fait ellemême défaut chez les mâles de beaucoup de Formicidés, hien qu'ils soient traités avec une certaine sollicitude par le personnel ouvrier ( $\mathrm{I}$ ).

Mais, en règle générale, chez tous les Aculéates sociaux, les mâles, bien qu'étant produits souvent en nombre considérable, sont simplement tolérés pour un certain

(1) Ainsi, quand un nid de Lobopella elongata est dévasté, les ouvrières saisissent les mâles et les emportent; dans ce cas, les mâles adoptent une attitude rigide, restent immobiles avec leurs appendices et leurs ailes repliées dans la même position que chez la nymphe, et ils sont transportés sous le corps des ouvrières, ahsolument de la même façon que les larves et les cocons. 
temps comme des parasites dans la colonie. Etant indispensables pour la fécondation, cependant, on ne peut les considérer comme absolument inutiles!.

Celte conception anthropomorphique ne doit point, évidemment nous conduire à considérer comme une erreur, de la part des Aculéates sociaux, la moindre socialisation du mâle en regard de la femelle. Nême de notre point de vue humain, il ne paraît pas probable qu'une telle utilisation sociale du mâle aurait amélioré les sociétés d'abeilles, de guêpes et de fourmis. Ceci nous est suggéré par les termites, qui réalisent véritablement ce que les Aculéates n'ont pu accomplir, ou tout au moins n'ont pas accompli ; puisque, dans les colonies de termites, les diverses spécialisations morphologiques et les diverses aclivités (division du travail) sont supportées également par les individus des deux sexes. A cet égard, ils nous paraissent des insectes sociaux plus parfaits que les Aculéates sociaux ; et pourtant je me demande si, pour quelque future métempsychose, il était offert à l'un de nous do choisir entre le sort d'une fourmi et celui d'un termile, si le dernier serait préféré.

Un examen superficiel pourra faire croire que les termites présentent des ressemblances frappantes avec les Aculéates sociaux et surtout avec les fourmis. Comme les Formicidés, ils sont tous sociaux, et ils offrent un polymorphisme très prononcé parmi les individus de la colonie, certains étant aptères et se comportant comme les ouvrières et les soldats des fourmis. Dans les cas typiques, il y a une seule mère reine, et, bien qu'elle soit ac- 
compagnée d'un roi, les deux perdent leurs ailes avant de fonder la colonie et après un vol de dissémination qui ressemble au vol nuptial des fourmis. On retrouve un mème parallélisme entre certaines fourmis el les termites supéricurs, en ce qui concerne les façons de récolter et d'emmagasiner des graines et de cultiver des champignons. Mais, si intéressantes et suggestives soient-elles, ces ressemblances, et quelques autres moins frappantés, sont simplement le résultat de développements parallèles ou convergents. Lorsque nous en venons à une étude plus serrée des termites, nous découvrons un certain nombre d'idiosyncrasies fondamentales, dont l'une des plus significatives est celle que nous avons indiquée ci-dessus : la composition bisexuelle de la colonie dans son ensemble.

Les termites constituent un ordre indépendant, les Isoptères de Brullé. Parce qu'ils sont presque tous des insectes Iropicaux, et qu'ils ne constituent pas des spécimens intéressants pour les collections, ils ont été très négligés par les entomologistes jusqu'au début du siècle actuel. Depuis ce moment, par contre, ils ont attiré un certain nombre de chercheurs éclairés, si bien que nous nous trouvons maintenant, en possession d'une littérature abondante, concernant leur anatomie, leurs møurs et leur taxonomic. Des travaux d'ordre plus général commencent aussi à paraître, tels, les traités de Hotmaren (rgogigr3), Escherich (igog, rgi i), Banks el Syyder (ig20), et Hegil (rg22) ; ils contiennent une telle quantité de renseignements que je n'essaierai même pas de concentrer, dans celte leçon, un résumé, même des plus importantes de ces données. Le récent volume de Hegh "Les Termites $),\left(1^{\text {re }}\right.$ partie), travail très étendu et illustré à prof 11 . 
sion, conslitue la lecture souhaitée pour tous ceux que cefle question intéresse. Comme dans les leçons précédentes, je m'en tiendrai, pour ma part, à la discussion de certains problèmes de l'évolution.

Jusqu'ici, on a décrit un peu plus de 1.200 espèces de Termites; la majorité appartient aux régions tropicales les plus chaudes, et spécialement, pour un tiers environ, à la région éthiopienne, c'est-à-dire au sud du Sahara africain. Aux ElatsUnis, nous comptons quelque 34 espèces; une ou deux seulement dépassent les frontières nord des Etats-Unis et pénèIrent dans l'Amérique britannique. L'Europe ne paraît posséder que deux espèces limitées au centre el au sud de la France et au littoral méditerranéen (1).

Présentement, la délimitation des groupes principaux et leurs noms sont quelque peu instables. Howmonen reconnait trois familles, qu'il a appelées les Protermitirlés, les Mésotermitidés et les Mélalermitidés (2).

Ces familles embrassent respectivement les formes les plus primitives, celles qui sont peu spécialisées et celles qui sont le plus hautement spécialisées; mais, malheureusement, ces noms de famille sont mal venus et ont été abandonnés, parce qu'ils ne correspondent à aucun nom générique (3).

(1) Le Dr Alfred Emerson m'a aimablement fourni quelques certitudes sur le nombre total de termites décrits aujourd'hui, pour les diverses régions du globe. Sa liste comprend un certain nombre d'espèces qu'il a décrites, provenant de la Guyane anglaise et du Congo, mais elle n'est pas encore publiée. Il y a 123 genres et sous-genres, comprenant 1.237 espèces. Si l'on exclut les espèces cosmopolites et celles dont l'habitat est inconnu, les autres se distribuent de la façon suivante: Ethiopie, 452 ; Madagascar, 38 ; Indo-Malaisie, 272 ; Papouasie, 30 ; Australie, 111 ; zones paléarctiques, 22 ; néarctiques, 34 ; néotropicales, 28?.

(2) C'esl là la classification qu'Howngren a publiée en 1910 dans le "Zoologischer Anzeiger ", el dans laquelle le groupe des Mastotermes est considéré comme une sous-famille des Protermitidés. Dans sa monographio de 1911, il accepte les vues de Sirvfstri (1909) et place au rang de famille les Mastotermitidés.

(3) Il existe $u$ genre Protermes, mai- appartenant aux Métatermitidés de HoLmirex ! 
Ictuellement, ses Protermitidés sont divisés en deux familles, les Mastotermitidés et les Calotermitidés, et B.NKS et SNYDER ont réuni les Miéso- et Métatermitidés, en une famille, les Termitidés. Si l'on veut conserver la distinction de HolMGREN en deux familles, les Mésoternitidés pourraient prendre le nom de "Rhinoternitidés ", celui de "Termitidés " étant réservé alors aux Métatermitidés. Les Mastotermilidés et les Calotermitidés, étant les termites les plus primitifs, reguièrent une attention toute spéciale, au point de vue de la phylogénie de l'ensemble des Isoptères. Dans la leçon précédente, j'ai négligé de montrer qu'il y a, chez les Formicidés, un accroissement très net et très significatif dans l'importance de la colonie et dans son polymorphisme, comme dans la complexité des mœurs, lorsqu'on passe des genres primitifs, surlout chez les Ponérinés, aux genres plus spécialisés de cette même sousfamille ou des autres sous-familles. Il en est de même, en général, pour les Termites, les colonies de Mastotermitidés et de Calotermitidés étant habituellement très réduites, et d'un polyInorphisme moins défini, que celles de Termes ou d'Eutermes, les genres les plus élevés des Termitidés.

Les Mastotermitidés sont représentés par une seule espèce vivante, Mastotermes darwiniensis, de l'Australie du Nord, décrite par FrogGatT $(1896,1905)$. Les caractères spécifiques sont tirés des formes sexuées, qui ont une longueur de $2 \mathrm{~cm}$; mais Froggatr a décrit aussi le soldat (1897) sous le nom de Termes errabundus. Les colonies sont petites et vivent dans des troncs d'arbre, sans faire de véritables nids. Elles s'établissent souvent dans les charpentes des maisons et y provoquent, comme d'autres termiles, des dommages considérables. Desneux (1904); puis Silvestri (1909) et HoLMüren (1911) ont très soigneusement étudié les Mastotermes; ils ont découvert que ces insectes constituaient un véritable type synthétique du plus grand intérêt. Quoiqu'étant sans aucun doute de vrais termites, ils possèdent néanmoins des caractères si nombreux des Blattoödés, qu'ils doivent en être certainement les descendants phylogénétiques. Holmgren a montré qu'ils pouvaient dériver des Protoblattoĩdés, groupe qui, dans la mesure où peut nous renseigner l'étude des fossiles, se serait êteint dans le Permien, en donnant naissance à deux rameaux, l'un conduisant aux Blattoïdés, ou blatles, l'autre aux Isoptères. Les Mastotermes måles et femelles, ont IIn lobe anal bien développé à l'aile postérieure, caractère qui a disparu chez tous les autres termites; la nervation de l'aile est identique à celle des Protoblattoïdés, les tarses ont cinq articulations distinctes, les tibias sont épineux, il n'y a pas de 
glande frontale, et la femelle possède des gonapophyses distinctes. Suivant Silvestri, il y a une véritable caste de soldats, mais pas de caste ouvrière proprement dite. It décrit une forme analogue à l'ouvrière, niais la considère comme un stade nymphal. Bien qu'Horngren considère cette forme comme une ouvrière vraie, l'opinion de Silvestri parait confirmée par les faits observés chez les Calotermitidés. HoLmares et Jucci (1924) ont montré que l'hypoderme des Mastotermes a la mème structure très particulière que celui des Blattoïdes, et Jucci a découvert que les réserves graisseuses renferment des cellules à bactéries contenant des bactéries symbiotiques analogues à celles qui ont été décrites par Blochusix, moi-même et d'autres, chez les Blaltes. Clevel.1xn (1923b), et Jucci ont aussi montré que l'intestin postérieur du Mastotermes contient des Infusoires flagellés identiques à ceux qui se trouvent dans tous les autres groupes inférieurs de termites. On ne connaît pas les œufs de Mastotermes, mais, si l'on en juge d'après la petitesse des colonies et d'après la taille des œufs de Calotermitidés, ils doivent être de grande dimension. Ceci constituerait également un caractère de Blattoïdés.

Les Calotermitidés, qui sont un peu plus spécialisés que les Mastotermitidés, comprennent un certain nombre de genres, dont plusieurs forment de petites colonies dans le bois, en bon état de préférence, et ne construisent pas de vrais nids. Miss Thompson (1919) a montré que leurs œufs sont de beaucoup plus gros que ceux des termites supérieurs (Termitidés). Elle donne les mesures suivantes pour les oufs de quatre des genres:Termopsis, 1,3 a 1,7 mm. ; Calotermes, 1,2 à 1,4 mm. ; Cryptotermes, 1,2 à $1.3 \mathrm{~mm}$. Neotermes, 1,6 mm. Je n'examinerai, et très brièvement, que les trois genres les plus primitifs: Archotermopsis, Termopsis et Calotermes.

Archotermopsis uroughtoni, l'unique représentant du genre, habite le nord-ouest de l'Himalaya, a des altitudes variant de 4.000 à 9.000 pieds; il a été étudié très consciencieusement par Imms (1919). Les colonies entièrement développées comprennẻnt, en plus du couple royal, des femelles et des mâles ailés, des soldats, des formes analogues aux ouvrières, des nymphes a des stades divers, mais non de vraies ouvrières. Toutes les formes ont des yeux; toutefois ils sont petits chez les soldats. Les œufs sont en petit nombre et de grande taille $(0,95$ a 0,98 millimètre de long. 0,45 a $0,50 \mathrm{~mm}$. de large). "Les soldats comptent parmi les plus grands que l'on connaisse et sont surtout remarquables en ce qu'ils présentent des caracteres secondaires externes bien définis, particularité qui a disparu en 
grande partie chez tous les autres termites. Dans le système reproducteur, on n'observe pas trace de dégénérescence ou d'arrêt de développement; il est dans les mêmes conditions que dans les formes sexuées ailées, avant l'essaimage. "Les larses d'Archotermopsis présentent cinq articulations comme chez Mastolermes, Termopsis et Hodotermes, caractère qui ne se retrouve chez aucum autre termite; les styles et les cerques sont très longs, leś derniers ayant six ou sept articulations. lms allire l'attention sur plusieurs caractères de Blattoïdés que présente $A$. wroughtoni. Cutlen (1921) a appelé l'attention sur lc fait que les Protozoaires intestinaux d'Archolermopsis sont d'un type plus primitif que ceux qui vivent dans l'intestin des termites supérieurs (1).

Le genre Termopsis est représenté par trois espèces, limitées au Pacifique et aux états du Nord-Ouest de l'Amérique du Nord. L'une de ces espèces, $T$. anguslicollis, a été bien étudiéc par Heath $(1902,1907)$, qui a constalé que ses colonies peuvent parfois atteindre des dimensions considérables (2).

Il n'existe pas, dans le genre T'ermopsis, de véritables castes ouvrières et les soldats peuvent, au moins dans certains cas, devenir fertiles et pondre des oufs donnant des descendants viables; toutefois on n'a pas suivi leur développenent complel. Au moment où Нвıтн a rédigé son ménıoire, il avait trou-

(1) “ Il est très intéressant de voir ainsi associés des parasites, ou bien des symbiotes primitifs, avec un hôte également primitif, el ce fait suggere que les deux groupes d'organismes ont dù être ainsi associés l'un à l'autre aı cours d'une longue période, sans avoir évolué vers une complexité plus grande, comme cela s'est produit pour d'autres termites et pour les Protozoaires qui leur étaient associés. " (Imws.)

(2) " Lorsque la colonie est accoinpagnée d'un couple royal primaire et qu'elle dure depuis un à trois, quatre ou peut-être cinq ans, elle compte de 50 à 1.000 membres. Lorsque le couple royal primitif est mort et que plusieurs formes royales de substitution se sont développées, le processus de formation des œufs s'accélère rapidement, et conduit à la formation de communautés très vastes. Une telle colonie, occupant un énorme tronc de pin, comptait 3.221 membres, et, à en juger par les milliers d'œufs déposés dans les galeries, ce nombre se serait vite accru, et fortement. Toutefois, dans les conditions normales, le nombre habituellement atteint est sans doutc d'environ $2.000)$ ). (НЕ.TH). 
vé trois de ces soldats fertiles ayant des ailes assez bien développées. Dans une lettre récente, il m'annonce qu'il én a trouvé quatre autres, et il m'en a envoyé un. Celui-ci possède de faibles vestiges d'ailes, ressemblant a ceux des Archotermopsis fertiles, reproduits par Imss, et aux soldats de Calotermes occidentis. (Vide infra). Toutefois, Miss -Tномpson (1922), qui a également ètudié Termopsis, croit que les soldats sont toujours inféconds; mais cela me parait insuffisamment prouvé.

Les Calotermes ont été étudiés par Grassi et Sandias (1893), IIEATH (1902), JuCCI (1924) et d'autres. Ce genre, dont on a décrit plus de cinquante espèces, est cosmopolite, et est représenté mème en Nouvelle-Zélande et dans les lles Galapagos. Les colonies sont petites et comprennent les mêmes castes que celles de Termopsis el d'Archotermopsis. La fécondité, au moins occasionnelle, des soldats est indiquée par les observations de Grassi et SNyder a élabli que, dans l'espèce la plus primitive et la plus répandue, Calotermes occidentis, de l'Ámérique centrale, les soldats ont tous des vestiges d'ailes. Retenons donc que le développement de la caste des soldats a précédé l'apparition de la stérilité. Imms l'avait vu clairement pour Archotermopsis, comme Heath pour les soldats fertiles de Termopsis, et les soldats, normalement subaptères, de Calotermes occidenlis, suggèrent la mème conclusion. On peut penser qu'une étude, pratiquée sur du matériel frais, de Mastotermes darwiniensis, montrerait aussi que, chez ces termites très primitifs, les soldats sont féconds, sinon toujours, au moins occasionnellement. Chez les Hodotermes africains, comnie chez les Hodotermopsis du Japon, qui sont aussi des Calotermitidés, il s'est développé également une caste d'ouvrières et une caste de soldats, et nous trouvons donc ici le nombre typique de castes des Termitidés, ou termites supérieurs. Mais chez ces derniers, les soldats et les ouvrières sont toujours stériles et, chez les Termes, il semble même qu'il ne se dévelopipe pas de formes royales complémentaires.

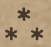

Si nous considérons la paléographie des termites, nous voyons qu'ils sont signalés dans presque toutes les formations tertiaires où l'on a retrouvé des fourmis, mais qu'ils sont encore inconnus dans les formations mésozoï- 
ques. Haxdurisci ( 1908 ) cite un grand nombre d'espèces fossiles. Plus récemment, Von Rosen ( 1913 ) a publié une révision des formes connues, qui a donné des résultats particulièremenl révélateurs.

Presque toutes les espèces de l'Eocène, de l'Oligocène el du Niocene de l'Europe et de l'Amérique du Nord se trouvent appartenir aux genres primitifs que nous avons examinćs, c'està-dire Mastotermes, Archolermopsis, Termopsis et Caloternies et à dès genres éteints très voisins. Ainsi, le seul termite signalé dans l'Eocène européen est Mastotermes bournemouthensis, de Bournemouth (Angleterre). Deux autres espèces, anglicus et batheri, ont été décrites dans l'Oligocène moyen de l'tle de Wight, et une autre, $M$. croaticus, est signalée, dans lo Miocène de Rahodoj. D'après von Rosen, l'Eutermes lossarum, du Niocène du Colorado, serait très probablement un Mastolermes. Il y a quelques mois, le Dr E. IV. Berry m'a présenté un dessin d'une aile de Mastotermes admirablement conservée, qu'il avait trouvé dans l'Eocène du Texas. Tout ceci est plein d'intérèt, car cela indique que les Mastotermes, actuellement représentés par une seule espèce confinée dans le Nord de l'Australie, formaient un genre cosmopolite florissant pendant le tertiaire inférieur et moven. Von Rosex attribue à un genre très voisin, Miotermes, qualre espèces du Miocène européen (Radohoj, OLiningen el Wiirtemberg) et une de Florissant, Colorado (M. coloradensis Scudder). Dans l'ambre de la Bătique, il se trouve une espèce d'Archotermopsis (A. tornquisti), genre qui, nous l'avons vu, est présentement limité à l'Himalaya, et trois genres éteints, voisins d'Archotermopsis et de Termopsis, qui sont: Xeslotermopsis, Proëlectrotermes et Electrotermes. En outre, les quatre espèces de Parotermes de Florissant, dont trois ont élé décrites par Scudner $(1883,1890)$ et une par CocKERELL (1913) sont très certainement rattachables au genre Hodotermes, actuellement réduit de l'Afrique, à l'Asie centrale et méridionale, et elles appartiennent donc aux Calotermitidé: Cockerell (1916h) a décrit un Termopsis swinhoi de l'ambre de la Burma (Miocène). Ce genre ne se trouve actueilement que dans l'Ouest de l'Amérique du Nord. Yon Rosen a reconnu un véritable Calotermes ( $C$. aeningensis) dans le Miocène d'OEningen. Ces faits montrent clairement que les termites primitifs étaient représentés, à l'époque tertiaire, par des espèces et des genres plus nombreux qu'à l'époque actuelle. Les espèces de 
lermites supérieurs sont rares dans le Miocène: Odontolermes pristinus de Radohoj, Eulermes fransi de Würtemberg et dewx especes de Termes, obscurus et rroaticus, de Radoboj, et chcore les références génériques en sont-elles, jour la plupart, douleuses. Pour les Termitidés inférieurs, le genre moderne leucotermes est représenté par une espèce ( $L$. harlingi) dans le Viocene de l'Europe et par une au Colorado (L. meadi). L'ambre de la Baltique contient trois espèces de Reticuliterme's, genre auquel appartiennent le plus grand nombre des termites de l'Europe et de l'Amérique du Nord (R. lucifugus et flavipes). La rareté des restes des Termitidés, dans le Tertiaire inférieur el moyen de l'Europe el de l'Amérique du Nord, semble prouver, ou que cette famille ajpparaissait tout juste a ce moment, ou que le climat de ces régions était trop froid pour elles. Mème aujourdhui, les Termitidés ne prospèrent que dans les parties les phus chaudes de l'Ethiopie el des régrions orienlatles et néotropicales.

Ainsi, les données paléographiques confirment les conclusions phỵlogénétiques générales, tirées de l'étude morphologique el laxonomique des termites vivants, et suggèrent que ces insccles ont paru dès les temps mésozoïques. Rien ne prouve qu'ils n'aient ipas commencé leur évolution pendant le Peraien, au moment où disparaissaient les derniers Protoblattoïdés. HANnlinscir (rgo3) montre les termites naissant de la souche des Blattoïdés, pendant le Crétacé, mais ce clébut doit èire plus ancien. Warren (I9I9) a fait ressortir certaines particularités de leur distribution géographique actuelle, montrant que les genres plus primitifs ont survécu plutôt dans les zones subtropicales nord et sud, et que les genres de termites les plus élevés sonl représentés surtoul dans les régions tropicales. Après avoir désigné comme les plus primitifs les genres Mastotemes, Termopsis, Archotermopsis, Calotermes, Leucotermes et Coptotermes, il dit: " $\mathrm{Si}$ on a joute les unes aux autres les espèces de ces genres conienues dans une région donnée, et qu'on compare ce total au 
nombre total des espèces de cette région, le pourcentage établi domera le facies général de la faune de termites ").

Le tableau suivint indique les résultats obtenus:

\begin{tabular}{|c|c|c|c|}
\hline nÉGION & $\begin{array}{l}\% \text { d'esp. } \\
\text { primitives }\end{array}$ & RÉGION & $\begin{array}{l}\% \text { d'esp. } \\
\text { primitives }\end{array}$ \\
\hline Amérique du Nord & 67 & Nord de l'Inde & 22 \\
\hline $\begin{array}{l}\text { Amérique du Sud et } \\
\text { centrale }\end{array}$ & 18 & $\begin{array}{l}\text { Péninsule de l'Inde } \\
\text { et Ceylan }\end{array}$ & 13 \\
\hline $\begin{array}{l}\text { Sud de l'Europe et } \\
\text { Nord de l'Afrique }\end{array}$ & 18 & Japon et Chine & 63 \\
\hline Ethiopie & 9 & Malaisie & 9 \\
\hline $\begin{array}{l}\text { Ethiopie } \\
\text { gascar }\end{array}$ & 7 & $\begin{array}{l}\text { Australie et Tasma- } \\
\text { nie }\end{array}$ & 30 \\
\hline
\end{tabular}

Je reproduis aussi les eonclusions générales de Wanrex, 'n ce qui concerne la dislribution géographique actuelle des Isoptères, parce qu'elles s'accordent bien, dans l'ensemble, avec celles que nous ont suggérées les Formicidés dans la leçon précédente.

$1^{\circ}$ Les portions nord el sud, de l'aire de distribution des Termites prises dans leur lotalité, ont un aspect ancien et primitif. Ainsi, l'Amérique du Nord, le Nord dé l'Inde, le Japon et la Chine, rqui forment la limile septentrionale, et l'Australie, dans le Sud, possèdent une faune ayant ce caractère.

2. L Afrique possède une faune de termites plus élevée, puisquielle contient un plus grand nombre d'espèces, et qu'elle a un plus fort pourcentage en espèces appartenant à des genres plus spécialisés que ceux d'aucune autre région du monde. On peut objecter que, pomr pouvoir faire celle comparaison, il fandrait tenir compte des aires comparées des différentes régions. Mais, ce faisant, les résultals globaux ne seraient pas modifies, puistgu'on peul constater que, dans l'aire relativement 
réduite de la région malaise, on a signalé queluue 185 espèces, tandis qu'il n'y en a que 77 pour l'Hindoustan et Ceylan, et 53 seulement pour toute l'Australie.

$3^{\circ}$ Immédiatement après, vient la faune de la région indomalaise, qui comprend la péninsule de l'Inde, l'Assam, la péninsule et l'Archipel malais. Ces régions renferment une plus grande proportion que la faune africaine, d'espèces appartenant à des genres moins spécialisés.

$4^{\circ}$ L'Amérique du Sud, avec l'Amérique centrale et les Antilles, a une faune un peu plus primilive que celle de la région indo-malaise ; et le noinbre des espèces signalées est beaucoup plus petit, variant de 188 à $26 \%$.

$5^{\circ}$ Les Termites de l'Amérique du Nord sont peu nombreux et de caractère primitif.

$6^{\circ}$ Les Termites dı Japon et du Sud de la Chine sont peu nombreux, mais renlerment un pourcentige élevé d'espèces non spécialisées.

$7^{\circ}$ En Australic, le nombre des espèces de lermites est un peu moindre, mais comple un haut pourcentage en formes non spécialisées et archaïques.

Les faits rapportés ci-dessus suggèrent l'hypothèse suivante: Si, dès l'époque tertiaire, les genres Termes et Eutermes étaient complètement formés, il nous faut admettre qu'aux temps préterliaires, alors que le dessin des grandes masses continentales n'était pas le même qu'aujourd'hui, une faune de genres non spécialisés de termites s'étendait sur des portions considérables de l'Australie, de l'Afrique, de l'Amérique, de l'Europe et de l'Asie. Dans l'absence de toute autre communication di- . recte entre l'Ancien ef le Nouveau Monde, nous devons supposer que la faune se continuait au nord, jusqu'au détroit de Behring mème. Pour expliquer la distribution des lermites, comme celle des mammifères, il faut admettre au moins l'existence d'un pont continental dans ces régions. Avec le refroidissement du climat, et les masses 
continentales prenant peu à peu leurs contours actuels, de nouvelles espèces évoluèrent, surtout d'un bout à l'autre des portions centrales les plus chaudes du monde (Amérique du Sud, Afrique, Inde et région malaise), landis qu'au nord (Amérique du Nord, Europe et Asie centrale) ef au sud (Australie), les survivants de l'ancienne faune de termites subsistaient, mais reculaient en s'éloignant de l'équateur.

Il est donc possible d'expliquer la distribution actuelle des termites sans l'hỵpothèse d'un pont réalisé par un continent antarctique.

Suivant cette opinion, le genre Porotermes, que l'on trouve au Chili et en Australie, serait une partie de l'ancienne faune cosmopolite, qui aurait persisté tout le long du bord méridional de l'aire de distribution des termites.

Le caractère relativement primitif de la faune malgache de termites proviendrait simplement de la préférence qu'ont les Calotermes pour les contrées forestières, mais ce fait est à rapprocher de la persistance, à Madagascar, de l'ancienne faune lémurienne, qui a été largement remplacée sur le continent is.

\section{$* *$}

Il est certain que les termites paraissent être des insectes très archaïques, bien plus même que les fourmis. Ceci n'est point pour surprendre, si nous considérons qu'ils sont les descendants de formes primitives comme les Protoblattoïdes. Nais les ordres vivants eux-mêmes auxquels ils sont étroitement alliés, ont un aspect archaïque analogue, ef constituent le rameau survivant d'une branche 
ancienne issue du fronc primitil des Hexapodes (Palarodictyoutères).

Il serait intéressant de faire la lumière sur les ordres voisins ; en effet, certains d'entre eux sont connus depuis peu de temps, et la plupart nous donnent à penser que les plus primitifs mème des insectes des âgés passés ont dî manifester des tendances sociales. Que des sociélés aussi compliquées et aussi complètes que celles des termites aient pu prendre naissance chez ces formes archaïques, ceci nous confirme la conclusion que les Aculéates, trop) richement doués, nous suggéraient moins aisément; à savoir que de simples activilés appétitives el physiologiques sont bien suffisantes pour produire' et perpétuer des organisations sociales très perfectionnées.

Les cinq ordres d'insectes vivants actuels qui nous paraissent se rapprocher le plus des Isoplères, sont les Blaltoïdés, les Embiidinés, les Dermatoptères, les Zoraptères et les Grylloblattoïdés ou Notoptères. Nous allons brièvement les passer en revue.

$1^{\circ}$ Les Blattoidés, étant dérivés des Protoblattoïdés, ont donc eu le même ancêtre que les termites, et, hien qu'ayant acquis l'habitude particuliere d'enfermer leurs nufs dans un oothèque, ils manifestent néanmoins quelquefois des soupçons d'habitudes sociales, rappelant celles des termites. Un des meilleurs exemples est Cryptocercus punclulatus, le seul représentant, dans l'Amérique du Nord, de la sous-famille des Panesthinés. On l'a trouvé dans les Etals de l'Atlantique, depuis les CalskillMountains jusqu'à la Géorgie et, vers l'ouest, jusqu'au Kenlucky, et, en outre, dans les Etats du Pacifique. Sa distribution est donc cliscontinuc. On connait une autre espèce au Japon. Les conditions dans lesquelles on trouve $C$. punclulalus sont toujours les mêınes; il vit en colonies comprenant les parents et leurs descendants, creusant des passiges, comme les termites, en dévorant l'aubier mort des trones et des souches, aux cndroits où le bois est mou, humide et pourri. Cruplacercus est, sans aucun doute, un survivant très ancien, analogue 
à plusieurs autres animaux de l'Amérique du Nord et de l'Asie orientale, comme la Limule, la Salamandre géante du Japon, le Necturus, le Menobranchus de l'Amérique du Nord, etc. J'ai relevé, en Nouvelle-Zélande, l'existence d'un autre cafard, Polyzosteria novæe-zelandia, qui a des mœurs très semblables, quoiqu'élant subaptère et applartenant à une sousfamille différente, celle des Blaltinés. Sxyon (1924) a attiré l'attention sur le fait que, chez les blatles des genres Salganea et Panesthia, " il se produit souvgnt une rupture, suivant en gé-

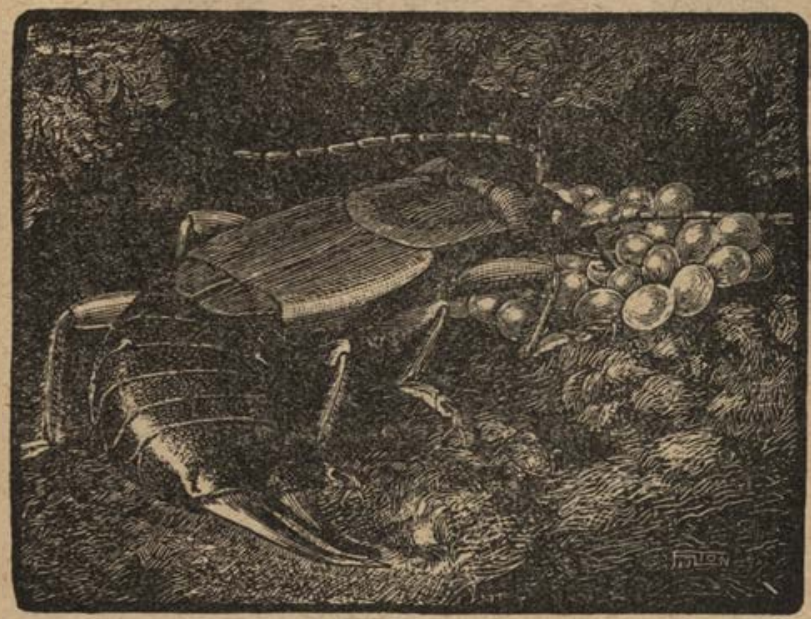

Fig. 19. -- Forficule femelle, avec ses œufs, dans ses fuarlier's dhiver. (diapres B.-B. Futrox.)

néral le sulcus anal, a lá base de l'aile, comme la suture humérale des termites, i l'endroit ou se détache l'aile, aprés le vol qui précède la fondation de la colonic; chez les termites primitifs, cette suture est souvent aussi très médiocrement indiquée. " L'auteur ajoute: " 11 est intèressant de noter que les blattes et les termites s'accouplent de la même façon \#, mais celle affirmation est quelque peu erronce, comme je volls le montrerai par la suite. 
$2^{\circ}$ I.es Dermapleres sont si connus que je ne juge pas necessaire de m'appesantir sur les mœurs. Je reproduis ici deux figures récemment publićes, et montrant la femelle d'une es-

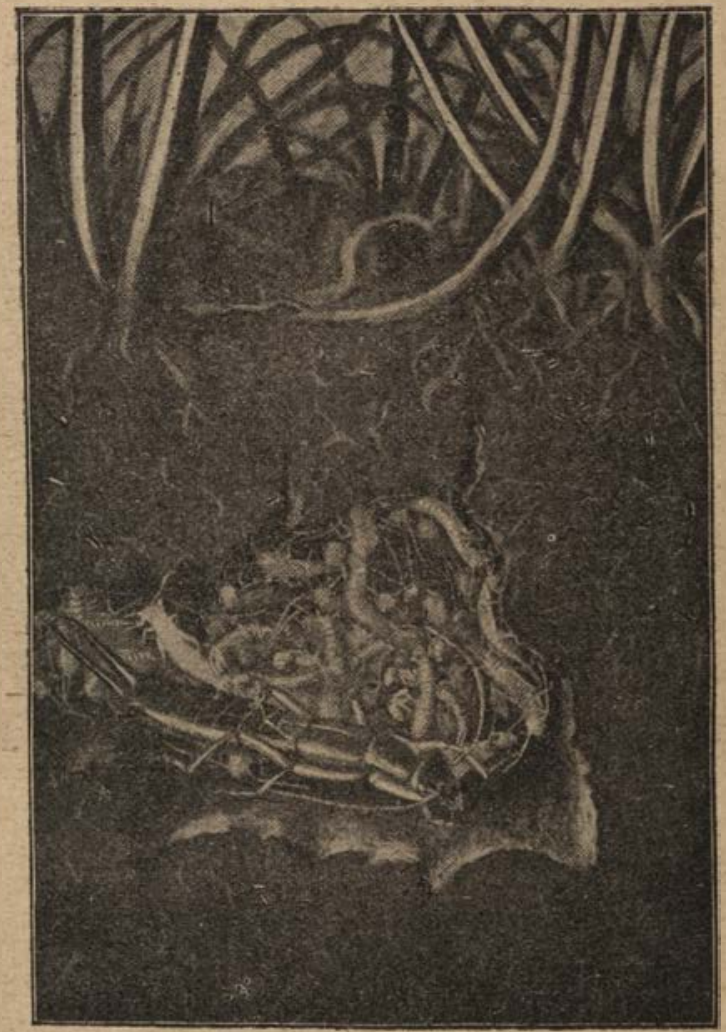

Fig. :"l. - Compe diune molle de gazon, montrant une forficule, aver ses jeunes, daus son nid soutertain. (d'apres I3.-B. Fur.ton.) 
pèce européenne très répandue, Forficula auricularia, soignant ses œufs et ses jeunes. Cette espèce a été introduite depuis peu aux Etats-Unis et s'est montrée très nuisible en plusieurs endroits. JoNes (1917) et Fulton 1921a et b) ont publié de bons Iravaux sur ses mœurs, qui rappellent beaucoup celles qu'à décrites BENNETT (1904) pour Anisolabis maritima, qui a été aussi introduite dans l'Amérique du Nord.

$3^{\circ}$ L'ordre des Zoraptères est demeuré inconnu jusqu'en 1913, époque à laquelle il fut créé par Silvfstri, pour une espèce unique du genre Zorotypus. Depuis, on a décrit plusieurs formes supplémentaires, provenant de diverses contrées tropicales, Afrique, Ceylan, Java, Costa-Rica et Floride. Le Prof. $\Lambda$. EMERsox en a trouvé une espèce dans da Guyane anglaise, et moi-même une autre dans l'Etat de Panama. On ne sait rien des mours de ces insectes, qui ont avec les termites plus de ressemblance qu'aucun des autres groupes mentionnés ci-dessus, sauf en ce qu'ils vivent en petites colonies, sous l'écorce oul dans le bois mort, et quelquefois à proxinité des termilières; de plus, leurs colonies comprennent des femelles ailcees el aptères et des mâles aptères, et les premières peuvent perdre leurs ailes comme les termites. Les adultes ailés ont des yeux bien développés, mais les adultes aptères sont aveugles. L'aile n'est pas du lout veinée comme celle des termites, et les tarses ont deux articulations.

$1^{\circ}$ En 1914, le Prof. E.-II. Walien a décrit sous le nom de Grulloblatta campodeiformis, un letit insecte aptère, ressemblant aux Thysanoures, qu'il trouva courant ça et là, comme un mille-paltes, sous les pierres, au revers d'un talus, à Banff (Nherta), à l'altitude de 6.000 pieds. Depuis, la même espèce, ou une espèce très voissine, a été découverte dans les montagnes de Plumas-County, Californie, et, plus récemment, une autre espèce a été trouvée aul Jajon. Ces insectes sont sans aucun doute des formes survivantes très anciennes, comme les Cryptocercus et les Termopsis, qui vivent dans les mèmes régions. Suivant Whiker, le Grylloblatta présente les caraclères combinés des Blattoïdés et des Dermaptères. Crampton (1915) le considère comme un chainon entre les Dermaptères et les Isoptères. WhLKEH en a fait le type d'une nouvelle famille d'Orthoptères, mais Brues et Melander (1915) ont créé un ordre, celui des Grylloblattoīdés, pour le situer. D'autres ont adopté pour l’ordre le nom de "Notoptères " ou "Grylloblattaria ". Comstock (192:1) ne se prononce pas sur la position taxonomique de cel insecte ; Il ANDLinsch (1924) place cette superfamille 
parmi los Orthoptères, entre les Locustariés et les Tridactylidés.

$$
*_{* * *}^{* *}
$$

Vous avez observé que, à l'exception des Grylloblattoidés, dont le mode de reproduction est inconnu, tous les ordres voisins des termites font montre de tendances sociales vagues, mais indiscutables. Il me semble que cela nous autoriec à en déduire que ces ordres, comme les lsoptères, quoique formés d'insectes archaïques et primitifs, indiqueraient une prédominance possible de la vie sociale chez leurs ancêtres, les anciens Orthoptérö̈des. Et, comme j'ai montré dans ma seconde leçon que nous avons maintenant de très bonnes raisons de supposer que les Hỵménoptères ne descendent pas des Orthoptéroïdes, suivant l'opinion de Handlirscri (1904, I908), - mais d'ancêtres très différents, les lécoptérö̈des, il nous faut donc nier tout rapport génétique entre les sociétés d'Aculéates et celles des termites. Les différences fondamentales qui séparent ces deux groupes ressorient fortement de leurs méthodes de formalion des colonies. Chez les Aculéates, la colonie est, typiquement, fondée par une femelle fécondée, soit seule, après le vol nuptial, soit accompagnée d'une compagnie, ou essaim d'ouvrières. Chez les termites, il y a aussi un vol de mâles et femelles ailés, mais chaque colonie est fondée par un couple (paire royale), après perte des ailes, ce dernier processus n'alleignant, chez les fourmis, que la femelle. Fuller (igr5), dans son important mémoire sur les termites de lifrique du Sud, a montré que les mâles el les femelles de chaque espèce ont une façon plutôt compliquée de se faire la cour après leur vol de dispersion. Je prends 
conıme exemple, parmi plusieurs récits qu'a donnés cet auteur, ceux qui ont trait à Termes natalensis et $T$. latericius :

"Les màles et les fenelles de $T$. natalensis s'èlèvent d'un vol soutenu et beaucoup planent assez haut. Les femelles redescendent les premières, en général; elles choisissent des brins tlherbe frais et rigirles: sur ceux qui s'élèvent à un pied environ ( 15 à 20 pouces au-dessus du sol), elles se renversent immédiatement, leur tête dirigée vers le bas. Léurs quatre ailes sont à demi ouvertes et l'extrémité de leur abdomen se gonfle visiblement. Quelques mâles volent à ce moment assez bas au-dessus do l'herbe; en peu d'instants, ils découvrent les femelles. Le màle descend alors directement sur le dos de la femelle, vers laquelle il vole avec la plus grande précision; vivement, il se place à ses côtés, les ailes entièrement repliécs et la tête soulevée, et il frotte, de ci de là, passant d'un cerce à l'autre, l'abdomen dilaté de la femelle. Pendant ce temps, la femelle reste immobile, mais, très vite, elle donne des signes évidents d'agitation; lorsque ses mouvements ont suffisamment stimulé le mâle, celui-ei monte sur le dos de la femelle. Elle s'avance alors en descendant le long de la tige; le mâle la retient en arrière avec ses organes buccaux, qui frottent sans cesse les plaques anales de la femelle. Pour réaliser cet accouplement, il est certain que le mâle, altiré vers la femelle dans d'autres cas par le sens de l'odorat, comme chez $T$. vulgaris, dans celui-ci voit sûrement la femelle, au moins à l'intéricur d'un certain rayon, comme le prouve la précision avec laquelle il descend vers elle; les rayons du soleil couchant jouant sur les ailes à demi éployées en font d'ailleur's un objet particulièrement visible. ")

Sur $T$. latericius, voici ce qu'écrit Full.ER : " J'ai observé l'accouplement de cette espèce à Prctoria, au crépuscule, les 4, 16 et 18 décembre 1914. Je vis les femelles descendre les premières, et se poser, en se renversant, sur les parties pendantes des herbes des prairies. Dès qu'elles eurent pris celte attitude, elles commencèrent à agiter violemment leurs ailes, et continuèrent jusqu'à ce qu'un màle vint les rejoindre. Je ne pourrais dire si une femelle peut rester ainsi longtemps sans qu'un mâle arrive, mais l'une d'elles altendit vingt minutes sans cesser d'agiter ses ailes el, à la nuit, elle ne s'ćtait pas encore accouplée. Les máles paraissent éprouver de grandes diflicultés pour trouver les femelles; ils volaient bas el sans arrèt 
sur les pointes des herbes, passant à plusieurs reprises tout près d'une femelle, et mème l'encerclant à quelques pouces de distance, puis s'envolant à nouveau au loin à travers champs. Après la réunion, qui se fait au crépuscule, on constate que le mâle volait en cercles de plıs en plus serrés autour de la femelle et redescendait près d'elle. Dès que le màle eut touché la femelle, leurs ailes à tous deux se détachèrent avec une soudaineté inexplicable, celles de la femelle paraissant tomber tandis qu'elles s'agitaient encore. Puis la femelle se met en route vers la terre, le màle la suivant de près. Ils creusent aussitôt le sol et forment une alvéole. Deux couples, que j'ai vus, creusant ainsi leurs cellules, le 16 décembre, produisirent l'un et l'autre quinze à vingt œufs en huit jours.

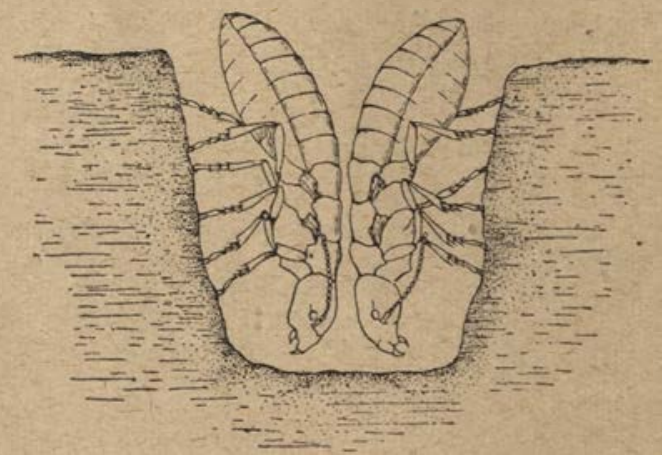

Fig. 21. - Jeunes roi et reine d'Hodolermes turlieslanicus commençant leur nid dans le sol, après le vol nuptial. (D'après G. JAcobsox.)

Ainsi, le couple royal ne s'unit pas tant que la cellule initiale du nid n'a pas été creusée par lui. Etant donné que le pénis du mâle fait défaut su est très réduit, la copulation se fait par simple apposition des orifices génitaux. Cet acte, observé pour la première fois par Grassi (1893-94), se répète souvent au cours de la vie du couple 
royal, comme l'ont vu Heath (I902) et Fertaud (IgI 2). Avec le temps, la reine des termites supérieurs devient, comme on le sait, très physogastrique. Elle est toujours accompagnée du roi, dont l'abdomen se développe très peli.

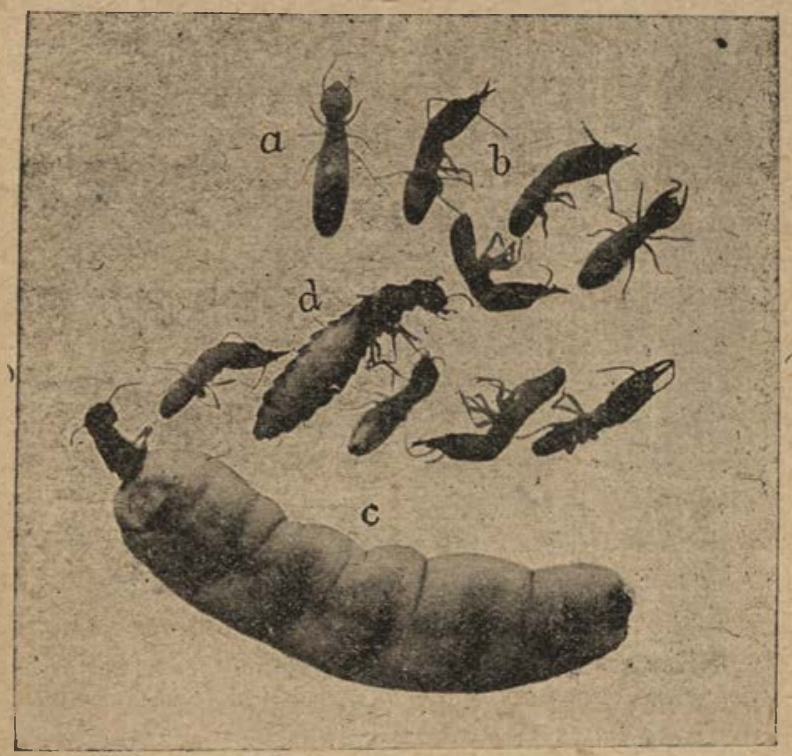

Fig. 22. - Leucolermes tenuis des Bahamas (Gr. 2). $a$, ouviere ; $b$, soldat; $c$, reine (physogastrique); $d$, roi.

Filler donne la description suivante des habitudes de celle majesté (Termes natalensis): "Cet animal fait preuve d'une dévotion extraordinaire à l'égard de son épouse; il ne $\mathrm{s}^{2} e n$ éloigne guère jamais de plus d'un pouce ; sans cesse, il approche d'elle sa tète et lui fait, semble-t-il, sa cour; puis, il ins- 
pecte ses flancs et ses cufs avec une vive altention; se comportant, en in mot, comme paraissent le faire la plupart des màles de termites, mais ne laissant jamais voir le but de son existence. Le mâle est toujours un peu gonflé par des masses graisseuses, qui distendent le tissu conjonctif des côtés de son abdomen, mais non les sclérites dorsaux et ventraux. ")

Les habitudes nuptiales décrites ci-dessus me paraissent fournir une nouvelle indication au sujet des affinités des Orthoptérö̈des et des Isoptères. On sait très bien que ces types de mœurs compliquées n'existent pas chez les Hyménoptères, bien qu'ils soient fréquents chez les Diptères, les Lépidoptères et les Orthoptères. J'ai publié récemment (1925) un récit de quelques cas assez singuliers survenant chez les mouches. Les séduisantes descriptions de Fabre sur les habitudes nuptiales des Mantides et des Locustides sont bien connues, et Haxcock (Ig) Engelhardt ( 1914 ) et Fulton ( 1915 ) les ont décrites chez les Gryllidés du genre $O E$ canthus. Chez ces derniers insectes, la femelle est attirée vers le mâle, grâce à une glande particulière placée sur la face dorsale du thorax et dont elle absorbe la sécrétion avant et après l'accouplement. Récemment, Wille (1920) a décrit en détail les mœurs nuptiales analogues et la copulation chez notre blatte commune, Phyllodromia germanica. Nans ce cas, la femelle absorbe la sécrétion du mâle, mais les glandes sont situées à la face dorsale de l'abdomen. Chez les termites, les rôles sont inversés, le mâle étant attiré par la sécrétion glandulaire émanant de la pointe de l'abdomen de la femelle, et la copulation est un processus bien moins perfectionné que chez les Blattidés. La remarque de SNyder rapportée ci-dessus exige donc quelque restriction.

Le passage suivant montre avec évidence que les observations de Fruter sur les habitudes nuptiales et la fonda- 
tion des colonies, chez les termites sud-africains, ont aussi une signification plus générale au point de vue des mœurs :

"Il semble bien certain que celte phase de l'histoire d'un termite est une succession d'événements qui se suivent dans un ordre régulier, et, si la chaine se rompt en un point quelconque, elle en reste là. Ceci peut être montré par plusieurs cas spécifiques. L'Eutermes trinervius, qui vole de nuit, descend, s'il est altiré par un lissu blanc éclairé, et les deux sexes perdent les ailes après s'être rencontrés. Si plusieurs d'entre eux sont capturés immédiatement avant quils se rencontrent et qu'ils soient placés dans un tube, ils ne se désailent pas. Plusieurs, ainsi capturés, ont été conservés vivants et enfermés pendant plus de quatre jours, et, tandis que quelques-uns perdaient leurs ailes, il n'y eut aucune tentative d'accouplement. Il en est de même avec Termes incertus, qui vole au crépuscule, mais avec quelques modifications. En outre, pour ces deux espèces et pour trois autres (non déterminées), il n'est fait aucune tentative pour creuser le sol, si les deux sexes sont isolés. Si on enferme deux mâles ensemble, l'un suit souvent l'autre comme si le second était une femelle. Ils sont alors poussés à creuser le sol ensemble, mais ils s'arrêtent bientôt, quoique s'excitant l'un l'autre à continuer pendant quelque lemps; enfin, ils meurent à la surface du sol. Autre cas: un rerlain nombre de couples de $T$. natalensis furent faits prisonnier's un soir et placés dans une petite boite; au matin, on Irouve les couples tout désorientés les uns en face des autres; placés sur une table, ils purent y circuler mais ils ne cherchèrent pas d'eux-mèmes à s'accoupler. Cependant, leur ayant préparé des terriers artificiels et les y ayant placés couple par couple, le milieu réagit sur eux immédiatement, et ils creusèrent vers le fond dı vase. En 1914, les mèmes résultals accompagnèrent les accouplements répétés de cette espèce. "

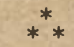

Je serai bref maintenant pour exposer le reste de mon étude sur les aspects archaïques des termites. Après les particularités sexuelles, déjà discutées, les traits primitifs les plus frappants peul-être sont présentés par l'ontogénèse 
et les habitudes alimentaires, qui ont des effets très loinlains sur toutes les activités sociales. Parce qu'ils ont conservé très strictement le type progressif, amétabolique, d'ontogénèse qui est si caractéristique de toute la série des Orthoptéroïdes, les termites ne réalisent pas des relations aussi intimes entre les parents, ou les autres membres adultes de la colonie, et les jeunes, que celles que nous avons découvertes chez les Aculéates sociaux. Chez ces insectes, les larves et les nymphes sont absolument impotentes; aussi les premières sont-elles soigneusement nourries, et les unes et les autres, chez les fourmis, sont Iransporlćes dans des conditions favorables de tempérafure, d'humidité, etc..., à l'abri de toutes ronditions défaforables, nuisibles. Chez les termites, de's soins de ce genre sont accordés seulement aux œufs et aux très jeunes nymphes. En outre, il y a. chez cos nymphes, une particularité, n'existant pas chez les Aculéates; c'est leur développement graduel avec sa série d'états actifs, séparés par des mues; ceci permet un arrêt permanent de la croissance à plusieurs périodes différentes du développement. Cet arrèt peut affecler à la fois le soma el les gonades, comme chez les soldats et les ouvrières des termiles: supéricurs; ou il n'atteint que le soma seul. Dans des cas de ce genre, la néoténie se produit, et on obtient des mâles el des femelles complémentaires, ou de substitution, qui, dans les colonies de certains termites, peuvenl remplacer, en tout ou en partie, le couple royal, s'il meurt de vieillesse ou d'autre cause. Chez les tculéates, le développement n'offre évidemment pas de telles ressources, quoiqu'il puisse se produire des formes adultes atyjiques (femelles orgatomorphiques, pseutogynes, ptérergales, elc.) Le développement progressif, amétabolique des 
termites, śajoulant à la présence en égale proportion des deux sexes dans le personnel de la colonie, fournit cerlainement la base d'un polymorphisme plus compliqué que celui que nous avons trouvé dans les colonies femelles d'Aculéates sociaux.

Les termites n'ont pas acquis cet extraordinaire ensemble d'instincts que nous observons chez les Aculéates so-

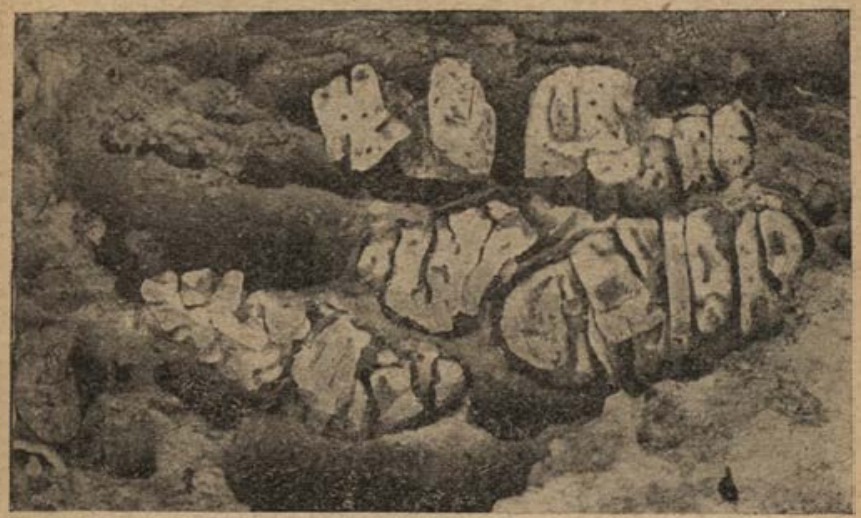

Fig. 23. - Jardin de champignons d Acanthotermes militaris

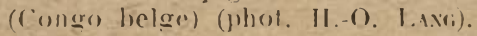

ciaux, sans doute parce que leurs ancêtres immédiats n'ont jamais traversé un stade d'habitudes carnivores et prédatrices. Différanl en cela de cos insecies agressifs et si batailleurs, ils ont vécu, et vivent en majeure partie encore an sein de leur nourriture, comme les Coléoptères qui creusent dans le hois. L'adresse ì récolter des aliments végétanx et ì les emmagasiner, n'existe même-que chez certains Calotermitidés el Termilidés, tels que les es- 
pèces d'Hodolermes, qui récoltent de l'herbe à la lumière du jour ; les espèces les plus hautement spécialisées du genre Eutermes, par exemple E. monoceros de Ceylan, qui, suivant Bugnos (1gog, 1914) récolle des lichens; diverses espèces africaines et australiennes du mène genre, qui récoltent de l'herbe morte, et Termes latericius, qui moissonne des graines (Fulber). Mais, mème les espìces de Termes, seules formes sachant élever des champignons sur leurs propres excréments et les utiliser pour la nourriture des jeunes et du couple royal, même ces espèces se nourrissent encore en grande partie d'humus, d'herbe morle et de bois, et sont, semble-t-il, bien loin d'avoir atteint l'habileté des Attinés, ces fourmis qui cultivent, propagent et utilisent si bien les champignons (voir MonsTATT, I922).

L'habitude ultra-conservatrice de dévorer le bois et de vivre dans sez cavités fournit la clef qui permet de comprendre la plupart des particularitís sociales des termites. Depuis longtemps, on a soup̧onné qu'il leur était impossible de digérer sans aide la cellulose et plusieurs chercheurs ont supposé que les Protozoaires ciliés qu'on trouve en grand nombre dans les espèces lignivores, chez les ouvrières lignivores el surlout dans la région inlestinale, où le hois est assimilé, sont les véritables agents de la digestion de la cellulose el la rendent assimilable à leur hôte (I).

(1) Voici ce qu'a pu établir Cr.firimn (1923a), en ce qui concerne la présence des Protozoaires chez les termiles. "Par un examen altentif du contenu intestinal de cing ouvrières de chacune des espèces de termiles représentées au U. S. Nalional Museum, on a constaté yue lorsqu'il y avait des Protozoaires, il y avait également du bois; et, mutatis mulandis, les protozoaires ne se trouvaient que là où il y avait du bois. 
Un ensemble considérable de-travaux ont élé publiés sur les Protozoaires des lermites ; heaucoup d'especces ont éli décrites, depuis qu'ils ont été découverts par Lespès en r 856 . Plus récemment, Buscaliovi et Cones (rgro); Imas (rg19), Jecci (r924) et Cueveland (1923 a, r 923 b, r 924 ) se sont consacrés, avec la plus grande attention, ì l'étude des problèmes inléressants que suggèrent ces or ganismes unicellulaires et leur rôle possible comme sym. biotes. Grassi, en rgir, chauffa des termites à envrion $35^{\circ}$ et réussit ainsi à détruire les protozoaires intestinaux sans léser les termites mêmes. Il affirme que ces termites purent vivre ainsi plusieurs mois, et en conclut qu'ils digèrent le hois sans le secours des protozoaires. Mais, Creveland, qui a poussé plus loin ses expériences de chauffage, sur Reticulitermes flavipes, Termopsis, et d'autres espèces nord-américaines, réussit à débarrasser complètement les termites de leurs protozoaires intestinaux en maintenant les insectes vingt-quatre heures à la tempéralure de $36^{\circ}$. Les individus ainsi traités, nourris au ré-

Ainsi est confirmé le postulat de Imss. On connaît quatre familles de termites. Chez les dix-huit genres et soixante-quatre espèces examinées, apparlenant à la famille des Termitidés, on n'a trouvé des Protozoaires et du bois que chez une seule les vingt et une espèces du genre Nasutitermes et chez deux des huit espèces dú genre Mirotermes; mais les Protozoaires et le bois étaient présents dans dix-huit genres et soixanteseize espèces, soit toutes celles qui furent examinées, des trois aulres familles (Mastotermitidés, Calotermitidés et Rhinotermitidés). Il y a donc une corrélation parfaite et positive entre l'alimentation stricte au bois, et la présence de Protozoaires intestinaux. "Cette répartition concorde avec celle qu'a donnée Grassi (1917) êt qu'a confirmée Jucci (1924, p. 209).

Dans un autre mémoire (1923 b), Crevelinn donne une liste taxonomiqque élendue des termites chez lesquels on a trouvé ou non des Protozoaires. 
grime normal du bois, moururent en dix à vingt jours ; mais, si on leur donnait du bois digéré (humus) ou de la cellulose digérée par des champignons, ils survivaient indéfiniment. Ils continuent aussi à vivre après avoir été réinfectés par des Protozoaires. Clevelaxd trouve en ouIre que, tandis que les autres organismes intestinaux des termites, bactéries et champignons, sont inaptes à digérer la cellulose, les Prolozoaires des genres Trichonympha et Pyrsonympha, si communs chez Reliculitermes flavipes, en sont capables. Mais cette faculté fait défaut chez plusicurs des autres Protozoaires intestinaux. Cleveland pense que les Protozoaires tirent leurs protéines de leur hôte, mais Jucci (1924) a trouvé que les Protozoaires sont sans doule digérés, si bien que les hôtes tireraient la majeure partie de leurs protéines des corps de leurs symbiotes. Cette méthode indirecte et dépendante d'obtenir les protéines et les hydrates de carbone semble avoir été abandonnée par les Termitidés plus élevés de PAncien continent, qui en sont arrivés à vivre d'humus (Cubitermes, Anoplotermes, etc.) et de champignons poussant sur leurs cxcréments (Termes).

La xylophagic a eu deux autres effets lointains sur l'organisation sociale des termites. En premier lieu, cette nourriture encombrante et d'une digestion extrêmement lente paraît être l'origine de l'habitude qu'ont ces insectes de se nourrir les uns les autres avec des aliments " stomodceal "), régurgités, et partiellement digérés, et avec des aliments "proctodceal ", évacués et complètement digérés, ce qui esi une forme de trophallaxis, et ce qui facitite, sans grande nécessité d'ailleurs, l'infection générale des divers individus de la colonie par les Protozoaires symbioliques. Brovfatu (igrof) a domné avec quelque rai- 
son le nom de "rumination sociale " à ce mode d'alimenlation des termites qui mangent ainsi le contenu intestinal, digéré en tout ou partie, d'autres individus.

En second lieu, l'habitude de saper et de parcourir l'intérieur du bois en le dévorant a amené tout naturellement

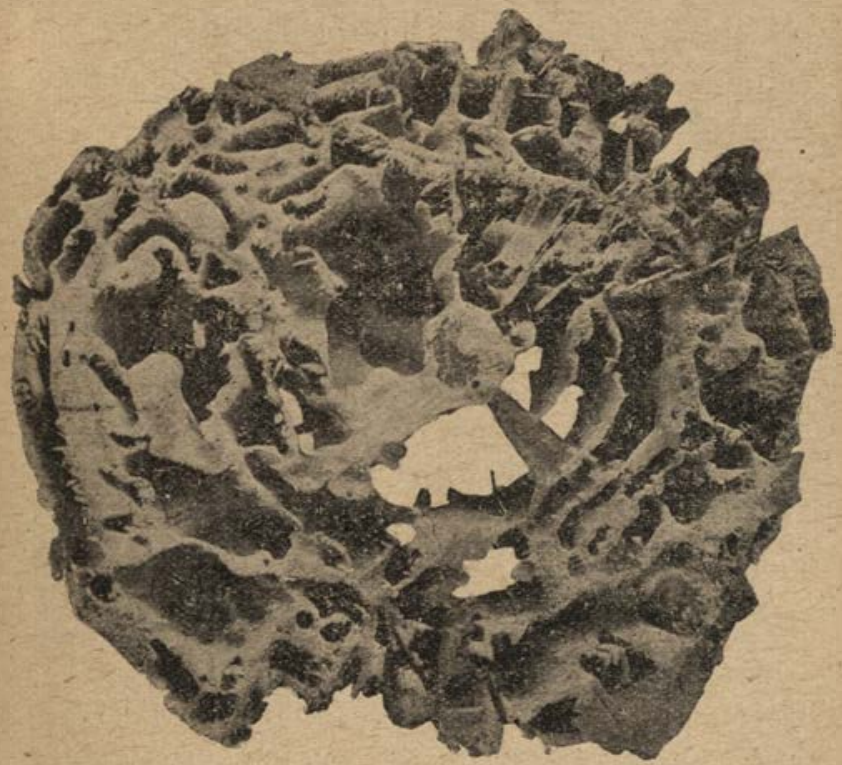

Fig. 21. - Coupe dune poutre rongée par Calotermes hubbardi (Arizona) (d'après Th. E. SNyder).

l'apparition d'une cryptobiose peureuse, et de tout ce que cela implique : dégénérescence ou perte des yeux, spécialement chez les castes d'ouvriers et de soldats; perte du pigment et amincissement du tégument, sauf chez les 
formes ailées et sur la tète et le thorax des soldats; et enfin tendances photophobes et hygrophiles très générales chez tous les membres d'une colonie. La fragilité ainsi acquise entraîne à son tour l'apparition de diverses formes de protection el de cerlaines habitudes de défense, comme les mandibules, le crâne résistant et les glandes céphaliques des soldats et, chez les-termites supérieurs, l'édification d'un nid solide ou termitière, avec de la terre, des excréments, etc., agglutinés.

$$
\text { ** }
$$

Cés adaplations protectrices sont rendues nécessaires par le nombre d'ennemis des termites, en particulier contre les fourmis, dont beaucoup vivent en grande partie ou même complèiement à leurs dépens. Ceci s'applique spécialement aux Ponérinés prédateurs, par exemple Megaporera fatens, Paltothyreus tarsatus et Euponera semuarensis cn Afrique, Nenponera commutala, N. crassinoda el Paraponera clavata dans l'Amérique centrale et l'Amérigue du Sud ct à un grand nombre d'espèces de Leptogeny's des tropiques des deux hémisphères. Dans un mimoire décrivant les divers modes de défense des termites, Brigiox ( I g23) dit: "Les lermites n'ont, dans les conIrées tropicales, pas d'adversaires plus redoutables, plus redoutés, que les fourmis. C'est, entre ces pelits êtres, une lutte incessante, une guerre implacable, poursuivie sans merci, depuis des milliers d'années. Aussi peut-on constater que les moyens de défense si ingénieux et si divers mis en cuvre par les termites sont, à peu près tous, destinés i les préserver des atlaques des fourmis, leurs ennemies héréditaires. " Fotons, en même temps, que plusieurs fourmis tropicales ne dévorent pas les termites. Ceci pa: 
rail exact pour nos énormes fourmis néolropicales (D)orylinés) du geure Eciton. Par contre, les Dorylinés d'Afrique, Anomma, Typhlopone, Rhogmus, ele., en semblent très friands. Il y a toute une armée d'espèces (Carebara, Poednlgus, Aëromyrmn, etc.), installées comme des roleurs dans les murs des termitières et pillant leurs habitants, et une autre série, encore plus importante, qui chassent, en partie ou tolalement, les lermites de leurs nids, et semparent de leurs galeries. C'est la fason d'agir accoutumée, en Australie, d'Iridomyrmex sanguineus et de beaucoup de fourmis des genres Opisthopsis, Calomyrmex, Polyrhachis, Camponolus, elc. D'autres, (rop nombreuses pour être nommées, se comportent de mème vis-à-vis des Termitidés africains. Lorsqu'on a été lémoin de cette hostilité incessante et implacable régnant sous les tropiques entre les fourmis et les termiles, on se demande avec étonnement si le sort des seconds n'est pas d'être exterminés par les premières.

Si, pour conclure, nous en revenons à la définition de De Gravge, faisant d'une société une collectivité cumulative, nous constatons que, au moins chez les termiles supérieurs, les activités cumulatrices les plus frappantes sont archilecturales et résultent primitivement du genre de leur aliment qui est abondant, d'une obtention aisée, mais d'une assimilation difficile. Cette alimentation spéciale facilite et simplifie aussi la formation de teurs colonies, qui devaient, à l'origine, ressembler à celles de divers Coléop'ères subsociaux (Passalus, Phrenapates, I'arandin, Coccidotrophus, Micromulthus, etc. ) (I).

(1) Après avoir rédigé ce paragraphe, j'ai relu un intéressant memoire de LAMEerre (1909) el j'y ai trouvé une tentative analogue, plus détaillée, d'expliquer l'origine possible de la colo- 
Tous ces insectes vivent dans des cavités des tissus végrétaux. Les développements, ou cumulations ultimes le: plus perfectionnées (polymorphisme, trophallaxis, mycétophagie, etc.) ont probablement été acquis de façon très nette, comme résultant d'interactions plus compliquées chez des termites pris individuellement, et naissant entre eux et le milieu ligneux qu'ils avaient élı.

nie de termites; la voici: "Rendons-nous compte, par l'imagination, de la situation de Blatles s'adaptant au régime xylophage, et forant, pour se nourrir, les troncs qui deviennent en même temps leur habital naturel; que d'énergic à dépenser, que de précautions à prendre pour assurer le sort des petits. L'oothèque peut disparaitre: elle est devenue inutile, puisque les œufs seront aussi bien protégés s'ils sont pondus dans les galeries creusées dans le bois qui forment maintenant un nid ; c'est donc une économie réalisable. Mais les jeunes, au sortir de l'œuf, sont incapables de se sustenter suffisamment en attaquant une nourriture peu réconfortante; ils sont alimentés par la mère, et l'on conçoit qu'il ait été fort utile que le père vienne à la rescousse. Nous aurions ainsi quelque chose de très analogue à ce que montrent les Passalus: ces Lucanides fouillent en tous sens le trone vermoulu des arbres pourris, le màie et la femelle màchant le bois pour l'alimentation des larves. ”

Nous n'aurions qu'à substituer dans ce passage le terme de "Proloblalloidés " à celui de " Blattes ». 


\section{CHAPITRE VI}

\section{LE POLYMORPHISME}

Cas divers. - La parthénogenèse et le sexe. - Différencialion d'une caste ouvrière chez les fourmis; son évolution. Caraclères adaptatifs des castes. - Polymorphisme des termiles. - Différenciation congénitale ou épigénétique des castes. - Les diverses formes de soldats. - Soldats (fourmis ou termites) gardant l'entrée du nid (phragmose).

Le polymorphisme a souvent été évoqué dans les leçons précédentes, mais j'ai évité toute discussion de ce sujet, car il se retrouve dans l'étude de presque tous.les insectes sociaux, et une juste vue d'ensemble n'en est possible qu'après avoir examiné toutes les idiosyncrasies de ces insectes. J'ai discuté ce sujet troublant il y a dixhuit ans $(\operatorname{I} 9 \cap \mp, x)$. Depuis, il a toujours été présent à ma - pensée et, naturellement, j'ai consulté tous les ouvrages compétents qu'il m'a été donné de rencontrer. A aucune époque, les études sur les insectes sociaux n'ont été aussi nombreuses, aussi intéressantes, aussi pénétrantes; jamais les travailleurs n'ont eu autant de facilités pour aborder ce sujet. Et pourtant, je ne suis point d'avis que nous ayons beaucoup ayancé vers la solution des nombreux problèmes que suggèrent les phénomènes du polymorphisme. Cette question est. effectivement très complexe ; et, bien qu'ayant réservé deux leçonš à sa discus- 
sion, je ne pourrai vous présenter rien de plus qu'un petit nombre de ses nombreux aspects el de ses difficultés. Pour plus de clarté, je me contenlerai, dans cette leçon, de passer en revue quelques-uns des faits principaux et les conclusions les plus évidentes; et, dans notre prochaine réunion, nous pénétrerons au sein des théories que tout cela nous suggère ef nous suggèrera sans doute encore longtemps.

Il est difficile de donner une définition du polymorphisme, parce que les phénomènes auxquels les zoologistes appliquent ce terme dans son sens le plus étendu, passent par des transitions insensibles aux phénomènes de variation, mutation et métamorphose, aux alternances de générations (hétérogonie), à la pæodogenèse, à l'hétéromorphose et à l'hétérochromatisme saisonnier ou aulres. Si nous restreignons ce terme aux cas dans lesquels deux (ou plusieurs) formes différentes coexistent dans la même espèce, il comprendra naturellement le dimorphisme sexuel, et les différences entre jeunes et adultes; mais, tel que l'emploient les entomologistes, il s'applique essentiellement à la coexistence de deux (ou plusieurs) formes adultes du même sexe ; c'est la " pœcilandrie " et la "pocilogynie " de Pexenmmorr (1897).

Il existe aussi des formes larvaires polymorphiques, exemples de ce que Giard (1891, 1894, r905) a appelé " pocilogonie ". J'aurai l'occasion de mentionner des cas de polymorphisme chez les insectes divers, mais mon intérêt ira principalement aux espèces sociales. Ces dernières sont constituées de façon si particulière que leur polymorphisme est souvent regardé comme lié nécessairement à certains autres phénomènes, lels que le déterminisme des sexes, la parthénogenèse, la néolènie, la stérilité et 
l'arrèt de développement; certains de ces phénomènes demandent à être examinés sommairement dans le but de les éliminer de la discussion générale. La question du sexe des descendants d'une femelle d'insecte parthénogénétique a été très embrouillée par l'interminable controverse qui s'est attardée, surtout en Allemagne, autour de l'abeille domestique, créature exceptionnelle, qu'il est très difficile de déloger des manuels de zoologie et des salles de cours, où elle représente le paragon des insectes sociaux.

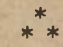

Il est certain, bien entendu, que les descendants d'un Hyménoptère non fécondé sont généralement des mâles ; mais les exceptions signalées deviennent si nombreuses, que la soi-disant " loi » de Dzı́́nzox doit ètre considérée comme la simple expression d'un phénomène habituel, chı̌ les espèces particulières où il a été observé. Ce n'est pas une loi, mais au plus une règle. Qu'il n'y ait pas de lien nécessaire entre ce phénomène et le polymorphisme des insectes sociaux, cela est suffisamment prouvé par les termites, chez lesquels les deux sexes proviennent d'œufs fécondés, et sont également polymorphiques.

Wisich $(1920, p .76 ; 108)$ a récemment pris la peine d'enregistrer les sexes produits par les femelles parthénogénétiques, chez les insectes el surtout chez les Hyménoptères. Chez les Phytophages, il a complé vingt-quatre espèces définitivement rlassées comme thélytoques, deux qui sont amphérotoques, et plus de quarante qui sont arrhénotoques. Mais quelques-uns de:s cas notés comme thélytoques ne le sont pas absolument, car un petit nombre de màles peuvent naitre parmi les femelles. Chez les Térébrants, en mettant à part les Cynipidés, chez lesquels il y a alternance entre lamphérotocie el Ja reprodiction bisexuèe, et en comptant les Bethylidès, il cite quatorze 
especes, représentant treize genres et plusicurs familles, qu sont certainement thélytoques et plusieurs qui le sont incomplètement, les couvées comprenant aussi quelques males. Les formes amphérotoques sont rares; le type dominant de parthénogénèse étant certainement le tỵpe arrhénotoque. On a signalé des cas particuliers rares, que l'on a découverts en Imérique, depuis la publication du travail de WiNkLER. En éludiant le développement de plusieurs milliers d'individus de Habrobracon brevicorne, Whiтivi (1921a) a démontré que les màles peuvent parfois être produits par des oufs fécondés. Il m'a fait savoir qu'il a obtenu des femelles à partir d'œuis vierges, et LEIBY (1922) affirme que, chez Copidosoma gelechia, Chalcidide polyembryonique, les œufs fécondés fournissent aussi bien des mâles que des femelles, tandis que les œufs vierges donnent seulement des mâles. M. S.-M. DониNisN, qui, au Federal Gypsy Moth-Laboratory de North-Melrose, près Boston, Mass., a expérimenté plusieurs années sur les. Hyménoptères parasites, me communique d'intéressants renseignements encore inédits. Un hyperparasite de Liparis dispar, Hemiteles tenellus Say, parasite d'Apanteles melanoscelus, s'est montré strictement thélytoque dans les lignćes pures réalisées au laboratoire (douze générations en trois ans) et dans l'élevage de plusieurs milliers d'individus, à partir de cocons d'Apanteles recueillis dans la campagne. Le mảle de cette espèce est d'ailleurs inconnu. Au contraire, chez l'espèce européenne, Hemiteles areator (si voisine de tenellus qu'il est difficile de bien les distinguer morphologiquement), le mâle est commun et DohıNisn en a obtenu à partir de femelles vierges. La meme opposition existe entre les deux espèces de Pleurotropis et celles d'Anastatus obtenues de cocons d'Apanteles, l'une des deux, pour chaque genre, produisant toujours des màles, l'autre des femelles, et les mâles des espèces thélytoques étant encore inconnus.

Picaro (1922) a aussi étudié deux des espèces européennes dHemileles, fulcipes et longicauda. La première est un parasite général, qui attaque les autres parasites, spécialement Apanteles spurius, difiérents Microgaster, quelques Tenthredinida (Blennocampa pusilla) et apparement. même les œufs des araignées. Les femelles vierges produisent seulement des màles. II. longicauda, qui est un hyperparasite de Apanteles glomeratus, est, par contre, régulierement, sinon exclusivement, thétytoque. Picırd dit: "Les mâles doivent être bien rares, car je n en ai pas obtenus en élevage et n'en ai pas rencontrés dans la nature. Les individus que je récoltais au dehors ne produi- 
sirent que des femelles et j'ai lout lieu de croire qu'ils n'étaient pas fécondés. Leur progéniture, en effet, qui ne l'était certainement pas, ne pondait aussi que des œufs femelles. »

En fin de compte, on a signalé chez les Aculéates sociaux plusieurs cas dans lesquels des femelles se sont développées a partir d'œufs vierges. Sans nous attarder à citer les travaux d'autres adversaires de Dzienzon, nous attirerons l'attention sur ceux de Onions (1912, 1914) et de JACK (1917), suivant lesquels l'ouvrière d'une race sud-africaine d'Apis mellifica (var. liafra ou intermissa) possede un spermathèque et produit parthénogénétiquement, non seulement des mâles et des ouvrières, mais mème des reines. Chez les abeilles solitaires et sociales, suivant les récentes et habiles recherches de Descy (1924) sur Osnia, de StöckHert (1923) sur Halictus, et de plusieurs observateurs sur Bombus, les femelles parthénogénétiques sont toujours arrhénotoques. Des résultats analogues ont été enregistrés pour les fourmis reines et ouvrières, non fécondées, par Forel (1874), LubBock (1888), Miss Fielde (1901), JANET (1909), Tavgury (1913) et moi-même; mais il y a des exceptions. TavNER (1892) affirme que, dans ses nids artificiels d'Atta cephalotes, la grande fourmi rongeuse de feuilles de la Trinidad, les ouvrières produisaient des mảles, des ouvrières et des reines; et Reichenbach (1802), Mrs. A.-B. Comstock (IVheelf.r, 1903a) et Crawley (1912) ont élevé des ouvrières a partir d'œufs vierges d'ouvrières de Lasius niger.

Les exemples que je vous ai présentés montrent clairement que les phénomènes n'ont pas un lien nécessaire avec le polymorphisme; et, quelle que puisse être la solution définitive du problème, pour les races communes de l'abeille domestique, soit que les ouvrières et les reines non fécondées produisent uniquement des mâles ou parfois aussi des ouvrières ; soit que les œufs fécondés puissent quelquefois donner des mâles, cela n'ajoutera au problème du déterminisme des sexes aucune donnée théorique importante qui n'ait déjà été fournie par d'autres Hyménoptères, chez lesquels de telles possibilités se réalisent sous des conditions bien plus favorables au contrôle expérimental. 


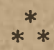

Il nous est difficile de parler de polymorphisme chez les Aculéates sociaux, tant qu'une caste d'ouvrières n'a pas fait son apparition, comme l'expression morphologique distincte de la division physiologique et éthologique du travail, entre les membres femelles, primitivement monomorphiques, de la colonie. Chez les abeilles, nous constatons l'existence d'une telle caste dans les sous-familles des Bombinés, Méliponinés, Apinés et Halictinés. Chez les Halictus sociaux, cette caste est représentée par la génération d'été des femelles, qui est quelquefois assez différente de leur mère, pour avoir été décrite comme une espèce distincte. (Stöcknert, I923). Les espèces sociales d'Allodape sont encore à un degré trop primitif pour avoir développé une caste d'ouvrières différenciées morphologiquement, quoiqu'on puisse dire qu'elle est représentée, à un degré primitif et seulement dans les mœurs de l'espèce, par les filles qui s'associent avec la mère, fondatrice du nid.

Chez les Vespidés, on peut suivre l'évolution graduelle de la caste ouvrière à travers les cinq-sous-familles sociales. Chez less Sténogastrinés et certaines Epinoninés on n'a pas signalé d'ouvrières; chez d'autres Epinoninés, la dissection seule révèle si une femelle fonctionne comme ouvrière, c'est-à-dire comme un individu stérile, ou nourrice. Chez les Ropalidiinés et Polistinés, les ouvrières diffèrent peu, extérieurement, des reines, mais, chez les Vespinés, les différences s'établissent bien, à la fois dans la taille et dans la coloration. Chez les fourmis et les termites, la caste ouvrière est aptère, différenciée généralement de façon tranchée, et souvent elle-même dimorphi- 
que ou pléomorphique, au second degré, par suile d'une division physiologique du travail plus profonde. Chez ces insectes aussi, la différenciation entre les castes d'ouvrières et de soldats n'apparaît évidemment au début que sur le plan fonctionnel, c'est-à-dire dans la physiologie et dans les mœurs ; puis elle devient aussi morphologique et les deux castes prennent les caractères qui nous permettent alors de les reconnaître sans hésitation ( $\mathrm{I}$ ).

S'il est exact, comme je l'ai supposé et répété dans mes leçons précédentes, que les fourmis, les termites et les divers groupes de guêpes et d'abeilles sociales sont des sociétés phylogénétiquement indépendantes, nous devons aussi admettre une indépendance analogue pour le développement de ces castes d'ouvrières; il n'y a pas moins de dix de ces groupes qui en possèdent, et elles tirent leurs caractères communs et convergents des conditions sociales, trophiques et autres, similaires, auxquelles ces groupes ont été constamment soumis au cours de leur ontogénie et de leur phylogénie.

Lne étude plus serrée nous montre que les dix sociétés indépendantes dont nous parlons ci-dessus peuvent se diviser en quatre groupes, suivant les idiosyncrasies de

(1) Comme caste physiologique, on peut citer, chez les fourmis, les plerergates ou "pols à miel 》 de diverses espèces de myrmecocystus, Prenolepis, Plagiolepis, Camponolus, Melophorus, Pheidole, etc., des régions arides. Ce sont des ouvrières ou des soldats qu'on ne peut distinguer d'abord des autres membres de leurs castes respectives; mais éventuellement, elles deviennent très lentes et subissent une forte distension du jabot et de l'abdomen, par suite de l'accumulation des aliments liquides. De même, les ouvrières gynœcoïdes, qui développent leurs ovaires et assument le rôla de reines dans les colonies qui en sont privées, peuvent être considérées comme constituant une amorce de caste physiologique. (EMERY, 1915.) 
leurs castes d'ouvrières : $I^{\circ}$ les Halictus, les bourdons et quatre sous-familles de guèpes; $2^{\circ}$ les abeilles des sousfamilles des Méliponinés et des Apinés ; $3^{\circ}$ les termites ; $4^{\circ}$ les fourmis.

Chez les Vespidés, Bombinés et Halictinés, la forme ouvrière, en se développant, voit croître sa stérilité, tandis que la reine conserve intactes la taille, la morphologie et la physiologie de la femelle des Vespidés ou Apidés solitaires. Dans ce cas, les conditions apparaissent très clairement; tous les phénomènes s'expliquent bien par ce que Marchal (1896, 1897) a appelé la " castration nutricielle " (de nutrix, nourrice). Rappelons qu'il a donné ce nom à l'atrophie fonctionnelle ou à l'arrêt de développement des ovaires chez les ouvrières destinées à assurer l'alimentation des larves. Il a montré qu'après l'élimination de la reine, dans une colonie de guêpes, plus d'un tiers des ouvrières deviennent fécondes. Le même effet suit la suppression complète, ou même l'arrêt temporaire, de la ponte de la reine. Marcial affirme, et cela est possible, que cette transformation est dûe à l'abolition des fonctions nourricières des ouvrières, lesquelles s'approprient les aliments qui, normalement, sont destinés aux larves. La castration nutricielle conduit naturellement à la castration des organes reproducteurs chez les larves qui donneront des ouvrières. Nous avons donc là un processus cyclique, dont les effets sont accentués par la tendance qu'ont les ouvrières adultes à distribuer la quantité limitée de nourriture disponible entre des larves beaucoup trop nombreuses pour pouvoir être ainsi nourries convenablement (voir Roubaud, r 9 r6). Le polymorphisme réduit des guêpes et aussi, Irès probablement, des bour-

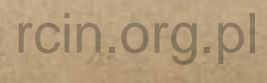


dons est dì à des processus instinctifs et physiologiques bien connus et spéciaux au milieu social.

Tandis que la caste ouvrière, chez les guêpes et chez les abeilles, parait acquérir sa stérilité graduellement et avant ou pendant sa différenciation morphologique, la caste correspondante des termites, et surtout la caste des soldats, paraît s'ètre développée morphologiquemeut avant que sa fécondité se fût atténuée ou eût disparu. Danṣ les espèces très primitives comme Archotermopsis et certainement aussi, en une cerlaine mesure, chez Termopsis, la caste des soldats est féconde, et il existe des mâles et des femelles néoténiques, mais pas d'ouvrières. Chez Archotermopsis, chaque sexe est trimorphique et représenté par trois formes fécondes, des individus parfaitement ailés, des individus néoténiques ailés incomplètement, et des soldats subaptères ou aptères. Avec le progrès du développement social, une caste ouvrière apparaît, par suite d'un arrêt précoce dans le développement de la nymphe, et de même le soldat devient stérile.

A première vue, les conditions paraissent être chez les Formicidés les mêmes que chez les guêpes, sauf que la casle ouvrière présente des modifications somatiques plus profondes et une stérilité plus prononcée. Cependiant, en faisant une étude comparative des fourmis primitives, spécialement des Ponérinés, Cérapachyinés, Dorylinés et Pseudomyrminés, on soupsonne que cette ressemblance n'est que superficielle. Comme nous l'avons vu, les fourmis sont heaucoup plus anciennes que les guêpes sociales et dérivent d'un groupe de Vespoïdés solitaires bien iplus rapprochés des ancêtres archaïques des Aculéates, les Béthylö̈dés, dont plusieurs genres ont conservé deux formes de femelles fécondes, l'une ailée, l'autre aptère. 
Dans ce cas, par conséquent, le polymorphisme est antérieur à la stérilité, même chez les ancêtres solitaires, et il n'est pas improbable que les premiers Formicidés aicnt conservé le dimorphisme de la femelle après être devenus sociaux, et s'en soient servi pour produire les reines ailées et les ouvrières aptères. La femelle ailée a gardé comme seule fonction de disséminer l'espèce et de fonder la colonie, tandis que ses filles aptères, mais encore fécondes, et restant auprès d'elle, se sont spécialisées en prenant soin de la couvée de la reine, puis de leurs propres couvées, et en allant en quête de la nourriture. La reine ailée a acquis la faculté de perdre ses ailes et les volumineux muscles vibratoires ont été alors supprimés et ont servi à alimenter ses œufs dans les ovaires et à prolonger sa vie jusqu'à l'apparition de ses descendants aptères. En effet, JaNet (Igo7) et C. P'Érez (I912, I920) ont montré que la perte des ailes se fait suivant un processus de ce genre chez les reines fourmis actuelles, après leur vol nuptial. Un pas de plus fut fait lorsque les femelles aplères fécondes furent réduites au rang d'ouvrières. Cette hypothèse pourrait expliquer les conditions observées dans certains genres de fourmis, comme le maintien d'une femelle aptère comme reine unique chez les Dorylinés, benucoup de Ponérinés et les Cérapachyinés, la présence à la fois de reines ailées et aptères dans une même colonie (Poneru), ou en des endroits différents (Harpagoxenus), l'existence assez fréquente de reines ergatomorphiques dans plusieurs genres (Leptngenys, Onychomyrmex, etc.).

A ce sujet, il me faut mentionner l'opinion de Mrazek (1916), qui s'est efforcé de montrer que, chez les Hỵménoptères sociaux, le polymorphisme est la conséqunce de générations 
alternantes dissimulées ou se lélescopant les unes les autres, comme il arrive chez les Cynipides. A première vue, une telle hypothèse parait difficile à soutenir, mais elle est au moins appuyée par les observations de StöckHert sur les Haliclus sociaux (1923). Evidemment, on peut faire dériver très justement Halicfus malachurus et les espèces voisines, d'espèces ayant deux générations annuelles (le genre en compte plusieurs), en tenant compte de la longévité croissante et de la survie des femelles d'automne qui passent l'hiver, et de la stérilisation progressive de la génération d'eté, qui finit par amener celle-ci à l'état de caste ouvrière. Si nous acceptons linterprétation que j'ai donnée plus haut, la colonie de fournis pourrait se concevoir comme naissint du " télescopage " de deux générations ayant, à l'origine, l'une des reines fertiles ailées, l'autre des reines fertiles aptères. Mais les guêpes sociales, les Epinoninés par exemple, me paraissent constituer un obstacle insurmontable pour l'hypothèse de Mrazek, car elles sont étroitement liées aux Euméninés solitaires, elles en dérivent trop naturellement, et rien n'indique dans ce dernier groupe, l'existence de générations alternantes. De même, les tentatives faites par Mrizer pour trouver trace de telles conditions chez l'abeille domestique ne me paraissent ni bien nettes ni bien heureuses, mais la mouche à miel parait destinée à confondre les biologistes. Autant que je puisse voir, il n'y a rien qui puisse faire soupçonner une alternance de générations dans la constitution des colonies de termites.

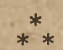

Vous avez remarqué qu'au lieu de rattacher le polymorphisme à une hétérogonie contractée ou " téléscopée ", j'ai préféré le considérer, au moins chez les fourmis et les termites, comme antérieur phylogénétiquemente aux conditions qui ont rendu stériles une ou plusieurs formes. Cette opinion implique que le polymorphisme est, par essence, identique à la poecilogynie et à la procilandrie de beaucoup d'insectes non sociaux.

Bien que beaucoup de ces derniers cas aient été passés en revie par de. Peyfrimhoff, il y a assez longtemps (1897), il me parait intéressant de mellre en lumière quelques-uns de ceux 
qui ont élé étudiés au cours de ces dernières années. Le plus familier est celui des Lucanidés màles, qui présentent souvent une ressemblance singulière avec les soldals et ouvrières pléomorphiques de certains lermites et fourmis, par la structure de la tête el des mandibules. Par exemple, chez les soldats du genre Eicilon $s$. str., nous pouvons reconnaitre des formes extrèmes tcholontes el priodontes, avec lous intermédiaires, comme che\% beaucoup de ILucanidés Odontolabinés, ainsi que les ont décrits Griffini (1905) et d'autres. Si j'en avais le temps, j'examinerais en délail celle ressemblance qui n'a pas échappé a Viennerer (1923). Les petits Coléoptères Psélaphides du genre By/lhynus fournissent un exemple rappelant la variabilité des yeux dans la série pléomorphique de fourmis ouvrières, ou dans les castes de termites. Chez B. algericus, suivant DE Peremianoff (1910), la femelle a de pelits yeux, landis que les males sont dimorphiques, l'une des formes ayant des yeux plutot petits, l'autre les ayant grands. Un polymorphisme des ailes, ressemblant it celui des formes néoténiques des termiles, est fréquent chez les Hémiplères des deux sexes, el a plé soigneusemenl éfudié par Porsson (192'i), chez un grand nombre d'eepeces aquatiques. Les insectes parliculiers aux liguiers (Chalcicidés) du genre Philotrypesis, décrits par Gr.wni (1921, 1923), fournissent un autre exemple, plus proche encore que les précédents du cas des fourmi-, bien quapparaissant seulement chez. le male. Les têtes des divers màles de $1 \mathrm{H} h$. erythriea et unispinosa val ornala, telles qu'elles sont représentées, pourraient très bien so confondre avec une série de tètes d'ouvrières plíomorphiques du genre Camponolus. Grani adopte les termes de BerLese pour les formes extrêmes et intermediaires, les màles "épimégétiques " étant les plus grands de la série, les "eumégéliques " faisant transition, et les "hypomégéticues " étant les plus petits. Mais la pœcilandrie du Philotrypesis est devenuc plus compliquée mime gue la pocilogynie des fommis : ainsi, cher. Ph. minulu, dont les màles forment deux sćries, une " acanthocéphale " et un " mutique ", chacune d'elles comprenant des formes eumégétiques et hyponégétiques, et en outre, des eumégétiques macrognathes (hétérodonles) \#. I.a ressemblance de cette espèce de Philolrypesis et des fourmis est accrue encore par son caractère subaptere poussé il l'extrème.

Que le polymorphisme relìve d'autres causes que celles qui déterminent le dimorphisme sexuel, cela est, je crois, 
netlement indiqué par les exemples précédents el par l'existence, toujours vérifiée, d'un polymorphisme identique dans l'un et l'autre sexe chez les termites. Cette conclusion devient une certitude, si nous considérons les cas de polymorphisme (" precilogonie ") chez les larves de certains insectes, qui, adultes, sont homomorphiques. II y a 20 ans, Grimd (rgo5) a passé en revue tous les cas connus, dans un important mémoire. Aussi n'en citeraije que deux, l'un découvert plus récenıment, et l'autre étudié depuis avec plus de précision. Silvestra (19o6) a découvert qu'il existe deux types très différents de larves chez le Chalcidide polyembryonique Litomastix truncalellus, l'un possédant des organes reproducleurs et éroluant en insecte adulte, l'autre ("larve asexuée ") n'ayant pas d'organes reproducteurs et mourant sans se développer davantage. Les mêmes types de larves furent ensuile découverts par PAtrenson (I9I8, I92I) dans un autre Chalcidide polyembryonique, Paracopidosomopsis florid.unus. Au nombre des exemples de poecilogonie, Giarn range succinctement les Chironomidés et les Cécidomyidés predogénétiques, comme " déterminés en partie par les facteurs primaires de température et alimentation ". Les éludes les plus récentes de Springer (igr5) et Harmis (1923, 1924) ont démontré que les larves des Cécidomyidés des genres Miastor et Oligarces sont nettement trimorphiques. Sprivger les désigne respectivement sous les noms de larves poedogénétiques typiques ", "larves errantes " et "larves nymphes " et croit que leur différenciation provient de conditions externes variables.

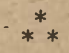

Le polymoriphisme a acquis un tel degré de com- 


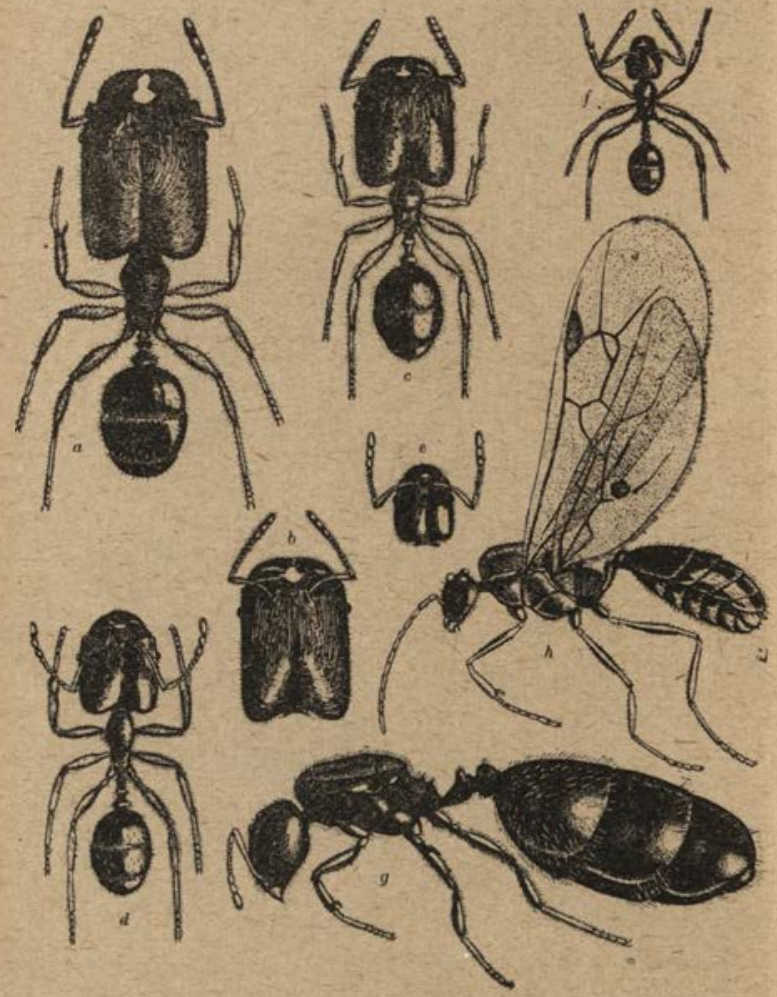

Fig. 25. - Myrmicine moissonneuse du Texas (Pleidole inslabilis) avec caste ouvrière polymorphe. $a$, soldat; $c$, ollvrierc; $b, e$, formes intermédiaires; $\%$, reine (désailée); $i$, male. Virme échelle pour loules les figures. 


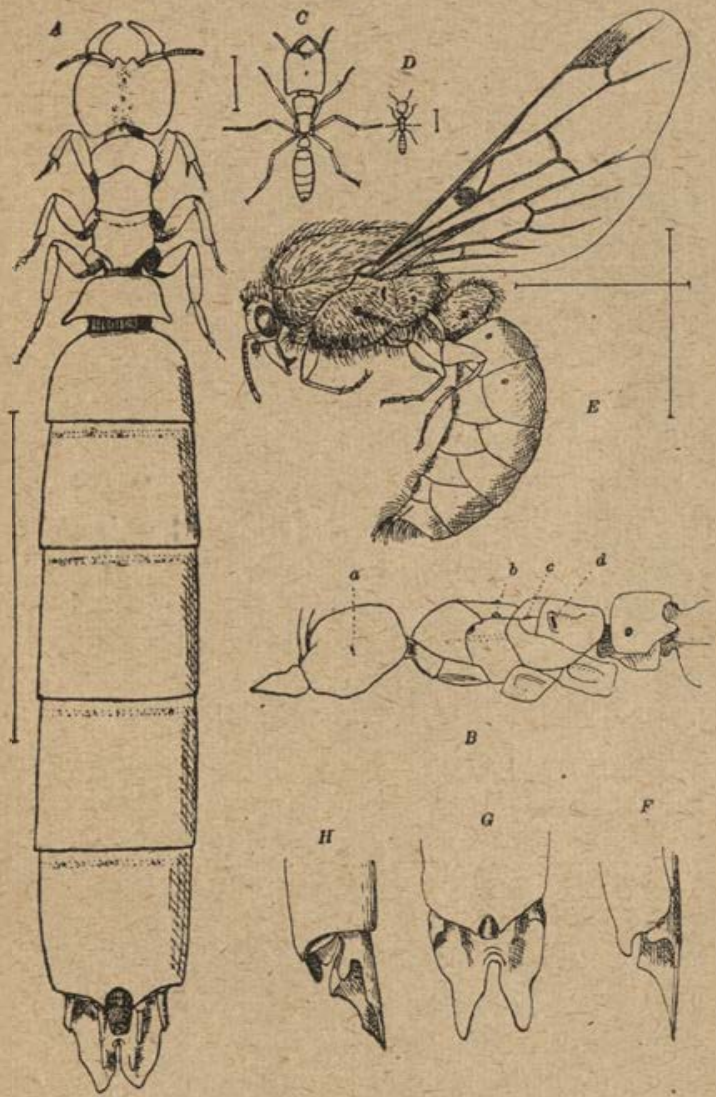

Fig. 26. - Castes de Dorylus helvolus (grossissement uniforme). Reine, vue dorsalement (A) et de profil (B); $b, c$, vestiges d'ailes; $C$, grande ouvriere; $D$, pelite ouvriere; $E$, mảle (d'après Emerr). 
plexité chez les fournis et spécialement chez les termites qu'il sera bon de concentrer notre attention sur ces groupes. La fourmi mâle est un organisme extraordinairement stable et conservateur; on sait pourtant qu'il est dimorphique, et qu'il présente une phase aptère chez de rares espèces (p. ex. Ponera eduardi) et qu'il est exclusivement aptère dans de rares genres (Anergates, Formicoxenus, Symmyrmicu, Cardiocondyla). En ce qui concerne au moins la taille, il peut êlre utilisé dans chaque espèce, ainsi que l'a montré Emrny, comme terme de comparaison, auquel ramener les reines et les ouvrières beaucoup plus soumises à des variations. L'extraordinaire degré auquel alteignent ces variations, dans le sexe femelle des Formicidés, est indiqué par le fait qu'on n'a pas reconnu moins de vingt et une formes différentes. De ce nombre, huit sont nettement pathologiques et proviennent soit de la présence de parasites dans les colonies, soit d'anomalies dans l'embryogénie ou la croissance de la larve (anomalies dans les chromosomes(?), dans la nutrition); qualre formes représentent simplement des excès ou des défauts de développement dans la taille (nanisme ou gigantisme), et les neuf formes restanles sont, on des formes normales se présentant de façon très générale, ou des formes spéciales particulières à certains genres. Si nous classons les vingt et une formes en typiques, atypiques et pathologiques, nous trouvons qu'il existe seulement trois formes ou phases typiques de la femelle: la reine, le soldat et l'ouvrière, et que chacune d'elles peut être regardée comme le centre de développement de formes atypiques et pathologiques, dont j'étudierai un certain nombre dans la prochaine leçon.

Une étude comparative de centaines d'espèces et de 
lous les genres connus nous a permis de dresser la liste suivante, groupant les degrés supposés de l'évolution dans la procilogynie des Formicidés (I).

$1^{\circ}$ Le mâle, la reine et l'ouvrière sont de la même taille ou presque, et l'ouvrière diffère de la reine uniquement par l'absence d'ailes, les ocelles et les yeux quelque peu plus petils, un thorax moins compliqué et des organes reproducteurs plus ou moins avortés. Tous les degrés suivants dérivent de celui-ci, ¡ui est représenté par les sous-familles de fourmis les plus primilives, les Ponérinés, Cérapachyinés et Pseudomyrminés.

$2^{\circ} \mathrm{La}$ reine a disparu, l'ouvrière est féconde et peut s'accoupler avec le mâle. Ce degré se présente chez nombre de genres de Ponérinés (Diacamma, Rhytidoponera, Dinoponera, etc...), probablement aussi dans le genre Leptomyrmex parmi les Do. lichodérinés et le genre Ocymyrmex parmi les Myrmicinés.

$3^{\circ}$ La reine augmente de taille, tandis que l'ouvrière conserve ¿ peı près la taille du mâle (Lasius, Brachymyrmex, Crematoyasler, Iridomyrinex, eic.).

$4^{\circ}$ La reine et l'ouvrière sont fortement différenciées; la première augmente de taille, et la seconde devient très variable, très instable et est représentée par toute une série s'étageant d'individus grands et à grosse tête jusqu'à des individus petits, microcéphales. Dans les ouvrages taxonomiques, les membres de cette série sont décrils comme " ouvrières majeures " ou " mineures ", ou ouvrières " maxima ", " media " et " minima ». (Camponotus, Atta, Pheido'ogeton, Dorylinés, etc.).

$5^{\circ}$ De la séric d'ouvrières du quatrième degré, seules survivent les " maxima " et les "media ", les premières se converlissant en soldats. (Myrmecocyslus bombycinus.)

$6{ }^{\circ}$ Les media, ou desmergates, qui relient les maxima et les ninima du qualrième degré, sont si peu développées que seuls les extrêmes persistent. Les maxima sont désignées comme soldats, (miles, dinergates), les minima comme ouvrières proprcment dites. A ce stade, la femelle est donc netlement trimorphique (Pheidole, Ceratopheidole, Acanthomyrmex, Ischnomyrmex, Oligomyrmex, etc.).

$7^{\circ} \mathrm{La}$ reine disparait et le soldat, devenant fertile, prend sa

(1) Ces degrés el leur ordre de groupement diffèrent quelque peu de ce qu'a publié Evem en 189' et de ce que j'ai publić moi-mêıne *n 1967. 
place, tandis que la caste ouvrière est représentée par les " minima " seules. Ce degré est hypothétique, mais il semble exister dans quelques espèces de Pheidole et Oligomyrmex (Ph. lamia et $O_{\text {a }}$ panamensis).

$8^{\circ}$ Les formes reine et soldat persistent seules, les ouvrières élant supprimées. C'est le cas, semble-t-il, des fourmis spéciales, faisant des esclaves, des genres Polyergus et Strongylognathus.

$9^{\circ}$ Toutes les formes ouvrières du quatrième degré disparaissent, sauf les " minima ", si bien que nous avons des reines énormes, et des ouvrières d'un type unique très petiles (Carebara, Predalgus, beaucoup de Solenopsis, etc.).

$10^{\circ} \mathrm{La}$ caste ouvrière tout entière disparaît, si bien que l'espèce retourne à un simple dimorphisme sexuel, c'est-à-dire qu'elle est représentée par des formes mảle et femelle uniques, comme dans la plupart des insectes et les autres Métazoaires. A ce degré, qui se présente seulement chez certaines fourmis parasites de façon permanente (Anergates, Whecleriella, Epocus, etc.) la femelle est à peine plus grosse que le male.

Nous ne devons pas considérer ces degrés comme se succédant l'un à l'autre en formant une série linéaire définie, mais comme rayonnant à partir du premier degré qui forme la base. Les degrés (2) à (10) ont évidemment pris naissance indépendamment et à plusieurs reprises, dans des genres éloignés. On peut en outre prouver que la plupart de ces types constituent une réponse à des exigences spéciales, extérieures on éthologiques, lesquelles sont le plus souvent reconnues en dernière analyse comme de nature trophique. Ceci est peut-être particulièrement clair dans les degrés (9) et (10), que nous illustrerons par les exemples des genres Carebara et Anergates.

Les espèces de Carebara snnt des "fourmis voleuses ", qui vivent dans les parois des termitières des termites africains et sud-américains. Le nid est relié aux chambres des terınites par des galeries très fines, trop ténues pour permettre le passage 
des termites, mais suffisantes pour laisser les pelites ouvrières de Carebara pénétrer dans les chambres et dévorer les couvées des termites. La réserve de nourriture est si abondante que les ouvrières peuvent élever des reines géantes, mais ellesmêmes sont condamnées à un perpétuel nanisme, à cause des nécessités de leur vie de rapine.

Chez Anergates atratulus, qui est monotypique, et qui vit en parasite de façon permanente dans les colonies du Tetramorium cæspitum, espèce commune en Europe, il n'est pas besoin de caste ouvrière, parce que les couvées des deux espèces sont ólevées par les ouvrières de l'espèce hòte. Aussi les ouvrières d'Anergates ont depuis longtemps disparu, et les reines sont de taille plus réduite. $\mathrm{Du}$ fait de ce retour secondaire au dimorphisme sexuel des Aculéates solitaires, le nombre de femelles pouvant se développer est fortement accru, avantage évident pour un parasite, et leur taille réduite leur permet d'être plus facilement adoptées par les colonies de Tetramorium. Ces dernières semblent réellement préférer les petils parasites à leur propre reine qui est très grosse, tout comme certaines dames préfèrent, pour des raisons esthétiques ou économiques, les minuscules chiens pékinois aux énormes dogues.

Ces quelques faits, et bien d'autres que l'on pourrait produire, montrent que, dans la colonie de fourmis, le nombre et le caractère des castes femelles sont réglés absolument comme le sont le nombre et le caractère des cellules dans le corps d'un Métazoaire. Cette régulation, qui est visible aussi bien dans le développement que dans l'abolition des castes, a un aspect ontogénique et phylogénétique à la fois, et constitue un processus déterminé, très plastique à certains égards, à d'autres assez. rigide, et paraissant dépendre de l'âge phylogénétique des casles dans l'espèce particulière que l'on éfudie.

Il est difficile de douter du caractère adaptatif que présentent les diverses castes femelles, puisque ces castes apparaissent dans la colonic comme l'expression d'une divi. sion, physiologique et éthologique, du travail. Une confusion considérable paraît s'être élablic dı fait des tra- 
vaux récents sur la morphologie $d u$ cerveau des fourmis et autres Aculéates sociaux, et on n'a pas suffisamment tenu compte des particularités des mœurs. Il semblerait. que la femelle féconde, a été choisie comme le type fondamental auquel se raltachent les diverses formes d'ouvrières et de soldats, qui en seraient des expressions ou des différenciations partielles ou plus on moins modifiées. Les instincls de la reine embrassent tous les ins. lincts essentiels des castes stériles on substériles. Ceci n'a jamais été discuté pour Vespa et Bombus, et il est indubitable que les reines de ces insecles ont un cerveau plus développé et mieux organisé que les ouvrières de la même espèce (Von Altex rgio). Beaucoup d'auteurs ont soutenu que la différenciation des corpora pedunculata de Dujardix, ou " corps fongueux" (" mushroom bodies "), comme on les appelle souvent, el qui sont situés dans la portion antéricure du profo-cerebrum, est directement proportionnelle aux capacités instinctives de l'insecte; ceci s'applique bien aux cerveaux de la reine, de l'ouvrière et du mâle des guêpes. Mais, chez l'abeille domestique, Jonescu (rgog) a montré que le cerveau de la reine est inférieur à celui de l'ouvrière. Il est vrai que, chēz cet insccte, la reine est rellement un parasite sur sa propre colonie et n'est pas, comme chez la guèpe, capable d'ćlever ses descendants sans le sccours des ouvrières. Chez les fourmis, heaucoup de reines se comportent comme chez les guêpes el ont un cerveau aussi développé; mais certaines reines, particulièrement celles des espèces parasites d'autres espèces, sont moins bien douées au point de vue instinctif, et possèdent des cerveaux plus petits et moins hautement différenciés que ceux des ouvrières de la même eṣpèce. D'aiprès mes obscrvations, ce dernier 
type est exceptionnel, le premier prévaut de beaucoup ; et c'est la base du désaccord qui sépare d'une part Miss Thompson et moi-même, d'autre part Forel (1874), Pietschien (igio). Zieglen (1912, ig20) et Brex (ig23a). Le premier, Fonel a présenté comme un fait général la supériorité du cerveau de l'ouvrière sur celui de la reine chez la fourmi, au cours de son étude sur Lasius fuligi. nosus, connu maintenant pour être un parasite social temporaire de $L$. umbralus, lequel à son tour est parasite de $L$. niger. Et, bien que j'ai appelé l'attention sur cette particularité, Brun a récemment produit la même conclusion que Fonel, après avoir étudié principalement $L$. umbratus! Les recherches de Miss Tıомpson sur trois espèces et genres de fourmis non parasites, Camponotus pennsylvanicus, formica schaufussi, et Lasius americanus, confirment les idées que j’ai émises en Ig1o, bien qu'elle ait constaté que le cerveau de la reine de $F$. schaufussi est dégénéré en comparaison de celui de l'ouvrière.

Les différences entre les résultats de Niss Thompson et les miens d'une part, et ceux de Forei, Pietschier, Ziegler el Brun d'autre part, sont difficiles à expliquer. Deux des espèces étudićes par Pietschier el Miss Thompson sont en réalité identiques (Camponolus pennsylulcanicus et ligniperda étant des sousespèces de $C$. herculeanus el Lasius americanus étant une variête de $L$. nigger), et tous ces auteurs, comme moi-même, ont ulitisé leur matériel au mème degré de développement, c'est-àdire qu'ils ont employé des nymphes très avancées, avant la pigmentation. Te mentionne ce fail, parce que HoLmgren (1909) a montré que le cerveau de la reine physogastrique âgée, chez Eutermes chaguima!yensis est réduit aux deux tiers de la taille qu'a cel organe chez la reine vierge, ce qui est l'indice de changements, d'une involution se produisant pendant la phase imago. Il n'esl pas improbable que des modifications similaires puissent survenir dans les cerveaux des reines fourmis perdant leurs ailes, au moment oit se dissout leur musculalure thora- 
cique. Ainsi donc, la comparaison des cerveaux de ces reines àgées avec ceux de leurs ouvrières semblerait apluyer l'opinion de Forel, Pietschier et Brun. Il sera donc opportun, dans les travaux futurs sur le cerveau des fourmis, de faire grande attention à l'état dans lequel se trouvent les reines. Il est très singulier que je n'aie jamais découvert une reine de $F$. schaufussi en train de fonder indépendamment une colonie, bien q e cette espèce soit une des plus abondantes de nos fourmis nord-américaines. Si, comme cela se pourrait, les jeunes reines élablissent leurs colonies après avoir émigré avec des corr pagnies d'ouvrières, elles réalisent ainsi les mêmes conditions que la mouche à miel (oı plutôt celles des Méliponinés), et nous pourrons admeltre l'existence d'un cerveau plus petit et dégénéré chez la reine (1).

(1) Je me suis attiré les critiques de Forel (1921-23) et Bruv (1923), par suite de mon scepticisme. à l'égard des rapporls étroits qui uniraient les conditions psychiques et le développement des corps pédonculés chez les fourmis (1910). Il y a plus de vingt-cinq ans, un de mes élèves, feu le $D^{r}$ C.-H. TunNer, entreprit l'étude comparative des corps pédonculés chez les Annélides et les Arthropodes inférieurs; il publia en 1899 un mémoire préliminaire, mais non définitif, sur ce sujet. Comme ce mémoire n'a jamais été consulté, semble-t-il, par ceux qui ont étudié le cerveau des insectes, il me paraît bon de mettre en lumière son contenu. Avant sa publication, HAmaken (1898) avait montré qu'il exislait des corpora pedunculala dans le cerveau de l'Annélide Polychète Nereis virens, el I)IETL (1876), BEnGen (1878), et Viallaves (1893) avaient noté leur présence chez les Crustacés décapodes. Tunner en trouva aussi chez les Annélides (Nereis, Polynoë, Lepidonolus) et les Décapodes (Cambarus). Les figures de son mémoire indiquent que ces corps sont beaucoup plus développés chez Polynoë et Lepidonolus, que chez. Nereis, ou chez les insectes inférieurs, comme, par exemple, Tomocerus et Lepisma, d'aptès Kuenvile (1913), Bottri:R (1910) et Bretschneider (1915). Mais ces corps se présentent dans des conditions particulièrement surprenantes chez les Limules, où, suivant Patren (1891) et Turner, les corpora pedunculala sont si gros et si compliqués, qu'ils rappellent les hémisphères cérébraux dun vertéhré supérieur. En fait, ils sont, dans ce cas, au point de vue du volume relatif et ce la différencialion morphologique, bien plus avancés, en comparaison des corpora pedunculala de la fourmi, que ne le sont ces derniers vis-à-vis de ceux des Orthoptéroïdes inférieurs. Je demanderai donc ent- 


\section{$\stackrel{*}{* *}$}

Le polymorphisme des termites, que nous allons examiner maintenant est plus compliqué que celui des four. mis, à la fois parce qu'il affecte également les deux sexes et parce qu'il est, dans chacun d'eux, plus compliqué. Il nous faut distinguer les huit castes suivantes, comprenant seize sortes différentes d'individus :

$1^{\circ}$ Première forme de mâles et de femelles (rois et reines viais).

$2^{\circ}$ Deuxic̀me forme de måles et de femelles (rois et reines néoléniques).

$3^{\circ}$ Troisième forme de mâles et de femelles (rois et reines ergatoïdes).

$4^{\circ}$ Grandes ouvrières mâles et femelles.

$5^{\circ}$ Petites ouvrières máles et femelles.

$6^{\circ}$ Grands soldats mâles et femelles.

$7^{\circ}$ Soldats moyens màles et femelles.

$8^{\circ}$ Petits soldats mâles et femelles.

Il est probable qu'une même colonie de termites ne produit pas toujours toutes ces formes, mais on en ren-

core: que fait une créalure comme la Limule, si stupide et si archaïque, de corps pédonculés, qui sont les plus développés de tout le rameau des Arthropodes? Fores dit qu'il ne sait rien de la Limule et il ne parait pas y ajouter d'importance, tant il est convaincu de la fonction précise des organes en question ; mais si ces derniers sont réellement, comme l'affirme Brun, un index infaillible " des capacilés mnémoniques individuelles et plastico-psychiques chez les insectes ", nous devrions admettre, soit que la Limule, quelque part dans le fond des iners et sans que nous en sachions $r^{\prime} e n$, fait étalage d'une richesse extraordinaire, au point de vue de la " mentalité plastique ", soil que ses corps pédonculés surprenants sont un dépôt merveilleux de "substance mnémique " acquis peut-être avec granrl'peine par les Protolimules actifs de l'époque palézoïque, et transmis comme un meuble inutile a leurs descendants modernes si stupides. 
contre souvent cinq ou même six. En outre, les soldats peuvent appartenir à deux types très différents, la plupart des espèces possédant des formes à grosse tête dites "mandibulées ", alors que certains genres de Termitidés ont un type très différent, dit " nasuti ", à tête petite, à région buccale réduite, et avec un long museau, à l'extrémité duquel s'ouvre la glande frontale. La question se complique du fait que les ouvrières, soldats et individus nćoléniques, peuvent être, surtout chez les termites inférieurs, à différents degrés de différenciation, et qu'ils diffèrent aussi par le nombre des articulations des antennes ou d'autres petits caractères. On suppose généralement que le polymorphisme a tendance à s'accroître lorsqu'on passe des termites inférieurs aux termites supérieurs ; mais Holmgnen est d'avis que c'est en sens inverse qu'il y a développement du polymorphisme. Il donne la série suivante comme formée des degrés les plus communs, série qu'il est intéressant de comparer avec celle que nous avons énumérée ci-dessus pour les fourmis.

$1^{\circ}$ Soldats (mandibulés) fortement po!ymorphiques, d'un type unique; néoléniques el ergatoïdes fréquents; pas d’ Juvrières proprement dites (Calotermes, Termopsis).

$2^{\circ}$ Soldals (mandibulés) polymorphiques, d'un seul type; néoténiques et ergatoïdes fréquents; ouvrières d'une forme unique (I.elucolermes).

$3^{\circ}$ Soldats polymorphiques, de deux lypes (mandibulés) et ì museau fourchu); probablement des ouvrières proprement diles, d'un seul lype. (Rhinolermes).

' Soldats (mandibules) dimorphiques, de deux types, grands et. petils, pas d'individus connus néoténiques et ergatoïdes; ouvrieres dimorphiques, de deux tailles. (Acanthotermes, Termes).

$5^{\circ}$ Soldals (mandibules), monomorphiques, d'un seul t!ppe, très grands; pas de néoténiques ni d'ergatoïdes; outrières dimorphiques, de deux tailles (Syntermes, Odontolermes). 
(i) Solduls (mandibuless), monomorphiques, d'un seul lype, plus petit; pas de néoténiques ni d'ergatoïdes; ouvrières dimorphiques, de deux tailles (Odonlotermes).

$i^{-}$Soldats (mandibulés) el ouvrières monomorphiques; néoténiques el ergatoüdes peuvent exister parfois. (La grande majorité des termites supérieurs.)

$8^{\circ}$ Les soldals manquent secondairement; ouvrières monomorphiques. (Anoplolermes.)

$9^{\circ}$ Soldats (" nasuti ») Secondairement dimorphiques, d'un seul lype; ouvrières monomorphiques; pas de néoténiques ni d'ergatoüdes (Eutermes bivalens, aquilinus, groupe de E. trinervius.)

$10^{\circ}$ Solduls ("-nasuti ") secondairement trimorphiques : ou vrières monomorphiques. (E. diversimiles, castaneiceps.)

$11^{\circ}$ Soldats (" nasuti )) secondairement dimorphiques; ouvrières monomorphiques (E. heleroplerus, velox et autres.)

$12^{\circ}$ Soldats (" nasuti 》) secondairement monomorphiques; ouvrières monomorphiques. (E. cyphetgaster, tenuirostris, hospitalis, etc.).

A celle liste, Houmgren ajoute le commentaire suivant: "L'évolution paraît se produire ici d'un polymorphisme très étendu vers des conditions plus simples. La simplification atteint son maximum chez les Anoplotermes, chez lesquels les soldals disparaissent vite et dont les ouvrières sont monomorphiques. Dans le degré (7), dans lequel existe un lrimorphisme simple, apparait, par différencialion secondaire, ure nouvelle caste, ce qui conslitue le degré (ı), suivi lui-même d'un second accroissement du nombre de castes. Ce polymorphisme secondaire, plus pénétrant (pentamorphisme) est à son tour réduit ensuite à un simple trimorphisme ( $12^{\circ}$ degré). L'évolulion du $1^{\text {er }}$ an F $^{e}$ degré se fail des condilions les plus lahiles (lluc'manles) aux plus slables; en effel, dans les promiers degrés de cetle série, la classe des soldats provient 
du développement d'un plus grand nombre de formes larvaires différentes 1$)$.

Puisque nous avons parlé du cerveau des Aculéates, il sera intéressant d'examiner celui des termites, d'autañt plus que certaines observations très significatives à propos de là différenciation des castes ont été failes sur cet organe. Il a été éludié par Holmgnen (Igog), KuennLe (rgr3), Miss Trompson (Igr3, rgi6, I917, rgig), von Rosen (19r3) et Jucci ( 1923 ). Après avoir étudié comparativement les diverses castes de Reliculitermes flavipes, Miss Tiompson résume de la façon suivante ses observations sur les corpora pedunculata . "Les corps pédonculés diffèrent très peu comme taille, d'après les mesures effectuées, et d'après le nombre supposé des cellules. Leur taille est maxima chez les ouvrières, minima chez les soldats et de dimension intermédiaire chez les formes sexuées : toutefois ces corps, chez l'adulte vrai, sont pres. que aussi grands que chez le's ouvrières. Si l'on conpare les corps pédonculés des Reticulitermes et ceux des fourmis et des abeilles, on voit, comme on pouvait s'y attendre que ces corps sont,' chez les termites, beaucoup plus simples et plus primitifs. Ce caractère primitif apparaît dans la dimension réduite et uniforme de toutes les cellules nerveuses "n, etc.

Ceci est en parfait accord avec les affinités des termites et des Orthoptéroïdes archaïques, quoique, d'après Kuemne, le cerveau des lermites possède des corps pédonculés beaucoup plus développés que ceux du cerveau des Dermaptères.

Dans ses derniers mémoires, (1916, $\left.9^{1} 7,1919\right)$, Miss THомpson décrit le cerveau et les organes reproducteurs des formes sexuées, comme étant nettement plus grands 
que ceux des ouvrières sortant juste de l'œuf. Ces intéressants résultats sont résumés daus un mémoire qu'elle a publié avec Siynlin (1919).

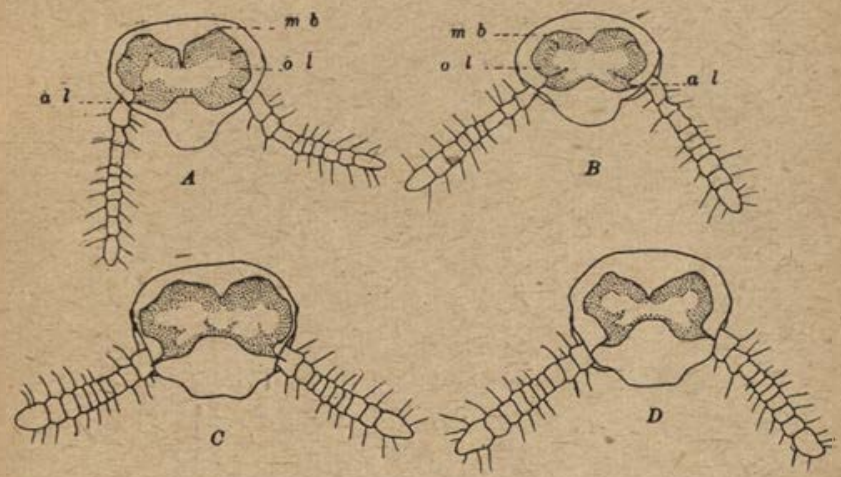

$f$
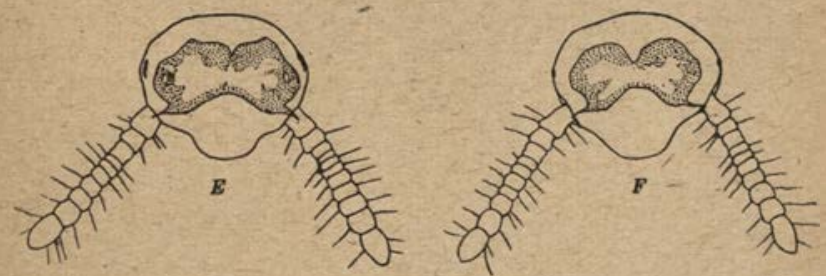

Fig. 27. - Coupes de tètes de jeunes termites, montrant la taille relative du cerveau et dés yeux. - A, C, E, formes sexuées venant d'éclore. - $B, D, F$, ouvrières et soldats venant d'éclore; $m, n$, corps fongueux du cerveau; ol, lobes optiques; al, lobes antennaires (d'après Caroline B. Thonrson).

Les mêmes auteurs concluent que les individus sexués adulles de la première forme (royale), possèdent en réalité un cerveau plus développé que les autres castes, que 
le cerveau des seconde et troisième formes est plus grand que celui des ouvrières, et que le cerveau des soldats est plus petit.

Evidemment, la découverte d'une différenciation intrinsèque des casies fertiles et stériles dans l'œuf même, est d'un intérèt tout particulier. Bugniox ( 9 I2, Ig 3 b, 1914) a décrit et figuré les soldats de Eutermes lacustris comme ayant le muscalı allongé (nasuli) dès la sortie de l'suf ; mais hiowen ( 189 ) a affirmé que les soldats de E. pilifrons ne présentent pas celte forme au moment de l'éclosion. En éludiant de nouveau cette question, Miss THompson (rgrg) est arrivée à la conclusion que Bugnion s'est absolument mépris et a confondu le labre en saillie de l'E. lacustris naissant, avec la corne céphalique des formes " nasuti ". Ses observations, portant sur les nym: phes juste écloses de E. pilifrons, morio et sanchezi, confirment celles de KNower, et, dans son mémoire de 1919 , clle a révélé l'existence, chez treize espèces appartenant i neuf genres de termites, de cette mème différenciation, observée par elle précédemment chez les nymphes naissantes, différenciation en deux formes correspondant aux deux séries de castes d'adultes fertiles et d'adultes stiriles. Mais, plus récemment, ses résultats ont été sérieusement mis en doule par Jucci $\left(1_{92} 3\right)$ et Hearu (1924). Pour le premier de ces auteurs, une étude soigneuse des formes sexuées néoténiques de Reticulitermes lucifugus l'amène à soutenir l'opinion de Grassi, suivant laquelle, les termiles, au moment de l'éclosion, sont lous identiques, el acquièrent, grâce à leur alimentation, les caractires distinctifs des castes. L'exposé de Jucci est très positif, pour ne pas dire dogmatique, mais la partie de son travail actuellement publice et qui contient des mesures 
faites sur les cerveaux des castes d'adultes ailés et néotoniques, ne parle pas des soldats et des ouvrières, ni des nymphes durant les premiers stades; il déçoit donc et n'apporte rien au problème que nous discutons ici. Quant aux recherches de Heati, dönt les' résultats ont été présentés au Congrès de Washington de l'Association américaine pour l'Avancement des Sciences (1923), elles n'ont pas encore été publiées Elles paraissent abso. lument en contradiction avec les résultats de Miss Tirompson, si nous en jugeons d'après une analyse sommaire parue dans l' "Anatomical Record " (1924, p. I15).

$$
\text { ** }
$$

En terminant, je désire ajouter quelques remarques sur les différents types de soldats qui présentent un intérêt extraordinaire, chez les termites comme chez les fourmis. Bien qu'apparaissant indépendamment dans les deux groupes, its ont entre eux des ressemblances accentuées, et constituent, dans ces deux groupes, une caste défensive ou protectrice, au moins primitivement. Tandis que celte caste est présente de façon constante chez tous les termites, sauf dans le genre Anoplotermes oì elle disparaît secondairement, chez les fourmis, par contre, elle ne se développe de façon définitive que dans des genres beaucoup moins nombreux. Et, tandis que chez les termites, comme on l'a vu pour les genres Mastotermes, Archotermopsis, Termopsis et Calotermes, la caste des soldats précède dans la phylogénie l'apparition des ouvrières, chez. les fourmis cette caste s'est certainement différenciée à partir de la caste ouvrière plus ancienne. Chez les fourmis, il n'y a qu'un type général de soldats, les " mandibulés ", qui est aussi développé de 
façon très générale chez les termites, mais chez certains Termitidés supérieurs (Eulermes), il est remplacé par un second type, dit "nasutus ". Il y a, en plus, une autre différence entre les fourmis el les lermites; chez les premières, les soldats diffèrent moins des reines et ouvrières de même espèce, dans la taille et la conformation de la léte el des mandibules, que les soldats des termites ne diffèrent des ouvrières et des formes sexuées de la même espèce.

Les méthodes de défense peuvent différer beaucoup suivant les espèces, et, par suite, nous trouvons, aussi bien chez les fourmis que chez les termites, des soldats adaptés à des offices spéciaux, à des "professions " par. ticulières. Dans l'une des formes, qu'on rencontre chez les deux groupes, le soldat peut se servir de sa tête pour clore el obturer presque entièrement la galerie d'enIrée du nid, ceci pour l'inlerdire aux intrus. Mais, dans le cas le plus fréquent, le soldat possède un crâne énorme, des mâchoires puissantes, dont il use pour attaquer les envahisseurs. Grâce au développement excessif de leurs mandibules et des muscles qui les actionnent, développement qui entraîne la forme particulière, la grande taille et la dureté du crâne, les soldats sont employés, chez certaines espèces de fourmis, à broyer les graines ou les parties les plus résistantes des insectes qui servent d'aliment, et qui, sans celte aide, ne pourraient être utilisées par la caste la plus faible des ouvrières (Pheidole, Pheidologeton, etc.). Chez certains termites et fourmis, les soldats paraissent aussi diriger en quelque sorte l'office d'approvisionnement (I).

(1) Hivgstov (1922) à décrit d'une façon très vivante un cas de cetle espèce chez Pheidole indica. Je cite une partie de son 
Chez beaucoup de soldats mandibulés, parmi les termites, et chez lous ceux du type " nasuti ", il existe une grosse glande fronlale qui produit un liquide glıant, ou latex, qui peut être projeté contre les ennemis, particulièrement contre les fourmis en maraude, et les paralyse. Chez certaines espèces, par exemple chez Rhinotermes taurus, suivant Holmgren (igog) el chez Copto-

récit: "Dès qu'une ouvrière découvre une chenille ou tout autre materiel pouvant servir comme aliment, elle commence par examiner soigneusement sa proie. Elle tourne autour de la chenille, l'explorant avec ses antennes sensibles, la secouant avec ses måchoires et essayant de la trainer jusqu'au nid. S'étant ensuite convaincue que sa découverte est bonne à être mise en réserve et jugeant que le transport de sa proie excède ses forces, elle se hâte vers le nid, très agitée, et par le plus court chemin. Qu'elle rencontre une autre ouvrière sur son chemin; les deux unissent leurs antennes, et la seconde, saisie du mème enthousiasme que la première, ayant appris la découverte, se håte aussi vers la proie. Une troisième, une quatrième ouvrière, plus si c'est possible, sont ainsi renseignées sur la route à prendre, et accourent de loin pour porter secours. Mais celle qui a fait la découverte se hảte vers le nid, très excitée. La voilà à l'entrée. Elle y pénètre et on la perd de vue. Quelques secondes après, un essaim de fourmis, s'excitant, se bousculant, surgit en hâte et pêle-mêle, du nid. Elles étaient là dans la galerie, juste auprès de la sortie, et toutes prêtes, et elles émergent toules en même temps en un seul corps, comme si elles attendaient qu'on les appelât à l'aide. Mais je suis certain, que, chez ces fourmis, le travail est divisé entre elles, certaines ouvrières ayant comme attribution spéciale la recherche de la nourriture; d'autres sous la conduile des soldats, doivent être toujours à l'entrée du nid, prêtes à courir au dehors et a porter secours lorsqu'elles apprennent qu'une trouvaille a été faite. Que la nouvelle arrive, l'essaim se précipite au dehors, en une foule serrée, précédée par les soldats. Sans la moindre hésitation, elles se hâtent sur le sol; se montant les unes sur les autres dans leur hâte folle. De tous cotés elles se pressent autour de la chenille, qui tenle vainement, par de violentes contractions, de rejeter au loin ses ennemis. La bataille 
termes ceylonicus, suivant Bugsior et Poporf (rgio) et Bugnion (1914, I923), cette glande est tellement déreloppée qu'elle s'étend en arrière dans l'abdomen et orcupe une grande partie de la cavité abdominale. D'autres conformations curieuses de la tête, chez les termites soldats, sans aucune ressemblance avec les adultes et les ouvrières, s'ohservent aussi chez les lypes mandibulés; ainsi, chez les Capritermes, les mâchoires sont longues, étroites,

se poursuit, toujours plus ardente et furieuse. En luttant, la chenille parvient parfois à dominer, mais les fourmis, surgissant en nombre toujour's croissant, la maitrisent peu à peu. De temps en temps, des ouvrières se retirent de la bataille et la quittent pour courir au nid avec la plus grande célérité, afin d'appeler de nouveaux renforts qui, entraînés par elles, se rendent au combat. La chenille faiblit: elle ne peut faire face i la puissance sans cesse croissante de son adversairc. EHe est accablée sous le nombre de ses ennemis, bientôt elle est épuisée, et gît à la merci des fourmis, qui, se cramponnant en un seul bloc autour de leur victime impuissante, la trainent lentement vers le nid. )

Hingston décril aussi les coutumes particulieres des soldats au moment ou se fait la migration vers un nouveau nid : "Ce sont les ouvrieres, plus petites, qui sont chargées du plus lourd travail; ce sont elles seules qui transportent les larves et qui conduisent souvent leurs compagnons d'un nid a l'autre. Les soldats ne transportent rien. Ils ne sont point d'humbles Iravailleurs; mais ils dirigent simplement le transport. Ce sont les aristocrates dans la socièté des fourmis. Ils sortent en hàle du nid, isolément, et à intervalles, avec une troupe de fourmis chargées marchant à leur suite, et chacun de ces puissants soldals, s'empressant le long de la troupe en marche re:semble à un officier conduisant et dirigeant sa compagnic d'hommes. Les soldats ne retournent jamais au vieux nid. Les petiles ouvrières, après avoir déposé les larves qu'elles portent dans le nouveau nid, reviennent en hâte chercher un second fardeau, mais jamais on ne voit un soldat revenir ainsi. Ils ont certainement d'importants devoirs à remplir dans le nouveau nid, mais ils ne s'occupent pas davantage de la troupe en voyage ». 
tordues et forlement asymétriques, et permettent à leurs possesseurs de tondir en l'air (I).

Très curieux est aussi le type du soldat, fourmi ou termite, dont la tête est modifiée de façon à pouvoir s'adapler à l'entrée dı nid et à en rendre l'accès plus difficile ou impossible. Le seul exemple de moi connu parmi les lermites est offert par le genre Cryptotermes, quoique les soldals du genre voisin Calotermes puissent se servir de leur têle de la même façon. En général, cette modification ne s'observe jamais dans les nymphes des Cryptotermes qui jouent le rôle d'ouvrières, ni dans les formes sexuées, dont les têtes ont toujours la conformation très simple ordinaire. Chez les fourmis possédant ce type de soldats, la tête de la reine est conformée presque de même mais moins parfailement adaptée. Les exemples les plus connus, parmi ces insectes, sont les Camponoti du sous-genre Colobopsis, dont plusieurs se rencontrent en Eurasie et dans l'Amérique.

Leur lête est courte, cylindrique et tronquée nettement à la partie antérieure; la surface tronquée, comprenant les mandibulcs, est circulaire, indurée, et plus grossièrement sculptée que le reste du coms, si bien qu'elle s'adapte très aisément dans l'entrée circulaire du nid qui est creusé dans du bois dur, dans des galles ligneuses ou dans des liges de roseaux. Quand un Colobopsis ouvrière désire quitter te nid, elle frôle de ses

(1) "Les Capritermes (soldats) ont des mandibules d'une forme très particulière, tordues à !a manière des cornes de bouc, adaptées à la fonction du saut. Prenant appui sur le sol, agissant comme un ressort, ces organes servent au termite d̀ projeter son corps en l'air. L'insecte qui retombe à 20 ou 30 centimètres de distance parvient, en sautant de côté et d'autre, à échapper à ses ennemis. Je ne puis dire toutefois (mes observations ètant encore incomplètes) si la singulière catapulto dont usent les Capritermes est spécialement destinće à les garer des fourmis. " (Bugnion, 1923.) 
antennes l'abdomen du soldat; la porte vivante se retire et, dès que l'ouvrière a franchi l'ouverture, elle reprend sa première position. Au retour, l'ouvrière heurte de ses antennes la surface tronquée, tournée vers l'extérieur, de la tête du por-

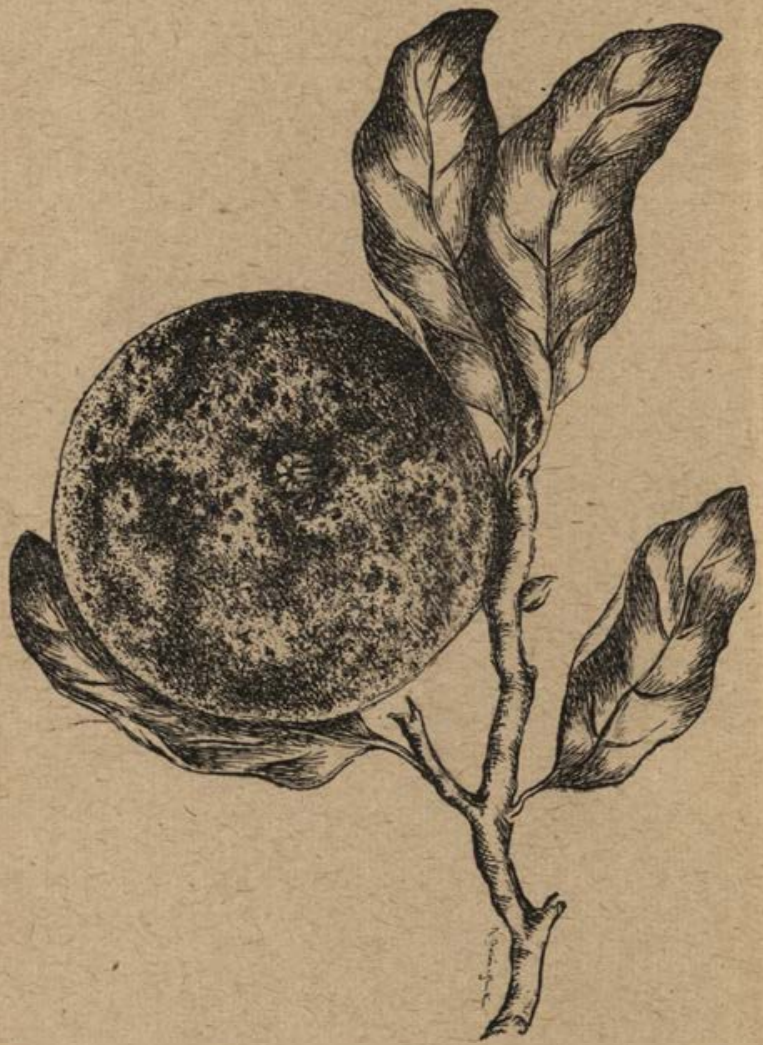

Fig. 28. - Galle d'Holcaspis cinerosus (sur chêne vert) occupée par une fourmilière de Colobopsis etiolata, dont un soldat ferme l'entréc avec sa tèle (phragmose). 
lier; l'effet produit est le même et l'ouvrière peut rentrer. J'ai Irouvé ce même type de tête dans les seules espèces exotiqués de trois autres genres éloignés, Pheidole ( $\mathrm{Ph}$. colobopsis (li Brésil), Cremalogaster (Colobocrema subg. nov. des Philippines, et Colobostruma (gen. nov.) de l'Australie, qui ont très probablement les mèmes habitudes. Des modifications analogues de la léic s'observent aussi dans les reines et les ouvieres les plus grandes de quelques sous-genres lignicoles de Camponotus (I'aracolobopsis, Pseudocolobopsis, Manniella, Neomyrmamblys). Dans beaucoup d'espèces de Cryptocerus, qui vivent aussi dans du bois dur, les têles des soldats sont larges, et en forme d'écu; elles servent aussi à fermer l'entrée du nid.

Des adaptations très voisines, permettant l'occlusion de l'entrée des terriers, etc., existent non seulement chez un grand nombre d'autres Arthropodes, mais même chez les animaux appartenant à d'autres embranchements. Dans certains cas ćest la tête, dans d'autres l'extrémité postérieure du corps qui subit la modification et qui s'adapte; dans l'un et l'autre cas, il y a une étrange similitude dans les caractères acquis : surface circulaire tronquée à tégument durci. Parfois, comme dans les larves de Cicindèles et chez les abeilles à terrier du genre Halictus, la tête cntière est presque circulaire et a l'air d'un tampon; dans d'autres formes, comme les Scolytidés (Ipidés, Plalypodidés) el les chenilles d'un phalène de l'Amérique du Nord, Cicinnus melsheimeri, et dans une espèce voisine de l'Amérique du Sud, Perophora sanguimolenta, qui habite de grands fourreaux tubulaires, faits avec des feuilles, l'extrémité postérieure du corps est tronquée nelfement et a une surface rugueuse ou épineuse (I).

(1) Voir les figures et les descriptions de Cicinnus données par Harris (1862, p. 416, fig. 206) el de Perophora données par SH.IRP (1899, p. 379 , fig. 188). 
Chez certaines Annélides tubicoles (Maldanidés, Amphictenidés), et chez un singulier crapaud de l'Inde orcidentale (Bufo empusus) qui vit dans les terriers, la tête a la forme d'un tampon ( 1 ).
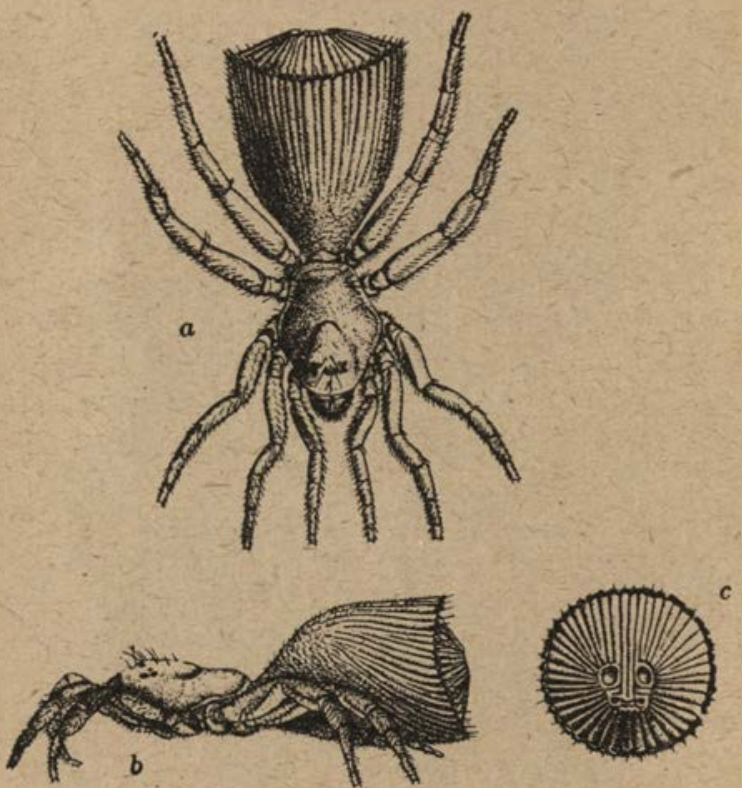

Fig. 29. - Chorizops loricutus, a. vue dorsalement; b. latéralement; $e$. extrémité postérieure (d'iurés PeTRLNiEwitch).

Je reproduis ici la figure que donne Petrinkewitcu d'une araignée spéciale, une Théraphosidée (Chorisops

(1) Voir Barboun 1914, p. 242, $243 ; 1919$, p. 100, pl. J, (fig. 3). 
loriculus), qui, au lieu de construire une porle ì piège comme les espèces voisines, ferme son nid avec l'extrémité postérieure, modifiée, de son corps. Le $\mathrm{D}^{\mathrm{r}}$ Thomas Barbour m'écrit que " certains serpents des Indes ont, au lieu de la forme normale, l'extrémité de la queue finissant en un bouclier ovale, effilé, en biseau, et couvert de tubercules. Ces sexpents creusent aussi des terriers et les ferment avec leur queue qui fait un superbe opercule $)$.

Puisqu'il n'existe pas de terme général englobant toutes ces modifications particulières, sporadiques et convergentes, grâce auxquelles les extrémités du corps sont employés pour clore des cavités tubulaires, je propose le mot "phragmose" (de soxy"u. défense ou barricade). Si l'on considère du point de vue de l'évolution et des moours ce phénomène qui représenfe l'une des méthodes de protection et de défense les plus frappantes et les plus efficaces, on pensera qu'il mérite un examen plus précis que celui dont il a été l'objet. Au lieu de secréter ou de construire une fermeture, comme l'opercule ou épiphragme des escargots et les portes ou barricades en terre ou en soie édifiées à l'entrée de leurs nids par les fourmis, les guêpes et les araignées à trappes, l'insecte phragmotique emploie rée:lement dans ce but une partie spécialisée de son propre corps, fournissant ainsi une preuve de l'impossibilité qu'il y a de tracer une ligne de démarcation rigoureuse et solide entre les mœurs d'une part et les processus morphogéniques et physiologiques d'autre part. Le développement phylogénétique de la phragmose est obscur. Les fourmis et les termites, du moins, paraissent montrer que ce phénomène ne provient pas d'une mutation, ou variation hrusque, sans transi- 
tions, mais'qu'il a dû se développer graduellement, puisque nous trouvons, chez beauroup de Camponoti et d'espèces de Calotermes lignicoles, des approximations variées, à rapprocher des conditions perfectionnées observées chez les Colobopsis et les Cryptotermes.

J'ai insisté sur les cas sporadiques de phragmose, parce qu'ils montrent très clairement le caractère adaptatif de l'un au moins des types de soldats existant chez les fourmis et les termites, laissant supposer que, très probablement, cette caste n'est pas née d'une mutation, d'un caprice. La signification de ces faits sera établie dans le chapitre suivant. 


\section{CHAPITRE VII}

\section{LE POLYMORPHISME (Suite).}

Prédétermination ou épigenèse ? - Role de l'alimentation: guêpes, bourdons, abeilles, fourmis. - Action des parasites: mermithergates, röle des Lomechusinx. - Parasitisme de fourmis sur d'autres fourmis. - Principe d'EMERY et de Vienmeyer. - Le gradient axial de Cuild. - Discussion des hypothèses trophogénique (EMERY) el blastogénique (WeISMaNi).

Si, après avoir examiné les faits de polymorphisme tels que les établissent l'observation et l'expérience, nous passons à leur interprétation, nous nous trouvons aussitôt plongés dans les problèmes physiologiques de l'ontogénie et de la croissance, de la phylogénie ou évolution, et des mœurs ou activités de l'organisme, en fait dans tous les problèmes biologiques véritablement fondamentaux. Aussi n'y a-t-il aucune raison a priori pour supposer qu'il faille chercher une explication spéciale, neuve et extraordinaire pour le développement des castes chez les insectes ; car, même chez les Aculéates sociaux, les activités physiologiques et instinctives l'emportent de beaucoup sur l'activité intelligente, celle-ci, par contre, jouant un rôle si considérable et si particulier dans l'interprétation des sociétés humaines et offrant un vaste champ à la multiplicité des opinions diverses qui partagent les so- 
ciologues et les philosophes. Dans mon ménoire sur le polymorphisme, publié en rgo-7, j'ai essayé de traiter ce sujet en me plaçant à des points de vue nombreux ; mais ici il me paraît préférable de réduire le problème à un choix à faire entre la prédétermination et l'épigenèse. Ce problème, comme vous savez, a longtemps divisé les biologistes en deux camps comparables à ceux qui divisèrent les philosophes en platoniciens ou aristotéliciens, idéalistes ou réalistes, nativistes ou empiristes, elc. Le morphologiste, qui est surtout un observateur, incline de préférence, naturellement, vers l'idée de la prédétermination; le physiologiste, surtout expérimentateur, vers lidée de l'épigenèse. Chacun a tendance à donner des faits une interprétation unilatérale, et tous deux ont raison, nous pouvons en convenir maintenant; il $y$ a, en effet, prédéterminisme, et épigenèse aussi, dans le développement organique; mais il y a encore des occasions nombreuses de voir naître des opinions divergentes, en s'en tenant à ces deux facteurs, au sujet de chaque cas particulier. En fait, les généticistes et les physiologistes actuels sont presque aussi profondément séparés que le furent les anciens préformationistes ou évolutionnistes et les épigénétistes.

\section{$*^{* *}$}

Il est difficile de fixer dans quelle mesure la différenciation des deux ou trois castes femelles des Aculéates sociaux, et des cinq castes (ou plus) des termites, est intrinsèque, ou prédéterminée dans l'œuf, ou dans quelle mesure elle peut dépendre des conditions extérieures; celte difficulté provient du fait que les castes se dévelop- 
pent seulement dans un ensemble de conditions extérieu. re: Irès particulières, standardisées, pour employer le mot de Cuncd (1924), et qui ne peuvent être aisément soumises au contròle expérimental. Elles n'apparaissent que dans les sociétés vivantes, dont les activités sont si multiformes, si interdépendantes el si déticatement équilibrées, que les divers facteurs n'en peuvent être isolés que mentalement, en théorie. Il est même très difficile de conserver des colonies dans des çonditions artificielles pendant de longues péricdes, et la petite taille des insectes présente des obstacles presque insurmontables à toute enquète physiologique s'adressant, avec les méthodes dont nous disposons actuellement, à la plupart de ces modes d'aclivités. Bien plus, loutes leurs habitudes familières les plus significatives ne se manifestent que dans la complète obscurité, et la scule intervention de la quantité de lımière nécessaire à l'observation de ces mours subtiles est déjà un grand facteur de trouble. Néanmoins, dans notre étude des causes de la différenciation des castes, nous devons exclure dès le début les facteurs extrinsèques, tels que température, humidité, obscurité, non qu'ils soient inopérants, mais parce qu'ils agissent uniformément sur toutes les casles, aux divers stades de l'ontogénie. Il est, de plus, évident, que les facteurs extrinsèques efficients doivent avoir leur origine dans la caste ouvrière adulte; en effet, chez tous les insectes sociaux, une fois fondée la colonie, cette caste, toujours et de beaucoup la plus riche en individus, est, en outre, la seule à intervenir dans l'acquisition et la répartition de la nourriture et dans l'éducation de toutes les castes, y compris elle-même naturellement. Ainsi donc, si les facteurs de différenciation ne sont pas blastogéniques, ou 
présents dans l'œuf, ils doivent être trophogéniques, ou provenant de différences dans le mode d'alimentation pratiqué par les ouvrières, qui constituent le milieu social. Mais, même avec ces restrictions, le problème du polymorphisme conserve encore une complexité exaspérante, puisqu'on en peut donner au moins six interprétations différentes que l'on peut formuler ainsi :

$1^{\circ}$ Les œufs sont tous identiques; les castes sexuées, ouvrière et soldat sont produites, différenciées, de par le milieu social, et grâce à l'alimentation des larves. (Grassi, МarchaL, et tous les mélittologistes).

$2^{\circ}$ Les diverses castes sont prédéterminées dans des œufs différents, mais la prédétermination est si faible, ou si incomplètement établie, que la nourriture des larves peut l'intervertir ou l'abolir, les résultats étant essentiellement identiques à ceux de $1^{\circ}$, (Bouvier).

$3^{\circ}$ Les castes sont prédéterminées, comme telles, dans les œufs; c'est-à-dire que chaque caste provient d'une sorte particulière d'œufs, et l'influence trophique du milieu social a des effets non morphogéniques, mais tout au plus quantitatifs, (mégétiques) en déterminant la taille. (Bugnion, Thомpson, Imms, etc.).

$4^{\circ}$ Les castes sont prédèterminées dans une certaine mesure ; les œufs, quoique lous identiques, possèdent chacun, ou:

a) une collection de déterminants, ides ou gènes, qui peuvent être aclivés par une espèce particulière de nourriture et qui produisent une caste spécifique (Weismans);

b) une sensibilité différentielle, une potentialité qui réagira spécifiquement à une sorte particulière de nourriture (EMERY).

$5^{\circ}$ Les castes seraient blastogéniques $\left(3^{\circ}, 4^{\circ}\right)$ dans certains groupes d'insectes sociaux et trophogéniques $\left(1^{\circ}, 2^{\circ}\right)$ dans d'autres.

$6^{\circ}$ Dans une même espèce, certaines des castes seraient blastogéniques, les autres étant trophogéniques.

Je vous ferai remarquer que certaines des distinctions entre ces interprétations possibles sont assez subtiles et obscures, spécialement en ce qui concerne la nature du 
déterminisme blastogénique. Comme ces causes déterminantes doivent relever de la biochimie ou de la physiologie, et qu'elles sont encore inconnues, je n'abuserai pas de votre temps en discutant les hypothèses essentiellement morphologiques que les généticistes ont mises en avant pour expliquer ces phénomènes, hypothèses qui, à mon avis, sont par essence des répliques des erreurs de Wersmaxs, et tout au plus de simples transpositions du problème dans un langage pseudomathématique. Tout ce que nous savons avec certitude, c'est que l'œuf de chaque espèce d'organisme a une constitution colloïdale spécifique, et donne naissance à un organisme qui est déterminé en partie par cette constitution, en partie par l'action du milicu dans lequel il est maintenu durant sa vie et dans lequel il se développe. Et, bien que les oufs de mêmes parents soient sans aucun doute différents de constitution, il est aussi certain que la nature précise de ces différences n'a, jusqu'à présent, pas été découverte par nos généticistes, dont les tendances sont trop statiques et morphologiques. Tournons-nous donc vers les facteurs extrinsèques, vers les divers modes d'alimentation des larves, qui, bien qu'extrêmement variés, permettent néan. moins, jusqu'à un certain degré, l'observation directe et l'expérience.

\section{**}

L'alimentation des larves peut varier soit quantitativement, soit qualitativement, cela est évident; on admet généralement que la première sorte de variations influe uniquement sur la croissance en grandeur, en taille, tandis que la seconde peut déterminer des différences mor- 
phologiques. Si nous considérons la nutrition au sens physiologique le plus large, nous distinguerons dans les aliments ceux qui sont utilisés seulement dans le métabolisme de la croissance, de l'équilibre et du remplacement, et ceux qui ont un effet stimulant, excitant, sur les processus métaboliques. Ces derniers seraient nettement morphogéniques, même à doses extrêmement faibles, comme les vitamines et les hormones. D'autre part, des aliments qualitativement différents peuvent n'avoir pas d'effets appréciables sur la différenciation des formes, tandis que, chez les plantes, des différences morphologiques très marquées peuvent résulter de différences quantitatives dans la nourrilure : ainsi, par exemple, des formes diverses de feuilles portées par des rameaux plus ou moins vigoureux. Bien qu'il soit difficile de découvrir des exemples aussi peu équivoques chez les animaux, il en est probablement de même.

Il faut noter, en outre, que ce n'est pas la nourriture administrée qui est cffective, mais celle qui est réellement assimilée et l'expérience nous a montré que cette dernière dépend de l'état physiologique et de l'appétit de l'organisme.

Tous les entomologistes savent que des dillérence- dans lit quantité de nourriture consommé pitr la larve aflectent la taille de l'imago. Weismaxi (1892) a élevé des larves de la mouche à viande avec des quantités différentes de nourriture, et a obtenu des individus tris petits, mais féconds. Il est douteux pourtant que ces individus aient fourni aulant d'œufs que des individus abondamment nourris. Des experienres similaires ont été poursuivies par Herms (1907) el Wurrivi, (1914) sur les mouches ef par Nelsox el Stinteint (19?) sur libeille. Poroviri-Bazostav $(1910 a, 19106)$ a réllssi i élever des alıeilles du genre Osmia ayant environ $1 / 6$ de la tiille normile, en leur fournissant seulement de petites portions du " pain ", que les meres avaient 
mis en reserve auprès des œufs. Ces exemples montrent bien que, dune fagon tres générale, des larves d'insectes soumises au jeùne produisent des adultes nuins, mais normaux à tous autres points de vue. Une résistance bien plus remarquable à linanition a élé constalće dans les expériences de WonsednIFK (1917) sur Trogoderma tarsale, un fléau très répandu dans les muscés. Il a réussi à conscrver vivantes les larves de cet insecte pendant cinq ans et deux mois environ sans nourriture. Pendant ce temps, les individus qui avaient atteint leur pleine croissance $(8 \mathrm{~mm}$. de long), devinrent de plus en plus petits, et finirent par reprendre la taille qu'ils avaient au moment de léclosion (1 mm.) ce qui représente $1600^{\circ}$ de leur laille du début. En nourrissant ces larves, elles recommencèrent à croitre.

Quoiqu'il n'y ait rien d'analogue, au point de vue résistance à l'inanition, chez les larves des Aculéates sociaux, on a observé des cas très fréquents où la nymphe se formait précocement, la quantité de nourriture absorbée étant alors très faible. Ainsi, chez certaines fourmis des genres Dorylus et Carebara, les nymphes des petites ouvrières sont quelque mille fois plus petites que celles des reines. Quelle que soit la cause déterminant ces différences, il est certain que la quantité de nourriture administrée à la larve peut, à elle seule, être un facteur essentiel dans la croissance de cette larve.

On sait parfaitement que les Vespidés sociaux nourrissent leurs larves avec des boulettes formées d'insectes malaxés el parfois humectés avec de la salive ou autre sécrétion buccale. On n'est pas certain que le miel soit consommé par les larves, même chez les guêpes qui cn cmmagasinent habituellement dans leurs rayons. Mais les portions molles d'insectes malaxés peuvent être de très inégale valeur au point de vue alimentaire. Les différences entre ouvrières et reines sont si faibles, et les formes intermédiaires si nombreuses, que des différences 
quantitalives dans l'alimentation pourraient expliquer lá production des unes et des autres. La stérilité coutumière des ouvrières paraìt dûe à une accéléralion dans le développement des tissus somatiques, à un retard dans la formation des tissus reproducteurs, ce qui réalise une condition inverse de la néoténie, dûe probablement à la formation précoce de la nymphe et par suite à la nature de la prothélélie. Les ovaires des ouvrières sont inhibés, non avortés ; ils peurent produire des œufs viables, si l'insecte adulte est en état de se procurer une nourriture suffisante, et ne subit, par la castration nutricielle, par suite d'un défaut excessif de nourriture. Sans aucun doute, les reines guêpes proviennent des larves les plus abondamment nourries. Ainsi donc, chez les Vespidés sociaux, il ne paraît pas nécessaire de faire apppel à des différences qualitatives dans la nourriture des larves, pour expliquer le faible dimorphisme du sexe féminin.

Chez les bourdons, malgré de grandes différences dans la qualité de la nourriture, le développement des castes ressemble tellement à ce qu'il est chez les Vespidés, que nous sommes naturellement tentés de lui attribuer des causes identiques, d'ordre simplement quantitatif. Chez l'abeille domestique et les abeilles sans aiguillon, par contre, la question se complique quelque peu. La plupart des auteurs semblent admettre que les différences somatiques entre la reine et l'ouvrière, chez la mouche à miel, sont déterminées directement par des différences qualitatives dans l'alimentation de la larve; mais, si l'on compare avec les Méliponinés, on soupsonne que les différences d'alimentation agissent, en premier lieu, sur la fécondité des deux castes, et que les différences somatiques dépendent du développement des tissus ovariens ou lui sont 
liées. Silvestri (rgoz) et H. von Ihering (rgo3) ont monIré qu'il existe deux manières d'être différentes chez les Méliponinés. Dans le genre Meliponn, les cellules dans lesquelles se développent les mâles, les reines et les ouvrières sont toutes identiques, et de même taille. Elles sont approvisionnées avec la même_espèce d'aliment, pollen et miel, et dans chacune un œuf est déposé. Elles sont ensuite closes; et, bien que la larve soit nourrie, non point jour après jour comme chez l'abeille ordinaire, mais, comme chez les abeilles solitaires, grâce à des provisions emmagasinées, ce traitement uniforme entraîne néanmoins la production de deux castes femelles netlement dilférenciées. Mais, au moment de l'éclosion, la reine a des ovaires très petits avec des œufs non encore mûrs. Dans les espèces du genre voisin Trigona, au contraire, qui diffèrent des Méliponinés en ce qu'elles construisent pour les reines de grandes cellules qu'elles munissent d'une provision de miel et de pollen plus grande que celle des cellules à ouvrières, la reine naît avec des ovaires pleins d'œufs mûrs. Ces faits sembleraient indiquer que la grande dimension des cellules de reines et leurs provisions plus abondantes sont de simples adaptations destinées à accélérer le développement des ovaires. Pour l'abeille domestique, nous pouvons peut-être adopter une explication similaire du mode d'alimentation de la larve de la reine avec une substance spéciale, la "gelée royale 1 . On sait depuis longtemps que les larves de reines et d'ouvrières, chez la mouche à miel, sont nourries de substances différentes, dès le deuxième ou troisième jour de leur vie post-embryonnaire. A partir de ce moment, la larve d'ouvrière reçoit pollen et miel, tandis que 
la larve de reine reçoit une pâtée spéciale, la " gelée royale $"(\mathrm{I})$.

La reine éclòt environ seize jour's apris que l'ouf a été pondu, tandis que l'ouvrière, plus petite pourtant et ayant des ovaires imparfaits, exige quatre à cinq jours de plus pour parfaire son développement. Cette interprétation du développement de la reine est certainement exacte, comme l'indique aussi le fait que cette reine peut pondre dans les dix jours qui suivent son éclosion. II semblerait donc que l'alimentation qualitative spéciale de la larve de reine intervient principalement dans la croissance des gonades et non dans la morphogenèse. La reine diffère des ouvrières par certains caractères de dégénérescence, tels que la tête et la langue plus courtes, les ailes plus réduites, les pattes postérieures simplifiées, l'absence de certaines glandes salivaires, etc. Ces déficiences résultent peut-être de l'hypertrophie ovarienne, si l'on en croit la loi d'Emery, que nous discuterons plus tard (2).

(1) ScHIEMENy (1883) croil que c'est une sécrélion salivaire des ouvrières nourrices; PLANTA (1888) et SCHÖNFELn la considèrent comme des aliments partiellement digérés et régurgités hors de l'estomac chylifique. Plus récemment, Metzcier (1910) et Zander (1911) ont montré que la structure du proventricule interdit toute régurgitation đu conlenu stomacal ; si bien que la gelée royale doit être regardée comme une sécrétion salivaire (voir aussi von Buttel-Reepen (1915), Nelson el Sturtevant (1924) el LineBUng (1924).

(2) Dans une intéressante discussion des effets de la salive sur la différenciation des castes chez les insectes sociaux. Jucci (1924, p. 221, en note) indique que Aeppler (1924) a trouvé de la cystine en quantités extraordinairement grandes parmi les constituants proléiques de la gelée royale, el que, "chez. les abeilles, suivant Bishor, l'absorption de cette salive peut exclure les facleurs qui inhibent le développement des organes re- 


\section{Les éleveurs d'abeilles ont expérimentalement démon-} tré le fait qu'un œuf ou une jeune larve, transportés d'une cellule ouvrière à une cellule de reine, où la larve reçoit comme aliment de la gelée royale, ou de la salive, produit une reine. Ces expériences ont été récemment répétées par Kuetr (rgo/) el von Butrel-Reepex (Igri).

Ce dernier cite les résultats de kices comme suit: "Si on prend dans une cellule d'ouvrière une larve ảgée d'une demi journce a un jour et demi, et quion la place dans une cellule de reine, cette larve donne une reine parfaite, la larve d'ouvrière ayant reçu jusqu'à ce moment la mème espèce de nourriture. Mais si l'on pratique la même expérience avec des larves àgées de deux jours et demi a trois jours et demi, on observe certaines petites différences, les reines obtenues élant légèrement plus petites, et leurs pattes, qui sont toujours plus claires de teinte chez les reines que chez les ouvrières, étant tachées de noir, surtout les tibias postérieurs, qui présentent, dans la dernière caste, une concavité, la corbula... Avec les larves qui ont vécu quatre jours et demi dans la cellule d'ouvrières, et qui sont ensuite transportées dans les cellules de reine, il y a une différence très nelte (c'est-à-dire que les

producteurs, facteurs consistant en un excès de certaines protéines ». Pézıro (1919), qui semble partager cette opinion, dit à propos de la castration alimentaire chez les poules exclusivement nourries de viande: "Le régime exclusif à la viande produit dans l'organisme une lente intoxication, à laquelle sont particulièrement sensibles les glandes reproductrices, et à la suite de laquelle, ces glandes s'atrophient graduellement ou ne se développent pas. "Jucci cite également quelques autres cas, dont on pourrait inférer que les ovaires des larves d'abeilles orvrières seraient inhibés par un régime trop riche en protéines (pollen), tandis que, pour la larve de reine, la présence de cystine dans la gelée royale neutralise cette action inhibibrice des protéines et assure le déve'oppement des ovaires. Il adopte une explication analogue pour le développement des termites néoténiques. Une telle explication ne convient point au cas des Méliponinés, sauf si nous sulposons qu'une quantité considérable de salive est mèlée au pollen el au miel emmagasinés dans les grandes cellules des reines. 
individus obtenus ressemblent plus aux ouvrières), ceci provenant de la nourriture des larves, qui, pour la reine, a une composition- différente à partir du second jour. "KLEIN a, en outre, transporté une larve âgée d'une demi-journée à un jour et demi, d'une cellule d'ouvrière à une cellule de reine, l'a laissée dans celte dernière pendant deux jours, puis l'a de nouveau retransportée dans la cellule primitive où elle a été nourrie comme une ouvrière fendant un jour à un jour et demi. L'adulte obtenu a les caractères de l'ouvrière bien prononcés, mais ses pattes postérieures portent des poils plus courts, et ont la forme, en une certaine mesure, de celles de la reine. On peut penser qu'en faisant varier ces expériences d'alimentation, il serait possible de produire artificiellement toute une série, à degrés très rapjrochés, de formes intermédiaires entre la reine et l'ouvrière typiques, comparable aux séries offerles normalement par les colonies de guêpes ou de bourdons.

$$
*_{* *}^{*}
$$

Chez les fourmis, on n'a pu jusqu'à présent démontrer l'existence d'une dépendance nette entre l'alimentation, et la différenciation des castes, parce que ces insectes, se nourrissant eux-mêmes avec une grande variété de substances, alimentent aussi de façon très variée toutes leurs larves, même les plus jeunes. Ces substances comprennent : des portions d'insectes les plus divers, des parties molles de graines, le nectar des plantes, les excreta sucrés des Aphides, Coccides, etc., et les hyphes de champignons. Certaines espèces sont strictement entomophages; d'autres s'attachent particulièrement aux Aphides et aux Coccides; d'autres sont exclusivement mycétophages, quelques-unes, très rares, comme Solenopsis geminata, peuvent être qualifiées d'omnivores. Et malgré cette extraordinaire variété dans le choix de l'aliment, et sauf quelques exceptions dues all parasitisme, la famille entière présente uniformément un dimor- 
phisme du sexe féminin, ou pléomonphisme. Ceci apparait clairement par les exemples suivants:

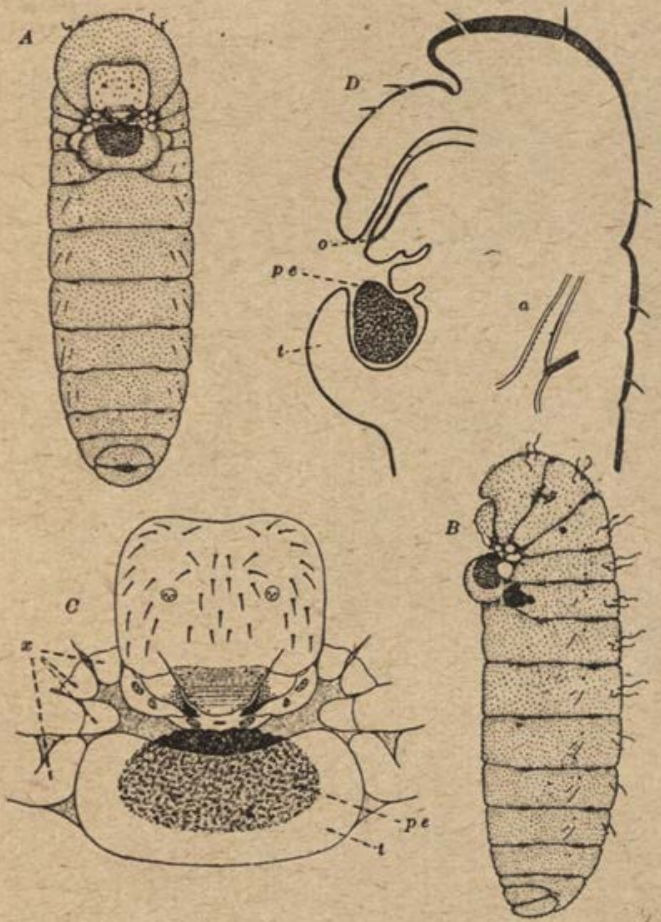

Fig. 30. - Larve de Pseudomyrma gracilis. A, vue ventralcment; $\mathrm{B}$, lintéralement; $\mathrm{C}$, tète et portions voisines grossies; $\mathrm{D}$, coupe sagittale de la région antérieure: o, houche; $x$, exsudatoire ; t, trophothylax, pe, pelote nourricière drposce par une ouvrière. 
I. -- Dans la sous-famille tropicale des Pseudomyrminés, comme je l'ai montré $(1918 a, 1920 a, 1922 a$, WHEeler et BAYLEY 1920,

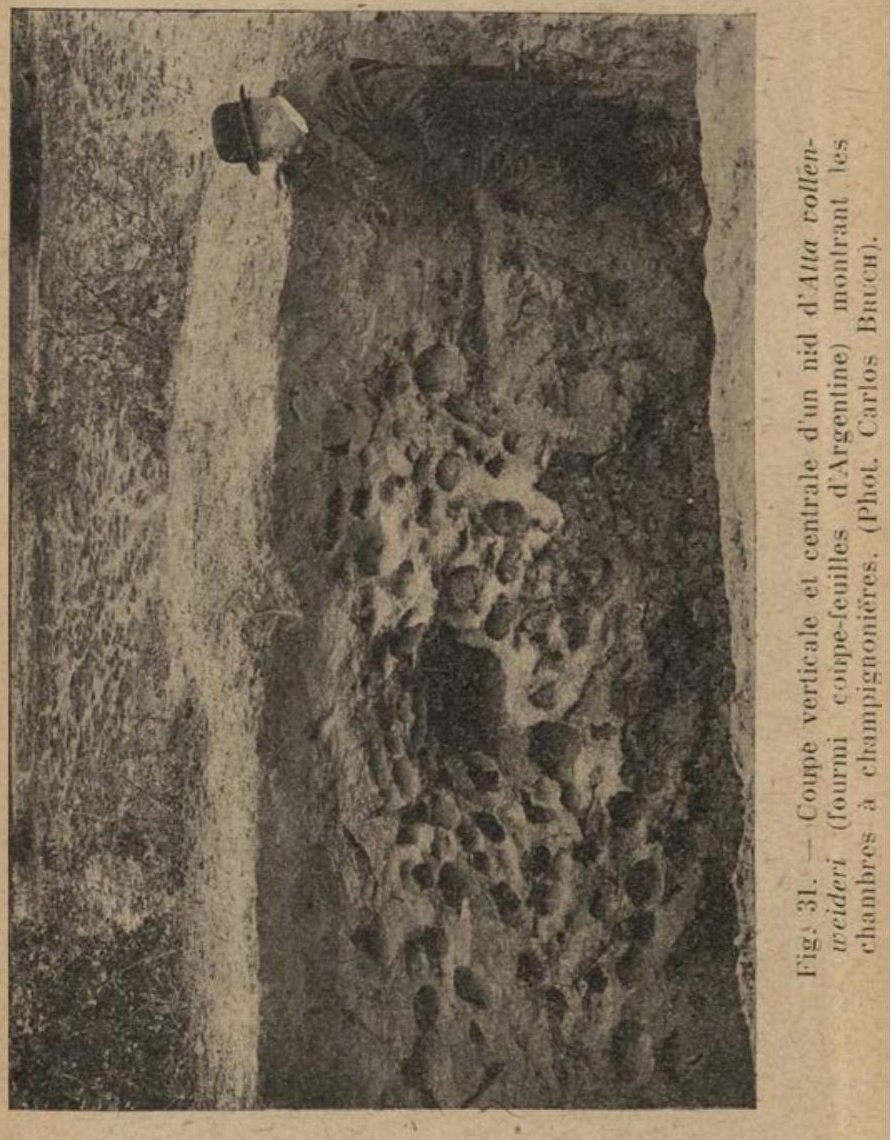

les larves ont comme particularilé de posséder, juste en arrière de la bouche, à la face ventrale du premier segment abdo- 
minal, une poche, te trophothylar, dans laquelle les ouvrières

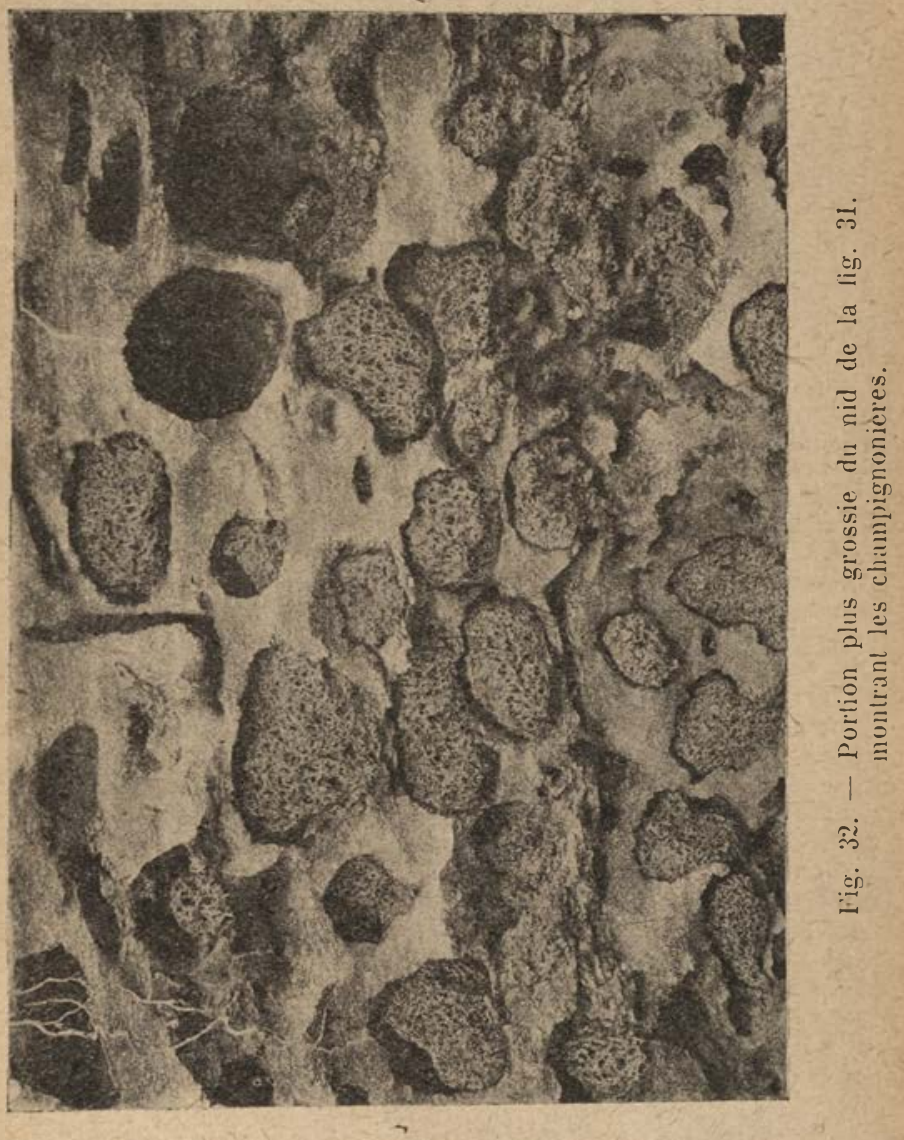

nourrices rleposent lit nourriture, qui consiste en une boulelte 
façonnée dans la propre poche infrabuccale des ouvrièrcs. Cette boulette est en réal:té du rebut; clle consiste en fragments triturés d'insectes, dont l'ouvriere a extrait tolls les sucs, plus des spores de champignons, des grains de pollen et autres particules de détritıs, que l'ouvrière a ratissées sur son corps, au moyen des peignes de ses pattes antérieures. Les fourmis des autres sous-iamilles rejettent cette boulette sans valeur, mais les-Pseudomyrminés sont si parcimonieuses qu'elles en nourrissent leur's jeunes; c'est absolument comme si nous en arrivions dans nos ménages à un lel degré d'économie que nous nourrissions nos enfants exclusivement avec ie contenu des boîtes à ordures et des seaux de toilette. Bien qu'une telle méthode d'alimentation semble exclure toute régulation qualitative du régime des larvès, il existe cependant, chez toutes les Pseudomyrminés, deux castes, ouvrières et reines, nettement différenciées, quoiqu'à peu près de même taille (1). Les toutes jeunes larves sont nourries de salive, jusqu'à ce que leurs mâchoires soient assez fermes pour triturer les boulettes; et peut-être certaines larves âgées reçoivent-elles aussi celte même substance, ce qui déterminerait leur transformation en reines; mais ceci n'est pas prouvé.

II. - Comme deuxième exemple, nous pouvons choisir les Attines, fourmis de l'Amérique tropicale; c'est une-tribu de Myrmicinés, qui utilise comme aliment, pour les larves comme pour les adultes, le mycélium de champignons spéciaux cultivés sur un substratum formé de lissus foliaires triturés ou d'excréments de chenilles, ou de Coléoptères. Dans le genre $A t t a$, le plus hautement spécialisé, les colonies sont très populeuses, les reines sont de très grande taille, et la caste ouvrièr présente une longue séric de formes intermédiaires, s'étageant depuis les soldats à tête énorme jusqu'aux formes très petites qui ne quiltent jamais le nid mais ont pour office de soigner les cultures de champignons. Chez Acromyrmex, les reines sont plus petites, et le pléomorphisme des ouvrières moins prononcé; et dans les genres inférieurs, Trachymyrmex, Cyphomyrmex, Sericomyrmex, Apterostigma, etc., les colonies sont très réduites, les ouvrières monomorphiques, et souvent presque aussi grosses que les reines.

Dans les derniers genres, suivant mes récentes observations, faites sur les colonies de Sericomyrmex de Panama, la reine

(1) Sauf chez Vilicicola tessmanni, qui, pour une raison encore inconnue, possède des formes intermédiaires entre la reine et l'ouvrière. (WhHELER, 1922 a.) 
éparpille les œufs parmi les champignons, et les larves naissintes dévorent le mycélium qui se trouve à portée de leur bouche, laquelle est parfaitement adaptée comme chez d'autres Allini (Weelen et Bailey 1920) à pratiquer des ponclions sur les hyphes et à aspirer leur contenu protoplasmique. Parfois les ouvrières transportent les larves sur des pâtures fraiches. mais jamais on ne les voit nourrir directement ces larves avec des champignons qu'elles auraient recueillis. Cependant, suivant TiNwer (1890), les Atta nourrissent leurs jeunes suivant cette dernière méthode. Mais, quelle que soit la méthode suivie, l'alimentation des larves chez les Attini est quantilative; cependant, on ne peut exclure absolument la possibilité d'une addition de salive au régime de certaines larves, ce qui assurerait le développement de ces dernières en reines.

L'alimentation des larves de fourmis avec des liquides régurgités a été observée par un grand nombre de myrmécologistes (Miss Fielde igoi, Newell i gog, Newell et Barber igr 3 , EMery i 9 i 6 , moi-même et d'autres), et dans cerlaines espèces (Dolichoderinés, Formicinés), c'est peut-être le seul mode d'alimentation employé. Chez heaucoup de Ponérinés, et certaines Myrmicinés (Aphoenogaster) et Formicinés (Lasius), les larves reçoivent des fragments bruts d'insectes; celles de Messor et de Pogonomyrmex, des fragments de graines. Mais les larves les plus jeunes sont toujours alimentées avec des aliments régurgités et les observations d'EMERY montrent que les larves plus âgées de reines et de soldats sont traitées de même. Il est difficile cependant de préciser la composition du liquide régurgité. Il peut consister en salive, ou en aliment liquéfié conicnu dans le jabot, ou en un mélange des deux. Il y a un cas pour lequel nous savons que les larves sont nourries durant leur vie larvaire avec de la salive pure, c'est celui de la première ponte de la reine qui fonde une colonie. On sait très bien que cette reine, qui ne peut absorber de nourriture pendant huit 
ou neuf mois après avoir quitté le nid maternel pour son vol nuptial, est cependant en état d'élever une couvée avec sa seule salive. Mais cette couvée ne renferme que peu d'individus, qui, de plus, sont toujours de petite taille, et toujours des ouvrières! Dans ce cas, le régime

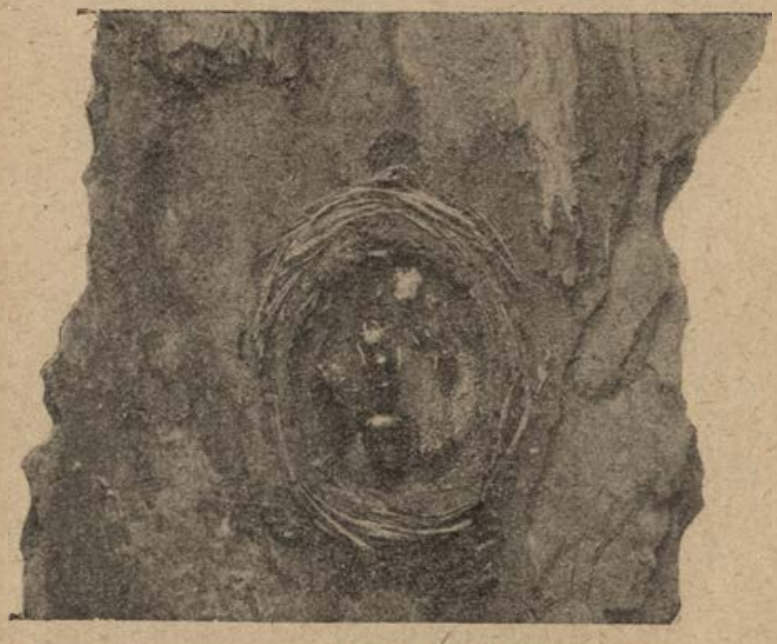

Fig. 33. - Début de colonie de Camponotus pennsylvanicus, dans un ancien cocon de Rhayium lineatum (Coléoptère), sous l'écorce diun Pin. La reine a élevé quelques ouvrières el a fait une seconde ponte (amas de larves, à la partie supérieur é de la figure).

exclusif de salive ne produit certainement ni soldats ni reines, et la faible grandeur de la couvée et de ses membres montre que la quantité de la nourriture importe plus que sa qualité. Cet aspect particulier n'est point dû 
à quelque incapacité constitutionnelle de la reine; ceci est bien prouvé par le fait que, chez les fourmis parasites, dont les reines pondent leurs premiers oufs dans les colonies d'autres espèces, la taille des adultes qui en proviennent n'est nullement réduite, puisqu'elles sont nourries convenablement pendant leur vie larvaire par les ouvrières de l'hôte.

La dépendance étroite qui lie la différenciation des castes reine et ouvrière et l'alimentation est parfaitement révélée dans le développement des colonies d'Aculéates qui sont établies par une reine unique. La première ponte donne toujours de petites ouvrières; celles des pontes suivantes ont une plus grande taille et ce n'est qu'après la production des plus grandes ouvrières qu'apparaissent les reines. Ceci se voit clairement chez les fourmis ayant des ouvrières polymorphiques, comme dans les genres Atta, Pheidologeton, Camponotus, etc. (I).

La même succession très lente de petites ouvrières, de grandes ouvrières, de soldats, de formes néoténiques et royales, s'observe aussi dans les colonies en formation de termites. Le phénomène est si constant et apparaît comme dépendant si nettement de l'amélioration graduelle des conditions trophiques dans la colonie de fourmis et de termites, que c'est là un des arguments les plus frappants

(1) Pricen (1908) a étiıdić le développement des colonies de Cumponolus pennsylvanicus et a ell les résultats suivants: les ouvrieres de la première ponte sont du type le plus petit (ouvrieres minima) et les ouvrières les pliss grandes ou maxima ne sont formées que la troisième annéc, alors que la colonic comple environ 900 a 1.200 individus. Jusqu'a la troisième et mème it la sixieme année, la population d'ouvrières s'ćlevant alor's à 2.000 individus, on ne voit ni mâle ni reine. 
en faveur de l'hypothèse trophogénique de la différenciation des castes.

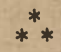

En dehors des observations sur les modes d'alimentation des larves de fourmis, et des expériences d'élevage de ces larves dans des conditions soigneusement contrôlées, il existe une troisième méthode d'aborder le problème qui nous occupe : c'est l'étude des effets produits par certains parasites sur les larves isolées, et des troubles introduits dans les mœurs alimentaires de la colonie, prise dans son entier, à la suite de la présence dans son sein de certains myrmécophiles. Je décrirai brièvement ici quatre exemples de ces " expériences natureHes $\|$ :

I. - Dans ma seconde leçon, j'ai insisté sur les Chalcididés Eucharinés, dont les larves planidium, introduites dans les nids de fourmis, s'attachent aux larves ou aux nymphes à demi formées, et aspirent leurs humeurs. Dans le cas de Orasema viridis, que j'ai observé au Texas (1907a), et qui infeste les colonies de Pheidole instabilis, les planidia se fixent d'ellesmémes au cou de la larve ou de la nymphe en formation; lorsqu'elles en ont retiré une nourriture suffisante, telles les sangsues ou les vampires, et lorsqu'elles ont atteint elles-mêmes le stade de la nymphose, elles se détachent, s'èloignent, et achèvent leur développement comme des organismes indépendants. La larve ou nymphe de fourmi attaquée ne périt pas immédiatement; elle peut achever sa nymphose, mais elle ne parvient jamais au stade adulte. Par suite de cette soustraction qu'elle a subie d'une telle quantité de sa propre substance, la nymphe a un aspect particulier, des caractères d'infériorité, tels qu'une moindre dimension de la tête, du thorax et des yeux, si on les compare à ceux des nymphes qui se sont développées à partir de larves non parasitées. Cette forme correspond a une nouvelle caste " phthisergale", non viable, résultant du retrait purement mécanique des sucs du corps et de la capacité que 
possède l'organisme larvaire de réaliser un tout symétrique mais microcéphale. Si l'adulte élait en état de naitre el de prendre part à l'activité de la colonie, von Buttel-ReEpen el biell des généticistes de l'école néo-darwinienne diraient sans doute que ses caractères singuliers dérivent de déterminants spéciaux, de gènes présents dans l'œuf qui l'a produit; mais une telle déduction serait bien erronée et n'expliquerait nullement l'extraordinaire pouvoir régulaleur de la larve de fourmi. II

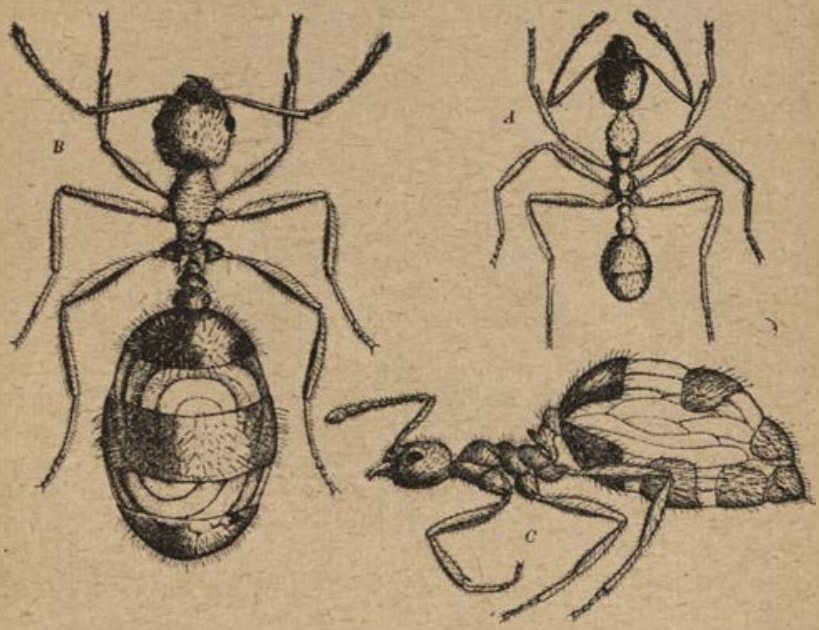

Fig. 34. - Pheidole communata. A, ouvrière normale ; $B$, et $C$, mermithergate (vues dorsale et latérale).

faut ajouter que des formes inférieures analogues (phthisodinergates et phthisogynes) semblent apparaitre dans les nymphes sous l'influence des Orasema, lorsque les larves parasitées auraient normalement produit des soldats ou des reines.

II. - Il y a un certain nombre d'années (1901a, 1907b, 1910b), j'ai décrit un autre cas de parasitisme d'une fourmi du Texas, Pheidole commulata. Les larves de cet insecte sont parfois 
infestées par un ver Némalode du genre Mermis, et produisent des formes aberrantes, que j'ai appelées mermithergates. Elles sont beaucoup plus grandes que les ouvrières normales, qui leur ressemblent néanmoins par leur structure et la petite taille de leur tète, quoique possédant des ocelles très petils. Par la structure du thorax, ces formes se rapprochent des soldats, tandis que l'abdomen est fortement distendu par le Mermis, les organes reproducteurs, comme les autres visceres et comme les réserves grasses, ayant presque entièrement disparu. Les mœurs des individus ainsi parasités sont aussi trés particulières; ils ne creusent jamais le sol, et ne s'occupent pas de la couvée comme les ouvrières normales, mais ils s'en vont de còté el d'autre, toujours affamés, quémandant de la nourriture auprès de leurs compagnons restés normaux. EuEr $(1890,1904)$ a décrit des mermithergates analogues dans toute une série de fourmis, y compris Pheidole absurda et divers Ponérinés des genres Odonlomachus, Neoponera, Ectalomma, P'achycondyla et Paraponera.

Comme toutes les anomalies observées atteignaient les ouvrières des espèces néotropicales, on a supposé que les larves d'ouvrières élaient seules altaquées par un Mermis spécial à l'Amérique. Mais j'ai décrit récemment (1922 a) un mermithergate de Camponolus (Dinomyrmex) cassius du Congo, et Mrizek (1908) a montré que les reines vierges du Lasius aliemus, espèce commune en Europe, peuvent ĉtre infestées par des vers el que ces individıs, quil a appelés mermithogynes, possèdent des ailes anormalement petiles. Ces mermilhogynes avaient déjà èté remar(pućs en 1898 par CRIWLEY (1919 a) qui les avait décrits, comme brachypteres, pour les distinguer des fenielles ordinaires macroptères. J'ai également trouvé quelques mermithogynes chez. une espèce de l'Amérique du Nord, Lasius neoniger $(1910 \mathrm{~b})$.

En 1!:?n, un myrmécologisle anglais, Donisthonpe (1921), a découvert de nombreux mermilhogynes dans une colonie de I. niger typique, el, la même année, Criwley el Birlis (1921) oul jublić un mémoire très détaillć sur un certain nombre de mermithogynes de L. Llavus et alienus, avec une description du lermis (M. murmecophila Baylis), Euny (1924) a decril deux mermilhogrres de lasius umbralus, var. meridionnlis, de l'Italie.

Le caractere brachyptere des mermilhogynes est très probablement dù à l'absorplion, par le Mermis, au cours de sa vie larvaire, de substances constituant le thorax, mais il est moins farile d'interpréter le cas des divers mermithergales. 
Dans les deux espèces de Pheidole (absurda et commutata) qui possèdent trois castes femelles bien neltement définies, reines, soldats à grosse tête et petites ouvrières, les mermithergates présentent un mélange singulier de leurs divers caractères. Nous devons sans doute en conclure que le Mermis exerce un double effet sur son hôte $; 1^{\circ}$ en augmentant l'appétit de la larve, et en stimilant par là les soins alimentaires des ouvrières nourricières; $2^{\circ}$ en modifiant les proportions des différentes parties du corps, peut-être grâce à quelques sécrélions du parasite, agissant comme une hormone sur les tissus en vole de croissance de la larve. Mais, quelle que soit l'interprétation adoptée, la fusion des caractères des trois castes, chez l'adulte mermithergate, permet de supposer que ces trois castes sont également en puissance chez la jeune larve.

III. - Le troisième exemple intéressant, sur ce point, est celui des Coléoptères Loméchusinès, Colćoptères de la tribu des Staphylinidés, qui ont été étudiés par Wasmann pendant plus de trente ans et sur lesquels il a publić presque autant de mémoires. Comme le dernier de ces mémoires (1920) contient une bibliographie complète, je ne citerai pas le détail des travaux parus, et je m'en tiendrai ici à exposer un court résumé des observations que j'ai publiées dans ma "Social Life among the Insects ». Les Loméchusinés ne renferment que trois genres: Lomechusa et Atemeles spéciaux à l'Europe et au nord de l'Asie, et Xenodusa, connu seulement aux EtatsUnis et au Mexique. Les espèces de Atemeles et Xenodusa ont deux hôtes, celles du premier vivant pendant l'été et se reproduisant dans les colonies de Formica, et hivernant dans les colonies de Myrmica; celles du second se reproduisant aussi chez Formica, mais hivernant parmi les fourmis du genre Camponolus; Lomechusa; par contre, n'a qu'un seul hôte, Formica sanguinea, sur lequel il vit durant toute l'année. Les adultes de ces trois genres se ressemblent beaucoup entre eux. Ils sont colorés en rouge gras, possèdent de longues antennes mobiles, des élytres courtes et un abdomen volumineux qui peut se replier sur le thorax, et qui porte, de chaque côté et sur le dessus, une séric segmentaire de superbes trichomes dorés. Les Atemeles et les Yenodusa quêtent leur nourriture auprès des fourmis en leur frottant les joues avec les pattes antéripures. Leurs larves sont très actives, elles ont de longues pattes et emploient la même méthode que les adultes pour obliger les fourmis à régurgiter. Elles dévorent également les larves sans défense des Formica. Le Lomechusu 
adulte est plus passif dans sa façon de faire et emploie sez antennes pour demander sa nourriture. Ses larves ont des palles très courtes, el, étant incapables d'aller et de venir, elles restent immobiles au milieu de la couvée de fourınis. Elles dévorent cette couvée et mangent aussi des aliments régurgilés. Il est très probable qu'elles secrètent des exsudats grais-

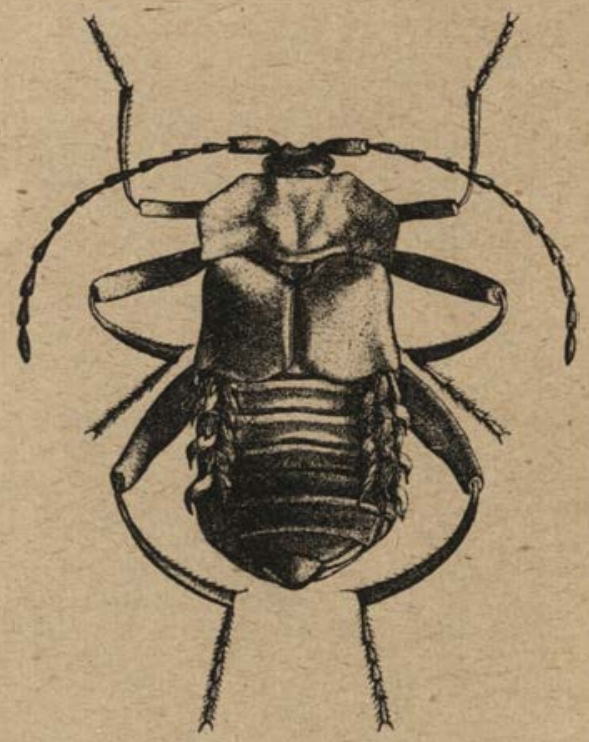

Fig. 35. - Xenodusa cava (Noter les deux paquets de trichômes sur les côtés de l'abdomen).

seux qui sont vivement appréciés par les fourmis. Quoi qu'il en soit, les fourmis semblent préférer ces larves parasiles à leurs propres larves, ou peut-ètre les considèrent-elles comme destinées à produire des fourmis extraordinaires. Cet engouement a pour conséquence que les larves de Lomechusa détruisent souvent la plus grande partie de la couvée, si bien que, 
dans les colonies de $F$. sanguinea fortement infestées, les larves de reines ont un développement anormal. Ou bion elles sont négligées, ou bien les fourmis s'efforcent de les transformer en ouvrières, sentant que celte caste est insuffisamment reprécentée dans la colonic. Mais, quel que soit le traitement subi par ces larves, elles produisent des adultes pathologiques, connus sous le nom de "pseudogynes ", individus avortes, ressemblant aux ouvrières par leur taille et par le dessin de la tète et de l'abdomen, mais ayant un thorax volu-
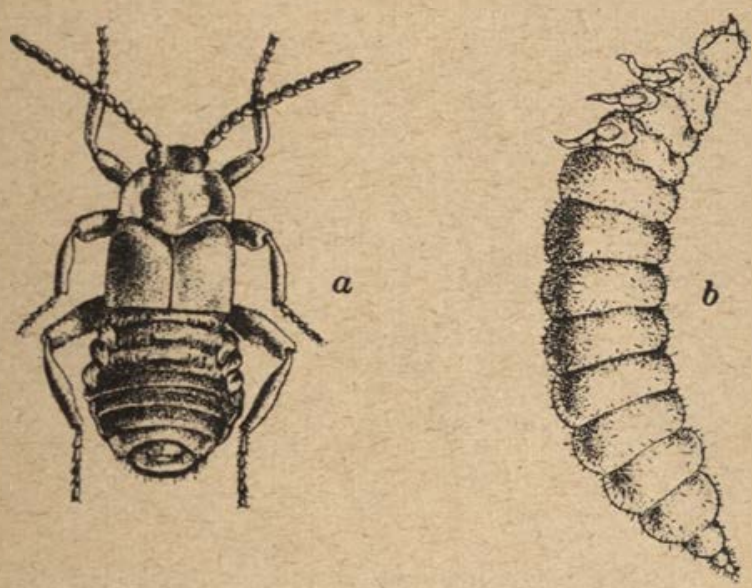

Fig. 36. - Lomechusa slinmosa: $a$, adulte; $b$, larve.

mineux et convexe, se rapprochant de celui de la reine. Elles sont de teinte plus pâle que les ouvrières normales, et très paresseuses, peureuses et ma'adroites. En général, elles forment cinq à sept pour cent du personnel de la colonie de sanguinea infestée, moins souvent, elles arrivent à atteindre vingt pour cent ou plus. Leur apparition dans le nid indique un état pathologique de la colonie qui est en voie d'extinction. Des pseudogynes analogues se produisent dans les colonies de Formica infestées par les Atemeles et les Xenodusa, mais non 
dans les colonies de Myrmica et de Camponotus où hivernent les Coléoptères, parce que ces derniers ne se reproduisent pas parni leurs hotes d'hiver, el qu'ils ne peuvent donc interférer avec le développement normal des couvées de ces hôtes.

On voit, qu'a l'opposé des Orasema et Mermis, le Lomechusa nest pas parasite sur ou dans la larve de son hòle prise isolément; c'est plutòt un parasite social, qui vit aux dépens de la communaute, en influençant en premier lieu la façon dont le personnel ouvrier s'occupe d'élever la couvée, lui faisant négliger les larves de reines ou l'entrainant vivement à modilier le mode normal dalimentation de ces larves. Wismax a montré que ces effets peuvent persister dans les colonies de sanguinea longtemps apres la disparilion de ces parasites, sous li forme d'ume tendance quiont les ouvrières à élever encore des pseudogynes, jusqu'a ce que la colonie succombe sous l'influence d'une véritable maladic sociale. VienMeyer (1904) a démontré expérimentalement que la production des pscudogynes est véritablement dùe à une modification, d'origine parasitaire, des nıœurs des ouvrières et non à un état pathologique quelconque des cufs pondus par la reine. Dans une de ses colonies de sanguinea, la production des pseudogynes a persisté pendant trois ans en dehors de la présence de Lomechusa. Durant la quatrième année, il a séparé la reine de ses ouvrières et a réussi à la faire adopter par un groupe d'ouvrières d'une colonic qui n'avait jamais élé infestée par les parasites, et qui n'avait donc jamais acquis l'habitude d'élever des formes anormales. Le nouveau personnel ouvrier éleva immédiatement les larves issues des œufs de la reine et en fit des individus normaux.

IV. - Lorsque les fourmis elles-mênies deviennent parasites d'autres fourmis, les habitudes des deux espèces s'en trouvent forcément modifiées, parce que les conditions sociales de chacune sont altérées. Laissant pour une leçon ultérieure l'examen détaillé de ce sujet, je donnerai ici seulement un exemple qui montre que les modifications des habitudes alimentaires des parasites se refletent dans la structure de leur reine et de leurs ouvrieres. Il y a quelques années (1901 $a, 1903 c$, 1917 c), j'ai découvert, dans les hauteurs de la Nouvelle Angleterre et plus tard dans les montagnes du Colorado, une petite fourmi, Leplothorax emersoni, qui est toujours associée avec une Myrmicinée plus grosse, Myrmica canadensis. Le Leplothorax occupe de petiles chambres, au voisinage de la surface du nid de Myrmica et les réunit par de fines galeries aux cham- 
bres de ses voisins. Les ouvrières du Leptothorax passent presque lout leur lemps dans le nid de Myrmica; elles grimpent sur le dos des ouvrieres de Myrmica, et leur léchent assidiment le corps, particulierement la tête et la région buccale. Les M!̣rmica paraissent apprécier vivement ces façons, et, de temps i autre. récompensent leurs petils cavaliers avec des gnultelelles dialiments regurgités. Mais, landis que les Leplothorax sarrogent le droit de se mêler en toute liberté aux Myrmira, et savent les amener a régurgiter leurs aliments, elles sopposent a lintrusion de ces Myrmica dans leurs propres habitations, et persistent a maintenir leurs couvées en un isolement absolu. Grâce à l'abondante nourriture qu'elles reçoivent et grace aussi, peut-être, à la grande quantité de salive que celle nourriture contient, les couvées de Leptothorax produisent en proportion considérable des formes intermédiaires entre les reines ailées et les ouvrières du type réglementaire. Une de mes premières élèves, Miss Margaret HoLLIIIY (1903), à la suite d'une étude trés soignée de 1.000 spécimells de $L$. entersoni, put distinguer non moins de onze formes diffèrentes, sèlageant depuis la reine ailće jusqu'aux plus pelites ouvrières, les micrergates. Des dissections des organes reproducteurs montrèrent que " les onze types possédaient lous des ovaires bien développés, contenant des œufs mûrs. Tous les spécimens disséqués avaient des ovaires mieux développés que la reine. Tous, sauf deux, avaient un spermathèque. Des formes de transition analogues entre les reines et les ouvrières sont connues également dans certaines espèces de L.cptothorax, non parasites, mais elles sont toujours rares (1).

(1) Miss Hor.tidiy a observé que, "chez Leptothorax longispinosus Rog., L. curvispinosus Mayr., L. obturator Wheeler et L. canadensis Prov., les phases sexuées sont représentées par les mâles, les reines, les macro- et microergates. Dans ces espèces, on n'a pas trouvé de femelles ergatoïdes. Chez $L$. canadensis, on constata la présence d'une ouvrière triocellée, d'une bi- el d'une uniocellée. Les reines el les ouvrières différaient par leur structure externé. Les ovaires des ouvrières possédaient un tube unique de chaque côté; et il n'y avait point de spermathèque! ” Cependant, Wasmax (1895) a trouvé des formes intermédiaires (" Mischformen »), microgynes et ergalogynes, ces dernières nombreuses dans une colonie de L. acervortum, et ADLERz (1881), WASMINA, STLMPEen (1921) ont observé des ergatogynes chez Formicoxenus nitidulus. Ce dernier vit comme hòte dans les nids de Formica rufa, et $L$. acer- 


\section{***}

Avant d'aborder d'autres questions, il faut altirer l'attention sur les deux principes d'Emeny ( 1904, Ig2 r) et de Vieirmeyer ( 1923 ), les lois du développement larvaire et. prénymphal chez les fourmis et autres insectes métaboliques. Emery appelle sa loi " loi du contraste entre la tête et l'abdomen "); il l'a établie, à la suite de son étude des mermithergates, de la façon suivante:

"La larve a pour fonction principale d'accumuler des provisions pour le travail de la métamorphose, sous forme de corps adipeux, et de préparer les ébauches des plaques imaginales des organes internes. Ces ébauches s'apprêtent à la concurrence qui a lieu dans la période prénymphale, lorsque le corps de la fourmi se divise en tète, thorax et abdomen. Les organes essentiels à la vie, notamment l'intestin, qui siège dans l'abdomen, ont la préférence toutefois pour les organes de la lête (ouvrières à petite tête). Quand les provisions sont plus abondantes, les plaques imaginales de la tête, qui sont plus voraces, tirent à elles le surplus (ouvrières à grosse tête, sol-

vorum fait souvent ses nids au voisinage d'autres fourmis plus grandes, vivant ainsi en plésobiose ou parœcisme, tout comme le canadensis de l'Amérique du Nord, qu'on regarde généralement comme une sous-espèce d'acervorum.

Cette plus grande fréquence des formes de transition, chez L. emersoni, peut, je crois, provenir des conditions trophiques extraordinairement favorables dans lesquelles les larves sont élevées. On peut s'attendre, dans ce cas, à voir l'évolution conduire à l'élimination totale des ouvrières stériles, et à un retour à deux types très semblables de femelles fertiles, l'un ailé et nomade, fondateur de colonies, l'autre aptère, combinant la fécondité de la reine à des habitudes voisines des ouvrières. Les exemples précédents suffisent à montrer que, chez les Formicidés, la larve femelle est beaucoup plus plastique que celle des guêpes ou des abeilles sociales. Cecr ressort également bien, en général, du plus grand nombre de castes femelles et de leur différenciation plus prononcée dans la plupart des genres et des especes. 
(dats). Enfirr, lorsque les provisions surabondent, les ébauches des ovaires et les plaques imaginales des ailes entrent en jeu et le thorax el labclomen croissent aux dépens de la tête (femelle ailée). )

Sans aucun doute, cetle loi a une valeur considérable comme description générale de l'ontogénie des diverses castes, non seulement chez les fourmis, mais aussi chez l'abeille domestique et les termites. Ce n'est là, cependant, qu'un cas particulier et assez frappant d'un phénomène plus général, que Cumo (rg2/4) a appelé le "gradieñt axial " et son inverse. Les tissus de la prénymphe présentent une différentielle dans la localisation de la croissance, laquelle est antéro-postérieure chez les soldais et les grandes ouvrières, et postéro-antérieure chez. la reine. Dans les deux cas, la décroissance est rapide. Dans le langage de Cumn, le gradient axial des soldals et des grandes ouvrières est l'inverse de celui de la reine, et peut-être dirait il que l'alimentation de certaines larves ne permet pas les variations du gradient originel: dans ce cas, il se produirait des reines; ou bien, elle peut inverser ce gradient, ce qui, suivant la puissance dies facteurs déterminants, peut amener l'apparition d'un soldat. Dans les petites ouvrières, la décroissance antéro-postérieure est faible. Tout ceci est purement descriptif et constitue les préliminaires à une connaissance certaine des processus réels du métabolisme que nous ignorons encore.

La loi de Vienmerer pourrait peut-être être considérée comme une application de la conception du gradient à la phylogénie comme à l'ontogénie. Cet auteur considère la plupart des organes de la fourmi fernelle, comme itant dans un état de variation, de flux, mais il les répartit en 
Irois catégories: ceux qui sont relativement fixes, ou stables, ceux qui tendent vers un maximum de développement, et ceux qui sont en voie d'atrophie. Sa loi s'applique aux deux dernières catégories d'organes qui sont seules influencées par l'alimentation de la- larve et elle affirme qu'il y a une tendance à accélérer ces deux processus dans le polymorphisme. Vienueyer dit:

"Il me semble qu'une longue série d'irrégularités dans la disposition des organes de la femelle et dans l'acquisition des caractères de l'ouviène peut s'interpréter comme une accélération ou un relard de leur développement. Partant du mème principe, peut-être pourrions-nous expliquer aussi beaucoup de différences existant entre les petites et les grandes ouvrières, ou entre les ouvrières vraies et les soldats, dans la mesure où ses différences impliquent le non-développement d'un organe. »

Comme exemples spécifiques, Viennexer cite la disparition très rapide des vestiges de dents mandibulaires chez les fourmis mâles (Formica), et des vestiges d'yeux chez les ouvrières (Lasius flavus et myops), sous l'influence des conditions trophiques défavorables où se trouvent les colonies.

\section{$*_{*}^{*}$}

Au cours des dernières années, la vive discussion de la différencialion des castes chez les insectes sociaux s'est déplacée des Aculéates aux Termites. Grassi est arrivé à la eonclusion que les termites qui viennent d'éclore ont tous la même constitution el que les castes sont le résullat d'une alimentation qualitative. L'absorption de salive par les nymphes, dans les premières phases, accélère le développernent des organes reproducteurs, si bien qu'il en résulte l'apparition de formes sexuées fertiles des pre- 
mier, second et troisième type. Les individus du second et troisième type sont néoténiques parce que le développement de leurs caractères somatiques (ailes, yeux, pigmentation, etc.) a été inhibé tout à la fois (I).

Les ouvrières ef les soldats sont des formes inhibées à la fois du còté somatique et du côté sexuel, à cause d'un régime perpétuel de bois, brut nu partiellement digéré, et d'excréments. Celte conception de la différenciation des castes chez les lermites a été acceplée par Feytain (1912), Wasmax (igo8a), Heiti, Escherich, Jucci el d'aulres. Howmak (rgo8) l'acceple aussi, mais il pense yue la production d'exsudats graisseux par la peau doit ilre le slimulus qui déterminc l'alimentalion différentielle des divers individus. Ics larves, à lous les stades et de toutes les castes, produisent de tels exsudats, mais en quantité très variable. La quantité, et peut-être aussi la (fualité des exsudats " est l'objet d'un choix amical ". Les individus qui produisent le plus d'exsudats reçoivent plus de nourriture el le tissu qui donne ces exsudats, s'accroît par là, puisque le tissu adipeux emmagasine les aliments superflus et les rejette ensuite sous forme d'exsudats. Donc, quand des larves, supposées toutes semblables, naissent des œufs de lermites, certaines d'entre elles reçoivent dès le début un peu plus de nourriture. Ces larves étant mieux nourries, secrètent des exsudats en plus grande quantité que les autres et ceci

(1) L.a question de la castration par les Protozoaires de l'inlestin posterieur s'est trouvée alre une complication inutile, pitisqu'on a montré que ces Protozoaires n'étaient pas des parasites, mais des symbiotes digerant le bois, qui disparais. saient naturellement des intestins d'individus nourris seulement de salive. 
indique pour elles un développement différenl de celui des larves qui sont plus pauvrement nourries, ce qui apparaît très tôt dans la distinction possible en larves dites "à pelite tête ", el en larves à "grosse tẻte ", cellesci représentant les individus moins bien nourris. Les "petites lêtes .) sont les larves des individus sexués, les " grosses têtes " celles des individus asexués. Holmarex pense que la différenciation ultérieure des premiers en formes néoléniques et royales, des seconds en ouvrières et soldats esı aussi dûe à des différences quantitatives dans la stimulation par les exsudals.

Très différentes sont les opinions de Bugrios, de Miss Thompsox et Sxyder et d'Imss, qui regardent les castes comme blastogéniques, ou prédéterminées dans l'œuf. Des observations de Bugvior sur les Eutermes, on peut su moins dire, comme nous l'avons vu, qu'clles sont peu nettes. Miss Thompsox el Siyder admettent que les termites sortant de l'œuf sonl lous identigures extérieurement, mais ils affirment que l'on peul distinguer intérieurement deux types, suivant les dimensions du cerveau et des organes reproductcurs, et que la différenciation ultérieure en castes définies est dûe également à des facteurs intrinsèques on blastogéniques et non extrinsèques et alimentaires. Ils considèrent donc les castes comme des mulations, comparables à celles qu'a découvertes de VRIEs chez l'OEnothère, et ils sont portés à interpréter le pelymorphisme comme un phénomène mendélien. Mais il est évident que le caractère mendélien des castes ne peut être prouvé que par des expériences de reproduction. Celles-ci seraient peut-ètre possibles, puisqu'il existe trois castes fertiles et peut-être quatre, si nous 
POLYMORPIISME

comptons les soldats, qui peuvent parfois produire des descendants, chez les termites inférieurs (r).

Voilà où en est actuellement la question et nous pouvons seulement, comme Goctur inourant, réclamer plus de lumière.

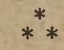

S'il est exact, comme je l'affirme, que le polymorphisme soit né et se soit développé indépendamment dans les divers groupes d'insectes sociaux, et si ces groupes sont, comme c'est probable, d'âges géologiques très différents, si, de plus, les castes, apparues tout d'abord comme des résultats fonctionnels - c'est-à-dire ayant trait à la fois à la physiologie el aux mœurs - d'une division du Iravail, n'ont acquis que progressivement leur différenciation morphologique, nous pouvons penser que ces castes diffèrent entre elles au point de vue de leur déterminisme héréditaire dans le plasma germinatif. Tandis qu'il paraît très possible que, dans les deux groupes récents des abeilles et des guêpes sociales, la reine et l'ouvrière, si faiblement difiérenciées, se développent à partir d'œufs de même espèce, il est plus probable que, dans les deux groupes plus anciens, les fourmis et les termites, les castes bien tranchées, - sexuée, ouvrière et soldat, - sont blastogéniques. Celte dernière considération ne peul s'appliquer à la caste des soldals, qui est évidemment d'âge très différent chez les fourmis et les termites. Chez les termites, elle est complètement diffé-

(1) Silvestri (1901) a décrit également des ouvrières gynécoïdes fécondes (peut-être des adultes diune troisiòne forme?) chez un termite de l'Amérique du Sud. 
renciée dès l'(Oligacène, et, puisquion a trowvé des spécimens ailés de Mastotermes dans l'Focène, il y a toute raison de penser que les soldats étaient aussi présents et aussi parfaitement développés que chez les $M$. darwiniensis actuels.

La reine est évidemment la caste de fourmis la plus ancienne et la plus stable et correspond à la femelle typique des Aculéates solitaires. Mème si nous admettons qu'à l'origine les fourmis avaient deux reines fécondes dimorphiques, les ouvrières sont une forme postérieure, secondaire, et les soldats, étant simplement des ouvrières particulièrement spécialisées, constituent forcément la plus récente des castes. Cette caste correspond donc plulòt aux ouvrières des termites, phỵlogénétiquement sinon fonctionnellement. L'existence de soldats chez les fourmis, au début du Tertiaire, n'est pas établie de façon salisfaisante. La plupart des genres de l'Oligocène inférieur qui ont survécu jusqu'à l'époque actuelle n'ont pas de soldats, et, chez d'autres, comme Pheidologeton, nous ne connaissons que des ouvrières mineures (les plus petites). Si ce genre a élé exactement identifié, il devrait posséder dess soldats, mais cela n'est même pas sûr, car il y a des genres vivants très voisins, comme Solenopsis, dont cerlaines espèces, peu nombreuses $(S$. geminata, $s a-$ vissima) ont à la fois des soldats et des ouvrières pléomorphiques, tandis que la majorité d'entre elles ont seulement de petites ouvrières monomorphiques. La caste des soldats ne serait donc différenciée des grandes ouvrières qu'à partir du Terliaire moven. Cette caste semble, en outre, avoir pris naissance indépendamment dans la plupart des genres où elle se présente, et n'avoir pas encore acquis une représentation définitive dans le plasma ger- 
minatif. Comme suite de ma tentative, exposée dans les précédentes leçons, de faire dériver les fourmis d'ancètres solitaires ou subsociaux d̀ femelles dimorphiques, et les termites d'ancètres solitaires ou subsociaux, ayant des màles dimorphiques comme les femelles, nous pourrions admettre que les reines et les ouvrières chez les Formicidés, les formes royales et les soldats chez les Isoptères, sont véritablement blastogéniques, mais que les autres caste's et surtout les formes pléomorphiques d'ouvrières et de soldals sont trophogéniques.

Les considérations précédentes en amènent d'autres qui rendent douteuse l'orignine Irophogénique uniforme des castes dans tous les insectes sociaux el spécialement chez lés fourmis el les termites. Quoique assez générales, ces considérations sont de prime abord très frappantes; suffisamment pour que Forkl (Igo2) ait eu recours à cerlaines d'entre elles pour appuyer l'hỵpothèse blastogénique de Weismax, en l'opposant à l'hypothèse principatement trophogénique soulenue par Emery dans plusicurs mémoires $(1894,1896,1904$, I 906 , rgio, 1918, 1921). Les considérations dont il s'agit sont: $I^{\circ} l^{\prime} e x-$ traordinaire stabilité des rastes typiques chez les espèces existantes de fourmis el de termites $; 2^{\circ}$ leur extraordinaire ronstance au cours des âtges géologiques ; $3^{\circ}$ la difficulté qu'il y a à expliquer les formes de fourmis gynandromorphiques par l'hypothèse trophogénique, et $4^{\circ}$ la difficulté quiil y a à expliquer les caractères adaptatifs des soldats el des ouvrières.

$1^{\circ}$ Le myrmécographe qui manie sans cesse des fourmis de loutes les régions du globe, et qui compare minutieusement des milliers de spécimens provenant de nombreuses colonies, ne peut manquer d'etre impressionné par l'extraordinaire sta- 
bilité des castes, malgré les innombrables et très faibles diffèrences qui créent les variétés, les espèces et sous-espèces et les genres.

Certes, la caste ouvriere fail defaut dans ccrtains gcnres parasites, et la reine dans certains autres; mais de tels cas proviennent de conditions éthologiques inaccoutumées, et sont assez exceptionnels pour confirmer plutôt la règle.

Les plus grandes différences climatiques de température n'ont elles-mêmes aucun effet perturbateur sur les castes dont on pense qu'elles sont si facilement modifiées par l'alimentalion. lit notre scepticisme s'accroît encore, devant la difficulté qu'on éprouve à produirc des modifications morphologiques comparables i celles des castes, au moyen d'une alimentation spéciale, chez. les autres insectes et chez nos oiseaux et mammiferes domestiques.

2 Encore plus extraordinaire est la fixité des castes de fourmis el de termites au cours des âges géologiques. Il m'est absolument impossible de distinguer les ouvrières de Formica llori de l'Oligocène el celles de $F$. fusca de l'époque actuelle, sauf par les différences dues aul long séjour des premières dans une enveloppe résineuse; et les ouvrières des autres espèces fossiles different des reines de mème espèce par des caractères qui sont précisément identiques à ceux qui les différenciaient dans les espèces acluelles. Les termites ouvriers et soldats, représentés par von Rosex, ont le mème aspect tout moderne. Il nous faut donc conclure que les mêmes castes se sont produites et reproduites au cours de plusieure millions d'années et dans les régions du globe les plus diverses et les plus éloignées.

Les objections que nous venons de faire it l'hypothèse trophogenique se retrouvent lorsquion considere que la constance remarquable des castes implique une constance similaire des espèces auxquelles elles appartiennent durant les longues périodes des époques conozoïque et tertiaire, dans loutes les parties du monde, el il devrait donc ètre de même pour le milieu alimentaire, dans lequel les castes se sont produites de toute nécessité, àge après àge. On peut admettre cette derniere supposition, mais cela ne prouve pas que les castes sont enlièreinent trophogéniques. car les blastogénistes admettront. que ces rastes quoique prédéterminées dlans l’œuf, ont été produites dans un milieu parliculier et grace i une alimentation speciale. 
$3^{\circ}$ On a décrit un nombre considérable de fourmis gynaniromorphiques. Ce sont des individus peu communs, anormaux, dont le corps est fait de deux moitiés, l'une droite, l'autre gauche, de deux portions antérieure et postérieure, ou d'une mosaïque de parties, appartenant à des sexes différents (IVueeler, $1903 \mathrm{~b}$ ). Les spécimens les plus curieux sont ceux qui possedent un corps, dont la moitié gauche est mâle, la moitié droite femelle; ou vice versa. Il existe en réalité deux lypes pour chacun des ras de gynandromorphisme, latéral, antéropostérieur et en mosaïque, suivant que le composant fomelle correspond ì la reine ou à l'ouvrière. J'ai appelé le premier type, gynandromorphisme proprement dit, le second IYpe, "ergalandromorphisme ". Les différences sont presque toujours nettement tranchees, et il n'y a que peu de cas connus vii lon puisse avoir quelque doute sur la caste femelle qui s'est combince avec le composant màle (1).

Comme on a reconnu que les gynandromorphes sont formés dans l'œuf, il semble que les composants reine et ouvrière doivent etre blaslogéniques; mais cela n'est pas une consćquence nécessaire (2).

Bien que les composants màle et femelle soient très probiblement flétermines au moment de la fécondation de l'œuf, si non avant, on conçoil que des différences dans l'alimentation Iarvaire puissent influencer le composant femelle tout coinme elles déterminent les castes des adultes. Néanmoins, nous pour-

(1) En 1919, jiii décrit un gynandromorphe de Camponofus (Colobopsis) ulbocinctus Ashm., des Philippines, dont la moitié droite de la tête était d'un mâle, la moitié gauche étant d'un soldat. Suivant EMEnY (1921), celte moitié gauche présenterait seulement des caracteres généralisés de reine et d'ouvrière et non les caracteres spécifiques des soldats. Je demeure convaincu que ce composant ne peut être d'une reine, vu l'absence d'ocelles, et qu'il possède distinctement la structure de la carène frontale, du front, des joues et des mandibules, qui est. celle de la caste des soldats.

(2) Kominsiy (1924il et 1924b) affirme avoir produit des " gynandromorphes " et des formes intersexuées, chez les Phalénes, en soumettant les larves et les nymphes à des températures anormalement élevtes ou basses, et en nourrissant les larres avec une nourriture inaccoulumée; mais son mémoire est tres court et on n'est pas bien lixé sur ce qu'il entend par un " gynandromorphe ». 
rions trous allendre à ce que la forme résultante, dans sa partie femelle, soit plus souvent une forme intermédiaire, c'est-itdire une reine ergatoïde ou pseudogyne, et non point, d'une façon aussi nette, soit une gynandromorple vraie, soit une ergatandromorphe vraie, ce qui est le cas de presque toutes les anomalies décrites jusgu'it ce jour. Eмгнy (1924) a décrit récemment deux gynandromorphes antéro-postérieurs de Myrmica ruginodis, qui présentaient un mélange particulier des caracteres de louvriere, de la reine et du mile. C'était, en fait, comme dit cet auleur, " des pseudogynes, avec des organes

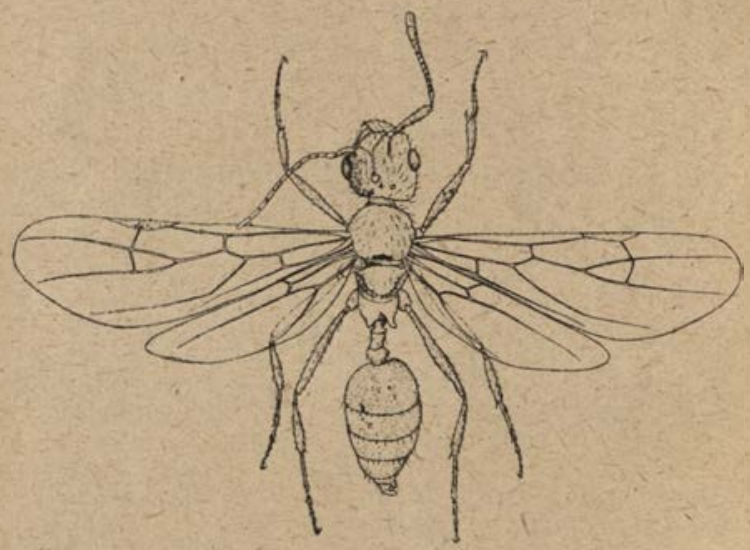

Fig. 3i. - Epipheidole manilina Ginnandromorphe. (mile it srauche, femelle is droite).

génitax miles ". Que ladimentation de la larve puisse delerminer la casle du composant femelle du gynandromorphe, ceci est prouvi également par les nombreux cas de ce genre obsaves chez les abeilles domesliques (voir von Sirbor.d, 186í). BOVER (1915) et Nlle Mruma (1915), car ils semblent se produire tous dans les callules d'ourrieres et donc etre ergatandromorphes.

4. Lobjection la plus grave faite a l'bypothèse blastogéniyute 
est li structure nellement adaptative, ou plutôt adaptée, des diverses castes, en particulier des ouvrieres et des soldats, telle que l'indiquent l'ahsence d'ailes, la structure de la tète el des mandibules, les yeux, etc., el les mœurs particulieres qui y sont liées (différence de professions) (Pnicer, 1908, Miss Buckingham 1911, Hingstox 1922, etc.). Et la difficulté s'accroit du fait que, dans les espèces les plus hautement spécia-
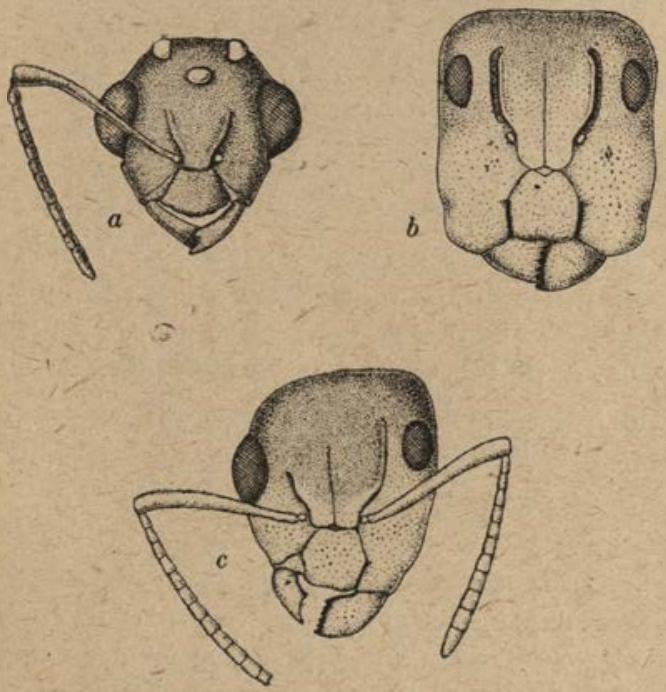

Fig. 38. - Camponolus (Colobopsis) albocinctus: $a$, tête le mile; $b$, têle de soldat; $c$, lète de gynandromorphe (mì̀le it gatuche; femelle à droile).

lisées, qui révèlent ces adaptations dans leur forme la plıs parfaite, les soldals et les ouvrières sont stériles, ou, sils sont fertiles, we pondent des reufs que dans des conditions exceptionnelles (ahondance de nourriture, haute tempéralure, elc.). Comme, chez les termites, le problime se pose de facon tris semblable, il me seinble que nous somnies contraints d'adopler l'une des trois lypotheses suivantes: 
1. Les castes stériles sont fertiles beaucoup plus souvent gu'on ne l'a supposé, et peuvent transmettre leurs caractères particuliers a travers leurs descendants males (fourmis) ou inailes et femelles (lermiles) et finissent donc par èlre représentés dans le plasma germinatif de l'espèce.

$2^{\circ}$ Les castes se produisent comme des varialions flucluantes, ou comme des mutations qui sont passées au crible par une séleclion naturelle, n'agissant pas sur les castes slériles, mais seulement sur la reine pondeuse et sur ses decendants reines, comme l'ont affirmé Darwin et Weismann.

$3^{\circ}$ Il existe quelque facteur finaliste, mélaphysique, une entéléchie spéciale qui initie et guide le développement adaplatif des castes stériles.

Il est inutile de dire qu'aucune de ces hypothèses n'est satisfaisantc. Evidemment, nous nous sommes trop fortement attachés aux aspects statiques, morphologiques du polymorphisme, et trop peu à ses aspects dynamiques, physiologiques et éthologiques. Cependant beaucoup de physiologistes sont capables de concevoir ou tout au moins d'amoindrir les particularités régulalrices et adaptatives des processus biologiques et celles-ci sont certainement Irès significatives dans la precilogynie el la pocilandrie des insectes sociaux. On a suggéré que la production des ouvrières et des soldats dans la colonie est une sorte de tératogénie expérimentale provoquée par les ouvrières nourrices, mais il est fort étrange que les monstres áinsi produits soient, par leur structure et leur fonction, aussi parfaitement adaptés à leurs professions particulières, comme les soldats portiers du Colobopsis chez les fourmis, et les soldats "nasuti ) chez les termites. Et cela nous oblige à croire que de telles formes prennent naissance dans des genres spéciaux, surgissant on ne sait d'où dans le „plasma germinatif, ou bien qu'elles se sont développées graduellement sous la conduite d'une sélection naturelle, à parlir de formes, qui, aulanl gue nous puissions le 
POLYMORPIISME

267

voir, ont une valeur ou une puissance de survie égale ou mème plus grande. Aboutissant à uñe telle impasse, nous n'embrasserons pas l'entéléchie aristotélicienne, cette aîeule de plus de 2.000 étés, qui est aujourd'hui si en faveur de l'autre côté du Rhin ; nous ne nous unirons point non plus aux apôtres de la théorie de la survivance des plus aptes, qui répètent sans fin "sélection naturelle ", mais il est de beaucoup préférable de s'arrêter, dans le laboratoire ou dans la nature, et se taire ou ne plus prononcer que le mot "ignoramus ", jusqu'à ce que nous ayons achevé, de ce phénomène, une étude parfaite, tant au point de vue des mœurs que de la physiologie. Ainsi que dit Roger Bacos: "Nullus sermo in his potest cerlificure, tolum enim dependet ab experimentia"). 



\section{CHAPITRE VIII}

\section{LE MILIEU SOCIAL ET LA TROPHALLAXIS}

Imporlance prépondérante de la caste ouvrière dans la conslilution du milieu social. - Champ trophoporique. - Trophallaxis (échange de nourriture). - Exemples de ce processus dans les diver's groupes d'insectes sociaux. - Rôle des perceptions gustatives et olfactives.

L'élude du polymorphisme nous a conduits à concevoir avec exactitude la signification du milieu social. Dans la société humaine, ce milieu est d'une telle complexité qu'ils sont sans doute rares, ceux qui en ont une notion précise, funique nous connaissions tous son imporlance. Les sociétés d'insectes étant beaucoup plus simples, elles nous permetlent d'acquérir une idée plus nette de la nature de ce milieu social. Dans mes précédentes leçons, j’ai souligné le fait que les activités des insectes sociaux sont de simples modifications des habitudes de leurs ancêtres solitaires. Ceci esı évident chez les Aculéates et est certainement exact aussi pour les Termites, quoique nous ne sachions rien des habitudes de leurs antécédents solitaires, les Protoblattoüdés. Dans l'exposé qui val suivre, je moccuperai principalement des Aculsates. I.es formes d'activité qui nous inléressent sont : $\mathbf{r}^{\circ}$ le vol nuptial ou de dissémination ; $2^{\circ}$ la nidification $; 3^{\circ}$ la récolte ef l'emmagasinemenl de la nourriture et sa répar- 
tition entre les membres de la colonie, et $4^{\circ}$ la défense. Dans les espèces solitaires, ces activités sont exclusivement réalisées par les femelles, sauf le vol nuptial ou son équivalent. Chez les espèces sociales, ces quatre formes d'artivité deviennent des phénomènes collectifs et sont donc intensifiées, et modifiées, le vol nuptial faiblement, la nidification et la protection forlement et, plus que toules, les habitudes trophiques, dont le but est de fournir l'aliment aux membres de la colonie, larves et adultes. Au sens très général, le milieu social peut être défini comme l'ensemble de ces activités modifiées et intensifiées. Chez les Aculéates sociaux, la nidification et la protection ont subi une évolution si longue et si compliquée qu'il faudrait une série spéciale de leçons pour l'exposer convenablement. Le temps dont je dispose me permèt seulement d'examiner les habitudes trophiques, qui dominent et tiennent sous leur dépendance toutes les autres activités de la colonie.

Chez les insectes sociaux, nous connaissons deux types primitifs pour la création des colonies; celles-ci naissent, soit des seuls parents (reines des Aculéates, couples royaux des termites), soit de l'essaimage, c'est-à-dire d'individus sexués accompagnés d'un certain nombre d'ouvrières de la colonic mère. Le premier type peut encore se subdiviser en deux sous-types: indépendant et dépendant. Dans le sous-type indépendant, la reine, ou le couple royal, est en état de fonder la colonie sans l'aide d'ouvrières ; c'est ce qui se produit chez les Polistinés des régions tempérées, les Vespinés, les Bombinés des régions tempérées et boréales, les Haliclinés et la grande majorité des fourmis et des termites. Le sous-type dépendant est représenté par de rares Vespinés, les Bombinés para- 
sites et un certain nombre de fournis, ordinairement parasites, dont les reines sont absolument incapables de fonder indépendamment les colonies et doivent être adoptées par des colonies de leur propre espèce ou d'espèces différentes, ou bien doivent s'assurer de la couvée d'une autre espèce qui leur fournira le concours d'ouvrières indispensable à l'élevage de leurs propres enfants. Je m'occuperai de ces cas dans une prochaine leçon. Les groupes qui fondent leurs colonies par essaimage sont les Epiponinés (Polybiinés) et quelques Polistinés des tropiques chez les guêpes, les Bombinés, Apinés et Méliponinés des tropiques chez les abeilles, et quelques termites (p. ex. Reticulitermes lucifugus en Italie). Remarquons que l'essaimage n'existe pas dans les régions tempérées, sauf pour l'abeille domestique, qui est en réalité un insecte indien.

Les types de formation dépendante et par essaimage peuvent être regardés l'un et l'autre comme des modifications du type de formation indépendante des colonies; ils expriment en réalité de façon plus précise la dépendance de la reine vis-à-vis de la caste ouvrière. Mais le type indépendant lui-même n'est qu'un intermède dans la vie sociale des reines et des couples royaux qui le réalisent, puisque les formes sexuées vierges de tous les insectes sociaux demeurent quelque temps dans le nid maternel avant le vol nuptial de dissémination et mènent ensuite une vie latente, jusqu'ì ce qu'ils soient à leur tour entourés des ouvrières auxquelles ils ont eux-mêmes donné naissance. L'insecte social est donc, comme l'homme,, social de façon permanente, les ouvrières durant toute leur ve, et les formes sexuelles du type indépendant aussi, sauf pendant une courte période, au cours de laquelle mûrissent leurs organes reproducteurs. Dans ces conditions, 
il devient forcément très difficile, ou même impossible, de déterminer dans quelle mesure les mœurs d'un insecte, pris irdividuellement, résultent du milieu social où il est plongé et qui agit sur lui de façon constante, ou dans quelle mesure ces mœurs dépendent de mécanismes reçus héréditairement. Cette difficulté est cause de différences dans l'interprétation qu'ont donnée de ces mours les divers savants. Le physiologiste qui étudie les insecles sociaux seulement en lant qu'organismes individuels, isolés expérimentalement de leur milieu social, a tendance à conclure que leurs actes sont entièrement réflexes, sont l'effet de tropismes, (Bethe, I 898 , 1900, 1 902 , et Henning, rgr6) ; tandis que ceux qui les observent dans leur ambiance sociale arrivent à une conclusion toute différente, et, tout en admettant que beaucoup de leurs activités soient réflexes (actes "automatiques" de Forel), ils insislent néanmoins sur les marques indiscutables que donnent ces insectes, de mémoire, d'appétits, d'émotions, d'imitation, et d'une faible intelligence, ou faculté de modifier leurs réactions conformément à des expériences antérieures et aux variations ambianles (acles "plastiques" de Fonel). L'accord général existant sur ces sujets laisse encore une grande place aux divergences cans leur interprétation, suivant la culture, les prédilections et les goûts philosophiques du chercheur. Fonel et Brun, qui ont une formation de neurologistes, sont frappés fortement par la mémoire organique, et font un conslant usage de l'hypoThèse mnémique de Semon el de sa Terminologie en décrirant les mœurs des insectes sociaux. Le père jésuile Wasmav, formé par la philosophie scholastique, opère avec des instincts, des vertus, des facultés (Vermögen) à la manière de saint Trovas n'Aorsis; el, avec celle habileté, 
pour laquelle son ordre est si réputé, à forger des arguments spécieux et à ignorer les faits pertinents, il enveloppe la question tout entière de la myrmécologie et de la myrmécophilie dans un épais brouillard de téléologie, de "Fremddienlichleit ", de sélection amicale, de mimélisme el de casuistique théiste. Ces dernières années, j'en suis venu à éviter de plus en plus le mot "instinct " et à lui préférer le mot "appétit ", ou "appétition ", aù sens de Foulllée (Wheeler r 92 I ). Il n'y a évidemment là aucune nouveauté, puisque ce mot était employé à peu près dans le même sens par les philosophes scholastiques (appetklus sensitivus, "sinnliches Begehrungsvermögen " de Wasmasx). C'est bien ce qu'Aristote appelait ópęıs et le synonyme du " libido " et du " désir " (craving) des psychanalystes modernes.

Pour exposer, comme il conviendrait, les facteurs cenIraux (cérébraux) intervenant dans le comportement des insectes sociaux, en les opposant aux facteurs périphériques (sensoriels ou récepteurs), il faudrait beaucoup de temps et cela n'entre point dans mes plans actuels, qui sont d'insister sur le fait que le milieu sacial est entièrement et nécessairement créé par la caste ouvrière. Cetle caste se développe en si grand nombre qu'elle constitue la presque totalité de la colonie et elle a seule la prérogalive de toutes les activités importantes, nidification, protection, approvisionnement et soins à donner à la reine et aux jeunes, sans compter sa propre subsistance. Ces aclivités sont ininterrompues, sauf dans des conditions climatiques défavorables et s'étendent non seulement à l'intérieur du nid, mais sur une aire Irès étendue que les insectes parcourent pour récolter la nourriture. Ce territoire, qui a un périmètre circulaire et qu'on pourrait ap. 
peler le "champ trophoporique ", est déterminé par les excursions des individus qui font la récolte et il s'accroît nafurellement avec l'agrandissement de la colonie. Le champ trophoporique d'une colonie qui débute, et qui compte un nombre très faible d'ouvrières de petite taille, est extrêmement restreint ; mais les fourrageurs augmentent en nombre et en dimensions avec les couvées successives, et le champ trophoporique s'élargit, jusqu'à ce qu'il atleigne son extension maxima, lorsque la croissance de la colonie est achevée. Sauf quand elles sont peu nombreuses et sporadiques, les champs trophoporiques des colonies d'une espèce ou d'espèces différentes empiètent plus ou moins les uns sur les autres. La quantité de nourriture est donc limitée et l'exploitation du champ est intensive. Aussi toute source convenable de nourriture est-elle scrupuleusement explorée et utilisée méticuleusement. Suivant les habitudes de l'espèce considérée, les ressources nutritives comprennent tous les insectes pouvant servir de proie, tous ceux dont les excreta sucrés peuvent être récoltés (Homoptères), toutes les plantes dont le nectar et le pollen sont accessibles. Bien que les champs trophoporiques des abeilles sociales soient bien plus étendus que ceux des fourmis, les ressources alimentaires sont précaires et soumises à des fluctuations saisonnières très grandes. Cerlains termites fourrageurs (Eutermes, Hodotermes, Termes, etc.) ont des champs trophoporiques plutôt étendus, d'autres en ont de très restreints. Chez les Dorylinés, ces fourmis entomophages si voraces qu'elles ne peuvent demeurer dans des nids permanents, le champ trophoporique se déplace conme celui d'un peuple nomade on chasseur.

Comme l'importance de la colonie est une fonction de 
la quantité de nourriture que peut fournir le champ trophoporique, celui-ci détermine et règle la croissance de la population. Les ouvrières sont-sans cesse occupées à recueillir le plus de nourriture et à élever le plus de jeunes possible. Aussi pratiquent-elles l'économie et l'épargne à un haut degré, surtout quand les champs trophoporiques de plusieurs colonies se superposent. Les formes sexuées et surtout les reines, qui exigent une quantité considérable de nourriture durant leurs stades larvaires, ne peuvent être élevées que pendant la saison la plus favorable de l'année. Des conditions trophiques défavorables poussent l'économie à un tel point que la couvée peut être dévorée toute entière ou en partie, et que des portions de la population adulte sont sacrifiées ; ainsi sont massacrés les faux-bourdons chez l'abeille domestique, ou les soldats chez certaines fourmis du genre Pheidole. Notre Ph. militicida de l'Amérique du Nord récolte des graines, et ses soldats à grosse tête sont employés à les broyer; mais quand vient l'hiver et que les services de ces broyeurs de graines deviennent inutiles, ceux-ci sont décapités, et leurs restes sont jetés hors du nid. Naturellement la caste ainsi éliminée sera rapidement reconstituée avec de jeunes larves, au printemps et à l'été suivants.

Les ouvrières de tous les insectes sociaux sont bien des formes inanitiées, comme le montrent leur voracité, leur stérilité, leur développement atrophique et, surtout, chez les fourmis et les termites, leur apparence d'adultes rachiles fourmis of les termites, leur apparence d'adultes rachiliques et apleres. Flles sont affamées et voraces comme un prolétariat stérile, qui n'a aucun intérêt à produire, maís 
qui trouve un vif intérêt à élever les larves et à assurer la nourriture nécessaire pour mener à bien tous les travaux.

$$
\text { ** }
$$

Il est évident que le milieu social comprend non seulement les activités habituelles des ouvrières dans le nid, et principalement la récolte de la nourriture et sa répartition entre ces ouvrières, les reines, les mâles et les larves, mais aussi les rapports avec les insectes el les plantes fournissant la nourriture dans le champ trophoporique, et avec les divers hôtes qui sont quelquefois présents à l'intérieur du nỉd même, en tant que parasites sensu latissimo. L'absence fréquente de ces derniers, leur petit nombre, quand ils sont présents, leurs relations exclusives avec les ouvrières qui sont normalement stériles, tous ces faits conslituent des objections presque insurmontables à l'idée de Wasmax, qui altribue à des "instincts " blastogéniques spécifiques la conduite des ouvrières vis-à-vis de leurs commensaux parliculiers. On peut admettre, soit qu'il y a là une hérédité de caractères acquis, - et ceci est excessif - à travers les màles issus d'œufs pondus à l'occasion par des ouvrières; soit que leur conduite vis-à-vis des commensaux est une modification purement ontogénique ou une perversion des habitudes threptiques coutumières que suivent les ouvrières à l'égard les unes des autres, ou ì l'égard des reines, des mâles el des jeunes. Je soutiens que cette dernière hypothèse est la plus probable, la plus économique et la seule qui soit en accord ¿troil avec les faits généralement observés dans les habitudes organiques. Nême dans l'étude des mœurs humaines, beaucoup mieux connues que celles d'aucun 
animal, la tendance actuelle est de réduire le nombre des postulats "instinctifs ", à un minimum, ou à les éliminer complètement par un examen sérieux (I).

Dans un mémoire publié en $19 \mathrm{r} 8$, j'ai essayé de souligner l'importance des habitudes trophiques et threptiques des insectes sociaux et de construire, à partir des nombreux faits disparates, une conception générale à laquelle j’ai appliqué le terme de trophallaxis (de iposn nourriture et jiv่jso(1), j'échange). Celle notion a été favorablement recue par les physiologistes et les éthologistes, mais elle a été traitée d'innovation scandaleuse par les Jésuites, labricants d’instincts (1).

(1) En examinant te dernier mémoire de hico (192.1), on yeut glaner quelques indications sur les conceptions que se font des instincts et de leur hérédité les élhologistes de laboratoire. Quoiquexrrimées dans un langage excessif, les vues de l'auteur ont une valeur indubitable, car elles fixent l'attention sur les assertions extraordinairement imprécises, peu scientifiques et dogmatiques de Wisminx et d'autres, en ce qui concerne les bases héréditaires des mœurs.

(2) Je pouvais mattendre it voir mes eflorts, comme tous les essais de ce genre, mal compris ou mal interprétés; mais je n'avais pas conscience de ma profonde ignorance concernant la biologie des Formicidés et de leurs hòtes. Dans un traité de 176 pages, le Père Wasmavi (1920) a promptement démontré ma depplorable ignorance et, comme si je m'étais aussi rendu coupable du péché mortel d'orgueil, il a très modestement placé comme devise sur la converture de son écrit polémique les lignes bien connues de "Faust " "IV'as glänzt ist für den Augenblick geboren, Das Echte bleibt der Nachwelt unverloren ". Le premier vers se rapporte, évidemment, à ma fille la Trophallaxis, le second sux propres rejelons immortels de Whsmaxx, les "Inslinels symphiliques \#. Comme on craignait dans le camp de la Societé de Jisus que son obusier ait manqué le but, j'ai subi quelques tirs de mitrailleuses dans les mémoires de l'Académie pontificale et romaine des "Nuovi lincei » (1923 b). En mème temps, Herr Reichensperger accourait à l'aide de son mentor outragé, et, avec sa propre bien 
Nous avons vu que la colonie ou société d'insectes peut ctre regardée comme un superorganisme, donc comme un tout vivant et tendant à assurer son équilibre mouvant et son intégrité. Les individus composant la colonie doivent donc être en communication les uns avec les autres. L'exactitude de cette affirmation ressort bien de l'observation d'une société quelconque d'insectes, mais les méthodes de communication employées diffèrent tellement les unes des autres que leur interprétation précise et même leur détection présentent d'énormes difficultés. Sans aucun doute, les insectes conmuniquent au moyen de signes, c'est-à-dire par des mouvements du corps et de ses appendices, des antennes en particulier (Forel, Wasman, ('rawiey ( $g$ gob), Doflety (Ig2o), etc.), par des vibralions, ou stridulations (Wheeler, rgy 4 ), des odeurs (vov Friscu, 1 $9^{2} 3$ ) et des saveurs. Tout ceci appartient à la catégorie biologique générale des stimuli et des réponses, et, comme les actes sont en grand rapport avec la nourriture et sa distribution, les stimuli sont principalement olfactifs et les réponses dépendent des chémorécepteurs et des appétits. Bien des critiques soulevées contre la tro-

veillance toute chrétienne, émeltait un petit nuage de gaz asphyxiant (1921), sous forme de commentaires à ma "recht liindliche Analogiebeweise ", etc. Tout ceci montre combien il est dangereux de mettre en doute même les dogmes biologiques des révérends pères et de se permettre de plaisanter leurs élucubrations favorites. Comme je n'ai pas célébré les charmes de la Trophallaxis dans tous les mémoires écrits depuis 1918, Wisman conclut que je dus l'abandonner comme un pauvre enfant mort-né. Ici sa perspicacité lui fait certainement défaut, car je suis si éloigné d'une telle intention que je me propose de consacrer à ce sujet le reste de celle leçon et quelques parties des deux suivantes, afin de montrer que c'est lá un enfant mieux portant et plus viable que je ne l'avais supposé. 
phallaxis sont immédiatement dissipées, si l'on fait ressortir qu'en employant le mot " nourriture ", je ne désigne pas seulement la matière même introduite dans le tube digestif et assimilée, mais aussi son rôle comme stimulus, ou comme excitant des chémorécepteurs. Ce dernier rôle a évidemment une signification majeure dans les organismes sociaux.

Je n'avais pas exposé des considérations aussi évidentes dans mon mémoire sur la trophallaxis, parce que je l'avais écrit pour les biologistes et non pour des théologiens. Ces derniers, en effet, reconnaissent deux sortes de nourriture, - une licite, qui est employée seulement dans la mesure nécessaire pour assurer la conservation du corps et de l'âme, et une autre qui est stimulante et peut être considérée comme "Leckerbissen " (friandise) et mène à la gourmandise ("Naschhaftiglieit ") ; c'est cette distinction que Wasmans a introduite dans la discussion sur les mours des insecles, comme un argument de poids contre la trophallaxis, après que j'eusse expressément indiqué (1918a) que les substances échangées ne sont pas quantitativement égales, par exemple dans des cas comme Formica sanguinea et son commensal Lomechusa, mais que les exsudiats du commensal, bien que produits en très petite quantité, peuvent néanmoins susciter des réactions intenses. On croirait que la nourriture servie dans un réfectoire de Jésuites doit être moins savoureuse que celle qui se sert dans un restaurant de cinquième ordre à New-York.

D'autres critiques ont imaginé que, par trophallaxis, j'enlendais, non seulement un échange de quantités égales de nourriture comme simple aliment, mais un échange nécessairement et immédiatement réciproque. D'autres 
encore, comme Monstatr (1922), croient que la trophallaxis peut se surajouter au soin de la couvée (Brutpllege), quoique je considère cel acte comme la manifesation d'activité la plus primitive et la plus caractéristique. Kemser ( $g_{2} 3$ ) a également critiqué la trophallaxis, en se fondant sur quelques observations intéressantes mais mal interprétées, faites sur un phalène myrmécophile de Java. Je discuterai ce cas dans la prochaine leçon. Parmi les entomologistes, Bron ( $9_{2} 23 \mathrm{~b}$ ) parait ètre le seul à avoir saisi le véritable sens de mon mémoire. Sa connaissance de la psychiâtrie l'a rendu capable de montrer que la trophallaxis comprend le principe de "plaisir " en tant qu'opposé au principe de "réalité », et d'attirer l'attention sur le rôle déterminant que joue le premier, mème dans la vie des insectes sociaux. Puisque tel est l'état des opinions concernant la trophallaxis, je m'excuse de passer rapidement en revue les faits concluants. II me faudra y ajouter quelques considérations, dont jespère qu'elles préviendront les objections vraiment sérieuses, tout en rendant ce principe d'une plus grande utilité pour l'explication et la découverte des faits.

La trophallaxis s'observe très aisément chez les guêpes, les fourmis et les termites. Du Bursson (1903) el Janet (1903) furent je crois, les premiers à montrer que les larves de Vespa el de Polistes, après avoir été nourries par les ouvrières, ou lorsqu'on excile leurs parties buccales, secrètent, par leurs glandes salivaires, des gouttes d'un liquide clair, ou douceatre, qui sont aussilot absorbées avidement par leurs nourrices. J'ai ohservé le mème fait à plusicur's reprises chez. notre Polistes mefrica de l'Amérique du Nord. C'est Roubıud $(1916,1924)$ cependant, qui lit, le premier, une étude détaillée de l'alimentalion réciprofjue chez certaines guêpes africaines (Belonogasler) et qü en saisit la signification sociale. Il appela cela "acotrophobiose ", lerme qui me parait gauche el peu adéquat. Je l'ai done remplacé par le terme " trophallaxis ". Dans son dernier 
mémoire (1924), Roubıun a adopté le mot " trophœecisme 》 et a, par suite, appelé la colonie de guêpes un " trophoecium ». Dans mon etude des larves de fourmis $(1918$ a), je mentionne un certain nombre de phénomènes quion ne peut interpréler aulrement que conme la trophallaxis des Vespidés. Chez cerlaines Ponérinés (Pachycondyla), qui sont des fourmis primitives, analogues aux guêpes, les larves, lorsqu'elles sont nourries, produisent une sécrélion salivaire que les nourrices absorbent en la léchant. Certaines larves de Myrmicinés

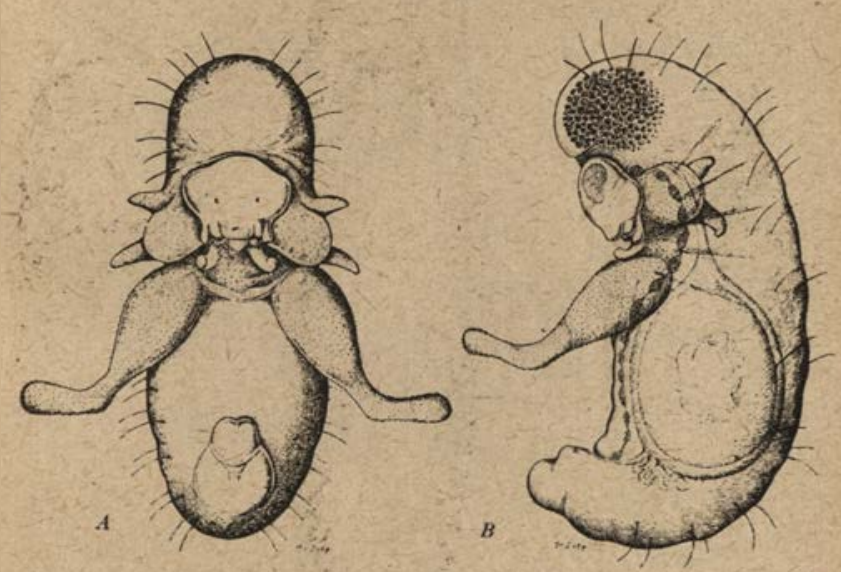

Fig. 39. - Pachysima latilrons (d Ethiopie) 1er stade larvaire (trophidium). A, vu ventralement; B, dorsalement, montrant les exsudaloires (appendices entourant la tête et appartenant aux trois segments thoraciques el all premier abdominial).

(P'oxlutyus) ont d'enorines glandes salivaires, dont la sécrélion ") tris probablement une fonction analogue, puisqu'elle n'est pas utilisée pour filer un cocon, une enveloppe de ce genre "chant jamais fabrifure par aucune des espèces de celte souslamille. Chez les litrves de toutes les Pseudomyrminés, lit lonche est enlourée d'une grappe de papilles particulières, ou d'appendices qui produisent cerlainement des exsudats, très 
analogues à ceux de certains ternilophiles (Spirachtha). Ces formations sont extrêmenent développées chez les très jeunes larves (trophidia) de deux especes du genre africain Pachysima (wethiops et latifrons), mais, au cours des derniers stades, clles
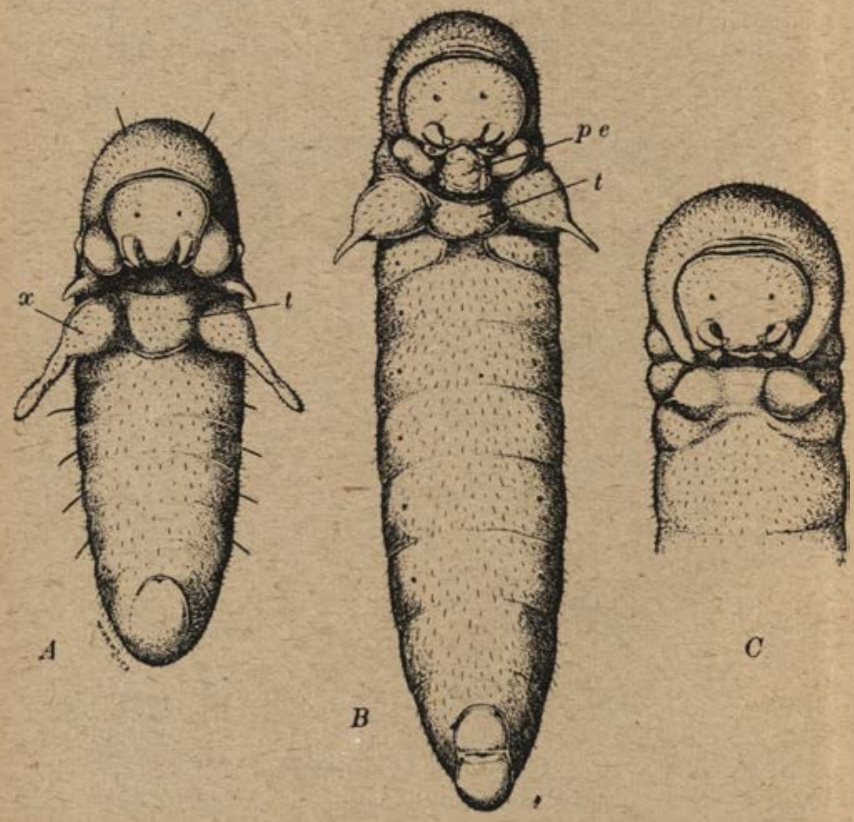

Fig. 40. - Pachysima latilrons. A, B, C, 2e, $3^{\text {e }}$ et qe stades litraires, montrant la régression graduelle des exsudatoires $x: p e$, pe'ote nulritive dans le trophothylax $t$.

décroissent rapidement, quand se développent les glandes salivaires, qui doivent probablement s'emparer de leur fonction sccrétoire. En outre, toutes les larves de fourmis accumulent plans leur corps une énorme quantité de graisse, dont une par- 
tie exsude ḋ traver's l'épaisse cuticule chitineuse que lèchent souvent les fourmis ouvrières. La surface des larves de fourmis est, en fait, toujours recouverte par une substance lipoïdique hydrofuge, qui doit provenir du sang ou des masses graisseuses. Les observations de Pantel (1909), Rocb.ıu (19:1) et d'autres, sur les jeunes larves de Tachinidés montrent bien que des substances de ce genre peuvent-bien traverser une chitine assez épaisse. Bien que ces larves soient entièrement enveloppées dans un $\mathrm{sac}$ de chiline produit par la région interne des trachées de leurs hòtes, elles se procurent néanmoins leur nourriture, par filtration ou osmose, à partir du sang de l'hòte. Il est donc probable que toutes les larves de fourmis produisent de petites quantités d'exsudats, qui sont bien appréciées par leurs nourrices. Mais même si cela n'est pas certain, on peut admeltre que la trophallaxis se produit d'une façon générale chez les fourmis, puisque la reine, après avoir élevé sa première couvée, est nourrie avec des aliments régurgités par celle-ci, c'est-d-dire par ses descendants ayant alteint leur état d'adulte. L'emploi quasi universel de celle forme d'alimentation réciproque par les ouvrières des fourmis sunérieures (Myrmicinés, Dolichoderinés el Formicinés) est un fait trop hien connu four quil soit nécessaire de le dévelopjer davantage. Nais, en outre, je considere comme indubitable que les fourmis adultes produisent aussi des exsudats graisseux ou des sécrétions glandulaires attractives et que l'habitude qu'ont ces insectes de se lécher les uns les autres peut ètre altribuée à la présence de substances de ce genre sur leurs corps et non a un sentiment affectueux ou à un goùt particulier pour la propreté. Les opérations de toiletle auxquelles se livre chaque fourmi n'obéissęnt pas à de tels motifs, mais sont des réactions réflexes suivant l'irritation que cause un corps étranger a la surface du corps ou des appendices.

L'échange mutuel d'aliments liquides trouve son expression li plus frappante chez les fourmis à miel, lesquelles appartiennent, comme je l'ai montré $(1908 b, 1914,1923)$, à un certain nombre de genres el d'espéces confinées dans les régions désertiques ou a température sèche, où l'aliment liquide est chose rare. Habituellement, certaines ouvrières accumulent les aliments dans leur jabot, qui est lellement distendu que l'abdomen devient très vaste et sphérique (Myrmecocystus, Camponotus inflatus, Melophorus bagoti), mais, dans beaucoup d'espèces de Pheidole, Pheidologeton et Oligomyrmex, les soldats se comportent comme des pols à miel et l'abdomen s'agrandit de facon moins visible. Il y a plusieurs années, EMEry (1898) a 
observé, chez les ouvrieres de rertaines fourmis dẹ déserls de l'Isic mineure et du Nord de. J. Afrique (Crmmonolus fedschenlii et aflontis), un développement parliculier des masses graisseuses abrominales. Il observa que celte "adipongustric ", comme il l'appela, étail plus dévelopjée chez les espéces nocfurnes et que celle wraisse represente des aliments emmagitsinés, non seu'ement pour la subsislance de l'ouvrière ellemême, mirs aussi jour celle des litres. apres que ces allimenls

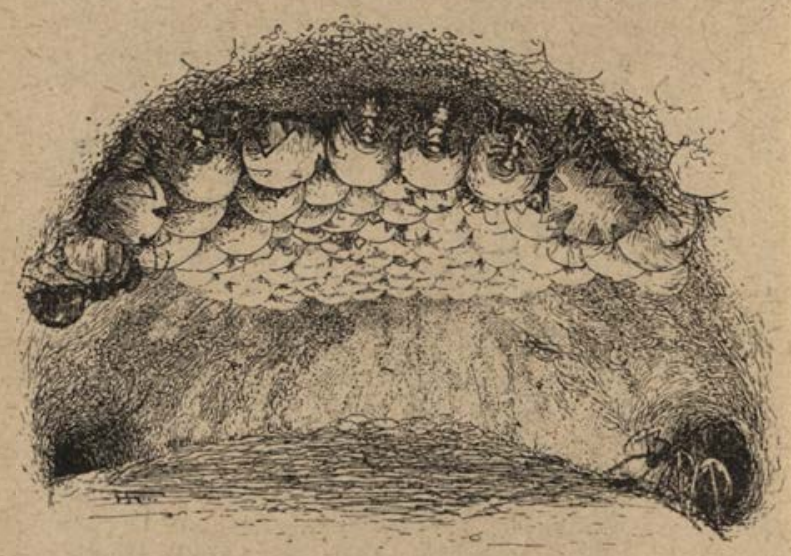

Fig. 41. - Chambre à miel de la fourmilière de Myrmecocyslus horti-deorum (d'après Mc Conk). Les ouvrières en réplétion sont suspendues à la voute rle la chambre.

ont été métabolisés. Une auipogastrie très similaire s'observe souvent chez beaucoup de fourmis hypogéiques, spécialement chez les Lasius de l'Amérique du Nord, appartenant au sousgenre Acanthomyops, qui vivent presque uniquement de Coccides et d'Aphides des racines. Les formes noclurnes jaunes de Camponolus castaneus en Nouvelle-Angleterre et de C. Maculalus des déserts de l'Arizona présenteraient aussi la même adiposilé (" Fellleibiglieit »). Comme l'a montré Ottramare (1919), il y a évidemment un lien très significatif entre l'absence de lumière 
el l'accumulation de graisses. Ceci apparail aussi dans la phyangastrie graisseuse des termites et des termitophiles.

II y a longlemps de cela, Ginssi a decrit le développement Ires élendu de la trophallaxis chez les termiles, et lous les cher-

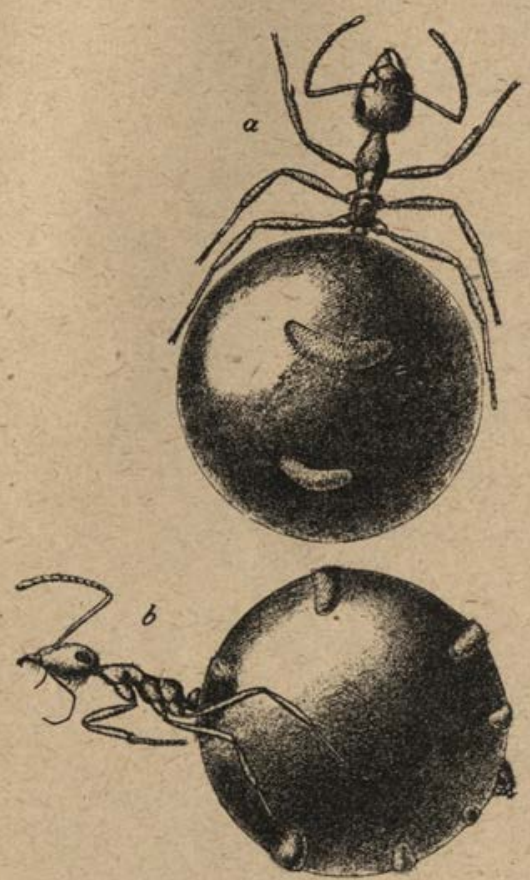

Fig. 12. -- Fourmi à miel, Myrmecocystus horli-deorum (d’après VC Соок). Ouvrières ¿̀ l'état de réplétion (vues dorsalement. et latéralement).

cheurs qui l'ont suivi ont confirmé ses observations. Les membres de la colonie se nourrissent l'un l'autre avec de la salive, rles aliments partiellement digérés et régurgités (stomodéaux), 
et des excréments ou scybalum (aliments proctodéaux). Cette singulière coprophagic a jour lésullat l'infection mutuelle des ouvrieres par les Protuzoaires symbiotiques, gui digerent le bo:s. Comme nous l'atons dit antériemrement, Holmguen (1909); a montré que toules les castes de termites, mais surtout les reines, ont de volumineux lissus i exsudats, formés par les couches périphériques des masses graisseuses abdominales. Les trophocytes de ces couches ne contiennent pas de graisse, mais do nombreux granules minuscules qui sont rejelés dans le sang et se convertissent en un exsudit qui traverse les nombreux poils très fins, ou lacunes, de la cuticule chitineuse, jusqu'd la surface, où ils sont léchés par les membres de la colonie. L'étendue de ces lissus a exsudats differe considérablement, non seulement dans les diverses castes, mais aussi aux diver's degrés de leur développement, et nous avons vu dans la leçon précédente que, d'après Howankx, la différenciation des castes suit l'absorption ou la non-absorption d'exsudats. Escherich (1911) a donné un récil encore plus vivant, pour ne pas dire plus imagé, de la voracité des termites. Chez les Termes rede. manni de Ceylan, les ouvrieres sont si avides des exsudats de leur grosse reine physogastrique, qu'clles vont jusqu'a déchirer rle petits lambeaux de sa culicule, afin d'atteindre plus aisćment le liquide. Lischerich observa que les reines âgées ont parfois leur abdomen blanc tacheté de petites cicatrices brunes, provenant des blessures que leur a ainsi infligées leur progéniture. Dans ce cas, le mode d'alimentation de la mère ct des enfants est linverse de celui qui se pratique dans les colonies de fourmis à leur début, puisque les reines sont nourries avec des aliments régurgilés par les ouvrières et nourrissent ces dernières avec leurs exsudals; mais il en est trés probablement de même dans les colonies de fourmis développées, quand les ouvrières sont venues à maturité et que la reine ne nourrit pas plus longtemps sa couvée.

II est plus difficile de mettre en évidence la trophallaxis chez les abcilles sociales, mais le cas n'est peut-être pas aussi désespéré que je l'avais supposé $(1918 a)$. Les abeilles ouvrières adultes nourrissent la reine et se nourrissent entre elles du miel régurgité (von Fisch, 1921), mais on ne parait pas avoir jamais observé si c'est là une pratique constante chez les. Bombinés et les Méliponinés. Lineburg (1924) a fait une étude altentive de la larve de l'abeille domestique, dans le but de s'assurer s'il existe dans ce cas une alimentation réciproque, comme chez les guêpes el les fourmis. "L'alimentation réciproque, dit-il, n'a jamais cté observée chez la mouche à miel, mais comme la 
mourriture donnće aux larves igées nest pas dun caractere complexe el nexige sans doute pas beaucoup de travail, de la part des nourrices, lour son claboration, il est difficile d'expliquer le lemps considerable que passent les nourrices danf les cellules, en croyant simplenient que lout ce temps est employé a donner aux larves leur nourriture. Comme on ne trouve jamais d'alimenls en quantité appréciable sur le fond ou sur les cotis des cellu!es de larves itgées, on peut penser que, diune fitcon générale, l'alimentation se fait directemenl par la bourhe de la larve. En déterminant le poids des résidus de nourriture trouves dans les cellules, on a constaté quï: est très constant pour les divers ages. Si on a la hardiesse d'admeltre l'existence de l'alimentation réciproque, celle-ci pourrait peutitre rendre compte du dévelopuement particulier de l'abcille ouvriere, de la régression de ses organes sexuels el de ses modifications morphologiques. "Ces remarques montrent que lalimentation réciproque entre les ouvrières et les larves d'ouvieres, chez labeille domestiqur, n'est pas absolument improbible. Les ouls et les larves d'ouvrieres possedent certainement quelque puissance diattraction, car les ouvrieres leur rendent d'innombrables visites et passent dans les cellules un temps considérable. " En prenant la moyenne des risultats trouvés lour des oeufs el des larves de lous iges, on a vu que celle moyenne s'éleve it plus de 1.300 visites en vingt-quatre heures. Le dernior jour avant la fermeture, les abeilles nourrices ne font pas moins de 2.85 visites a une seule cellule ; et ce jour-lä, les ouvrieres passent environ quatre heures trois quarts it l'intérieur de la cellule... Plus de 10.000 visites sont faites par chaque abeille adulte an cours des huil jours qui s'écoulent. entre la ponte de l'ouf el l'occlusion de la cellule. »-

Dans une leçon précédente, j'ai attiré votre attention sur la sécrétion de la cire chez les abeilles sociales et sur celle d'une substance analogue chez certaines espèces solilaires (Tetralonia, Eucera, etc.). La cire, qui est en majeure partie constituée par un palmitate de myricile, est véritablement un exsudat lipö̈lique lit aux graisses (.Iatuews, I j? I). Sa production serait peut-être le ré. sultat de la grande absorption de sucre par les abeilles, puis(gue des exsudats semblables sont produits par les 
Coccides, les Aphides, les Fulgorides, etc. qui vivent de la sève des plantes ; ils ingèrent tant de sucre qu'ils en éliminent eux-mèmes en grande quantité dans leurs excréments. Ce qui est assez curieux, c'est que beaucoup de larves de Coccinellidés (par exemple Brachyacantha), qui mangent exclusivement ces Homoptères, produisent aussi en abondance des exsudats cireux (WheELer, ig I Ib). Suivant Friess (1923), les exsudats graisseux des abeilles solitairess "représentent de toute évidence une énergie en excès dérivant des aliments et qui distend le corps de la larve, sous forme du corps adipeux blanchâtre, avant la nymphose. Cette énergie chimique emmagasinée qui est utilisée dans les trophocytes pour conserver la vie de la nymphe et, pendant quelques années, celle de l'imago, n'est en général pas entièrement consommée mais produit les exsudats graisseux déjà signalés chez les abeilles solitaires tuées depuis peu de temps. Ces exsudats ressemblent aux u matelas d'une subslance analogue à la graisse ou a la cire ") que l'on trouve entre les quatre segments médians, sur la face dorsale de l'abdomen de l'abeille à longue corne, Tetrulonia ruficollis $"$ ( $\mathrm{r}$ ).

Bien qu'actuellement la cire soit employée exclusivement pour la construction des alvéoles dans lesquels les

(1) En connexiou avec le fait que, seules, les jeunes mouches it micl secrètent la cire, Friese remarque: "Nous voyons donc; quion a ajouté une faible attention au lien organique existant contre les masses griisscuses de la larve et la production de rire de l'abeille adulte. Je suis d'avis que la sécrétion de cire difend entierement de la yuantite d'energie fournie par les yraissas de lat larve. En se plaçant à ce point de vue, on peut aisénent expliquer les conditions dans lesquelles se trouvent ley abeilles àgces, en ce qui concerne la production de cire, comme alussi l’origine phylogénétique des écailles et des glindes i cire. ") 
larves sont élevées et les aliments emmagasinés, on peut se demander si elle n'était pas utilisée, dans une certaine mesure, pour la nourriture dès larves chez les ancêtres immédiats des Bombinés, Méliponinés et Apinés. Deux des anciens, et au moins l'un des modernes observateurs des bourdons (SWammendam 1737 , Ríacmur 1742 et Haerter, i $89^{\circ}$ ), ont en effet affirmí que la " pàte de pollen ", ou mélange de pollen et de cire, dans laquelle la reine dies Bombus pond ses œufs, est dévorée par les larves. Cependant, la plupart des chercheurs récents ont été incapables de confirmer cette observation. Comme la larve de la mite des ruches, bien connue, (Galleria mellonella) peut digérer la cire, bien qu'elle ne puisse achever son développement sans l'addition d'autres substances (Dönioff 1882, et Sieber et. Metaliikow 1904, BiederIANN I9I I), il n'est pas invraisemblable que les larves de hourdons soient capables de produire quelque ferment apte à rendre assimilable la cire. Ce serait de la spéculation pure de supposer que les ancêtres des trois groupes les plus élevés d'abeilles sociales auraient nourri leurs larves avec des exsudats graisseux, mais nos connaissances sur les habitudes cachées de ces insectes sont si rudimenlaires, qu'il n'est pas mauvais d'attirer l'attention sur cette possibilité.

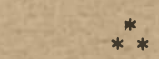

Ftant donnée la grande place que tíent; chez les divers insectes-sociaux, la trophallaxis, ou échange de nourrilure, le principe est suffisamment élastique pour couvrir $u n$ nombre de phénomènes beaucoup plus grand enrore, si nous l'élendons, des substances qui sont socia- 
lement excilatrices par les récepteurs gustatifs, à celles qui affectent les autres sens chimiques, l'olfaction en particulier. A première vue, ceci paraît improbable, sinon absurde, mais je crois que les remarques suivantes indiquent qu'il y a de nombremses raisons de concevoir le goût et l'odorat, chez les insectes, d'une façon très différente de celle qui est communémenl retenue, pour ces seus, par la physiologie de l'homme et des mammifères. Chez les mammifères, les récepteurs gustatifs et olfactifs sont nettement séparés dans leur structure et dans leur position, bien que nous sachions parfaitement que l'olfaction infervient très fortement dans ce que nous appelons les sensations du goût et on sait que, chez certains poissons, les papilles gustatives sont dispersées sur la surface dı corps (I).

Dans les deux genres de récepleurs, les terminaisons nerveuses sont affectées par les substances chimiques se dissolvant dans la couche liquide ou muqueuse qui les recouvre. On a l'habitude d'appliquer aux insectes notre

(1) Parmi les poissons, Hrrrick (1903) a relevé plus de 35 espèces, chez lesquelles des papilles gustatives ont été trouvées à la surface externe de l'animal aussi bien qu'à l'intérieur de la bouche. Le poisson-chat, Amiurus, est remarquable- à cet égard, en ce que la totalité de la surface externe y est munie de tels organes, qui sont abondants en particulier sur les barbillons. Lorsqu'une parcelle de viande est mise en contact avec les barbi'lons id'un de ces poissons, l'animal cherche aussitôt à s'emparer du morceau et l'avaler. Il en est de même quand l'aliment est mis en contact avec le flanc du poisson (PAnKER, 1922). Herrick a démontré que les réactions produites lor squ'on laisse écouler d'une pipette du jus de viande contre le flanc du poisson, sonl dûes au sens du goût et non au toucher, et Paniran (1912) a montré qu'après lésion du septième nerf crânien qui innerve les papilles gustatives dans le flanc de l'Amiurus, les réponses aux excitations gustatives cessent vite de se produire. 
nolion des récepteurs olfactifs et gustatifs distincts ; pourtant, mème chez les Verlébrés, le goût n'est pas un sens chimique unitaire et, chez les insecles, on ne peut le distinguer de l'odorat par la structure des organes de ces sens.

Nous avons tout simplement pris l'habitude de considérer les chémorécepteurs (sensillo) comme gustatifs dans la région buccale et comme olfactifs sur les antennes. Mais Mrisici (1921, 1922 a, I922b, 1924) a récomment prouvé, par des expériences conduites avec le plus grand soin, que les papillons goûtent avec leurs pattes (avec les quatre porlions terminales, tarsales et distales, des articulations du basitarse des seconde et troisième paire de pattes). Cette forme de réception des sensations est probablement très fréquente chez les insectes. Il est de même inutile de distinguer des récepteurs à distance pour l'olfaction et des récepteurs par contact pour le goût, car les insectes utilisenl leurs antennes d'une façon et de l'autre, comme pour les sensations tactiles.

L'étude des chémorécepteurs des insectes est troublante et déconcertante, à cause de la diversité des appareils sensoriels et de leur large distribution sur le corps. Et la confusion s'accroit du fait des divergences d'opinion, à l'égard de leur structure, qui parcourt tous les degrés depuis les sensillir, qui peuwent chte ou tactiles ou olfaclives, en passant par dles sensilla très diverses qui sont probablement olfactives et guslatives, jusqu'aux sensilla de Hicks, qui ont êté interprétées de façon variable, comme des organes sensoriels pour la pression, la temperature, l'humidité ou les vibrations. Tandis que beaucoup d'auleurs croient que les organes sensoricls ollictifs sont localisés exclusivement ou en grande partie dans les antennes, MAC INDoo (1914 $a, 1914 b, 1914 c, 1915,1916,1917$, $1918,1920)$ pense, pour quelque raison inconnue, que les orgitnes olfactifs sont réparlis sur presque toutes les régions dii corps, sauf les antennes, et il croit avoir fourni lit preuwe. expérimentale indéniable de celte assertion. Berlese (1909), 
dans un Iravail remarquablement clair sur les organes de l'olfaction, a décrit ccux-ci comme contenant toujours des cléments glandulaires en plus des cellules sensorielles. Il rappelle les anciens mémoires d'Enichson (1817) el de Saulcy (1891), qui trouvèrent sur les antennes des insectes une mince couche de liquide. Suivant Berlese, celte pellicule est la sécrétion des éléments glandulaires et forme avec la subslance olfactive une solution qui agit sur les terminaisons des cellules sensorielles. Il est, en effet, difficile de concevoir comment l'olfaction peut se produire sans un tel solvant. Hochneuter (1912), DeEgenen (1912), Dewoll (1917), MAc Indoo, elc, interprèlent les éléments glandulaires de BenLese comme des cellules sensorielles et ne disent rien ou presque rien au sujet d'un liquide olfactif ou de son origine. A certains égards, les études physiologiques sont plus satisfaisantes que les morphologiques el permetlent presque sùrement de croire que les chémorécepleurs sont largement distribués sur les antennes, la région buccale et peut-être d'autres portions des téguments et que les réactions aux substances chimiques, soit a distance, soit par contacl, sont de beaucoup les plus importanles réactions sensorielles des insectes. Ceci est vrai surtout pour les espèces sociales, et de nombreux chercheurs ont montré que beaucoup de leurs actes sont déterminés par de telles réactions. Chez les fourmis, les abeilles et les termites, des odeurs d'individu, de casle, de colonie, d'espèce et de nid ont pu être distinguées expérimentalement, par Fonel (1910 a), Bethe (1898), Brun (1917), Wasmann (1909 a, Miss Fielde $(1904,1905)$ et autres. L'odeur de la couvée des fourmis, surlout des nymphes et des cocons, est nettement perceptible, mème pour le grossier odorat humain. Beaucoup de fourmis adultes ont une odeur intense, souvent très différente de celle de l'acide formique; ainsi par exemple, Acanthomyops, Crematogaster, Eciton, Megaponera, beaucoup de Pheidole, Tapinoma, Atta, etc. Il en est de même aussi pour les abeilles et les termites. Les violentes odeurs des Méliponinés sont souvent très agréables; certains Trigona néotropicaux sentent la noix de coco, d'autres le citron, d'autres la rose, etc. Le siègede l'odeur, chez l'abeille domestique, paraît avoir bien été localisé dans la glande de Nasson off [SlAdEN (1902), von Friscr (1923)], entre les cinquieme el sixième segments abdominaux. Chez les fourmis, l'odeur provient peut-être des glandes épinotales spéciales, décrites par JANET (1898 a, 1898 b), qui existent dans toules les castes et qui sont admirablement construites pour produire, conduire à l'extéricur et volatiliser les sécrétions 
odoriféranles. D'autres glandes comme les glandes à poison ou les glandes à acide formique des formicinés et les glandes anales des I)olichudérinés (Tapinoma, Asleca, etc.) produisent des sécrétions à ondeur tris forte, el, d'aprés Fonel, il en serait de mème pour certaines glandes de la tète. On sait depuis Iongtemps que les substances odoriférantes sont aisément absorbées el retenues par les graisses. Toute ménagère est accoulumée à cette propriété du.beurre, et, dès les temps les plus anciens, des onguents ont été utilisés comme véhicules pour des parfums. On emploic encore les graisses et les paraflines dans "l'enfleurage ", procédé d'extraction des parfums de fleurs. Evidemment, l'enduit lipoüdique des surfaces des fourmis, larves, nymphes ou adultes, et le revêtement pileux si dense des abeilles relient aisément les odeurs de l'individu et celles qui lui viennent du dehors, ou du nid, el retardent leur diffusion.

Les sécrétions glandulaires des insectes sociaux sont cerlainement émises en plus grand volume sous l'influence d'une excitation; mais les odeurs permanentes des individus, des castes, des colonies ou des nids étant des moyens importanls de reconnaissance et de comınunication, il n'y a aucune raison pour ne pas considérer les odeurs, aussi bien que les stimuli gustatifs, comme trophallactiques. Dans un livre très intéressant, von Friscu (1921) a montré récemment que les abeilles domestiques emploient vruiment pour communiquer les odeurs adventices dı nectar et du pollen adhérant à leur corps. Quand les abeilles chargées de nectar retournent à la ruche, elles se livrent à une danse particulière ("Rundtanz»), qui attire liallention des ouvrières inactives et il en est de même pour les abeilles revenant chargées de pollen. Ces dernières exé"utent une danse différente ("Schwänzeltanz»). D'après les deux cas, les individus inactifs sont informés de l'espèce des lleurs qui ont fourni le nectar et le pollen en abondance, et sont stimulés à prendre leur vol à leur recherche. Les abeilles déposent aussi parmi les fleurs les odeurs de la glande de Nassonoff, et les individus qui font la récolte peuvent ainsi plus aisément diriger leur vol. Feu M. F. W. L. Stanev, une autorité célèbre en matière de Bombinés, me fit voir un jour au Canada, comment les mâles de certains de nos bourdons découvrent les femelles vierges. Ces dernieres volent dans les creux des racines de grands arbres el y déposent une odeur particulière, nellement perceptible mème par l'odoral humain; celte odcur est si lourde et diffuse si lentement que les mâles peuvent la suivre d'arbre en arbre el atteignent ainsi leurs 
épunses. Le même fait s'olserve aussi chez les màles el les femelles des abeilles solitaires, sauf que les odeurs sont dépovées sur les fleurs ou le feuillage, non dans les cavités des troncs d'arbrc. Tous ceux qui ont chissé des femelles d'Andrena sont familiers avec leurs odeurs violentes, qui semblent émaner des grosses glandes des fovear faciales, dont j'ai parlé dans une leçon précédente.

Il est peut-être exact que, dans mon mémoire de I 918 , jai négrligé d'insister suffisamment sur le rôle de l'olfaction dans les rapports réciproques des insectes sociaux. Il semblerait pourtant, d'après l'exposé précédent, que c'est une question purement scolastique que celle de savoir si une fourmi ou une abeille goûte ou sent avec ses antennes, sa nourriture, une larve, une nymphe, ou une autre fourmi on abeille; cetle question n'a, en tous cas, qu'une signification physiologique bien faible. Puisque les termes de " govît " el d' " odorat " sont entachés d'anthropomorphisme, el que, dans les deux cas, les stimuli sont chimiques, pourquoi n'emploierait-on pas plutôt le mol de "chémoréception "? Et puisque, en outre, les stimuli alimentaires sont nécessairement chimiques, je ne vois pas de raison de changer le terme de "trophallaxis ", parce que heaucoup des actes des insectes sociaux sont, comme nous avons dit, "olfactifs". Le fail que certains de ces actes soient dépendants de stimuli autres qu'alimentaires, n'est pas non plus un argument valable contre la trophallaxis, car je n'ai jamais affirmé que celte trophallaxis comprit toutes les activités sociales. Je crois néanmoins qu'elle constitue la caractéristique essentielle du milicu social. Si nous comparons la distribution de la nourriture dans la colonie regardée comme un super-organisme avec le courant circulatoire (" milieu intérieur ") d'un insecle ou d'un vertẹhré, la 
trophallaxis, qui est l'échange réciproque de nourriture entre les individus de la colonie, peut être comparée aux f́changes chimiques entre les éléments des tissus et le sang, ou entre les diverses cellules elles-mêmes. Ce proressus comprend nécessairement, non seulement le transport el la distribution des matériaux nutritifs, mais aussi la transmission des stimuli, ou excitations, au sein des éléments vivants. Et, attendu que les divers organismes étrangers qui sont établis dans les nids des insectes sociaux font, dans la plupart des cas, partie intégrante de la colonie, tout comme certains de nos animaux domestiques forment partie intégrante des sociétés humaines, les commensaux et les parasites des insectes sociaux doivent aussi être compris dans le circuit trophallactique. ( $\mathrm{I}$ ).

Dans les deux chapitres suivants, j'exposerai les faits el les arguments qui confirment cette opinion.

(1) Cf. De Graxir: (1923): " Les phénomènes sociologiques ne sont jamais exclusivement humains. Les animaux inférieurs furent fréquemmen! une partie intégrante du groupe primitif. Le chiell, par exemple, ne fut en aucun sens un concurrent sauvage, subjugué par la ríse el par la force, ni un simple objel de luxe, mais un véritable allié, un socius. ” 

CHAPITRE iX

\title{
EVOLUTION DES ASSOCIES ET DES PARASITES DES INSECTES SOCIAUX
}

\author{
SOMMAIRE
}

Calégories diverses: Sphẻcophiles, Mélittophiles, Myrmécophiles, Termitophiles. - Exemples de synœeètes et de synechthres. - Associés vrais ou symphiles. - Parasites. - Termitophiles. - Parallélisme de l'évolution des associés des fourmis et des lermites (physogastrie, etc...). - Critique des interprétations de WASMINN.

Les Insectes sociaux ayant réussi à se créer à euxmêmes un milieu particulier, se sont trouvés exposés à l'invasion d'une armée a'associés et de parasites pleins d'avidilé, qu'il est de notre devoir d'étudier maintenant. Presque tous ces intrus sont des Arthropodes, la grande majorité sont des Hexapodes appartenant aux ordres et aux familles les plus diverses. Nous comprenons sans peine que des espèces étrangères viennent en si graud nombre s'installer dans les nids, ou dans les champs trophoporiques qui les entourent, car le milieu spécial des insectes sociaux leur offre bien des attractions et des conditions très favorables. En premier lieu, les galeries el les chambres de leurs nids fournissent des retraites très commodes; en outre, elles sont à une température un peu plus élevéc que lextéricur, surlout par temps froid, 
du fait de l'entassement de leur population. En second lieu, les ouvrières de rebut, affaiblies et mouranles, ou mème les bien portantes, représentent une abondante provision de nourriture, et, plus encore, les larves et les nymphes, inertes el sans défense. Et troisièmement, comme les insectes sociaux ont une tendance plus ou moins prononcée ì combattre les ennemis de toutes sortes qui les attaquent, cette protection s'étendra aussi à tous les petits organismes qui pourront éviter l'hostilité des propriétaires du nid. Les inconvénients sont moins frappants ; ils existent néanmoins, car les ouvrières, mème dans un nid donné, présentent souvent des variations considérables dans leur tolérance vis-à-vis des intrus. Et, même si la plupart des ouvrières d'une colonie consentent à tolérer ou à adopter un insecte étranger, celui-ci peut ètre éventuellement chassé ou détruit, pour peu qu'il y ait quelques rares ouvrières s'obstinant dans leur hostilité et leur intolérance, car, tòt ou tard, elles arriveront au contact de l'intrus. Ainsi, la tolérance unanime ou l'adoption, de la part de la colonie, doit être assurée pour chaque insecte, s'il survient un associé régulier, et c'est beaucoup plus difficile qu'on pourrait le supposer. Il est done surprenant de trouver un si grand nombre de satellites dans les nids des divers groupes d'insecles sociaux sans exception. Nous élablirons une division naturelle parmi ces intrus, en les séparant en deux groupes, comprenant, le premier d'autres insectes sociaux (parasites sociaux), el le second, des espèces non sociales, solitaires, sporadiques. Je parlerai aujourd'hui de ces dernières, renvoyant à la prochaine legon l'étude des divers parasites sociaux.

L'allraction exercée sur les insectes étrangers par les 
rolonies des espèces sociales est intéressante aussi à certains points de vue sur lesquels Cuénot (IgIr) a attiré l'attention. Cet auteur pensait que les aires inhabitées, les "places vides " comme il les appelait, ont tendance d̀ altirer les éléments de la faune plus fortement que les aires déjà pourvues d'êtres vivants. Mais Rabacd (rgir,

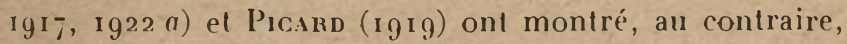
que plus une aire est peuplée, plus elle tend à attirer de nouvelles espèces, tandis que les " places vides " n'exercent nullement une telle attraction, et souvent restent complètement lésertes pendant des périodes indéfinies. Les nids des insectes sociaux, bien qu'étant au nombre des aires les plus fortement peuplées dans la nature, tendent néanmoins, de façon constante, à attirer et à retenir des habitants étrangers supplémentaires provenant du milieu externe général; ils peuvent done être considérés comme une démonstration frappante de l'exactitude dés opinions de Rabavd et Picarn.

II n'est nullement facile de classer les Arthropodes qui envahissent le milieu social. Le mode de groupement général le plus commode serait peut-être la division en Sphécophiles, Mélittophiles, Mýrmécophiles et Termitophiles, suivant qu'ils sont associés aux guêpes, aux abeilles, aux fourmis et aux termites. Nous pourrions aussi les classer suivant le type des rapports qu'ils entretiennent avec leurs hôtes. Wasmaxi, après des années de recherches laborieuses: a adopté les cinq catégories suivantes :

$1^{\circ}$ Synechlhres, on prédateurs ; c'est-ii-dire espèces qui font leur proie des insectes sociaux ou de leur couvée, et ne sont pas, en général, tolérés, mais persécutés.

$2^{\circ}$ Synoecetes ou commensaux et nelloyeurs, qui sonl, ou 
ignorés, oll indifléremment tolérés. Evidemment ils sont out inodores, ou porteurs d'odeurs neutres, qui n'éveillent pas la curiosité ou l'animosité de leurs hôtes.

$3^{\circ}$ Trophobiontes, ou insectes tels que les Iphides, les Coccides, les Membracides, etc., qui excrètent de la miellée, et les larves de certains Lépidoptires (Lycrenidés), qui produi sent des sécrétions sucrées; ils sont, de ce fait, recherchés ou même nourris par leurs hotes. Ces trophohiontes vivent le plus souvent en dehors des nids, dans le champ trophoporique, mais ils peuvent éventuellement se trouver dans les nids (sur les racines des plantes, à la surface inférieure des pierres, elc.).

$1^{\circ}$ Symphiles, ou associés vrais, qui entretiennent avec leurs holes les relations les plus intimes et les plus amicales, et présentent des adaptations structurales spéciales (trichomes, exsudatoires, etc.) à leur mode particulier d'existence.

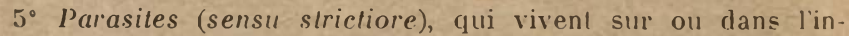
secte social pris individuellement, et peuvent xlone etre classés en externes ou internes.

D'une façon générale, les groupes de Wasmun représentent des catégories suivant lesquelles l'intimité va croissant entre l'intrus et l'hòte. Les symphiles et les parasites sont évidemment des synechthres ou des synacètes évolués, cl, tout au moins pour les Hexapodes, les parasiles sont le plus souvent des parasiloüdes, au sens qui a été donné à ce terme dans liume des premières leçons. Bien que la classification de Wasmaxu soit la meilleure qui ait été établie jusqu'aujourd'hui, il est difficile de la regarder comme satisfaisante ou définitive. Yon seulement certaines formes possèdent successivenent les habitudes distinctives de différentes catécrories à leurs stades larvaire et adulte, mais quelques-unes combinent les habiludes de plusieurs catégories, à un stade unique de leur existence. I'autres encore se comportent de facon si.aberrante qu'il est difficile de les situer dans l'une des 
catégories de Wasmans. L'une au moins de ces catégories, celle des symphiles, n'existe que chez les myimécophiles et les termitophiles, et, bien que les guêpes et les abeilles absorbent la miellée des Aphides et des Coccides, il esı très rare qu'elles sollicitent directement cette sécrétion (I).

Ainsi donc, les sphécophiles et les mélittophiles sont lous des prédateurs on des parasitoïdes qui attaquent la couvée, soit des nettoyeurs (synocètes) qui dévorent les matériaux du nid ou les détritus. Il n'est pas douteux que les plus intéressants, et de beaucoup les plus nombreux des intrus, sont ceux qui vivent avec les fourmis el les termites. Yalheureusement, personne n'a encore cherché à faire, des sphécophiles et des mélittophiles, une étude d'ensemble; mais on en sait suffisamment à leur sujet pour être sûr que serait amplement récompensé de ses efforts, toul jeune investigateur qui les soumettrait à une étude aussi patiente que celle à laquelle Wasmavi, Dovisthorpe, Silvestri, Mavi, Reichensper(iER ct autres, se sont livrés sur les termitophiles et les myrmécophiles.

Comme j'accorderai une très grande attention, dans celte leçon, aux myrmécophiles et aux termitophiles, mon étude des sphécophiles et de mélittophiles sera très brive.

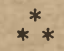

Les sphécophiles appartiennent a plusieurs ordres; l'un des phis curieux, celui des Strepsiplères, comprend des parasiles internes des Vespidés, solitaires aussi bien que sociaux. Dans

(1) Bezt (1874) a décrit une guêpe du Nicaragua qui recherche les pucerons et sollicite leur micllée, de la même façon que les fourmis. 
l'Amérique du Nord, les espèces solitaires d'Odynerus, d'Eumenes el de Zelhus sont occasionnellement stylopiscees par un genre spécial, qui est actuellement étudié par un de mes élives, M. George S.aLt. Les espèces de Xenos, en Europe comme dans l'Amérique rlu Nord, sont particulières aux guêpes sociales du genre Polistes. Il arrive que près de $25 \%$ des gruêpes d'une localité donncé soient infestées par ce Strepsiplère (voir Wheelen, 1910). Suivant Roubuin (1924), les guêpes sociales d'Afrique du genre Belonogaster ne sont que très rarement stylopisćes, évidemment par quelque genre spécial de Strepsiptère. D'autres sphécophiles intéressants, voisins des Strepsiptères, appartiennent ì une famille de Coléoptères, les Rhipiphoriclés, dont Сновит (1891) a décrit une espèce, Emenadia llabellata, qui se nourrissait, au stade larvaire, de la couvée d'Odynerus simulator; et Chapmin (1870), Murray (1870) et d'autres ont décrit le développement, à peu près identique, de Metocus paradoxus, dans les nids de V'espa. Il y a aussi plusieurs IIyménoptères sphécophiles. J'ai indiqué, dans ma deuxième leçon, le parasitisme des Trigonalides sur les larves de diverses guêpes, et, récemmenl, BEovient a découvert un jntéressant Chalcidide, Polistomorpha, dans les nids de cerlaines Epinoninés de l'Honduras. Ed. AnnRÉ, qui, dès 1881, a publić une appréciable revue des sphécophiles européens, mentionne un Ichneumonide, Typhon vesparum, comme parasite fréquent de Vespa germanica et vulgaris, el plusieurs autres espèces (Cryplurus argiolus, Mesostenus gladiator et Ephialles extensor) comme parasitant les larves de Polistes. Mutilla brutia et littoralis envahissent aussi les nids de cette dernière guêpe. Briuer (1869) signale un Névroptìre, Trichoscelia, allié aux Mantispa, comme se développant dans les nids d'une espèce de Polybia sud-américaine qui fahrique du miel. Les larves d'un petit Tinéide, Melissoblaples anellus, sont connues pour détruire les malćriaux des nids de lespa. Parmi les Diptères, il y a au unoins trois groupes remarquables de Sphécophiles, à savoir: diverses espèces de Volucella (zonaria, inanis, etc.), dont cerlaines ont été parfaitement étudiées, il y a longtemps, par Kunciel d'Henculis $(1869,1870,1875-81,1895)$; certaines espèces de Conopidés, el certaines espèces d'un genre de Tachinidés, Anacamptomyia. Roubıun (1921) a publié un beaı et suggestif mémoire sur ce dernier genre, qui comprend des parasites internes de larves de Belonogaster. Divers auteurs ont altiré l'allention sur les nombreux Coléoptères, qui sont, sq̨it prédateurs, comme un Staphylinide, Quedius dilatalus, dans les nids de guêpes européennes et Triacrus superbus, dans les nids 
de Polybia vicina, au Brésil (Whasmanx, 1904), soil simplement nettoyeurs, vivant des détritus du nid (petites espèces de Staphylinidés, Cryptophagidés, Dermestidès, Phoridès, etc.). Les larves d'un Syrphide, Microdon, hien qu'étant le plus souvent ossociées avec les fourmis, ont été trouvées également dans les nids de Ve"pa.

Tout aussi multiformes sont les mélittophiles, qui sont associées avec tous les groupes d'abeilles sociales, sauf peut-être les espèces d'Allodape de l'Afrique du Sud. Les Halictinés, qui, nous l'avons vu, font leurs nids en groupes, sont infestés par de nombreux parasites et nettoyeurs (mouches, Coléoptères, Mutilidés, etc.) comme l'ont montré Melander et Brues (1903), dans le cas de l'espèce nord-américaine, Halictus pruinosus. On sait depuis longtemps que les Bombinés sont parasités par des Mutillidés, des Conopidés, des Tachinidés, des Méloïdés, el par un ver nématode particulier, Sphærularia bombi, qui a élé étudié par Réaumur (1742), Dufour (1837), von Siebold (1836) ; LubBock (1861), SchNeider (1883, 1885), Leuchilrt (1886) et Dıмmock (1886). Leurs cellules à cire sont dévorées par des larves de phalènes, Aphomia colonella en Europe et Nephropteryx edmondsi dans l'Amérique du Nord (Packird, 1865), tandis que les débris du nid sont mangés par des larves de Coléoptères du genre Antherophagus, qui, au slade adulte, visitent les fleurs' et qui se fixent d'eux-mêmes aux pattes et aux antennes des abeilles et sont ainsi transportées à l'intérieur du nid (voir XVheeler (1919c). Le genre Volucella fournit une série remarquable de parasites des couvées de bourdons, ces parasiies élant souvent colorés comme leurs hôtes. Ils sont communs en Europe, mais on n'a encore rien publié sur les habitudes die nos espèces américaines. Gabritchevsix (192/) a donnó récemment un intéressant mémoire sur la génétique et le mimétisme chez $V$. bombylans, hoemorrhoidalis, flava, plumala et caucasica. Il a trouvé que ces cinq formes sont simplement des vitriétés d'une espèce unique, car elles s'hybrident aisément et produisent des descendants suivant les proportions mendéliennes. Wasmanx (1901) a signalé un Staphy.linide chasseur, Belonuchus mordens, el quatre espèces d'un genre de Silphides, Scolocryplus, trouves par Gorm dans les nids de quelques espéces de Melipona du Brésil, el un Silvanide, Nausibius clavicornis, gui, avec sa larve, vivait dans un nid de Trigona ruficrus. On connait depuis longtemps un grand nombre d'insectes infestant les mouclies à miel (voir Assmus 1865). Les plus célèbres sont la mite des ruches, si répandue, Galleria mellonella (Pur.Lu's, 1907), qui détruit les rayouns; un Cléride, Tri. 
chobius apiarus, et certaines espèces de Mcloe, qui, en Europe, s'emparent de la couvéc; un Diptère aberrant, aptèrc, de très petite taille, Braula coeca, lo " pou d'abeille ", qui est un cctoparasite de l'abeille et Mermis albicans, qui en est un entoparasite. Evidemment, les bactéries, les moisissures el les microsporidies, auxquelles sont dúes diverses maladies des abeilles, doivent aussi être comprises dans les parasites des: $\Lambda$ pinćs (voir IVhite 1906).

$$
\text { ** }
$$

Le nombre des Myrmécophiles paraît être très élevé. 2.000 espèces au moins ont été décrites à l'heure actuelle, et un examen attentif des colonies de fourmis, surtoul des tropiques, révèle sans arrêt l'existence de nouvelles formes remarquables. Bien que le plus grand nombre de ces espèces soient fournies par l'ordre des Coléoptères, presque tous les ordres d'insectes, et quelques-uns de ceux des autres classes d'Arthropodes sont représentés. Les moenrs de ces organismes sont si extraordinaires qu'il faudrait des volumes entiers pour les décrire convenablement. Je ne peux exposer ici que quelques exemples choisis pour montrer la diversité des relations qu'ils entretiennent avec leurs hôtes.

Les synechthres, ou intrus persécutés, sont principalement des Staphỵlinidés, qui se cachent dans les galeries les moins fréquentées des nids, évitant autant que possible le contact des fourmis, et s'emparant des couvées ou des adultes débilités. Comme cetle catégorie ne présente pas un intérèt extraordinaire, je citerai seulement un exemple, celui d'un Coléoptère assez commun dans les nids de notre Formicu exsecloides, de l'Amérique du Nord, Megastilicus formicarius, qui ressemble à son hôte par la coloration el par la forme. Si on l'enferme 
avec des fourmis dans un nid artificiel, il est invariablement tué en l'espace de quelques heures, mais, daus les nids naturels, il évite adroitement son hôtc, de la même façon que les espèces européennes de Myrmedonia : lorsqu'une fourmi essaie de s'en saisir, il relève la pointe flexible de son abdomen, el émet un liquide blanchâtre, qui oblige la fourmi à reculer, comme si un flacon d'ammoniaque avait été subitement débouché à sa face ; celte manœuvre donne naturellement au Coléoptère le temps de s'échapper.

Une grande proportion de myrmécophiles sont synoecètes, ou tolérés, mais il se comportent de façon si diverses, qu'ils défient toute classification, et, dans certains cas, ils passent même au groupe des symphiles. Beaucoup appartiennent à un groupe que j'ai appelé synoecètes neutres, parce qu'ils vivent sans que les fourmis les remarquent, sur les déchefs ou les matériaux du nid.

Des exemples typiques sont: un petit Podure panmyrmécophile, blanc, du genre Cyphodeira, un Isopode européen, blanc de neige, a mouvements très lents, Platyarthrus holfmannseggi, beaucoup de petits Coléoptères, des Acariens, des larves de Phoridés, de Lépidoptères et de Diptères, et la curieuse larve de Vicrodon. L'aliment de ces dernières fut pendant longImmps ignoré, jusqu'à ce que Donisthorpe $(1912,1921)$ montra "fu'il consiste en petites pilules (" boulettes de nettoyage )) rejetées par les fourmis de leurs poches infrabuccales. Plus récemment, Borgimeter (1923) a trouvé que les larves d'une espèce hrésilienne dévorent réellement les larves de son hôte, la fourmi de fou, Solenopsis soevissima. Le phalène Nurthia auririllei, que Kenner (1923) a décrit comme vivant dans les parois soyeuses des nirls de Polyrhachis bicolor, à Java, et qu'il regarde comme représentant un type nouveau de myrmécophiles, " hỵphænosymphiles ", est neltement un synœcète, comme tant d'aulres Tinéides (1).

(1) Je ne m'explique pas pourquoi Kexner regarde cet insecte comme une sorte de symphile. Comme je n'ai jamais af- 
Parmi les synœcètes, on doit placer aussi un grand nombre de Staphylinidés el d'Histéridés, associés aux Dorylinés, Eciton, dans les tropiques du Nouveau Continent et Dorylus dane les tropiques de l'Ancien. (Whismans, 1910a, 1915a, etc.). Ces Coléoptères s'emparent probablement de portions de la proie recueillie par leurs hôtes et transportée dans leurs nids teinporaires. Wasmaniv a supposé que certains Staphylinidés sont protégés contre leurs hôles par leur forme qui rappelle celle des fourınis et le dessin particulier de leur tégument qui res-

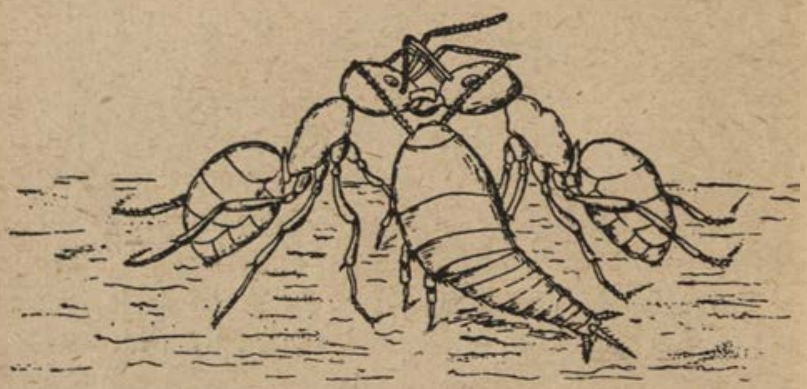

Fig. 13. - Atelura formicaria happant une gouttelette nutrilive régurgitée par une ouvrière de Lasius mixtus pour une autre ouvriere (d'après Ch. JANFr).

semble étroitement à celui des Dorylinés; d"autres le seraient par leur corps arrondi, convexe, et leurs surliaces dures et glissantes. Un autre groupe comprend les myrmécocleptiques, dont les meilleurs exemples sont fournis par les nombreuses espèces du genre de Thysanoures, Atelura, dont la distribution est mondiale. Atelura formicaria observé tout d'abord par

firmé que tous les insectes simplement tolérés dans les nids de fourmis entretiennent avec leurs hotes des relations trophallactiques, il m'est impossible de découvrir quelque intérêt aux observations qu'il a publices en 1918, au sujet de mes propres idées. Il parait accepter l'interprétation erronée et préjudiciable que Wisuans a donnce de mon arlicle, sans même avoir pris la peine de le lire attentivement. 
JiNer (1896i), pourrait êlre considéré comme un synechlhre, puisqu'il est quelquefois menacé par les fourmis, qui ne peuvent s'en eniparer à cause de son tégument glissant et de son ahdomen très effilé. Il se déplace avec circonspection parmi les fourmis (Lasius mixlus) et happe des gouttelettes de nourrilure, pendant que les fourmis sont occupées à régurgiter entre elles. Un myrmécocleptique encore plus intéressant est lit larve d'une petite Phoride, Metopina pachycondylie, que j'al ohservée au Texas (1901a), dans les nids d'un Poneriné, Pachycondyla montezumia. Les larves de cette fourmi sont nourries par les ouvrières avec des fragments d'insectes placés sur la surface ventrale-large et plate de leur abdomen; et la larve

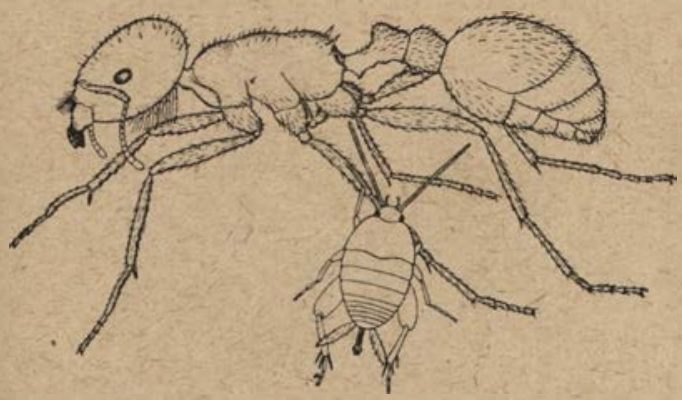

Fig. 11. - M!yrmecophila nebrascensis (Orthoptere) grignolant sur le tibia d'un Pogonomyrmex moleiaciens.

le Melopina qui encercle le cou de la larve de fourmi, comme un collier de la reine Elisabeth, tend sa tête en avant et prend sa part du festin. Quand tes deux larves, celle de la fourmi el celle dı commensal, sont entièrement développées, la première lile un cocon à l'intéricur duquel la seconde forme sil pupe, au polo postéricur. La fourmi adulte émerge en premier, laissant à l'extrémité antéricure du cocon une ouverlure, d travers laquelle la mouche peut s'échapper.

II exisle enfin un groupe de synœecetes qui passe la majeure partie du temps rongeant nu lèchant la surface des fourmi.: irdultes el tirant certainemeñt quelque aliment des sécrétions ou exsudals qui recouvrent leurs corps. Les criguels presque 
aveugles du genre Myrmecophila (Wheeler (1900b), Wasmixi (19016), Schimer $(19(19,1910))$ et le Staphylinide Oxysoma oberIlıüri du Nord de l'Afrique appartiennent à ce groupe (Escheucn 1902); aussi les petites blattes subaptères du genre Attaphila, qui vivent dans les jardins de champignons des Allini rongeurs de feuilles. J'ai décrit en 1900 le type de ce genre, Allaphila lungicola; des nids d'Atta texana et j'ai récemment trouvé la même espèce, en Guyane anglaise, dans les jardins le champignons de $A$. cephalotes et dans ceux d'Acromyrmex oclospinosus, dans l'Etat de Panama. A la même époque, d'autres especces du mème genre ont été trouvées dans l'Amérique du Sud avec d'autres Ittini. Shelforn $(1906,1907)$ a décrit une

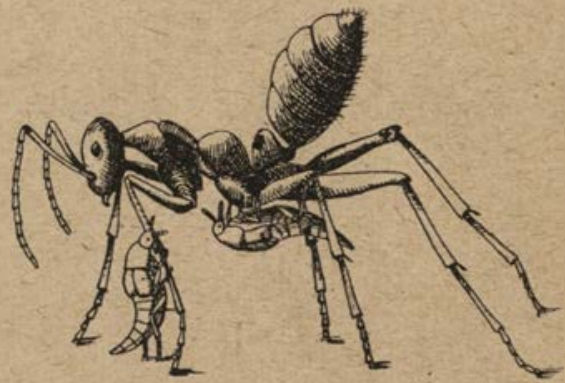

Fig. 15. - Oxysoma oberthuri (staphylinida) sur Myrmecocystus vialicus (d'après Escherich).

blalle voisine, Sphecophila polybiarum, vivant dans les nids d'un Eppiponiné, Polybia pygmzea, dans la Guyane anglaise. o! une seconde espèce, $S$. termitium des termitières de l'Afrirjue. occidentale.

C'est ici le lieu d'étudier aussi quelques synœcètes et synechlhres spéciaux, peu nombreux, qui ont été observés tout récemment et sont d'un Irès grand intérêt, soit parce qu'ils vivent entièrement hors des nids de fourmis; dans le champ trophoporique, soit parce qu'ils sont récllement importés de ce champ dans le nid par les fourmis; ils y ont alors un compor. tement tout ì fait diffèrent. Ce qui est remarquable, e'est qu'ils appartiennent aux ordres des Diptères, Lépidoptères el Hélé. 
roptères, ordres qui ne nous semblent guère capables d'acquérir, avec les fourmis, des relations aussi spéciales.

$1^{\circ}$ On connait certains Calliphorinés paléotropicaux appartenant aux genres Bengalia et Ochromyja, qui se postent près des files de fourmis émigrant ou cherchant leur nourriture ${ }_{2}$ ct fuii se jettent sur leurs proies ou leurs couvées. Ces mouches different nettement des autres Calliphorinés par la structure de leur trompe, qui est rigide et chitinisce et munie d'ume pointe à fortes dentelures, dirigée en avant. Ces mouches ont été élıdiées en premier par Naxcle (1905) dans l'Inde et par E. E. Crusex, à Ceylan $(1906,1908)$; les observations de ces auteurs ont èté ensuite confirmées par Beculert (1922), qui a examiné les mêmes insectes au Congo (1).

(1) Les mouches observées par Nixgle et Green élaient Bengalia obscuripennis et elles chassaient les termites ailés volant i la nuil. "J.-IV. YerburY vit celte mème espèce " essayant de Iransporter une grosse fourmi (d'une espèce de Lobopella). ") J.-W. Thoupson fit l'observation suivante sur B. jejuna (Fabricius) de l'Inde : "J'ai, de tout lemps, remarqué des spécimens de ette espece sur le sol, ou sur une pierre ou une feuille, près d'un nid de fourmis. En les épiant, je les vis se jeler sur une fourmi transportant un nuf ou un larve, prendre cet œuf ou celte larve à la fourmi, la transporter à quelque distance et se mettre à la sucer. " Bengalia latro de Meijere, i Java, se posle au voisinage d'une colonne de Pheidologelon diversus (Jendon); quand une fourmi nuvriere s'avance portant sa proie, la mouche se jefle au milielı de la colonne en marche, prend vivement la proie au porteur et retourne à son perchoir où elle dévore a loisir sa caplure (JiCOBSon, 1910, DE. Meruene, 1910). Plus tard, G.-R. Dutt, dans son intéressant ouvrage "Life Hislories of Indiun Insects " (1912) écrit les dignes suivantes a propos de Monomorium indicum Forel: "Un matin, je vis les locataires diun nid en sorlir avec des jeunes. Tout prés du nid, élait posée une mouche, une Muscidée (unc espece d'Ochronyja), qui s'attaquait de temps en temps aux larves et aux nymphes uni Iransportaient les ouvrières. I. a mouche nenlevait jamais sa victime a la fourmi qui la tenait, mais elle lit "lochinil " simplement, de sa place avec sa trompe qui, en se retirant, laissait la larve ou la nymphe entièrement ralatinée. "Les Bengalia africaines onl donc heaucoup des habitudes de leurs congéneres indiens. Suivant W.-A. Lamions (1913, 1919) Bengalia depressa (Walker) de la Nigéria du Sud, 
$2^{\circ}$ Plilocerus ochraceus est un IIétéroptère javanais très intéressant, qui vit sur les fourmis dans le champ trophoporique. Sa conformation et ses mours ont élé décrites par KinkaLim (1911) et JACOBson (1911a). Au milieu de la face ventrale, il porte une glande particulière avec une touffe de poils fauves (trichomes). Quand l'insecte est posté sur le bord d'une file de fourmis allant en quête de nourriture, fourmis communes dans l'Inde, Hypoclinea bituberculata, il surveille la venue d'une d'elles et dès qu'elle approche, il relève la portion antérieure de son corps de fagen a découvrir ses trichomes. Leur odeur allire la fourmi et l'incite ì les lecher et à les mordiller. Le Plilocère s'abaisse doucement, repliant simplement ses paltes: antérieures sur la tête de la fourıni, comme s'il était sûr d'eil faire sa proie. La fourmi mordille souvent si avidemment les trichomes avec ses mandibules qu'elle agite le Ptilocère de haut en bas. Mais la sécrétion de la glande a un effet toxique paralysant sur la fourmi. Jees que la pauvre bête retire ses palles el veut s'y poser, le Ptilocère la saisit avec ses patte: intéricures, enfonce sa trompe a travers l'une des sutures tho raciques ou de prélérence au point dinsertion d'une antenne et aspire le contenu du corps. La paralysie est bien due à unc substance de la glande absorbéc par la fourmi et non à la bles sure faite par la trompe du Ptilocire; ceci, d'après Jaconsox est " prouvé par le fait que, lorsqu'un grand nombre de fourmis ont léché quelque temps la sécrétion du trichome, elles s'ecartent quelque peu du Plilocere. Mais elles sont très vite saisies par la paralysie, mème si elles nont été nullement touchées par la trompe du Ptilocère. De celte manière, un bien plus grand nombre de fourmis est détruit qu'il n'en est utilisé pour la nourriture des Ptilocires el il faut s'émerveiller de !a fécondité des fournis qui permel au Ptilocèe de prélever un si lourd tribut sur la population d'une communauté. " Les nymphes de Plilocires, lout comme les adultes, attaquent indistinctement les fourmis et quoique ces IIémiptères soient confinés en un seul endroit, ils peuvent infester en grand 11:11bre les routes de fourmis.

$3^{\circ}$ Les fourmis, dans le champ trophoporique, sont également soumises it l'exploilation, bien plus bénigne d'ailleurs, d'un moustipue paléotropical, superbement coloré en noir

suit Inujours les armées en maraude de Dórylus nigricans, pour leur déroher leurs proies. "Voir aussi Suncour (1912), Crigg (1917, 1918) el Firquhirson (1919). 
ćtoilé d’argent et apparlenant àu genre Harpagomyia. Sa distribution et sa taxonomic ont élé étudiées par Murr (1918), EDWMins (19:2) el aulres, ses métamorphoses par be Meusere $(1909,1911)$ et ses mœurs curieuses par Jicobson $(1909,1911 b)$, ḋ Java, Jumes (1914) à Ceylan, Bixks aux Philippines et FinQLHARson $(1918,1922)$ dans la Nigéria. Harpagomyia se reproduit dans les cavités remplies d'eau des troncs d'arbres, et, sur ces troncs, il fréquente les routes suivies par diverses espèces de Cremalogaster, importunant les fourmis jusqu'à ce qu'elles régurgilent des aliments, ce qui s'obtient sans peine. Leur mode d'alimentation a été très bien décrit par JACobson et FarQUHARson. Voici ce que raconte ce dernier auteur pour $H$. farquharsoni (1918, p. X.XXIV): " Tandis qu'ils vont et viennent du haut en bas des troncs d'arbres, les Crematogaster sont sans cesse en train d'effectuer entre eux de "petits échanges d'aliments régurgités » et c'est cet acte trophallactique qu'exploite le moustique. L'insecte voltige à un pouce, ou mime moins, de la ligne de fourmis (parfois se posant sur le tronc et s'écartant du chemin quand cela est nécessaire), jusqu'à ce qu'il avise quelque fourmi qui soit sans doute de son goùt. Si la fourmi chemine vers le bas, le moustique se laisse tomber, en plein vol, également vers le bas, se maintenant un peu en avant de la fourmi, aussi près d'elle que possible sans la tou. cher. La fourmi cherche à l'éviter, mais le moustique, en général, l'empêche de s'évader et la fourmi finit par s'arrêter. Le moustique se pose vivement et l'échange habituel commence, très rapide, le moustique projetant sa trompe, - qui, au repos, est recourbée en-dessous du corps tout comme le rostre d'un Réduviide, - de lelle sorte que l'extrémité renflée se place pratiquement à l'intérieur des màchoires de la fourms. J'ai vu vibrer sur lui les palpes (non les antennes) de la fourmi. " Farquharsen $(1918,1922 a)$ a décrit également les mœurs d'autres petites mouches du Niger (Milichia argyraloides, proectes, prosietes et dectes) qui sollicitent et reçoivent leurs aliments des fourmis. Une autre espèce, un Cécidomyidé, Farquharsonia rostrata (Collix, 1922), a été vue volant au-dessus des nids en carton de Cremalogaster alligalrix, s'approchant des fourmis en train de se nourrir l'une l'autre et enlevant adroitement quelques portions des gouttelettes régurgitées, ce qui constitue un type de mours analogues i celui qu'a décrit JiNet pour Atelura.

$1^{\circ}$ Encore plus extraordinaires sont les mœurs d'autres mouches décriles par Jiconson (1909) el Finquhinsox (1918, 1922b). 
Le premier auteur a observé un Milichiidé, Prosielomilichid m!yrmecophila (de Mejiere, 1909), qui va et vient aulour de. fourmis (II!) poclinea bilurberculala) et lèche l'extrémité de leurs abdomens, absorbant évidemment leurs sécrétions anales. Dans quelques cas, on vit aussi cette mouche absorber les excreta (mielléc) des nymuhes de Membracides. La mouche observéc par Funquinsox est une Ephydridé, Rhynchopsilopa apicalis (Cortix 192:). Il la vit tout d'abord (1918) s'alimenlint à l'anus d'ouvrières mortes de Cremalogaster; mais plus tard (1922b), i' la vit tournant autour des pointes des abdomens de fourmis vivantes, et les lèchant, tout comme les Prosatomilichia javitnaises. Les deux observateurs disent que les fourmis paraissent être sensibles aux atlentions persistantes des mouches.

Les deux cas suivants se rapportent à des chenilles de Lépidopteres, qui se trouvent en relation avec les fourmis apres aroir élé transportées par elles à l'intérieur de leurs nids.

5n F.-P. Dodo (1912) a découvert que la larve d'un petit phalène gris du Queensland (Cyclolorna monocentra), est, à son premier stade, un ectoparasite d'un IIomoptère Jasside, qui =c nourrit sur certains arbres el qui est accompagné et exploile par Iridomyrmex sanguineus. La fourmi transporte le parasite, mais non le Jasside, dans son nid. Là, le parasite file un cocon temporaire, et en sort plus tard sous la forme d'une larve all second stade, plate, rouge, munie de deux longs appendices anaux. A ce stade, elle ne subsiste que "sur les larves de fourni, en aspirant leurs sucs ", mais, comme dans le cas des Lomechusa vivant dans les nids de Formica sanguinea, en Europe, la lourmi est particllement récompensće de la destruction de sa couvée. Dodp dit: "On a rapporté que les chenilles, mème celles qui sortent du cocon, tiennent soulevés leurs segments terminaux. Ceci m'a suffi pour lenter quelques recherches. J'ai donc, à divers moments, placé une chenille dans un vase en verre avec des fourmis et des larves; je pouvais ainsi les observer facilement el sans les troubler. Quand la partic anale était projetce en avant, quelque fourmi s'en aperceviat vite el on la voyait y porter la plus grande altention. J'observai bientòt qu'une liquide élait émis, souvent parfailemeut transparent (il avait celte apparence sur le fond bleu-vert, mais yeut-être était-il bleu pâle), el ce liquide était bu avidement par les fourmis. A muinles reprises, avec ou sans loupe, j'ai vu celle émission el sa rapide absorption par les foumnis. Cerlaines fourmis, peut-être à jeun, ou plus entreprenantes Iue diaures, cherchaient un supplément aupris d'une: seconde 
chenille. Si une fourmi n'est pas satisfaile de la quantilé émise, elle saisit délibérément la partie saillante et la pince doucement; on voit très bien les mandibules presser sur la chair jutcuse; si la demande ne reçoit pas de réponse immédiate, une plus vigoureuse étreinte est donnée et les appendices peuvent être saisis el comprimés. Ce manège est extrêmement comique; on ne peut se méprendre sur le désir de la fourmi et-la chenille le saisit si bien, qu'clle obéit toujours a la seconde injonction. Dond vit aussi des chenilles lécher el neltoyer les fourmis! A maturité, la chenille quitte le nid, émigre jusqu'au tronc le plus proche, en compagnie des fourmis en quête de nourriture; elle file son cocon dans une fente de l'écorce et forme la pupe. Au bout de vingt jours environ la phalène éclot.

$6^{\circ}$ La seconde chenille est celle de Lycrena arion, qui a été étudiée par Chapman $(1915 a, 1915 b)$ et Frohawk (1915) en Angleterre. Le papillon pond ses œuis sur le thym ou d'autres plantes. La larve s'y nourrit et elle est souvent recherchée par les fourmis, parce qu'elle possède une glande à miel, comme beaucoup dautres larves de Lycænidés. Quand elle a atteint le troisième, ou dernier stade, elle rampe sur le sol et, quand elle rencontre un ouvrier fourrageur de Myrmica lævinodis ou scabrinodis, elle soulève les segments antérieurs de son corps d'une façon toute particulière. Frонаwк considère ce geste comme un signal qui amène la fourmi à saisir la chenille et à la transporter à l'intérieur du nid. Chipmin a pu constater que la chenille se nourrit avec des larves de Myrmica. Pendant celte période de son existence, on ne la voit point produire la sécrétion de sa glande ḋ miel, et elle est traitée par les fourmis comme un associć loléré el indifférent, ce que Wasmax appelle un synœcète.

Je me suis occupé de ces cas particuliers, - et bien l'autres pourraient être indiqués aussi, - parce qu'ils montrent que les prédateurs et les synoecètes ne limitent pas hécessairement leur aclivité au nid, et parce qu'ils illustrent la diversité vraiment surprenante des inéthodes employées pour exploiler les activités normales des fourmis. Les Bengalia sont, ordinairement, de vérilables pré- 
daleurs; les Harpagoruyia el les Milichiidés pourraient itre désignés comme mỵrmécocleptiques ou symphỵloïdes ; le Ptilocère est un prédateur, mais ses trichomes paralysants rappellent plutôt les conditions de la symphilie vraie dont nous allons parler; le Cyclotorna est parasite d'un Homoptère à son premier stade larvaire, et un symphile prédateur d'une fourmi à son second stade; tandis que la chenille de Lycarna arion est un trophobionte à son premier stade larvaire ef un synoecète prédateur à son dernier stade ( $\mathrm{I})$.

\section{***}

Les associés vrais, ou symphiles, comprennent seulement 300 à 400 espèces, surlout des Coléoptères (Loméchusiens, Clavigéridés, Paussidés, Histéridés, etc.) et constituent l'élite des Myrmécophiles; ils ne sont pas seulement adaptés à leurs hôtes au point de vue de leurs actes, comme les prédateurs et les synœecètes; mais ils présentent aussi des adaptations structurales particulières. D'une façon générale, ces dernières adaptations sont: le développement d'un tégument spécial, rouge, huileux; la présence, sur divers points du corps, de trichomes jaune doré, et de glandes produisant des sécré-

(1) La littérature concernant les chenilles de Lycænidés associées aux fourmis est trop élendue pour pouvoir être indiquée ici. Il m'est impossible, cependant, de ne pas signaler l'important mémoire de Lamborn (1914), sur les Lycanidés de l'Afrique Occirentale et autres trophobiontes, car il donne une tmage tris instructive du nombre el de la diversité des insectes recherchés par les fourmis dans une aire tropicale bien délimitée. Voir aussi les publications intéressantes de Dono (1902 a, 19026) et ChapMax (1902), sur Liphyra brassolis. 
tions éthérées agréables; des modifications de la région buccale : réduction de la langue et du nombre des articulations des palpes; des modifications des antennes telles que la fusion de leurs articulations qui se soudent souvent el s'adaptent à caresser l'hôte (Clavigéridés) ou qui s'élargissent et s'épaisissent, servant alors comme de poignées, par lesquelles les fourmis saisissent leurs associés et les transportent à travers le nid (Paussidés). Was-
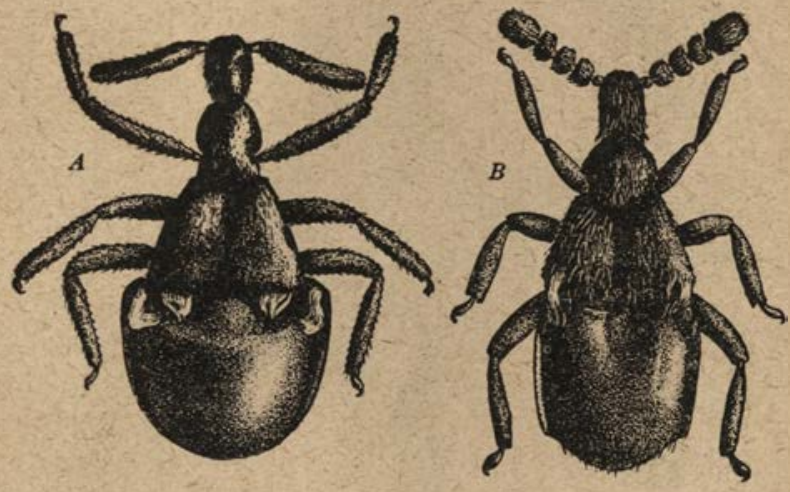

Fig. 46. - A, Adranes lecontei (Amérique du Nord). - B. Claviger testaceus (Europe), avec trichômes jaune d'or au sommet des fourreaux alaires et i la base de l'abdomen.

Mavx a fait une étude détaillée des symphiles curopéens, et surlout des Loméchusiens ( $195 a$ ) ; mais nos connaissances sont encore très réduites en ce qui concerne les mœurs des associés des fourmis tropicales. J'ai parlé des Loméchusiens dans une leşon précédente, à propos de la production de pseudogynes dans les colonies où se 
reproduisent ceś Coléoptères. J'ajouterai ici quelques indications au sujet de tomechusa strumosa, l'un des plus typiques et cerlainenent le mieux connu des symphiles.
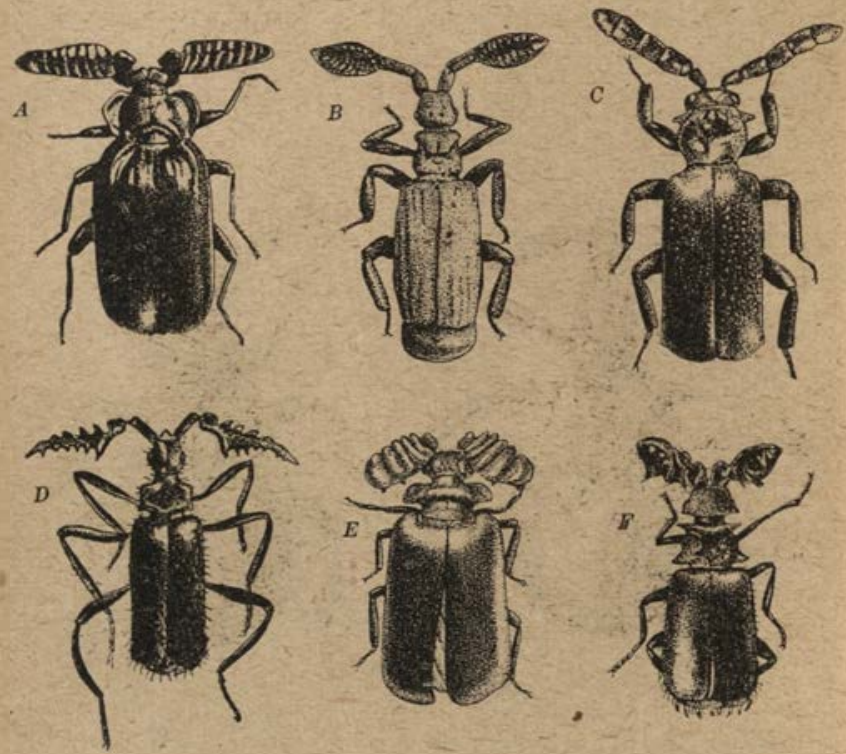

Fig. 17̄. - Diver's Paussidæ: A. Pleuropterus brevicornis ; B. Paussus hora; C. Pantaplatarthrus natalensis; D. Paussus dama; E. Labrocerus goryi; F. Paussus spiniceps (d'après WASMLN).

A son slade larvaire, ce Staphylinide est vérilablement un parasite prédateur des nids de Formica sanguinea, y dévorant les larves de la fourmi. Le tissu graisseux de la larve de lomechusa fournit sans doute aux fourmis un exsudat agréable et l'adute leur procure certainement une sécrétion aussi 
agréable, provenant des glandes abdominales à trichomes. quand ces larves, qui sont traitées absolument comme des larves de fourmis, sont entierement développćes, elles sont cufouies dans le sol, de la mème manière que les larves de fourmis, atin qu'elles s'y transforment en nymphes. Les pupes sont ensuite déterrées, comme les pupes de fourmis, apré: qu'elles ont filé leurs cocons, mais ce traitement est fatal pour le parasite, et seules les pupes qui ont été négligées ou ou-

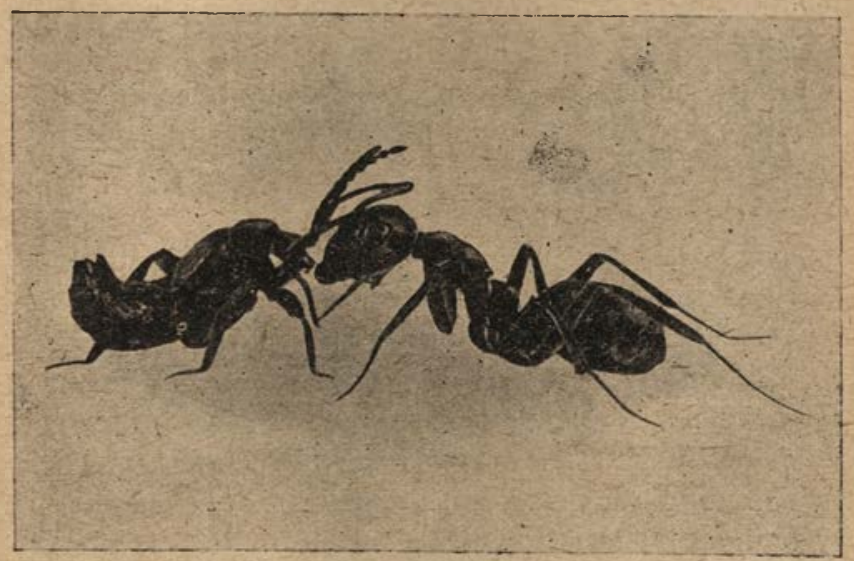

Fig. 18. - Ouvrière de Formica sanguinea, nourrissant I.omechusa strumosa (d'après DoNisthoRPE).

bliées (?) et laissées dans le sol peuvent se transformer en adultes. Lorsque la majeure partie de la couvée des fourmis a été détruite par les larves de Lomechusa, beaucoup des larves reines mal soignées donnent, comme nous l'avons vu, des psetidogynes, ou formes pathologiques intermédiaires entre les ouvrières et les reines, et incapables d'accomplir les fonctions d'aucune des castes qu'elles représentent imparfaitement. Telle est, dans ces grandes lignes, l'histoire des Lomechusa (1).

(1) Pour plus de détails, je vous renvoic aux nombreux méinoires de IVasmaxix (voir la biblingraphie, dans ses mémoires de $1915 a$ et 1920 ). 
Faute de temps, je ne pourrai étudier les myrméco. philes parasites. Dans les précédentes leçons, jai décrit déjà certains d'entre eux, des plus intéressants, tels que les Eucharidés et les Mermis, qui provoquent des modifications nettes de la structure chez les fourmis qu'ils infestent; beaucoup de formes, telles que les acariens, les champignons parasites, etc., sont tellement semblables à ceux qui infestent les insectes solitaires, qu'ils n'offrent que peu d'intérêt pour l'élude générale faite ici.

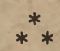

Nous considérerons donc maintenant les termitophiles, qui peuvent être grounés, très sommairement, suivant les mêmes catégories que les myrmécophiles, c'est-à-dire en synechthres, synœè̀tes, symphiles et parasites. La catégorie des trophobiontes n'est pas représentée, les termites ne sachant, en aucun cas, réclamer les excrétions sucrées d'Homoptères ou des chenilles de Lycænidés.

Quoique les termitophiles aient été moins étudiés que les myrmécophiles, on en connaît néanmoins de 600 à 700 espèces. $70 \%$ au moins d'entre elles sont des Coléoptères, les $30 \%$ restant appartiennent à un grand nombre d'autres ordres d'insectes. Warnex a dressé le tableau ci-après, montrant la distribution des termitophiles connus en igrg. Depuis cette époque, on a pu ajouter quelques représentants d'autres ordres, particulièrement des Hỵménoptères Braconides. parmi lesquels Cusumav (1923) a décrit deux espèces d'Ypsisterocerus de Bolivie, et Bruns (Ig23 a) une espèce de Termitobracon $T$. emersoni) de la Guyane anglaise. Dans sa liste, Warren indique que le nombre de termilophiles éthiopiens $(319)$ excède de beau- 


\section{Distribution des Termitophiles}

\begin{tabular}{|c|c|c|c|c|c|c|c|c|c|c|c|}
\hline FAUILLES, elc. & 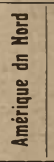 & 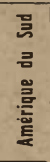 & 总 & & 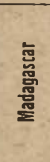 & 递 & $\frac{5}{3}$ & 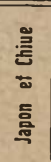 & 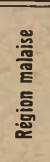 & 奠 & 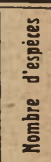 \\
\hline \multicolumn{12}{|l|}{ Coléoplères } \\
\hline Cicindelida. & - & 5 & - & & & - & & & - & & \\
\hline Staph & $\overline{3}$ & 1 & $=$ & 10 & $\overline{3}$ & I & 3 & - & 1 & - & 16 \\
\hline Psela & - & ${ }^{47} 8$ & - & $\begin{array}{r}40 \\
3\end{array}$ & 3 & 21 & 30 & $2^{2}+10-1$ & 9 & $?$ & 167 \\
\hline $\begin{array}{l}\text { Scyd } \\
\text { Siloh }\end{array}$ & - & 2 & - & - & - & - & - & - & - & - & 2 \\
\hline Histeridx & $=$ & $i^{2}$ & $\bar{I}$ & 12 & $\bar{z}$ & $\bar{x}$ & $\overline{2}$ & $\bar{z}$ & $=$ & - & 1 \\
\hline Lathridiidx. & $=$ & - & - & $\mathrm{I}$ & - & - & - & - & - & - & I \\
\hline $\begin{array}{l}\text { Scarabæidæ.... } \\
\text { lymexylonidx }\end{array}$ & $\bar{z}$ & $\begin{array}{l}8 \\
2\end{array}$ & = & 22 & - & $\underline{6}$ & 5 & - & - & 2 & 43 \\
\hline Tenebrionida. & - & - & - & 5 & - & 1 & I & - & $\overline{-}$ & $\sqrt{1}$ & $\begin{array}{l}2 \\
8\end{array}$ \\
\hline & - & - & - & 2 & - & - & - & - & - & - & 2 \\
\hline $\begin{array}{l}\text { Khysse } \\
\text { Curcul }\end{array}$ & 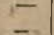 & - & $\bar{z}$ & $4 \mathrm{r}$ & 9 & 3 & - & 1 & 7 & - & 52 \\
\hline & - & 1 & - & $\bar{z}$ & $\overline{-}$ & $\bar{z}$ & $I$ & $\overline{-}$ & $\overline{-}$ & $\overline{1}$ & I \\
\hline \multirow{3}{*}{ Erotylida .. } & - & - & - & - & - & 1 & 1 & $\bar{z}$ & = & - & \\
\hline & - & - & - & 3 & - & - & - & - & - & 1 & 2 \\
\hline & 3 & 80 & 1 & $14^{3}$ & 3 & 34 & 43 & 3 & 21 & 17 & 348 \\
\hline \multirow{10}{*}{ 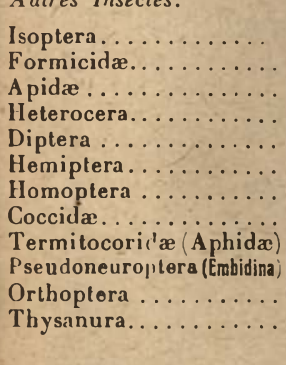 } & - & 2 & - & 3 & - & - & & - & & - & \\
\hline & - & $?$ & - & 8 & 2 & - & 8 & - & - & - & 25 \\
\hline & $=$ & - & - & $\overline{2}$ & $\overline{-}$ & $\overline{-}$ & $\bar{x}_{1}$ & $\bar{z}$ & $=$ & 二 & $\begin{array}{l}6 \\
3\end{array}$ \\
\hline & - & 5 & - & 6 & 2 & 3 & 3 & - & 3 & - & 22 \\
\hline & $\bar{z}$ & I & - & - & - & $\mathrm{I}$ & - & - & - & - & 2 \\
\hline & $=$ & - & $\overline{-}$ & $\bar{s}$ & 1 & z & $\bar{z}$ & ב & - & - & $x_{3}$ \\
\hline & Z & 2 & $\overline{-}$ & 1 & $\overline{-}$ & $\overline{-}$ & $\overline{-}$ & $\overline{-}$ & $\overline{-}$ & - & 3 \\
\hline & - & - & - & i & $\bar{z}$ & $\overline{-}$ & 二 & - & $=$ & - & 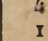 \\
\hline & - & ${ }^{2}$ & - & 2 & - & - & 1 & - & - & - & 5 \\
\hline & $=$ & 3 & $=$ & 22 & - & 7 & 5 & $=$ & $\mathrm{I}$ & - & 38 \\
\hline \multirow{2}{*}{$\begin{array}{l}\text { Arlhropodes } \\
\text { (antres que les insectes) }\end{array}$} & - & 30 & - & 46 & 5 & II & 21 & $\overline{-}$ & 4 & 1 & 118 \\
\hline & & & & & & & & & & & \\
\hline \multicolumn{12}{|l|}{ Arachnida (autres que } \\
\hline Acari & 1 & 5 & $=$ & $\frac{2}{5}$ & $=$ & $\bar{z}$ & 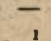 & $\bar{z}$ & 1 & - & \\
\hline & & - & - & 5 & - & - & - & - & - & - & 5 \\
\hline & & 3 & - & 4 & - & - & 2 & - & - & - & \\
\hline \multirow[b]{2}{*}{ Total des espéćcs.. } & 1 & 9 & - & 16 & 8 & - & 3 & - & $\mathrm{I}$ & - & 30 \\
\hline & 4 & 119 & $\mathrm{I}$ & 205 & 8 & 45 & 67 & 3 & 26 & 18 & $\longdiv { 4 9 6 }$ \\
\hline
\end{tabular}


coup celui de loute autre région; mais tant d'espèces onl été découvertes récemment dans l'Amérique du Sud el l'Amérique centrale, que nous devons sans doute regarder la région néotropicale comme possédant le plus grand nombre de formes. Ceci est exact également pour d'aulres organismes; la richesse de la faune d'insectes, en particulier, est presque inépuisable dans l’Amérique Iropicale. On connaît très peu de termitophiles proveлlant des nids des termites primitifs (Calotermitidés); la plupart proviennent des termitières si populeuses des Termitidés. De même, les myrmécophiles sont rares dans les petites colonies des sous-familles de fourmis les plus primitives (Ponérinés, Cérapachyinés, et Pseudomyrminés) et nombreux, comme espèces et individus, dans les seules colonies des fourmis les plus spécialisées, Dorylinés, Myrmicinés, Dolichodérinés et Formicinés. En d'autres termes, les associés et les parasites sont adoptés plus aisément et mieux tolérés dans les colonies des espèces, fourmis et termites, les plus hautement spécialisées, - fait très suggestif, sur lequel on n'a pas suffisamment insisté dans la littérature.

Comme presque tous les termites sont tropicaux, on sait actuellement très peu de chose des mours des termitophiles. Vivant comme ils le font dans les galeries somhres et fraîches; entassés avec leurs hôtes, ils ont certainement un milieu plus hautement-spécialisé et moins variable que celui des myrmécophiles. Dans presque Inus les cas, on a déduit avec plus ou moins de certitude les ielalions avec l'hôte de la structure du termitophile, et non d'olservations réelles ou d'expériences. Warnev (I y rg) a si clairement résumé nos connaissances acluelles sir ces relalions que je cite ici ses remarques : 
"La conformation des mandibules montre clairement que beaucoup de termitophiles attaquent les jeunes des termites et aspirent les fluides de leurs corps; aussi, lorsque les termit'jphiles sont nombreux dans un nid, ils sont très nuisibles à la communauté. Il est par suite nécessaire que le termitophile ai? quelque moyen de plaire aux termites, puisque le termitophile ne pourrait, de toute évidence, résister à une altaque concertée d'un groupe nombreux de soldals ou d ouvriers. Les divers moyens adoptés peuvent actuellement se résumer comme suit:

$1^{\circ}$ En adoptant un contour très sinuple, arrondi, le corps se rend inaccessible à toute attaque facile, el il peut, en outre, etre uni et aplati. C'est la le type défensif de Wismano ("Trutzlypus n).

$2^{\circ}$ Plus rarement, le corps est muni de poils raides, qui en rendent l'approche difficile ou désagréable aux-lermiles.

$3^{\circ}$ Il est tris probable que cerlains termitophiles peuvent se protéger cux-mêmes contre toute attaque en dégageant quelque odeur déplaisant aux ternites, ce qui conduit ces derniers a éviler autant que possible les intrus.

$4^{\circ}$ D'autre part, il $y$ a des termitophiles que les termites ne fuient, tout au moins, pas, et qui vivent dans les rapports les plus étroits, avec leurs hotes. Ces lermitophiles peuvent ne pas posséder des organes exsudateurs, mais ils doivent avoir des glandes à parfum bien développées et on peut supposel que ce parfum est agréable aux termiles.

$5 \cdot$ Chez d'autres termitophiles, le corps est muni d'excroissances très bien visibles, dont la structure inicroscopique indique qu'elles laissent exsuder des liquides. Par analogie avec les myrmécophiles, on peut être certain que ces liquides s'évaporent en donnant un parfum agréable, ou bien sont léchés par les lermites.

$6^{\circ}$ Dans quelques, rares cas, les corps des termitophiles portent des touffes de poils jaunes brillants. De tels poils existent très souvent sur les myrmécophiles, et on a vu les fourmis les lécher avec une grande assiduité. Le Dr Brauns a attiré mon attention sur la présence occasionnelle de ces poils chez les termitophiles, et nous pouvons penser qu'ils exercent sur les termiles une attraction du même genre.

$7^{\circ}$...Il arrive parfois que les corps des termitophiles sont forlement distendus, ou physogastriques, comme on dit; et ceci résulle d'une imitation manifeste des termites jeunes 0.1 adultes. Cette ressemblance avec les termites peut être utile en diminuant les attaques auxquelles sont exposées les termi- 
tophiles; mais ceci ne suffit cerlainement pas at explicjuer complètement l'origine du physogastrisme. »)

Il est clair que l'évolution des termitophiles et celle des myrmécophiles se sont déroulées parallèlement. Dans les deux cas, on trouve à l'origine des synechthres et des synocètes, qui, en pénétrant plus avant dans l'intimilé de leur hôte, „prennent peu à peu les caractères spéciaux des symphiles. Wirrex ne considère pas les parasites internes et externes, ni les Protozoaires symbiotiques dont il a été question dans une leçon précédente (I).

(1) La note suivante, de Cutber (1921), sur les symbiotes intestinaux des lermites est intéressante à rapprocher des éludes d'autres auteurs sur le parallélisme phylogénétique entre hôtes et parasiles, notamment, des travaux de Metcalf (1923) sur Opalina et de Kellog $(1913,1914)$ sur les poux des oiseaux et des mammifères : "Chez lous les Prolozoaires examinés, provenant de l'intestin d'Archolermopsis wroughtoni, la division nucléairc est Irès différente de ce qu'elle est chez les espèces voisines. En outre, chez Ditrichomonas termitis, la division n!lcléaire du complexe locomoleur est de nature plus primitive que celle qui a été décrite pour d'autres Trichomonadés: ce fait doit s'appliquer sans doute aussi à Joënopsis polytricha et Pseudotrichonympha pristina. Il semble que les Protozoaires hospitalisés par Archolermopsis wroughtoni sont en général plus primitifs que ceux habitant d'autres espèces de Termites. Imss décrit $A$. wroughtoni comme " un des termites les plus primitifs ". Ainsi, l'association de parasiles (ou d' "associés " suivant le cas) primitifs, avec un hôte primitif est extrêmement intéressante, el suggère que les deux groupes d'organismes sont restés associés l'un à l'autre pendant une longue période, comme ceci s'est produit avec d'autres termites et les Protozoaires qui leur sont associés. "

Fig. 19. - Termitophiles du type défensif, dans trois régions zoologiques.

1. Horyloxenus transfuga Wasm. (2.0 mm.). - Discoxenus lepisma Wasm. (2.0 mm.). - C. Hæmilopsenius caudatus Wasm. (1.3 mm.). - D. Termilodiscus heimi Wasm. (1.3 inm.). - E. Pygostenus pubescens Wasm. (2.8 mm.). - F. Termitodiscus splendidus Wasm. (1 mm.). - G. Termitonan- 


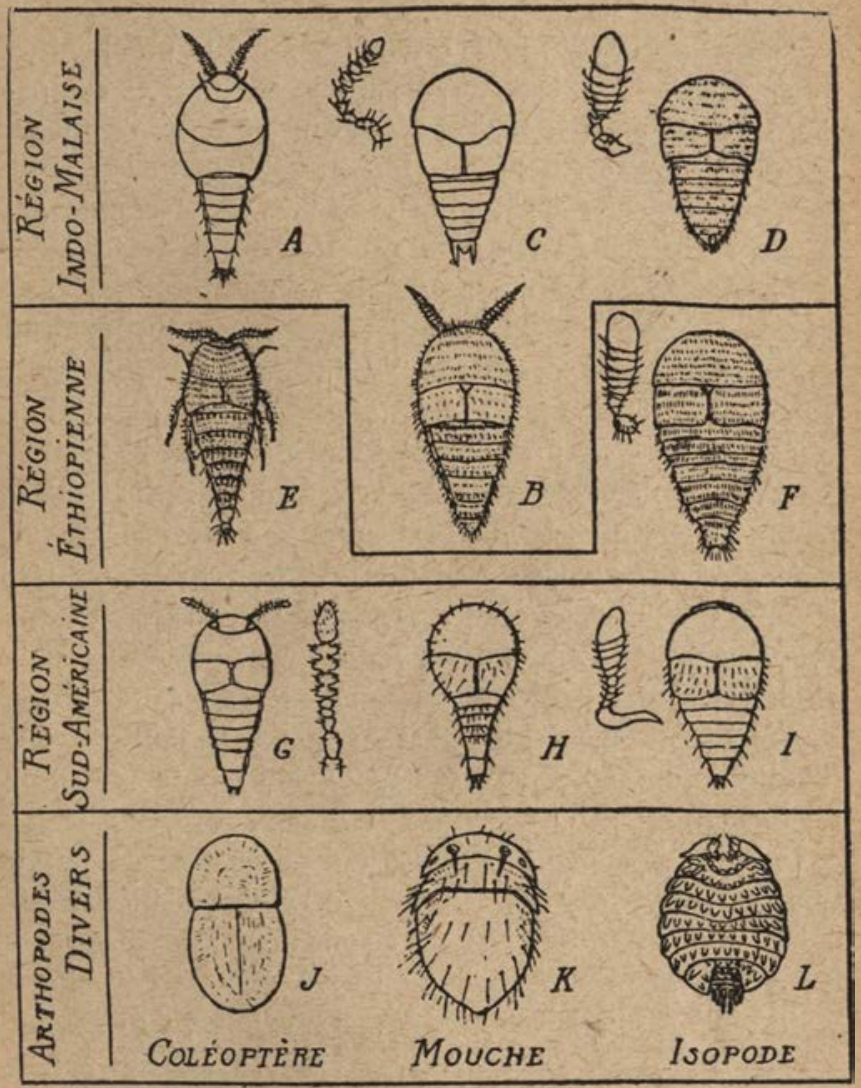

nus m.ijor Wasm. (1.8 mm.). - H. Termitopsenius limulus Silv. (2 mm.). - I. Eupsenius clavicornis Silv. (1.2 mm.). J. Endostomus sudanensis Wasm. (1.8 mm.). - K. Thaumaloxena wasmanni (B. et B.) (2 mm.). - L. Termiloniscus fulleri Silv. (4 mm.). - (F. Original ; G, H, I, L d'après Sir-

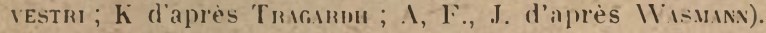
(D'ilprès E. W MrHEN.) 
WARREN appelle pourtant l'attention sur un fait intéressant: c'est que les modifications adaptatives particulières caractérisant les sept catégories de termilophiles se sont développées, de façon indépendante, dans chacune des zones à termites, pour lesquelles on a convenablement étudié les termitophiles, c'est-à-dire les régions indo-malaise, africaine et sud-américaine. Parmi les figures de Warrex, la première, montre le développement du type défensif, des Staphylinidés dans les trois séries supérieures et, en bas, un Coléoptère (Termitodiscus), une mouche extrêmement aberrante (Thaumaloxena) et un Crustacé Isopode (Termitoniscus), lous ayant la même forme générale. La figure suivante montre des larves de Coléoptères (A. B.), des chenilles (C. D.) et des larves de Muscidés (F. G.), avec des organes à exsudats spéciaux tubulaires et quelquefois articulés. Tragardi (1907) a ćludié la structure de ces organes chez des čhenilles de Tinéidés, Warrex (igzo) chez les larves de Paracorotoca (Slaphylinidés) el Silvestri (Ig20) dans toute une série de formes.

Le caractère le plus frappant de beaucoup de termitophiles est la physogastrie, ou développement excessif de l'abdomen, qui ressemble étroitement à la physogastrie de l'hôte et surtout des reines âgées. Ce développement relève de plusieurs causes, telles qu'un grand

Fig. 50. - Structures exsudatoires des Termitophiles.

A. Coléoptère (Coroloca ?), Larve $(8 \mathrm{~mm}$.), Natal, a vec renflements abdominaux latéraux. - B. Coléoptère (Termilomimus ?), Larve (2 mm.), Natal, avec renflements latéraux. C. Lépidoptère (Tinéide), Larve ( $15 \mathrm{~mm}$.), Nalal, avec appendices abdominaux non articulés. - D. Lépidoptère (Tinéide), Larve $(14 \mathrm{~mm}$.$) , Congo, avec appendices abdominaux arti-$ culés. - E. Appendice articulé de D. - F. Diplère (Mlusei- 


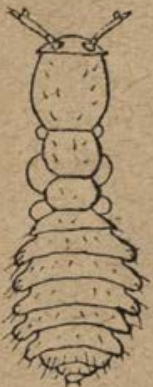

A
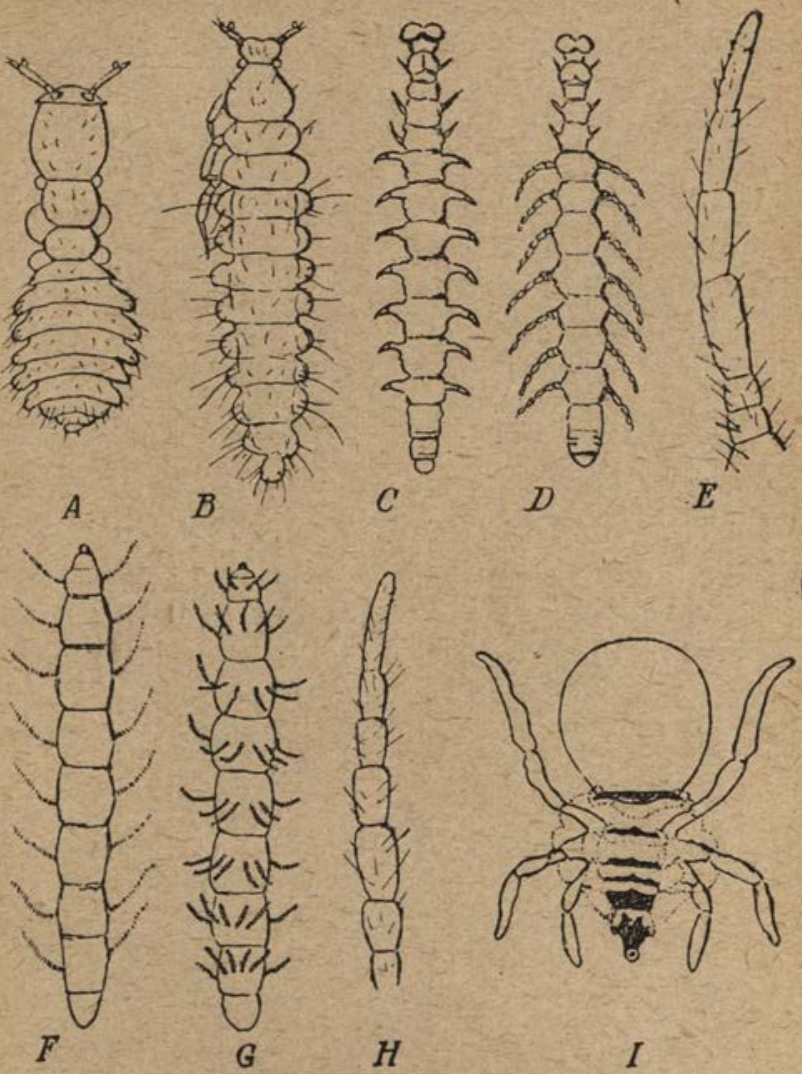

(lx), Larve (il mm.), Madagascar, avec appendices latéraux, pairs, a nombreuses articulations. - G. Diptère (Muscidae), l.arve (33 mm.), Transvaal, avec appendices arliculés disp?sés en anneaux. - II. Ippendice articulé de G. (cf. E.). I. Surface dorsale de l'abdomen $(2 \mathrm{~mm}$.) du Coléoptère Spirechlhu du bresil, avec appendices abdominaux i trois articles (fig. 1). F modifićes dapress Wismox; fig. I d'aprés SCUÜDTE). 
accroissement des tissus gras ou des exsudats, un élargrissement du tube digestif ou des organes reproducteur's [(Wasmanix (Igo3), Tragardu (1907) et Warren (I9I9), I920)]; il est particulièrement remarquable chez certaines larves de Coléoptères, des Staphylinidés adultes de nombreux genres, et chez d'étranges Diptères, appartenant aux genres Termitoxenia, Termitomyia, Ptochomyia, Timeparthenus, etc. Chez les larves de certains Coléoptères prédateurs, comme celles d'un Carabide, Glyplus punctulatus, qui vit dans les nids d'une espèce africaine, Termes bellicosus, la physogastrie est moins prononcée, mais, chez les Staphylinidés, elle se produit ì tous les degrés et atteint son point culminant chez certaines formes remarquables, qu'on peut véritablement qualifier de monstrueuses. Warren a établi un diagramme illustrant les trois modes indépendants du développement phỵlogénétique, pour la physogastrie de ces Coléoptères, dans les trois grandes régions géographi-

Fig. 51. - Coléoptères termitophiles physogastres de trois régions zoologiques.

A. Myrmedonia (condition non modifiée). - B. Jacobsonella ler. mitobia Silv. - C. Disticla capritermitis Wasm. - U. Asticta butteli Wasm. - E. Termilotima assmuthi Wasm. - F. Termitoptochus indicus Silv. - G. Termitoprdia kohli. Wasm. - H. Termitobia physogastra Wasm. - I. Termitotecna braunsi. Wasm. - J. Idiogaster escherichi Wasm. - K. Paracorotoca aliermani War. - L. Termitomimus entendveniensis Träg. - M. Timeparthenus regius Silv. - N. Termitophic. heyeri Wasm. - O. et Ol, Xenogaster inflala Wasm. P. Coroloca melantho Schiödte. - Q. Spirachtha eurymedusu Sch. (Fig. F, M, d'apres Silvestri, K original, L simplifiée d'ajprès Triginde, $P$ el Q d'après Scmöntr. le restant simplifié (d'apres Wasmini).

(D'après E. WanRE.) 


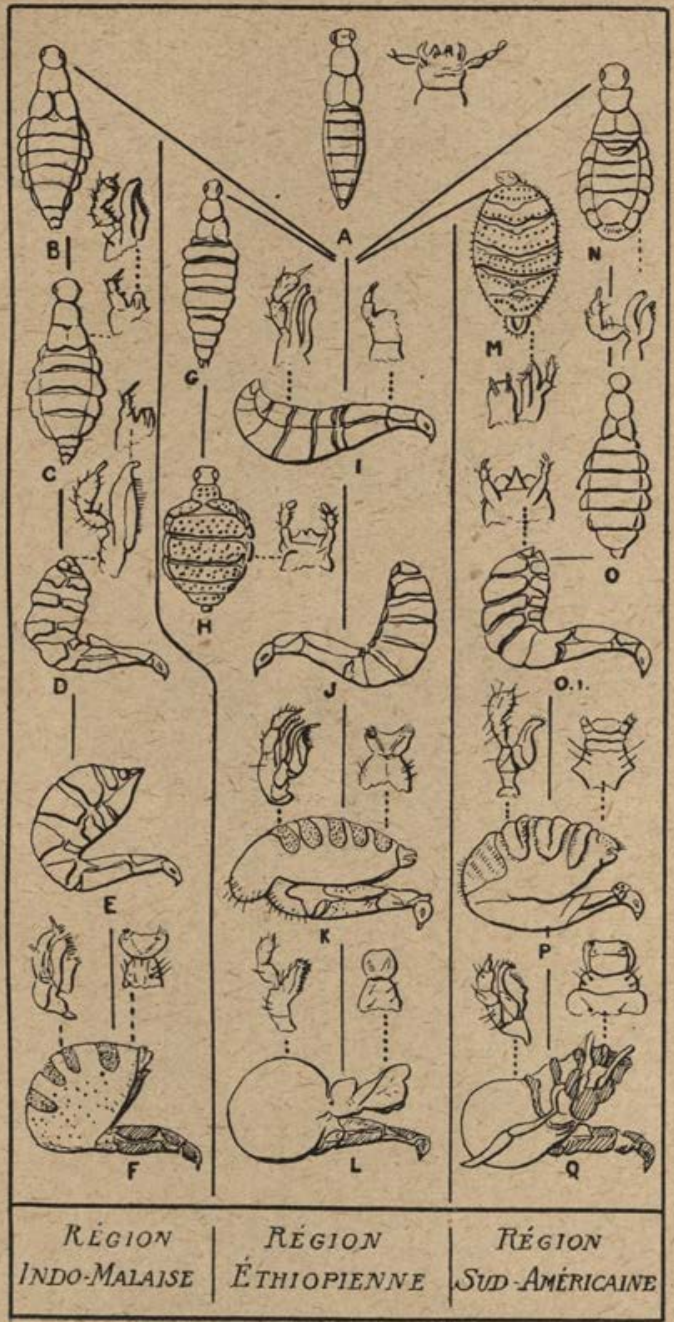


ques, en prenant comme exemple un Staphylinidé prédateur commun, comme Myrmedonia.

Parmi un nombre considerable de formes, nous choisirons comme exemples de physogastrie très développce: Corymbo.
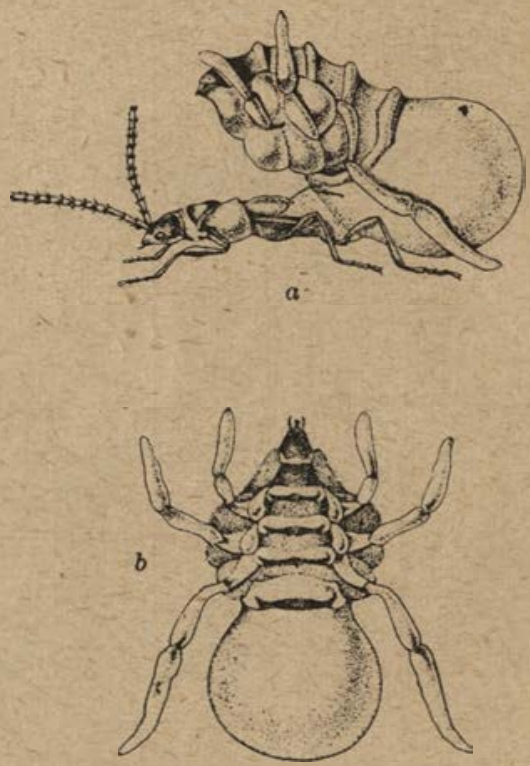

Fig. 52. - Spirachtha eurymedusa, Staphylinide physogastrique : $a$. profil; $b$. vue dorsale de l'abdomen, montrant les trois paires d'exsudatoires en forme d'appendices (d'apris SсHı̈̈DTE).

gaster miranda de la Guyane anglaise, Coroloca melantho el phỵlo du Brésil, Oediprosoma mirandum de lifrigue, et Thyj. rioxenus pulchellus de la Guyane anglaise. Ces dernières formes sont si ałerrantes que seul un coléoptériste très averti 
peut y reconnaître des Staphylinides. Mais il exisle des types encore plus bizarres, qui non seulement sont fortement physogastriques, mais possedent en outre de longs appendices exsildaloires; ainsi l'especce vivipare Spirachtha eurymedusa, du

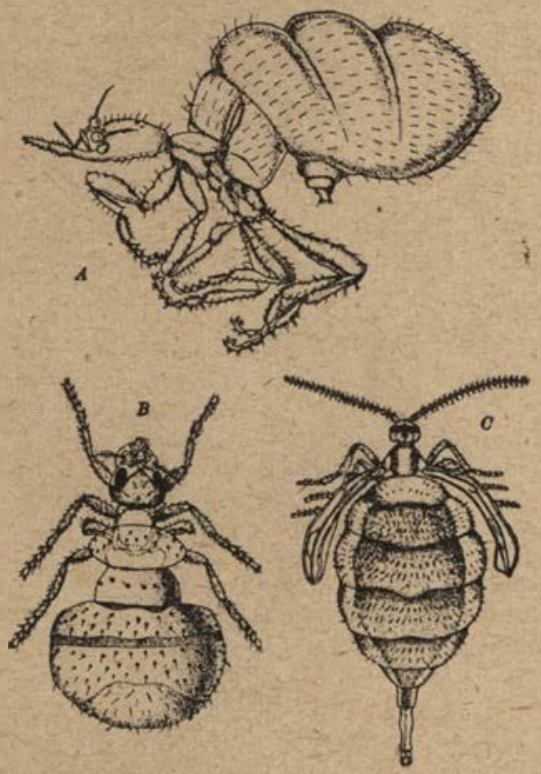

Fig. 53. - Nouches termitophiles physogastriques: A. Termitoxenia heimi, Phoride des nids de Termes obesus (Inde) (d'apres Wasmox); B. Plochomyia afra, Phoride des nuls d'Ancistrolermes crucifer (Afrique occidentale) d'apres Sir.vEstuI); C. Termilomuslus leploproclus, Nématocéride denids d'Anoplotermes relonditus (Amérique du Sud) (d'apris Silvestri).

Brésil, décrile il y a longtanps par Scmönte (18533, 18;6), el li forme plus extraordinaire encore, Spirachtha mirabilis, ricemment découverte par Emensox dane la Guyane anglaise "t 
décrite par Manv (1923). La structure particulière de ses trois paires d'exsudatoires a été élucidée par Mac Innoo (1923). Je

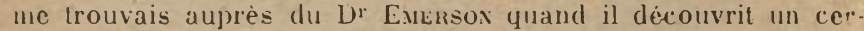
lain nombre de spécimens de ce termitophile, dans un nid de Nasutitermes (Constrictotermes) cavifrons, dans la superbe forêt de Kalacoon. Lorsque les termites et leurs associés eure it été placés dans le laboratoire, nous vîmes les premiers transporter les Coléoptères et lécher souvent, non seulement leurs exsudaloires, qui semblent présenter des surfaces gluar. les ou visqueuses, mais aussi d'autres portions de leur corps. Il est très suggestif de comparer les exsudatoires de cet étrange insecte avec ceux des larves d'une fourmi africaine, Pachysima, décrite dans une leçon anlérieure.

Les Diptères physogastriques mentionnés plus haut sont à certains égards encore plus aberrants que les Staphylinidés, parce qu'ils ont en outre des ailes et des yeux plus réduits. L'un des genres, Timeparthenus, appartient au sous-ordre dos Nématocères, et a été découvert par Silvestri (1903) dans l'. Imérique du Sud. Les autres genres, Termiloxenia, Termitomyia el Ptochomyia, se trouvent dans les régions orientale et éthopienne, et ont été étudiés par Wasmans (1900, 1901a, etc.), Assmuth (1913), Bugnion (1913 a) et Silvestri (1920). Ces caracfires, déja notés chez les termiles, et qui se retrouvent semblables chez ces mouches et ces Coléoptères: physogasırie, subaptérisme, microphthalmie, nous paraissent s'expliquer très naturellement comme résultant d'une adaptation aux habitudes trophallactiques particulières des termites, et aux mêmes conditions physiques et trophiques, en particulier, l'entassement dans des cavités obscures, pauvres en oxygène et de température plutòt basse, en comparaison de l'air et du sol environnants, l'abondance de la nourriture, etc. Ces conditions produisent, on le sait, des résultals absolument identiques chez d'autres insecles (comparer par exemple la physogastrie et l'accumulation de graisses, la microcéphalie et l'absence d'yeux chez les larves parasites d'Hyménoptères, de Diptères, etc., l'absence d'ailes et la cécité de beaucoup d'insectes hypogéiques, etc.).

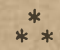

Pour conclure, je parlerai quelque peu des opinions avaneées pour expliquer, d'une part. les moeurs extra- 
ordinaires et les idiosyncrasies morphologiques des myrmécophiles et des termitophiles; d'autre part la façon dont se comportent vis-à-vis d'eux leurs hôtes. Malheureusement, la question considérée dans son ensemble, a été tout enveloppée dans lés nuées de la spéculation, en grande partie à cause des efforts toujours croissants qu'a faits Wasmaxx pour la transporter, du champ des recherches biologiques légitimes, dans celui de la philosophie néothomistique que cultivent si assidument, aujourd'hui, les Jésuites. Il a exposé ses opinions dans une longue série d'articles, dont on trouvera la bibliographie dans ses Iravaux de igio, ig15 el rg2o. Comme son dernier article est consacré en grande partie à une polémique contre mes propres idées de 1918 , el comme je continue à n'être mullement convaincu par ses arguments, ou plutôt par ses affirmations, je répéterai ici quelques-unes des idées que j’ai déjà exposées.

Ayant constaté que des symphiles particuliers vivent seulement avec certaines fourmis ou certains termites, Wasmaxx conclut: I $^{\circ}$ que ces derniers ont acquis, au cours de leur phylogénie, des instincts symphiliques spéciaux, par différenciation on modification de leurs instincts primitifs de nourrices ou de mères adoptives ; el. $2^{\circ}$ que les associés vrais des termites et des fourmis ont été produits par ces instincts symphiliques, grâce à un processus appelé " sélection amicale " que Wasmaxx compare à la sélection artificielle consciente employée par lhomme pour perfectionner les variétés si nombreuses el souvent si bizarres de ses plantes et animaux domestiques. Ces vues n'ont jamais été acceptées ni par Escue-

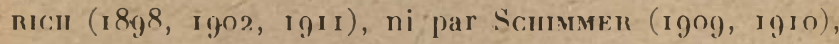
et je ne vois nullement que Wismanx ait heureusement 
réfuté nos arguments. Le sujet tient entièrement dans les réponses à deux questions: les termites et les fourmis possèdent-ils des instincts symphiliques spéciaux? et : L'hypothèse de la sélection amicale est-elle nécessaire pour expliquer les faits? A mon idée, les deux questions doivent recevoir des réponses négatives.

Il est inutile d'examiner tous les divers symphiles que WisM.ıN a étudiés si longuement et si assidument. J'ai donné déja un court exposé du cas de Lomechusa strumosa, son grand cheval de bataille et, suivant lui-mème, l'un des symphiles les plus typigues; c'est li un exemple suffisant. Wasunns pense que l'hôte de cet insecte, Formica sanguinea, a acquis, au cours de sa phylogénie, un instinct symphilique spécial, qui 1. pousse à nourrir Lomechusa au détriment de ses propres colonies et donc au détriment de son espèce; il considère que ce cas fournit un argument crucial contre la sélection naturelle. Il applique naturellement le même raisonnement, mutatis mu tandis, à Claviger teslaceus qui vit avec Lasius flavus, aux Paussidés qui vivent avec différentes espèces de Pheidole, aux Staphilinidés physogastrigues qui vivent avec divers termites, etc., etc.

Pour que l'argument semble plausible, il ne faut point lui appliquer une critique bien profonde. Lorsque nous demandons comment s'établissent chez sanguinea, les instincts particuliers gui poussent a nourrir le lomechusa, comment ils deviennent héréditaires, nous voyons que Wismaxi a considéré en grande partie les points en litige comme déji donnés. Nous ne savons évidemment rien de la phylogénie de sanguinea dans ses rapjorts avec Lomechusa. Dans les colonies assez. àgées pour être infestées par le Colćoptère, la reine de sanfluinea et ses descendants femelles, n'accordent pas une attention particulière au parasite, el ne pourritient donc acquérir que par inspiration un instinct comme celui que Wasman sup. pose. Les ouvrières, qui s'occupent du Coléoptère, se reproduisent rarement et ne se reproduisent sans doute jamais dans les colonies infestées; elles ne sont donc point en état de transmeltre un instinct, en admeltant qu'elles l'aient açuis. Fit comme la couvée de sanguinea est nu hien largement divonrie ou bien convertic en pseudogynes infécondes, si hien que la colonie tend à disparaitre, il n'y a plus rien qui puisse 
cngendrer et transmeltre un instinct aussi spécialisé que celui qui a trait à un symphile donné, sauf quelque circonstance extérieure favorable (1). Ajoutons à cela le fait que le Lomechusa est un parasite local ou sporadique ; cela rend le cas de Wasmann absolument désespéré. Il pourrait évidemment prétendre que primitivement le Lomechusa était un parasite universel de sanguinea, ou tout au moins, un parasite beaucoup plus abondant et distribué plus uniformément qu'à l'époque actuelle, mais si cela était, comment sanguinea aurait-elle pu survivre, si les ravages avaient èté aussi importants que l'affirme Wismin, surtout si nous considérons que cetle fourmi est elle-même, comme nous le montrerons dans la prochaine lecon, parasite d'une autre fourmi, Formica fusca, et donc dépendante d'un hote ?

La lecture des mémoires de Wasmaxi laisse l'impression que cet auteur est d'avis de voir dans la symphilie in phénomène biologique unique, et nous serions conduits à admettre a priori un instinct spécifique pour expliquer toute particularité des moeurs des fourmis. Si, parmi mes tantes, il se trouve trois vieilles demoiselles avant. quelque animal favori, et préférant, l'une des chats, l'autre les perroquets, el la troisième les singes, je ne suis guère renseigné si le médecin de la famille, me prenant à part, m'annonce sentencieusement que ma lante Elise a certainement minstinct alurophile, ma tante Varie, un inslinct psittacophile et ma tante Jeanne III instincl pithécophile, et que toute leur conduite s'ex-

(1) Daprès Lonncril (1921), les màles formés dans les colonies de san!luinea infestées par le Lomechusa, peuvent aussi être alfectés, alleints de nanisme, (" micraners "), n'ayant plus en longueur que la moitié environ de la taille des individus normaux. En ardmetlant que de tels males puissent peut-être descendre des ouvrières, ils ne constiluent certainement pas un matériel favorable à la transmission des instincts de symphilie acquis par ces ouvrieres. 
plique de façon satisfaisante par l'existence de ces inslincts. Il est visible que le médecin a simplement baptisé de noms grecs les stimuli qui affectent chacune de mes trois tantes, en y ajoutant un suffixe indiquant le " penchant $n$; il a supposé l'exislence de ces stimuli comme des entités dans l'esprit de mes tantes, et les fait ressortir naïvement comme une "explication ". On s'étonne que tunt de siècles soient nécessaires pour déraciner ce mode scolastique de raisonner, déjà si finement raillé par Mourène, qui alléguait la "virlus dormitiva " comme explication des effets soporifiques de l'opium.

Les faits et considérations rapportés dans cette leçon et la précédente me paraissent former un argument formidable contre l'existence d'instincts symphiliques spécifiques; en particulier, si les rapports entre parents et enfants, ou entre les ouvrières nourrices et la couvéc, sont trophallactiques, il en est de même, par essence, des rapports entre l'hôte et le symphile. Il n'est donc pas nécessaire de supposer que, chez les fourmis et les termites, l'instinct nourricier primitif, qui est une alimentation muluelle, a été spécialisé ou modifié au cours de la phy̆logénie en s'adaptant à des symphiles particuliers. De légères modifications ontogéniques, restant dans les limites des actes plastiques, ou "intelligents ", des fourmis, apparaissant comme une réponse à l'organisation et aux habitudes spécifiques des symphiles, paraissent amplement suffire à expliquer tous ces phénomènes.

Mais les instincts symphiliques et la sélection amicale ne sont que deux des nolions particulières introduites par Wasmann dans l'étude générale des associés des fourmis et des termites. II se sert aussi d'autres conceptions con- 
lestables, telles que le mimétisme (I), l'hyperlélie, la "Freunddienlichlieit ", et les rapports de son Dieu personnel avec les fourmis et les myrmécophiles. Je ne souhaite point discuter de telles idées, mais seulement indiquer que le biologiste qui subit leur charme risque d'être tôt ou tard conduit à des spéculations qui outrepassent de beaucoup les faits d'observation, ou qui tendent à les déformer. Wasmann a été incapable d'éviter ce piège, comme le montrent ses considérations sur Termitoxenia et les genres voisins, ces Phoridés particuliers, subaptères et physogastriques dont je vous ai parlé. Quand il les découvrit pour la première fois, il émit cette affirmation sensationnelle qu'ils étaient hermaphrodites ; qu'ils ne pondaient pas d'œufs et n'avaient pas de larres, comme les autres Diptères, mais qu'ils étaient vivipares, ou plutôt imaginipares et produisaient des jeunes de la forme adulte; que leurs ailes spéciales étaient utilisées comme organes d'équilibration, ou comme poignées permettant aux termites de les transporter; qu'ils étaient nourris comme des parasites sur les couvées de termites, etc. Après que ces suppositions ont été répétées par Wasmani, Assmutir et autres, pendant quelques annćes et qu'elles ont été mentionnées comme des faits dans divers ouvrages populaires, il est prouvé maintenant qu'elles sont dénuées de tout fondement. Brues ( $1998 \mathrm{~b})$, Bugvion (r913) et Silvestri (ig?o) ont montré que Termiloxenia n'est pas hermaphrodite, et Kenver (1922) a trouvé qu'il pond des oufs, qu'il a des larves du type normal des Diptères, qu'il mange les oufs de termites, que l'adulte

(1) Voir la discussion des idées de Whasmann sur le a mimélisme \# chez les associés des fourmis, et de la façon incorrecte dont il use de ce terme, in IIenkentingen (1919). 
est très probablement nourri par régurgitation, que les ailes ne servent ni de balanciers ni de poignées, etc. En présence de l'écoulement de cet édifice aux conséquences sensationnelles, érigé sur une collection restreinte de spécimens conservés dans l'alcool, sommes-nous blâmables si nous soupçonnons que quelques autres des constructions compliquées dressées par le savant Jésuite peuvent se révéler aussi peu solides lorsqu'on les examinera un jour plus attentivement? 


\section{CHAPITRE $\mathrm{X}$}

\section{L'EVOLUTION DES PARASITES SOGIAUX}

Guêpes et abeilles parasites. - Fourmis parasiles. - Cleptobiose, Lestobiose, Parabiose, Xénobiose, etc. - Esclavage (dolose), ses variétés : cas de Formica sanguinea, des Polyergus (fourmis amazones), des Strongylognathus, des Harpagoxenus. - Parasitisme temporaire. - Parasitisme permanent.

Nous allons aujourd'hui considérer les insectes sociaux qui ont adopté cux-mêmes l'existence de parasites dans les colonies d'aulres insectes sociaux; sujet qui a été exIrèmement étudié durant le dernier quart de siècle. Des cas de parasitisme social ont été observés chez tous les groupes de guêpes, abeilles, fourmis et termites, mais ils sont surtout fréquents, et ont été plus soigneusement étudiés, chez les fourmis. Les rapports entre les parasites sociaux et leurs hôtes sont très divers, à la fois dans leur caractère et dans le degré d'intimité. Un parasite et un hôte qui sont étroitement parents par leur structure et par leur éthologie, et qui représentent des espèces d'un même genre, ont entre eux des rapports beaucoup plus étroits que des espèces ayant des relations lointaines ou que celles qui appartiennent à des sous-familles ou à des ordres différents. Ainsi, bien que des colonies de fourmis et de termites puissent contracter des rapports les 
unes avec les autres, ces rapports ne sont jamais très inlimes et consistent soit en prédatisme de la part des fourmis, soit en un simple parocisme, ou tolérance indifférente. Du point de vue de l'évolution, il est intéressant de noter que les nombreux cas actuellement connus de parasitisme social sont tous des développements convergents et sporadiques; et si nous laissons de côté, comme nous en avons l'intention, les termites, du fait de l'insuffisance de nos connaissances et de notre temps trop limité, si nous fixons notre attention sur les seuls Aculéates, nous trouverons que l'infestation de leurs colonies par d'autres Aculéates est, par beaucoup de ses caractères, très différente de tous les types connus de parasitisme. Les seuls phénomènes qui s'en rapprochent sont la parasitisation de groupes et de races humaines par d'autres groupes de races humaines, c'est-à-dire, une parasitisation se produisant entre sociétés de même espèce, sujet qui n'a pas attiré comme il le méritait l'attention des sociologues depuis qu'il fut mis en lumière, il y a de nombreuses années, par Massart et Vandervelde (1 893 ). Cerles, Giarn avait parfaitement saisi la signification du parasitisme hu. main, puisqu'il donna aux travaux des savants helges une place dans son "Bulletin Scientifique ") (I).

(1) Des cas analogues à ceux que rapporient Massart et VANdervelde dans les paragraphes suivant et beaucoup d'autres, peuvent se présenter à tout observateur, non prévenu, des sociétés humaines actuelles ou à tout historien: "Le parasitisme social, caractérisé par l'emprunt des moyens d'existence, n'occasionne pas de perturbations aussi graves que le parasitisme de nutrition dont nous venons de décrire les effets. Cependant il arrive que les parasites sociaux se multiplient de telle sorte, ou acquièrent un tel degré de nocuité, qu'ils épuisent conplètement les travailleurs qui les font vivre. SPEnren rapporte qu'en Orient, la rapacité des monarques a élé que!- 


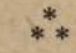

Nous parlerons rapidement des guêpes et des abeilles qui sont des parasites sociaux, avant de passer en revue. les cas de parasitisme social offerts par les fourmis. Les guêpes solitaires parasites sont elles-mêmes rares et limitées, autant qu'on sache, aux espèces des genres Ceropales, Nysson, quelques Pompilus peu nombreux (peclinipes, campes/ris et aculeatus), et quelques Stizus (uni. cinctus). Chez les espèces sociales, nous connaissons seulement deux formes parasites, Vespa austriaca et $V$. arctica. La première est connue depuis longtemps en Europe, où elle vit dans les nids de $V$. rufa [Morawitz (i 864), Robson (i 898), Carpenter et Pack-Beresford (rgo3), etc.] Tout récemment, Bequaert (igi6) et StaDEN (I918)-ont trouvé austriaca aux Etats-Unis, au Canadia et à l'Alaska, mais on ignore encore quel est son hôte nord-américain, bien qu'on le suppose être $V$. con-

quefois jusqu'a prendre une si grande partie dı produit des sujels, qu'il fallait ensuite leur rendre de quoi faire les seinailles.

L'Espagne, à la fin du siècle dernier, peut être considérće comme le type de la communité réduite à une sorte de consomption économique par le développement effrayant des parasites et des travailleurs insuffisamment productifs. Sous Philippe III, on comptait 988 couvents de femmes et 32.000 moines mendiants; le nombre des monastères avait triplé pendant les cinquanle années qui précédèrent 1624, et le nombre des moines s'était accru dans une proportion plus forte encore. Le recensement de 1788 donna un tolal de 1.221 .000 prêtres, soldals, marins, nobles, avocats, employés du fisc, étudiants el domestiques sur environ 3.800 .000 hommes, desquels il faut rléduire encore une masse de mendiants, de vagabonds, cle. (Rochin, Principes d'Econ. Pol. II. p. 144, 145. Trad. Noln(WSKI) 》. 
subrina. Flétchen ( 1908$)$, Taylon et moi ( 1921$)$, nous avons prouvé que $V$. arctica vit dans les nids de l'espèce si commune, $V$. diabolica. Austriaca et arctica ont l'une et l'autre complètement perdu leur caste d'ouvrières, et ne sont donc représentées que par des mâles et des femelles fécondes. Il fut un temps, certainement, où elles n'étaient pas parasites, tout comme leurs hôtes actuels ; mais aujourd'hui, elles sont élevées et nourries par les ouvrières des hôtes, absolument comme les propres formes sexuées, plus favorisées', de ces derniers. Il résulte Ue ce mode d'alimentation que ces insectes sociaux, primilivement indépendants, ef possédant deux castes dis. tinctes de femelles, sont actuellement revenues au statut des insectes solitaires, n'ayant qu'un type unique de femelle.

Comme nous connaissons plusieurs genres d'abeilles solilaires parasites, (voir WheEIER 1919a), nous ne sommes point surpris de découvrir des Apidés parmi les parasites des abeilles sociales. En fait, toutes les sous-familles possèdent de tels salellites, sauf les $\Lambda$ pinés, et, dans chaque cas, il est évident que le parasite dérive du mème genre que l'hote. Brauns (1902) a décrit une espère, Eucond!lops lionowi, comme parasite de l'Allodape, mais il n'a recueilli aucune observation sur ses mours. Les abeilles banales du genre Sphecodes, ont été étudiées par un grand nombre de chercheurs: W'arckeNafr (1817), Mrsmifi. (1835), Lepeletifn (1811), Spinoli (1851), Taschenberi: (1866), F. Simtir (1851), Sirhel (1865), Bneitenbach (1878), J. Pítiez. (1881), Penkins (1887, 1889), Morice (1901), Fenton (1890, 1898. 1905), Manchar. (1890, 1894), Stanen (1895), Nielsen (1903), Friese: (I!)?3), mais il nous manque encore des données définitives sur leurs relations avec leurs hoiles, les abeilles du genre Haliclus. Un mémoire de Stöcknent, qui prometlait d'éclaircir la question, n'a pas encore élé publié. D'après les oloservations de la plupart des auteurs indiqués plus haut, il semblerait que les fromelles àgées d'Haliclus qui veillent à l'entrée du nid, s'opposent violemment à l'invasion des Sphécodes. Fenton (1905) a vu un $S$. subquadralus faire irruption dans un nid de IIalic- 
lus malachurus : "Ne pouvant saisir la sentinelle qui lui barrait le passage avec sa tête, il creusait un trou contre le terrier des Abeilles; il parvint ainsi à saisir et à tuer la gardienne, qu'il rejela derriere lui en dehors du conduit. Un second, puis un troisième Halictus vinrent successivement remplacer le premier, et curent le même sort. "Après avoir conquis une entrée du nid, le Sphécode paraît détruire les oufs des cellules achevées par les Halictus, et pondre ses propres œufs à leur place, de sorte que les larves qui en naissent n'ont point à entrer en compétition avec les propriétaires légitimes du pollen et du nectar emmagasinés. Le Sphécode est donc un parasite prédateur. Cependant, quelques auteurs (Heselhuus 1923, elc.) ont récemment soutenu que celle abeille construit et approvisionne ses propres alvéoles à l'intérieur des nids d'Halictus.

Les parasiles les plus intéressants des abeilles sont incontestablement les especes de Psithyrus (Apathus), qui sont depuis longtemps connues pour vivre et se reproduire dans les colonies de Bombus. Les espèces européennes ont été étudiées par F. SMith (185j), Hoffer (1881, 1888), Friese $(1888,1923)$, SlaneN (1899, 1912), Wigner (1907), etc. ; certaines espèces de l'Amérique du Nord par Slidin (1915), Frisox (1916, 1921) et Platu (1922). Le genre Psilliyrus est évidemment un rejeton du genre Bombus, et, comıne les guêpes parasites, il a perdu sa caste d'ouvrières. Il est très probable que les c-pèces de ce genre sont polyphyléliques, c'est-à-dire qu'elles dérivent de plusieurs espèces différenles de Bombus. Conme chez d'autres abeilles parasites, les femelles n'ont point développé l'appareil collecteur des paltes postéricures, mais, ì tous autres égards, elles ressemblent alosolument a leurs hôles. Dans quelques espèces, celle ressemblance est-remarquablement frappante, surlout dans la coloration, el on l' a interprétée comme une forme de mimétisme; mais, d'apris la théorie de la dérivation polyphylélique, elle serait véritablement génétique (PL.1TH, 1922). La femelle de P'silh!n'us a quelque difficulte i penetrer dans un nid de Bombus; mais, lorsquelle y est installée, elle ne tolère plus la présence d'une autre femelle de Psithyrus. Suivant Sranex, le parasite tue la reine de lhote, mais Smitn, IIofren, et Pr.utu onl niontré que cela ne se produit pas loujours, ni même d'une faģon générale. Plith a découvert, pour une espèce nordatméricaine, Ps. laboriosus, qu'après avoir réussi a pénétrer dans le nid de Bombus vaglans, le parasite intimide les ouvrieres en les bousculant avec violence, mais il ne les pique pas. Après celle période de "prise de contacl", les membres de la colonie de tagans-laboriosus ne présentent entre eux pas plus 
d'hostilité que les membres d'une colonie non infestée. La reine parasite ne pond pas toujours (si même cela lui arrive) ses œufs dans une masse de pollen contenant déjà des œufs ou des larves de l'hóte; mais, comme la reine des Bombus, elle construit ses propres alvéoles et, comme cetle dernière, elle les fixe à un ou à plusieurs des cocons déjà existants. Néanmoins, suivant Pцiтh, la femelle de Psithyrus détruit les œufs el les jeunes larves de son hôte, ce qui lui assure l'élevage de sa propre couvée par les ouvrières de Bombus. Ainsi donc, le parasile se comporte bien comme un prédaleur.

Friese (1923) a publié de courtes notes, extrêmement inléressantes, sur une abcille néotropicale prédatrice de la famille des Méliponinés. Il considère cette espèce, précédemment appelée Trigona limao, comme le type d'un nouveau sous-genre, Lestrimelitta (Friese 1903), parce que les ouvrières n'ont point le corbula aux pattes postérieures, caractère prouvant qu'elles ne récoltent pas de pollen. Il soutient que limao est bien une abeille voleuse, qui subjugue les colonies de quelques espèces industrieuses de Trigona, chasse ou tue les proprićlaires légilimes du nid, s'inslalle dans ce nid et se nourrit du pollen et du miel qui y sont accumulés, puis s'en va conquérir le nid de quelque autre colonie pacifique.

Ces quelques indications sommaires sur les abeilles sociales parasites montrent très nettement qu'elles sont loutes plus ou moins prédatrices. La Lestrimelitta est, par le fait, si fortement prédatrice qu'on peut à peine la considérer comme un parasite. Psithyrus dénote aussi un caractère nettement prédateur dans sa façon de pénétré dans le nid, d'assurer par force son adoption et de détruire les œufs et les larves de son hôte. Les mœurs des Sphécodes paraissent similaires, d'après ce que nous en savons. Celles de Vespa austriaca et arctica n'ont pas été étudiées, mais ces parasiles ne doivent certainement pas être adoptés par leurs hôtes sans qu'il y ait conflit.

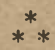

La littéralure se rapporlant aux parasites sociaux des 
fourmis a pris une extension si considérable qu'il m'est impossible de la mentionner toute. La majeure parlie en cst consignée dans mes ouvrages ( $\left.1910,19^{2} 3\right)$ et dans le premier volume de Wasmanx, "Gesellschaftsleben der Ameisen " ( $1915 c)$. Comme on pouvait s'y attendre, les fails se compliquent singuliêrement quand un superorganisme social, comme une colonie de fourmis, s'associe intimement avec une autre colonie. Il y a longtemps déjà, Wasmann (I $89 \mathrm{r}$ ) divisa ces associations en deux catégories, les " nids composés " et les "colonies mixtes ». Quoique cette division soit encore assez correcte, la distinction n'est pas aussi tranchée que Wasmann 'e supposait; je le montrerai par la suite. Les nids composés sont des associations entre deux ou plusieurs colonies entières de fourmis, de fourmis et de termites, ou de termites, vivant dans une contiguïté étroite, mais en réalité dans des nids différents, et élevant séparément leurs couvées respectives. Les colonies mixtes de fourmis (on n'en connaît pas chez les termites) représentent une fusion du personnel de deux ou rarement plusieurs colonies, qui occupent le même nid et élèvent leurs convées en commun. A quelques rares exceptions près, seule l'une des colonies est complète, c'est-à-dire possédant une reine fertile aussi bien que des ouvrières. Les fourmis qui forment des nids composés peuvent être divisées en plusieurs sous-catégories, les cleptobiotiques, lestobiotiques, parocismiques (plésiobiotiques), parabiotiques et xénobiotiques, ou myrmécophiles. Tous ces sous-groupes comprennent un certain nombre de cas, allant de la simple contiguité, basée sur le prédatisme actif de l'un des composants, jusqu'à l'état de symphilie pure et simple, en passant par la tolérance indifférente ou une coopéra- 
tion modérée. Cette série, dont je décrirai quelques cas, se développe donc parallèlement à la série progressive des myrmécophiles et des termitophiles, passant danj leurs relations avec leurs hôtes, des synechthres aux symphiles, par les synœcètes.

$1^{\circ}$ Différentes colonies de fourmis, mồme d'une seule espèce, sont si hostiles entre elles que leur simple existence còte à côte implique que l'une des espèces exploite plus ou moins sa voisine. On donne à juste titre le nom de brigands (cleplobiotiques) à certaines espèces, de petite taille, mais ilgressives, qui s'àssurent une partie all moins de leur subsislance en guetlant au passage les fourrageurs d'une autre espèce et en s'emparant de leur proie. De telles fourmis édifient naturellement leurs nids auprès de ceux des espèces qu'elles dépouillent. Ainsi, Dorymyrmex pyramicus, des Etats du SudOrest, contruit souvent ses nids dans les environs immédiats, ou mème sur les larges talus des fourmis moissonneuses du genre Pogonomyrmex.

$2^{\circ}$ Dans le cas de lestobiose, ou de rol, l'exploitation est plus subtile et plus efficiente. Les fourmis voleuses, qui-vivent toules dans le sol et ont des ouvrières très petites, nichent dans les cloisons en terre des fourmilières ou termitières bien peuplées. Les chambres des deux nids sont reliées par des galeries extrêmement étroites, permettant aux fourmis voleuses d'envahir les nids et de dévorer la couvée de leurs voisins plus gros, mais ne permeltant pas à ces dernicrs de pénétrer dans les nids de leurs voleurs, qui sont, ou ignorés, ou négligés, a cause de leur petite taille el de leur odeur neutre. L'abondance de la nourriture qui leur est ainsi assurée leur permet d'élever des reines et des mâles très gros et souvent forlement colorés, mais les ouvrières même sont condamnées à un nanisme perpétuel et à une coloration très atténuée, à causc de leur mode de vie criminel. Les fourmis voleuses les plus remarquables appartienent au genre Carebara et ont été Irouvées dans les énormes termitières des tropiques. Les ouvrierres de ces fourmis sont minuscules, jaune pàle et aveligles; les reines et les màles fortement colorées et plusieurs millicr's de fois plus gros que les ouvrières. AnNold (1916) a suggéré récemment que ces différences extraordinaires dans la taille emprecheraient absolument la reine de nourrir sa première 
couvéc d'ouvrières et d'élablir par suite sa fourmilière de la façon indépendante qui est le type normal chez les autres fourmis. Pour ces raisons, lorsque la reine abandonne le nid maternel pour accomplir son vol nuptial, elle emporte, fixćes par leurs mandibules aux masses pileuses de ses pattes, quelquesunes de ces minuscules ouvrières qui l'accompagnent ainsi

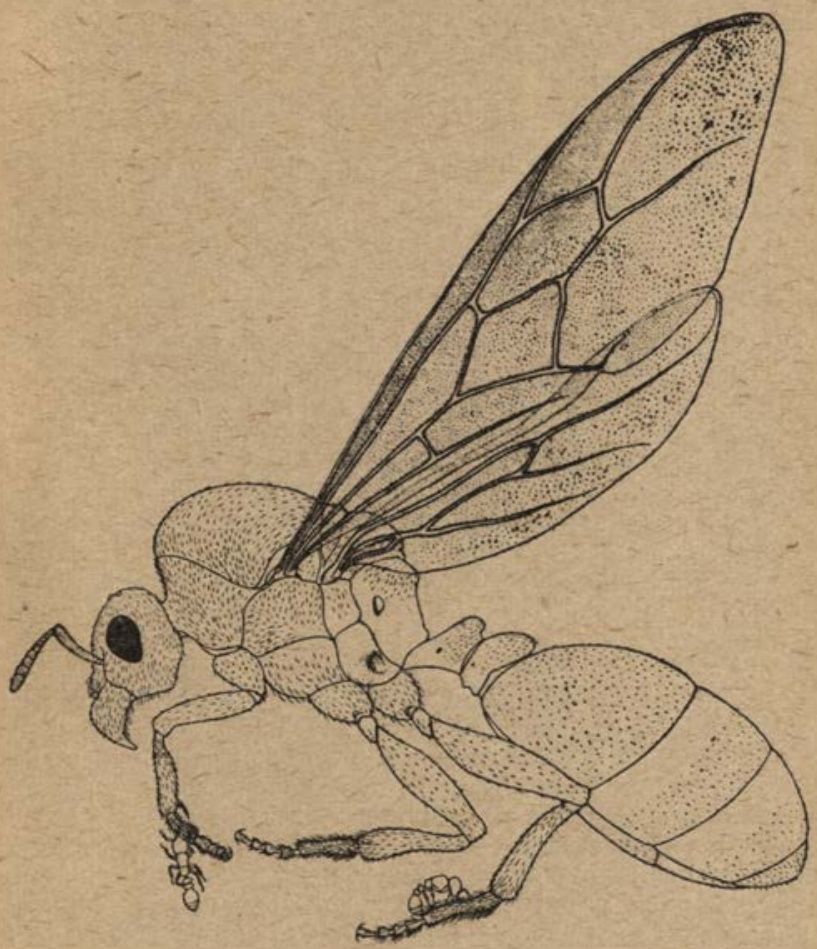

Fis. F. - Reine ailee de Carebara vidua, portant de petiles ouvrieres aveugles de son espèce sur les poils de ses tarses. 
jusqu'à ce qu'elle ait édifié sa cellule dans quelque termilière et qui assurent alors l'élevage de sa première couvéc.

$3^{\circ}$ La plésiobiose ou parœcisme ("voisinage amical ») est un type très hanal de relations entre deux ou plus rarement trois ou même quatre espèces de fourmis, vivant dans des nids ayant souvent des galeries entremêlées, mais jamais des galeries communicantes, sous la même roche ou dans la même prairie. En général, quand les fourmis des différentes colonies se rencontrent, elles manifestent une certaine hostilité. Si l'une des espèces est petite et faible, elle se trouve sûrement quelque peu protégée en vivant simplement dans le champ trophoporique de sa voisine plus grosse et plus puissante; ou bien la plus faible se nourrit en une certaine mesure des déchets de la plus forte. Lorsque les deux espèces sont de grande taille et agressives, elles peuvent peut-être trouver quelque avantage à présenter un front hostile combiné aux altaques d'alltres fourmis.

$1^{\circ}$ Ce que Fonel (1898) a baptisé " parabiose \#, (ce que l'on pourrait traduire par " lutelle »), parait être un type de relation mieux défini, consistant en une protection mutuelle ou uni. litérale. Dans un cas typique que j'ai récemment observé dans la Guyane anglaise (1921), nous avons deux espèces, un petit Cremalogaster noir, (C. parabiotica), et un gros Camponotus brun (C. iemoratus) habitant ensemble dans une grosse boule de terre qu'ils ont agglutinée autour d'une branche d'arbre. Dans ce nid, qu'on peut appeler " jardin de fourmis " puisqu'il porte de nombreux épiphyles, le Crematogasıer occupe uniquement les portions superficielles et le Camponotus les portions centrales. Si le nid est légèrement attaqué, les patites fourmis noires se précipitent au dehors pour lutter contre les intrus; mais si le danger est plus grave, aussitôt apparaissent les bataillons de l'espèce brune, plus forte et extrèmement belliqueuse. Les Cremalogaster seraient donc comme les lignes avancées prolégeant les Camponolus. Bien que les galeries communiquent librement entre elles, et bien que leurs ouvrières aillent ensemble quêter leur nourriture, formant de longues files communes, parmi les végétaux environnants, les deux espèces maintiennent une séparation absolue entre leurs couvées.

50 Les rapports entre le: fourmis occupant les nids composés deviennent encore plus intimes dans les cas de xénobiose, dont le meillcur exemple est fourni par Leplothorax emersoni et 
Myrmica canadensis. Comme nous l'avons vu dans une précsdente leçon, ce Leptothorax est véritablement un assocjé ou symphile, s'ilimentant des sécrétions superticielles de son hòte et de nourriture régurgitée par ce dernier (Wneelen $1901 \boldsymbol{c}$, $1903 c, 1907 c$ ). Bien que les deux espèces élèvent séparément leurs couvées, il est possible, dans les nids artificiels ne contenant pas de terre, de les amener ḋ fusionner leurs couvées et à former une colonie mixte. Au même groupe de fourmis associées appartient probablement une autre espèce nord-américaine, Symmyrmica chamberlini, qui vit dans les nids de Neomyrma mutica, dans l'Utah, et qui a des mâles aptères; et aussi l'espèce européenne, Formicoxenus nitidulıs qui vit dans les talıs de Formica rufa et pratensis et a aussi des màles aptères, mais nettement ergatomorphiques. Bien que $F$. nitidulus ait été étudiée par Anlerz. (1884), Wasmanx (1891, 1915c), StaeGER (1918) et STUMPER $(1918 a, 1918 b, 1921)$, on a encore quelques doutes sur la nature de son aliment. Stumper pense qu'elle se nourrit de l'eau de pluie chargée de matières végétales, après sa Tiltration à travers les matériaux du nid de son hôte, mais ceci semble peu probable. Suivant Brun (1924), Staeger a indiqué que liormicoxenus est bien nourri par son hôte comme Leptothorax emersoni.

J'ai trouvé récemment, dans une petite clairière pratiquée pour la construction du laboratoire tropical de l'ile BarroColorado dans le lac de Gatun, Panama, un nouvel associé des fourmis, très spécia', dont je donnerai ici une description succincle, en attendant la publication de mes mémoires sur sa taxonomie et ses mours (1925). Pendant la saison des pluies, on trouve dans celle île au moins quatorze espèces de fourmis cultivant des champignons, appartenant à la tribu des Attini, et représentant à peu près tous les genres décrils; beaucoup d'entre elles sont présentes dans la petite clairière en question. J'ai découvert p'us de douze nids d'une forme minuscule, Sericomyrmex amabilis, sp. nov., à quelques pas du lahoratoire, chacun renfermant un ou plusieurs jardins de champignons dans de petites chambres et souvent suspendus à des racines laissées intactes par les fourmis quand elles creusèrent le sol. Il y avait cent à trois cenls ouvrières par nid, une seule reine très nonchalante et une nombreuse couvée, élevée dans les jardins de champignons, qui avaient seulement deux a trois pouces de diamètre. Dans presque lous les nids, j'ai rencontré une colonie d'une fourmi associée, appartenant ḋ un genre néotropical très singulier, Megalomyrmex, mais représentant une espèce et un sous-genre nouveaux, que j'ai appe- 
lée Cepobrolicus symmetochus. C'est une fourmi menue, plus petile et plus grêle rue son hôte, rouge et hérissé de
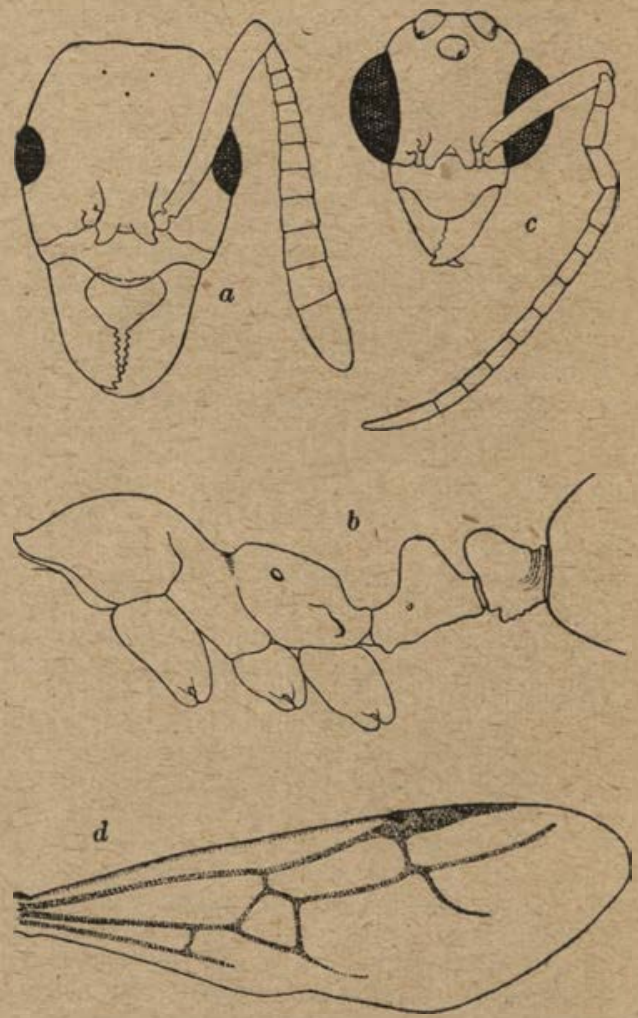

lig. 5ૅ. - Megalomyrmex (Cepobroticus) symmetochus Wheeler.a. Lèle d'ouvrière; $c$. tête de mảle.

poils. En oulre, ses colonies sont plus peliles, comprenant seulement de quirante à cinquante individus, et une reine uni- 
que, pas plus grosse que les ouvric̀res. Les deux espèces vivent ensemble amicalemeut, et, ḋ l'occasion, se lèchent entre elles, mais elles n'échangent aucune autre allention. Quand on les transporte dans les nids artificiels, on voit les ouvrieres des deux espèces vivre et se nourrir sur les jardins de champignons qui sont cultivés exclusivement par les Sericomyrmex. Les reines, qu'on trouve souvent côte à côte sur les champignons, éparpillent leurs œufs parmi les bouquets d'hyphes. Les larves et les pupes sont donc mélangées les unes aux autres, mais, à ce que j'ai vu, chaque espèce s'occupe uniquement de sa propre progéniture. Ce cas est exceptionnellement intéressant, d'abord parce que la fourmi associée appartient a un genre particulier, dont les autres espèces connues ne sont nullement mycétophages, et, d'autre part, parce qu'il se présente dans des conditions qui forment nettement un intermediaire entre les nids compusés et les colonies mixtes de IV asmax. J'ai désigné cette forme nouvelle et unique de symbiose sociale du noin de "mycétométochie".

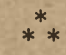

Les fourmis vivant dans les divers nids composés ne sont pas très proches parentes, mais appartiennent à des genres différents ou mème à des sous-familles différentes; ceci explique qu'elles occupent des nids séparés et qu'elles n'élèvent pas en commun leurs couvées, les soins réclamés par la couvée étant extrêmement délicats et variant beaucoup d'une espèce à l'autre. Nous nous attendons par suite à trouver que les colonies mixtes soient formées seulement par des espèces très rapprochées, c'est-à-dire apparlenant soit au même genre, soit à des genres très voisins; cela a bien été reconnu exact. Mais avant d'exa. miner les divers types de colonies mixtes, il nous faut insister sur deux faits :

$I^{\circ}$ On a découvert que beaucoup de fourmis volent et mangent les larves et les pupes appartenant à d'autres colnnies, de leur propre espìce ou d'espèces différentes. 
Toute une sous-famille, celle des Dorylinés, pratique très nettement et de façon constante ce genre de rapt.

J'ai été souvent témoin de telles expéditions accomplics par nos très petites espèces d'Ecilon (Acamatus), el j'ai observé lout récemment les mêmes habitudes, mais à plus grande échelle, dans des espèces plus grosses ( $E$. burchelli et hama(um). J'ai, en outre, observé, en Australie, que les membres d'un genre, au moins, de Cérapachyinés, Phyracaces, font des ravages du mème genre dans les colonies d'aulres fourmis, dont elles prennent la couvée et la transportent comme une proie dans leurs propres nids. M. J. CLiRK, de Perth (Australic occidentale) m'a raconté qu'il a été récemment témoin d'une expédition de ce genre, exécutée dans une colonie d'Iridomyrmex gracilis, par une espèce non décrite de Phyracaces (1).

11 arrive fréquemment que les jeunes ainsi volés soient. dévorés, mais, dans les colonies bien approvisionnées, on leur laisse achever lear développement et les ouvrières ainsi formées sont adoptées comme membres bonâ fide de la colonie, même si elles appartiennent à une espèce différente. Il est ainsi possible de produire expérimen. talement une colonie mixte, en fournissant à la colonie la couvée, à maturité, d'une autre espèce. Par ce moyen, Miss Fielde (Igo5) parvint à amener des espèces appar-

(1) Rappelons que Darwin faisait dériver de ces mœurs l'habitude de faire des esclaves, que possède Formica sanguinea, et, en dépit de tous les arguments dont Wasminn appuie l'hypothèse inverse, je crois encore qu'il y a une très grande part de vérité dans l'hypothèse phylogénétique du grand naturaliste. $F$. sanguinea a été primitivement une fourmi prédntrice, descendant très probablement de formes ayant des habitudes très voisines de celles d'Eciton; mais actuellement, elle en est venue à limiter ses incursions à certaines espèces, abondantes et prolifiques, du même genre qu'elle (variétés de $F$. fusca, cinerea, neogagales, pallidefulva, elc.). Elle dévore toujours la plus grande partic des larves el des pupes dont elle s'est emparée. 
tenant mème à des sous-familles différentes, à vivre $\mathrm{en}$. semble dans une entente parfaite. Il est intéressant di: constaler, en outre, que les fourmis élevées ainsi dans la colonie d'une espèce étrangère peuvent se montrer très hostiles vis-à-vis de leurs propres sœurs qui sont restées et se sont développées dans le nid maternel.

$2^{\circ}$ Le second point, sur lequel je désire insister, est le suivant: les colonies mixtes rencontrées dans la nature ne sont point produites, dans le premier cas, simplement par le vol de la couvée d'une espèce étrangère, mais aussi par une jeune reine d'une espèce parasite, qui n'est point capable d'établir une colonie indépendante et qui enva. hit le nid d'une autre espèce, laquelle devient ainsi son hòte. L'envahisseur et l'hôte se comportent différemment suivant les espèces; mais, dans presque tous les cas observés, la reine de l'hôte, si elle existe, est tuée, et l'intruse prend sa place. Comme la reine des fourmis est véritablement l'organe reproducteur de la colonie considérée comme un superorganisme, on peut dire que la colonie envahie est castrée et son personnel d'ouvrières stériles est contraint de consacrer toutes ses forces à élever la couvée que produit aussitôt le parasite fécond.

Ayant présentes à l'esprit ces considérations générales, uous allons examiner maintenant les divers types de colonies mixtes, parmi lesquelles nous distinguerons trois catégories: celles des faiseurs d'esclaves, et celles des parasites sociaux, temporaires ou permanents.

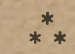

I Le phénomène particulier connu sous le nom d'esclacage, on "doulose ", se produit dans deux genres de 
Formicinés, Formica et Polyergus, et deux genres de Mỹrmicinés, Strongylognathus el Hurpagoxenus; il présenle trois slades phylogénétiques: un stade primitif, (a) chez Formica sunguinea, un stade culminant (b) chez Polyergus et un stade évanescent (c) ou dégénéré (d) chez Strongylognathus et Harpagoxenus.

a) Formica sanguinea est une fourni rouge esclavagiste, commune mais plutôt localisée et ayant un abdomen nour ou brun; elle est représentée par de nombreuses sous-espèces et variétés, occupant le nord de l'Europe, l'Asie et le Nord de l'Amérique. Elle est facile à distinguer des autres espèces du genre, au moins dans l'Ancien Continent et les Etats-Unis de l'Est, par la découpure très accentuée du chaperon. Sanguinea est l'une des fourmis les plus intelligentes, et par suite l'une des plus intéressantes à conserver dans les nids artificiels. Ses mours furent étudiées en premier lieu, il y a plus d'un siècle, par Pierre IIunen (1810), le fils de François Hr̈вг", l'aveugle, et, depuis, elle a toujours attiré l'altention des myrmécologistes, parce que ses armées d'ouvrières font des incursions périodiques dans les colonies d'une espèce noire très cominune, Formica fusca; elles enlèvent les larves el pupes d'ouvrières, les transportent dans leur nid, en laissant beaucoup se développer et les conservent comme membres de leur colonie. Ainsi la colonie est mixte, et les individus noirs ont èté appelés esclaves à cause de leur provenance et de leur couleur. Il est évident loutefois, que ce terme est peu approprié, car un esclave est " un homme qui esl la propriélé d'un intre homme, occupant politiquement et socialement un nivean inférieur à celui des ciloyens ordinaires, et contraint par la lorce au travail " (NiEßoER); et aucun de ces caractères dislinctifs n'est applicable aux ouvrières fusca dans le nid de sanguinea. On devrait plutôt les appeler des "auxiliaires " (Hilfsameisen). L'une au moins des sous-espèces de sanguinert (aserva) ne fait pas d'esclaves, et-les colonies de quelques inrmes perdent cette habitude au bout d'un certain temps; la colonie de sanguinea, une fois étahlie, peut donc parfaitement mener une vie indépendante.

I)iRwin el d'autres ont fourni des explications variées de celte habiude particuliere aux sunguinen, mais la signification en est restée obscure, jusqu'à ce que j'aie découvert (1905, $1906,1907 a, 1908 b)$ qu'elle a son origine dans les mœurs de la 
jeune reine. Celle-ci est absolument incapable de fonder seule une colonie; aussi, après son vol nuptial, doit-elle adopte. l'une des trois méthodes suivantes: ou retourner au nid ou elle a été élevée, ou pénélrer dans quelque autre nid de sanguinea, ou envahir un nid de Formica fusca. Comme le premier et le second moyens sont quelquefois adoptés par d'autres fourmis et ne conduisent point à la formation de colonies mixles, nous ne nous y arrèterons pas davantage et nous étudierons seulement le troisième. Dès que la reine sanguinea

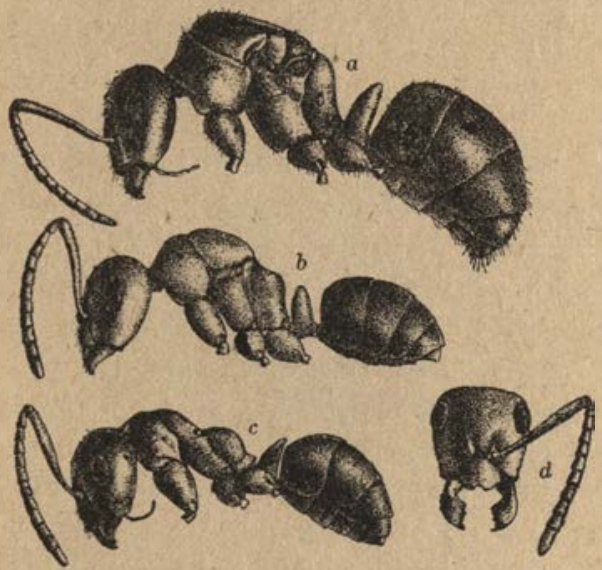

Fig. 56. - Formica sanguinea (profil). a. reine (ailes el pattes supprimées) $; b$. pseudogyne ; $c$. ouvrière ; $d$. tête d'ouvrière, avec l'échancrure caractéristique sur le clypeus.

it envahi une colonie de fusca, elle devient très agitée, elle s'intéresse beaucoup à la couvée, prend et réunit en une pile autant de pupes qu'elle en peut saisir el monte la garde autour d'elles. Elle tue quelques ouvrieres lusca qui sont assez hardies pour tenter de reprendre leur hien el elle est bientôt laissie en toute tranquillite, maîlesse du produit de son larcin. l'arfois, des ouvrières fusca sortent des cocons et adoptent immédialement une altilude amicale à l'égard de la reine, là 
nourrissent par régurgitation et la traitent comme si elle étaıt leur propre mère. Elle commence à pondre des œuls et les larves qui en sortent sont nourries et élevées par les ouvrièré noires, si bien que l'apparition des ouvrières sanguinea coïncide avec la formation d'une colonie mixte. Ces ouvrières prouvent qu'elles ont hérité dle leur mère les tendances à voler 'id couvée des colonies voisines de fusea, mais elles pratiquent ce vol en groupe et transportent la couvée de fusca dans leur nid. Dans certaines colonies, comme je l'ai indiqué, celle lendance au vol el à l'esclavage peut disparaître après un cerlain temps et, chez aserva, elle semble s'effacer très précocement, ou peut-être même n'est-elle pas transmise aux ouvrières. Dans de tels cas, le personnel des colonies âgées peut done ctre uniquement formé de sanguinea, après que soient mortes de vicillesse les ouvrières fusca du premier lot volé et éleví par la reine.

Ces mœurs, que j’ai observées chez les jeunes reines de sous-espèces américaines de sanguinea, ont été plus tard retrouvées chez les formes européennes typiques, par Vienmeyer (rgo8, igog, igio a, igro b), Wasmain

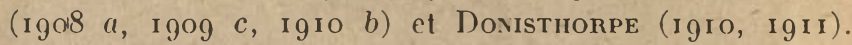
Il est évident que l'esclavage est au fond une forme de prédatisme et tire son origine de l'impossibilité où se trouve la jeune reine de fonder une colonie sans l'aide d'ouvrières. A l'encontre de la plupart des reines de four. mis, elle n'a pu accumuler dans son corps assez de nourriture pour supporter un long jeûne et alimenter sa première couvéc. A un autre point de vue, elle est certainement un parasite, et les ouvrières fusca en sont. l'hôte. La colonie pouvant éventuellement cesser d'accroître son personnel ouvrier par le vol de couvées de fusca, c'est là une raison suffisante pour donner à ce type d'esclavag'e le qualificatif de temporaire, aigu on facultatif.

b) Les espèces de Poluergus, ou " amazones " comme les a appelées Pierre Huber (1810), ont à peu près la même distribution que sanguinea et ont pour esclaves les mêmes espèces 
de Formica; mais leur façon de s'assurer ces esclaves est extrêmement perfectionnée. Les amazones sont de très belles fourmis rouges (sauf l'espèce japonaise, $P$. samurai, qui est noire); elles ont des mandibules minces, en forme de faucille, parfaitement adaptées pour le combat, mais inutilisables pour creuser le sol ou capturer une proie. Ces insectes ne peuvent donc, ni faire des nids, ni se nourrir eux-mêmes, ni soigner leurs jeunes; ils dépendent absolument de leurs esclaves. Comme sanguinea, les amazones font des incursions périodiques, qui ont toujours lieu, pour on ne sait quelle raison, l'après-midi; mais leurs armées ont une organisation et une tactique des plus perfectionnées; la soumission et le pillage

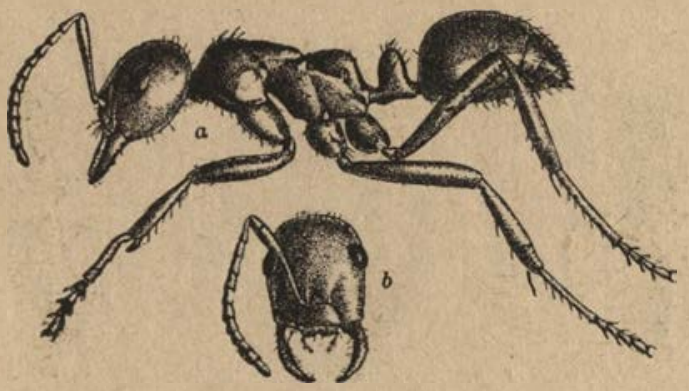

Fig. 57. - Polyergus lucidus. a. ouvrière (profil); b. tête avec mandibules en faucille.

des colonies de fusca s'effecluent avec rapidité et précision, on peut dire même avec l'éclat le plus consommé. En général, à l'approche des amazones, les ouvrières fusca fuient terrifiées; mais si elles tentent quelque résistance, les amazones leur transpercent la tête de leurs mandibules recourbées. Au sorlir des pupes volées, les jeunes ouvrières creusent le nid, nourrissent les Polyergus, et élèvent leur couvée, mais elles ne suivent pas les armées dans leurs raids. Les premiers stades de la fondation des colonies ont été étudiés par EMery $(1908 b, 1909 a, 1909 b, 1911 a, 1911 b)$, qui trouva que les jeunes reines Polyergus se font adopter par quelque petite el faible colonie de fusca, aptès en avoir tué la reine d'une blessure à la 
lête. Elle donne ensuite naissance à la couvée qui se livrera plus tard à la conquète et à la soumission des colonies de fusca. Ces tendances aux expéditions ne disparaissant jamais, inème dans les colonies àgées, on peut regarder polyergus comme un faiseur d'esclaves chronique, ou obligatoire.

c) En Europe, il y a plusieurs espèces du genre Strongylognalhus, fort intéressant; ces fourmis ont, comme Polyergus, des mâchoires en faucille, elles appartiennent toutefois a une sous-\{amille toute différente, celle des Myrmicinés, et elles vivent toujours dans les colonies de la fourmi des pavés, Tetramorium cospitum. Nos connaissances fragmentaires
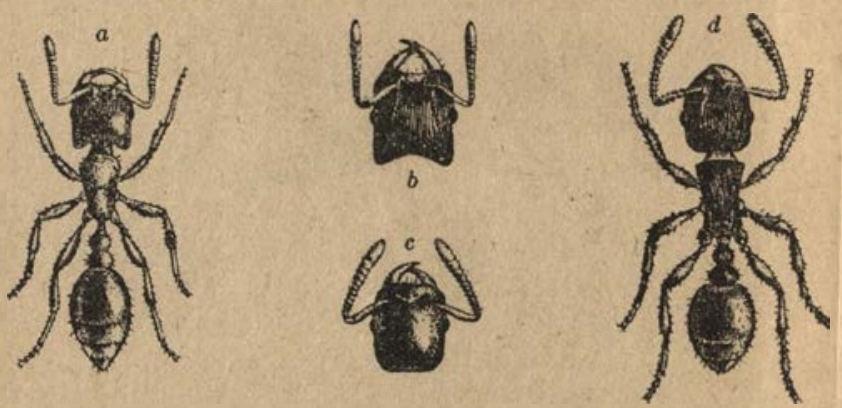

Fig. 58. - Strongylognathus testaceus. $a$. ouvrière; $b$. tête d'ouvrière; $c$. tête d'ouvrière de Str. huberi; $d$. ouvrière de Tetramorium cæspitum.

nous indiquent que ce cas représente un type d'esclavage dégénéré ou évanescent. Les ouvrières de $S$. rehbinderi et huberi paraissent faire encore des incursions dans les colonies de Tetramorium et transporter les couvées prises (Forel, 1900), KutTER $(1920,1923)$ a observé aussi des raids nocturnes accomplis par alpinus, une sous-espèce de huberi que j'ai découverte, il y a plusieurs années (1909), près des sources de Visp, en vue du Matterhorn; dans ces raids, elle est accompagnée par ses esclaves, qui prennent une grande part au combat et ramènent au nid la couvée de leur propre espèce. Dans ce 
cas, les esclaves sont vraiment les maitres et ils semblent cmployer les Strongylognalhus simplement pour déconcerter ou effrayer les colonies de caspitum, dont ils veulent voler la couvée. En dernier lieu, l'espèce du genre la mieux connue, $S$. testaceus, ne fait aucune expédition, et sa caste ouvrière tend ì disparaître. Wasminn (1891, 1915c), Mrazer, Foner. et moi (1908), nous avons découvert que les colonies de cxs. pitum infestées par cette espèce conservent leur reine. Il est donc probable que pour fonder sa colonie, la jeune reine testaceus s'associe avec une jeune reine cæspitum, au moment où celle-ci établit son nid. Dans les colonies mixtes d'autres espèces de Strongylognathus et de Tetramorium, la reine de l'hôte parait.ètre éliminée, comme dans les colonies de Polyergus.

d) On peut compter encore au nombre des fourmis faiseuses d'esclaves ou doulotiques, un autre genre de Myrmicinés, Harpayoxenus, comprenant seulement deux espèces, $H$. sublievis de l'Europe centrale et septentrionale, et $H$. americanus des Etats de l'Est. Il y a plusieurs années (1906), je découvris cette dernière espèce dans une localité isolée de l'Etat de NewYork; elle vivait au sein des colonies de Leplothorax curvispinosus, dans des troncs creux de sureau; mais jamais on ne l'a retrouvée en cet endroit. L'espèce européenne sublævis a élé étudiée par Iolerz (1896) en Suède, où elle n'a que des reines ergaloïdes, et par Vienuever $(1906,1912,1921)$ en Allemagne, où elle a, en plus, des reines ailées. Ces deux chercheurs ont trouvé que les ergatoïdes et les ouvrières font irruption dans les nids de Leptothorax acervorum ou muscorum, prennent la couvée et l'emportent dans leur nid. Suivant Vienneyer, cet Harpagoxenus se comporte comme Polyergus et présente une dépendance absolue vis-à-vis de ses esclaves. Cet auteur décrit comme suit le mode de formation de la colonie, qui rappelle celui de sanguinea: "Comme chez loutes les fourmis parasites, la colonie se fonde suivant le type dépendant. La fondation a lieu sans doute pendant les cxpéditions de conquète. Les femelles ergatoïdes participent a ces raids el restent en arrière dans le nid dépouillé, afin d'y élever pour leur propre comple un premier lol d'auxiliaires provenant des races pupes de Leptothorax qui y ont été laisscees ou qui leur sont échues en partage. J'ai découvert deux fois de telles colonies, récemment fondées (Dresde 1908, et Oberrittergrün 1915). Flles consislaient en une femclle solitaire, ergatoïde, avec, dans un cas, une seule, dans l'autre 
deux, ouvrières Leptothorax et quelques jeunes des auxiliaires. I)es expériences ont, en outre, prouvé qu'une femelle isolée parvient tres bien a piller un nid et voler les pupes. Mais, dans le cas des femelles ergatoïdes, ce type de fondation est improbable, car la réussite en est moins sùre que lorsque a colonie se fonde aul cours d'un raid. D'ailleurs, les deux jeunes colonies paraissaient avoir élé élablies dans les nids pillés. ")

\section{$*^{* *}$}

$2^{\circ}$ En I 904, j’ai découvert un autre mode de formation des colonies mixtes, que jai appelé parasitisme temporaire, et qui pourrait aussi être appelé parasitisme aigü, ou protélien. Ce type se rencontre chez un certain nonbre d'espèces appartenant au moins à trois sous-familles de fourmis ; plusieurs Formica et Lasii, parmi les Formicinés, certaince espèces de Bothriornyrmex chez les Dolichoderinés, el quelques rares espèces d'Aphoenogaster et de Crematogaster chez les Myrmicinés.

Je l'ai découvert d'ahord chez Formica consocians, qui appartient à un groupe d'espèces (groupe de microgyna) représentées seulement dans l'Amérique du Nord et caraclérisćes par les reines très pelites, souvent plus petites ou du moins pas plus grosses que les plus grandes ouvrieres, et parfois très différentes par la couleur ou la pilosité, - jaunes ou rouges, avec de longs poils dorés. Ces caractères sont trés significatifs, puisqu'ils sont justement ceux des myrmécophiles symphiles. La jeune reine de ces fourmis pénètre dans le nid d'une autre Formica, ajpartenant aul groupe de fusca, ou de pallidifulva; elle se fait adopler facilement, sans doute à cause de sa pelite taille et de son odeur atlirante. On ne connait pas avec cerlitude le sort de la reine de l'hòte, dans les nids ainsi enrahis, mais elle est probablement tuee par ses propres ouvrières. Le parasite commence alors a produire sa convée, qui est soignće par les ouvrières de l'hôte, et il en résulte une colonie mixle. Comme les descendants de la reine nont aucune tendance it piller d'autres nids de l'espèce hôte, et, comme les ouvrieres de l'hote meurent naturellement au 
cour's de quelques années, il ne reste qu'une colonie pure de l'espece parasite, qui peut s'accroitre jusqu'a être très populeuse el très agressive ; cela rappelle de superbe façon certaines institutions humaines, qui, après un stade de parasilisme humble et servile, ont acquis, au cours des siècles, une puissance exubéranle et insolente. Par la suite, ce mode de

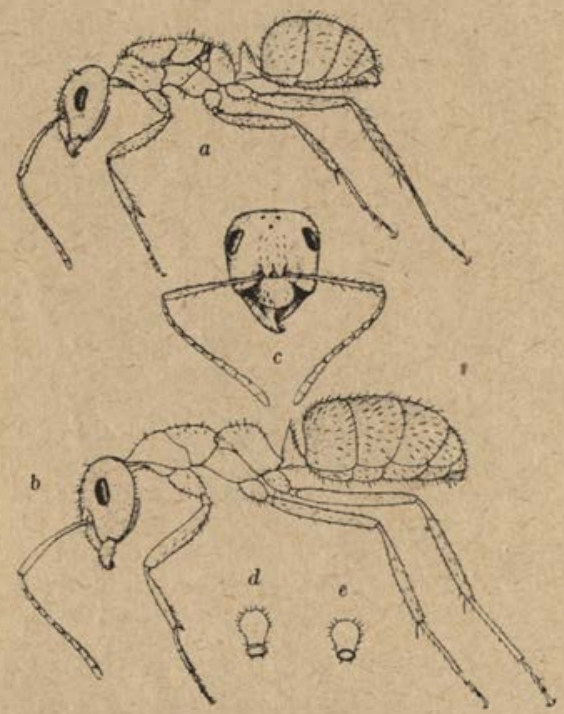

Fig. 59. - Formica microyyna. a. reine (ailes supprimées); b. grande ouvrière (à la mème échelle); $c$. sa tête; $d$. et é. petioles d'ouvière et de reine, face postérieure.

parasitisme temporaire fut decouvert dans deux autres groupes, dont les types sont $F$. rufa et exsecta. Ces fourmis $(F$ rufa, pratensis, obscuripes, exsectoides) dressent souvent des nids en monticule alteignant des dimensions considérables, soit en Europe, soit dans l'Amerique du Nord. La méthode suivie pour fonder leurs colonies a été étudiée par Wismans, Vienmeyer? 
(1908), Donisthorpe (1909, elc.), Brun (1910, 1912 a, 1912 b, 1913 a, 1913 b, 192/), Cimwley et Dovisthorpe (1913), Kutteh (1913 $a, 1913 b, 1917)$, TANoUary (1911), moi-même (1908 c, 1909) et d'autres (1).

On a découvert aussi que les diverses sous-espèces el variétés de Lasius umbralus, d'Europe et d'Amérique du Nord, sont des parasites sociaux temporaires de $L$. niger et de ses variétés (Crawley 1909, Crawley et Donisthorpe 1913, WasmanN 1909b, 1923a, TANQuary 1911, Wieeler 1917a, Rushkimp 1924), et, ce qui est plus remarquable, que l'espèce européenne aberrante Lasius (I)endrolasius) fulig̣inosus élablit ses colonies avec l'aide d'ouvrières umbralus et bicornis. (DONIsthorpe 1897, 1922, etc., Crawiey 1910a, Cnawiey el Donisthorpe 1913, DE Lannoy 1908, Forel 1908, EMery 1908a, WisMaNy 1909, BönNeR 1915, Lomicki 1922). I. fuli(jinosus est done un type hyperparasite social temporaire. Taxouny a ohservé un cas d'adoption d'une reine de $L$. umbralus minutus par une colonie de $I$. niger var. americanus, et, en 1917, il m'a été donné de voir de nombreuses jeunes reines de $L$. umbratus subumbratus rechercher les colonies de $L$. niger var. neoniger et sitkaënsis et se faisant adopter par elles, à l'altitude de 9.000 pieds, à Cloudcroft, dans les Monls Sacramento, au Nouveau Mexique. La femelle de subumbratus est couverte de longs poils jaunes et a une odeur suave particulière. On observe une pilosité analogue chez la femelle de L. cilialus du Cashmir. Les espèces nord-américaines L. (Acanthomyops) latipes el murphyi, dont les femelles ont aussi des poils dor's ressemblant ì des trichomes, sont peut-ître des parasites temporaires, ou des hyperparasites de quelques-unes de nos formes de $L$. niger ou umbratus.

On ne connait pas avec cerlilude le sort exact des reines de l'hote dans les colonies mixtes de Lasius, mais il est probable que la reine de nịjer est luée par la reine umbratus. Dans le cas de Bolhriomurmex decapitans, le massacre de la reine de l'hôte a parfaitement été observé par Sintschi $(1906,1920)$, i 'Tunis. La jeune reine redescendant après son vol nuptial, erre sur le sol, jusqu'à ce qu'elle rencontre le nid d'une colonie de

(1) Plus de vingl espexes de Formica, des groujues de rufu, ersectu et microm!nna, comprenant un grand nombre de soll:espèces el de virictés, peuvent ctre aujourdhui considérés comme des parasites sociaux lemporaires, d'espices et dis variétés des groupes de fusca el pallidefuleu. 
Tapinoma nigerrimum; elle se laisse prendre et arrêter par les ouvrières; celles-ci se meltent ell devoir de l'entrainer dans leur terrier en la tirunt par ses pattes et ses antennes. Après avoir pénétré dans le nid, le parasile est parlois altaqué par les ouvrières; mais elle se rélugie alors sur la couvée ou sur le dos de la grosse reine de Tapinoma. Dans l'une ou l'autre de ces positions, elle paraît absolument à l'abri de toute altaque, sans doute parce que sa propre odeur est dońince par celle de la couvée ou de la reine de l'hôte. Santschi a observé que le parasile passe souvent de longues heures sur le dos de la reine de Tapinoma, et que, dans cette position, elle s'occupe à couper la tête de son hôte. Tout en accomplissant ce cruel exploit, elle acquiert peu à peu l'odeur du nid et elle est adoptée par les ouvrières Tapinoma, à la place de leur malheureuse mere. A partir de ce moment, le parasite s'occupe de leur faire élever sa couvée. Il arrive que les ouvrières hôtes meurent de vieillesse et le nid devient alors la propriété d'une colonie pure et prospère de Bothriomyrmex decapitans.

\section{$*^{* *}$}

$3^{\circ}$ Il n'y a pas moins de 14 genres et $\mathrm{I}_{7}$ espèces de fourmis, de diverses parties du monde, qui puissent être classées au nombre des parasites sociaux permanents ou chroniques. Elles ont toutes complètement perdu leur ('astc ouvrière, et ressemblent étroitement, à cet égard, à Vespa austriaca et arctica et aux espèces de Psithyrus.

Nous distinguons deux types parmi ces parasites permanents, ceux chez lesquels mâles et femelles ont une même taille et même struclure et qui ont des mœurs normales ou peu modifiées (Epipheidole, Sympheidole, Wheeleriella, Epocus, etc.), et ceux chez lesquels l'un des sexes, ou les deux, présentent des modifications dégénératives neltes (Anergates, Anergatides, Bruchomyrma).

Les jeunes reines pénetrent dans les nids d'autres fourmis, et se font adopler, comme les reines des parasites temporai- 
res. Il semble que la reine de l'hôle soit régulièrement assassinéc par ses propres ouvrieres. Ceci a du moins été observé par SinTschi (Foner. 1966) dans le cas de Wheeleriella santschii, qui vit dans les nids d'une espece nord-africaine banale, Monomorium salomonis. Apres la fécondation, la reine de Wheeleriella erre de ci de lit a la surface du sol, cherchant un nid de Monomorium. (Duand elle s'apploche de l'entrée d'un tel nid, elle est " arrîlée ", comme dit SiNTs'Hi, par une bande d'ouvrieres de Monomorium, qui s'atlachent i ses paltes el a

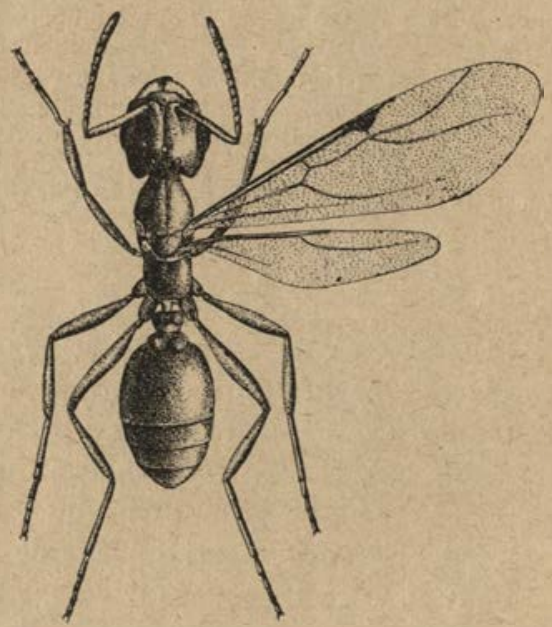

Fig. 60. - Wheeleriella sanstchii, femelle (Tunis).

ses antennes et l'entraînent ainsi dans leurs galeries. Quelquefois, on l'a vue s'élancer subilement, et de son propre mouvement, dans l'entrće dı nid, et être arrètée à l'intérieur. Les Monomorium ne donnent aucune marque dirritation et la reine peut bientôt aller et venir le long des galeries sans être molestée. Les ouvrieres commencent à la nourrir et l'adoptent, et en peu de jours, elle pond ses premiers œufs qui sont acceptés et soignés par les hóles. Le parasite n'accorde aucune atten- 
lion à la reine, heaucoup plus grosse, de Monomorium, mais celle-ci est parfois tuée par ses propres ouvrières.

D'autres espèces, comme le fameux Anergates atralulus d'Europe, l'Anergatides liohli (Wasmaxi 1915b), récemment découvert au Congo, et le Bruchomyrma d'Argentine (Sintschi), sont bien plus fortement modifiés et représentent les stades IIltimes de la dégénérescence par parasitisme. Chez Anergates, qui vit avec Telramorium crespitum et a été étudié par de nombreux chercheurs, la reine est petile et ailée, mais lorsqu'elle

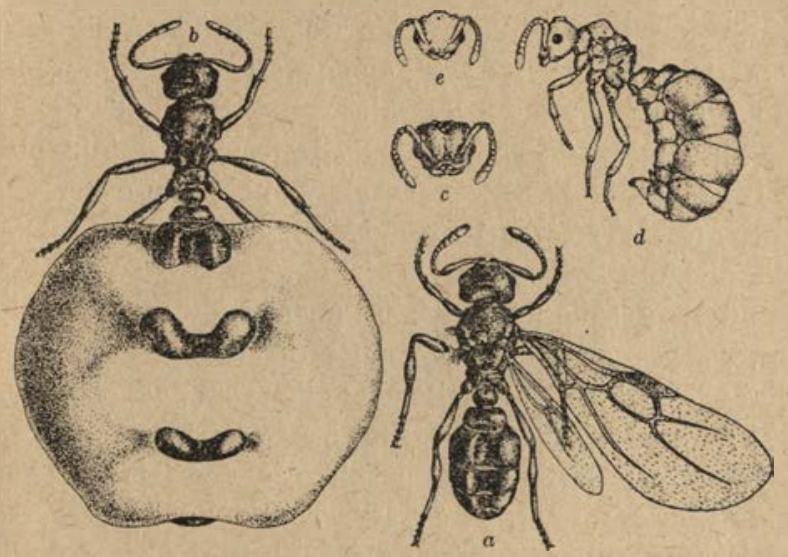

Fig. 61. - Anergales atralulus, $a$. reine vierge; $b$. vieille reme pondeuse à abdomen renflé ; $c$. sa lête vue de face; $d$. màle ajtère et pupoïde; $e$. sa tète.

a perdu ses ailes et a élé adoptée, son abdomen devient démesurément gonflé par les œufs, si bien qu'elle ressemble à une reine àgée de termites. Le màle est aptère et ressemble ì une nymphe; il est incapable de quitter le nid ; aussi l'accouplement se fait-il entre frères et sœurs (" adelphoyamie " de Fonfi.). On retrouve des conditions analogues chez. Anerifulides et Bruchomyrma, qui vivent dans les nids de Pheidole. Chez toutes ces espèces sans ouvrières, les descendants de li 
reine sont, tous, forcément des mâles et des femelles et sont formés pendant la durée de vie des ouvrières de l'hôte. Les colonies sont donc mixtes durant toute leur existence qui s'achève nécessairement par la mort de l'hôte.

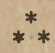

Bien que le précédent exposé des diverses formes de parasitisme social ait été fortement condensé, afin de le réduire aux limites d'une seule leçon, fort peu de temps me reste pour terminer sur quelques courtes remarques. On a pu noter que tous les hôtes sont très abondants, ont une aire très vasle et sont donc des espèces dominantes. Ceci est particulièrement exact pour les fourmis, For. mica fusca, Tetramorium caspitum, Tapinomn sessile. Monomorium salomomis, Leptothorax acervorum, etc... En outre, presque tous les hôtes forment de grandes colonies, parce qu'ils ont des reines prolifiques et des ou. vrières réussissant parfaitement l'élevage des couvées. Tout au contraire, les parasites sont très rares et sporadiques. C'est particulièrement le cas des faiseurs d'esclaves dégénérés, et des parasites permanents sans caste ouvrière, dont certains comptent parmi les fourmis les plus rares. Pendant plus de quarante ans, Forel ne put trouver en Suisse qu'un très petit nombre de colonies d'Anergates atratulus, bien que ce pays ait une faune de fourmis plutôt exubérante, et cette espèce n'a été décou. verte que tout récemment en Grande-Bretagne. Dans l'Amérique du Nord, Epœecus pergandei a été Irouvé une première fois il y a de nombreuses années, et ni Maxv ni moi n'avons pu le rencontrer de nouveau, quoique nous ayons examiné des centaines de colonies de son hôte, la banale Monomorium minimum. Presque tous les 
autres parasites ne sont connus que par des spécimens trouvés dans des colonies uniques de leurs hôtes pour. tant si abondants. Il faut aussi remarquer que certains de ces derniers, par exemple Formilca fusca et Tetramorium cospilum, sont parasités par plusieurs espèces de fourmis. Il est vrai, en outre, que seules les fourmis ayant des colonies vigoureuses et appartenant à des espèces dominanles abritent des myrmécophiles nombreux et variés.

Cette sensibilité parliculière à l'infestation parasitaire, de la part des espèces vigoureuses de Formicidés est intéressante à rapprocher des conclusions analogues de $\mathrm{RAI}_{\mathrm{Al}}$ NES (I922) au sujet des rapports entre la vigueur végétalive d'une plante hôte et sa sensibilité aux maladies.

Tous les chercheurs sont d'avis que les parasites permanents, sans ouvrières, représentent le stade final, dé. généré et évanescent de l'évolution dans la série des parasites sociaux ; mais les opinions sont divergentes en ce qui concerne le stade initial ou primitif. Wasmans et Pićron (rgro) soutiennent qu'il est représenté par les parasites temporaires et font dériver les mœurs de ces derniers de celles des reines fourmis, qui, après la fécondation, recherchent et s'assurent l'adoption des colonies de leur propre espèce (pléométrose secondaire). Suivant ces auteurs, les types de parasitisme doulotique et permanent dériveraient l'un et l'autre de ce parasitisme temporaire. Viehmeyer, Emery, Brun et moi, nous soutenons au contraire, que les habitudes prédatrices du type réalisé par Formica sanguinea représentent plus probahement le stade initial, et que le parasitisme conciliant, temporaire et finalement servile et permanent représente le développement naturel ultérieur de cette forme violente et agressive des relations entre hôte et parasite. Cette af- 
firmation est basée sur les considérations suivantes : $1^{\circ}$ d'une façon générale, le parasitoïdisme et le parasitisme sont dérivés évidemment du prédatisme, chez la plupart des insectes solitaires; la même succession se retrouve chez les myrmécophiles, les parasites sociaux des abeilles, les faiseurs d'esclaves dégénérés (par exemple Strongylognathus), les fourmis qui forment les nids composés, etc.; el. $2^{\circ}$ plus particulièrement, les reines de certains parasites temporaires, ayant de grosses reines, comme Formica rufa, se comportent quelquefois comme $F$. sanguinea, quand elles fondent leurs colonies et les espèces à petites reines, de couleur jaune ou rouge et ayant des trichomes, sont évidemment des formes dérivées, etc.

Les relations entre hôte et parasite, considérées ci-dessus, ont aussi une grande importance au point de vue de la " sélection amicale " et des " instincts symphiliques " de Wasmans. On peut admettre que les parasites temporaires et permanents, et au moins plusieurs espèces dulotiques (Strongylognathus, Polyergus), se comportent comme des myrmécophiles symphiliques, puisqu'ils profitent comme ceux-ci des tendances progénitophiles de leurs hôtes et ont avec eux des relations trophallactiques. Nous avons vu que certains parasites temporaires ont véritablement acquis des caractères symphilitiques (petite taille, coloration jaune ou rouge, trichomes, habitudes de conciliation ou de sollicilation). En outre, les parasites permanents, qui ont perdu leur caste ouvrière, sont aussi réellement des insectes solitaires et peuvent être considérés comme des symphiles. La disproportion considérable entre l'abondance de ces parasites et celle de leurs hôles montrent bien que les premiers ne peuvent tirer leurs caractères particuliers d'une sélection amicale accomplie 
par les derniers, et le fait que les parasites provoquent réellement la castration de la colonie qu'ils infestent, ou y conduisent, et qu'ils ne tolèrent jamais la formation de descendants mâles provenant des ouvrières, ce fait prouve qu'il n'existe aucune base héréditaire pour le développement d'instincts symphiliques de la part de l'espèce de l'hôte. Comme dans le cas des myrmécophiles, les adaptations particulières, soit de structure, soit de moeurs, à des hôtes spéciaux, débutent et se développent entièrement sous l'action des parasites sociaux eux-mêmes, et rien n'indique que ces adaptations exigent quelque explication biologique différant de façon fondamentale de celles qui ont été proposées pour les cas innombrables de parasitisme se produisant chez les insectes solitaires, chez les autres animaux ou chez les plantes. 


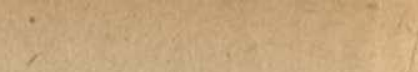




\section{CHAPITRE $\mathrm{XI}$}

\section{CONCLUSIONS}

Si les conclusions auxquelles nous sommes parvenus dans les leçons précédentes sont exactes, il est possible d'esquisser dans ses grandes lignes le plan probable de l'évolution des insectes vraiment sociaux, appartenant à l'ordre des Isoptères et au sous-ordre des Aculéates, chez les Hyménoptères.

Nous avons vu que les premiers sont issus des Protoblatloïdés, peut-être dès la période permienne, et, après avoir traversé des stades primitifs analogues à ceux des Maslotermitidés et Calotermitidés actuels, ils ont atteint leur point culminant chez les Termitidés spécialisés et puissants de l'Ethiopie et de l'Orient. Il est évident que le caractère et la direction de l'évolution des Isoptères ont été, dans leur ensemble, déterminés principalement par le mode d'alimentation spécial à ces insectes. Un régime de cellulose ou d'humus est responsable de leurs particularités les plus frappantes : leur tégument vulnérahe, leur microphthalmie et leur photophobie, leur habilude d'employer leurs excréments comme matériaux de construction, les relations trophallactiques qu'ils ont entre eux, leur utilisation des Protozoaires symbiotiques dans la digestion de la cellulose, etc. Seuls, les termites supérieurs ont acquis une habitude nouvelle, celle de 
cultiver et de manger des champignons, habilude qui est aussi liée étroitement avec leur régime et leur milieu d'existence, qui sont l'un et l'autre la cellulose. Le choix d'un aliment abondant, mais de digestion difficile, et l'existence confinée dans de petites cavités aux cloisons résistantes, déterminent une prolongation de la vie, comme chez la plupart des insectes qui creusent le bois, et une lendance au groupement des adultes, au rapprochement des parents et des enfants. Nous trouvons dies transformations semblables, mais plus atténuées, subsociales, chez beaucoup de Coléoptères, appartenant aux familles des Scolytidés, Platypodidés et Passalidés, chez Phrenapates, parmi les Ténébrionidés el chez Parandra, parmi les Cérambycidés.

L'évolution des Aculéates sociaux présente une bien plus grande diversification, un plus grand rayonnement des caractères d'adaptation, une intensité et une accélération plus grandes. Nous l'avons indiqué précédemment pour leurs ancêtres, les Béthyloïdés, qui sont dérivés des Térébrants prinitifs, descendant eux-mèmes de Phytophages inconnus. Les premiers Vespoïdés et Sphécoïdés provenaient probablement des Béthyloïdés ; ils forment actuellement un complexe, hautement différencié, de petites formes archaïques. Les abeilles, qui sont les plus élevées des Apidéo sociaux, ont certainement des ancêtres Sphécoïdés, et les Vespidés et Formicidés sociaux descendent certainement des Vespoïdés primitifs. Là aussi, comme chez les Isoptères, le caractère de l'aliment a été le facteur déterminant de l'évolution, en même temps que l'indépendance et les initiatives du sexe femelle. Cette indépendance résulte naturellement elle-mène de l'existence d'un spermathèque très spécialisé 
el permetlant une longue rétention du sperme, conservé vivant. L'évolution des Hyménoptères débute par des formes comme les Protohyménoptères permiens, découverts par Tillyard et rattachés à nos Mécoptères modernes ; ce groupe avait des potentialités d'évolution beaucoup plus grandes que les Protoblattoïdés et il a conduit aux Phytophages rongeurs de feuilles et de bois. Certains d'entre eux, comme les Oryssidés, devinrent parasitoïdes et ouvrirent ainsi la voie à l'extraordinaire floraison des Térébrants ; tous conservèrent des habitudes entomophages, sauf quelques rares familles (Cynipidés, certains Chalcidoïdés). Les Béthyloïdés conservèrent aussi les mêmes habitudes dans leur essence, mais quelques genres (Bethylus, Scleroderma) devinrent plus plastiques et établirent des relations plus intimes les uns avec les autres et avec leurs descendants. Les Vespoïdés et Sphécoïdés primitifs présentaient pratiquement les mêmes mœurs que les Béthyloïdés, mais avec l'accroissement dı nombre des plantes à fleurs durant le Crétacé, quelques groupes (Apidés, Masaridés) devinrent exclusivement anthophiles, d'autres le devinrent partiellement (au stade adulte). La plupart des fourmis, issues également d'ancêtres Vespoïdés (Tiphiidés ou Scoliidés), sont encore entomophages, mais beaucoup de genres sont devenus de plus en plus végétariens.

Il est intéressant de noter que les changements de régime qui se sont produits au cours de l'évolution des Aculéates, apparaissent d'abord chez la femelle adulte et sont transmis ultérieurement à la larve. Ainsi, chez les Phỵtophages, les adultes et les jeunes vivent les uns et les auIres d'aliments végétaux grossiers (feuilles ou bois), mais les adultes de quelques Tenthrédinidés sont devenus en- 
tomophages. Chez les Oryssidés et les Térébrants, ces habiludes on tété acquises aussi par la larve. Chez les Sphécoïdés et les Vespoïdés, les adultes sont devenus plus ou moins nectarivores, cependant que chez les abeilles et beaucoup de 'Masarinés, les larves, aussi bien que les adultes, se nourrissent exclusivement de nectar et de pollen. Comme nous l'avons déjà indiqué, les fourmis présentent des tendances semblables. Sans aucun doute, la consommation du nectar, de graines, de champignons, et de miellée (cette dernière substance étant simplement des sucs végétaux modifiés par la traversée des intestins d'Aphides, Coccides, etc.), était, à l'origine, limitée aux fourmis adultes. Mais quelques espèces granivores nourrissent aussi leurs larves de graines, et les larves de toutes les espèces mytécophages ont le même régime que les adultes.

Une intéressante ohservation, que m'a communiquée Mr. IN'. F. Fiše, indique que même l'habitude de manger les excréments sucrés des Homoptères est en train de se transmettre aux larves. Il y a quelques années, dans l'Afrique orientale anglaise, il a vu des fourmis transportant leurs larves hors du nid, en les hissant sur un tronc d'arbre, dans le feuillage, jù elles les maintenaient, la bouche fixée à l'orifice anal des pucerons, afin que ces larves puissent boire la miellée. Comme Mr. Fiske est un entomologiste très compétent, je ne doute nullement de l'exactitude de son observation, tout en regrettant qu'il ail négligé de conserver quelques spécimens des fourmis, qui nous auraient permis d'identifier l'espèce et d'étudier de nouveau plus aisément ces curieuses habitudes.

L'un des résultats, et des plus intéressants, de notre étude de l'évolution est la conclusion suivante à laquelle on se trouve amené : c'est que le même type d'organisation et de mours sociales a été atteint indépendamment par au moins douze groupes différents d'insectes. Il n'y 
a peut-ètre pas, dans tout le règne animal, d'exemple plus frappant de ce qu'on appelle un développement "parallèle " ou "convergent". En outre, les diverses manifestations du type social se sont arrêtées dans leur phylogénie, à des stades Irès différents et il y a toute raison de supposer que ces arrêts sont dûs en partie à une stabilisation ou à une standardisation des conditions extérieures, et en partie à une standardisation constitutionnelle concomitante, ou à l'incapacité où se trouvent les insectes mêmes de se développer davantage. Même les formes supérieures, les plus spécialisées (fourmis, termites, abeilles domestiques) paraissent ainsi avoir depuis longtemps achevé leur évolution. Peut-être se forme-t-il encore lenlement de nouvelles variétés ou sous-espèces, mais ce sont là de faibles oscillations qui n'introduisent point de déviations significatives dans les caractères essentiels de l'organisation sociale.

\section{$*^{*} *$}

Dans les leçons précédentes, je me suis permis, à l'occasion, d'établir des comparaisons entre les insectes sociaux et l'homme, et je voudrais consacrer une grande partie de cette leçon à une tentative plus approfondie dans celte direction. Ce n'est point là, à mon avis, une entreprise futile, quoique présentant seulement un intérêt théorique. Bien entendu, on a fait de nombreuses comparaisons, et, pour toutes sortes de motifs, aussi hien sérieux que saliriques, et sur la base de conceptions souvent inadéquates ou erronées des caractères qu'offrent les sociétés des hommes ou des insectes. Dans l'ensemble, les résultats en ont été si peu satisfaisants que les sociologues 
s'en sont détournés avec dédain. Mais, quoique l'homme, à cause de sa structure, de son hérédité et de ses mœurs très particulières, ne puisse tirer des insectes sociaux aucun renseignement applicable avec profit à la solution des problèmes sociaux complexes qui le concernent, il ne faut pas oublier que cet homme ne cessera jamais d'être un animal et que ses activités ont des assises puissantes et irrévocables dans les fonctions de nutrition, de reproduction, de relation et d'appétition, communes à tous les autres organismes vivants. Aussi, quand bien même le sociologue tiendrait peu compte des sociétés animales dans ses études spéciales, le biologiste, qui doit toujours considérer l'homme comme un Primate, a parfaitement le droit de considérer la société humaine comme une société animale. Et le sociologue n'a aucune objection à faire, puisqu'il est le premier à admettre que la sociologic est encore une science rudimentaire et théorique.

$I^{\circ}$ Les sociétés animales et humaines représentent-elles des entités, ou des ensembles comparables, quoique d'un ordre plus élevé, à l'organisme individuel, celui-ci étant, comme on le sait, une colonie de cellules? Cette queslion a été souvent discutée, spécialement par Herbert Spencer, Espinas, Staeckel, Ribot, Lilienfeldt, De Greef, Fouillée, Novicow, Waxweiller, René Worms, Bartir, etc. Récemment Ferrière (I १i5) a repris toute la question et l'a soumise à un examen approfondi. L'intérêt est évidemment concentré sur la société humaine. Les différences entre celle-ci et les sociétés animales sont si grandes, que la plupart des sociologues ont abandonné la question comme étant simplement verbale et scolastique. L'humanité est en réalité une association très intri- 
quée de groupes liés les uns aux autres, dans leurs coopérations et leurs antagonismes, par des aclivités en partic conscientes et en partie inconscientes, sans limites spatiales définies, mais s'effectuant dans'le temps, et ayant un caractère si mobile et si compliqué, que chaque individu est simultanément membre fonctionnel de plusieurs groupes.

Ainsi, Ferriène se refuse à regarder la société humaine comme un organisme au sens statique, quoiqu'il admette que ce soit un organisme au sens dynamique. Il paraît cependant obscurcir le problème en négligeant de fournir une juste estimation de sociétés telles que les sociétés d'insectes. Celles-ci sont certainement des entités distinctes, comme les simples Métazoaires ou Métaphytes, possédant une limite, une taille, une structure et une ontogénie, toutes bien définies, et consistant en éléments polymorphiques, en dépendance mutuelle. Elles peuvent donc être appelées superorganismes et constituent un stade intermédiaire très intéressant entre le Métazoaire solitaire et la société humaine. Chaque société d'insectes est une famille, comme la famille humaine ou la horde qui constitua probablement la société humaine à son premier stade postanthropoïde. On pourrait donc regarder la société humaine actuelle comme une fusion de sociélés, un hyper-hyperorganisme, ou hyperorganisme du second degré.

La distinction élablie par FouilléE et Ferrière, d'après laquelle les individus, dans les sociétés animales ou humaines, conservent leur mobilité et sont isolés spatialement, tandis que les éléments histologiques de l'organisme sont fixes et contigus, est peut-être moins significative que le pensent ces auteurs. Les globules du sang 
sont aussi une partie de l'organisme et les distances séparant les éléments des tissus sont relatives. Bien mieux, les cellules, le sérum, la lymphe, elc., sont des masses d'électrons séparés par des distances si énormes que, si le corps humain pouvait ètre comprimé jusqu'à ce que tous ses électrons fussent au contact les uns des autres, il aurait un volume total n'excédant pas quelques millimètres cubes. L'inter-relation, ou ce qui se passe entre les électrons, les atomes, les molécules, les micelles, les cellules et les organismes individuels, est la chose significative et c'est dans les zones entre les éléments que les scientistes concentrent de plus en plus leur attention.

$2^{\circ}$ La caractéristique distinctive de la vie sociale a été définie différemment par les différents sociologues; pour Herbert Spencer c'est la "coopération " entre les membres; pour TARde (I92I), c'est leur "imitation " les uns par les autres, pour Durckneim (1922) c'est leur " contrainte "; pour DE GRange (1923) leurs " activilés "cumulatives". Indubitablement, toutes ces caractéristiques, si frappantes dans les sociétés humaines, ont été aussi relevées chez les guêpes, les abeilles, les fourmis et !es termites. L'imitation et la contrainte, au moins si on entend par ce dernier mot la dépendance biologique, sont moins apparentes, mais on ne peut nier la coopération et surtout la cumulation, telles qu'elles apparaissent dans la mise en réserve de la nourriture, la prise de possession d'une territoire, qui est le champ trophoporique avec son troupeau d'Aphides, de Coccides, etc., le développement de la couvée et l'acquisition de mỵrmécophiles, de termitophiles et de myrmécophytes, la construction du nid, etc. Celte réelle cumulation, conséquence fatale des ac- 
tivités de nombreux individus associés, et source de surproduction ou de suraccumulation, est d'une importance fondamentale dans toute la vie sociale, comme l'a suggéré De Grange et aussi Le Dantec (I918) dans les intéressantes remarques qui suivent : "La seule formule générale qui puisse s'appliquer à toutes les associations, quelles qu'elles soient, est la suivante : il faut, pour que l'association continue, que chaque associé retire de l'association des avantages compensant, et au-delà, les inconvénients qui résultent de la concurrence des appétits spécifiques. Dans tous les cas, quand il s'agira d'associations d'individus libres, on aura à évaluer l'excès de la production individuelle sur la consommation individuelle, et non, comme dans le cas des individus pluricellulaires, la collaboration de chaque cellule à un travail d'ensemble, qui seul est capable d'assurer la vie de l'agglomération. C'est surtout par cette particularité très nette que les associations d'individus libres sont différentes des agglomérations de cellules fixées réduites à l'état de simples éléments histologiques ").

Il est non seulement exact, comme dit de Grange, que " la première des conditions de l'opération du processus sociétal est l'accumulation des produits matériels, mais aussi la suraccumulation qui en résulte naturellement, surtout dans les sociétés humaines, a des conséquences d'une importance considérable par ses réactions sur les individus $"(\mathrm{r})$.

(1) Bien entendu, les accumulations immatérielles sont ausci importantes, même des accumulations aussi surprenanles que la théologie et la philosophie scolastique (voir Rougren 1925). l.es differeneses entre ces accumulations et celles de l'industric moderne ont été bien mises en lumnière par Tu.guen (1921) dans sa crilique de Ferreno: " C'est l'ère de ce que Guglielmo 
Dans nos sociélés civilisées, la surproduction et la suraccumulation sont si considérables, les produits de nos activités, les excreta de notre métabolisme social si abondants, non seulement sous la forme d'objets matériels, mais aussi sous la forme moins tangible de mœurs, rites, superstitions, modes, lois, institutions, etc., que les générations, en se succédiant l'une à l'autre, éprouvent une difficulté croissante, non point même à progresser, mais seulement à vivre. En outre, l'accoutumance à ces condilions a créé en nous un tel respect du passé et de ce qui est vieux, que, de quelque nom que s'appellent nos gouvernements : monarchies, républiques, aristocraties ou démocraties, tous pourraient plus exactement s'appeler gérontocraties. Certes, des parties complètement usées s'éliminent constamment, mais c'est là un processus très lent. De temps en temps, quand la société arrive à être complètement intoxiquée par cette accumulation, la guerre ou la révolution peuvent seules briser la croûte des dépôts sociaux et fournissent l'occasion d'un progrès ultérieur. Ces douloureuses méthodes resteront sans doute notre unique ressource, jusqu'à cc que nous ayons acquis un savoir et un courage suffisants pour rejeter (" to scrap ", comme nous disons en Amérique), un lot de nos héritages sociaux, traditions et

Fennero appelle la civillì quanlitativa, (la civilisation quantitative), à laquelle il oppose, comme civilisation de type con. Iraire (civiltà qualitativa), (civilisation qualitative), ayant pour idéal la qualité, la perfection, l'achèvement) la civilisalion anlique, prouvint ainsi qu'il n'a pas compris que cette derniere, plus encore que la civilisation moderne, marchait vers l'illimité, et que la seule différence entre les deux réside dans l'objel divers de leurs aspirations: la dumination absolue de sni-même pour l'une; du monde extérieur pour l'autre », 
habitudes, - jusqu'à ce que nous nous refusions à accor der aux vieillards, dont le seul désir est de ne pas challger, une prépondérance indue, et que nous nous tournions vers les jeunes qui possèdent au moins l'amour de la nouveauté et de l'expérimentation. La vérité, " crue, nue, verte et sans phrase ", c'est ce que nos corps politiques, nos universités, nos académies, nos églises et autres institutions, renferment beaucoup trop d'insensés et, comme disait Guetue à Eckermans, nos funérailles nationales ne se suivent pas avec une fréquence suffisante. Et la situation devient d'autant plus intolérable, que la science médicale progressant prolonge la vie humaine et accroît la population en général et le nombre des vieux fous en particulier ( $\mathbf{r}$ ).

Comme le problème se résout différemment, chez les insectes sociaux! Quand la colonie est parvenue à l'apogée de ses accumulations, elle produit des individus frai: et jeunes, qui fondent de nouvelles colonies, tout comme le corps adulte du Métazoaire émet de jeunes cellules germinatives, et elle se laisse ensuite mourir avec une résignation pleine de modestie, ce qui est parfait au point de vue cosmique. Cette méthode ne peut évidemment pas permettre une accumulation phylogénétique rapide et excessive, mais elle conduit à une stabilité plus uni forme durant de longues périodes, comme nous l'avons vu dans l'évolution des insectes sociaux, des temps pré. tertiaires à l'époque actuelle; et elle offre aussi le retour plus fréquent de circonstances permettant certaines

(1) Ce langage est tres choquant, mais il serait facile de dresser une liste de cinquante réactionnaires chargés d'ans, ayant a leur tête William Jennings Bryan, qui, au cours des dernières années, ont fait plus de mal à la civilisation qu'un nombre égal de n'importe quels criminels, au choix. 
adaptations nouvelles, comme celles qui sont exigées par un milieu extérieur variant graduellement.

$3^{\circ}$ Autrefois, Espinas a divisé les sociétés animales en nutricielles et reproductives; les premières étant représentées par des formes telles que les Siphonophores, les secondes par les Insectes sociaux. Ciette distinction est toujours valable. Dans les sociétés humaines, les acti. vités les plus caractéristiques sont évidemment psychi. ques, mais, dans chaque type de société, le plus élevé renferme les caractères saillants de celui qui lui est in. médiatement inférieur ; ainsi, dans le vieux schème aristolélicien, la nutrition caractérise les plantes, la nutrition et la sensation caractérisent les animaux, et la nutrition, la sensation et la raison, l'homme. Il ne fait aucun doute que la raison d'être des sociétés d'insectes est la reproduction. Leur fin naturelle, si elles en ont une, est de produire le plus de jeunes et le plus de colonies possibles. C'est là un fait manifeste et qu'il n'est pas néces saire de décrire en langage téléologique. II s'ensuit, naturellement, que le sexe femelle est prééminent, et cela est exact aussi pour les sociétés de termites, comme le montre l'énorme dévelopipement somatique et gonadique des reines dans les espèces supérieures. Les sociétés d'Aculéates sont franchement femelles, les mâles étant róduits à une simple action fécondante temporaire. Ainsi, dans son développement, tant ontogénique que phylogénique, la colonie d'insectes dépend d'une élite de femelles fertiles, condition qui diffère beaucoup de celles que nous trouvons dans les sociétés humaines, dont les progrès dépendent d'une élite d'individus intellectuels des deux sexes. Les biologistes savent parfaitement qu'il y a une 
sorte d'antagonisme entre les activités sexuelles et inte!lectuelles, et certains historiens (p. ex. TOWNEk, 1923) ont relevé aussi ce fait dans les civilisations humaines. Les sociétés dépérissent intellectuellement ou plutôt se rapprochent du type de l'insecte social quand elles ont des tendances excessives à la reproduction.

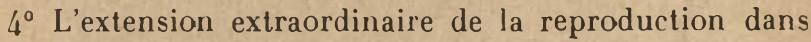
la société d'insectes a conduit directement au dévelop. pement de castes et au polymorphisme, quelques rares individus féconds, les reines, se spécialisant dans la production des œufs, tandis que la majorité des individus, les ouvrières, très mal doués au point de vue sexuel, se spécialisent dans la récolte de la nourriture nécessaire et le soin des jeunes. Dans les espèces prolifiques, ces dernières fonctions sont si exigeantes qu'il se forme une classe supplémentaire, celle des soldats, qui assure la défense de toute la société ou s'acquitte d'autres devoirs que ne pourraient accomplir convenablement les ouvrières nourrices ou approvisionneuses. Ces diverses différenciations sont si anciennes et sont si strictement éta. blies, au moins chez les insectes sociaux supérieurs (fourmis et termites), que les mœurs et la structure spéci fiques des individus appartenant aux différentes castes sont fixées définitivement dès leur sortie de la nymphe. Chez l'homme, le développement des castes, bien que dépendant aussi d'une division du travail, est, naturelle. ment, très différent, et a une origine purement professionnelle. Ce sujet a été étudié si parfaitement par Durcкиегм (г922) qu'il est inutile de l'examiner ici. Il suffira de noter que l'individu humain normal, quoique naissant dans la société avec des dons héréditaires particu- 
liers, est néanmoins si généralisé et si plastique, qu'il leut, gràce d̀ l'éducation, se transformer en un mem. bre plus ou moins efficient d'une caste quelconque ou de plusieurs castes (professions). Et, comme nous le savons Ious, ajprès àvoir exercé sa profession pendant des années, il acquierl dans ses actes, les idiosyncrasies de son métier particulier ou même des stigmates physiques spé ciaux. L'acteur lui-mème, dont la profession consiste en une imitation neuromusculaire d'individus appartenant à de nombreuses castes, acquiert souvent une expression caractéristique, dans son visage et dans ses attitudes.

$5^{\circ}$ Conme les caştes résultent d'une spécialisation pro noncée dans la structure et dans les mours, leurs membres deviennent nécessairement des représentants par. ticls, plus ou moins avortés, de leur espèce, et présen. tent donc des caractères qui ont souvent été qualifiés de "dégénératifs". Mais ce terme est toujours pris daus une acception pathologique. Pourtant, comme les carac. tères dont il s'agit ont persisté pendant des centaines et des milliers d'années, non seulement sans porter atteinte ì l'espèce, mais comme des adaptations nécessaires à un milieu particulier, il est plus exact de les regarder comme des spécialisations. Il est évident qu'elles peuvent mettre en péril la vie de l'organisme, si le milieu social ou exté. rieur varie, mais il en est de même pour toute adaptalion précise, serait-elle d'une sorte dont on ne pourrait jamais dire qu'elle est "dégénérative " ou "pathologique ". La confusion est née de notre anthropomorphisme indéracinable et de notre habitude de toujour; tenir compte de "valeurs", surlout dans tout ce qui concerne la vie et la mort. La meilleure voie, pour sor 
lir de ces difficultés, est sans doute d'éviter les termes tels que "dégénératifs " et de les remplacer par des expressions descriptives neutres comme " atrophique " " hypertrophique ", "régressif ", "involué ", etc.

$$
* *
$$

II y a grand profit à examiner en détail les faits se rapportant à la spécialisation des caractères dans les castes d'insectes. Il existe des signes indéniables d'involution morphologique ou de régression, chez l'insecte qui a subi une adaptation au milieu social et ces signes se multiplient et s'aggravent, quand nous passons des sociétés les plus primitives aux plus avancées. Ces signes comprennent non seulement des caractères comme la plus grande simplification des dessins de l'aile et des pièces buccales chez les abeilles sociales comparées aux abeilles solitaires (I), l'absence d'ailes, la microphthalmie, la simplification du thorax, des organes génitaux, etc., des ouvrières et des soldats comparés aux formes reproductrices, chez les fourmis et les termites, lorsqu'on

(1) Suivant L.hounfer (1897), les pièces buccales de l'abeille domestique et du bourdon sont moins développées que shez des abeilles solitaires comine Trachusa et Anthidium, et Tosı a découvert que Te proventricule de l'abeille domestique est moins développé que chez Bombus. Friese (1923) admet comme cerlaine la réduction de nervation de l'aile chez les abeilles sociales. Chez les Formicidés, ces dessins présentent une simplification croissante, quand on passe des Ponérinés et des Dorylinés primitifs aux Formicinés plus haulement spécialisés. II en est de mème pour la réduction de l'aiguillon qui est très développé chez. les groupes inférieurs et dont il n'y a plus qu'un vestige chez. les Dolichoderinés et Formicinés, comme aussi chez les Méliponinés parmi les abeilles sociales. 
passe des espèces primitives, ayant des colonies réduites. à des espèces hautement spécialisées ayant des colonies très populeuses. Les organes internes présentent aussi des altérations régressives croissantes pendant la vie adulle des formes sexuclles, et surtout de la reine, qui représente le type complet ou parfait de l'espèce chez presque lous les insectes sociaux.

Les observations de Holmanen (1909) et de von Rosen (1913b), sur les termites, sont particulièrement instructives à ce point de vue. Le premier a découvert que, chez les reines âgées, 'e cerveau se réduit au tiers de ses dimensions primitives et cette réduction, qui se produit en quelques jours après la fondation de la colonie, est dùe principalement à l'atrophie des lobes et nerfs optiques et des ommatidies. Mais il y a aussi des variations hypertrophiques concomitantes dans beaucoup d'organes. Ainsi, les ganglions du système nerveux sympathique augmentent de volume, même chez le roi. La section transversale du ganglion postcéphalique, chez le roi comme chez la reine jeunes, ne mesure que $67 \mu$, chez le roi âgé, sa taille croît jusqu'à 135-162 $\mu$, chez la reine âgće, jusqu'à $175-190 \mu$. Le cœur, les masses graisseuses, le tube digestif s'agrandissent aussi, surtout chez la reine àgée, cet accroissement étant dù pour l'intestin à un régime uniquement composé de salive. Les organes respiratoires aussi sont transformés, les stigmales s'agrandissanl, et les ramifications des trachées devenant plus nombreuses, ceci étant sans doute une adaptation directe au confinement dans l'étroite chambre royale, et à l'hypertrophie des masses graisseuses et des autres viscères. Von Rosen a découvert une atrophie idenlique de l'appareil visuel adulte des formes royales néoténiques de Calolermes flavicollis. On trouverait probablement des modifications de la même espèce chez les reines de fourmis. Nous avons rappelé dans une lecon antérieure l'extraordinaire involution de la volumineuse musculature thoraci(jue, découverte par J.ANET et Ch. PÉrEz, chez les reines de fourmis après leur vol nuptial. A côté de ces variation onlogéniques, et d'autres, analogues, qui se produisent chez. les insecles sociaux, a mesure ru'ils s'adaptent it des fonctions parliculieres dins la dernière partic de lour exislence, il en est d'autres qui se sont fixées dans la phylogénie vu au cours des slades larvaires, en corrélation avec d'autres 
raracteres. Ainsi, chez les reines de l'abeille domeslique et des fourmis parasiles, le cerveall est moins développé, inême chez la nymphe, que celui des ouvrières, et, chez l'abeille domestiyue, il y a aussi une atrophie des pièces buccales, de l'aiguillon, des ćperons des pattes postérieures, des glandes salivaires, elc. Chez quelques castes rles Formicidés, comme les plérergales des fourmis a miel, le jahol shypertrophie an stade adulte. Mais, chez l'abeille domestique, quoique l'ouvrière soit, par sa structure, tris proche du type femelle primitif de l'espèce et présente une atrophie plus marquée, en ce qui concerne les organes reproducteurs, elle fait preuve néanmoins de mœurs régressives, dans sa dépendance vis-à-vis des autres ouvrières. Suivant von BuTTEL-REEPEN (1905), elle meurt en peu d'heures, si on l'isole dans la colonie, tandis qu'une ouvrière de fourmi peut être conservée en vie dans l'isolement pendant six mois (Miss FifLide, 1902, p. 599). Quand la température tombe all-dessous d'un cerlain niveau, liabeille domestique isoliee ne peut conserver assez de chaleur pour rester en vic. IIais la difference entre les fourmis et les abeilles domestiques est seulement une question de degré, en ce qui concerne la dépendance de l'ouvriere vis-i-vis de ses sœurs.

Holmgren a découvert que les termites ont, dans l'ensemble, une structure corporelle assez uniforme, et que. malgré l'acquisition évidente de caractères morpholo giques au cours de la phylogénie, la disparition de cerlains caractères est encore plus évidente. Les termites inférieurs renferment toute chose in nuce, et bien que des spécialisations aient pris naissance à tous les degrés du développement phỵlogénétique, il n'est apparu rien de véritablement nouveau, mais seulement des modificalions ou des réductions de ce qui existait primitivement. Holmgrex conclut done de la façon suivante: "Une ílude de la vie en communaulé nous suggère, théorique. ment que re mode de vie conduirait graduellement ì une dégénérescence des membres parlicipants, puisque lia sélection naturelle accomplirait non la différenciation maxima des individus, mais seulement celle des 
colonies. Puisque, très probablement, la différenciation des casles est le résultat de la vie sociale, el la division du travail, très vraisemblablement, la cause immédiate de la différenciation des castes, les effets dégénératifs de la vie sociale pourraient être attribués à une division toujours croissante du travail et à la différenciation toujours plus marquée des castes. Ainsi, sommie toute, nous pourrions admettre a priori l'existence d'un développement régressif des termites. "Une étude d'ensemble des Formicidés amène à la même conclusion. Le développement des diverses castes existant chez les sousfamilles supérieures était présent, en puissance, chez les Ponérinés primitifs, et si nous comparons par exemple une ouvrière de Pheidole, de Camponotus ou de Dorylus, avec une ouvrière du genre primitif australien Myrmecia, nous sommes contraints d'admettre qu'en tant qu'in dividu, la dernière est un insecte plus puissant et d'une organisation bien plus élevée.

Il est intéressant d'observer que l'on peut déceler aussi chez l'homme civilisé, comparé au sauvage ou au barbare, des effets analogues à ceux que nous venons d'examiner chez les insectes. Comparons par exemple, l'indépendance et les ressources individuelles dont disposait un grand général de l'époque homérique, Acuilte ou Hector, se livrant à des combats singuliers, avec la dépendance d'un Focr ou d'un Persurng vis-à-vis de leurs armées et du reste de la société, au cours de la récente guerre. Nul Homère ne pourra peindre ces gentlemen si civilisés, provoquant Hindenbourg ou Ludendorfa en combat singulier pour décider dı sort d'une bataille. L'ourrière de Nyrmecia, avec ses grands yeux, part seule à l'attaque d'autres insectes, tandis que l'ouvrière aveu- 
gle de Dorylus est obligée de se maintenir en communication constante, par le toucher et l'odorat, avec ld colonne en maraude, ou alors elle est désespérément impotente. On retrouve des contrastes analogues dans les sociétés civilisées, même entre les divers groupes; ainsi entre voleurs et employés de banque. La place qu'on donne dans les journaux aux premiers, comparée à celle qu'on accordle aux seconds, indique à qui va notre intérèt. Et si nous nous intéressons tellement aux voleurs, ce n'est point parce qu'ils sont criminels, mais parce que nous ne pouvons nous empêcher d'admirer leur har. diesse, leur ingéniosité et peut-être parce que nous avons une vague conscience de la servile dépendance dans laquelle nous vivons nous-mêmes, vis-à-vis des lois et de l'opinion publique.

On a constaté depuis longtemps les signes d'involution que la vie sociale imprime sur l'individu humain: c'est là, actuellement, le sujet d'une littérature abondante, scientifique et historique, tout comme ce fut, dans la dernière partie du xvıI ${ }^{\ominus}$ siècle, le sujet d'une littérature aussi abondante, mais ni historique, ni scientifique.

Les recherches de Le Bon (1925), Martin (ig20), Mac Dougall (1920), Freud (1921), et autres, sur les mœurs de ces associations temporaires appelées foules, ont mis en lumière des manifestations nettement régressives, dans le domaine psychologique. Comme dit Freud : "Un individu d'un groupe esl soumis à l'influence de ce groupe, qui altère souvent profondément l'activité menlale de l'individu. Les émotions de celui-ci s'en trouvent extraordinairement intensifiées, landis que ses capacités intellecluelles diminuent fortement, les deux processus 
teridant: évidemment à rapprocher l'individu des autres: membres du groupe ; et le résultat ne peut être atteint que far la substilution de ces intibitions aux instincts qui sont particuliers à chaque individu et par l'abandon des manifestations de toutes préférences personnelles ") (I).

Bien entendu, la foule étale sous une forme aiguë et exagérée des tendances qui sont présentes sous une forme chronique, mais moins apparente, dans les masses ou groupes de loute la société. Mais il y a des exemples plus surprenants des effets régressifs de la vie sociale. Les psychologues ont découvert que peu de nous attei. gnent un àge intellectuel de dix-huit ans et que la plupart conservent durant toute leur vie la mentalité d'enfants de $1 \nmid$ ans, ou moins. Cela même est trop flatteur, et les psychanalystes attirent l'attention sur la grande difficulté que les meilleurs d'entre nous éprouvent à conserver une attitude đ̆adulte ou même d'adolescent en face de la réalité et sur la facilité extraordinaire avec laquelle nous glissons à l'infantilisme. Ils montrent aussi la prédominance effrayante, dans nos civilisations, des névroses el des psychoses, caractérisées de façon habituelle ou chronique par ces façons enfantines. Et le sociologue prouve par des statisliques la grande extension du suicide et ses liens de dépendance intime avec la

(1) Il est intéressanl de noler que les insectes sociaux supérieurs, surtout les guĉpes, les abeilles domestiques et les fourmis, font montre de phénomènes, analogues à ceux que révèlent les foules humaines, forle émotivilé, suggestibilité, violence, etc. En outre, ces manifestations ne se produisent que dans les colonies populeuses, puisqu'une foule implique un arand nombre d'individus cooperint; des colonies faibles ou naissanles, de gucepes, d'abeilles ou de fourmis sont foujour: tres limicles. 
civilisalion (DünckneIv). D'innombrables individus se donuent la mort, simplement parce que la société les lasse et les pousse ì la mort. Ces considérations et bien d'autres ont conduit différents chercheurs tels que Didis et Juppoyt, BoAs et StaERcke, sans compler les historiens comme Spfichen et Towxen, à exprimer des vues également pessimistes sur les civilisalions et les capacités humaines. Suivant Dide et Juppoxt (1924), " une considération propre à susciter l'humilité humaine simpose. Les formes subalternes de la conscience, liées étroitement à l'instinct, demeurent encore vibrantes d'énergie formative, alors que les fonctions perceptives, pourtant de date ontogénique bien plus récente, non seulement paraissent ne plus évoluer, mais encore marquent chez l'homme une certaine régression. Et ceci concorde avec ce que nous révèle la cytogénèse comparée. Les cellules mal différenciées sont dotées d'une prodigieuse activité reproductrice, au lieu que les cellules hautement différenciées, particulièrement celles de la corticale cérébrale, qui président aux fonctions perceptives et gnostiques, ont perdu toute activité reproductrice. L'un de nous s'est atlaché depuis longtemps à cette étude spéciale et, ayant constaté encore quelques traces de division directe dans les cellules pyramidales des Rongeurs, il a vainement recherché pareille tendance chez l'homme, au lieu que, dans des travaux encore inédits, il a pu noter la constance du processus amilosique dans les cellules du sympathique, agent des fonctions viscérales. Ces faits, désormais indisculables, laissent peu d'espoir sur les deslinées Inintaines des races biologigues supéricures. " 


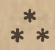

Suivant Boss (1924), "lorsque nous cherchons la signification des différences de races, nous devons nous souvenir que les races humaines ne peuvent se comparer aux formes sauvages de la vie animale, mais que l'homme est la forme domestiquée la plus ancienne. L'usage d'une nourrilure préparée artificiellement, qui s'est établi avec l'invention du feu, à l'époque glaciaire, marque le début de la période de domestication; celle-ci peut d'ailleurs être encore plus ancienne et débuter en même temps que l'emploi d'outils. Cette opinion fut exprimée en premier par Le Salles en 1849 . Hann la rappela plus tard; je m'en préoccupai en igri, et, en rgi3, Eugen Fischer, puis KLATT, ont repris cette question du point de vue des preuves anatomiques. Les cheveux blonds, les yeux bleus, la peau claire, sont des caractères de domestica. tion, aussi bien que les cheveux bruns et frisés. Aussi la réduction des dimensions de la face et son allongement. La permanence de la poitrine chez la femme, des ano malies dans les habitudes sexuelles, labsence d'une saison pour l'accouplement, apparliennent à la même catégorie. "Le psychanalyste Stakncke (1921), frappé comme heaucoup d'autres du caractère névrotique prédominant de notre civilisation, va jusqu'à la considérer comme une maladie spréciale qu’il baptise "métaphrénie ", qui " est imposée à une certaine partie de la société, afin d'obtenir un certain gain anormal dont tous tirent avan. tagre 1 . Et il conclut son chapitre sur la métaphrénie par l'exposé suivant: "Les sublimations et la formation des réactions, chez l'être humain social, suivent les mécanismes des névroses par obsession (il y a bien une res- 
semblance entre la contrainte morale logique et esthéthique et la contrainte de névroses). Le côté matériel subjugué lend à reprendre le dessus. Nous voyons la civilisation d'un peuple ou d'une race s'édifier en cycles, sui. vant les mécanismes des névroses par obsession, jus. qu'au jour où elle ne peut plus être tolérée davantage : elle parvient alors à une limitation de l'effet utile par le retour sous une forme déguisée des parties matérielles comprimées, et des choses interdites se font jour, par la guerre et la révolution, suivant les principes des psy. choses maniaques, sans que fassent défaut divers "ismes" analogues aux champs paranoïdes. "

La notion de "domestication " que, dans un autre passage, Staercke voudrait remplacer par celle de " sublimation "), est applicable aussi aux insectes sociaux. Mais la domestication est proche parente du parasitisme. La différence entre la faible contribution personnelle de l'iridividu et les avantages considérables qu'il recueille comme membre de la société, pour la nourriture et la protection seules, cette différence est si énorme, qu'on peut la comparer ì bien des égards à la différence entre l'hôte et le parasite. Par suite, les caractères de régression physique el mentale des individus ressemblent a ceux des parasites, comme nous le voyons pour nos animaux domestiques. Mais la domestication produit des effets opposés sur différents organismes. Chez certains, elle amène la stérilité, tandis que chez d'autres elle en. Iraîne l'atrophie des organes de relation (système sensoriel et neuromusculaire), et l'hypertrophie des gonades, du lube digestif el d'autres viscires. Cher les insectes sociaux, ces deux effets peuvent étre observés, le premirr chę les ouvrières et à cerlains degrés chez les sol- 
dats, le second chez kes reines. Il y a là des analogies évidentes avec la société humaine. Cet effet bivalent de la domestication a été dissimılé, au moins en ce qui concerne les animaux domestiques, par le fait que l'homme peut choisir et retenir seulement les espèces fécondes, et en ce qui concerne les plantes, parce qu'elles peuvent avoir une reproduction asexuée.

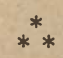

$6^{\circ}$ Mais, malgré lous les caraclères régressifs que nous avons mentionnés comme caractérisant l'individu social on peut admettre que les sociétés, considérées chacune comme un tout, sont des agents très puissants, comparés à n'importe quel organisme solitaire, non social. Les seuls rivaux dangereux des espèces sociales sont donc d'autres espèces sociales. Elles constituent en effet les forces les plus virulentes de toute la faune, puisqu'elles sont capables de se détruire entre elles comme elles détruiseni. les espèces solitaires qui les entourent, soit par la lutte, soit par la rapine. Yous devons donc attendre des organismes sociaux que leurs activités, si clles ne sont contenues par des facteurs externes, climaliques ou autres, amènent éventuellement l'extermination des espèces solitaires et la survivance d'un petit nombre d'espèces sociales très puissantes et très prolifiques. Il est à peine nécessaire d'attirer l'attention sur la façon dont agit l'homme à ce point de vue. Ayant exterminé presque toutes les sociélis humaines primitives, el presque fous les mammifìres, sauf les races quili a été en état de domestiquer, il a entrepris maintenant, la destruction de presque tout ce qui resle encore vivant sur la planète. Et les insectes sn- 
ciaux assument le même ròle parmi les Arthropodes terrestres, surtout dans les régions des tropiques, où ils ont la prépondérance absolue et ne sont pas encore entrés en contact avec l'homme civilisé. Mais avant de développer ce thème, disons quelques mots de la mort des sociétés.

Les plus pessimistes des penseurs eux-mêmes (SPENGLER, etc.) ne croient pas que les sociétés humaines périssent par les effets de leur culture et de leurs guerres exterminatrices. Après une floraison superbe, elles retournent à un état de somnolence, de paresse, comme celui des Egyptiens, des Chinois et des peuples des âges des ténèbres en Europe, et la race humaine ne peut être exterminée que par quelque catastrophe cosmique. Les sociétés d'insectes ressemblent plus à des organismes simples, parce qu'elles meurent. Malheureusement nos connaissances sur la durée, le déclin et l'extinction des sociétés pluriannuelles sont beaucoup moins précises que nous le désirerions. Nous connaissons, évidemment, les condilions d'existence des guêpes, bourdons et Halictus des régions tempérées, dont les colonies vivent seulement six ì huit mois, puis disparaissent, laissant les jeunes reines fécondées qui fonderont de nouvelles colonies l'année suivante. La vie d'une colonie de fourmis, ne contenant qu'une seule reine fécondée, doit prendre fin peu après la mort de celte reine. Pour certaines de nos espèces, on sait que la reine vit au moins quinze ans, ce qui doit être aussi, à peu près, la durée d'existence de la colonie, tandis que les ouvrières ont une vie bien plus courte (trois a quatre ans). Mais, quand les filles fécondées de la reine sont adoptées, année après année, par la colonie, cetle dernière doil vivre bien plus longtemps - quarante ì cinquante ans, ou peut-être plus - mais sur ce point 
comme sur la longévité des colonies de termites, nos connaissances sont limitées. Cependant, puisque la vie de l'espèce ne dépend pas de la vie de la colonie seule, un autre problème, plus obscur, se pose, à savoir comment se fera l'extinclion des espèces d'insectes sociaux. Comme nous l'avons vu, leurs colonies sont bien plus puissantes et persistantes que des organismes individuels, et leur aire de distribution est si vaste, qu'elles ne peuvent guère ¿̀tre détruites, mème par des animaux plus gros (oiseaux, fourmiliers, etc.) ou par de simples conditions climatiques locales ou temporaires; de sorte que l'extermination des espèces d'insectes sociaux doit provenir d'autres causcs. Elle s'est produite cerlainement et à plusieurs reprises au cours des époques géologiques, et il est fort probable qu'elle s'effectue encore. Dans les régions tempérées et boréales, les conditions climatiques contraires ont certainement les plus cruels effets sur les colonies de guêpes el de bourdons. Pour ce qui est des causes d'extinction agissant sur les Vespinés, les Apinés et les Méliponinés des tropiques, nous n'en savons rien, quoique ces colonies soient beaucoup moins nombreuses que celles de fourmis et de termites. Leur croissance et leur déclin sont probablement réglés par les aliments qu'elles exigent, plantes ou insecles. Les fourmis de la famille des Dorylinés sont certainement de redoutables ennemis pour les guêpes. Les plus grands ennemis des termites sont les fourmis; el ceux des fourmis sont d'autres fourmis; et, sans aucun doute, l'homme civilisé est l'ennemi le plus implacable de ces deux groupes d'insecles sociaux. L'ahondance des espèces et la survivance de beaucoup de types primitifs dans certaines parties de l'Inde, de l'Australie et des régions néotropicales, qui n'ont pas encore 
été touchées par l'homme civilisé, indiquent qu'en ces points s'est conservé, au cours des àges, un équilibre biocœnotique très perfectionné ; mais parlout où l'homme štablit, cel équilibre est promptement bouleversé. La suppression de la végétation indigène et l'introduction diz plantes étrangères cullivées et d'animaux domestiques, sont, on l'a bien constaté, les facteurs les plus graves de l'extermination des fourmis et des termites. Les effets de la civilisation sont donc, pour une grande part, indirects, C'est bien visible dans l'Amérique dı Nord, où toutes les fourmis les plus primitives comme les Ponérinés, qui; pendant des siècles, s'étaient accomodées de conditions édaphiques ou autres, précises, disparaissent aujourd'hui rapidement. Seules, les Myrmicinés et les Formicinés, indigènes, plus plastiques et s'adaptant mieux, arrivent i survivre dans les régions colonisées, mais elles tendent ì disparaître, à mesure que s'accroît la densité de la population humaine. Dans les tropiques, une destruction plus rapide encore est amenée par certaines espèces de fourmis, ayant une facilité d'adaptation extraordinaire, capables de vivre même sur des bateaux et qui ont été ainsi transportées dans toules les régions du monde où elles ont pris rapidement possession des aires ayant la même lempérature annuelle moyenne que leur habitat originel. Nous allons examiner succinctement deux de ces espèces, une Myrmicinée, Pheidole megacephala et une Dolichoderinée, Iridomyrmex humilis.

On suppose que Pheidole megacephala est originaire de Nadagascar, parce que cette île renferme le plus grand nombre des variétés et des espèces voisines. Au cours du siècle dernier, clle a été transportée dans toutes les régions tropicales et elle y a exterminé toute la faune autochtone 
de fourmis, partout où l'homme avait préparé la voie à ses conquêtes, en détruisant la végétation indigène et en introduisant de vastes cultures de plantes étrangères. Ces cultures ont été rapidement infestées par des insectes et spécialement par des Coccides qui sont les protégés favoris de megacephala. Heer a montré qu'en r 852 , cette fourmi a pris complètement possession de l'île de Madère. Elle a aussi exterminé toutes, ou presque toutes les fourmis de certaines des Antilles, particulièrement les Bermudes, les Bahamas, Saint-Thomas et Culebrita, près de Porlo-Rico, et elle a fait des progrès considérables à Cuba et aux Hawaï. Dans les aires plus vastes, surtout sur les continents, ses progrès sont plus lents, parce qu'il en est de même pour celui qui la précède, l'homme civilisé. J'ai été témoin de son invasion graduelle le long de la côte orientale de l'Australie, nù les colons anglais ont fondé de nombreuses cités en des points très éloignés les uns des autres. Ph. megacephala est apportée dans ces villes par les bateaux de commerce; elle se confine d'abord dans les rues et les jardins, remplaçant complètement la faune indigène de fourmis, élablissant ses nids partout, autour des racines des arbres, dont les feuilles et l'écorce nourrissaient les Coccidés. En avançant quelque peu à l'intérieur des terres, dans une zone défrichéc et mise en culture, on peut voir megacephala en conflit avec les fourmis indigènes, envahissant leurs nids, enlevant leurs couvées, et massacrant les adultes. J'ai vraiment assisté à la destruction d'une colonie de Podomyrma femorata, une superbe fourmi, de grande taille, mais assez slupide, qui occupe des cavilés dans le bois dur de troncs sur pied. Ces fourmis étaient parfaitement incapables de se défendre elles-mêmes contre les hordes des 
petites ouvrières de Pheidole. La colonie fut complètement détruite, les larves et les adultes tués, et emportés lente ment. Plus avant dans l'intérieur des terres, on atteint Ta forêt primitive intacte, où, grâce aux rapports parfaitement équilibrés qui lient les divers composants de la faune et de la flore, megacephala ne peut pénétrer, tant que l'homme n'y a pas porté la dévastation.

L'histoire d'Iridomyrmex humilis est identique. Elle semble originaire d'Argentine, aussi l'a-t-on appelée la fourmi d'Argentine. Après avoir pénétré dans la NouvelleOrléans, elle s'est beaucoup accrue numériquement et s'est répandue rapidement sur tous les Etats du Sud et la Californie, exterminant partout les fourmis indigènes. Elle traversa l'Atlantique et infesta des régions aussi éloignées l'une de l'autre que la colonie du Cap et le Portugal. Suivant Stour. (1898), elle s'installa aussi à Madère et réussit à y supplanter Pheidole megacephala, qui était restéc maître de cette île depuis l'époque de Heer. Plus récemment, humilis a fait son apparition en France et aux îles Canaries. Le fait que, parlout où cette fourmi entre en contact avec megacephala, celte dernière lui laisse la place, est très intéressant à rapprocher de mes remarques sur le contraste existant entre la puissance d'une colonie et la faiblesse de ses membres. I. humilis est une Dolichodérinée au corps mou, dépourvue d'aiguillon, tandis que megacephala a un tégument dur, un aiguillon, et des soldats à la grosse tête el aux mandibules puissantes. Quoique la fourmi d'Arontine soit individucllement la plus faible, elle surpasse Pheidole par la férondité de sa reine el l'imperlance de ses colonies, par son activité entreprenante et son ingéniosité, tous caractères qui la rendent supérieure à Pheidole, comme 
celle dernière est supérieure aux autres fourmis, lorsqu'elle s'introduit dans une faune qui a été plus ou moins affaiblie sous l'action de l'Homme.

Il est à prévoir qu'avec les progrès de la civilisation, l'homme exterminera la faune et la flore terrestres, sauf tout ce dont il peut tirer quelque profit. Mais les fourmis les plus prolifiques et les plus ingénieuses resteront comme ses rivaux et son lourment, et seront sans doute au nombre des insectes qui disparaîtront les derniers. Et dans sa lutte contre ces fourmis, l'homme triomphera surtout grâce à des méthodes indirectes, c'est-à-dire en modifiant ou en supprimant les conditions externes indispensables pour leur survie. 


\section{INDEX BIBLIOGRAPHIQUE}

Adberz (G.). - 1884. Myrmekologiska Studier. I. Formicoxenus nitidulus Nyl. Ofv. Vet. Akad. Förh. 8, p. 13-61, 2 pls. - 1896. Myrmekologislia Studier III. Tomognathus sulrlævis Mayr. Bihang K. Svensk. Vet. Aliad. Ilandl, 21 p. 3-76, 1 pl.

Alten (F.) (von). - 1910. Zur Phylogenie des IIymenoplerengehirns. Jen. Zeitschr. Naturw. 46. p. 511-590, 4 pls., 28 tigs. A.NDRÉ (Edm.). - 1881. Species des Hyménoptères d'Europe et d'Algérie. 2.

André (Ern.). - 1892. Voyage de M. Chaper à Bornéo. Mém. Soc. Zool. France 5, p. 46-5̌, 5 figs - 1895. Notice sur les fourmis fossiles de l'ambre de la Baltique el description de deux espèces nouvelles. Bull. Soc. Ent. France 20, p. 80-81. - 1903. Famille Mutillidie in Wrisman, Geneva Insectorum. p. 1-77, 3 pls.

Armbruster (L.). - 1914. Problème des Hummelstaales. Biol. Centralbl. 34,' p. 685-707, 1 pl., 1 fig. - 1916. Zur Phylogenie der Geschlechtsbestimmungsweise bei Bienen. Zool. Jahb. (Abt. Syst.) 40, p. 328-388, 1 pl., 6 figs.

Ashmead (W. H.). - 1890. Neolarra pruinosa n. gen. n. s. p. Colorado Biol. Ass. p. 8. - 1893. Monograph of the North American Proctotrypiclie. Bull. 15, U. S. Nat. Mus. Washington, 472 pp., 18 pls. - 1896. The Phylogeny of the Hymenoptera. Proc. Ent. Soc. Washinglon, 3, p. 323-336, 2 tigs. 1902-03. Classification of the Fossorial, Predaceous and Para. sitic Wasps, or the Super-family Vespoidea. Paper no. 10. Canad. Ent. p. 287-291; Paper no. 12, Ibid. p. 39-11.

Assuess (E). - 1865. Die Parasiten der Honigbiene und die durch dieselben bedingten Krankheilen dieses Insektes. Berlin, E. Schotte et Co. 56 pp., 3 pls.

Assmutu (J.). - 1913. Termitoxenia assmuthi. Wasm. Nova Acta Leop. Carol. Akad. Naturi. 98, p. 191-316, 11 pls. 
Audouin (J. V.). - 1842. Histoire Naturelle des Insecles Nuisibles, p. 189.

Aunivillius (C). - 1896. Ueber Zwischenformen zwischen sucialen und solitären Bienen. Feslschr. Wilk. Lilljeborg. Lu. sala, p. 69-77.

13.ker (C. F.). - 1896. On the Affinitics of Neolarra. Proc. Iint. Soc. Washington 4, p. 20-23, 1 fig.

liıks (N.). - 1904. (On Social Spiders), ibid. 6, p. 118.

BanKs (N.) et SNyder (T. E.). - 1920. A Revision of Nearclic Termites with Notes on Biology and Geographic Distribulion. U.S. Nat. Mus. Bull. 108, 228 pp., 35 pls., 70 figs.

- Barbour (T.). - 1914. Zoogeography of the West Indies. Mem. Mus. Comp. Zoology 44. - 1919. The Herpelology of Cuba. Ibid. 47.

Becher (E.). - 1917. Die fremddienliche Zweclimässighicit der Pflanzengallen uns die Hypolhese eines überindividuellen Seelischen. Leipzig. 1:19 pp.

Bhit (T.). - 1874. The Naturalist in Nicaragua. London, Bumpus.

Brinetr (C. B.). - 1904. Earwigs (Anisolabis marilima), Psyche, 11 , p. 47-53, 2 figs.

Bequaert (J.). - 1916. On the Occurence of Vespa alstriacn Panzer in the Northeastern United States. Bull. Broolilyn Ent. Soc. 11, p. 101-107. - 1918. A Revision of the Vespidic. of the Belgian Congo, elc. Bull. Amer. Mus. Nat. IIisl. 39. p. 1-384, 267 figs., 6 pls. - 1922. (On Bengalia) in Wheeler Ants of the Belgium Congo. Ibid. 15. p. 281. - 1924. Galls. that secrete Iloney_dew. Bull. Broolilyn Iint. Soc. 19, p. I0I 124.

Bergen. - 1878. Untersuchungen über den Bau des Gehirns und der Retina der Arthropoden. Arb. Zool. Inst. Wien 1.

Brirlese (A.). - 1909. Gli Inselti. I. Milano, Soc. Edit. Libr.

Bithe (A.). - 1898. Dürfen wir den Ameisen und Bienen pșychische Qualitälen zuschreiben. Arch. Gesam. Physiol. 70, p. 15-100, 2 pls., 5 figs. - 1900. Noch einmal üher die psychischen Qualiäten der Ameicen.

Ileimkrhefähigkeilen der Imeisen und Bienen, zum Teil nach ueuen Versuchen. Biol. Cenlralbl. 2?. 1. 193-215, 23!-238.

Biedermann (W.). - 1911. Die Aufnahme, Verarbeilung und Assimilation der Nahrung. Winterslein's IIandbuch d. vergleich Physiol. 2.

Bıschoff (H.). - 1909. Neue Beilräge zur Lebensweise der Tri. gonaloiden, Berlin. Enl. Zeitschr. 54, p. 76-80, 1 fig. 
Blanchard (R.). - 1889. Pseudoparasites. Dictionn. Encyclopé dique des Sciences Médicales (2) 27, p. 704-709.

LöNNER (W.). - 1915. Der temporäre soziale Hyperparasitismus von Lasius juliginosus und seine Beziehungen zu Clatiger longicornis Müll. Zeitschr. wiss. Inselilenbiol. 11, p. 11-20.

Börser (C.). - 1919. Stammesgeschichte der Haulflügler. Biol. Zenlialbl. 39 , p. 145̆-186, 6 figs.

Böttger. - 1910. Das Gehirn eines niederen Insekis. Jen. Zeılschr. f. Naturiv, 45.

liutrimertik ('l'.). - 1923. Beitrag zur Biologie der Feuerameise. und ihrer Gäste (Solenopsis geminata saevissima) Zeilschr. deutsch. Ver. Wiss. Kunst, Sầo Paulo 3, p. 1-9, 1 fig.

Botvier (E. L.). - 1918. La Vie psychique des Insectes. Paris, Flammarion.

Boveri (T.). - 1915. Ueber die Entstehung der Eugsterschen Zwitter-Bienen. Arch. Entwiclilungs mech. Ory. 11, p. 26:1311,2 pls.

Bradley (J. C.). - 1922. The Taxonomy of the Masarid Wasps, including a Monograph of the North American Species. Univ. Ciala. Publ., Techn. Bull. 12 p. 369-464.

liminen (J. C.). - 1900. Ants as Geologic Agents in the Tropics. Journ. Geol. Chicago 8, p. 151-153, 3 figs.

Brauer (F.). - 1869. Beschreitung der Werwandlungsgeschichle der Mantispa styriaca Poda und Betrachtungen über die sogenannte Hyprermetamorphose Fabres. Verh. Zool. bol. Ges. Wien 19, p. 831-840, 1 pl. - 1883a. Zwei Parasiten des lihizotrogfus solstilialis aus der Ordnung der Dipteren. Sitzl). K. Aliad. Wisss. Wien 88, p. $865-877,2$ pls. - 1883b Ergänzende Bemerkungen zu A. Hindurnsci's Mitteilungen über Hirmoneura obscura Meig. Wien. Ent. Zeitg. 2, 1883, p. 26. Bumins (I.). - 1902. Eucondylops n. g. apidarum. Zeitschr Sys!. Hym. Dept. 2, p. 377-380. - 1910-11. Biologisches über südalifritianische Hymenopteren. Zeilschr. wiss. Inselitenbiol. ’े, p. $384-387,445-147 ; 7$, p. 16-19, 90-92, 117-120, 238-240.

Brettenbach (W:). - 1878. Ueber Halictus 4-cinclus $\mathrm{F}$. und Sphecodes giblus L. Stellin. Enl. Zeilg. 1). 241-213\%.

Bretschneider (F.). - 1915. Neuere Untersuchungen über das Gehirn der Inseliten. Wochenschr. n. f. 14, p. 17-24, 17 ngs. Burnweli (J. C.). 1917a. Noles on a Peregrine Bethylid. Proc. Hawaii Ent. Soc. 3, (1916), p. 276-279. - 1917b. A Note o'n an Epyris and its Prey. Ibid. 3 (1916) p. 262-203. - 1917c. Noles on Bruchidx and their Parasites in the Hawaiin Island:. Ilid. 3 (1916) p. 465-500. - 1919. Some Noles on Hawaiian and olher Bethylidse, with Descriptions of new Species. Ibid. 4 
(1918) p. 21-38. - 1920. Some Notes on Hawaiian alld utiler Bethylidxe, with the Description of a new genus (second paper). Ibid. 1 (1919) p. 291-314.

Browne (F. B.). - 1922. On the Life Hislory of Meliltobia acaslu Walk., a Chalcid Parasite of Bees and Wasps. Parasilolog!! 14.

Bnuch (C.). - 1917a. Observacion:s sobre Hirmoneura exolica IViedem. Physis 3, p. $427-430,2$ figs. - 1917b. Nuevas capturas de insectos mirmecofilos. Physis 3, p. 40ั8-465, 3 figs.

Brues (C. T.). - 1906. Fossil Parasitic and Phylophagous Hymenoptera from Florissant. Bull. Amer. Mus. Nat. Hist. 22, p. 491-498, 7 figs. - 1908a. New Phytophagous Hymenoptera from the Tertiary of Florissant, Colorado. Ibid. 51, p. 259-276, 10 figs. - 1908b. Some Stages in the Embryology of certain degenerate Phorid:e and the supposed hermaphrudilic genus Termitoxenia. Science n. s. 27. - 1908c. The Correla tion between Habits and Structural Characlers among Parasilic Ilymenoptera. Journ. Econ. Ent. 1. p. 123-128. - 1910a. The Parasitic Ilymenoplera of the Tertiary of Florissanl, Colorado. Bull. Mus. Comp. Zool. 54, p. 3-125, 1 pl. 88 figs.$1910 b$. Some Notes on the Geological History of the Parasitic Hymenoptera. Journ. N. Y. Ent. Soc. 18, p. 1-22. - 1919a. A New Chalcid Fly Parasitic on the Australian Bull-Dog Anl. Ann. Ent. Soc. Amer. 12, p. 13-21, 2 pls. - 1919b. The Clas sification of Insects on the Characters of the Larva and Pupa. Biol. Bull. 37, p. 1-21. - 1921. Correlation of Taxonomic Affinities with Food Habits in Hymenoplera, wilh Special Reference to Parasitism. Amer Natural. 55, p.131-161. 1922. On the Hymenoplerous Genus Harpagocryplus and its Allies. l'syche 29, p. 101-109. - 1923a. Termilobracon, a Termitophilous Braconid: from British Guiana Zoologica 3, p. 427-432, 2 figs. - 19236. Some New Fossil Parasitic Hyme. noptera from Baltic Amber. Proc. Amer. Acad. Arts. Sc. 58, p. 327-346, $1 \mathrm{fig}$. - 1923c. A Fossil Genus of Dinapsince from Ballic Amber. Psyche 30, p. 31-55, 1 lig. - 1924a. Triungulins of a Meloid Beetle borne by Xylocopa, with Remarks on the Type of Larva in the Coleoptera and Strepsiptera. Zoolo. gica j, p. 125-136, 5 figs. - 1924b. Some South African Parasitic Hymenoptera of the Families Evaniidx, Braconidx. Alysiidx and Plumariidx, in the South African Museum, with a Catalogue of the Known Species. Ann. S. Afric. Mus. 19, p. $1-150$

Brues (C. T.) et Melander (A. L.) - 1915. Key to the Fami- 
lies of North American Insects. Boston, Mass el Pullman Wash.

Brun (R.). - 1910. Zur Biologie und Psychologie von formica rufu und enderen Ameisen. Biol. Cenlrabl 30, p. 5:44.545.

1912a. Zur Psychhlogie der künstlichen Allianzkolonien bei den Imeisen. Ibid. 32, p. 308-322. - 1912b. Weitere Beiträge \%ur lrage der Koloniegründung bei den Ameisen mit besonlerer Berücksichtigung der Phylogenese des socialem Parasitismus und der Dulosis bei Formica. Ibid., 32, p. 15/-180. 216-266. - 1913b. Zur Biologie von Formica rufa und Camponotus herculeanus i. sp. Zeilschr. wis.s. Inselitenbiol. 9, p. 1519. - 1913b. Ueber die Ursachen der künstlichen Allianzen bei den Ameisen. Journ. Psych. u. Neurol. 20, p. 171-181. -1917. Die moderne Ameisenjsychologie, ein anthropomorphischer Irrtum. Biol. Centralbl. 37, p. 357-372. - 1923a. Vergleichende Untersuchungen üher Insektengehirne. mit besondere Berücksichtigung der pilzförmigen Körper (Corpora pedunculata Dujardin). Schweiz. Arch. Neurol. Psychiall. 13. p. 144-172, 5 figs. - 1923b. Selektionstheorie und Lustprinzip. Internat. Zeitschr. Psychoanal. 9, p. 183-200. 1924. Das Leben der Ameisen. Leipzig, Berlin, Teubner.

Bristell (G.). - 1904. Collezionismo e Ibernazione nell'Origine degli Istinti delle Api Solitarie e Sociali. Revist. Ital. Sc. Nat. 21, p. 3-7.

Buckingham (E. N.) - 1911. Division of Jabor among Anls Proc. Amer. Acad. Arls. Sci. 46, p. 425-507, 1 pl.

Bicion (E.). - 1909. Le termite noir de Ceylan (Eutermes monoceros Koen.) Ann. Soc. ent. France 78, p. 271-281, 3 pls. - 1910. La structure anatomique du Trigonalys Hahni Spin. Mitteil. schweiz. Ent. Gesell. 12, p. 14-20, 4 pls. - 1912. Observations sur les Termites. Différentiation des castes. C. R. Séances Soc. Biol. 72, p. 1091-1094, 2 figs. - 1913 a. Termiloxenia, étude anatomo-histologique. Ann. Soc. Enl. Belg, 57, p. 23-44, 3 pls. - 1913 b. La différentiation des castes chez les Termites. Bull. Soc. Ent. France p. 213-218. 1914. La Biologie des Termites de Ceylan. Bull. Mus. d'Hisl. Nal. Paris p. 170-204, 8 pls. - 1923. La Guerre des Fourmis el des Termites, la génèse des instincts expliquée par cell: guerre. In Fonel's "Le Monde Social des Fourmis ", 1923, p. ]-55, 8 pls.

Bligivion (E.) et Popoff (N.). -- 1910. Le termile il lalex de Ceylan, Coptotermes tratians IInviland. Mem. Sor. Zool. France p. 11) $7-123,2$ pls., 1 fig. 
Burke (II. E.). - 1917. Oryssus is Parasilic. Proc. Enl. Soc. IVashington 19, p. 87-89.

Buscalioni (L.) el Comes (S.). - 1910. La digestione delle membrane vegetali per opera dei Flagellati contenuti nello intestino dei Termitidi e il problema della Simbiosi. All. Acoad. Gioenia Sc. Nat. (5) 3, 16 p.p., 4 figs.

Butrel-Reeren (H. von). - 1903. Die stammesgeschichtliche Entstehung des Bienenstaates, sowie Beiträge zur Lebensw'eise der solitären und sozialen Bienen (Hummeln, Melipouen, etc.) Leipzig, G. Thieme. XII et 138 pp., 20 figs. - 1905. Soziologisches und Biologisches von Ameisen-und Bienenstaal. Wic entsleht eine Ameisen-Kolonie. Arch. Rassen. Gesell. Biol. 2, p. 1-16, 1 fig. - 1906. Apistica, Beiträge zur Systematik, Biologie, elc. der Innighiene (Apis mellifica L.), ihrer Varietäten und der übrigen Apis Arten. Mitteil. zool. Mus. Berlin 3, p. 121-201, 8 figs. - 1911. Atavistischo Erscheinungen in Bienenstaat (Apis mellifica L..), elc. 1er Congr. Internat. d'Ent. Bruxelles, p. 113-132. 1 pl., 3 figs. 1915. Leben und Wesen der Bienen. Braunschweig, Viehweg u. Sohn.

Buysson (R. du). - 1903-05. Monographie des Guêpes ou Vespa. Anл. Soc. Ent. France 72 , p. $260 ; 74$, p. 485.

Caffrey (D. J.). - 1921. Biology and Economic Importance of Anastatus semi-flavidus, a recently described Egg Parasite of Hemileuca olivx. Journ. Agric. Research 21, p. 373-38.1, 1 pl. 3 figs.

Cinpensen (G. 1). H.). - 1919. The Fly Bengalia depressa Walk., altacking a wingless Termite. Proc. Ent. Soc. London, p. LVIII.

Cirpenter (G. H.) et Pick-Beresforn (D. R.).- 1903. The Relationship of Vespa austriaca to Vespa rula. Ent. Month. Ma! (2) 4, p. 230-244, 1 pl.

Caullery (M.). - 1922. Le Parasilisme el la Symbiose. Paris. Doin.

Chapmin (T. A.). - 1870. Some Facts towards a Life-History of Rhipiphortls paradoxus. Ann. Mag. Nat. Hist. 6, p. 314-326. 1. pl. - 1878. On the Economy, etc. of Bombylius. Ent. Month Mag. 14, p. 196. - 1902. On the Larva of Liphyra brassolis Weslw. Enlomologis/ 35. p. 225-228,252-255, 1 pls. - 1915 a. What the Larva, of Lycena ario does during its last Instar. Trans. I:nt. Soc. London p. 291-297, 9 pls. - 1915. b. Observalions completing an Oulline of the Life History of Lyfeienu arion I. Ibid. p. 298-312, 2 pls. 
Chisp (C. М.). - 1924. Physiological Foundations of Behavior. New York, Henry Holt et Co.

Сновıыт (А.). - 1891. Mceur's et métamorphoses de Emenudia flabellata F., insecte Coléoptère de la famille des Rhipiphorides. Ann. Soc. Ent. France 60, p. 447-456, 6 figs.

Culsex (C. P.). -- 1923. The Biology of Schizaspidia tenuicornis Ashm., a Eucharid Parasite of Camponotus. Ann. Ent. Soc. Amer. 16, p. 19ð-217, 2 pls.

Culitelind (L. R.). - 1923a. Symbiosis belween Termites and their inlestinal Protozor. Proc. Nul. Acul. Sci. 9, p. 424-4?8. 1923l. Correlation between the Food and Norphology of Termites and the Presence of Intestinal Protozoa. Amer. Journ. Hygiene 3, p. 441-461. - 1924. The Physiological and Symbiolic Relationship belween the intestinal Protozoa of Termites and their Host, with special reference to Fieliculilermes flavipes Kollar. Biol. Bull. 46, p. 177-225.

Cockiretu ('T. D. A.). - 1906a. A New Fossil Ant. Ent. News, p. 27-28. - 1906 b. Fossil Hymenoptera from Florissant Colorado, Bull. Mus. Comp. Zool. 50, p. 33-58. - 1909a. Descriptions of Hymenoptera from Baltic Amber. Schrift. Physili. ökon. Gesell. Königsberg 50, p. 1-25, 14 figs. - 1909 b. Some European Fossil Bees. Entomologist. p. 313-317. - 1913. The Fenera Parotermes and Hodotermes. Ent. News 24, p. 6-8. 1915. British Fossil Insects. Proc. U. S. Nat. Mus. 49, p. 469-499, 6 pls. - $1916 a$. Some American Fossil Insects. Iola, 51, p. $89-106,1$ pl. - 1916b. Insects in Burmese Amber. Amer. Journ. Sci. 42, p. 135-138, \& figs. - 1917. Descriptions of Fossil Insects Pror. Biol. Soc. Washinglon 30, p. 79-81, 4 figs.

1920 a. Fossil Arthroporls in the British Museum I, Ann. Mag. Nat. Hist. (9) 5, p. 273-279. - 1920 b. Eocene Insects from the Rocky Moumtains. Proc. U. S. Nat. Mus. 5i. p. 233. $26 j_{0}, 5$ pls. - 1921 a. Some Encene Insects from Colorado and Wyoming. Ibid. 59, p. 29-39, 1 pl. 9 figs. - 1921 b. Fossil Arthroporls in the British Mureum, V. Oligocene Hymenoplera from the Isle of Wight. Ann. Mag. Nat. Hist. (9) 7. p. 1-25. 32 figs. - 1923 $\alpha$. Fossil Tnsects from the Eocene of Texas. Amer. Journ. Sci. 5. p. 397-100). 1 lig. - 1923 b. The Earliest known Ponerine Ant. Eintomologist 56, p. 31-5̃. 1 fig.

Corr.s (J. E.). - 1922. Deseription of a new Fenus and lwo new -pecies of Ceridomuidide, and six new snecies of Acalyplrate Vuscidre (Ephydridie and Vilichiidie). Trans. EnI. Soc. London, p. 501-517, 4 pls. 
Comstock (J. C.). - 1924. An Introduction to Enlomology. Comstock Publ. Co. Ithaca, N. Y.

Cring (W.). - 1918. Appetites and Aversions as Constituents of Instincts. Biol. Bull. 34, p. 91-107.

Crugg (F. W.) - 1917-18. The Mouthparts of Ochromyia jejuna, a Predaceous Muscid. Ind. Journ. Med. Hesearch う, p. 516,1 ll.

Crimpton (G. C.). - 1915. The Thoracic Sclerites and the Syslematic Position of Grylloblatla campodeiformis Wallier, a Remarkable Annectant "Orhopteroid" Insect. Ent. News. 26, p. $337-350,1 \mathrm{pl}$.

Crawley (IV. C.). - 1909. Queens of Lasius umbralus Nyl. accepted by Colonies of Lasius niger L. Ent. Month. Mag. (2) 20. p. 91-99. - 1910 a. Summary of Experiments with Fertile Females of Several Species of $\mathrm{n}$ (s. Ent. Rec. 22. - 1910 b. IIow Ants greet Members of the Same Colony. Ibid. 22, p. 13-11. - 1912. Parthenogenesis in Worker Ants. Wilh Special Reference to two Colonies of Lasius niger, Linn. Trans. Ent. Soc. London. p. 657-663.

Crawley (IV. C.) and Biylis (H. A.). - 1921. Mermis parasilic on Ants of the Genus Lasius. Journ. Roy. Micr. Soc. p. 353372,12 figs.

Chiwley (W. C.) and Donisthorpe (H.). - 1913. The Founding of Colonies by Queen Ants. Intern. Ent. Congr. Oxiord (191?) 2 , p. 5-55.

Culiot (L.). - 1911. La Gènèse des Espèces animales. Paris, F. Alcan.

Cuntis (J.). - 1845. Description of the Nests of two Ilymenopterous Inserts inhabiting Brazil, and of the Species by which they were constructed. Trans. Linn. Soc. 19, p. 249-259, 1 pl. Cusumin (R. A.). - 1913. The Calliephialles Parasite of the Codling Molh. Journ. Agric. Research 1. p. 211-237, 1 pl., 15 figs. - 1917. Noles on the Biology of Schizonolus sieboldii Ratz. Proc. Ent. Soc. Washington 19, p. 128-129, 1 pl. - 1923. A New Subfanily of Braconidie from Termile Nests. Ibid. 5.). p. $5 / 1-55,1$ pl.

Citren (IV.). - 1921. Obrervations on the Protozoa parasitic in Archotermopsis Wrol:ghtoni D. Part. III. Pseudotrichonympha pristina. Quart. Journ. Mirr. Sci. 65, p. 217-261, 10 pls., 8 figs.

Divinson (A.). - 1913. "Masaria respoirles ". Bull. Soulhern Cala. Aerad. Sci. 12, p. 17.

Divis (J. J.). - 1919. Contributions to a Knowledge of the 
Natural Enemies of Phyllophaga. Bull. 13̈. Nal. List. Surt. Illinois p. $533-138,13$ pls., 16 ligs.

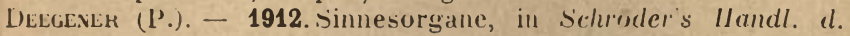
Ent. Bd. I, p. 142-158. - 1918. Die fiormen der Vergesellschaltung in Tierreiche. Leipzig, Veit el lo.

De Graxge (Mc Q.) -- 1923. La Courbe de Nouvement Societal. Paris.

Je Litidere (J. C. H.). - 1909. Drei nyrmeliophile Dipleren aus Java. Tijdschr. $v$. Lint. 52, p. $165-174,1$ pl. -1910 . Ueber drei von Jacobson auf Java bei Pheidologeton diversus Jerdon beobachtele Fiegen. Ibid. 53, p. 336-340. - 1911. Zur Metamorphose der myrmetiophilen Culicide IIarpagomyia splendens de Meij. Ibid. 5.4, p. 162.-16ī, l pl.

Demoll (R.). - 1917. Die Sinnesorgame der Arthropoden, ihr Bau und ihre Funklion. Braunschweig.

Descy (A.). - Recherches sur la Sexualite et I'Instinct chez les Hymenoptères. Bull. Biol. France Belg. 58, p. 1-37.

I)esneux (J.). - 1904. A propos de la Phylogenie des Termilides. Ann. Soc. Ent. Belg. 48, p. 278-286, 2 figs.

Dide (M.) and Juppont (P.)- - 1924. La Métaphysique Scientilique. Paris. Alcan.

Dietr (M. J.). - 1876. Die Organisation des Arthropodengehirns Zeitschr. f. wiss. Zool. 27, p. 489.

Diguet (L.). - 1909a. Sur l'araignè Mosquero. C. R. Acad. Sc. Paris, 148, p. 735-736. - 19096. Le Mosquero. Bull. Soc. Nal. Acclim. Paris, 56 , p. 368-375.

Diмmoск (G.). - 1886. Sphaerularia in America. Amer. Natural. 20 , p. $73-75$.

Distant (W. L.). - 1898. Zoological Rambles in the Tranvaal. Zoologist, (4) 2, p. 249-260, $1 \mathrm{pl}$.

Dudd (F. P.). - 1902a. Notes on the Queensland Green Tree Ants (OEcophylla smaragdina Fabr. ?). Victorian Nalural. 18, p. 136-140. - 1902b. Contribution to the Life History of Liphyra brassolis. Westw. Entomologist 35, p. 153.1566. - 1912. Some Remarkable Ant-friend Lepidoptera of Queensland. Trans. Ent. Soc. London 10, p. $577-590_{2} 1 \mathrm{pl}$.

D)̈̈ноғт (Е.). - 1882. Ein Wachsspaltungsferment in Darm der Larve der Wachsmolle. Alch. Anat. Phys. Abl. p. 163.

Dofleis (F.). - 1920. Mazedonische Ameisen. Jena.

DoNistuorpe (Н.). - 1897. Myrinecophilous Coleoplera in 1897. lint. Record 9 p. 216. - 1910. Some Experiments with Ants' Nests. Trans. lint. Soc. London, p. 112-150. - 1911. Further Observations on Temporary Social Parasitism and Slavery in 
Inls. Ibid. p. 175-183. - 1912. Mymecophilous Noles for 1911 lint. Record :2, p. 4-10, 3-140. - 1915. British Anls, their Life IIislory and Classification. Plymoum, Brendon el Son. 1920. British Oligocene Ants. Ann. Mag. Nat. Hist. (9) 6, p. 81-9.', I pl. - 1921 a. Myrmecophilous Noles lor 1920. Lint. liecord 33, p. 21-25. - 1921 b. The subfamilies of liormicid'u. Proc. Ent. Soc. London, p. XL-XLVI, 5 figs. - 1922. The Colony Founding of Acanthomyops (Dendrolasius) [uliginosus l.atr. Biol. Bull. 42, p. 173-184.

Dork (S. B.). - 1911. Concerning the Relation of Food 10 Reproductive Activity and Longevity in Certain Hymenoplerous Parasites. Techn. Bull. 78. Agric. Exper. Slat. Inic. vevada. p. $7-30,10$ pls.

Uhever (J.). - 1917. Instinct in Man, a Contribution to the P-ychology of Education. Cambridge Univ. Press.

Dinyling (L.). - 1905. Die wachsbereitenden Organe bei den gesellig lebenden Bienan. Zool. Jahrb. (.4bt. Anut.) 22, p. 289330,2 pls. 1 fig.

Ducke (1.). - 1902. As Especias Paraenses do Genero Luglossa Latr. Bol. Mus. Para 3, p. 561-5̃ 17 pl. - 1903. Biologische Notizen über einige südamerilianische Hymenoptera. Allgm. Zeilschr. Ent. 8, p. $36 \overline{7}-372,5$ figs. - 1905. Biologische Nolizen über einige südamerikanische Hymenoptera. Zeitschr. wiss. Inselilenbiol. 1, p. 175-177. - 1910. Revision des Guêpes Soriales Polygames d'Amérique. Ann. Mus. Nalion. Hungarici8, p. 449-544, 17 figs. - 1914. Ueber Phylogenic und Klassifi. kalion der sozialem Vespiden. Zool. Jahrb. (Abt. Sys.) 36, p.

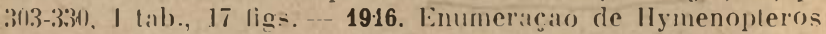
Colligidas pela Commisao. Revisao das especias de abelhas do Brasil. Com. Lin. Telegraph. Estraleg. Mallo Grosso ao Ama:onas. No. $35,175 \mathrm{pp} .7 \mathrm{pls}$.

Dufoun (L.). - 1837. Recherches sur quelques Entozoaires et larves parasites des Insectes Orthoptères el Hyménoptères. Ann. Sc. Nat. Zool. (2), 7 p. 5-20, 1 pl.

Dunbir (C. O.). - 1924. Kansas Permian Insects. Part. I. The Cienlogic Occurence and the Environment of the Insects. Amer. Journ. Sci. (5) 7. p. 171-209, 4 figs., 1 pl.

Dirckilim (E.). - 1922. De la Division du Travail Social (10 edit.) Paris, Alcan.

I)rtt (G. R.). - 1912. Iife IIislories of Indian Insects. $1 / \mathrm{em}$. Dep. Agric. India. Ent. Ser. 11 No. 4, p. 251.

Fowanns (F. IV.). - 1922. A Revision of the Genus Harpagnmyin de Veij. Trans. Ent. Soc. Lonilon p. 496-503, pl. 16, ligs. 5-12. 
Emers (C.). - 1890. Studii sulle formiche della fauna neotropica. Bull. Soc. Ent. Ital. 22, p. 38-40, 5 pls.- 1891. Le formiche dell' Ambra Siciliana nel Museo Mineralogico dell' Lniversita di Bologna. Mem. R. Accad. Sc. Ist. Bologna (5) ], p. 141-165, 3 pls. - 1894. Die Entslehung und Ausbildung des Arbeiterstandes bei den Ameisen. Biol. Centralbl. 14, p. 53-59. - 1895. Die Gattung Dorylus Fabr. und die sysiemalische Eintheilung (ler Formiciden. Zool. Jahrb. (Abt. Syst.) 8, p. 685-778, \& pls., 41 figs. - 1896. Le Polymorphisme des Fourmis et la Castration alimentaire. C. R. 3e Congr. Intern. Zool. Leyden p. 395410. - 1898. Beiträge zur Kenntniss der paläarklischen Ameisen. Ofvers. Finsli. Vet. Soc. Förhandl, 20, 28 pp. - 1904. Zur Kenntniss des Polymorphismus der Ameisen. Zool. Jahrb (Suppl. VII.) p. 587-610, 6 figs. - 1905. Deux fourmis de l'Ambre de la Baltique. Bull. Soc. Ent. France p. 187-189. 2 figs. - 1906. Zur Kenntniss des Polymorphismus der Ameisen. Festschr. 1. J. Rosenthal, Leipzig, G. Thieme p. 35-40. 1908 a. Remarques sur les observations de M. de Lanior touchant l'existence de I.asius mixtus dans les fourmilières de L. \{uliginosus. Ann. Soc. Ent. Belg. 52, p. 182-183. - 1908 b. Osservazioni ed esperimenti sulla Formica Amazzone. Rendic. R. Accad. Sc. Ist. Bologna p. 49-62. - 1909 a. Ueber den Ursprung der dulotischen, parasitischen und myrmekophilen Ameisen. Biol. Centralbl. 29, p. 352-362 ; Rendic. R. Accad. Sc. Ist, Bologna p. 36-50. - 1909 b. Nuove osservazioni ed esperimenti sulla Formica Amazzone. Ibid. p. 31-36. - 1910. Il polimorfismo e la fondazione delle societa negli inselti sociali. Scientin 7, p. 336i-319. -... 1911a. Llleriori osservazioni ed esperienze sulla Formica Amazzone. Rendic. R. Accad. Sc. Ist. Bologna p. (00-7.). - 1911b. Benbachlungen und Versuche an Polyergus rufescens. Biol. Centralbl. 31. p. 625-642. 1915 a. La Vita delle Formiche, Torino, Fratelli Bocca. 1915b. Können weisselose Ameisenvölker die fehlendle Muller aus eigenen Mitteln erselzen? Biol. Centralbl 35. p. 252-25)4. 1916. La londazione delle società di Myrmica. Considerazioni sull alimentazione delle formiche. Rendic. R. Accad. Sc. Ist. Bolognna p. 45-50. - 1918. Esperienze intorno a l'. produzione di individui sessuadi nella societa della formiche. Ibid. p. 6.57 . 1920. I a distribuzione geografica alluale delle formiche. Rerl. Acad. Lincei (o) 13, p. 3-98. - 1921. Quels sonl les farleurs du polymorphisme du sexe feminin chez les fourmi-? Rev. Gén. Sciences 32, p. 737-7亿1. - 1924. Casi di anomale e di parassitismo nelle formiche. Rendic R. Acead. Sc. IsI. Bolognu 1). 82-89, 4 ligs. 
Exgelinntit (vox). - 1914. Ueher die Hancoclische Drüse von Oricanthus pellucens Scop. Zool. Anzeig. 44, p. 219-2227.

Enicuson (W. F.). -- 1847. De fabrica et usu antennarum in insectis. Berolini.

Escherich (K.). - 1898. Zur Analomie und Biologie von Paus. sus turcicus Fauv. Zool. Jahrb. (Abl. Syst.) 12, p 27-70. 1 pl., 11 figs. - 1902. Biologische Studien über algerische Myrnickophilen, zugleich mi allgemeinen Bemerknugen über die Entwiclilung und Bedentung der Symphilie. Biol. Centralb/. 22, p. 638-663, 4 figs. - 1909. Die Termiten oder weissen Amersen. Leipzig. - 1911. Termilenleben auf Ceylon. Jena. 1917. Die Ameise, Schilderung ihrer Lebensweise. 2te Aufl Braumschweig, Viehweg.

Lssinis (A.). - 1877. Des sociétés animales, élude de psychologie comparée. Paris, G. Baillière et Cie.

Evensmax (E.). - 1946. Brutzellen des Hylacus quadricinclus und Pelopoeus destillatorius. Bull. Naturf. Ges. Mosliau 19, p. 188-193.

Fabre (TT. H.). - 1879-80. Etudes sur les moeurs el la parthonogénèse des Halictes. Ann. Sc. Nat. Zool. (6) 9, j. 1-27, 1 1). 1790. Souvenirs Entomologiques 3 e sér. 2e édil. - 1903. Ibid. 8 e ser. chap. $7-9$.

Finringer (J.). - 1914. Ueber den Nestbau zweier Bienen. Zeitschr, wiss. Inselstenbiol. 10 p. 16-20, 5 figs.

FARQuharson (C. O.). - 1918. Harpagomyia and olher Diptera led by Crematogasier Ants in Southern Nigeria. Proc. Ent. Soc. London, p. XXIX-XL. - 1919. The Tachinid Fly Bengalia attacking Termites. Ibid. p. LII-LVIII. - 1922 a. Five Years Observations (1914-1918) on the Bionomics of Soulhern Nigerian Insects (edited by E. B. Poulton). Trans. Ent. Sor. London p. 319-148. - 1922 b. The Pursuit of Living Ants by the Ephydrid Rhynchopsilota apicalis Collins sp. n. Ilid. p. 413-444.

Ferrière (A.). - 1915. La loi du progrès en Biolngie el ell Sociologie et la question de l'Organisme Social. Paris, Giard et Brière.

Ferton (C.). - 1890. L'évolution de l'instinct chez les Hymimoptères. Rev. Srient. p. 45, 496-498. - 1898. Sur les morur: des Spliecodes I atr. et des Haliclus Iatr. Bull. Soc. Er.'. France 1). 75-77. 1901-21. Noles détachées -urr linstinct de: Hỵménoplères Mellifères et Raviseurs. 9 séries. Ann. iro?. lint. France 70-89. 
Feytad (.J.). - 1912. Contribution a l'étude di retmite lic:i.:ze Areh. d'Anal. Micros. 13, p. 181-607, 3 pls. 31 iggs.

Filloe (A. M.). - 1901. A Study of an Ant. Proc. Acal. Nal. Siei. Phila. 53, p. 425-449. - 1902. Noles on an Alnt. Ibid. 54, 1. 599-625, 2 figs. -- 1904. Power of Recognition among Ants. Biol. Bull. 7, p. 227-250, 4 figs. - 1905 a. The Communal Lile of Ants. Nature Study Review, 1, p. 239-250, 4 figs. - 1905 b. Observations on the Progeny of Virgin Ants. Ibid. 9, p. 35j360. - 1906. The Progressive Odor of Ants. Ibid. 10, 1. 1-16. F'rske (J.). - 1874. Cosmic Philosophy I.

Fletcher (J.). - 1908. Vespu boreulis an Inquiline? Ann. Iint. Soc. America 1, p. 30 ; Psyche 15, p. 16.

Fonbes (H. O.). - 1885. A Naturalist's Wanderings in the Easter'n Archipelago. London.

Forbes (S. A.). - 1908. On the Life History, Habits and Economic Relations of the White-Grubs and May-beelles (Lachnosterna). 24th Rep. State Ent. Nox. Benef. Insects, Illinnis. p. $135-168,3$ pls.

Fond (N). - 1922. An Undescribed Planidium of Perilampus from Conocephalus. Canad. Ent. p. 199-204, 1 fig.

Funf. (A.). - 1890. In parasite de la Myrnecia forjicata F. Ann. Soc. Ent. Belg. 20, p. 8. - 1894. Ueber den Polymorphismus und Ergalomorphismus bei den Ameisen. Vers. deutsch. Naturl. Wien p. 142-147. - 1898. La Parabiose chez les Fourmis. Bull. Soc. Vaud. Sc. Nat. (4) 34, p. 380-381. 1900. Fourmis de Japon. Nids en Toile. Strongylognalhus hubveri et voisins. elc. Mitteil. schweiz. Ent. Ges. 10, p. 267287. - 1902. Beispiele phylogenetischer Wirkungen und Rück: wirkungen bei den Instinkten und dem Körperbau der Ameisen als Belege für die Evolutionslehre und die psychophysiologische Identitätslehre. Journ. Psych. Neurol. 1, p. 99-110. - 1904. Ueber Polymorphismus und Variation bei den Ameisen. Zool. Jahrb. (Suppl. VII), p. 571-586. - 1906. Mœurs des fourmis parasites des genres Wheeleria el Bothriomyrmex. Pev. Suisse Zool. 14, p. 68. - 1908. Lettre à la Société Entomologique de Belgique. Ann. Soc. Ent. Belg. 52, p. 180-181. 1910a. Das Sinnesleben der Insekten, München. - 1910 b. Aperçu sur la distribution géographique et la phylogénèse des fourmis. 1er Congr. Internat. d'Ent. p. 81-100. - 1920. Les Fourmis de la Suisse $2^{\circ}$ édit. (1re éd. 1874). - 1921-23. L.e Tonde Social des Fourmis. 6 vols. Gehève, Libr. Kundig. Fouillée. (1.). - 1920. L'évolutionnisme des idées-lorces. $6^{\circ}$ éd. Paris, F. Alcan (1re édit. 1890). 
Fox (IV. J.). - 1893. Observations on the IIymenopterous genus Neolarra Ashmead. Ent. News 4, p. '92:293, 1 lig.

Liteud (S.). 1921. Group Psychulogy and the Analysis of the Ego. Transl. by James Strachey. New-York, Boni el Liveright.

Finese (H.). - 1888. Dic schmarolzerbienen und ihre Wirle. Zool. Jahrb. (Abt. Sysl.) 3, p. 847-870. - 1891. Beilräge zur Biologie der solitären Blumenwespen (Apidie). Ibid. 5, 1). 751 . Stio, I pl. - 1903. Neue Meliponiden II, Zeitschr. Hymen. Dipt. 3, p. 359-361. - 1905. Ein Bienennest mit Vorratsliam- mern (Lithurgus dentipes Sm.), Zeitschr. wiss. Insclilenbiol. 1, p. 118-119. - 1923. Die europäischen Bienen (Apidie). Das Leben und Wirken unserer Blumenwespen. Berlin u. Leipzig, de Gruyter et Co.

Frisch (K. von). - 1921. Ueber den Sitz des Geruchsimnes bei Insekten. Zool. Jahrb. (Abt. Zool. Physiol). 38, 1). 449-516. 1923. Ueber die "Sprache » der Bicnen. Jena, G. Fischer.

Frison (Th.). - 1916. Note on the Habits of Psilhyrus variabilis. Cress. Bull. Brooklyn Ent. Soc. 11, p. 16-47. - 1921. P'sithyrus laboriosus Fabr. in the Nests of Bumblebees. Canad. Ent. 53, p. 100-101.

Froggate (W. W.). - 1895-97. Australian Termilida Pts. I-Ill. Proc. Linn. Soc. N. S. Wales. p. 420, 455, 721. - 1905. White Ants. Misc. Publ. No. 874 Dep. Agric. N. S. Wales p. 1-47. 2 pls. 12 figs.

Fнонишк (F. W.). - 1915. Further Observations on the Last Stage of the Larva of Lycæna arion. Trans. Enl. Soc. London, p. $313-316_{2} 2$ pls.

Fullaway (D. T.) - 1920. New Species of Sierola, with Explanatory Notes. Occus. Paper's Bernice Pauahi Bishop Mus. 7, p. $57-159,1 \mathrm{pl}$.

Fuller (C.). - 1915. Observations on Some south African Termites. Ann. Natal Mus. 3, p. 329-504, 11 pls. 16 figs.

Fulron (B. B.). - 1915. The Tree Crickets of New York : Life History and Bionomics. Techn. Bull. No. 12, New York Exper. Sta. 17 pp, 6 pls, 21 figs. - 1924a. Some Habits of Earwigs. Ann. Ent. Soc. Amer. 17, 1. 357-367, 1 fig. - 1924b. The European Earwig. Bull. 207 Oregon Agric. Coll. Exper. Sta. p. 5-29, 6 figs.

Gabritschevsiy (E.). - 1924. Farbenpolymorphismus und Vererhung mimetischer Varietäten der Fliege Volucella bombylans und anderer " hummelähnlicher " Zweiflügler. Zeilschr. indulit. Abstam. Vererbl. 32, p. 321-353. 
Ginnd (1.) - 1889a. Sur une galle produjte chez le Typhlocyba rosic L. par une larve d'Hyménoptère. C. R. Acad. Sc. Parts, 10!), 1). 79. - 1889/). Sur la cistrition parasilitire de Typhlocylue par une larve d'Hyménoptère (Aphelopus melaleucus Dalm.) et par une larve de Diptère (Alcloneura spuria Meig.) Ibid. 1). 708-710. - 1905. La P'uccilogonie. Bull. Sc. France Belg. 39, p. 153-187.

Giradd (J.). - 1871. Notes sur les mœurs du Céramius lusilunicus Klug. Ann. Soc. Ent. France (5) 1, p. 37ॅ-379.

limun (G.). - 1921. Ricerche sul genere Philotrypesis lürs:., Boll. Lab. Zool. Gen. Agrar. Portici 15, p 33-190. 41 lig-.

1923. Gli Inselti dei Caprilichi. Rivist. Biol. 5, p. j-26, 15 figs. Grassi (B.) et Sandias (A.). - 1893-94. Conslituzione e sviluppo delli società dei Termitidi. Alti. Acead. Ciiocnia (1) 6-7, $151 \mathrm{pp}$. 5 pls.

Gurasi (A.). - 1905. Sludi sui Lucanidi. ]e Considerazione generali sulla grande variazione di caratteri nei maschi dei Lucanidi. Torino, P. Gerbone, 40 pp., 1 figs.

Green (E. E.). - 1906-07. Spolia Zelanica 3, 1. 220. 1 p. 183-181.

- 1908. Tongue of an Ochromyia. Proc. Ent. Soc. London. p. XXVI-XXVII.

Gutbier (A.). - 1914. Ueber einige Hymenoplerennester aus Turkestan. Zeitschr. wiss. Insektenbiol. 10. p. 339-315, 6 figs.

Harten. - 1890. Biologische Beobachtungen an Hummeln. $2 \bar{\tau}$. Ber. Oberhess. Gesell. Natur. Heilk. p. 59-75.

HALidiy (A. H.). - 1835. Noles on the Bethyli and on Dryinus pedestris. Ent. Mag. 2, p.219-220.

HAMner (J. J.). - 1898. The Nervous System of Nereis virens Sars. Bull. Mus. Comp. Zool. 32, p. 89-124, 5 pls.

HiNcock (J. L.) - 1905. The Habits of the Striped MeadowCricliet (OEcanthus fasciatus) Amer. Natural. 39, p. 457.

Handlirsch (Adam). - 1882-83. Die Melamorphose und Lebens. weise von Hirmoneura obscura Meig., einem Vertreter der Dipteren-Familie Nemestrinidie. Wièn. Ent. Zeilg. 1, p. 221228 ; II. Ibid. 2 , p. 11-15, 1 pl.

Huxnlirsch (Anton.). - 1887-95. MLonographie der mit Nysson und Bembex verwandten Grabwespen.SB.K.Akad. Wiss. Wien 8 partes. - 1903. Zur Phylogenie der Hexapoden. Ibid. 112, p. $1-23,1$ pl. - 1904. Zur Systematik der Hexapoden. Zool. Anzeig. 27, p. 733-769. - 1908. Die fossilen Insekten und die Phylogenie der rezenten Formen. 2 vols. Leipzig. W. Engr.mann. - 1910. Fossile Wespennester Ber. Senlienber!. Naturl. Gesell. Franli, a. M. p. 265-266, 1 fig. - 1913a. Bei- 
träge zur exacten Biologie SB. K. Aliad. Wiss. Wien, 1:2, p. 3h1-481, 6 fig. - 1913b. Ueber einige Bezichungen zwischen l'aliontologie, geographische verbreitung und Phylogenie der Insekilen. Intern. Congr. Enl. Oxford 2 (1912) p. 248-270, 3 pls. - 1924. Systematische Uebersicht. Kap. 9 in $C$. Schröder's Handbuch der Entomologie. Jena, G. Fischer, 1. $712-825,112$ figs.

IIinnis (R. G.) -- 1923. Occurrence, LifeCycle and Maintenance under Artificial Conditions of Miaslor. Psyche 30, p. 95101. 1924. Sex of Adult Cecidomyidx (Oligalces sp.) arising Irom Larvie produced by Padogenesis. Ibid. 31, p. 148-154.

Hinnss (T.). - 1862. Treatise on Some of the Ihisects Injurious to Vegetalion. Boston, Crosby and Nichols; New York, O. S. Fel!.

Hiuser (G.). - 1880. Physiologische und histologische Untersuchungen über das Geruchsorgan der Inseliten. Zcilschr. uiss. Zool. 34, p. 1-3ī, 3 pls.

Heitн (H.). - 1902. The Habits of California Termites. Biol. Bull. 4, p. 47-63, 3 figs. - 1907. The Longevily of Nembers ti the Different Castes of Tcrmopsis angusticollis. Ibid. 13, p. 161-164.

ILeEr (O.). - 1849. Die Insektenfauna der Tertiärgebilde von OEningen und von Radoboj in Croatien II. Neue Denlischr. allgem. Schweiz Geol. Ges. Nalurw. 11, p. 1-264, 17 pls. 1852. Ueber die Hausameise Madeiras. Züricher Jugend, v.d. naturf. Gesellsch. 54.

IIEgh (E.). - 1922. Les Termiles. Partie Générale. Brussels. 1.60 pp. 461 figs.

Heikertinger (F.). - 1919. Die metöke Myrmekoidlie. Tatsachenmaterial zur Lösung des Mimikryproblems. Biol. Zentralbl. 39 , p. 65-102, 13 figs.

HeNNing (H.). - 1916. Der Geruch. Leipzig.

Henbst (P.). - 1922. Revision der Halictus-Artán von Chile. Ent. Mitteil. 11.

Heums (W. B.). - 1907. An Ecological and Experimental Slurly of Sarcophagidx with relation to Lake Beach Débris. Journ. Exper. Zool. 4, p. 45-83, 7 figs.

Ilerrick (C. J.). - 1903. The Organ and Sense of Taste in Fishes. Bull. U. S. Fish Comm. (1902) p. 237-372.

Heselhius (F.). - 1922. Die Hauldrüsen der Apiden und verWiandter Formen. Zool. Jahrb. (Abt. Anat.) 43, p. 369-464, $11 \mathrm{pls}$.

Hrymoss (R.). - 1907. Die verschiedenen Formen der Inselilell. metamorphose und ihre Bedeuung im Vergleich zur Meta- 
morphuse anderer Arthropoden. Ergebn. u. Forlschr. Zool. 1. p. 137-188. - 1908. Süsswasser-Hymenopleren aus der Lmgebung Berlins. Deutsch. Ent. Zeilschr p. 137-150, 4 'figs. Hingston. 1922. A Naturalist in Himalaya. Boston. Small, Maynard et Co.

Hochreuter (R.). - 1912. Die Hautsinnesorgane von Dyliscus marginalis L., ihr Bau ind ihre Verbreitung am Körper. Zeitschr. wiss. Zool. 103, p. 1-114, 102 figs.

Hoffer (E.). - 1881. Biologische Beobachtungen an Hummeln und Schmarotzerhummeln. Nitlh. Nalurw. Ver. Sleiermarli 18, p. 68-92. - 1788. Die Schmarotzerhummeln Steiermarlis, Lebensgeschichte und Beschreibung derselben. Ibid. 25, p. 8:159,1 pl.

Hollidiy (M..) - 1903. A Study of Some Ergatogynic Ants. Zool Jahrb. (Abt. Syst.) 19 1. 293-327, 15 figs.

Holmgren (N.). - 1909-13. Termitenstudien, 4 parts. K. Siensli Velensk. Handl. 44,215 pp. 3 pls. 76 figs.; 46,86 pp. 6 pls. 6 figs. ; 48,166 pp. 4 pls. 88 figs. ; 50,276 pp. 8 pls. 14 figs.

Hoon (J. D.). - 1913. Notes on the Life-History of Rhopalosoma poeyi. Proc. Ent. Soc. Washington 15, p. 145-148.

Howard (L. O.). - 1891. The Methods of Pupation among the Chalcididæ. Insect Life 4, p. 193-196, 6 figs. - 1905. The Insecl Book. Doubleday. Page et Co. - 1908. The Importalion of Tetrastichus xanthomelana Rond. Journ. Econ. Ent. 1, p. 281289. - 1910. On the Habit With Certain Chalcidoidea of Feeding at Puncture Holes made by the Ovipositor. Ibid. 3, p. 257-260.

Howes (P G.). - 1917. Tropical Life in British Guiana (Beebe) Ent. Pait III p. $371-450,2$ pls. 15 figs.

Huber (P.). - 1810. Recherches sur les mœurs des Fourmis indigènes. Paris et Genève.

Huntington (E.). - 1914. The Climatic Factor as illustraled in Arid North America. Washington, D. C. Carnegle Inst. 311 pp. 90 figs.

Hrslop (J. A.). - 1916. Prislocera armilera (Say) parasitic on Limonius agonus (Say). Proc. Ent. Soc. Washington 18. p. $169-170,1 \mathrm{pl}$.

Inering (H. von). - 1896. Zur Biologie der socialen Wesjen Brasiliens Zool. Anzeig. p. 419-453. - 1903. Biologie der stachellosen Ilonigbienen Prasiliens. 7nol. Jahrb. (Abt. Sysl.) 19 , p. $179-287,13$ pls., 8 figs.

IhERing ( $R$. von). - 1903a. Biologicche Beobeachtungen an brasiliensischen Bombus-Nestern. Allgem. Zeitschr. Ent. 8. p. 
117-153, J figs. - 1903b. Contributiuns à l'élude des Vespides. Ann. Soc. Ent. France p. 144-155. - 1903 c. Zur Frage nach dem Ursprung der Staatenbildung bei den socialen Hymenupteren. Zool. Anzeig. p 113-118. - 1904. Biologia das abelhas solitarias do Brazil. Revist. Mus. P'aulista 6, p. 461-481, j figs.

Imus (A. D.). - 1919. On the Structure and Biology of Archo. termopsis, together with Descriptions of New Species of Intestinal Protozoa and General Observations on the "Isoptera ». Phil. Trans. Roy. Soc. London (B) 209, p. 75-180, 7 pls.

Jick (R. W.). - 1917. Parthenogenesis amongst the Worliers of the Cape Honey Bee. Trans. Ent. Soc. London. p. 396-409, 2 pls.

JicoBson (E.). - 1909. Ein Moskito als Gast und diebischer Schmarotzer der Cremastogaster difformis Smith und eine andere schmarotzende Fliege. Tijdschr. v. Ent. 52, p. 158-161.

1910. Pheidologeton diversus Jerdon und eine myrmeliophile Fliegenart. Ibid. 53, p. 328-335. - 1911 a. Biological Note on the Hemipteron Ptilocerus ochraceus. Ibid. 51, 1. 175-179. - 1911 b. Nähere Mitteilungen üher die myrmekophile Culicide Harpagomyia splendens de Meij. Ibid. 54, p. 158-161, 3 pls.

Jimaunathin (N. S.). - 1908. The Habits and Life History of a Social Spider (Stegodyphus sarasinorum Karsch). Madura, Vicloria Press. 14 pp.

Jimes (S. P.). - 1914. Summary of a Year's Mosquito Work in Ceylon. Ind. Journ. Med. Research 2, p. 233-234.

IANET (C.). - 1896. Sur les rapporls des Lépismides myrmécnphiles avec les Fourmis. C. R. Acad. Sc. France 122, p. 79?. 1 fig. - 1898 a. Etudes sur les Fourmis, les Guêpes et les Aheilles, $19^{\circ}$ note. Analomie du corselel de la Myrmica rubra reine. Mém. Soc. 7ool. France 11, p. 393-450, 1 pl., 25 figs. 1898 b. Sur une cavité du tégument servant, chez les Myrm. rinés à élaler, aı contact de l'air, un produit de sécrélion. C. R. Acad. Sc. Paris 126, p. 1168-1171. - 1903. Observations sur les Guêpes. Paris, Carré et Naurl. - 1904. Observations: sur les Fourmis. Limoges. - 1907. Anatomic du corselet el histolyse des muscles vibrateurs, après le rol nuptial, chez. la recine de la Fourmi (lusius nieger). Limoges, Ducourtieux et foutt. pl). $149+20,13$ pls. 11 figs. - 1909. Sur la partheinoginese arrhénotoque de la Fourmi nuvrière. Mém. Sor. Oise. $8 \mathrm{pp}$.

Ja:innel (R.). - 19t3. Sur des larves de Proctullupida parasites 
dHomoplères. In Voyage de Ch. Alluaud et R. Jeannel en Afrique Orientale. Hymenopt. 1 p. 36-39, 1 1).

Soñes (D. W.). - 1917. The European Earwig and its Control. Bull. 566, U. S. Dept. Agric. 12, pp. 8 figs.

Joxescu (C. «.). - 1909. Vergleichende Untersuchungen über das Gehirn der Honighiene. Jen. Zeilsch:. Naluru. 15, 1). 111-180, ; pls.. 13 figs.

Jucci (C.). - 1924. Su li differenziazione de le casle ne la societa dei Termitidi I. I neotenici. Mem. R. Acal. Naz. dei Lincei (5) 14, p. 5-238, 4 pls. 23 figs.

Kimin (D.). el Thomsson (W.R.). - 1915. I. cycle evolutif des Dryinidat. C. R. Soc. Biol. Paris 78, p. 83-87, 10 figs.

Kellogr. (V.). - 1913. Distribution and Sjecie-Forming of EcloParasites. Amer. Nalural. 47, p. 129-158. - 1914. Ecloparasites of Mammals. Ibid. 48, p. 257-279.

Kifll (E. O. G.). - 1914. A New Sarcophagid Parasites of Grasshopper's. Journ. Agrie. Research. Dep. Agric. 2, p. 435$1: 45,1 \mathrm{pl}$.

Kemer (N. A.). - 1922. Die Larve der Termitoxenien entdeckl! Ent. Tijdschr. p. 58-61. - 1923. Hyphaenosymphilie, eine neue, merkwürdige Art von Myrmeliophilie bei cinem neuen myrmecophilen Schmetterling (Wurthia aurivillii n. sp.) aus Java, beobachtet. Ark. f. Zool. 15, p. 1-28, 4 pls.

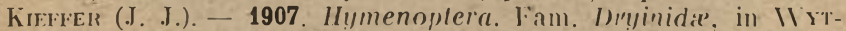
sux's Genera Insectorum, 33 pp., 2 pls. - 1908. Hymenoplera. Fam. Bethylidæ, in Wrtsman's Genera Insectorum 50 pp. 3 pls. -- 1909. Descriptions de nouveaux Microhyménoptères dı Brésil. Ann. Soc. Ent. France 78, p. 287-348.

Kixg (J. L.). - 1916. Observations on the Life History of Pleroclontia flavipes Gray. Ann. Ent. Soc. Amer. 9, p. 309-321. 2 pls. linsir (A .C.). - 1919. Fossil Cynipids, Psyche 26, p. 11.19. 2 figs.

lirkituy (G. W.). - 1911. Some Remarks on the Reduviid Subfamily Holoptilina, and on the Species Ptilocerus ochra. ceus Montandon. Tijdschr. v. Ent. 54, p. 170-174, 1 pl.

h́lebs (R.). - 1910. Ueber Bernsteineinschlüsse im allgemeinen und dic Coleopteren meiner Bernsteinsammlung. Schrịll. |lhyssili. ölonom. Ges. Köni!!sher!y 51, 1. ?17-2/2.

litux. - 1904. Fullerbrei und wriblielie Bienenlarve. Die Bienenpllege 26.

Kiower (H. McE.). - 1894. Origin of the "Nasulus ") (soldier) of Eutermes. Johns Hoplins Univ. Circ. 13. p. 58-59.

Kúber (L.). - 1921. Der Bau der Erde. Berlin, Gebr. Bornträger. 
Konl (F. F.). - 1896. Die Gattungen der Sphegiden. Ann. K. K. naturh. Hofmus. Wien 11, p 233-516, 7 pls., 90 ligs.

Kolbe (H. J.). - 1913. --Die Differenzierung der zoogeographischen Elemente der Kontinente. Trans. 2nd Intern. Congr. Ent. (1912) 2, p. 433-4i6.

Kominsky (P.). - 1924a. Ueber Erzeugung von Intersexen bei Stilpnotia salicis L. im Temperalur-Experiment. Biol. Zentralb. 44, p. 15-16. - 1924b. I)er (jynandromorphismus bet Lymantria dispar. L. unter der Einwirkung äusserer Einflüsse. lbid. 14, p. 66-68, 3 figs.

Liominusen (S. J.). - 1919. The Sexual Characteristics of the Membracid Thelia bimaculata (Fabr.) I. Exlernal Changes Induced by Aphelopus theliae (Gahan). Journ. Morph. 3?, p. 531-635, 54 figs.

Kroner (R.). - 1913. Zweck und Geselz in der Biologie. Tühin. gen, J. C. B. Mohr. - 1919. Das Problem der historischen Biologie. Heft 2. in Scuixel's Abhandl, theorel. Biol. Berlin, Gebr. Bornträger.

KüнNLE. - 1913. Untersuchungen über das Gehirn, die Koplnerven und die Kopfärüsen des gemeinen Ohrwurms. Jen. Zeitschr. 1. Nalurw 50 , p. 147-276, 5 pls.

Kï̈NÇKeL-D'Herculıis (J.). - 1869. Observations relalives aux Volucella zonaria, inanis, pellucens et bombylans. Ann. Soc. Ent. France (1) 9, Bull. p. 17, 20, 23. - 1870. Observations sur les mélamorphoses des Conops. Ibid. (1) 10, p. 63. - 1875-81. Recherches sur l'organisation et le développement des Vo:ucelles, insectes I)iptères de la fimille des Syrphidés. 1re prarlie 1875 p. ]-208, 12 pls; 2e partic 1881, 13 pls. - 1395. Notices sur les titres el travaux de M J. Künchel n'Herculais. Paris. Ed. Crété.

Kuo (Z. Y.). - 1924. I Psychology without Heredity. Psychol. Review 31, p. 127-418.

Kutrer (H.). - 1913a. Zur Biologie von Formica ruia und Formica fusca i. sp. Biol. Centralbl. 33, p. 703-707. - 1913b. Ein weilerer Beitrag 7.11 Frage der sozialparasitischen Kolonic gründung von $F$. rufa L. Zeitschr. wiss. Insektenbiol. 9, 1). 193-196. - 1917. Myrmekologische Beohachlungen. Biol. Zen. tralbl. 37, p. 429-437. - 1920 Strongylognathus huberi v. alpr. mus Wh. eine sklavenraubende Ameise. Ibid. 10, p. 528. 1923. Der Sklavenräuber Stron!n!nloynathus huberi Forel s. sp. alpinus Wheeler. Rer. Suisse Zool. 30, p. 397-1?'1.

I.ABоnx (W. A.) - 1913. The Fly Bengalia depressa Walk (Calliphorinæ) robbing Dorylus nigricans on the march. Proc. 
Ent. Soc. London, p. CXXV-CXXXII. - 1914. On the Rela tionship between certain West African Insects, expecially Ants, Lycinidie and Homoptera. Trans. Ent. Soc. London p. $436-524,4 \mathrm{pls}$. - 1919. Further Notes on the Habits of the Fly Bengulia. Proc. Ent. Soc. London p. LII-LVIII.

Lamerne (A.). - 1909. L'origine des sociètés d'Insecles. Ann. Soc. Enl. Belg 53, p. 507-515.

L.inghofer (A.). - 1897-98. Beitrãge zur Kenntnis der Mundleile der Hymenoptera. I $\Lambda$ pidæ. Arbeit. Akad. Wiss. Kunst. Agram 80, p. 25-93, 3 pls ; 81, p. 609-613.

Linvoy (F. DE). - 1908. Notes sur le Lasius niger et le Lasius fuliginosus. Ann. Soc. Ent. Belg. 52, p. 47-53.

LE Bon (G.). - 1925. Psychologie des Foules. 31॰ Edit. Paris, F. Alcan.

Le Dantec (F.). - 1918. L'Egoïsme, seule base de toule sociélé. Paris, E. Flammarion.

Leconte (J.). - 1884. Elements of Geology. New York, D. Applelon and $\mathrm{Co}$.

LEGEWIE (H.). - 1922. Beiträge zur Biologie der Bienengattung Halictus. Mitteil. Bad. Landesv. Naturk. Naturschulz Freiburg i. Br. n. f. 1, p. 235-237.

LEIBY (R. W.) - 1922. The Polyembryonic Development of Copidosoma gelechire, with Notes on its Biology. Journ. Morph. 37 , p. 195-284, 18 pls.

Lepeletier de Saint-Fargeau (A. L. M.). - 1827. Encyclopedie Méthodique 10, p. 183.

LEspìs (C.). - 1856. Recherches sur lorganisation el les mœurs du Termite lucifuge. Ann. Sc. Nat. Zool. (4) 5, p. 227-282, 3 pls.

Leuckart (R.). - 1887. Neue Beiträge zur Kenntniss des Baues und der Lebensgeschichte der Nematoden. Abh. math. phys. Cl. Kgl. Schs. Gessels. Wiss. 13 p. 567.704, 3 pls.

I.ichtenstein (J. L.). - 1921. Sur la biologie d'un Chalcidien. C. R. Acad. Sc. Paris, 173 p. 733-735, 1 fig.

Lichtenstein (J. L.) et Picard (F.). - 1917. Etude morphologique et biologique du Sycogaster Lavagnei Pic. et Licht. Bull. Biol. France Belg. 51, p. 410-474, 33 figs.

Lichtenstein (J. L.) el Rabaud (E.). - 1922. Le comporlement des "Polysphincta ), Ichneumonides parasites des araignées. Bull. Biol. France Belg. 55, p. 267-287, 11 figs.

Lineburg (B.). - 1924. The Feeding of Honey-Bee Larva. Bull. No. 1222 U. S. Dep. Agric. p. 25-37, 2 figs.

I.omickı (J.). - 1921. Sur la micrandrie chez la fourmi sanguine. Kosmos, Bull. Soc. Polon. Nalural. Leopol. p. 98-99:- 
1922. Leber den Anfang der Kolonien der glänzend-schwarzen Holzameise. Lint, Anzeig. 2, p. 79-80.

Longman (H. A.). - 1923. The Loogeography of Marsupials, with Notes on the Origin of the Australian Fauna. Mem. Queenslund Mus. 8, 1. 1-15.

Luvisuy (A. O.). - 1922. The Lenglh of Human lufancy in Ligh_ teenth Century Thought. Journ. Philos. 19, p. 381-385.

Lubвock (Sir J.). - 1861. On Spharularia bombi, Natural llisl. Review I, p. 44-57, 1 p.I - 1863. On two Aquatic Hymenol) lera, one of which uses its wings in swimming. Trans. Linn. Soc. London 24, p. 135-141, 6 ligs. - 1888. Ants, Bees and IV asps. London.

Lucis (H.). - 1878. (Une nole sur la nidification d'un Hyménop)tère du genre Luglossa) Bull. Soc. Lint. France 8, p. CXLIICXLIII.

Lubwig (F.) - 1904. Nesl und Vorratskammern der Lonalap von Ponape. Allgem. Zeilschr. Enl. 9, p. 225-227, 1 fig.

Lüdenw:ILd (H.). - 1911. Nestbau von Neocorynura erinnys Schrollky. Zeitschr. wiss. Inselitenbiol. 7, 1). 94-96, 1 lig.

Mac GillivraY (A. D.). - 1914. The Immature Slages of the Tenthredinoidea. 44 th Ann. Rep. Ent. Soc. Ontario (1913) p. 54-75, $1 \mathrm{pl}$,

Maxw (W. M.). - 1923. New Genera and Species of Termilophilous Coleoptera from Northern soutn America. Zoologica 3, p. 323-366, 13 figs.

Minchal (Р.). - 1890. Formation d'une espèce par le parasitisme. Etude sur le Sphecodes gibbus. Rev. Scient. 45, p. 199. 204. - 1894. Le parasilisme des Sphecodes. Bull. Soc. Ent. France 63, p. CXV. - 1896. La reproduction et l'évolution des Guêpes Sociales. Arch. Zool. Expér. Gén. (3) 4, p. 1-100, 8 figs. - 1897. La castration nutriciale chez les Iyménoptères Sociaux. C. R. Soc. Biol. 2 pp. - 1900. Sur un nouvel Ilyménoptère aquatique, le Limnodytes gerriphagus. Ann. Soc. Ent. France 69, p. 171-176. - 1905. Observations biologiques sur un parasile de la Galèruque de lOrme, le Tetras/lchus xanthomelxnae (Rond.) Bull. Soc. Lint. France p. 64-GS. - 1906. Recherches sur la biologie et le développement des Hyménoptères parasites II. Les Platygasters. Arch. Zoor. expérim. (1) 4, p. 485-640, 8 pls. - 1909. La ponte des Aphelinus et l'intérêt individuel dans les actes liés à la conservalion de l'espèce. $C$. R. Acad. Sc. Paris 148, p. 1223-1225.

Munsuar (G. A. K.). - 1898. Notes on the South African Social Spiders (Stegodyphus). Zoologisl (1) 2, p. 417_422. 
Mirtin (E. D.) - 1920. The Behavior of Crowds. N. Y. and London, Harper Bros.

Missimt (J.) el VANubrvelue (E.). - 1893. Parasilisme Organique et P'allusitisme Social. Bull. Se. France Bely. '25, 1'. 1.tis. Mitueson (R.) et Crosisi (C. R.). - 1912. Aquatic Hymenoplera in America. Ann. Ent. Soc. Amer. 5, p 6̆̃-71. 3 figs

Mathews (A. P.). - 1921. Physiological Chemistry. N. Y., $1 \mathrm{~mm}$. Wood el Co.

Mitthew (W. D.). - 1915. Climale and Evolution. Ann. ․ I. Acad. Sc. 24, p. 171-318.

Mitracsch (I.). - 1909. Gynandromorphic Membracidx. Joun. N. Y. Ent. Soc. 17, p. 165, 1 pl. - 1911. The Effects of Paritsitic Castration in Membracidx. Ibid. 19, p. 194-196, 1 pl.

MIYR (G.). - 1867. Vorläufige Studien über die Radoboj-Formiciden. Jahrb. K. K. Geol. Reichsmus. Wien 17, p. 47-62, 1 pl. -- 1868. Die Ameisen des baltischen Bernsteins. Schrift. physili. ölionom. physik. Gesell. Königsbery 1, 102 pu. 5 pls.

IC Iougith (IV.). - 1920. The Group Nind. New York el L.ondon G. P. Putnam's Sons.

IIC Indoo (N. E.). - 1914a. The Olfaclory Sense of Insects. Smithson. Misc. Coll. 63, p. 1-63, 6 figs. - 1914b. The Olfactory Sense of Hymenoptera. Proc. Acad. Nat. Sc. Philn. p. 294-341, 2 pls. 3 figs. - 1914e. The Olfactory Sense of the Honey Bee. Journ. Exper. Zool. 16, p .265-346, 21 figs. 1915. The Otfactory Sense of Coleoptera. Biol. Bull. 28, p. 407_ 160,2 pls. 3 ligs. - 1916. The Sense Organs of the Mouthparts of the Honey Bee. Smithson. Misc. Coll. 65, p. 1-55, 10 figs. - 1917. The Olfactory Organs of Lepidoptera. Journ. Morph. 29, p. 33-54. - 1918. The Olfactory Organs of Diplera Journ. Comp. Neurol. 29, p. 457-484. - 1920. The Olfactory Organs of Orthoptera. Ibid. 31, p. 457-484. - 1923. Glandular Structure of the Abdominal Appendages of a Termite Guest (Spirachtha). Zoologica 3, p. 367-380, 3 figs.

Menling (E.). - 1915. Ueber die gynandromorphen Bienen des Eugsterschen Stockes. Verh. phys. med. Gesell. Würzburg, 43. p. $173-236,8$ pls.

Nehlis (G.). - 1915. Lehrbuch der Geschichtsphilosophie. Berlin. J. Springer.

Vielinder (A. L.) et Brues (D. T.). - 1903. Guests and Parasites of the Burrowing Bee Ilalictus. Biol. Bull. 5, p. ]-27, 7 figs.

Virtcilf (M. M.). - 1923. The Opalinid Ciliate Infusorians. Bull. 120 U. S. National Mus. 484 pp. 258 figs.

Metzer (C.). - 1910. Die Verbindung zwischen Vorder-und 
Vilteldarın bei der Biene. Zeitschr. wiss. Zool. 96. p. 33\%571,2 pls. 2 figs

Mrnuletun (IV.). - 1917. Nutes un the Larvas of Sonte Cephidie Proc. Ent. Soc. Wash. 19, p. 17'1-179.

Mı (J.). - 1882. Zur Biologie von Gonulopus pilosus Thoms. Wien. Ent. Zeitg. 1, p. 215-221, 1 pl.

Millikex (F. B.). - 1921. Results of Wiork on Blister Beelles in Kansas. Bull. 967 U. S. Dep. Agr. 26 pp. 22 figs.

Mrsich (D. E.). - 1921. An Experimental Study of the Tarsal Chemoreceptors of two Nymphalid Butterflies. Journ. Exper. Zool. 33, p. 173-203, 6 figs. - 1922a. The Chemical Sensitivity of the Tarsi of the Red Admiral Butterfly, Pyrameis atalanla Linn. Ibid. 35. p. 57-81. - 1922b. A Quantitalive Study of Tarsal Sensitivity to Solutions of Saccharose, in the Red Admiral Butlerly, Pyranteis atalanta Linn. Ibid. 36, p. 445̄-45̄, 1 fig. - 1924. The Olfactory Sense of the Cabbage Bulterfly, Pieris rapx Linn., an experimental Study. Ibid. 39, p. 33?$356,1 \mathrm{fig}$.

Möвius (K.). - 1856. Die Nester der geselligen Wespen Abhandl. Ver. Hamburg. 3, p. 121-171, 19 pls.

IIORAIETz. (F.). - 1864. Leber Vespa austriaca Paz. und drei neue Bienen. Bull. Soc. Imp. Nat. Moscou 37, p. 439-149.

MORg LN (C. L ). - 1923. Emergent Evolution. London, Williams et Norgate.

MORICE (R. F. D.). - 1901. Observations on Sphecodes. Enl. Month Mag. 12, p. 53-58.

Mortey (C.). - 1910. On the Position of the Rhopalosomidic with the Description of a Second Species. Trans. Ent. Soc London. p. 386-387, Pl. 50, Fig. 15.

Nonstatt (H.). - 1922. Ueber Pilzgärlen bei Termiten. Ent. Mitleil. 11, p. 94-99

Mrizek (1.). - 1908. Myrmekologiche poznamky III Brachỵterni mermithogyny $\|$ I.asius alienus. Act. Soc. Ent. Bohem. ¿ p. 1-8, 1 figs. - 1909. Fleischfressende Blaltwespen. Zeitschr. wiss. Inselitenbiol. 5, p. 245. - 1916. Der Polymurphismus der sozialen Hymenopteren. Sbornik, Zool. 1 p. ?T$5 \overline{7}$.

MüLler (H.).. - 1872. Anwendung der Darwinschen Lehre auf Bienen. Verh. naturh. Ver preuss. Rheinl, u. Weslf. 29, p. 196, 2 pls. - 1881 Die Entwicklung der Blumenthatigkeil hei den Insekten. Kosmos 5, p. 258-272, 351-370, 415-432.

Müllter (IV.). - 1889. Ueber Agriolypus almatus. Zool. Jahrb. (Abt. Syst.) 4, p. 1132-1134. 
Iurn (F.). - 1918. (Harpagomyia recorded from Formosa) Proc. Ent. Soc. London p. XXXVIIII.

ILnRAy, - 1870. Note on the Egg of Rhipiphorus parudoxus. Ann. Mag. Nat. Hist. 6, p. 326-328.

Mrers (J. G.). - 1925. Biological Noles on Arachnocoris albomaculalus Scolt. Journ. N. Y. Enl. Soc. 33 p. 136-140. $1 \mathrm{pl}$.

Ningle. - 1905. Food of Predaceous Flies. Journ. Bombay Nat. Hisl. Soc. 16, p. 717.

Nelson (J. A.) et Sturtevant (A. P.). - 1924. The rale of Growth of the Honeybee Larva. Bull. 1222. U. S. Dep. Agiir. p. 1-24, 11 figs. 4 tabl.

Newell (W.). - 1909. The Life Hislory of the Argentine Ant. Joutn Econ. Ent. 2, p. 174-192, 3 pls. 4 figs.

Newell (W.). el Barber (T. C.). - 1913. The Argentine Ant. Bull. 122, Bur. Ent. U. S. Dep. Agric. 98 pp. 12 pls. 13 figs:

Niflsen (J. C.) - 1902. Biologiska Sudier over danske cnlige Bier og deres Snyltere. Vidensk. Weddel. Natur. For. Kï̈ben. hatn. p. 75-106, 16 figs. - 1903. Om Bislaegten Sphecodes Latr. Enl. Meddel. (2) 2, p. 21-30. - 1903-05. Om Perisemus fulvicornis Curt. En Overgangsform mellem Snylte-og Fravehvepsene. Ibid. (2) 2, p. 105-109.

ONions (G. W.). - 1912. South African "Fertile Worker Bees ". Algric. Journ. Union S. Africa, 3, p. 720-728. - 1914. South African "Fertile " Worker Bees. Ibid. 7, p. 11-16.

Ottrimire (J. H.). - 1919. Quelques réflexions à propus de l'action de lobscurité sur les êtres vivants. C. R. Soc. Biol. Paris 82, p. 190-191.

Pickinn (A. S.). - 1865. The Humble Bees of New England and their Parasites. Proc. Essex. Inst. 4, p. 107-140, 1 pl.

PiNret (J.). - 1909. Recherches sur les Diptères à larves ento. mobies. I. Caraclères parasilíques aux points de vue biologiques. ethologiques et histologiques. La Cellule 26, p. ?ৃ-216, 5 pls. 26 figs.

Parker (G. II.). - 1912. The Relation of Smell. Tasle and the Common Chenical Sense in Vertebrates. Journ. Acad. Val. Ser. Pliila. 15 p. 221-231 - 1922. Sniell, Taste and Allied Sen. ses in the Vertebutes. Philadelphia et I.omdon, I.ippinentt el (o. - 1924. Some Implications of the Evolulion Hypolluesiz. I'hilos. Revieu 33, p. 593-603.

P.ruker (J. B.) el Böving (A. G.). - 1924. The Blister Beclle 
Trierania sunguinipennis. Biology, Descriptions of Diflerent Stages and Systematic Relationship. Proc. U. S. Irl. Mus. 64, p. 1-40, 5 pls.

Pattin (IV). - 1894. On the Morphology and Physiology of the Brain and Sense Organs of Limulus. Quart. Journ. Mier. Sc. 35, p. $1-96,5$ pls.

Pittrarson (J. T.). - 1918. Sludies on the Biology of Paracopidosomopsis IV. The Asexual Larva. Biol. Bull. 35. 1). 36?376,3 pls. - 1921. The Development of Paracopidosomopsis. Journ. Morph. 36, p. 1-68, 12 pls.

PLirz (R.). - 1924. Studies on Human Biology. Ballimore, IVil. liams et Willins, Co.

Píhez (C.). - 1912. Rapport sur les Iravaux exécutes en 1911. Caisse des Recherche- sci. Rilpporls. r. (693-70.4. - 1920. Tilres et travaux scienliliques. Laval. Barnooud el Co. p. T1)-il. I'inzz (J.). - 1884. Les apiaires parasites, au point de vue de la théorie de l'évolution. Bordeaux. 1895. Sur ia frélendue parthènogénèse des Halictus. Act. Soc I.inn. limileari: 18 , 1). 145-157.

Pin: ('T. DE STltin). 1902. Osservazioni biolngiche sopra un Braconide acqualio, Giardinaiu urinalor, e descrizione di due altri Imenotteri nuovi. Zool. Jahrl). (Abl. Syst.) 15, p. (1925-(i3', $1 \mathrm{pl}$.

Perkins (R. C. I..). - 1887. Noles on Some Habils of Sphecodes Latr. and Nomada. lint. Month. Mag. 23, p. 271-27'́.

1889. Is Sphecodes Parasitic? Ibid. 25, I. ? ?jo 305. 1905. Leaf-Hoppers and their Natural Enemies. Part I Dr!yinidae. Bull. 1 lixper. Stat. Hau aii Sugar Plant. Assne. 69 j!. Perris. - 1857. Nouvelles excursions dans les grandes landes. Ann. Soc. Linn. Lyon (2) 4, 1. 172-173.

Plisuce (R.). - 1906. Origine polyphyedique. homolypic al non conparabilite directe des socieles animales. Noles el Mem. Inst. Solva!! Fasc. T, $126 \mathrm{pp}$.

Pl:minuof (P. DE) - - 1897. La Variation sexuelle chez leArlhropodes. Ann. Soc. Ent. France 66, 1. 215-?6r) - 1910. Sur un cas de Poecilandrie discontinue chez un Bylhinus. Bull. Soc. Ent. France p. 287-290, 3 figs.

Phistirs (E. F.). - 1907. Wax Mollis and American Foul Brond Bull. 75, Bur. Ent. J. S. Dep. Agrrie 1). 19-22. 3 pls.

Presin (F.). - 1919. Contribution is lelude du peuplement diun vigetal. I a faume entomolngique rlu figuier. Ann. Serv. I:prphut. 6, p. 35̌-174. 36 figs. - 1922. Contribulion it l'étude de: parasites de Pieris brassieg I.. Bull. Biol. Frunre Belry. jiti. 1. 5i-13u. - 1923. Recherches biologiques el analomigues 
sur Melillobia acasta IIalk (Hymenoptere Chalcidien). Mhid. ธт, Р. 469-э08.

Plizon (II.). - 1910. La génése des instincls esclavagisles et parasilaires che\% les fummis. Rev. Gén. Sciences 21, 1. i2(j$736,769-779$.

Pietschies (H.). - 1910. Das Gehirn der Ameise. Jen. Zeilschr. Naturw. 47, p. 1-72, 3 pls. 16 figs.

Pr.inta-Reichenau (A. von). - 1888. Ueher den Fullersafi del Bienen. Zeitschr. physiol. Chem. 12 p.

P.iтн (O. E.). - 1922. Notes on Psilhyrus, with Records of IWo American Ilosis. Biol. Bull. 43, p. 23-44, I pl.

Pocork (R. I.). - 1903. Notes on the Commensalism subsisling hetween a Gregarious Spider, Slegodyphus sp. and the Iolh Batrachedra steyodyphobius Wisn. Ent. Monlh Mag. (2) 1'́, p. $16 \overline{7}-170$.

Pousson (R.). - 1924. Contribution a lélude des Itémipleres Irquatiques. Bull. Biol. France Belg. 58 p. 49-305, 13 pls. 3 figs.

Porovici-Bnzmosanu (А.). - 1910a. Experimentelle Untersuchungen über Osmia rufa. Zeilschr. wiss. Inselitenbiol. 6, p. 22'1228,3 figs. - 1910b. Relation entre la taille de l'adulte et la quantité de nourrilure absorbée par les larves chez l'Osmiø rufa el Osmia cornuta. C. R. Soc. Biol. Paris 68, p. 480-48I.

Pricter (J. L.) - 1908. The Life-History of the Carpenler InI. biol. Bull. 14 p. 177-218, 7 figs.

RuBuIn (E.) - 1911. I.e déterminisme des changemenls d, milieu. Bull. Scient. France Belg. 45, 169-185. - 1917. Es:ini sur la vie et la mort des espèces. Ibid. 50, p. 287-380. - 1922a. L'adaplation et l'évolution Paris. E. Chiron. - 1922b. Le conIraste entre le régime alimentaire des larves et celui c'cs adultes chez divers insecles. Bull. Biol. France Belg. ifo. 1). $230-2 \cdot 13$.

Ruins (M. A.). - 1922. Vegelative Vigor of the Host as a facIor influencing Susceptibility and Resistance to Certain Rus: Diseases of the Higher Plants. Amer. Journ. Bolan!l 9, p. 18:3238. 2 pls.

Rıтн (O. von). - 1894. Ueber die Nervenendigungen der Haulsinnesorgane der Arthropoden nach Behandlung mit der Methylenblau-und Chromsilbermethode. Ber. Naturl. Gesell. Freiburg i. $\mathrm{Br}$. 9, 1). 1-?8, $1 \mathrm{pl}$.

Récirur (R. A. F. DE). - 1742. Mémoires pour servir à thisloire des insecles. Tome VI. Paris. Impr. Roy.

Rerchiasich (H.), - 1902. Ueber Parthenogenese bei Ameisen 
Ind andere Beobachlungen an Ameisenkolonien in kïnstlichen Neslern. Biol. Centralbl. 22, ए. 461-165.

libuten (O. .1) - 1918. Lebensgewohnheilen und Inslinlite der Insekten bis zum ErWachen der sozialen Inslinkte. Berlin, I: Friedländer et Solnn.

Richindson (C. H.). - 1913. Studies on the Habits and Development of a Hymenopterous Parasile, Spulanyia muscidarum Richardson. Journ. Morph. 24, p. 513-5̌i, 1 pls.

Richert (H.). - 1921. Die Grenzen der naturwissenschalllichen Begriffsbildung. 3 u. 4 Aufl. Tübingen, J. C. B. Mohr.

Riley (C. V.) - 1893. Parasitism in Insects. Proc. Lint. Soc. II'ash. 2, p. 1-35.

Robson (C.). - 1898. Vespa austriaca. a Cuclioo Wasp. Science Gossip (N. S). 5, p. 69-73.

RöHLer (E.). - 1905. Beilrage zur Kenntnis der Sinnesorgane der Insecten. Zool. Jahrb. (Abt. Anat. Ont.) 22, p. 225-288, 2 pls.

Romwen (S. A.). - 1912. Studies oir the Wooúwasp Superfitmily Oryssoidea a, with Descriptions of New Species. Proc. U. S. Nat. Mus. 43, p. 141-158, 2 pls. 6 figs. - 1913a. Noles on the Feeding Habils of two Adult Sawflies, Proc. Ent Soc. II ash. 15, p. 148-149. - 1913b. (Remarlis on Mr. Hood's Patjet in Rhopalosoma pocyi) Ibid. 15, p. 14i-118. - 1915. A Remarkable New Genus of Cephidse. Ibid. 17, p. 114-117, 1 1)l. -- 1916. Vespoidea in "The Hymenoplera of Connecticul ". Stute Geol. et Nal. Hist. Survey. Bull. 22, p. 606-61'.

RoHwen (S. A.) el Crshmix (R. A.). - 1917. Idiogasıra, a New Suborder of Hymenoptera, with Noles on the immature Stages of Oryssus. Proc. Lint. Soc. Wl ashing. 19, p. 89-98, 2 pls.

Roor (F. M.). - 1924. Parasitisnı among Insecls. Scienl. Month. 19, p. 479-495.

Rosex (K. von). - 1913a. Die fossilen Termiten: eine kurze Zusammenfassung der bis jelzt bekannten Funde. Trans. and Intern. Congr. Enl. (1912) p. 318-335, 6 pls. - 1913b. St11rlien am Sehorgan der Termiten nebst Beiträgen zur Kennt. nis des Gehirns derseben. Zool. Jahrb. (Aht. Anal. Ont. 3\%, p. $62 \mathrm{j}-664,3$ pls. 11 figs.

Rolbaud (E.). - 1908. Gradation è perfectionnement de linslincl chez les guèjes solitaires d Vfrique ru genre Synatgris. C. R. Acad. Se. Paris 147, p. 695-697. - 1910a. Evolution de linstinct chez les guêpes sociales d Afrique du genre Belon norgasler Sauss. Ibid. 1.̈l, p. 5j3-jöfo e-1910b. Recherches sur li) biologie des Synagris. Evolution de linstinct chez. les guepes solilaires. Ann. Soc. Lnt. France 79, p 1-21; Trad. Ann, 
Rep. Sinithson. Inst. p. 507-525, 4 pls. - 1916. Recherches hi: logiques sur les guêpes solitaires et sociales d'Afrique. Ann Sc. Nal. Zool. (10) 1, p. 1-160, 34 figs - 1917. Observations sur Nasonia brevicornis Ashm., Chalcide parasite des Pupes du lluscides. Bull. Sc. France Belg. 50, p. 425-1j9, 1 fig. - 1918. Le Venin et lévolution paralysante chez les Hyménoplères prédateurs. Bull. Biol. France Bely. 51, p. 391-419. - 1924. Ulisloire des Anacamptomyies, mouches parasiles des Guêpe: sociales d'Afrique. Ann. Sc. Nat. Zool. 7. p. 197-248, 2 pls. 15 figs.

Rorriter (I.). - 1925. La Scolastique et le Thomisme. Pari:, Gauthier-Villars et Co.

Rousseau (E.). - 1907. Les Hyménoptères aquatiques, avec des. criplion de deux espèces nouvelles par IV. A. Schulz. Ann. Biol. Lacust. 2, p. 388-401.

Reschlimp (F.) - 1924. Instinkimodifikation in einer AmeisenAdoptions Kolonie. Zeitschr. wiss. Insetitenbiol. 19, p. 176-178

Stivsch (F.). - 1906. A propos des mours parasitiques des fourmis du genre Bothrionulymex. Ann. Soc. Ent. France ij, v. 363-392. - 1920. Fourmis du fenre Bolhriomyrmex. Emery (Systématique el mœurs.) Rev. Zool. Agric. 7, p. 201-22.4, 2 pls. 2 figs.

Sillcy (F.). - 1891. Ueber die Antennen. Ann. Soc. Ent. Frarire (6) 10. Bull. p. 154-155.

Stussure (A. DE). - 1852, Monographie des guèpes solitaires ou de la tribu des Euméniens. Paris, Masson, 6 parties. 286 pp. ?2 pls. - 1853. Note sur la tribu des Masariens et princip,a. lement sur le Masaris vespiformis. Ann. Ser. Frit. France (3) 1. p. 17-21. - 1875. Synopsis of American Wasps. Mise. Coll. No. 254. Smilhson Inst. pp. XXXV-39i, i ils.

Srrixel (J.) - 1922. Grundzüge der The urienhi'dung der binlogie. 2 Aufl. Jena. G. Fischer.

SchifMfaz (P.). - 1883. Ueber das Herkommen des Futlersalles und die Speicheldrüsen der hiene, nehst einem Anhange über das Riechorgan. Zeitschr. wiss. Zool. 38, p. 71-135, 3 pls.

Schmmer (F.). - 1909. Beitrag zu einer Monographie der Gry]Iodeengattung Myrmecophila L.alr. Zellschr. wiss. Zool. 9? р. 409-534, 3 pls. 29 figs. - 1910. Ueber die Wasmannsche Hypolhese des "Duldungsinstinkles der Ameisen gegenüber syn̈̈ker Myrmekophilen. \%ool. Anzeiq. 36, n. 81-95.

ScriöDre (M.). - 1853. On some Staphylinidx, found in the Nests of Termites. Proc. Zool. Soc. London \&1, p. 101-103. 
1856. Observations sur des Staphylins vivipares qui habitent che\% les Termites. Ann. Sc. Nat. Ziol. (4) 5, p. 169-183, 1 pl. SchMieneкNecht (O.). - 1907. Die Hymenoptereu Milteleuropas. Jena, G. Fischer.

Schmid (H.). - 1910. Beitrag zur Biologie der Steinobst-Blall. wespe (Lyda nemoralis L.) Zeitschr. uciss. Insetienbiol. 6, p. 17$23,86-92,4$ figs.

Schneider (A.). - 1883-85. Ueber die Entwickelung der Sphaerularia bombi. Zool. Beitr. I p. 1-9, 1 pl., Il p. 247-251.

Schönfeld (P.). -- 1886. Die physiologische Bedeutung des Magenmundes der Ilonigbienne. Arch. Anat. Physiol. (Physiol. Abth.), Jahrg. 1886, p. 451-458. 1 fig.

Schrotтку (C.). - 1922. Soziale Gewohnheiten bei solitären Insckiten. Zeitschr. wiss. Insekienbiol. 17, p. 49-5̄.

Schuchent (C.). - 1914. Climates of Geologic Times. Chapt. ?l in E. Huntington, "The Climalic Factor as illustrated in Arid America ». Carnegie Inst. Washington. D. C. $341 \mathrm{pl}$. 90 figs.

Scrultz (IV: A.). - 1905. Hymanopteren-Studien. Leipzig. IV. Engelmann. 13 figs. - 1907a. Family Trigonaloidde in VyTsuv's Genera linsectorum. 24 pp. 3 pls. - 1907 h. Schwimmende Braconiden. Ann. Soc. Ent. Belg. 51, p 164-173. - 1910a. Neuer Beitrag zur Kenntnis der Wasserimmen. Ann. Biol. Lacust. 4. p. 187-193, \& figs. - 1910b. Süsswasser-Hymenopteren aus dem See von Overmeira. lbid. 4, p. 194-210, 2 pls. 2 figs.

Scurvarz (E. A.). - 1904. (On the Social Spider Uloborus remublicanus Simon in Cubal. Proc. linı. Soc. Wash. 6. 1) 147-148. Scott (H.). - 1920. Noles on (I) the Parasilic Staphylinid Aleochara algarum Fauvel, and its hosts, the Phycodromid Flies. (II) A case of Supposed Parasitism in the Genus Homalota. Ent. Month. Mag. (3) 6. p. 148-157, 2 figs.

Scunder (S. HI.) - 1890. The Tertiary Insects. Hayden s Rep. U. S. Geol. Surı. Terr. 13, 734 pp.. 28 pls.

Sellars (R. IV.). - 1922. Evolutionary. Naturalism. Chicago, Open Court Publ. Co.

Srarichox (L.). - 1912. Parasitisme provoqué entre deux larves d'Halictus quadricinelus Fabricius. Bull. Sor. Finı. Fance D. 90-92.

Srverin (H. C.). - 1920. The Plum Web-spinning Sarfly. Techn. Bull. 1, Agric. Exper. Stal. South Dakola.

Share (D.). - 1899. Insects. V.ol. II Cambridge Nal. Hi=l. Jum. don. Macmillan et Co.

Shrtford (R.) - 1906. Studies of the Blaltidre V-VII. Trans. 
Ent. Sor. I.mdon. p. 487-519. 1 pl. - 1907. Blaltoidea in Sdïstrur s Kilimandiaro-Meru Expedil. No. 17, 2, ю. 13-48, 2 pls.

Shet.fond (V. E.). -- 1913. The Life-Hlistory of a Bee-Fly (Spogostylum ancle Say) parasite of the Larva of a Tiger Beelle (Cicindela scutellaris Sin, var. Lecontei IIald.) Ann. Ent. Soc. Amer. 6, p. 213-225.

Sicire. (J). - 1865. Revision monographique, critique el synonymique du genre Mellifère Sphecodes I.atr. Ann. Soc. Ent. France (1) 5. p. 397-166.

Suber (N.) et Merusukny (S.). - 1904. Veher Ernihhrung und Verdauung der Bienenmotte (Galleria mellonella). Arch. Ges. Ph!lsiol. p. 269-286.

Sieboln (C. T. E. von). - 1836. Berjcht über die Leislungen im Gebiete der IIelminthologie wïhrend des Jahres 1837. WIEcMun's Arrhiv. 4, p. 305. - 1858. Ueher Agriotypus armatus in Trichosloma nicimone. Ber Versam. Naturf. Carlsruhe p. 211. - 1864. Ueher 7.witterbienen. Zeitschr. wiss. Zool. 14 p. $73-80$.

Sir.vestni (F). - 1901. Operai ginecoidi de Termes, con osservazioni interno l'origine delle varie caste nei Termitidi. Real. Acead. Lincei (5) 10, p. 479-48' - 1902. Contribuzione alla conoscenza dei Meliponidi del Bacino del Rio de la Plala. Biv. Patol. Vegev. 10, p. 121-170, 3 pls. 19 figs. - 1903. ConIribuzione alla conoscenza dei Termitidi e Tedmitofili dell' America Meridionale. Redia 1, p. 1-235, 6 pls. - 1904. Contribuzione alla conoscenza della metamorfosi e dei costumi della Lebia scapularis Fourc. Ibirl. ? p. 68-81. 5 pls. - 1906. Contribuzione alla conoscenza biologica degli Imenotteri Parassiti. L Biologia del Litomaslix truncatellus (Dalm.) Ann. R. Scuol. Sup. Agric. Portici. 6. p. 1-51, 5 pls., 13 figs. - 1907. Contribuzione alla conoscenza degli Insetti dannosi all'Olivo. La Tignola dell'Olivo. Ibid. 2 p. 83--18', 68 figs. - 1909 Isoplera, in Michaelsen el Hirtmeyer : "Die Fauna Südwest-Aus. traliens ». 2. n. 279-314. 6 pls. - 1910. Contribuzione alla conoscenza degli insetti dannosi e dei loro Simbionti, I Galerucella dell'Ormo (Galerucella luleola F. Müll.) Boii. Lab. Zool. Gen. Agrar. Porlici 4 p. ?46-288, 25 figs. - 1911. Contribuzione alla conoscenza degli Inselti dannosi e dei lorn simbionti. II Plusia gamma (L) Ibid. 5. p. 287-319, 26 figs. 1913. Descrizione di un nuovo ordine di Insetti, Ibid., 7, p. 193209. - 1920. Contribuzione alla conoscenza dei Termitidi e Termitofili dell'Africa occidentale II Termitofili. Ibid. 1', p. 265-319, 32 figs. 
Sinox (E.). - 1891. Oloservalions biologiques sur les Arachnides, $1^{\circ}$ Araignées Sociables. Ann. Soc. Ent France 60, p. 5-14 1 pls. - 1892-95. Histoire Naturelle des Araignées ( $2^{\circ}$ édit.) ? vols. Paris, Roret. - 1909. Sur l'araignée Mosquéro. C. $R$. Acad. Sc. Paris, 148, p. 736-737

Stadex (F. IV. L.). - 1895. Observations on Sphecodes rubicundus v. Hag. Ent. Month Mag. 31, p. 256. - 1899. Bombi in raptivity and IJabits of Psilhyrus. Ibid. 35, p. 230-234. 1902. A Scent-producing Organ in the Abdomen of Worker of Apis mellifica. Ibid. 38 , p. 208-211. - 1912. The Humblebee, its Life History and how to domesticate it. London, Macmillan et Co. - 1915. Inquiline Humble-bees in British Columbia. Canad. Ent. 47, p. 84. - 1918. The Genus Vespa in Canada. Ottawa Naturalist. 32, p. 71-72.

Sirtu (F.). - 1855. Catalogue of British Hymenoplera in the Collection of the British Museum. Part. I. Apidæ. London. 1866. Notes on Some Hymenopterous Insects collected by Mr. Peckold at Catagallo, South Brazil, Trans. Ent. Soc. London (3) 5, p. 323-327.

Sirı (H. S.). - 1912. The Chalcidoid Genus Perilampus ana its Relations to the Problem of Parasitic Introduction. Techn. Ser. No. 19. Pt. IV. U. S. Dep. Agric. Bur. Ent: p. 33-69, 8 figs. -1916 . The Habit of Leaf-Oviposition among the Parasitic Hymenoptera. Science N. S. 44, p. 925-926. - 1917. The Habit of Leaf-Oviposition among the Parasitic Hymenoptera Psyche 24, p. 63-68, 4 figs.

S.мiтн (J. B.). - 1901. Notes on Some Digger Bees. Journ. N'. Y. Ent. Soc. 9, p. $29-40,52-72,3$ pls. 3 figs.

Smulyan (M. T.). - 1916. The Hyperparasitic Chalcidoid Planidium on Aphides. Journ. Econ. Ent. 9, p. 510.

Snyder (T E.). - 1924. Arlaptalions to Social Life : The Termiles (Isopterv). Smithson Misc. Coll. 76, No 12, p. 1-11, 3 pls.

Springer (F.). - 1915. Ueber den Polymorphismus bei den Larven von Miastor metraloas. Zool. Jahrb. (Abt. Syst.) 10. p. 57118,2 pls.

Staerer (R.). - 1918. Myrmeliologische Beobachlungen aul Belalp. Ber. Berner Naturf.

Staercke (A.). - 1921. Psychoanalysis und Psychiatrie. Internal. Journ. Psychoanal. 2, p. 361-415.

StöckHert (E.). - 1923. Ueber Entwiclilung und I.ebenswe s der Bienengattung Halictus Lalr, und ihrer Schmarolzer. I Teil. Die Biologie der Galtung Halictus Lats. Konouvia 2, ค. $48-61,146-165,216-247$. 
Sroul (O.). - 1898. Zur Kenntnis der geographischen Verbreil. ung der Ameisen. Mitteil. Schueiz. Ent. Gesell. 10, p. 127-151, Strichlind (E. H.). - 1912. The Pezomachini of North America. Ann. Ent. Soc. Amer. 5, p. 113-140, 5 figs.

Stumper (R.). - 1918a. Zur Kenntnis des Polymorphismus der Formiciden. Bull. Soc. Nalur. Luxemburg, 8 figs. - 1918 b. Formicoxenus nilidulus Nyl. 1. Beitr. Biol. Zentralbl. 38, p. 160-179, 14 figs. - 1921. Etudes sur les fourmis III. Recherches sur l'éthologie du Formicoxenus nitidulus Nyl. Bull. Soc. Ent. Belg. (7) 3, p. 90-97, 1 fig.

Suricouf (F.) et Guyon (L.) - 1912. Nouvelles espèces de Calliphorines de lAfrique occidentale. Bull. Mus. ditist. Nat. Paris, p. 418-428.

Swammerdam (J.). - 1737-'38. Biblia Naturæ, sive Historia Insectorum, etc. 2 vols. Leyda, Severin etc.

SWENK (M. H.). - 1911. Notes on Some Insects Injurious in Nebraska in 1910. Journ. Econ. Ent. 4, p. 286.

SWEEZY (O. H.). - 1903. Observations on Hymenopterous Parasiles of Certain Fulgoridæ. Ohio Naluzalist $3_{2}$ p. 444-45l, 2 pls. - 1915. A Preliminary List of the Hymenopterous Para. sites of Lepidoptera in Hawaii. Proc. Hawaii, Ent. Soc. 3, p. 99-109.

Tanner. - 1892. OEcodoma cephalotes. Second Paper. Trinidad Field. Naturalists' Club. 1, p. 123-127.

Tinguniy (M.). - 1911. Experiments on the Adoption of Lasills, Formica and Polyergus Queens by Colonies of Alien Specir:Biol. Bull. 20, p. 281-308. - 1913. Biological and Embryolo. gical Studies of Formicidx. Bull. Illino:s State Lab. Nal. Hist. 9 , p. $417-477,8$ pls.

Tinde (G.). - 1921. Les Lois de l'Imitation, 7e édit. Paris, Alcan.

'TAYLOR (G. W.). - 1898. Nole on Trigonalys canadensis Hargln. Canad. Ent. 30, p. 14-16.

Tuompson (C. B.). - 1913. A Comparative Sludy of the Brains of three Genera of Auls, with Special Reference In the Mushroom Bodies. Journ. Comp. Neurol. 23, p. 515-5ī0, 2 pls. 1916. The Brain and the Fronlal Gland of the Castes of the "White Int " Leucolermes flavipes, Kollar. Ibid. 26, p. 55j. 602,5 pls. - 1917. Origin of the Castes of the Common Termite, Leucotermes flavipes Koll. Journ. Morph. 30, p. 8.3152, 42 figs. - 1919. The Development of the Castes of Nine Cienera and Thirteen Species of Termites. Biol. Bull. 36, p. 
379-398, 10 figs. $^{-1}$ 1922. The Castes of Termopsis. Journ. Morph. 36, p. 495-535, 2 pls. 9 figs.

Thompson (C. B.) et SNYDen (T. E.). - 1919. The Question of the Phylogenetic Origin of Termite Castes. Biol. Bull. 3̈(j, 1. 115$13: 2,2$ pls., 5 ligs. -1920 . The "Third form ", the Wingles: Reproductive Type of Termites . Reticulitermes and Prorhlnoiermes. Journ. Morph. 34, p. 591-632, 3 pls. 20 figs.

Thompson (W. R.). - 1915. Contribution à la connaissance de lit larve Planidium Bull. Sci. France Belg. (7) 48, 1. 1-31, 5 figs.

THurstone (L. L.). - 1924. The Nature of Intelligence. New York, Harcourt, Brace et Co.

Timuen (A.). - 1921. La crisi mondiale e Saggi Critici di Marxismo e Socialismo. Bologna, N. Zanichelli.

Tillyard (R. J.). - 1918. The Panorpoid Complex. Pt. I. Proc. Linn. Soc. N. S. Wales 13, p. 265-319, 2 pls. 16 figs. - 1923. The Lower Permian Insects of Kansas. Prelim. Announce. ment. Ent. News 34, p. 292-295. - 1924. Kansas Permian Insects. Pt. 3. The New Order Protohymenoplera. Amer. Journ. Sci. (5) 8. p. 111-122, 1 pl., 5 fige.

Timberlake (P. H.). - 1912. Experimental Parasilism, a Sludy of the Biology of Limnerium volidum (Cresson). Techn. Ser: No. 19. U. S. Dep. Agric. Bur. Ent. p. 71-72, 10 figs.

Tosı (A.). - 1895. Osservazioni sulla valvola del cardias in varii generi de la familia delle Apidi. Fice: Lab. Anal. Lnir. lioma 5, p. 5-26, 3 pls. 1896. Di ın muovo genere di Apiaria fossile nell'ambra di Sicilia (Meliponorycles succini - 11 . sicula). Rivist. Ilal. Paleont. i plu., 1 jl. 9 figs.

Towner (R. H.). - 1923. The Philosophy of Civilization. 2 vols. London and N. Y. Putnam's Sons.

Trifirnh (I.). - 1907a. Descriplion of Termitomimus, a new Genus of Termitophiloıs, Physogastric Aleocharini, with Noles on ils Anatomy. Zool. Studier, Upsala. p. 172--19!. 1 pl. 10 text-figs. - 1907 b. Notes on a Termilophilous Timeid Larva. Alk. Zool. 3, p. 1-7, 1 pl.

Trouvelot (B.). - 1921. Observalions biologiques sur lHabro. bracón johanseni C. R. Soc. Biol. Paris, vol. 85, p. I(1):1024.

Tunner (C. H.). - 1899. Notes on the Mushroom Bodies of the Inverlebrales. Zool. Bull. 2, p. 155 160, 6 figs.

Turner (R. E.) et Witrinston (J.). - 1917. Noles on the IIymenopterous Families Bethylidx and Rhopalosomidie. Ann. Mag. Nat. Hist. (8) 20, p. 101-108, 1 fig. 
Limsn. - 1912. Die Trichopteren des Baltischen Bernsleins. Beilr. Nalurli. Preussens, herausg. physili. ölion. Gesells. Königsberg 10, 380 pp., 180 figs.

$\checkmark$ brnutr (C.). - 1891. Biologische Aphorismen über einige Hymenopteren, Dipteren und Coleopteren. Verh. Naturh. Ver. Preuss, Rheinl.48, p. 1-80, 3 pls.- 1892. Beiträge zur Biologic rler Ilymenoptera. Zool. Jahrb. (Abl. Sysl.) 6, p. 680-751, 2 pls.; 7 figs. - 1897. Zur Lebensgeschichte der Gattung Halictuls (Anthophila), insbesondere einer Uebergangsform zu sozi:1len Bienen. Zool. Anzei!). 20, 1). 369-393, 21 ligs

Vinllanes (H.). - 1893. Etude histologique et organologique sur les centres nerveux et les organes des sens des animaux Arliculès. Ann. Sc: Nal. Zool. Pal. 14, p. 405-455, 2 pls.

Viahueyer (II.). - 1904. Experimente zu Wismaxis LomechusaPseudogynen-Theorie. Allgem. Zeilschr. Lint. p. 331-314. 1906. Beiträge zur Ameisenfauna des Königsreiches Sachsen. Abhandl. nalurw. Gesell. Isis. Dresden, p. $5 \overline{5}-69,1$ pl. 1908. Zur Koloniegründung der parasitischen Ameisen. Biol. Centralbl. 28, p. 18-32. - 1909. Beobachtungen und Experimente zur Koloniegründung von Formica sanguinea. Zeilsch:". wiss. Insetitenbiol. Ј, p. 353-356, 390-394. - 1910 a. Bemerkungen zu II Asmaxx's neuester Arbeit: Ueber den Ursprung des sozialen Parasilimus, der Sklaverei und der Myrmekophilie hel den Ameisen. Zool. Anzei,y. 35, p. 150-457. - 1910 b. Ont'sgenelische und phylogenelische Belrachtungen über die paritsitische Kolon,iegrüudrung von formica sanguinea, Bibl. Centralbl. 30 p. 569 -580. - 1912. Ueber die Verbreilung und die geflügelten Weibchen von Harpogoxenus sublaevis Nyl. Ent. Mitleil. 1, p. 193-197. - 1921 Die milleleuropäischen Beobacl. lungen von Harpagoxenus. Biol. Zentralbl. 41, p. 269-2i8. 1923. Polymorphismus und Ernährung bei den Ameisen. Arch. 1. Nalurg. 89, p. 1-12.

Vourissovitci (P.) - 1924. Sur la biologie d'un Ichneumanide (Dicielotus erythrostoma) parasile de l'Eudemis (Polychrosis) bolrana sichifi.) Bull. Biol. France Bel!l. 58, 1. 495-499. 2 lig-:

Winswontu (J. T.). - 1915. On the Life-history of Aleochard bilineata, Gyll., a Staphylinid parasite of Chorlophila brassicæ, Bouché. Jouin. Econ. Biol. 10, p. 1-27, 2 p's., 1 fig.

IVIGIER (WV.). - 1907. Psychobiologische Untersuchungen an Ilummeln mit Bezugnahme auf die Frage der Geselliglieit im Tierreiche. Zoologica 19, p. 1-239, 1 pls. 136 figs.

Wilchenier $(C, A, D E),-1817$, Némoires pour servir à l'histoile 
nulurelle des abeilles solitaires qui composent le gente Halicte. Paris, Didot. 90 pp. 1 pl.

Wilken (E. M.). -- 1914. A New Suecies of Orlhoplera, forming a New Genus and Family. Canad. Lnt. 16, p. 93-99, 1 pl. Whlier (F.). - 1836. Agriotypus armatus. Einl. Mag. p. 412.

Wilsingham (Lord). - 1903. Description of a New Species of Batrachedra associated with Spiders in South Africa. I:nt. Month. Mag. (2) 4, p. 166-167.

Wirren (E.). - 1919. Termites and Termitophiles. South Afric. Journ. Sc. p. 1-20, 3 pls. - 1920. Observations on the Comparative Anatomy of the Termilophilous Aleocharine Paracoro. loca aliermani (Warren). Ann. Nalal Mus. 4 p. 297-366. 6 pls. 5 figs.

Wismanin (E.). - 1891. Die zusammengesetzten Nester und gemischten Kolonien der Ameisen. Münster, Aschendorli. 262 pp. 16 figs. - 1895. Die ergatogynen Formen bei den Ameisen und ihre Erklärung. Biol. Centratbl. 15, pp. 606-640. - 1900-'01 a. Telvmitoxenia, ein neues flügelloses, physogastres Dipterengenus aus Termilenneslern. 1. Theil. Zeitschr. wiss. Zool. 57, p. 599-617, 1 pl., 2 Theil. Ibid. 70, p. 289-298. 1901 b. Zur Lebensweise der Ameisengrillen (Myrmecophila: Nalur. u. Offenbar. 47. - 1901 c.-'02. Neues über die zusammengesetzlen Nester und gemischten Kolonien der Ameisen. Allgent. Zeitschr. Ent. 6, p. 23-2! ; 7, p. 1-21. - 1903. Zur näheren Kenntnis des echten Gastverhältnisses (Symphilie) bei den Ameisen-und Termitengästen. Biol. Centralbl. 23, 1. (ii)- 72, 195-207, 232-218, 261-276, 298-310. - 1904. Contribuiçio para o estudio dos hospedes de abelhas brazileiros. Revist. Mus. Paulista 6, p. 482-487, 6 figs. - 1905. Ursprung und Entwickelung der Sklaverei bei den Ameisen. Biol. Centralbl. 25 , p. 117-127, 129-141, 161-169, 256-270, 273-392, 2 figs. 1908a. Zur Kaslenbildung und Systemalik der Tormilen. Biol. Centrallw. 28, p. 68-73. - 1908b. Weitere Beiträge zum soziillen Parasilismus und der Slilaverei bei den Ameisen. Biol. Centralbl. 28, p. 257-271, 289-306, 323-333, 353-382, 417-411. 1909 a. Die psychischen Fähigkeiten der Ameisen. 2 Aufl. Stultgart. - 1909b. Ueber gemischte Kolonien von LasiusArlen. Zool. Anzeig. 35, p. 129-141. - 1909 c. Zur Geschichte der Sklaverei und des sozialen Parasilismus bei den Ameisen. Nalurw. Wochenschr. N. F. 8, p. 401-407, 5 figs. - 1910 a. Ueber das Wesen und den Ursprung der Symphilie. Biol. Cenlralbl. 30, p. 97_102, 129-138, 161-181. - 1910b. Nachträge z.um sozialen Parasitismus und der Slilaverei bei den Ameisen. Ibid. 30, p. 453-46.1, 175-496, 515-524. - 1911. Die Imeisen 
III)d ihre Gäsle. 1er Congr. Intern. d'Ent. (1910) 1'. 209-23'1, (i pls. - 1915a. Neue Beiträge zur Biologie von Lomechusa und Atemeles, mit kritischen Bemerkungen über das echte Gastverhältnis. Zeitschr. wiss. Zool. 11.t, p. 233-402, 2 pls., 3 figs. - 1915 b. Anergatides Kohli, eine neue arbei'erlose Schmarotzerameise vom oberen Congo. Ent. Mitteil. 4. p. 27!)98, 2 pls. - 1915c. Das Gesellschaftsleben der Ameisen. $2^{\text {ta }}$ Aufl. 1 Bd. Münster i. w., Aschend.orff. - 1917. Neue Anpassungstypen bei Dorylinengästen Afrikas. Zeilschr. wiss Zool. 117. p. 257-360, 4 pls. -- 1920. Die Gaitpflege der Ameisen. ilıre biologischen und philosophischen Probleme. Ileft. 1, Schaxel's Abhandl. zur theoret. Biol. pp. XVII + 176, 2 pls. 1923a. Eine interessante Instinklregulation bei Ameisen (Lasius mixlus Nyl.). Atti Pontif. Accad. Romana p. 255-259. - 1923 b. Die Larvenernährung bei den Ameisen und die Theorie der Trophallaxis. Mem. Pontif. Accad. Rom. Lincei (2) 6. p. 3-23, 1 fig.

Webster (F. M.). et Reeves (G. I.). - 1909. The Wheal Strawworm (Isosoma grande Riley). Circ. No. 106, L. S. Dep. Agric. 15 pp. 13 figs.

IVfismin (1.). - 1892. Das Keimplasma. Eine Theorie der Vererbung. Jena, G. Fischer.

WFsmat (C.). - 1835. Observations sur les espèces du genre Sphécode. Bull. Acad. Roy. Sc. Bruxelles. 2, p. 279-287.

Westwoon (J. O.) - 1874. Thesaurus Entomologicus Oxoniensis, or Illustrations of New, Rare and Interesting Insecls. Oxford, Clarendon Press. - 1881. Observations on the Hymenopterous Genus Scleroderma, Klug. and Some Allied Groups. Trans. Ent. Soc. London p. 117-140, 4 pls.

Whefe:R (E. W.). - 1923. Some Braconids Parasitic on Aphids and their Life Ilistory. Ann. Fnt. Soe. Amer. 16, p. 1-29, 9 figs. IVhfter (G. C.) el F. H. -1924. A New Species of Schizaspidia (Euchroride) with Notes on a Eulophid Ant Parasile. Psuche 31, p. 49-56, 2 figs.

IVhifi.fr (IV. M.). - 1900a. A New Myrmecophile from the Mushroom Gardens of the Texan Leaf-cutting Anl. Amer. Natural. 31, p. 851-862, 6 figs. -1900 b. The Habils of Mlurmecophila nebrascensis Bruner. Psyche 9, p. 111-115. 1901a. An Extraordinary Ant-Guest. Amer. Nalural. 35, i. 1007-1016. 2 figs. - 1901 l. The Parasitic Origin of Macroërgates allong Anls. Ilid. 25, 1). 877-886, 1 fig. - 1901r. The Compromel and llixed Nests of American Ints. Itid. 3.. 1 . 115-131. - 1903a. The Origin of Female and Worlier Anls from the Eggs of Porthogenetic Workers. Science N. S. 
18. p. 830-833. - 1903b. Some New Gynandromorphous Ants, will a Review of the previously recorded cases. Bull. Amer. Mus. Nat. Hist. 19, p. 653-683, 1$]$ figs. - 1903c. Ethological Observalions on an American Ant (Leptolhorax emersoni Wheler). Arch. I'sychol. Neurol. 2, p). 1-31, 1 pl. - 1904a. The Phylogeny of the Termiles. Biol. Bull. 8, 1. 29-3i. - 19046. I New type of Social Parasilism among Ants. Bull. Amer. Mus. Nat. Hist. 20, p. 347-375. - 1904c. Three New Genera of Inquiline Ants from Liah and Colorado. Ibid. 20, p. 1-17, 2 1)1s. - 1905. An Interpretation of the slave-Viaking Instincls of Ants. Ibid. 21, p. 1-16.-1906. Onthe Founding of Colonies by Queen Ants, with Special Reference 10 the Parasilic and slave-making species. Ibid. '22, p. 33-105, 7 p's. - 1907a. 'The Polymorphism of Ants, with an Account of Some Singulat Ibnormalities due to Parasitism. Ibid. 23, p. 1-93, 6 pls. 1907b. The Origin of Slavery aturng Ints. Popul. Sic. Month. p. 35u-359. - 1907e. Notes on a New Guest Ant. Leptothoras ylacialis, and the Varieties of Myrmica brevinodis Emery. Bull. W'isconsin Nal. Hist. Soc. j, p. 70-83. - 1908a. Comparalive Elhology of the European and American Ants. Journ. Psych. Neurol. 13, p. 404-435. -. 1908b. Honey Ants, wilh a Revision of the North American Myrmecocysti. Bull. Amer. Mus. Nal. Hisl. 21, p. 315-397, 28 figs. - 1908c. The . Inls of Casco Bay, Maine, with Observalions on lwo Races of Formica sanguinea Latr. Ibid. 24, P. 619-645. - 1909. Observaliohs on Some European Ants. Journ. N. Y. Ent. Soe. 17, p. 173-187, 2 figs. - 1910a. Ants, their Structure, Development and Behavior. New York, Columbia Univ. Press. - $1910 \mathrm{~b}$. The Elfecls of Parasilic and wher Kinds of Castration in Insects. Journ. Exper. Zool. 8, p. 377-438, 6 figs. -- 1911a. The Ant Colony as an Organism. Journ. Morph. 22, p. 307-325. 1911b. In Ant-Nest Coccinellid (Brachyacantha quadripunrtala Mels.) Journ. N. Y. Ent. Soc. 19, p. 169-174, 1 fig. 1914. The Ants of the Baltic Amber. Schrifl. physili. ölion Gesell. Königsberg, ว̌, p. 1-142, 66 figs. - 1916. Four New and Interesting Ints from the Mountains of Borneo and Luzon. Proc. New Engl. Zool. Club 6, p. 9-18, 4 figs. 1917a. The Temporary Social Parasitism of Lasius subumb. vilus Viereck. Psyche 2'- p. 167-176. - 1917b. The Plén. melrosis of Myrmecocystus. Ibid. 24, p. 180-182, - 1917c The Mountain Ants of Western North America. Proc. Amer. Acad. Arts Sc. 52, p. 457-569. - 1918a. A Sludy of Some Ant Larvæ, with a Consideration of the Origin and Meaning of the Social Habit among Insecls. Proc. Amer. Philos. Soc. 5i, 
p. 293-343, 12 figs. - 1918b. The Ants of the Genus Opisfliop. sis. Bull, Mus. Comp. Zool. 62, p. 343-362, 3 pls. - 1919a. The Parasitic Aculeala, a Study in Evolution. Proc. Amer. Philos. Soc. 58, p. 1-10, 6 tables. - 1919b. Two Gynandromorphic Ants. Psyche 26, p. 1-8, 2 figs. - 1919c. The Phoresy of Antherophagus. Hid. 26, P. 145-152, 1 fig. - 1920. The Subfamilies of Formicidie, and other Taxonomic Noles. Ibid. ?i. p. 46-5̌, 3 figs. - 1921 a. A New Case of Parabiosis and the ". Ant Gardens " of Brilish Guiana. Ecology 2, p. 89-103, 3 figs. - 1921b. Chinese Ants Collected by Prof. C. W. Howani, - Psyche 28, p. 110-115, 2 figs. - 1921 c. A study of Some Social Beetles in British Guiana and of their Relations to the Ant-Plant Tachigalia. Zoologica 3, p. 35-126, 5 pls. 1: ligs. 1922a. Ants of the American Ifuseum Congo Expedition. A Contribution to the Myrmecology of Africa, will the collaboration of J. Bequiert, I. W. BuILFY, F. SANTSChI and W. M. Manv. Bull. Amer. Mus. Nat. Hist. 45, $1139 \mathrm{pp}$. 45 pls. 17 maps and 103 figs. - 1922b. Observalions on Giganliops destruclor Fabricius and Qther Leaping Anls. Biol. Buli. 1:. p. 185-201, 3 figs. - 1923. Social Life Among the Insecls. New York. Harcourt Brace and Co. --1925. A New Guest InI and Other New Formicide from Barro Colorado, Panama. Biol. Bull. 49, p. 150-181, 8 figs.

Wheeler (W. M.) el Balley (I. W.). - 1920. The Feeding Habils of Pseudomyrmine and other Inls. Truns. Amer. Philos. Soc. N. S. 22, p. $235-279,5$ pls. 6 figs.

Wheeler: (II M.) et MoClendon (S. I... - 1903. Dimorphic Qucens in an American Ant (Lasius latipes Walsh). Biol. Bull. 4, p. 149-163, 3 figs.

M helele (W. M.) el MAN (W. M.). - 1923. A Singular Habit of Sawfly Larva:. Psyche 30, p, 9-13, 1 fig.

IVheelen (IV. M.) el Taylor (L. H.). - 1921. Vespa aretica Rohwer, a parasite of l'espa dirabolica De Sanssure. Psyche ?8, p. 135-144, 3 figs.

IIнате (G. F.). - 1906. The Bacteria of the Apiary, with Special Reference to Bee Disuises. Techn. Se.. No 14. Bur. Inl. I. S. Dep. Agric. $50 \mathrm{pp}$.

II hiș (P. W.). - 1914. Ob-ervaliuns on Blow-fies; Duratlion of the Prepupal Stage and Color Delermination. Biol. Bull. ?6, p. 184-194. - 1921a. Rearing Meal Moths and Para. sicic Il is ps for Experimen'al Puposes. Joun. Ilered. I?, 1. 255-261, 9 figs. - 1921b. Studies on the Parasitic Wasps. Habrobracon brevicornis (Wesmael) I. Genelics of an Orange. 
eyed Mulation and the Production of Mosaic Males from Fertilized Eggs. Biol. Bull. 41, p. 12-54.

Wieland (G. R.). - 1924. Fossil Plants as Evidence for the Resistence to Environment. Chapt. V in "Organic Adaptulion to Environment ". New Haven, Yale Universily Press.

Witr.e (J.). - 1920. Biologie und Bekämpfung der deutschen Schibe (Phyllodromia germanica L.) Mlonogr. angew:mitt. Ent. No. 5. Zeitschr. angeuandt. Ent. p. IV $+110,2$ pls. 53 figs.

WiLlians (F. X.). - 1919a. Epyris extraneus Bridwell, a Fossi)rial Wasp that preys on the larva of the Tenebrionid Gonocephalum serialum (Boisduval). Proc. Hutaii T:nl. Sor. i, p. 55-63, 2 pls. - 1919b. Philippine Wasp Studies. Bull. No 11. Exper. Stal. Hawaii Sugar Plant. Assoc. 186 pl). 196 figWillims (F. X.) el Hungerford (H. B.). - 1914. Notns on Coleoplera from Weslern Kansas. Enl. News 25. p. 1.9. ? pls. Winkien (H.). - 1920. Verbreitung und Ursache der Partheno. genesis im Pflanzen und Tierreiche. Jena, Guslay Fischer. Wodsedllek (J. E.). - 1917. Five Years of Starvation of Larvic. Science N. S. 46, p. $366-367$.

Jussi (H.). - 1922. A Classification of the Larva of the Tenthredinoidea. Illinois Biol. Mono!fr. T. $172 \mathrm{mp} .14$ pls.

7. inmin (E.) - 1911. Der Bau der Biene. Stuit rast.

Zititier (H. E.). - 1912. Die Gehirne der Insehten. Vulurusis: Wochenschr. N. F. 21, p. 133-112, 18 tigs. - 1920. Her Beorifl des Instinktes einst und jetzt. 3te Aufl. Iena, G. Fischer.

\section{Insecles eẗ Araignées Subsociales}

Binks, N. (1904), Bennett, C. B. (1904), Böttger (1910), Culfis. J. (1845). Diguet, I. (1909a, 1969b), Distant, W. L. (1898),

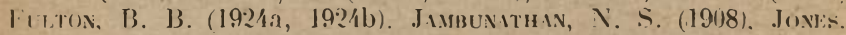
1). W. (1917), Kühnle (1913), MARshile, G. A. K. (1898), Pocorк, R. I. (191)3), Schivarz, E. A. (1904), Senichon (1909), Silvestm, I: (1913). Sinon. E. (1891, 1892-95, 1909). Whefler (1921c).

\section{II!minopleres Phylopha!ges}

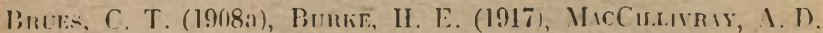

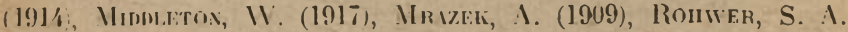
(1912, 1913a, 1915), Romwin S. 1. el Cismmix, R. A. (1917), 


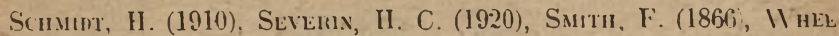
I.En, II. II el IIIN, IV. II. (1923), YUISI, H. (192?).

\section{Iyménoptères Térébranls}

Bischof, H. (1909), Browne, F. B. (1922), BRUES, C. Т. (19U1, $1908 \mathrm{c}, 1910 \mathrm{a}, 1910 \mathrm{~b}, 1921,1923 \mathrm{~b}, 1923 \mathrm{c}, 1924 \mathrm{~b})$, Bugnox, L. (1910), Caffrey, D. J. (1921), Cushmix, R. A. (1913, 1917), Dotex, S. B. (1911), Ford, N. (1922), Giand, A. (1889a, 1889b), GriNd, G. (19:1, 19:3), HeYMons, R. (1948), How:R1), L. O. (1891, 1948, 1910), Kelly, E. O. G. (1914), Kinsey, А. C. (1919), KonNhilestr, S. I. (1919), Leiby, R. IV. (1922), Lichtenstein, J. L. (1921), Lichtensten, J. L. el Picird, F. (1917), Lichtenstein, J. L. el Rabiun, Ł. (1922), Lubвоск, J. (1863), Mitausch, I. (1909, 1911), MAтheson, R. el Cllosbi, C. R. (1912), MarchaL, P. (1900, 1905, 1906, 1909), Müller, W. (1889), Patterson, J. 'T. $(1918,1921)$, Pérez T. de S. (1902), Picakd, F. (1922, 1923), Richandson, C. H. (1913), Roubaud, E. (1917), Rousseau, E. (1907), Schultz, IV. A. (1905, 1907a, 1907b, 1910a, 1910b), von SiEbold, C. T. E. (1858), Sildisstu (1906, 1907, 1910, 1911), Sмith, H. S. $(1912,1916,1917)$, Smulyan, M. T. (1916), Strickland, E. H. (1912), SWenk, M. H. (1911), Taylor, G. W. (1898), Тhompson, W. R. (1915), Тimberliki, P. H. (1912), Tkouvilot, B. (1921), Voukissovitch, P. (1921), Wilker, F. (1836), Webster, F. M. et Reeves, G. I. (1909), I heelfi, İ. IV. (1923), W Hiting, P. W. (1914, 1921a, 1921b).

\section{Hynénoptères Aculéales (Général)}

Von Altex, F. (1910), Ashue.ın, W. H. (1896), Bethe, А. (1898, 1902), Cockerely, T. D. A. (1906b, 1909a, 1916a, 1920b, 1917;, II Nolinsch, Anten (1887-95), von Iherivg, R. (1903c), Кон, F. F. (1896), Lepeletien de SilNT_Fargeid. A. L. M. (182i), Llubbock, J. (1888), McINdoo, N. E. (1914b); Mr.zeк, А. (1916), RАвıUи, L. (1922), Roubaud, E. (1918) SChMiEdelinecht (1907), Whetifn, IV. .I. (1919), ZiegLer, H. E. $(1912,1920)$.

\section{Bélhyloidès}

Ashue In (1893), Audoiv, J. V. (18:12), Bnibwild, J. C. (1917̄a, 1917b, 1917c, 1919, 1920) Fullawi, D. Т. (1920), HaıniY, А. II. (IS:35), Hoon, J. D. (1913), HYsLor, J. A. (1916), JEANNEL, R. (1913), 
Kisilin, D). et Thompson, W. R. (1915), KiefFer, J. J. (1907, 1908. 1909), Mik, J. (1882), Mortey, C. (1910), Nielsen, F. C. (1903-05), Periass, R. D. L. (1905), Perris (1857), Roniven, S. A. (1913b),

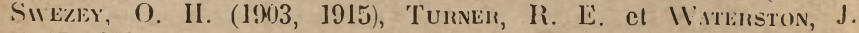
(1917), Westwoon, J. O. (187i, 1881), Williums, F. А. (1919a).

\section{Vespoidés}

Axori, Eirn. (1903), Ashueid, IV. H. (1902-03), Brovarnt, I. (1918), BRILLey, J. C. (1922), Brauns, H. (1910-11), ni Busson (1910:3-(5), Livinsox, A. (1913), Davis, J. J. (1919). Ducke, A. (1910, 1914). Fenton, C. (1911), Fonbes, Н. O. (1885), Fonbl: S.. 1. (19118), Ginien, J. (1871), IINnLInsen, Inlon (19111. Howes. P. G. (1917), Von IHERINi, H1. (189\%), von IHERING, R. (I993).), Janet, C. (1903), Marchal, P. (1896, 1897), Möbies, K. (18.76), Rоншт., S. A. (1916), Roubiun, E. (1908, 1910 a., 1910 b., 1916), de Silssinf, H. (1852, 1853, 1875), Willinis, F. X. (1919).

\section{Apidés}

Armbruster, L. (1914, 1916), Asmaeid, W. H. (1890!, АERIVIIIS (1896), BuKeR, C. F. (1896), BOVER, J. (1915), BHEITENBICH,

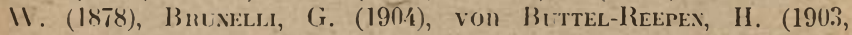
I!)(6), 1911, 1915), Cockemelt, 'T. D. А. (1909h), Dnfilisf, L. (1905), Ducke, A. (1902, 1903, 1905, 1916), Eversmix, E. (1846),

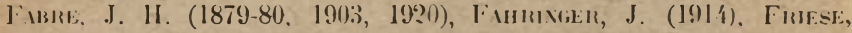
il. $(1888,1591,1903,1905,1923)$, von Fитсн, K. (19:21, 19:3), Fox. II. J. (1893), Gutbier, A. (1914), Harter (1890), Herbst, P. (19?2), Hiselhats, F. (1922), von lheriNg, H. (1903), von Ihemai, R.

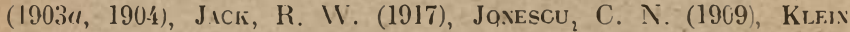
(1904), Linghofer, A. (1897), Legewie, H. (1922), LineblRG. B.

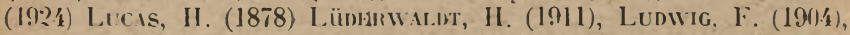
Me Innoo, N. E. (1914c, 1916), Mehling, E. (1915), Metzer, C. (1910), Mü̈len, H. (1872, 1881), Nelson, J. А. el StunteviNt, 1. P. (19:4), ONioNs, G. IV. (1912, 1914), PÉRlz, J. (1884), Von

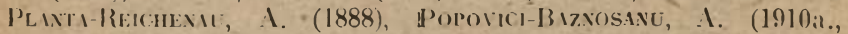

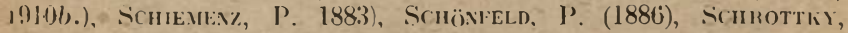
C. (19?2), Schulz, W. А. (1902), Semichox, L. (1912), von Sifiorn, C. T. (1861), Sillestri, F. (19112), Slidex, F. W. (1902. 1912), Simtн, F. (1855), Sмитн, J. В. (1901), Sтӧскневт, E. (1923), 'Tosı,

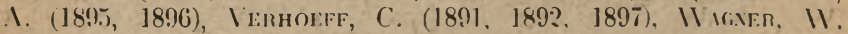

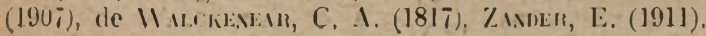




\section{Formicidés}

ANwri, Ern. (1892, 1895), Bethe, A. (1900), BhANen, J. ( (1900), Bnex, R. (1917, 1923a, 1923b, 1924), Búckinghiм, E. N. (1911), von Buttel-Reepex, H. (1905), Cockerell, T. D. A. (1906i, 1915, 1920a, 1921a, 1921b, 1923a, 1923b), CRIIVLEY, W. (. (1910a, 1910b, 1912), Doflfin, F. (1920), Donisthorpe, H. (191う. 1920a), EMERY, C. $(1890,1891,1894,1895,1896,1898,1904,1905$, 19166. 1910, 1915a, 1915l, 1916, 1918, 1920, 1921, 1924), Escherich, K. (1917), FIEI.IE, A. M. $(1901,1902,1904,1905 a, 1905 b, 1906)$, Fones, A. (1891; 1902, 1904, 1910h, 1920, 1921-23), Heer, O. (1849; 18:?), HingstoN (1922), Hollidiy, M. (1903), Huber, P. (1810), JINET, C. (1898a, 1898b, 1904, 1907, 1909), LOMNICKI, J. (1921),

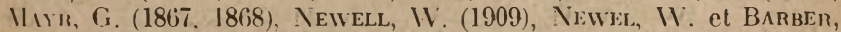
Т. (. (1913), Pimz, С. (1912, 1920), Pietschler, Н. (1910), Prich?,

J. L. (1908), Reichenibich, H. (1902), Scunner, S. N. (1890), Stol.L, 0. (1898), Stumper, R. (1918 $($ ) , Tanew: (1892), Tanquiry, II. C. (1913), Thompson, C. B. (1913), Viehmeyer, H. (1906, 1923), Wis-

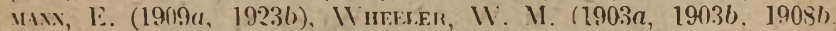
1910a, 1911a, 1914, 1916, 1917b, 1917c, I918a, 1918b, $1919 b$.

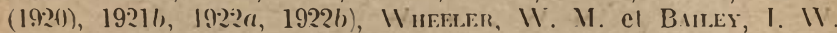
(1920), Whitetir, W. M. el McClendon (1903).

\section{Termites}

Bivis, N. el SNyoer, T. E. (1920), Bugiton, E. i1909, 1912 , 1913b, 1914. 1923, Brginon, E. el Popolf, N. (1910), Cockeneti.. T. D. А. (1913, 191(ib). Drssieux, J. (1901), Eschenich, K. (1909, 1911), Fe:tael, J. (1912), Froggatt, IV. W. (1895-67, 1905). Fui. LER, C. (1915), (irissi, B. et Sandias, A. (1992-91), Ifearu, II.

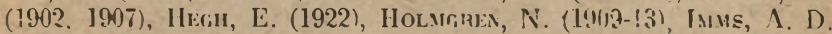
(I!)!9), JuCG, C. (1924), KNower, H. McC. (1891), Lfspìs, C. (18,i). Morstiti, H. (1922), von Roska. K. (1913a, 1913hi), Scti)-

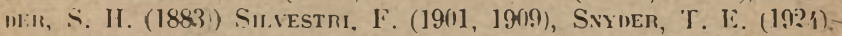
'Thomesos. C. 13'. (1916, 1917, 1919, 1922), Thompson. C B et STider, T. E. (1919, 1920), Wasmann, E. (1908), Whifeiter, W. $\mathrm{M}$. (I90!a).

\section{Sphécophiles et Melillophiles}

INuit, Edlu. (1881), Assmuss, E. (1865),-Butjes. C. Т. (1924u, Ciлрмах, Т. А. (1870), Сновает, А. (1891), Diммоск, G. $\bullet$ (1886), Fumb; J. H. (1890), Gibmitschevsky, E. (1921), Künckel-1'Her- 
CULAIS, J. (1869, 1870, 1875-81, 1895), LEUCKIITT, R. (1887), LuH Bock, J. (1861), Melinder, A. L. et Bruls, C. T. (1903), Millikis, F. B. (1921), Murray (1870), Nielsen, J. C. (1902), Packimi, А. S. (1865), Pinker, J. B. et Böving, A. G. (1921), Phillips, E. F. (1907), Rélumur, R. A. F. (1742), Roubald, E. (1924), Schneiner. A. (1883-85), Shelfond, R. (1906), von Siebold, C. T. (1836), WisMiNi, Е. (1901), Wheelen, М. М. (1919), Whiте, G. F. (1906), Wirmins, F. X. et Hungenfond, H. B. (1914).

\section{Myrmécophiles}

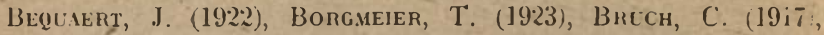

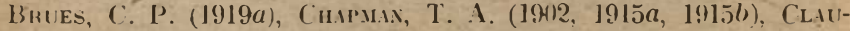
si:s, C. P. (1923), Collin, J. E. (1922), Chigg, F. W. (1917-18), Crawley, W. C. el Biylis, H. A. (1921), Dodd, F. P. (1902u, $1902 b, 1912)$, DoNisthonpe, H. $(1912,1921 a, 1921 b$ ), EmWALs, IF. IV. (1922), Escherich, K. (1898, 1902), Farouharson, C. O. (1918, 1919, 1922a, 1922b), Fonis, А. (1890), Frонашк, F. IV. (1915), Greex, E. E. (1906-197, 1908), Heiliertinger, F. (1919), Jicobson, ¿. $(1909,1910,1911 a, 1911 b)$, Jines, S. P. (191/), JiNet, C. (1896.

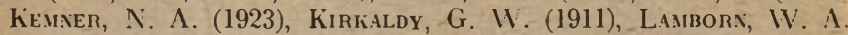
(1913, 1914, 1919), DE MEJERE, J. C. H. (1909, 1910, 1911), MRIZEli, А. (1908), Muir, F. (1918), Ningle (1905), Schimmen, F. (1999, 1910). Surcouf, J. el Guron, L. (1912), Virhmerer. H. (19(1)\}). IVAsMixx, E. $(1895,1901 b, 1903,1910 a, 1911,1915,1917,1920$ :, Wheelen, G. C. et E. H. (1924), W heelfir, W. M. (1900a, 1900b, $1991 a, 1901 b, 1907 b, 1910 a, 1911 b)$.

\section{Termitophiles}

Assmuth, J. (1913), Bries, C. T. (1908b, 1923a), Bugnos, l:. (1913a), Buscalioni, L. el Comes, S. (1910), Carpenter, G. I). H. (1919), Clevelinn, L. R. (1923a, 1923b, 1924), Cushim, R. A. (19:3), Cutler, W. (1921), Kemner, N. A. (1922). McInooo, N. l.

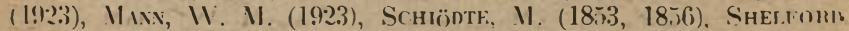
R. (1907), Silvestri, F. (1903, 1920), Trasirdo, I. (190ia, 1907h. WinRFi, E. $(1919,1920)$, Wısmix, E. $(1900-01 a)$.

\section{Parasiles Sociaur}

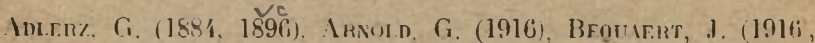

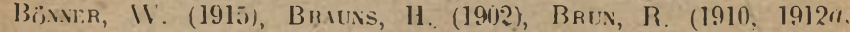
-191?b, 1913a, 1913b), Cinfesten, G. IJ. el Pick-Benesfond, D. R. 


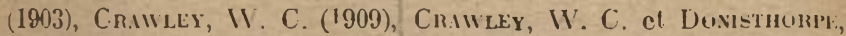

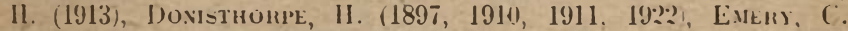
(19118a, 1908b, 1909a, 1909b, 1911a, 1911b, FЕКTUN, C. (1891), 1898, 1:901-21), Fllitcher, J. (1908), Fuliel, А. (1898, 1900, 1994, 1908), Fnison, 1. II. (1916, 1921), HoFren, E. (1881, 1888), KUTtEh, II. $(1913 a, 19136,1917,1920,1923)$, de LANivo , F. (1908), LoMNicki, J. (192:), Малсhal, P. (1890, 1894), Morawitz, F. (1864), Monice, R. F. Г. (1901), NizLsex, J. C. (1903), PÉnez, J. (1895), PenkiNs. R. C. L. (1887, 1889), PiEnox H. (1910), PLith, O. E. (1922), Robsox, C. (1898), Ruschilмi, F. (192), Sixtschi, F. (1906, 1920), Sichel, J. (1865), Sladei, I. IV. L. (1895, 1899, 1915, 1918;, STIEgiti R. (1918), Stumpre, R. (1918b, 1921, ThQuan, M. (1911). V'ІеHMEYER, II. (1908, 1909, 1910a, 1910b, 1912, 19?1), IV ISMAN.

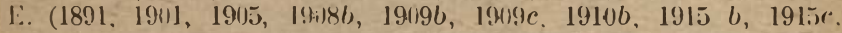
19:3a, Wesmael, C. (1835), Wheeler, IV M. (1901e, 1903\%',

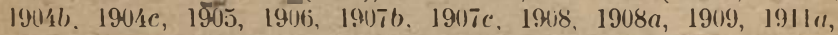
$19: 1 a, 1925)$, WHeELIR, W. V. el T'YYion, L. H. (19?1).

\section{Cilations Générales et Divers d'Entornologie el de Zoologie}

Bequiert, J. (1924), Birger (1878), Berlesf, $A$. (1969), BörNak, C. (1919), Bouvien, E. І. (1918), Briven, F. (1869, 1883a., 188.3b.), Britschneider, F. (191j), Bruch, C. (1917a), Brues, C. T. (1919b), Brots, C. T. et Memandr, A. L. (1915), Chamman, T. А. (1878), Comstock, J. C. (1924), Chinpton, G. C. (1915), Deforemen. P. (1912). Descy, A. (1924), Dfamole, R. (1917), Dieti, M. J. (1876), Dönioff, E. (1882), Dufolr, L. (1837), DunвıR, C. O. (1929). Dutt, F. R. (1912), von Engeliurmt (1914), Euchson, W. F. (1817), Forei, А. (1910a), Fulton, B. B. (1915), Griffini, А. (190こ),

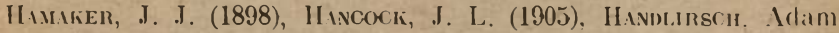
(1882-83), Hindlinsch, Anton (1903, 1904, 1908, 1913a, 19136 1921), Hinnis, R. G. (1923, 1924), Hanris, T. (1862), Hermons, R. (1907), Herms, W. B. (1907), Hochreutri, R. (1912), Howard, I. O, (1905), Kélogg, V. (1913, 1914), KiNG, J. L. (1916), Ki.frs, R. (I910), Kominsky, P. (1924a, 1924b), Limfere, A. (1909), Mc Indon, ‥ E. $(1911,1915,1917,1918,1920)$, Мімкіс, D. E. (1921, 1922a, $1922 b$, 1924), Myens, J. G. (1925), Pantel, J. (1909), Pitten, W. (1891), de Pryerimhoff. P. (1897, 1910), Picand, F. (1919), Poisson, R. (1924), von Rith (1894), Reuter, O. M. (1918), Riley, C. V. (1893), Rоот, F. M. (1921), Siulcr, F. (1891), Scott, H. (192). Shili, D. (1899), Shelfori, V. E. (1913), Sieber, N. el MetuiNikov, S. (1904), Silvestri, F. (1901), Srringeli, F. (1915), SwanMEIDIM, J. (1737-38), Tillyand, R. J. (1918, 1923, 1924), TURNEK, 
C. H. (1899), Uluer (1912), Vialianes, H. (1893), Wanswontu

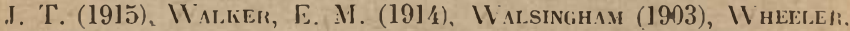
IV. II. (1923), WiLIE, J. (1920), WOnSLDALEK, J. E. (1917).

Oucrages Physiologfiques, l'sychologiques, Sociolongiques, ele.

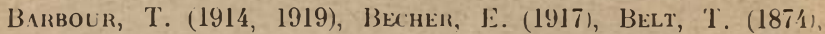
Bienemanin, W. (1911), Blanchiro, R. (1899), Cillegry, M. (1922), Chiln, C. М. (19:1), Craig. IV. (1918;, Júxot, L (1911), Diliti: Nl:k, P'. (1918), DE Gringe, MCQ. (1923), DHE, M. el JUPONT, P'. (1924), JREver, J. (1917), DuRkheim (1922), EsPiNis, А. (187i). Fermienf, A. (1915), Fiske, J. (1874), Foulllée, A. C. (19:0), Freld, S. (1921), Giard, A. (1905), Heniss, H. (1916), Неrnick. C. J. (1903), Huntington, E. (1914), Koner, L. (1921), KolbF, H. J. (1913), Krover, R. (1913, 1919), Kuo, Z. Y. (1924), LE BoN, G. (1925), Le DANTEC, F. (1918), Leconte, J. (1884), Longmin, H. A. (1923), Lovejor, A. O. (1922), McLolgal, W. (1920), MarTiN, E. D. (1920), Massikt, J. el Vandervelde, E. (1893), Mathews, A. P. (1921), Mitтhew, W. D. (1915), Mehlis, G. (1915), Metcalf, M. M. (1923), Morfin C. L. (1923), Otтrimike, J. Н. (1919), Palliek, G. H. (1912, 1922, 192/) Pelli, R. (1924), Petrucci, R. (1916), Ribiud, E. (1911, 1917, 1922a), Raines, M. А. (1922), Rickent II. (1921), Rougier (1925), Schıxel, J. (1922), Schuchent, C. (1911),

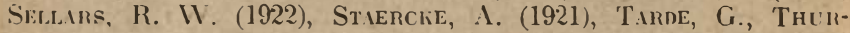
stone, L. I. (1921). Tilghfir, A. (1921), Towner, R. I1. (1923). Wrismix. A. (1892), Wielaxd, G. R. (1924), Winkier, H. (1920). 


\section{INDEX ALPIIBESTIQUE DES AUTEURS}

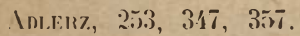

Aipri.ler. 206.

AITEN (von), 94, 208.

Axpne, Fdm. 302.

Мкиц, Ern. 121, 130, 138.

Aisistote, 273.

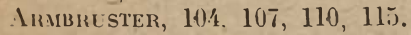

ARNOLD, 344.

AsнMe.n, $27,36,60-62,66,75$, 93.

Assuuss, 303.

Assmuth, 3э0, 335.

Aunolin, 66.

Aunivillius, 101.

BiCON Rloger, $26 \overline{\text { i }}$.

B.IILEY, 240, 243.

BALFOUR-BROWN, $5 \overline{7}$.

Balier, vi3.

Banks, C. S., 311.

BıNks, N.. 5, 7, 60, 159.

ASIRBER, H. S., 15.

Barber, T. C., 243.

BARBOUR, 224, 225.

B.IRTH, 374.

BAYLIS, 248.

BECHER, 9.

BEIT, 301.

BENNETt, 173.

BEQUAERT, 59, 75, 302, 309, 339.

BerGi:R, 210.

BerLest; . 20, 291, 292.

ВЕит, 165.

BЕTHE, $272, \alpha y 2$.
BHinelimini, 28:).

Bischofe, is.

BLANChIID, 35.

B1.UCHMAN, 162.

Boss, 390.

BÖNER, 360 .

Вӧнкен, 36, 37, 60, 62, 92, 12!).

BOETTGER, 210.

Böving, 19.

BOLGMEIER, 305.

Bolvier, 1, 7, 230.

BRADLAY, $27,36,59,60,6$ ? , (i3), T5, 93'.

Brinner, 20.

Brauer, 50, 51, 302.

Briuns, 74, 101, 103.

IBRETSCHNEIDER, 2$] 0$.

-BheiteNBiCh, 94, 104, 340.

BRIDIVELL, 62, 64, 66, 67, 72.

BRIschlie, 112.

ВRLCH, 50.

Brues, 33, u1, 49, 51, 53, 54 . 6?, $63,104,130,173,303,318$, 335.

Brutlé, 159 .

Brun, 122, 209, 210, 272, :80).

$292,34 \overline{7}, 360,365$.

Brunelli, 184.

BUCKINGHAM, 260.

Bugnion, 38, 48, 186, 216, 2:0.

221, 230, 258, 330, 33\%.

Burke, il.

liLnMister, 11:.

Buscilioni, 183. 
Busck, 66 .

IjUTtel-REFilex (Von), 101, 111, 11:, 113, 119, :36, :237, 21т 385.

Cifrerey, 57.

CARPENTER, 339.

Cillelery, 35 .

ChIPMAN, $50,302,313,314$.

CHILD, 255.

Сновіยт, $30 \%$.

ClLIRK, 350 .

Cliusein, 44, 4ī, 48.

Clevelaxd, 162, 18\%, 183, 184.

Cockerell, $34,38,95,111,112$, $133,134,136,137,165$.

Comes, 183.

Comstock, А. B., 193.

Constock, J. H., 27, 31, 59, 1i3 CONTE, $125,156$.

CONDORCET, 125.

('RHG, 9.

Cinlici, 310.

CRIWIEY, 121, 193, 248, 278. 360.

Crosby, 54.

ClĖot, 299.

Cuntis, 32.

Cusumin, $31,53,56,318$.

C'UTLER, 163, 322.

DIRIIN, 266, 3an, 352.

DIVIDSON, 71.

Divis, $\because 2$.

DEEGENFR, 4, 292.

De Gringe, $156,295,376,37 \pi$. Fabre, $1,50,51,104,105,106$,

DE: GHEEF, 374.

DEMOLL, 292.

UESCY, 193.

DESNEUX, 161.

DIUE, 389.

DIETL, $2^{10}$.

Dig.UF.T, 7,8 .

DimMock, 303.
D) ISTINT, $;$.

Donn, 312, 311.

- Dӧнанк, ?89.

DOFLEIN, 270 .

DOHININ, 192.

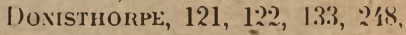
$301,305,351,360$.

[) OTL: 5657.

DHEVE凡, 9.

DUEYLING, 114.

I) BLYSSUN, 280.

DUCKE, $32,73,75,78,79,80$,

- 84, 99, 84, 116, 118.

JefotR, $1,303$.

DLJIRDiN, 2U8.

DUNB.11, 29.

DURCKHEIM, $376,381$.

I) UTT, 309.

Jzierzon， 191, 193.

Echermani, 379.

EnwiRds, 311 .

EMEnson, 160, 173. 329, 330.

EMEITON, 7.

EverT, 121, 122, 126, 130, 132. $139,146,14 \overline{1}, 148,195,204$. $230,236,243,248,254,261$. $? 63,261,283,355,360,365$.

ENGelhant, 178.

EnICHSON, 292.

Escherich, 1:22, 159, 257, 286, 308,331 .

EsPinis, 5, 374, 380.

Evensminn, 104.

\section{$107,110,178$.}

Fahinivger, $104,110$.

FARquharson, $310,311,312$.

lierrero, $3 i i$.

[PerRiére, 374, 375.

Ferton, 1, 63, 64, 72, 74, 94, 104, 340.

FEYTILD, 177, 257. 
[HILDE, 193, 2)13, 29:, 350, 385. JISCHER, 390.

FISKE, J., 11.

FISKE, W. F., 372.

Fletcher, 340.

lonbes, 72.

Fond, 42.

Fonfl, 4', 121, 122, 124, 130, $115,193,209,210,211,261$, $272,278,292,293,346,356$, $357,360,362,364$.

Fouilete, 9, 273, 37', 375.

Fox, 93.

FinELD, 387.

Firiese, 90, 94, 10', i13, 288. $3 \mathfrak{1 0}, 311,342,383$.

Fisch (von) 286, 292, 293.

Finison, 341.

Finogg.tT, 161.

FinoแIIK, 313.

FlLLAWIY, 66.

Fil.LI:R, 174, 175, 1Ti, 182.

FเLTON, 173, 178.

(i iBntchevsKY, 303.

(iпRо, 1, 69, 190, 201, 338 .

GiRILD, 74.

(ioethe, 259, 379.

(inแNm, $56,200$.

(insssi, 164, 183, 234), 256, 28i.

(intis, 309.

GIIFFINI, 200.

filtiter, 104.

IIHFITER, 289.

Huin, 390.

IIA.IDAY, 60. 62, 63, 64.

HIMIKER, 210.

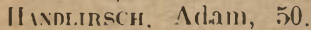

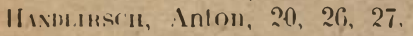

?9 $\because 9,33,21.36,59$, (i) 62 ,

(3) $92,95,9 ;, 110,125,1 \geqslant 9$.

130. $131,131,142,147,149$.

165, 166, 173, 17'1.
HiNcock, 178.

Hinkis, 1 had, -22'3.

Hinnis, R. G., 201.

HIISWELL, 150 .

НЕ.тT, 163, 164, 17т. 216, 217, $25 \%$.

HEIOLEY, 150 .

He.iH, 159.

IILILERTINGER, 35ั.

IIINNING, 272.

HI:RHST, 104 .

HerMs, 232.

Hernick, 290.

HF.SELHILS, 114, 341.

Hiniston, 218, 2605.

IIOINGREN, 159, 160, I61, 16:?, $209,212,213,214,-219,257$. 381, 380.

HeYmons, j].

Hochnelter, :92.

IlUFFER, 341.

HoLLIDIY, 253.

lloon, 6?.

IIOWIRI), 38, $5 \overline{7}, 58$.

HUWES, 78.

ILUBE,R, F., 35\%.

Tliber, P., 353, 354.

Heivgerfond, 49.

IILNTINGTON, 148.

HYstop, 61.

IเI:!ı, H. (von), 84, 116, 118, 149, 151, 235.

IHERING, R. (von), 83, 99, 101, 116,118 ,

IMns, 162, 164, 183, :30, 258.

J

Jicobsox, 309, 3in, 311.

JIMIBINIT:IN, 7 .

JiNIS 31$]$.

IINET 119, 131, 193, 198, 2811, $? 9^{\circ}, 30 i, 384$.

JONES, 173. 
Joxisetr. 2085.

Jucer, 162, 16:1, 18:, 181, 211, $216,236,237.257$.

JCPPONT, 389.

Kil:zLis, 69.

hisley, 43.

KELLOGG, 322 .

liemaer, 280, 305. 335 .

KiefeEr, $61,66,69$.

KING, 50.

KINSEY, 34.

KIRIILIY, 310.

liLitT, 390.

KLEBS, 138.

Ki.EIN, 237.

KNOWER, 216.

KOBER, 151.

КонL, 95.

KOLBe, 150 .

KOMINSKY, 263.

KorNhaLSER, 69, 70.

KORZYBSKY, 156.

KRONER, 19.

Ki EHNLE, 210, 214.

KUNCKEL d HERCUlAis, 302.-

Kuo. 277.

Kute1, $356,360$.

Limbons, 309, 314.

L.MMkRE, $18 \bar{T}$.

LINGHOFER, 94, 383.

L.Nivor (de), 360.

L.ITREILI.E, 1, 26. 60, 90, 99, 121, 1:28.

I.E Bon, $28 \bar{T}$.

L.E DNNTE. $3 \pi T$.

I. E:Gi:WIS. 10.'

Y.I:I3\%, I!)?

LIPELETIE:, $1,99$.

1.1: SMI.TS, 39u.

I.1:si:s, 183.

L.EUCKIIT, З30̈.

LACHTENSTLIN, $56,57$.
LILIINIFLIT, 374.

LINEBU

1.OCKE, 11.

I. OMNICKI, 333, 360.

LONGMAN, 150.

Love.Jor, 11.

цчввоск, јя, 19.3, 303.

Lucis, 99.

L.UDWIG, 104.

I.ÜDFRIII.DT, 104.

MicClendon, 130.

MacDouginl, 387.

MacGilliviriY，32.

MICINDOO, 291, 29:2, 3330.

IIETERLINCK, 23.

Ммк, 17, 32, 47, 121, 14t6, 311, 329,364 .

IIIRCHIL, $1,54.5 \overline{4}, 196,230$, 340.

MIRI.ATT, 66.

MARSHAL, $T$.

MARTIN, 387.

Missaht, 338 ,

Mitiusch, 69.

Mitueson, 54.

VITHEWS, 287 .

MATTheW, 150.

MYYR, 121, 133, 138.

MEHLING, 264.

MeHLIS, 19.

Meluere (1):), 309, 31], 31?.

MELANDEK, 104, 173, 303.

Netichliow, 289.

Metcilf; 322.

Vetzgen, 236.

MInnt.ETON, 31.

W/k, 8?.

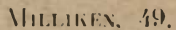

VINNICH. 2?!I.

Vïвичs, 99.

Vomilite, 339.

MOMleEY, 62.

VURGAN, ?3. 


\section{INDEX ALPIABÉTIQUE DES AUTEURS}

Morice, 340.

. Ionstatt, 182, 279.

NRAzEK, 32, 198, 199, 218, 35 7.

Mü̈lLer (H.), 93, 94.

Mǘler (W.), 54.

MUtR, 311.

MURray, 30 ?

MYERS, 6.

Navgle, 3 UY.

NeLson, 232.

Newell, 243.

Nieboer, 35\%.

Nizlsen, 63, 64, 94, 129, 340.

Nilander, UL.

ONıons, 193.

OTTRAMARE, 28'.

PackiRd, 303.

P'ack-Beresfoili, 339.

PARKer (G. H.), 23, 290.

PiRKer, 49.

PitTen, 210.

Pitterson, 201.

PEARL, 11.

PÉrez (C.), 198, 384.

Pérez. (J.), 1, 104, 340.

Perkins, 61, 69, 340.

PellRis, 69.

Petrucci, 8.

Peyerimhoff, 190, 199, 200.

PEZARD, 237.

Phillips, 303.

Plath, 341, 342.

PICARD, 36, 56, 58, 66, 192, 299.

PIERON, 365.

Pietschker, 209, 210.

Planta, 236.

Рососк, 7.

Porsson, 200.

J'OPENOE, 6 Ul.

Popoff, 220.

Popovici-Baznosanu, 232.
PIICER, 245, 265.

Ribaun, 56, 96, 299.

RuINes, 365.

Rénumur, 1, 23, 289, 303.

ReEves, 5ó.

Неichenbach, 193.

Reichensperger, 277, 301.

Rruter, 35.

Riвot, 374.

Rich.ARDSON, 49.

Rickert, 19.

RiLey, 38, 50.

RoвsOn, 339.

ROCHER, 339.

Rohiven, 27, $32,59,60,62,63$, 129.

Roor, 35.

Rosen (von), 165, 214, 262, 381.

RoubAud, 57, 78, 82, 81, 95,

$116,118,155,196,281,302$.

RUUGiER, 377 .

Ruusseiu, 11.

Ruschiamr, 360 .

SILT, 3U2.

SiNnIIS, 164.

SiNтschi, 121, 360, 361, 36\%.

SiUlCY; 292.

Sirssune (de), 74, 78.

SCHIRFF, 149.

S.HIXEL. 19, 20.

SCHIEMENZ, 236.

SCHIMimen, 308, 331.

SCHIÖDTE, 329.

SCHNIEDEKNECHT, 36 .

S.н.IDT, 31.

SchïNitro, 236.

SCHNEIDER, 303.

SrIUn̈̈DR, $\approx 6$.

SCHROTTKY, 99.

SCHUCHERT, 1/8, 150.

Sсhulz, 36, 37, 54, 99.

SСHWARZ, 7, 15. 
Sicort, j1.

SCUDDER, 136, 16.

SEllirs, 23.

SenichoN, T, 104.

SEverin, 31.

SHIRP, 62, 223 .

Shelford, R., 308.

SHELFORd, V. E., 50.

Sichel, 340.

Sieber, 289.

Siebold (von), 54, 264, 303.

Silvestri, 50, 56, 57, 64, 116, $160,161,162,173,201,235$, $259,301,324,330,335$.

गHON, $6,7,8$.

SLIDEN, 292, 293, 339, 341.

Siitth, F., 32, 104, 121, 340, 341.

S.мiтн, H. S., $40,42,43$.

Silth, J. B., 104, 105.

SNYDER, 159, 164, 171, 178, 215, 258.

SPENCER, 371, 376.

SPRINGER, 201.

STIECKEL, 371 .

Staegrer, 347.

Staercke, 389, 390, 391.

Stefani Perez (de), 54.

STÖCKHERT. 104, 105, 107, 109 , $110,118,193,194,198,340$.

Strickiling, 56.

STUMPER, 253, 347 .

Sturtevant, 232.

SUNDWICK, 115.

SuRCOUF, 310 .

SWAMMERDAM, 289.

SWENK, 43.

SWEZEY, 66, 69.

TANNer, 193, 243.

Tanguary, ic's 360 .

TIRDE, 376 .

TIYLOR, L. H., 37.

TAYI.OR, G. W., 340.

THOMAS D'AQuis, 272.
ToSI, 112.

Тиompson, С. B., 16:2, 164. 209, $214,230,258$.

Thompson, J. W., 309.

Thompson, IV. R., 42, 69.

Thurstone, 9.

Tiligher, 377 .

'Tillyard, 28, 29, 44, 371.

TOWNER, 381 .

Trigirdi, 324, 326.

Trouvelot, 57.

TURNen, 63, 210.

ULMER, 144.

Vindervelde, 330.

VERHoefF, 104.

Viallanes, 210.

Viehmeyer, 121, 200, 252, 255, $256,354,357,359,365$.

loukassovitch, 58.

V RIES (nE), 258.

WADSWORTH, 51.

Wagnen, 341.

IVAlchenaen, 104, 340 .

WaLKeP, 54.

W Walker, E. M., 173.

Walsinghim, 7.

WarRen, $16 j, 167,318,320$. $322,324,326$.

Wasmans, $249,252,253,257$, $272,273,276,277,278,279$, 292, 299, 300, 301. 303, 31ні. $308,315,317,326,330-335$, $343,347,349,350,354,357$. $359,363,365,366$.

Waterston, 63.

WAXWEILER, 374.

WEBSTER, 56.

Wrismanx, 230, 231, 232, 261, 206.

IVESALAF, 310 .

IVESTWOOD, 36, 61, 62, 63, 
Whelien, Ю. II.. 14, 49. 53. Wille, 178.

IVheeler, G. C. 44

Villidis, 49, 64, 78, 79, 80.

W HEELER, W: И., 32, 38, 91, 94. 121, 1?2, $130,133,138$. 139. $119,193,240.242,243$, $? 46,247,249,252,263,273$, $?-28,281,283,288,302,303$ $307,308,340,346,347,3.57$ $3(6)$.

IVHTE, 304.

WIITING, 57. 192, 23:.

WIELAND, 96.

MOJSEDILEK, 233.

WORMS, 374 .

YerBerY, 309.

I U.1Si, 30,31 .

ZINDER, $107,236$.

Z.IEGLER, 209. 



\section{TABLE AII'ILABÉTIQUE IDES UITIËRES}

deamatus, 350 .

Acanthomyops, 281, 292.

Acanthomyimex, 205.

Acanthotermes, 21?. - militaris, 181.

Icariens, 138.

Acrocéridés, 50,52 .

Acromyrmex, 242. - oclospinosus, 308.

Acronycta lobelize, 38.

Ictivités cumulatives, 376 .

Aculéates, $14,23,25,26,55$, $59,61,90,95,128,141,154$, $155,157,170,174,198,214$, $227,256,260,269,270,370$.

Idelphogamie, 363 .

Adipogastrie, 284.

Adranes lecontei, 315.

Aëromyrma, 187.

Agapostemon, 104.

Agriotypus, 54.

Aleochara, 51, 5 ?.

Alyoëlla, 62.

Aliments proctodaaux, 184 . stomodaaux, 184.

Allodape, 14, 100-103, 104, 194, $303,340$.

Amaurobius ferox, 7 .

Imazones, 355.

Amblyoponini, 126.

Imiurus, 290.
Ammophila, 14.

- Amphérotoquie, 32.

Amphicténidés, 224 .

- Imphotérosynhesmium, 4.

Ampulicidés, 60, 62, 71.

Anacamptomyia, 302.

Anastatus, 192. - semillavidus, 57 .

Ancistrolermes crucifer, 329. Andrena, 92, 95, 99, 107, 114, 29.4.

Anelosimus socialis, 6.

Anergates, 204, 206, 361. - ritratulus, :207, 363.

Lnergatides, 361. - Kohli, 363,364 .

Aneurelus, 121.

Inisolabis maritima, 173.

Annélides, 224.

Anomina, $18 \overline{\mathrm{T}}$.

Anoplotermes, 184, 213, 217.

- reconditus, 329.

Anoxus, 66.

Antherophagus, 303.

Anthidium, 95, 383.

Anthoboscincs, 60, 62, (33, 1:8, $129,130$.

Anthonomus grandis, 12.

Anthophiles, $60,90$.

inthophora, 92.

Apunteles glomeratus, 190. - 
melanoscelus, 18?. - spurius, I!!:

Ipalhus, 3a1.

Apenesia, 61, 166.

Aphienongsler, 136, :'13, :3.8. Aphelinus fuscipennis, דi. muliluspielis, $\mathbf{i \pi}$.

Aphelopus melaleuras, 69. theliel, (i)!, TI).

Aphirlés, I 11. :38, :288, :37?, 37 ti.

Aphomia colonella, 303.

lpides, 60,89 el ser., 90, 91, (17, 122, 125, 126, 1:8, 196, 3il. - gastréleges, 103 . parasites, 91, 93.

Ajinés, 14, 100, 110, 114, 115, $116,-194,196,271,370$.

Apis, 14, 111, 112. - adamilica, 11:. - dorsala, 118. iloréa, 118. - méliponoides, 11?. - mellifiea, 111,116 , 118, 199. - Var. cuifru, 193.

- Var. intermissa, 193. proava, 112. - Iormquisti, 112.

- Apjélition, 9, $: \div 3$.

Apoica, 62.

Apocriles, 26 .

- Approvisionnement en masse.

11). -- progressif, III.

Aplerogyna, 130.

Apterogynidés, 128, 130. 14:. Aplerostiyma, 24?.

Arachnocoris albomaculalus. bi.

Araignées sociales, 5.

Archiglossatu, 37 .

Archihymen, 130.

Archimyrmex rostralus, 136.

1.37.

Archotermopsis, 165, Jut, 197,

217. - lornquisti, 165. - urou!ghloni, 16:2, 163, 161, 3:?.). ;i):?

Aregtyrodes nephillie, 6.

- Mrrienotoguie, 32.

Aspidiolus ostreieformis, $\overline{i t}$. rapux, ji.

Asthenohymen dumbari, :9.

istiela bulleli, 3:7:

Alelura formicaria, 3116.

Alemeles, ?49, 25il.

$1 / 11, ?(1) 5,213,295,292 .-c e-$ phaloles, 193, 308 - texuna, 318. - vollenuceideri, 210, 211.

Allaphila, $308 .-$ fun!jicola, 3018.

Attinés, 20, 182, 242, 2:3.

Augochlora, 105.

Aulacidia, 3i.

A:teca, 393. - joveolala, 15.

Bareogonalos canadensis, $3 \overline{7}$. Balruchedra slegodyplobius, 7 .

Belonogasler, 14, 8?-85, 117 11!), ?80, 30?.

Belonuchus mordens, 3013.

Bembex, 14, 99.

Bembiciné- $14,19$.

Bengalia, 309, 313. - depressa, 30!. - jejuna, 3in.

lulro, 309. - obscuripennis. 3en?.

Belhylides, 14, 27, 3i, 60, (i). (j3.) $71,73,131,191$.

lielhyloüdés 60, $71,199, \quad 132$, $19 \overline{7}, 3 \overline{7}(), 3 \overline{\mathrm{I}}$.

Belhylus, (61, 6i3, 3il. - julvicornis, lit. - juscicornis, (i)́.

- Bieconose.

Blillicles, 178.

Blitloüdes, 14, lli1, l6tj, 170, 173. 
Blennocampa pusilla, 192. Bombinés, 11, 100, 110, 114, $115,116,194,196,270,271$, :93.

Bombus, 14, 109, 111, 114-119, $208,289,341,342,383$. intiquus, 111. - carbonarius, 111, 116. - cayennensis, 116. - crassipes, 111. grandievus, 111 . - jurinei, 111. - pusillus, 111. vagjans, 341.

Bombusoides mengei, 111.

Boinbylides, 50.

Bothriomyrmex, 358. - decapitans, 360,361 .

Brachyacantha, 288.

Brachymyrmex, 205.

Braconidés, 34, 53, 111.

Braula coeca, 304.

Bruchomyrma, 361, 362.

Bulo empusus, 224.

Bythynus, 200. - algericus, 200.

Calliephialles messor, 53.

Calliphorinés, 309.

Calomyrmex, 146, 187.

Calotermes, 162, 164, 165, 166, $169,212,217,221,221,226$. - flavicollis, 384. - hubbardi, 185. - aeningensis, 165. - occidentis, 164 .

Calotermitidés, 13, 161, 162, $181,183,320,369$.

Calyptapis, 95.

Cambarus, 210.

Camponotinés, 122, 126, 127.

Camponolus, 134, 146, 187, 195, $? 00,205,223,245,249,252$, 386. - americanus, 69 . ullantis, 281. - cassius, 218. - caslanells, 281. jedschenti, 281. - jemora- tus, 316. - inflatus, 383. japonicus, 11-16. - ligniperda, 209. - maculatus, 281. - pennsylvanicus, 209, 24, 245. - velus, 136.

Capitoniidés, 34 .

Capritermes, 220, 221.

Cardiocondyla, 204.

Calebara, 187, 206, 207, 233, 344. - vidua, 345 .

Castanea sativa, 44.

Castration alimentaire, 237. - nutricielle, 196, 234.

Cataphractus cinctus, 54.

Cecidomyidés, 6, 201.

Celonites, 74, 96.

Cephalonomia, 66.

Cephidés, 30, 31, 141.

Cepobroticus symmetochus, 348.

Cerambycidés, 12.

Ceramius, 14, 74, 96. - lichtensteini, 74.

Cerapachyinés, 123, 126, 12 $\overline{\text {, }}$ $131,140,205,197,320$.

Cerapterus, 138.

Ceratina, 95, 100, 101. - cllcurbitacea, 98.

Ceralininés, 14.

Ceratopheidole, 205.

Ceropales, 339.

Chalastogastres, 26.

Chalcididés, 54, 141, 200, 216.

Chalcidoüdés, 34, 49.

Chalcobombus, 95.

- Champ trophoporique, 271

Chartergus, 14, 80.

Chémorecepleurs, 277, 2ï8, 291.

Chémoreception, 29:.

Chirononidés, 201.

Chloraliclus, 10.4 .

(horizops loricallis, 224. 
Chrysididés, $27,33,52,55,60$, 61.

Chrysopa, 42.

Cicada septemulecim, 12.

Cicinnus melsheimeri, 223.

Cimbicidés, 31.

Cionus thapsi, 57.

- Civilisation qualitative, 378 . quantitative, 378.

Cladrastes amurensis, 14. floribunda, 14.

Claciger testaceus, 315, 332.

Clavigeridés, $314,315$.

Cleptidés, 27, 60, 62 .

Cleptobiose, 344 . -

Clistogastres, :6, $32,33,55$.

Contrainte, 376 .

Coccidés, 140, 238, 288, 372, 376.

Coccidotrophus, 14, 18, 187. - cordix, 17. - socialis, 15-17.

Coccinellidés, 288.

Conothele gregalis, 7,8 .

Coléoptères, 14, 15, 26, 50, 52, $138,181,187$.

Colletes, 92, 91. - succinclus, 98.

Colobocrema, 223.

Colobopsis, 141, 221, 226, 266. - albocincta, 263, 265.

etiolata, 222. - stricta, 134.

Colobostruma, 223.

Colonies mixtes, 313, 349.-

Comedo, 58.

Conocephalus fasciatus, 42.

Conopidés, 302, 303.

Coopération, 376.

Copidosoma gelechiæ, 192.

Cordia alliodora, 17.

Corotoca, 325. - melantho, $327,328$.

Corpora pedunculata, 208-210, 214.
Corynura, 104.

Copris, 14.

-Coprophagie, 286.

Coptotermes, 166. - ceulonicus, 219.

Cosilidés, 62 .

Crabro, 95.

Crabroninés, 95.

riralolechus, 58.

Crematogasler, 131, 115, 205, 992, 311, 312, 358. - alligaIrix, 311. - parabiolica, 346. Crioceris asparagi; 57.

Crustacés, 35.

Cryplobiose, 185.

Cryptocercus, 173. - punclulatus, 170.

Cryplocerus, 223.

Cryptophagidés, 303.

Cryplotermes, 162, 211, 226.

Crypturus argiolus, 302 .

Ctenoplectrella, 95.

Cubitermes, 184.

Cỵclotorna, 314. - monocentra, 312.

Cyllene, 72. - picla, 67.

Cynipidés, 34, 141, 191.

Cynipoïdés, 49.

Cyphodeira, 305.

Cyphomyrmex, 242.

Cyrtomyrma, 147.

Dasypoma, 14.

Dendrolasius, 360 .

Dermaptères, 14, 170, 172, 173, 214.

Dermestes, 66.

Dermestidés, 303 .

Développément convergent, 373.

Diacamima, 62, 200.5.

Dianthidium, 95.

Dicxlotus erythrostoma, 58.

Dichrogéniilés, 60. 
Diclyna muraria, 7.

Dielocerus, 32.

- Dimorphisme, 190.

Dimorphomyımex, 137, 138.

- Dinergates, 205.

Dinomyrmex, 116.

Dinoponera, 205.

Diploptères, 60, 73, 100, 128.

Diptères, 27, 35, 50, 178.

Discoxenus lepisma, 323.

Disticta capritermitis, 327.

Ditrichomonas termitis, 322 .

- Division du Iravail, 381 .

Dolichodérinés, 122, 123, 126,

$127,136,140,145,2.13,283$,

$320,383$.

Domestication, 390. -

Dorylinés, $122,123,125,126$, $127,130,131,137,140,145$ $148,197,205,274,306,320$, 3.) 0,383 .

Doryloxenus transiuga, 323.

Dorylus, 233, 306, 386, 387. helvolus, 203. - nigricans, 310.

Dorymyrmex pyramicus, 344 .

- Doulose, 351, 365.

Dryinidés, 55, 56, 60, 61, 63, 69, 71.

Echinopla, 146.

Eciton, 62, 187, 200, 292, 306, 3ก๊0. - burchelli, 350. - hamalum, 350.

Ectatomma, 248.

I:ctalommini, 126.

I:lasmus flabellatus, 56.

Electrapis, 95, 112. - lornquisti, 112.

Electrotermes, 165.

Elis, 131. - 4-notata, 72.

limbidaria, 14.

Embiidinés, 170.

Embotemidés, 60, 62 .
Emenadia flabellata, $30 ?$.

Endostomus sudanensis, 3?3.

Enléléchie, 23.

l:olormica eocenticu, li36, 137.

Incira bandelieri, 6.

lipeolus, 93.

Ephedrus incompletus, 53.

Ephemerides, 12.

Ephestia liuehniella, 56,5 .

liphialles extensor, 30?.

liphiallites, 34. -- jurussicus, 28.

Epigénise, 228.

lipiphridole, 361. -.. inquilnir, ?ót.

Epiponinés, 14, 74, 80, 84, 89, 194, $199,271$.

l:poecus, 206, 361. - pergandei, 364 .

Eptyris, 62-64. - extranells, $64,65$.

- Ergatandromorphisme, 263.

- Ergatogynes, 25.3.

Lisclavage, 351 et seq.

Eucamponotini, 146.

Fucera, 99, 113, 287.

Eucharides, 45, 48, 50, i1.

Eucharinés, 246.

lithcharis myrmecix, 14.

Eucondylops lionowi, 310.

liuglossa, 99. - nigrila, 99.

- surinamensis, 99.

Fulixma, 99.

liumenes, 78, 302.

Eumenidés, 20, 63.

Eumeninés, 14, 74, 78, 132, 199.

Eumorpha, 99.

Eunausibius, 14, 18. - wheeleri, 15-17.

Euparaginés, 74, 75.

liuplectrus bicolor, $57 . \quad-$ comstoclit, 57.

Euponera sennarensis, 186.

luupsenius clacicornis, 323. 
Eusphinetus, 131.

lintermes, 161, 168, :18, 258,

:T\{. - aquilinus, 213. - bivalens, 213. - castancicaps, I13. - chaquimayensis, 209. cyphergaster, 213. - diversimiles, 213. - fossarum, 16i). - fraasi, 166. heleropterus, 213. - hospilalis, 213. - lacustris, 216. - monoceros, 182. - morio, 216. - pilifrons, 216. sanchezi, 216 . - tenuirostris, 213. - trinercius, 179, 213 . - velox, 213.

Evaniidés, 34, 53, 141.

Evolution émergente, 23.

Exorista lobelix, 38.

Farqutharsonia rostrala, 311. Fedschentia, 130.

Fedschenkiidés, 128-130, 142 . Forficula auricularia, 171-173. Forficulidés, 14.

Folmica, 14, 119, 136, 249, 251, ?5t, 352. - aserva, 352, 354. - cinerea, 350 . - consociruns, 358. - eoptera, 136. exsecla, 359,360 . - exsectoides, 301, 359. - flori, $14 \overline{7}, 262$. - fusca, 147, 262, $333,350,352,353,354,358$, 360,365 . - heteroplera, 134. - microgyna, 358, 359, 360.

- neogagates, 350 . - obscuripes, 359. - pallidefulva, $3 \% 0,358,360$. pralensis, 317,359 . - rufa, 253, 347, $359,360,366$. - sanguinea, $219,251,252,279,312,316$, $317,332,333,350,352,353$, 354,366 . - schaufussi, 209, 210 .

Formicides, 14, 60, 62, 90, 121 el ser. 1:9, 141, 14: 143, 14i, 148, 149, 197, 198, :?14, ?(j1, 370, 383.

Formicina, 1:?).

Formicines, 122, 123, 126, 127, $138,110,145,213,383,3 ?$, 383.

Formicoxenus, 201. - nilidulus, 253, 317.

Fonisseur's, (3), 92, 93.

Fremddienlichkeit, $273,335$.

Fulgoridés, 69, 288.

Galertucella xanthomelıxna, jī.

Gialérurue de l'orme, 51, 57.

Galleria, mellonella, 289, 303.

Gesomyrmex, 138, 1:16. - chaperi, 138. - howardi, 11ti.

Giardinaia urinalor, 5'.

Gi!jantiops, 1't6.

Gigantisme, ?04. -

Giluplapis, 95.

Glyptus punclulatus, 3:6.

Gonocephalum serialum, 6', 65.

Foniozus, 66.

- Girégarisme, 3 .

Grylloblalta campodeiformis, 173.

Grylloblatlaria, 173.

Grylloblattoüdés, 170, 17! .

Gryllotalpa, 14.

Gyniecoïles, 195, 259.

Cynandromorphisme, 26:3.

Mabrobracon brevicorne, 19?. - johanseni, 57.

Habrocytus cionicida, 57, 58. Hiematopsenius caudalus, 323. Haliclinés, 14, 194, 196, ?70, 303.

Halictus, 14. 92, 100, 104-110. $115,117-119.193,194,19 t$, 
19?. 2923, 340, 341, 393. - cal. cealls, 105,108 . - clavipes, 107. - c!nlindricus, 105. erinnys, 101. - jodiens, 111.. - graminea, 101. humeralis, 10), 105. - immaculatus, 109. - immar!ninalus, $10 \%$. - lineolalus, 11ii. - longulus, 108, 10!. - maculalus, 107, 109. malachurus, $107-109, \quad 111$, 199. 311. - morio, 107. puixillus, 109. - pruinosus, $30 \%, 301$. puncticollis, 107 , 109. - quadricinclus, 105, 1119. scabiosie, 105, 110. sexcinclus, 105-166, 109. villosulus, I0T, I0!).

II amamelis viryinica. $\{?$.

II armolitu, jti.

Harpagomyja, 311, 311. - [arquharsoni, 311.

IIarpa!!oxenus, 198, 35:2, Зร̄. - americanus, 3.5. - sublievis, $35 \overline{5}$.

Homileuca olivie, $5 \bar{T}$.

Hémipteres, ?01\%.

llemileles areutor, 192. - julvipes, 19:. - longicauda, 192. - tenellus, 192.

Ileriades, 95.

Helérochromalisme, I90.

Uélérogonie, 190, $] 99$.

Helerogynà, $601,63,128,132$, 140, 142.

Heléromorphose, 190.

Hétéropteres, 1'́.

Hèlerosymphagopiedum, 4.

Helerosymporium, 1.

Ilètérosynepileium, 1.

Hicolea, $6 \overline{\text { I }}$.

Ilieracium, 108.

II:stéridés, 30ti, 314.
Hodolermes, 161, 165, 182, ?74. - Inrlieslanicus, 17ij.

Holcuspis cinerosus, 2.2.2.

IIoleptyris, bis, bí. - hrutcuiensis, tí.

Homoptèes, 69, :Т4. :288, 318, ;i:?.

IIylieodes, 94.

II!jmenochimara abnormis, 6:. IIyménopteres, 14, 18, 23, 25, 178.

- Hypermélamorphose, 51.

- IIypertélie, 335.

Hypharnosymphilie, 305.

Hyphantria textor, 10.

Hypoborus ficus, it6.

Hypoclinea beluberculata, 310, 311.

lchmeumonidès, 1:1l.

Ichneumonoïlés, 53.

Idiograsler escherichi, :3:7.

Idiogastres, :7.

Imitation, 376.

Infusoires, 35 .

- Insectes atrophaptiques, 10 ,

- 5i.3. - eutrophapliques, 11.

- dystrophapliques, 10, 53.3.

- - infrasociaus, 11. - quia-

- si-sociaux, 11. - subsocialux

- II.

Instincts, ?it. _ symphiliques, 2666, ?77.

Ipides, 1/, 18, 223.

Iridomyrmex, 205. - !nppperti, 138. - gracilis, 350. humilis, 395,397 . - san!nuinells, 187, 31:2.

Ischnomyrmex, 205.

Isoptères, 14, 18, 159, 166, 171), $173,178$.

Jacobsonella termilobia, 3?T. Jassidés, 69.

Juënopsis polytricha, 32.2. 
Knouiella, 130.

l.ubrocerus yoryi, 316.

Lasius, 281. - bicornis, 360. ciliulus, 360. - llavus, 332. - fuliginosus, 360 . latipes, $360 . \quad$ minutus, 360. - mixlus, 307. - murphyi, 360. - néoniger, 360. - niger, 360. - sitkuensis. 360 . - subumbratus, 360 . -nimbratus, 360.

Leptothorax acervorum, 357, $361 . \quad-$ curcispinosus, $35 \overline{\text {. }}$

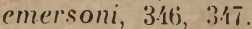

- Lestobiose, 311.

L.estrimelitta, limao, 3.12.

liphyra brassolis, 314.

lobopelta, 309.

- Lomechusa, 279, 316. - strumosa, 316, 317, 332, 333.

Lomechusinés, 314, 315 .

- Lifcana arion, 313, 314. Lycrenidés, 314, 318.

Maldanidés, 22:4.

Mâles eumégétiques, 200 . - hétérodontes, 200. - epi-

- mégétiques, 200. - macro-

- gnathes, 200. - hypomégé-

- liques, 200.

Mamestra brassica, 57.

Manniella, 223.

Mantidés, 178.

Mantispa, 50, 52, 302.

Masaridés, 73, 74, 371, 372.

Masaridinés, 14, 74, 75, 96.

Masaris, 74, 96.

Mastotermes, 160-163, 165, 166, 217. - anglicus, 165. - batheri, 165. - bournemouthensis, 165. - croalicus, 16... - darwiniensis, 161, 164,260 .
Yastolermitidés, 161, 183, 369

Mecoptères, 29, 37, 52, 371.

Mecopteroüdés, 17'́.

Meguchile, 92, 10:3, 11:2.

Menulomyrmex, 3:17.

Megalopleres, 5\%.

Végallyrides, 53.

Me!rustilicus formicarius, 304. Velanophthalma, 8.

Melasoma interruplum, 57.

Velipona, 14, 11\%, 118, 23ॅ, :30.3.

Meliponines, $13,14,100,110$ $11: 111-116, \quad 19.1,196,210$, :34, 235, 271, 292, 38.3.

Veliponoryctes sicula, 112. succini, 112.

Melissoblaptes anellıs, 302.

Velioma, 99.

Melillobia acasla, ¿8.

- Melillophiles, 299.

Velliferes, 90.

Veloë, jl, 301.

Meloüdés, 49, 52, 303.

1/elophorus, 195. - ba!joti, :83.

Meinbracidés, 69.

Menobranchus, 171.

Meraporus, 57.

Meria, 61.

Mermis, 218, 249, 262, 318 . albicans, 304. - myrmecophila, 248. - mermithogy- nes, 248.

- Mermithergates, 248.

Mesitius, 61.

Mesostenus gladiator, 302 .

Mesotermitidæ, 160, 161.

Messor, 145.

Metaphrénie, 399.

Métatermitidès, 160,162 .

Méthocidés, $60,128,130,1: 12$.

Methocinæ, 129.

Melocus paradoxus, 302. 
Melopina pachycondylæ, 307. Vliastor, 201.

Mierobacon juglundis, 56, 57. Microdon, 303, 305.

Microgaster, 192.

Microgynes, 253.

Vicromalthus, 187.

Milichia argyratoides, 311. decles, 311. - prœcles, 311. prosztes, 311.

Milichiidés, 314 .

. limétisme, 273, 335. -

Minotaurus, 14.

Miotermes, 165.

Mirotermes, 183.

Nonogamie, 116.

Monogynie, 116, 118 .

Monométrose, 116.

Monomorium indicum; 309. minimum, 361. - salomonis, $362,364$.

Morus ulbu, 44.

Mosquero, 8.

Muscidés, 324, 325 .

Mutilla, 129. - brutia, 302. littoralis, 302.

Mutillidés, $37,60,61,128,130$, $132,141,142,144,303$.

Mycétomélochie, 349 .

Mymaride, 54.

Myrma, 146.

Myrmecia, $138,-386 .-$ forfi.. cata, 44. - yulosa, 44.

Mrrmécocleptiques, 306.

Miyrmecocystus, 117, 119, 195, 283. - bombycinus, 205. horli-deorum, 284, 285. vialicus, 308.

V!yrmecophila, 308. -- nebrasrensis, 307.

Myrmécophiles, 299, 304.--

Myrmecopterina, 130 .

Myrmedonia, 305, 327, 328.

Myrmihopla, 147.
M!̣ınica, 136, 249, 252. - catnadensis, 253-254, 347. lavinodis, 313. - ruginodis, 26.4.

Myrmicinés, 122, 123, 126, 127, $140,145,205,243,281,283$, 320 .

Myrmorhachis, 146.

Myrmosidés, 60, 130, 112 .

Myrmothrinax, 147.

Myrmoturba, 146.

Mystrocnemis, 62.

Myzine, 62, 72, 131.

-Nanisme, 20\%, 333.

Nasonia brevicolnis, 57.

Nasutitermes, 183 - caviirons, 330.

Nausibius clavicornis, 303.

Necrophorus, 4.

Vectarina, 80, 8'. - leche!luana, 73.

Necturus, 171.

Nèmestrimités, 50 .

Neolarra pruinosa, 93.

Veomyrma mutica, 347.

Neonuyrmamblys, 223.

Veoponera, 145, 248. - commutata, 186. - crassinoda, 186.

Neoscleroderma, 66 .

Néoténie, 190.

Nephilla plumipes, כ.

Vephropleryx edmondsi, 303.

vereis virens, 210.

Névropteres, 50, 52.

- Nids composés, 3.13.

Vomada, 93. 129.

lomadina cisandina, 37.

Notoptères, $170,173$.

voromessor, 145.

Vurthia aurivillii, 30\%.

Nysson, 339.

Nyssoninie, 95. 
Ochromyia, 309.

Ocymyrmex, 205.

Odontolabinés, 200 .

Odonlomachus, 2'18.

odonlolermes, 212, 213 . prisllinus, 166.

Odynerus, 14, 71, 75, 94, 302. - dorsalis, 75. - simulalor, 302.

(Nicanthus, 178.

A:cophylla, 137, 146, 147. barloniana, 134.

- Otcotrophoniose, 280.

(K:diprosoma mirandum, 328.

Winothère, 258.

Oligarces, 201.

Oligomyrmex, 205, 206, 283. -.. panamensis, 206.

Onychomyrmex, 198, 283.

Opalina, 322 .

Ophion macrurus, 38.

Opisthopsis, 146, 187 .

Orasema, 47, 252. - viridis, $38,39, \mathbf{1} \overline{7}, 216$.

Orocharis saltator, 62 .

Orlhoptera, 14.

Orlhoptères, 17'́, 178.

Orthopléroïdés, 174, 178,211

Oryssidés, 2̄i $30,31,33,3 \bar{\jmath}$, $3 i: 2$.

()ryssus, 31.

Osmia, 99, 111, 193, 23:2. inermis, 113.

Oxysoma oberlhüri, 308.

Puchycentris, 99.

Pachycondyla, 2:18, 281. monlezumia, 307 .

Pachymelus, 113.

Pachyssima, 330. - ielhiops, 282. - Ialifrons, 281, 282.

Piedalyus, 187, 206, 281.

- Pardogrenése, 190.

Pulirobethylus, 6?.
Paléodiclyoptéroüdé:, $2 \overline{7}$.

Palthothyreus tarsalus, 186.

Pamphiliidés, 30, 1'11.

l'anesthia, 1i.

l'aniscomima erlan!feriuna, (0:?.

Panorpes, 29.

Panorpoïdés, 28.

P'antaplalarthrus natalensis, 316.

Panur!jus, 99.

P'aracharteryus apiculis, 37.

Parachrysis, (61.

l'arrcolobopsis, ?2:3.

l'aracopidosomopsis [loridanus, :2()].

l'alacoroloca, 3:!'s. - aliermani, $32 \bar{i}$.

Paraponera, ?:8. -- clavala, 186.

Parascleroderma, bi.

Parasierola, 66 .

- Parasites, 35, 300. - liumains,

- 338. - protéliens, 35. - sociaux, 337, el seq. - socianx, permanents, 351 sociaux temporaires, 351 , 358.

Parasitoïles, 35, 361.

Parocisme, 9!, 251, 3.16.

Parolermes, I6j.

- Parthénogénese, 32, 190, 19]. thélyto(que, 106, 191. amphèrotoque, 191. - arrhénolorpue, 100, 191.

Passalidés, 14, 370.

Prtssalus, 14, 18̄ 188.

Paussidés, 138, 314.

Puussus clamu, 316. - hovu, 316. - spiniceps, 310.

Pelecinidés, 111.

Pelecinoüdes, 26.

Pelecinus, 20.

l'emphredon, 6.'.

Pemphreclonine's, 95. 
Pentamorphisme, 213.

Perena, 14.

Perilampides $\quad 18,49,51$.

Perilampus, 12, 13, 17.

chrysopie, 43. - hyjalinus, 40, 41, 44.

Perisemus, 63.

Permohymen schucherli, 29.

l'erophora sanguinolenta, 223.

Pélioliventres, 26.

Pe:omachus flavocinctus, 56. Pheidole, 17, 205, 206, 218, $283,292,332,363,386 .{ }^{\circ}$ absurda, 248, 249. - colobopsis, 223. - commutata, 2'17, 2'8. - indica, 218. inslabilis, 38, 39, 202, 246. - lamia, 206. - megacephala, 395, 396. - militicidil, 275.

Pheidologelon, 137, ?05, 218, $245,260,283$. - diversus, 309.

Philanthinés, 95.

Philanthus, 99.

Phileremulus, 93.

Philotrypesis, 56, 200. - erylhriea, 200. - minula, 200. - unispinosa, var. ornata, 200.

Phoriclés, 303, 305.

Phragmose, 225.

Threnapates, 1'i, 187, 370 .

Phthisergates, 246.

Phthisogynes, 217.

Phlhisodinergales, 217.

Phullodromia germanica, 179.

Phylogénie, 19, 20.

Plyyracaces, 350.

Physocrema, 131.

Physogastric, ?85, 327,330 .

Phylophages, 26, 33, 55, 95, Polygamic, 116. 111, 37i), 3i1.
Pieris rupx, ī.

Pison, 95.

Pissodes strobi, (it.

Places vides, 299.

Plagiolepis, 195.

Planirlium, 39-11, 43, 11.

Patyarthrus holfmannseggi, $30 \overline{5}$.

Platygastridés, 49.

Platypodidés, 14, 18, 2:23, 371.

Plat!ypus, 14.

Platythyreini, 126.

- Pléoinétrose, $116,117$.

- Pléomorphisme, 200.

Plérergates, 195.

Plésiobiose, 346.

Pleuroplerus, 138. - brevicornis, 316.

Pleurotropis, 19:.

Plumariidax, 60, 128, 129, 142.

Plumarius, 129, 130.

Plusia gamma, $\overline{7}$.

- Poecilandrie, 190, 199, 200.

Pocilochroa convictrix, 8.

- P'œcilogonie, 190, -ul.

- Poecilogynie, 190, 199.

Pogonomyrmex, 1/5, 341. molefaciens, 307.

l'olistes, 1', T'4, 85, 117, 280, 302 . - canadensis, 37. metrica, 280.

Polistinés, 14, 74, 85, 89, 90, 194, 270, 271.

Polislomorpha, 302.

Polybia, 74, 82, 81, 117, 302. - alra, 82. - dimidiala, 37.

- p!y!mæa, 308. - vicina, 303.

Polybiinés, 74, 80, 271.

32\}, 326, - l'olybioüdes melana, 82.

l'olychrosis, bolrana, is.

Polyergus, 206, 352, 354, 3506, 
357, 366. - lucidus, 355. sumurai, 355.

- Polygynie, 116.

- Polymorphisme, $\check{5}, 56,189$ el seq., 381.

Polynoë, 210.

Polyrhuchis, 146, 147, 187. bicolor, 305. - dives, 44.

Polysphincla, 6, 56.

Polyzosteria, novæzelandiæ, 171.

Pompilidés, 57, 60, 71, 128.

Pompiloïdés, 60 .

Pompilus aculealus, 339. campestris, 339. - pectinipes, 339.

Ponera, 198. - eduardi, 204.

- hendersoni, 136.

Ponerinés, 13, 122-125, 127, $138,140,148,197,205,243$, $320,383$.

Porotermes, 169.

Prays oleellus, 56.

Prédateur's, 35.

Prédéterıninalion, 228 .

Prenolepis, 195.

Prestwitschia aquatica, 54.

Prionomyrmex, 138. - longiceps, 139.

Pristocera, 62, 63. - armife$r a, 61$.

Proceratini, 126.

Proctotrypidés, 61, 62.

Prodorylinx, 126.

Proëlectrotermes, 165 .

-Professions, 381.

Proponerinse, 126.

Prosatomilichia murmecophi-

$$
\text { la, 312. }
$$

Prosopis, 92-94.

- Prosymphyles, 30, 31.

Prolermes, 160.

Protermitidés, 160, 161.
Protoblatloïdés, 27, 161, 166, $188,269,369,371$.

Prolobombus, 95, 111.

Protodermaplères, 29.

Protoliymen permianus, 29.

Protohyinénoptères, 29, 371.

Protolimules, 211.

Provespa, 85.

P'rolozoaires, 182-184, 257, 286, $322,369$.

Psæenythia, 93.

Pseudochrysalide, 5l.

I'seudococcus brevipes, 15.

Pseudocolobopsis, 223.

P'seudogonalos hahni, 38, 18. Pseudogynes, 251, 317.

Pseudolasius, 137.

I'seudomasuris, Tí, 96.

I'seudomyrma, 145. - crucians, 15. - elegans, 145. - gracilis, 239. - maligna, 15.

Pseurlomyrminés, 123, 127, $145,197,205,240,242,281$, 320.

- P'seudoparasitisme, 35.

I'seudosirex, 28.

Pseudolrichon!mmpha prislina, 322.

Psilogaster fuciiventris, 44.

I'sithyrus, 341, 342. - laboriosus, 341 .

P'terodontia ilavipes, 50.

Pleromalus puparum, 57.

Plerombrina, 129.

Plery!joyenea, 20.

Plilocerus ochraceus, 310.

Plochomyia, 326, 330. - afra, $3: 9$.

I'y!gostenus pubescens, 323.

P!n'sonympha, 184.

\section{Quedius dilatatus, 30 ?.}


Ruclobitenidie, 1:99.

Regime aphidicole, 124. - enlomorphage, 12:. - mycé(1)phage, 124. - spermatopliage, 1?4.

Reticulitermes, 166. -. Ilavipes, 166, 183, 181, 214. lucijugus, 166, 216, 271.

Rha!fium lineatum, '211.

Rhinopsis, 6?.

lihinolermes, 2t:? - laurus, ?1?.

Rhinotermitidés, 161, 183.

Rhipiphorides, 19.

Rhogmus, 187.

Rhopalomastix, 124.

Rhopalosoma, 63. - poeyi, 62 . Rhopalosomatidés, 27, 57,60 , $62,63,129$.

Rhynchopsilota apicalis, 312 . Rihytidoponera, 205.

Ropralidia, 14, 84.

Ropalicliinés, 14, 94, 194.

Rumination sociale, 185.

Salıanea, 171.

Sap!nga quinque-punclala, 1:9. - similis, 129.

Sapygidés, 35, 55. 60, 63, 128, 1?9), 141, 142.

Sarcophaga, 12.

Scurabixidx, 14.

Scelephron cementarium, 129. - spirifex, 74.

Schizaspidia, 47. - polyrlinchicida, 11. - tenuicornis, 低-46.

Schizonolus sieboldi, 56 .

Seleroderma, 14, 66, 72, 73 , 132, 371. - immigrans, 67. - macrogaster, 67-69.

Sclerogibuidx, 60, 62.

soCIETES D'NSECTES
Scoliidés, (j)-(i:), $72,128,131$, 1.14. 371.

Seoliinie, 129.

scolioüdés, 127.

Scolytidés, 18, 141, 142, 293, 370 .

Scolocryplus, 303.

- Selection amicale, 366.

Seminola depressa, 37. - mejicana, $3 \bar{i}$.

Sericomyrmex, 212. - amabilis, 317-319.

Sessiliventres, 26 .

Sicilomyrmex corniger, 136.

Sierola, 66.

Sierolomorpha, 62.

silphidés, 303.

Silvanides, 14.

Siphonophores, 380 ).

Siricidés, 30, 31.

- Sociétés (mort des) , 393.

- nutricielles, 380. - reproductives, 380 .

Solenopsis, 119, 206. -- geminata, 238, 260. - saevissi$m a, 260,305$.

- Soldats mandibulés, 212, 213, 217. - nasuli, 212, 213, 216, ?18.

Sophrobombus, 95, 111.

sphécidés, 60, 70), 92, 93, 95, $97,128,132,141$.

Sphéciformiés, 60 .

Sphécinés, 14, 95.

Sphecodes, 92-94, 106, 310. subquadratus, $3 \mathbf{1 0 .}$

Sphecoïdès, 60, 93, 91, 370372.

sphecophila polybiarum, 308. - termitium, 308.

Sphécophiles, 299, 301.

Sphex procerus, $70,71$.

Sphinctomyrmex, 131.

Spinoliella, 99. 
Spirachtha, 282, 325. - eurymedusa, 327-329. - mirabilis, 32 ?.

Staphylinidés, 303, 304, 306, $324,326$.

Staphylins, 51 .

Stegodyphus, 7 .

Slenogaster, 11. - micans var. luzonensis, 81. - varipictus, $80,81$.

Slenogastrinés, 14, 74, 89, 194. Slephanidés, 53, 141.

Slizus, 99, 339.

Sirepsiptères, 49, 301.

Strong!nlo!nalhus, 206, 352, 356,366 . - alpinus, 356. huberi, 356. - rehbinderi, 356. - lestaceus, 356.

Sublimation, 390.

Sipperentéléchie, 23.

Superorganismes, 375.

Stjcogaster lavagnei, 56.

Stymm!ımica, 204. - chamDerlini, 347.

Sympheidole, 361.

Symphiles, $300,322$.

- Symphilie, 314, 334, 366.

Symphilö̈des, 314 .

Symphotium, 4.

- Symphyies, 26.

Synngris, 11, 89. - callida, 78. - cornula, 76. - spiniventris, 78.

Synechthres, 299, 304, 322.

- Synœcètes, 299, 305, 322.

Syntermes, 212.

Tachigalia paniculata, 15, 17. Tachinidés, 50, 283, 302, 303. Tapinogonalos pulchella, 38 . Tapinoma, 292, 293. - nigerrimum, 361. - sessile, 362.

Telea polyphemus, 38.

Tenebrionidie, 14.
Tenthredella, 3:.

Tenthredinides, 1', 30-3:, ;:, 141.

Tenthredo, 32.

Tenturia, 72.

Terebranlia, 111.

Térébrants, 25, et seq., 33, 5\%, 5), 95, 129, 15. 191, 371), $371,372$.

Termes, 13, 161, 1ti.1, 168, 18:2, 181, 212, 274. - bellicosus, 326. - croaticus, 166. - errabundus, 161. - incerlus, 179. - latericius, 175, 18?. - natalensis, $175,17 \%, 179$. -- obesus, 329. - obscurus, 166 . - redemanni, :85.) - vulgaris, 17\%.

Termites, ] 33 el ser., 25t. 269 , 38.1.

Termitides, 14, 161, 181, :36!.

Termilobia physonastra, 327.

Termitobracon emersoni, 318.

Termilodiscus. 324. - heimi, 323. - splendidus, 323.

Termitomastus leploproctus, 329.

Termitomimus, 325. - entendeeniensis, 3:7.

Termitomyia, 326, 330.

Termilonannus major, 323 .

Termitoniscus fulleri, 323, 321.

Termilophia heyeri, 32i.

Termitophiles, 299, 318 el ser., 32:).

Termilopsenius limulus, 32:;.

Termitotecna braunsi, 32:

Fermilotima assmulhi, 3:27.

Termiloxenia, 326, 330. 335, 336. - heimi, 329.

Termopsis, 162-166, 173, 18:3, $197,212,217$. - anguslicollis, 163. - swinhoei, 165. 
Telralonia, 113, 287. - ruii- - Trophallasis, 24, 269 el seq., collis, 988.

Telramorium, 119. - crespi- Trophidia, 282.

lım, 207, 3.76, 35i, 363-365. - Trophobiontes, 304, 318.

Tetrastichus usparagi, 57. - - Trophœcisme, 280.

ranthomeluenix, 57 Trophocium, 281.

Thuumatorena wasmanni, 323, - Trophothylax, 211. $3: 4$.

Thelia bimaculala, $69,70$.

Thélyloruie, 32 .

Theridion, 6.

Termophilie, 112, 1.13.

Thumalus fulgidus, $\mathbf{i} \overline{\text { T. }}$.

Thymnidés, $60,72,128-130,132$. $14:, 141,145$.

Thulreoxenus pulchellus, 328.

Thysanoures, 306.

Timeparthenus, 326,330 . renius, 327.

lincidés, $305,325$.

Tiphia, 61, 64, 7:2.

Tiphiides, 60, $62,72,128,131$, $1: i 2,371$.

\%omocerus, ?11).

Torllix, 64.

Trueluusa, 383.

Trarhymyrmex, 242.

Triucrus superbus, 302 .

Trichobius apiarius, 304.

Trichogrammide, 54 .

Trichomes, 366 .

Trichomonadés, 322.

Trichonympha, 18!.

Trichoscelia, 302.

rrichoptères, $5 ?$ ?

'Tridactylidés, 174.

Tripona, 11, 111, 113, 235, 292. - limao, 3\%?. - ruficrus, 303.

Trigonalides, $27,33,36,37$, $49,50,51,342$.

Trigonalys pervelus, 38 .

Trimorphisme, 213.

Trupoxylon, 14.

Trypoxyloninés, 14.

Tuhuliteres, $60,61$.

Typhlocyba rosæ, 69.

Typhlopone, 187.

Tilphon vesparum, 302.

Lloborus, b. - republicanus, 6. 7 .

Varichiela aldrichi, 41, 42.

Veramessor, 14ti.

lespa, 14, 85, 86. 89, 109, 116$118,208,280,320$. - arclica, $339,340,312,361 .-$ austriaca, 339, 340, 342, 361. ronsobrina, 340 . - diabolica, 340. - germanica, 302. occidentalis, 37. - rufa, 339 - vulyaris, 302.

1 espides, 37, 55 et seq., 60 , (j2) $73,74,89,90,96,97,100$, $122,125,126,128,141,191$, 196, 234, 281, 301, 370 .

lespilormies, 60, 128.

Vespines, 14, 74, 85, 90, 194, $: 70$.

Vespoildes, $60,61,128,142$, 197, 370-372.

Viticicola lessmanni, 2:2.

Volucella, 302, 303. - bombylams, 303. - caucasica, 303. - llava, s03. - bremorrhoidalis, 303. - inanis, 302. plumata, 303. - Eonaria,

Troyorlerma larsale, 233. 30 ?. 
- Jenubiose, 346, 3:17. - Senorlusa, 249, 251. - cavu, 25 (1).

Xenongaster inflata, $32 \overline{\text { in. }}$

Xenos, 302.

- Xorophilie, 142, 143. Veslotermopsis, 165 .

Xiphydriidés, 30, 31. Xýelidés, 30, 31.

lyloliorus, 14.

Villocopa, 95, 113.
Ypsisterocerus, 38.

Zasphinelus, 131.

Zethinés, 14, 74, 78-80, 89. 113. Zeluus, 14, 302. - cyanoplerus, 78,79 . - romanndinus, 78.

Zethusculus lobulatus. 79. Zoraptera, 1's. Zoraptères, $170,173$. Zorolypus, 173. 

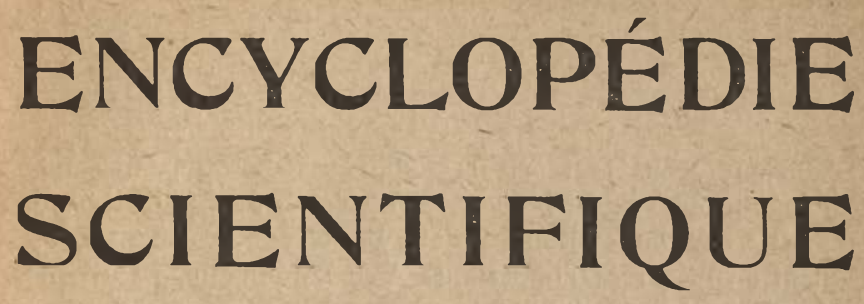

||I|||||||||||||||||||||||||||||||||||!||||||||||||||||||||||||||||||||||||||||||||||

\section{CATALOGUE \\ DES OUVRAGES PARUS \\ 25 JANVIER 1927}

PRIX NETS

Catalogue annulant les précédents.

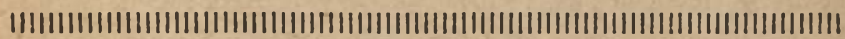
GASTON DOIN \& C Cie, ÉDITEURS, A PARIS Registre du Commerce Seine $n^{\circ} 3895$;

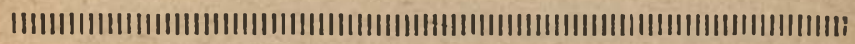


Nous avons entrepris la publication, sous la direction générale de son fondateur, le $\mathrm{D}^{r}$ Toulouse, directeur à l'École des Hautes Études, d'une Encyclopédio scientifique dont on mesurera l'importance à ce fait qu'elle est divisée en 40 sections ou Bibliothèques et qu'elle comprendra environ 1000 volumes. Elle se propose de rivaliser avec les plus grandes encyclopédies étrangères et même de les dépaszer, tout à la fois par le caractère nettement scientifique et la clarté do ses exposés, par l'ordre logique de ses divisions et par son unité, enfin par ses vastes dimensions et sa forme pratique.

LES VOLUMES, ILLUSTRÉS POUR LA PLUPART, SONT PUBLIÉS DANS LE FORMAT IN-16.

DiRgCTRUR : D' TOULOUSE.

Secretairi général : H. PIÉRON.

SEcretaIRE POUR LES SCIENCES TECHNIQUES: L. POTIN. 


\section{PATHOLOGIE MÉDICALE}

Directesir : Docteur M. KLIPPEL, Médecin des Hópitaux de Paris.

Les Maladies du Péritoine, par le Docteur A. Pissavy, médecin des bopitaux de Paris. 1 vol. de 420 pages............ 18 fr.

Les grands Processus morbides: Congestion. Inflammation. Suppuration. Ciangrène, par le D' Camille HanN, lice sciellcer, médecin-assistant à l'hòpital Saint-Michel. 1 vol. de 480 pages, avec figures dans le texte.............. $18 \mathrm{fr}$.

La Gontie ot l'Obésité, par les docteurs A. Florand, médecin de l'hðpital Lariboisière, et M. Françors, ancien interne des hópitaux de Paris. 1 vol. de 550 pages............. $18 \mathrm{fr}$.

Fièvre typhoỉde et fièvres paratyphoïdes, par H. Duroun, médeciu de l'hópital Broussais, et J. ThIERS, ancien interne des hópitaux de Paris. 1 vol. de 450 pages avec figures dans le texte............................... $18 \mathrm{fr}$.

Grippe, Coqueluche, Erysipèle, Oreillons, par H. BARBIer, médecin des hopitaux de Paris 1 vol. de 300 pages........ $18 \mathrm{fr}$.

Diphtérie. - Poliomyélite. - Encéphalite léthargique. Méningite cérébrospinale. - Zona, piar le Dr BARBIER: médecin des hopitaux de Paris. 1 vol. de 350 pages....... $18 \mathrm{fr}$.

Septicémies (Seplicopyohémies, Bactériémies), par E. VA VChER et P. WoringBr, chargé de cours et chef de laboratoire à la Faculté de médecine de Strasbourg. 1 vol. de 516 pages avec figures................................. $20 \mathrm{fr}$.

\section{NEUROLOGIE ET PSYCHIATRIE}

Directeur : Docteur ToulousB.

Thérapeutique des Maladies du Système nerveux, par le professeur Grasset et le $D^{r}$ L. Rimba Ud, $2^{\circ}$ édit. 1 vol. de 600 p. $18 \mathrm{fr}$.

Séméiologie des Maladies du Systemenerveux, par le $\mathrm{D}^{\mathrm{r}} \mathrm{Henri}$ Durour, médecin de l'hỏpital Brousżais. 1 vol. de 540 pages, avec figures dans le texte. .................... $18 \mathrm{fr}$.

Maladies de la Moelle of du Bulbe (non systématisées). Polyomyélıtes, sclérose en plaques, syringomyélie, par le professeur C. ODDO. 1 vol. de 400 pages, avfC 24 figures dans le text.e.. $18 \mathrm{fr}$.

Le Tabes et les Maladies systématiques de la Moelle, par le docteur E. DE MAssart, médecin des hópitaux de Paris. 1 vol. de 350 pages, avec 28 fig. dans le texte............ $18 \mathrm{fr}$.

GASTON DOIN et Cle, Editeurs. 
La Paralysie générale, par le professeur A. Jofrrot, et le docteur Roger Mignot, médecin en chef de la Maison Nationale de Charenton (épuisé).

L'Hystérie. Définition et Conception. Pathogenie. Traitement, par le D' H. Bernetis, professeur honoraire à la Faculté de médecine de Nancy. 1 vol. de 450 pages............... $18 \mathrm{fr}$.

\section{MICROBIOLOGIE ET PARASITOLOGIE}

Directeurs : Professeur A. Galmerta, Sous-Directeur de l'Institut Pasteur, ot Docteur F. Brzançon, Professeur à la Faculté de Médecino de Paris.

Le Micro-organisme de la Syphilis. Treponema pallidum (Schaudinn), par le docteur Ĺ́vY-BıNG, chef de Laboratoire de SaintLazare, lauréat de l'Académie de Médecine. 1 vol. de 350 pages, avec figures dans le texte et une planche en couleurs hors texte................................. $18 \mathrm{fr}$.

L'Étude expérimentale de la Rage, par le docteur A. Marir, chef de service a l'Institut Pasteur. 1 vol. de 400 pages, avec figures dans le texte et une planche en couleurs hors texte.................................. $18 \mathrm{fr}$.

Les Dysenteries. Étude bactériologique, par le Dr Ch. DoptsR, professeur agrégé au Val-de-Grace. 1 vol. de 300 pages, avec figures dans le texte et $12 \mathrm{pl}$. hors texte........... $18 \mathrm{fr}$.

Les Insectes piqueurs et suceurs de sang, par le $D^{r}$ Edmond Sergent, chef de laboratoire a l'Institat Pasteur de Paris. 1 vol. de 310 pages, arec 229 fig. dans le textc............ $18 \mathrm{fr}$.

\section{HYGIENE ET MÉDECINE PUBLIQUES}

\section{Directeur : Profosseur A. Calmetrz.}

L'Ouvrier (Son atelier, son hygiène, son habitation), par le docteur René Martial. 1 vol. de 425 p., avec fig. daris le terte.. $18 \mathrm{fr}$.

Hygiène scolaire, par L. Dupestź, médecin inspecteur des Bcoles de París. $2^{\circ}$ édition, 1 vol. de 460 pages, avec 72 figures dans lo texte................................ $18 \mathrm{fr}$.

GASTON DOIN et $\mathrm{C}^{\mathrm{le}}$, Éditeurs. 
Hygièno du premier áge, par P. Lassablière, chef de Laboratoire à la Faculté de médecine de Paris. 1 vol. de 352 pages et 18 figures dans le texte................... $18 \mathrm{fr}$.

\title{
THÉRAPEUTIQUE
}

\author{
Directeur : Docteur G. PoucheT, \\ Professeur à la Faculté de Médecine de Paris.
}

Les Médicaments, Action physiologique, formules, emplois, par le $D^{r}$ A. F. PlicQur, ancien interne, lauréat des Hópitaux de Paris. Préface du professeur G. Povchrt. 1 vol. de 400 pages... 18 fr.

\section{PHYSIOLOGIE}

\author{
Directeur : Docleur J. GAUTRELET, \\ Professeur aurégé des Fiacultés de Médecine, \\ Virecteur de Lahoratoire à l'École des Hautes Etudes.
}

La Fonction musculaire, par J. Joтвүко, docteur en médecine, chef de Laboratoire à l'Université de Bruxelles. 1 vol. de 410 p., avec 35 figures dans le texte. ................. $18 \mathrm{fr}$.

La Cellule nerveuse, par G. MARINesco, professeur à l'Université de Bucarest. Avec une préface de M. le professeur RAMon Y Cajal (de Madrid). 2 vol. formant 1.148 pages, avec 252 figures dans lo texte............................. $36 \mathrm{fr}$.

Les Fonctions nerveuses : les fonctions bulbo-médullaires, par W. BEснтвкEW, professeur à l'Université de Saint-Pétersbourg. 1 vol. de 400 pages, avec fig. dans le texte.......... $18 \mathrm{fr}$.

Les Fonctions nervouses : les fonctions bulbo-médullaires. Fonctions viscérales, sécrítoirbs, trophiques ot thermogeniques, par W. BECHTRREW. 1 vol. de 600 pages, avec 43 figures dans le texte................................. $18 \mathrm{fr}$.

La Fonction córébelleuse, par le docteur ANDRÉ-TномAs, ancien interne des hópitaux de Paris. 1 vol. de 350 pages, avec 89 fig. dans le texte............................... $18 \mathrm{fr}$.

Les Fonctions digestives, par le docteur E. BARDIRR, professeur agrégé d̀ la Faculté de Médecine de Toulouse. 1 vol. de 450 pages, avec 29 figures dans le texte................... 18 fr.

La Fonction sexuelle, par le docteur H. BusQUET, professeur agrógé à la Faculté de Médecine de Nancy. 1 vol. (épusisé).

8, Place de l'Odéon, Paris $6{ }^{\circ}$. 
La Croissance, par le docteur L. Dufestel, médecin inspecteur des Écoles de la Ville de Paris. 1 vol. de 310 pages avec 20 fig. dans le texte............................. 18 fr. $-$

\section{ANATOMIE ET EMBRYOLOGIE}

Directeur: R ANTHONy, Professeur au Muséum d'Histoire naturclle.

Anatomio plastique, par Edouard CoYER, peintre, professeur d'anatomie. 1 vol. de 350 pages, avec $146 \mathrm{fg}$. dans le texte... $18 \mathrm{fr}$.

\section{BIOLOGIE GÉNÉRALE}

Directeur : M. Caullery, Professeur à la Faculté des Sciences de Paris.

La Tératogenèse. Etude des variations de l'organisme, par Etienue Rabaun, maltre de Conférences à la Faculté des Sciences de Paris. I vol. de 360 pages, avec $9 \mathrm{x}$ fig. dans le texte... $18 \mathrm{fr}$.

L'œuf et les facteurs de l'ontogénèse, par A. Brachet. profes. seur à l'Université de Bruxelles. 1 vol. de 350 pages avec 57 fieures............................... $18 \mathrm{fr}$.

Le Parasitisme ot la symbiose, par M. CAULLERT, professeur a la Sorbonne. 1 vol. de 400 pages a vec 53fig. danale texte... $18 \mathrm{fr}$.

L'hérédité, par E. GuYénot, professeur à l'Université de Genève. 1 vol. de 470 pages avec 47 figures.............. 25 fr.

L'Adaptation, par L. Cú́NoT, correspondant de l'Institut, professeur a la Faculté des Sciences de Nancy. 1 vol. de $\$ 20$ pages avec 82 figures........................ $25 \mathrm{fr}$.

Les pigments dans l'organisme animal. Chimie. Morphologie. Physiulogie. Étiologie, par le Dr J. VERNE, docteur ès sciences, profenseur agrígé a la faculté de médecine de Paris. 1 vill. de 612 pages, avec 32 figures dans le texte........... $28 \mathrm{fr}$.

Les sociétés d'insectes. Leur origine, leur évolution, par le prifessuur William Horton WhEeLER. 1 vol. de 4 i2 pages, avec 61 figures dans le texte..................... $25 \mathrm{fr}$.

\section{ANTHROPOLOGIE ET ETHNOGRAPHIE}

Direeteur:P. RIVkT, Assistant d'anthropelogie au Muséum d'Histoire naturelle.

Les Peuples aryens. Leur origine en Europe, par ZABorowsKI, professeur à l'Ecole d'Anthropologie, ancien président de la Société d'Anthropologie de Paris. 1 vol. de 450 pages, avec figures dans le texte et une carte hors terte............... $18 \mathrm{fr}$.

GASTON DOIN et $C^{10}$, Editeurs. 
Le Paganisme contemporain chez les peuples celto-latins, par Paul SEBILLOT, ancion présıdeut de la socicté d'Anthropolngie de Paris, directeur de la Revue des traditions populaires. 1 vol. de $\$ 00$ pages.. ........................... 18 fr.

Le Folk-Lore. Littérature orale et Ethnographie traditionnelle, par P. Stibllot. 1 vol. de 493 pages............... 18 fr.

Les Blancs d'Afrique, par le Dr H. Wrisgrabra (épuisé).

Anthropologie anatomique (crâne, face, tête sur le vivant), par le Dr G. PAUl-Boncour. vicc-président de la Société d'Anthropologie, médecin en chef de l'Institut médico-psychologique, ancien interne des hópitaux de Paris. 1 vol. de 400 pages, avec 4 figures......................... 18 fr.

\title{
PALÉONTOLOGIE
}

Directeur: M. Boule, Professeur au Muséum d'Histoire Naturelle.

Paléontologio végétalo. Cryptogames cellulaires et cryptogames vasculaires, par Fernand PKLOURDE, docteur ès sciences, préparateur au Muséum d'Histoire Naturelle, préface de M. R. ZEILLER, membre de l'Institut. 1 vol. do 400 pages, avec 80 figures dans le texte............................. $18 \mathrm{fr}$.

\section{OCÉANOGRAPHIE PHYSIQUE}

\author{
Directeur : Doctour J. RICHARD, \\ Directeur du Musée océanographique de Monaco.
}

Les Dépôts marins, par L.-W. Collet, professeur à l'Université de Genève. 1 vol. de 325 pages, avec 35 figures dans le texte et une carte hors texte ....................... $18 \mathrm{fr}$.

\section{ZOOLOGIE}

Directeur : R. Anrhony, Professeur au Muséum d'Histoire naturelle.

Les Insectes, Anatomie et physiologie genérales. - Introduction a létude de l'enlomologie biolouique, par C. HovLBERT, prifesseur à l'École de Médecine dellennes. $2^{\circ}$ édition. I vol. de 400 p., avec 207 figures......................... $18 \mathrm{fr}$.

La Distribution géographique des animaux, par le $D^{2} L$. TnouesSART, professeur all Vureum natio al d histoire n iturelle de $\mathrm{Pa}$ ris. 1 vul. de 350 payes avec tigures dans le texio..... $18 \mathrm{fr}$. 
Les Batracions et principalement ceux d' Europe, par G.-A. BoULENGgR, D' Sc., D' Phil., membre de la Sociéte royale de Londres, vice-président de la Société de zoologie de Londres. 1 vol. de 320 pages, avec 55 flqures dans le texte........... $18 \mathrm{fr}$.

Mollusque; de la France et des régions voisines. - ToME I : Amphincures, Gastéropodes Opisthobranches, Hétéropodes, Marséniadés et Oncidiidés, par A. VAYssière, professeur à la Faculté des sciences de Marseille. 1 vol. de 430 pages, avec 42 planches hors texte............................ 18 fr.

Tome II : Gastéropodes pulmonés et Prosobranches terrestres et fluviatiles, par L. GERMAIN, docteur ès scieuces, préparateur au Muséum d'Histoire naturelle. 1 vol. de 380 pages, avec 25 planrhes hors texte............................... $18 \mathrm{fr}$.

Les Coléoptères d'Europe (France et régions voisines), par C. HOd LBERT, professeur à l'Ecole de médecine et de pharmacie de Rennes.

TOME PREMIER : 1 vol. de 350 pages, avec 104 figures dans le texte.................................. $20 \mathrm{fr}$.

TOMB DBUxIÈme : 1 vol. de 310 pages, avec 99 figures dans le texte et 30 planches.............................. $20 \mathrm{fr}$.

TOME TROISILMB : 1 vol. de 300 p., avec 30 planches.... 20 fr.

Les Thysanoures, Dermopteres et Orthoptères de la Faune européenne. Tome I, par C. Houlbert. 1 vol. de 382 pages avec 87 tigures dans le texte et 9 planches............. $20 \mathrm{fr}$.

TONE II (en cours d'impression).

Les Échinodermes des mers d'Europe. ToME I, par R. KoEHLBR; professeur de Zowlogie à la Faculté ue Médecine de Lyon. 1 vol. de 370 pages avec 9 planches hors texte........... $20 \mathrm{fr}$.

TOYE II (en cours d'impression).

Les Oiseaux, par M. Boubier, préśident de la Société zoologique do Genève. 1 vol. de 306 pages, avec 78 figures dans le texte et 10 planches........................... $28 \mathrm{fr}$.

\section{ZOOLOGIE APPLIQUÉE}

Direçeur : J. PELLEgriN, Assistant au Muséum d'Histoire Naturelle..

Les Vers à soie (Sériciculture moderne), par Antonin RoLET, professeur à l'École d'Agriculture d'Antibes. 1 vol. de 450 pares, avec 102 figures dans le texte.................. $18 \mathrm{fr}$.

La Pisciculture industrielle, par C. Raveret-W attel, ex-maître de Conférences de Piaciculiure à l'Ecole Nationale des Punts et Chaussées. 1 vol. de 400 p., avec 74 fig. dans le texie... $18 \mathrm{fr}$.

GASTON DOIN et $C^{10}$, Editeurs. 
Les Equidés domestiques, le Cheval, l'Ane et le Mulet, par A. GALLIER, médecin iétérinaire, inspecteur sanitaire de la ville de Caen. 1 vol. de 380 pages, avec $68 \mathrm{fig}$. dans le texte... $18 \mathrm{fr}$.

\section{BOTANIQUE CRYPTOGAMIQUE}

Directeur : L. MaNeIN, de l'Institut,

Direeteur au Muséum d'Histoiro naturelle.

Les Urédinées (Rouilles des Plantes), par Paul Hariot, assistant de cryptogamie au Muséum d'Histoire Naturelle.1 vol. de 400 pages, avec 47 figures dans le texte.................. $18 \mathrm{fr}$.

Les Champignons. Essai de classifacation, par le $D^{r}$ Paul VulLLBMix, professeur à la Faculté de Médecine de Nancy. 1 vol. de 425 pages............................... $18 \mathrm{fr}$.

Les Levures, par A. Guilliermond, docteur ès sciences. Préface du $D^{r}$ E. Roux, directeur de l'Institut Pasteur. 1 vol. in-18 jésus, cartonné toile, de 565 p., avec 63 fig. dans le texte.... $18 \mathrm{fr}$.

\section{BOTANIQUE APPLIQUÉE}

Directeurs : H. Lecoure, de l'Institut, Professeur au Muséum d'histoire naturelle, et L. MANGIN, de l'Institut, Directeur du Muséum d'histoire naturelle.

Les Bois industriels, par J. Brauvrais, chargé d'nn cours de botanique appliquée à la Faculté des sciences de Lyon. 1 vol. de 420 p., avec 53 figures dans le texte.............. $18 \mathrm{fr}$.

Les Plantes a tubercules alimentaires des climats tempérés et des pays chauds, par Henri JumzLLE, professeur à la Faculté des sciences de Marseille. 1 vol. de 380 pages, avec 35 figures dans le texte ........................... $18 \mathrm{fr}$.

Les Plantes à gommes et á résines, par H. JACOB DE CORDEMox, ducteur ès sciences, docteur en médecine, chargé dg cours à l'école de Médecine de l'Lniversité d'Aix-Marseille. 1 vol. de 420 pages, avec 15 figures dans le texte............ $18 \mathrm{fr}$.

Utilisation des algues marines, par C. SAUvageaU, professeur à la F'sculté des sciences de Bordeaux. 1 vol. de 400 pages, avec 26 figures............................. $18 \mathrm{fr}$. 
Les Palmiers, par C.-L. Gatin, docteur ès sciences, ingénieur agronome, préparateur de botanique à la Sorbonne. 1 vol. de 350 pages, avec 46 figures.................... $18 \mathrm{fr}$.

\section{PHYSIOLOGIE ET PATHOLOGIE VÉGÉTALES}

Directeur : Professeur L. MANGIN,

Directeur du Muséum d'Histoire Naturelle, Membre de l'Instótul.

Biologie florale, par F. PвCHо UTRE, docteur ès sciences, professeur au Lycée Louis-le-Grand. 1 vol. de 380 pages, avec 82 figures dans le texte........................ $18 \mathrm{fr}$.

Nutrition chez la plante, I. Echanges d'eau et de subslances minerales, $2^{e}$ édition, par M. MolliarD, doyen de la Faculté des sciences de l'Université de Paris, membre de I'Institut. 1 vol. de 400 pages, avec 46 tigures dans le texte........... $25 \mathrm{fr}$.

Nutrition de la plante, II. Formation des substances ternaires, par M. Molliard. 1 vol. de $450 \mathrm{p}$, avec $88 \mathrm{fig}$ dans le texte... $20 \mathrm{fr}$.

Nutrition chez la plante, III. Utilisation des substances ternaires, par M. MolliaRD. 1 vol. de 324 pages avec 54 figures dans le texte............................ $20 \mathrm{fr}$

Nutrition de la plante, IV. Cycle de l'azole, par M. Molliard. 1 vol. de 330 pages avec 56 figures.............. $20 \mathrm{fr}$.

\section{GÉOLOGIE ET MINÉRALOGIE APPLIQUÉES}

Directeur : L. Gaygux, Professeur de Géologie au Collège de France.

Les gisements de pétrole, par J. Crautard. 1 vol. de 350 pages avec 47 figures dans le texte................... $18 \mathrm{fr}$.

\section{PHILOSOPHIE DES SCIENCES}

Directeur : A. Rey, Professeur à la Sorbonne.

Les disciplines d'une science, la Chimie, par G. UnBain, membre de I'Instilut, professeur de la Faculté des S iences de Paris. 1 vol. de 340 pages avec figures................ $18 \mathrm{fr}$.

GASTON DOIN et $\mathrm{C}^{10}$, Editeurs. 
La Physique depuis vingt ans par P. LANGrvin, professeur au Collège de France. 1 vol. de 350 pages avec figures..... $25 \mathrm{fr}$.

Les nouvelles conceptions de la matière ot de l'atome, par A. BERThOUd, professeur à l'Université de Neuchatel 1 vol. de 330 pages avec 21 figures dans le texte........... $18 \mathrm{fr}$.

Le tombeau d'Aristoxène. Essai sur la musique, par G. UnBain, professeur à la raculté des Sciences de Paris. I vol. de 240 pages avec figures........................... $18 \mathrm{fr}$.

\section{PSYCHOLOGIE EXPÉRIMENTALE}

Directeur : Doctour TOULOUsR.

Technique de Psychologie expérimentale, par Toulouse, VasChIDs et PIBRon. Detuxième édition, entièrement nouvelle, par le docteur Ed. TOUlousk et H. PIÉRON. (Epuisé.)

L'Hypnotisme et la Suggestion, par le professeur Grasset, 4* édition. 1 vol. de 481 ) pages avec figures dans le texte..... $18 \mathrm{fr}$.

La Volonté, par Fr. Paulhan, 2 édition. 1 vol. de 332 p. 18 fr.

La Morale. Fondements psycho-sociologiques d'une conduite rationnelle, par G.-L. DUPRAT, docteur es lettres, lauréat de l'Institut, correspondant du Ministére de l'Instruction publique, associé de l'Institut international de sociologie, directeur du Laboratoire de psychologie expérimentale d'Aix-en-Provence. 2o édition. 1 vol. de 400 pages......................... $18 \mathrm{fr}$.

La psychologio sociale. Sa nalure et ses principales lois, par G.-L. DUPRAT, docteur ès lettres, lauréat de l'Institut. 1 vol. de 370 pages............................. 18 fr.

L'Inconscient, par A. HesnaRD, professeur à l'École de Médecine navale de Bordeaux. 1 vol. de 300 pages avec figures dans to texte.................................. 18 fr.

L'odorat, par H. ZWAARDEMAKER, professeur do physiologies à l'Université d'Utrech. 1 vol. do 312 pages avec figures. $18 \mathrm{fr}$.

8, Place de l'Odéon, Paris $6^{\circ}$. 


\section{PSYCHOLOGIE APPLIQUÉE}

Directeur : Docteur TOULOU8E.

L'Éducation des Sentiments, par le docteur V. BRIDou. 1 vol. de 410 pages.............................. $18 \mathrm{fr}$

La Pédagogie expérimentale, par Gaston RIC la Faculié des lettres de Bordeaux. 1 vol. de 350 pages. $18 \mathrm{fr}$.

La Pratique commerciale, par J.-H. Haendel. (Épuisé.)

L'éducation de la volonté et des facultés logiques, par G -L. DuPRAT, ducteur ès lettres, lauréat de l'Institut, ansocié de l'Institut international de sociologie. 1 vol. in-18 grand jésus, de 324 pages............................. $18 \mathrm{fr}$.

\section{SOCIOLOGIE}

Directeur: G. Richard, Professeur à la Faculté des Lettres de Bordeaux.

La Sociologie générale, par Gaston RICHARD, professeur de sociologie à l'Université de Bordeaux. 1 vol. de 400 pages... $18 \mathrm{fr}$.

Evolution des moours, par Gaston RICHARD, professeur à la Faculté des lettres de Bordeaux. 1 vol. de 400 pages...... $25 \mathrm{fr}$.

Les Types sociaux et le Droit, par Joseph Mazzarella, docteur en droit. 1 vol. de 450 pages, avec nombreux tableaux... $18 \mathrm{fr}$.

La Solidarité sociale, par G.-L. DuPrat, professeur au Lycée de Rochefort (ouvrage recoinpensé par l'Academie des sciences morales et politiques. Prix Saintour, 1906). Préface du professeur G. Richard. 1 vol. de 360 pages................. $18 \mathrm{fr}$.

Géographie sociale : La Mer. Populations maritimes. Migratuons. Péches. Commerce. Domination de la mer, par C. VAlLAUX, docteur es lettres, professeur de géographie à l'École navale. I vol. de full pages.................... $18 \mathrm{fr}$.

Gćographie sociale : Le Sol ot l'Etat. par C. Vallaux. 1 vol. do 120 pages avec 31 figures dans lo texte.......... $18 \mathrm{fr}$.

La Société et l'Ordre juridique, par Alessandro LEvı, professeur de philos .phie du druit à l'Université de Ferrure I vol. de $\$ 10$ pagre........................... $18 \mathrm{fr}$.

Lart ot la vio sociale, par Charles Lalo, docteur ès sciences, prolr de philosophie $x$ u lycée Hoche. 1 vol. de $390 \mathrm{p.} . \ldots 18 \mathrm{fr}$.

CrASTON IOIN et $\mathrm{C}^{10}$, Editeurs. 


\section{SOCIOLOGIE APPLIQUÉE}

Directeur : Th. RuYssen, Professeur la Faculté des Lettres de Bordeaux.

Les Régies municipales. Exploitation collective des services publics, par E. BoUvirR, professeur de science et de legislation financières à la Faculté de droit de l'Université de Lyon. 1 vol. de 450 pages............................ $18 \mathrm{fr}$.

La Protection des faibles (Assistance el Bienfaisance), par G. RONDEL, inspecteur général au Ministère de l'Intérieur, membre du Conseil supérieur de l'Assistance publique. 1 vol. de 300 pages........................... $18 \mathrm{fr}$.

Géographie économique. Exploitation rationnelle du globe, par Paul Clerget............................ (Epuise).

\section{ÉCONOMIE POLITIQUE}

Directeur; Grorges Renard, Professeur au Colldge de France.

La Monnaie, le Change et l'Arbitrage, le Crédit, par M. et A. MkLIOT. (Epuisé.)

Guerre et Paix internationales, par Eugène d'Eichthal, membre de l'Institut. 1 vol. de 350 pages avec graphiques...... $18 \mathrm{fr}$.

La Colonisation ot les Colonies, par Pierre AdBRT, docteur ès sciences juridiques, politiques et économiques. 1 vol. de 278 p., avec nombreux tableaux..................... $18 \mathrm{fr}$.

Le Commerce et les Commerçants, par Yves GuYot, ancien ministre, vice-président de la Société d'Economie politique. (Epuisé.)

L'Industrie ot les Industriels, par Yves GUYoT, 1 vol. de 400 p., avec rableaus dans le texte.................... $18 \mathrm{fr}$.

Le Blé et les Córéales. par Daniel Zolla, professeur à Grignon et à l'Écule libre aes sciences politiques. 1 vol. de 300 pages, avec cartes et graphiques dans le texte............ $18 \mathrm{fr}$.

Les Fibres textiles dorigine animale (Laine et soie), par D. Zulla, 1 vol. de 3511 pyzes, arec cartes et graph:ques. . 18 fr.

Syndicats, Trade-unions ot Corporations, par Genrges RENARD, prolesseur au Collège de France. 1 vol. de 420 pages... 18 fr.

Salariat et Salaires, par E. Levasseur, membre de l'Institut, adininistrateur du Collège de France. 1 vol. de 500 pages $18 \mathrm{fr}$. 
La Machine et la Main-d'cuvre humaine, par D. Beller, secrétaire perpétuel de la Société d'ecollomie politique, professeur à l'Ecole des Sciences politiques et. à l'Ecole des Hautes Etudes commerciales. 1 vol. de 300 pages. ............... 18 fr.

La Vie chère, par G. Renard, professeur au Collège de France. 1 vol. de 250 pages....................... 18 fr.

L'Économie politique ot les Économistes, avec une introduction sur I'Economique et la Guerre, par G. SCHELLE, vice-président de la Société d'Économie politique. 1 vol. de 400 pages $18 \mathrm{fr}$.

Le Luxe, le Bien-ätre et la Consommation, par André PINARD. Ouvrage couronne par l'Academie française (Prix Fabien 1919). 1 vol. de 480 pages......................... 18 fr.

Les Monopoles, par E. PAYRN. 1 vol. de 450 pages..... $18 \mathrm{fr}$.

Les Systemes socialistes, par H. Bourgix. 1 volume de 400

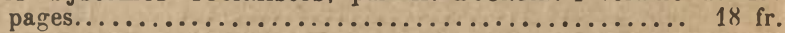

L'Enseignement commercial en France ot à l'Etranger, par M. FACY. 1 vol. de 330 pages.................. 18 fr.

\section{CHIMIE}

\section{Directeur : A. PIctet,}

Professeur à la Faculté des sciences de l'Université de Genève.

Zinc, Cadmium, Cuivre, Mercure, par A. Bоuch oxnzt, préparateur à la Faculté des sciences de l'Université de Paris. 1 vol. de 410 p., avec figures dans le texte.................... 18 fr.

Btain, Plomb ot Bismuth, par A. Boochonnet. 1 vol. de 380 pages............................... $18 \mathrm{fr}$.

Bydrocarbures, Alcools et Éthers de la série grasse, par P. CARRÉ, docteur ès sciences, professeur à l'Ecole des Hautes Etudes commerciales, préparateur à l'Institut de chimie appliquée. 1 vol. de 420 pages............................... $18 \mathrm{fr}$.

Phosphore - Arsenic - Antimoine, par A. Boutaric, agrégé de I'Université, docteur es sciences, maître de conférences de physique à la Faculté des sciences de Dijon, et A. RAYNAUD, ingénieur-chimiste, chargé des fonctions de chef des travaux de chimie à la Faculıé des sciences de Montpellier. 1 vol. in-16 de 420 pages, avec ngures dans le texte............. $18 \mathrm{fr}$.

La Chimie des matières colorantes organiques, par P. CAstan, docteur ès-sciencea de l'Université de Genève. 1 vol. de 460 pages, avec nombreuses formules.................. $36 \mathrm{fr}$.

Les sucres et leurs dérivés, par Marc СнAмBR, docteur ès sciences. 1 vol. de $3 \mathrm{~b} 0$ pages, avec nombreuses formules. $28 \mathrm{fr}$.

GASTON DOIN et $C^{1 e}$, Éditeurs. 


\section{CHIMIE BIOLOGIQUE}

Directeur: G. Bertrand, Professeur de Chimie biologique à la Sorbonne.

Le Parfam chez la Plante, par Eug. Charabet, docteur ès sciences physiques, inspectcur et membro du Conseil supérieur de l'Enseignement technique, et C.-L. Gatin, docteur ès sciences naturelles, ingénieur agronome, préparateur à la Faculté des Sciences de Paris. 1 vol. de 400 p., avec 21 fig. dans le texte... 18 fr.

\section{INDUSTRIES BIOLOGIQUES}

Directeur: Professeur G. BERTRAND.

Industrie des Parfums naturels, Les Principes odorants des Végétaux (Méthodes de dosage, d'extraction, d'identifcation), par Eug. Свававот. 1 vol. de 400 pages, avec figures dans le texte................................. 18 fr.

La Fabrication du sucre, par D. SIDERSKY, ingénieur-chimiste. 1 vol. de 360 pages, avec 37 figures dans le texte..... $18 \mathrm{fr}$.

\section{INDUSTRIES CHIMIQUES}

Directeur : J. Deròme, Inspecteur général de l'Instruction publique.

Les Produits pharmaceutiques industriels, par P. CARRE, doCteur ès sciences, préparateur à l'Institut de Chimie appliquée. 2 vol. formant 820 pages.................... $36 \mathrm{fr}$.

Industrie des Métaux secondaires et des Terres rares, par Pierre Nicolarvot, capitaine d'artillerie, chef du Laboraloire de Chimie de la Section tochnique. 1 vol. de 420 pages, avec 37 lig. dans le texte............................ $18 \mathrm{fr}$. 
Pierres ot Matériaux artificiels de construction, par Albert GRANGRR, professeur a l'Ecule d'applicatiulı de la Manulacture nationale de sèvres, chargé de cunférences a l'École de physique et de chimie industrielles de la Ville de Paris. I vol. de 350 p., avec 55 figures dans le texte................. 18 fr.

L'Eau dans l'industrie. Application. Epuration, par Georges BOURREY, inspecteur de l'Enseig»ement technique, ingénieur chimiste des cheurns de fer de l'État, professeur à l'École spéciale des travaux publics. Avec une préface dH M. MaX DE Nassouty. 1 vol. do 468 pages, avec 57 figures dans lo texte..... $18 \mathrm{fr}$.

Industries des Acides minéraur (Acides sulfurique, chlorhydrique et azotique), par $\mathfrak{E}$. BAUB, maltre de conférences à la Faculté des Sciences de Marseille. 1 vol. de 360 pages, avec 82 fig. dans le texte.............................. $18 \mathrm{fr}$.

Industries du Plomb et du Mercure. I. Métallurgie; II. Composés, par A. Bouchonnet, préparateur à la Faculté des Sciences de Paris. 2 vol. formant 660 pages, avec 57 figures dans le texte.................................. $36 \mathrm{fr}$.

Industries du Chrome, du Manganese, du Nickel et du Cobalt, par L. OUVRaRD, directeur du Laturatoire de chimie generalo de la Sorbonne. 1 vol. de 310 pages, avec 22 fig. dans le texte................................. $18 \mathrm{fr}$.

Alcool ot Distillerio. Production et consommation de l'alcool. Utzlisation des sous-produits. Analyse. Législation, par A. MoNvoisis, chef des travaux de physique et chimie à l'École nationale vétérinaire d'Alfort (préface de M. L. LINDRT, professeur a l'Institut nutional agronomique). 1 vol. de 450 pages, avec 112 figures dans le texte...................... $18 \mathrm{fr}$.

Caoutchouc et Gutta-percha, par E. TAssilly, docteur ès sciences, professeur agrégé à l'Ecole supérieure de pharmacie, chargé de conférences à l'Ecolo de physique et chimie industrielles. 1 vol. de 400 pages, avec 56 figures dans le texte......... 18 fr.

Industries des Métaux précieux : L'Argent et les Métaux de la Mine de Platine, par M. Molinite, ingénieur-chimiste, chef du Laboratoir des Essais du Comptoir Lyon-Altmand, et H. Dirtz, iıgénieur-chimiste, directeur de l'Usine d'uffinaze du Comptoir Lyon-Alemand. 1 vol. de 400 pages, avec 93 figures dans le texte............................... $18 \mathrm{fr}$.

Industries des Matières colorantes organiques, par André Wabl, docteur ès sciences. prolesaeur hu Conservatoire national des Arts et Métiers. $2^{\circ}$ Edilion: Tusz Phrmigr. les Produits intermédiaires, 1 vol. de 350 pages aiec 24 figures dans le texte............................... $18 \mathrm{fr}$.

GASTON DOIN et $C^{16}$, Editeurs. 
Industries des cyanures, par P. BRUN, 'licencié ès sciences physiques, plıarmarien de $1^{\text {re }}$ clasve, ingénieur chimiste, préparalrur a la Faculté des sciences de Montpellier. 1 vol. in-16 de 480 pages, avec 44 figures................ $25 \mathrm{fr}$.

Industries des 08 , des Déchets animaux, des Phosphates ot du Phosphore, par L. Vézıen, ingénieur-chimiste. 1 vol. de 425 pages, avec 50 fig. dans le texte............ 18 fr.

\section{PHOTOGRAPHIE}

\section{Directeur : A. SEYBWBTZ.}

Sous-Directeur de l’école de Chimie industrielle de Lyon.

La Photographie, par G. ChICANdard, licencié ès sciences physiques. 1 vol. de 350 pages.................... 18 fr.

Les Positifs en photographie, par E. Trutat, docteur ès sciences, directeur du Musée d'Histoire naturelle de Toulouse. $1 \mathrm{vol}$. de 300 pages, avec fig. dans le texte............... $18 \mathrm{fr}$.

Lo Négatif on photographie, par A. Seyewetz. $2^{e}$ édition. 1 vol. de 320 pages avec 4 ' higures dans le texte.......... $18 \mathrm{fr}$.

Les Reproductions photomécaniques monochromes. Photogravure, similigravure, phototypie, holiogravure, etc., par L.-P. Clerc, ingenieur, préparateur à la Faculté des sciences de l'Université de Paris. $2^{\circ}$ Edition. 1 vol. de 400 pages avec 61 figures.............................. 18 fr.

Les Reproductions photomécaniques polychromes. Sélections trichromes, Orthocromatisme, Procédés d'interpretation, par L.-P. Clenc. 1 vol. de 350 p., avec 73 fig. dans le texte... $18 \mathrm{fr}$.

La Photographie á la lumiére artificielle, par Albert LONDE, directeur honoraire des services de photographie et de radiographie à la Salpetrière. 1 vol. de 400 pages, avec 80 figures dans le texte............................... $18 \mathrm{fr}$.

La Photographie des couleurs, par J. Thovert, professeur à la Faculté des nciences de Lyo:ı. 1 vol. de 300 pages avec 93 figures daus le texte et 4 planches en couleurs hors texte.... 22 fr.

La Photographio des radiations invisibles. Rayons cathudiques, rayons anodiques, rayuns de Rontyen, du tubs de Crookss et de l'ampoule de Coolidge, rayons du radium et des substances radio-actives, rayons spectraux infra-rouges et ultra-violets, par M.-A. Chanoz, docteur en médecine, docieur ès sciences physiques, chef des travaux de physique médicale à l'Université de Lyon. 1 vol. de 424 pages avee 111 figures.......... 18 ir. 
La Chimie photographique, par H. BARbier et J. PARIS, chef mistes des Etablissements Lumière. 1 vol. de 350 pages. $18 \mathrm{fr}$.

Applications de la Photographie aérienne. Lacture des photographies aériennes, stréescopie de précision, appareils et méthodes pour la photıtopoyraphie aérienne, par L.-P. Clenc, ancien commandant d'uue section de photngraphie aérienne aux armér.s. 1 vol. de 350 pages, avec 136 figures dans le texte et 10 planchos hors texte...................... $18 \mathrm{fr}$.

Macrophotographie et microphotographie, par F. MonPILlard. 1 vol. de 682 pages avec 86 figures.............. $32 \mathrm{fr}$.

\section{ASTRONOMIE ET PHYSIQUE CÉLESTE}

Directeur : Professeur J. MABCaRT, Directeur de l'Observatoire de Lyon.

Les Observations méridiennes, Théoris ef pratique, par F. B0QUET, docteur ès seiences mathématiques, astronome à l'Observatoire de Paris. 2 vol. formant 650 pages, avec 162 figures dans le texte et 2 planches hors texte................. $36 \mathrm{fr}$.

Spectroscopie astronomique, par P. SALET, astronome à l'Observatoire de Paris. 1 vol. de $\mathbf{4 3 2}$ pages, avec 44 figures dans le texte et une planche hors texte................ $18 \mathrm{fr}$.

Les Théories modernes du Soleil, par J. BosLER, astronome à l'Observatoire de Meudon. 1 vol. de 380 pages, avec 49 figures dens le texte............................. $18 \mathrm{fr}$.

Calcul des Orbites et des Ephémérides, par Luc Picart, directeur de l'Observatoire de Bordeaux, professeur à la Faculté des Sciences. 1 vol. de 300 p., avec 23 figures dans le texte... $18 \mathrm{fr}$.

L'Astronomio. Observations, theorie et vulgarisation generale, par M. MOYE, professeur à l'Université de Montpellier. 1 vol. de 400 pages, avec 43 figures dans le texte et 4 planches hors texte................................... $18 \mathrm{fr}$.

Les ttoiles simples, par F. HenroteaU, docteur ès sciences de l'Université de Bruxelles, astronome à l'Observatoire de la Puissance du Canada à Otlawa. 1 vol. in-16 de 250 payes, avec fig. dans le texte ............................. 18 fr.

Histoire de l'Astronomie, par E. Dollblet, astronome à l'Observatoire de Bordeaux. I vol. de 596 pages avec fig.... 22 fr.

Évolution et Constitution de l'Univers, par A. VÉronnet, asıronome a l'Ubservarnire de Sirasbourg, chargé de cinferelices à l'Université. 1 vol. in- 16 de 4:6 pagees, avec 29 figuris dans le rexte............................ $28 \mathrm{fr}$.

GASTON DOIN et $\mathrm{C}^{10}$, Editeurs. 


\section{PHYSIQUE}

Directeur : A. Laduc, Professeur de Physique à la Sorbonne.

Oscillations et vibrations, par A. BuUtaric, agrégé de l'Université, chargé d'un cours supplémentaire de physique à l'Université de Montpellier. 1 volume de 429 pages, avec 139 figures dans le texle.................................. $18 \mathrm{fr}$.

Optique géométrique, par J. BLrin, professeur au Lycée SaintLouis. 1 vol. de 276 pages, avec 107 figures dans le texte $18 \mathrm{fr}$.

\section{PHYSIQUE BIOLOGIQUE}

Directeur : L. PECH, Professeur a la Faculté de Médecine de Montpellicr.

Rayons $\mathbf{X}$ et Radiations diverses, Actions sur l'organisme, par le $\mathrm{D}^{\mathbf{r}} \mathrm{H}$. Gullleminot, vice-président de la Société de Radiologie médicale de Paris. 1 vol. de 320 p., avec figures dans le texte $18 \mathrm{fr}$.

\section{INDUSTRIES PHYSIQUES}

\section{Directeur: H. Chaumat,}

Sous-Directeur de l'École supérieure d’Électricité de Paris.

Instruments optiques d'observation et de mesure, par J. RAIBAUD, capitaine d'artillerie. 1 vol. de 380 pages, avec 144 figures dans le texte............................ 18 fr.

Le Ferro-magnétismo. Applications industrielles, par R. Jovaust, chef de travaux au Laboratoire central d'électricité. 1 vol. de 420 pages, avec 55 fig. dans le texte.............. 18 fr. 


\title{
MATHÉMATIQUES APPLIQUEÉES
}

\author{
Directeur : M. D'OCAgNE, Professeur à l'École Polytechnique \\ et à l'Ecole des Ponts et Chaussées, Membre de l'Institut.
}

Calcul graphique ot Nomographie, par M. d'OCAGNe, professeur à l'Ecole des Ponts et Chaussées et à l'Ecole Polytechnique, membre de l'Institut. $3^{e}$ édition. 1 vol. de 410 pages ave.c 146 figures............................. $30 \mathrm{fr}$.

Calcul numérique. Opérations arithmétiques et algébriques, Intégrations, par R. DE MONTESSUS et R. D'ADH'́rMAR, docteurs ès sciences mathématiques. 1 vol. de 250 pages, avec figures dans le texte................................ 18 fr.

Calcul mécanique. Appareils arithmétiques et algébriques, Intégrateurs, par L. JACOB, ingénieur général de l'Artillerie navale. 1 vol. de 428 pages, a vec 184 figures dans le texte...... $18 \mathrm{fr}$.

Géodésio élémentaire, par le général R. BoURGerols, professeur à l'Ecole polytechnique. $2^{\circ}$ édition, revue, corrigée et augmentée avec la collaboration du Lieutenant-Colonel NoIreL, du service géographique de l'armée, répétiteur à l'Ecole polytechnique. 1 vol. de 470 pages, avec 138 figures.................. $22 \mathrm{fr}$.

Navigation. - Instruments, Observations, Calculs, par E. PERRET, lieutenant de vaisseau, professeur à l'Ecole Navale. 1 vol. de 360 pages, avec 57 figures dans le texie et 4 tableaus hors texte.................................. $18 \mathrm{fr}$.

Théorie et pratique des 0pérations financières, par A. BARrIol, membre de l'Institut des actuaires français, directeur de l'Institut des Finances et Assurances. $3^{e}$ édition (Prix Montyon). 1 vol. de 408 pages avec nombreux tableaux et graphiques...... 25 fr.

Théorie mathématique des Assurances, par P.-J. Richard et PrTit, anciens élèves de l'Ecole Polytechnique, actuaires. $2^{\circ}$ édition revue, corrigée et augmentée, par P.-J. RicBard. Ouvrage couronné par l'institut (Prix Montyon, 1922). 2 vol. formant 775 pages avec figures et tableaux dans le texte..... $36 \mathrm{fr}$.

Statistique mathématique, par Hermann LAURENT, membre de l'Institut des actuaires français, répétiteur à l'École Polytechnique. 1 vol. de 300 pages, arec figures et tableaux dans le texte................................. $18 \mathrm{fr}$.

Góométrie descriptive, par Raoul BRICARb, ingénieur des Manufactures de l'Etat, professeur au Conservatoire national des Arts et Métiers, répétiteur à l'B́cole Polytechnique. I rol. de 275 pages, avec 107 figures dans le texte................. $18 \mathrm{fr}$.

GASTON DOIN et $\mathrm{C}^{\text {le }}$, Editeurs 
Métrophotographie, par le capitaine du génie Th. SAconnex, chef du Labnratoire d'aérologie et de téléphotographie militaires de Chalais-Meudon. 1 vol. de 300 pages, avec 130 figures dans le textc............................... $18 \mathrm{fr}$.

Géométrie perspective, par M. Emanaud, chef des travaux graphiques à l'Ecole polytechnique. 1 vol. de 440 pages avec 168 figures................................ $18 \mathrm{ir}$.

\section{MÉCANIQUE APPLIQUÉE ET GÉNIE}

Directeur : M. D'OCAGNE,

Professeur à l'École polytechnique et à l'École des Ponts et Chaussées, Membre de l'Institut.

Balistique extérieure rationnelle (Problème balistique principal), par le général P. CaArbonnirr. (Epuisé).

Balistique extórieure rationnelle (Problemes secondaires), par le général P. Charbonniar. (Epuisé.)

Balistique intérieure, par le général P. СаARBonNirR. (Epuisé.)

Mécanique des Explosifs, par E. Jougurt, ingénıeur en chef au Corps des Mines, répétiteur à l'École Polytechnique. 1 vol. de 525 pages avec 120 figures.................. $18 \mathrm{fr}$.

Mécanique des Affûts, $2^{\circ}$ édition, par le colonel J. Challéat et le commandant Thomas. Toms I. 1 vol. de 370 pages a vec 97 fluures dans $1+$ texte.............................. 22 fr.

Tom B II. 1 vol. de 360 pages avec 62 figures.......... $22 \mathrm{fr}$.

Résistance et construction des Bouches à fou. Autofrettage, par L. JACOB, ingénieur général de l'Artillerie navale, conseiller technique aux Etablissements Schneider. $2^{\circ}$ édition. 2 vol. formant 600 p., avec 131 figures dans le texte et 10 grands graphiques hors texte.......................... 36 fr.

$\Delta r$ rillerie de campagne, par J. PALOQUE, lieutenant-colonel, professeur à l'Ecole supérieure de guerre. (Epuisé).

L'Artillerio dans la bataille, par le colonel J. PALOQUE, commandant le $18^{\circ}$ régiment d'artillerie. 1 vol. de 460 pages, ave.c $14 \mathrm{fig}$. dans le texte et une carte hors texte............ $18 \mathrm{fr}$.

Artillerie navalo, par L. JACoB, ingénieur général de l'Artillerie navale. 2 vol. formant 950 pages, avec 462 figures dans lo texto................................. 36 fr.

8. Place de l'Odteon, Paris, 60. 
Cinématique appliquée. Théorie des mécanismes, par L. JACoB, ingénieur général de l'artillerie navale. 1 vol. de 400 pages, avec 171 figures dans le texte...................... $18 \mathrm{fr}$.

Organes des Machines opératrices et des transmissions, par L. JaCoв, ingénieur général de l'artillırie navale. 1 vol. de 360 pages, avec 63 planches, contenant 372 figures...... $18 \mathrm{fr}$.

Lois mathématiques de la résistance des fluides. - Théorie de l'hélice, par H. WillutTe. insperteur général honorsire des Ponts et Chaussées. 1 vol. in- 16 de 300 pages, avec figures dans le texte................................ $18 \mathrm{fr}$.

La résistance de l'air et l'expérience. - Les conséquences, par L. JACOB, ing乌́nieur général de l'artillerie navale. 2 volumes formant 600 pages, arec 83 figures dans lo texte...... $36 \mathrm{fr}$.

Les commandès sont fournies dès réception de leur valeur, augmentée des frais de port.

Compte Chèques postaux Paris 201.74

Téléphone : Fleurus 08.00

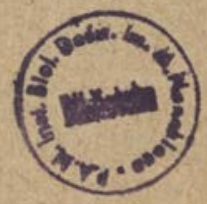

B - 3833 - L.-Imp. róun., 7, rue St-Benoft, Puris. 
NIORT - - IMPRIMERIE SAINT-DENIS

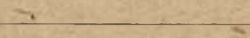


rcin.org.pl 



